\title{
Serge Moscovici
}

\section{La société \\ contre nature}

Union générale d'édition, Paris 1972

Collection 10/18

Un document produit en version numérique par Jean-Marc Simonet, bénévole, professeur retraité de l'enseignement de l’Université de Paris XI-Orsay Courriel: jmsimonet@wanadoo.fr

Dans le cadre de la collection: "Les classiques des sciences sociales" Site web: $\underline{\text { http://classiques.uqac.ca/ }}$

Une collection développée en collaboration avec la Bibliothèque Paul-Émile-Boulet de l'Université du Québec à Chicoutimi Site web: http://bibliotheque.uqac.ca/ 
Cette édition électronique a été réalisée par Jean-Marc Simonet, ancien professeur des Universités, bénévole.

Courriel: jmsimonet@wanadoo.fr

À partir du livre de

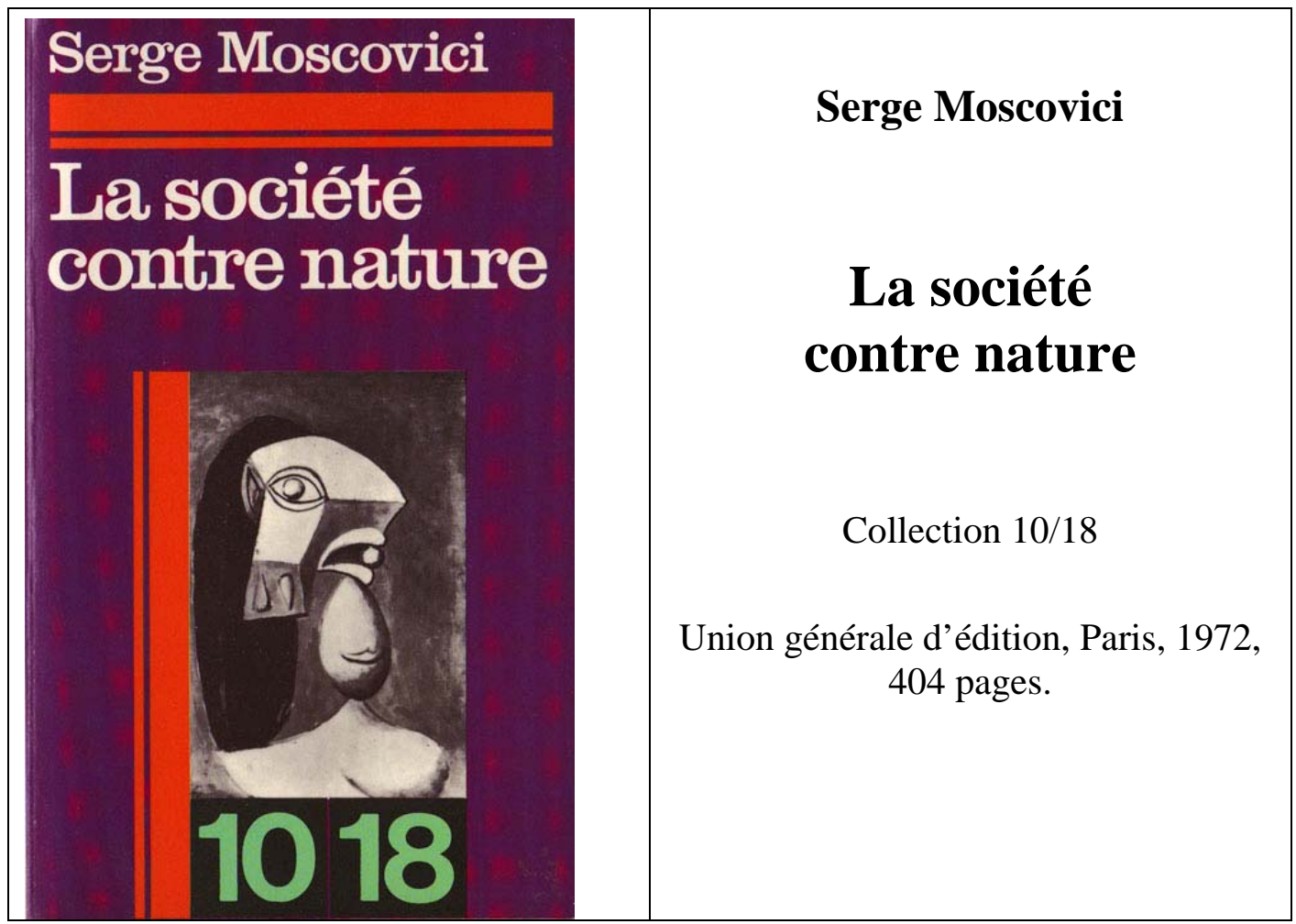

Polices de caractères utilisées :

Pour le texte: Times New Roman, 14 et 12 points.

Pour les notes de bas de page : Times New Roman, 10 points.

Édition électronique réalisée avec le traitement de textes Microsoft Word 2004 pour Macintosh.

Mise en page sur papier format : LETTRE (US letter), 8.5'’ x 11'’)

Édition numérique réalisée le 7 décembre 2007 à Chicoutimi, Ville de Saguenay, province de Québec, Canada.

\section{Fait avec}

Macintosh 


\section{Table des matières}

\section{Introduction}

I.

$\underline{\text { II. }}$

III.

$\underline{\text { IV. }}$

\section{Première Partie ÉVOLUTION ET HISTOIRE}

\section{Chapitre I. - Les premiers primates, promoteurs de l'histoire}

I. Mutation, adaptation et évolution : rappel succinct de deux mécanismes essentiels

II. La société adaptative

$\underline{\text { III. L'ancien et le nouvel art de survivre }}$

\section{Chapitre II. - Des sociétés sans paroles}

I. Les hiérarchies bien tempérées

II. Les prescriptions de la vie en commun

III. Comment réussir à combler le fossé qui sépare les générations

\section{Deuxième Partie \\ LA NATURE DE L'HOMME}

\section{Chapitre III. - Le nouveau monde animal}

I. Dans le no man's land : hominisation ou cynégétisation

II. Population, ressources et pressions sur l'environnement

1. Description liminaire de deux états stationnaires

2. Les mâles surnuméraires et le petit monde menacé de la forêt

III. La cueillette des animaux 
Chapitre IV. - Les deux naissances de l'homme

I. De la prédation à la chasse

1. La barrière des ressources principales

2. Une séparation maîtresse

3. Les arts de la ruse et de la mort

II. L’homme dénaturé

1. A l'école des anthropoïdes artificiers

2. La naturalisation des artifices

III. Remarques finales : l'élément humain et la structure humaine

Chapitre V. - De la sélection à la division naturelle

I. Sur l'inversion des rapports entre population et milieu

II. Faire des femmes, faire des hommes

1. Invention et croissance

2. Transmettre et conserver

$\underline{\text { III. Le processus de division est naturel }}$

\section{Troisième partie \\ SOCIETE ANIMALE ET SOCIETE HUMAINE}

\section{Chapitre VI. - Les scociétés qui viennent de nulle part}

I. La nature prise en défaut

II. Du désordre biologique et animal

III. Les sociétés « avec » et les sociétés « sans »

Chapitre VII. - La chasse et la parenté : premières constatations

I. Les trois dimensions de l'entreprise cynégétique

II. Le problème du mâle

1. Découverte de la paternité

2. Du célibat : le mariage et l'égalité des hommes

III. Le principe du partage : don et réciprocité

1. L'exogamie conjugale

2. L'endogamie sociale et le pouvoir généalogique

3. La généralité du partage exogamique

Chapitre VIII. - Les femmes dans la société des hommes : le problème de l'inceste

I. Pourquoi les femmes?

II. Loi naturelle ou règle sociale?

III. Le seul inceste vrai : celui de la mère

$\underline{\text { IV. Les règles de parenté, règles de domination }}$ 
Chapitre IX. - La lutte des sexes

I. Deux sociétés en une seule

1. La société du secret

2. Les discriminations sexuelles

II. Les hommes entre eux

1. Devenir homme

2. La ruse de la raison

Chapitre X. - La moitié-nature et la moitié-culture

I. La diflérence fondamentale

1. Deux phénomènes universels

2. Le sexe avant la classe

II. La place de la prohibition de l'inceste dans le partage exogamique et la division naturelle

Chapitre XI. — Éloge de l'ordre

I. L'inceste, menace de la culture

1. La Grande Peur

2. Edipe et Antigone

II. L'éternel présent

III. Conclusion

Chapitre XII. - Rétrospective

I. Le paradigme en question

II. Le thème de la rupture et de la conquête

1. L'artifice social

2. La pollution par l'humain

III. Le thème du changement et de la création

1. La nature historique

2. La société positive et négative

IV. Le retour dans la nature

Notes de la première partie

Notes de la deuxième partie

Notes de la troisième partie 


\section{INTRODUCTION}

\section{I.}

Pour se convaincre de sa singularité, le genre humain — ou la partie du genre humain qui s'arroge le droit de parler en son nom élève des barrières autour de soi, se pose par contraste avec le reste des êtres animés. Certes, il a un mérite : celui d'exister. Au vu des nombreux échecs qu'enregistrent des organismes désireux de vivre ou de survivre, ce mérite est grand. Il le renforce dans sa conviction d'avoir réussi un exploit, d'être allé plus loin que quiconque, d'occuper une situation privilégiée dans la longue chaîne des êtres. Pourtant se penser unique et distinct n'est pas une condition de tout repos. Aussi éprouve-t-on continuellement le besoin de motiver cette unicité, d'affirmer cette distinction, de s'assurer qu'elles reflètent le cours nécessaire de l'univers et qu'elles sont définitives. L'exploration des espaces lointains, par les rencontres qu'elle suscitera, modifiera peut-être un jour cet état de choses. En attendant les groupements humains ne cessent de se définir, de dire pourquoi ils sont ce qu'ils sont, humains et non pas animaux ou végétaux. Derrière le langage sobre des théories avancées à ce sujet, on pressent la fascination exercée par le problème des origines. La cause qui a déclenché l'éruption du genre humain en le séparant du monde animal et matériel, l'écart qui permet à l'homme de se hausser au-dessus des autres espèces - ou d'autres fractions de l'humanité, primitifs, femmes, enfants, etc., réputées plus proches de l'animalité — sont les facettes de ce problème. La sortie de la nature, la formation d'un ordre à part, artificiel, représente maintenant la substance de sa solution, que l'on s'efforce de démontrer de mille façons. En même temps, la quête de 
ce qui est le propre de l'homme, la rupture de la société et de la nature, le rapport d'exclusion par lequel on démarque leurs domaines exclusifs jouent un rôle capital. La société est le domaine des hommes, la nature, le domaine des choses. Notre civilisation, en particulier, s’appuie fermement sur cette séparation. Elle la conçoit intégrée à son armature, imprimée dans la structure du monde, s’imposant à l'ensemble du réel de manière permanente. Là se dissimule la ligne de partage entre le supérieur et l'inférieur, le spirituel et le matériel, le produit et le donné, ce qui existe avant l'homme et sans l'homme et ce qui existe après lui, avec lui. Ce rapport d'exclusion qui est tout à la fois différence et négation, autonomie et extériorité, se retrouve au fondement de nos sciences, façonne et organise nos conduites politiques, économiques et idéologiques. Le passage de l'animal à l'homme, de l'état de nature à l'état de société, y est un leitmotiv constant, signe d'un découpage effectif des phénomènes ordonnés dans l'espace et engendrés dans le temps. Certes, des doutes sont émis périodiquement sur la réalité de l'opposition tranchée des deux états. Le philosophe Hume conseillait de l'accepter à titre de fiction et soutenait qu'elle n'était rien d'autre ${ }^{1}$. Les réserves portant sur le détail des observations, sur l'enchaînement des raisonnements, n’ont cependant pas entamé les systèmes d'idées qui l'ont toujours reprise en sous-œuvre ou qui en découlaient, tant sa cohérence, son pouvoir de conviction et son usage sont grands. Il s'agissait en effet de sauvegarder l'essentiel : le caractère contre-nature de la société, le caractère exceptionnel de l'homme.

Mais nous vivons dans un siècle où l'espérance de vie d'une vérité s'est considérablement raccourcie et où des concepts que l'on estimait devoir durer indéfiniment portent les traces d'une érosion qui les rend méconnaissables, quand ils n’ont pas purement et simplement basculé dans le néant. Même les sciences qui nous sont familières sont appelées, à plus ou moins brève échéance, à se combiner, changer ou disparaître. Les savants y contribuent sans relâche, quand ils s'efforcent de mettre à rude épreuve et de démentir plutôt que de confirmer et de préserver les vérités et les théories consacrées. Les découvertes des sciences biologiques et préhistoriques font voir sous un éclairage différent de celui auquel nous sommes accoutumés le comportement et le

1 D. Hume : Treatise on Human Nature, Londres, 1758, vol. 2, p. 265. 
monde animal, la chaîne des événements qui ont conduit du primate à notre présente espèce : par suite, il semble que soit considérable le volume de ce qui est à désapprendre. De leur côté, les forces historiques propres à entraîner les civilisations dans de nouvelles directions, à produire de nouvelles pratiques économiques, politiques, culturelles, minent les notions conçues antérieurement en vue d'autres pratiques, rendent caduc l'esprit qui les a soutenues. La rencontre sur la scène de l'histoire de sociétés ayant probablement suivi un développement divergent, rejetées par nos soins vers l'extrémité « nature » de l'échelle dont nous occupons d'office l'extrémité " culture » est la plus manifeste de ces forces, et ses conséquences sont profondes. Par ailleurs, ce qui touche au déséquilibre écologique, à la croissance des populations et à l'amendement du milieu, bref notre question naturelle, n'est pas moins significatif. Savoir comment gouverner les forces matérielles, comment réduire les écarts entre l'expansion démographique et les ressources de l'environnement, quel rôle assigner au progrès scientifique, suscite des mouvements sociaux et nous oblige à réviser nos options fondamentales. Et notamment à mettre en doute l'idée que l'homme est maître et possesseur de la nature, qu'il conquiert, de l'extérieur, l'univers des choses. On en vient même à soutenir l'hypothèse contraire, c'est-à-dire que l'homme intervient dans l'univers mais de l'intérieur, en tant qu'une de ses parties. Last but not least, le plus souvent, théories, arguments, interrogations renvoient aux expériences, à la sensibilité, aux phénomènes propres à une époque et à une société, surviennent et s'estompent avec elles. Ainsi la naissance de l'individualisme, avec l'individualisation des actes, des intérêts et des rapports humains, a donné une impulsion vigoureuse à l'opposition de la société et de la nature. Tout est alors taillé sur ce patron : atome permanent insécable ou monade sans porte ni fenêtre, organisme luttant pour sa survie - le plus fort vaincra ! - animal agrégé à une horde, acheteur ou vendeur sur le marché, savant isolé aux prises avec les énigmes de l'univers. En physique, en biologie, en économie, en philosophie, partout l'individu est l'unité de référence. Expression la plus complète de l'essence des choses et de l'homme, il incarne la nature humaine et témoigne de son état originaire. En comparaison, la société ne saurait être rien d'autre qu'un état antagoniste, une association dérivée de volontés diverses et de molécules indépendantes, soumises à des contraintes. Déduits de cet antagonisme, les 
principes des institutions et des lois politiques et sociales qui nous guident aujourd'hui y sont fermement ancrés.

Pourtant la socialisation des intérêts, des actes, des rapports humains est une tendance fondamentale de notre présent. C’est une évidence à laquelle on ne peut guère échapper, même dans le domaine de la science. En physique : c'est par paquets que les atomes se transforment, ont une durée de vie, se meuvent. En biologie : la survie de l'espèce est fonction de la population et non pas de l'individu; les groupements animaux sont organisés, connaissent la hiérarchie et la convention. En philosophie : la communauté des savants en tant que telle est engagée dans le travail de découverte des lois de la matière. Comme autrefois, dans la production, le travailleur collectif a remplacé le travailleur individuel, on voit aujourd'hui le penseur collectif se substituer au penseur individuel. Pénétrant, sous la forme directe ou indirecte de population, de collection, d'ensemble statistique, notre vie et nos catégories de pensée, le social émerge unité de référence, paradigme du réel. Il suffit de fort peu de chose pour que, par analogie et à la place de l'individu, il aboutisse à manifester l'essence de l'homme, son état naturel. Dans ces conditions, l'opposition qui nous occupe, cessant petit à petit de trouver un écho dans nos expériences, nos attitudes mentales, perd de son acuité et de sa pertinence. Il restera la tâche de reformuler les principes des institutions, des lois politiques et sociales, à partir d'autres liens entre société et nature, processus déjà engagé qui ira s’amplifiant.

Ainsi, découvertes scientifiques et forces historiques, la rencontre n'est pas fortuite, nous amènent à remiser les faits et la logique qui ont servi à formuler le problème de nos origines, à dissocier notre monde social de notre monde naturel en leur conférant des propriétés antinomiques. Mais elles nous invitent aussi à les réordonner dans un cadre différent. N'allons cependant pas trop vite. Nous touchons là en effet à un système d'idées et à un langage qui, bien qu'ayant perdu le pouvoir de critiquer et d'éclairer, pour ne garder qu'une physionomie figée et opaque, restent gravés dans les esprits, résonnent aux oreilles, comme l'expression du vrai et du réel. Avant d'examiner leur valeur à cet égard, pour s'assurer de leur teneur, il convient de les rappeler encore une fois, comme on rejoue un disque, comme on repasse un film, sinon pour le plaisir, du moins pour être sûr que l'on parle bien du 
même morceau de musique ou du même personnage. Au fil des indications et des commentaires se dégageront spontanément les perspectives qui motivent le présent travail.

Retour à la Table des Matières

II.

Dans quelles circonstances l'homme est-il sorti de la nature ? Quels sont les facteurs décisifs de la coupure avec l'univers biologique et matériel ? Ceux qui posent ces questions et s'efforcent d'y répondre aspirent à fixer le seuil d'un commencement absolu, à résoudre l'énigme de notre singularité. A un moment du processus d'hominisation, semble-t-il, un changement anatomique et physiologique a eu lieu dans l'organisme pratiquement stabilisé. Ce changement cortical et soudain, du type du tout ou rien, analogue au saut d'un niveau quantique à un autre, a ouvert une brèche dans l'évolution. Pour le décrire, les anthropologues emploient l'image glorieuse de passage du Rubicon. La faculté de parler, d'abstraire, de combiner des moyens artifactuels s'est introduite par la brèche produite. De là, chez l'homme, son étonnante flexibilité, ses capacités d'inventer qui l'aident à profiter de la plupart des ressources existantes, à accumuler et transmettre les savoirs, à passer rapidement d'un entourage à un autre. Dans la plupart des espèces, lorsqu'il s'agit d'accéder à des milieux différents, de s'y développer, des modifications génétiques, soumises aux lenteurs de l'hérédité, sont indispensables. Pas plus qu'il ne dépend de telles modifications ou de telles lenteurs, le développement spécifiquement humain n’en connaît ni n’en entraîne. Ses arts seuls, surajoutés à sa structure organique, sont affectés. Certains y aperçoivent même une enveloppe, un vernis de surface appliqué sur un être qui demeure organiquement, par de nombreux côtés, un singe, à vrai dire nu : « Il y a une nature fondamentale, écrivait Henri Bergson ${ }^{2}$, et il y a des acquisitions qui se superposent à la nature, l'imitent sans se confondre avec elle. » C'est que, au cours du temps, les déterminismes généraux se sont vus supplantés par des déterminismes particuliers à l'homme, lui permettant de créer son cadre

2 H. Bergson : Les deux sources de la morale et de la religion, Paris, 1932, p. 289. 
de vie exceptionnel dans le milieu d'origine. La nature s'est dépassée en l'occurrence, le libérant des servitudes communes, lui donnant la possibilité de se retrouver à l'extérieur d'un monde qu'il a pu prendre pour objet, n'y participant que de manière résiduelle. Hors d'elle ou à côté d'elle, la barrière de l'animalité franchie, s'est instaurée une relation, résumée dans et par l'artifice ou l'intelligence, qu'aucune autre espèce ne connaît ni n'a eu l'avantage de connaître. Tel est du moins le schéma auquel on se rallie en général.

Les conjectures qui l'accompagnent sont valides aussi longtemps que l'on conçoit l'organisation biologique de l'homme comme une donnée invariante, son action sur le monde extérieur, par dérogation à la loi générale, demeurant sans répercussions anatomo-physiologiques sur elle, ses diverses opérations pratiques ou intellectuelles se bornant à reproduire artificiellement le milieu sans intervenir dans sa constitution. Or il n'en est rien. La forme du corps, du crâne et des membres, les propriétés spécifiquement humaines, la station debout, le volume du cerveau, le langage, nous en sommes aujourd'hui certains, sont les conséquences de l'activité de prédateur de l'homme, de son aptitude à employer les ruses et les outils nécessaires pour y réussir. Les modifications génétiques, sociales, qui lui sont propres, n’ont pas précédé cet état de choses : elles lui ont succédé. En gros, comme en détail, on ne le rappellera jamais assez, l'homme est son propre produit. Depuis ses premières ébauches comme entité autonome, sa réalité naturelle a toujours impliqué une connaissance, une habileté à faire, associées à un agencement finalisé de gestes et d'instruments appropriés. A aucune phase de son évolution, cette réalité n’a été limitée à un équipement purement organique ou instinctuel. Les paléontologues en conviennent qui interprètent de plus en plus sa biologie par sa technologie.

Il en est de même en ce qui concerne le milieu matériel. Les théories anciennes avaient tendance à le rapporter à l'individu, à le définir uniforme, constant, toujours semblable à lui-même, indépendant des influences exercées par les créatures qui l'habitent et l'exploitent. Le milieu matériel se confondait avec sa dimension géographique et géo- 
logique, à quoi se ramène la nature selon une opinion répandue ${ }^{3}$. Or l'écologie d'une espèce, les études approfondies le montrent, lui est particulière. Elle est relative à une population répandue dans une aire déterminée, aux modes opératoires de cette population qui la conduisent à s’approprier une ressource de préférence à une autre. Pour un observateur externe, la forêt semble être un milieu unitaire et distinct. Pour les animaux qui y cohabitent, elle est un univers structuré, diversifié, dont seule une parcelle leur apparaît familière et connue, le reste étant comme inexistant. Le milieu qui entoure l'être humain, comparé à celui qui entoure l'animal, n'en diffère pas simplement par sa variété et sa surface, puisqu'il couvre l'ensemble de la terre. Il contient des espèces physiques, végétales et animales que nous avons produites et qui interfèrent aussi bien entre elles qu'avec celles qui existaient déjà. La biosphère qui lui correspond est spécifique, étant donné les processus qui l'affectent et qui l'ont modelée.

Quand on jette un coup d'œil sur les facteurs internes et externes qui ont contribué à la genèse de l'homme, force est de constater qu'avec lui se dégage un rapport différent, un écart qualitatif. Ce nouveau rapport, il faut y insister, inclut d'emblée un faire et un savoir conçus par l'homme. Il n'en a pas connu d'autre ; il n'y a pas de rapport de l'homme à son milieu qui ne résulte de l'initiative humaine, non qu'il l'ait engendré, mais parce que l'homme s'est constitué ce qu'il est, physiologiquement, psychiquement, socialement, en l'engendrant. Dans un précédent ouvrage, Essai sur l'histoire humaine de la nature ${ }^{4}$, j'ai soutenu et démontré qu'il était possible de concevoir la coexistence et la succession de plusieurs rapports, tous également naturels, dans l'univers. Celui qui nous concerne pose l'homme à un pôle et les forces matérielles à l'autre pôle. J'ai étayé cette façon de voir par un examen de l'activité humaine qui engendre des éléments physiques, chimiques, génétiques, des combinaisons inédites de ces éléments, et non seulement, comme on l'affirme, des artifices. La différence classificatoire du naturel et de l'artificiel, à l'arrière-plan de la dichotomie d'une nature organique qui nous contient et d'une na-

3 « Le monde naturel est le monde géographique des phénomènes que nous percevons autour de nous. » P. BIDNEY: Theoretical Anthropology, New York, 1959, p. 18.

4 S. Moscovici : Essai sur l’histoire humaine de la nature, Paris, 1968 
ture inorganique que nous conquérons et transformons en technique, n'a pas la solidité qu'on lui suppose. La lutte de l'homme seul contre la totalité de la nature - à présumer qu'elle soit distincte de la lutte entre les hommes, et plus âpre - se laisse concevoir comme un affrontement dans la nature. A ce conflit, tout d'abord, la société, qui est une constituante décisive de notre complexion vitale, prend part. Ensuite s'y adjoignent, sans discontinuer, d'autres puissances matérielles. Avec les plantes «contre » les animaux, avec l'électricité « contre » les forces mécaniques, nous participons, d’une différenciation régulière du monde matériel, nous le mettons à jour en tant que système de relations. L'intervention de l'homme revêt la signification d'un rapport établi dans le système avec une de ses parties. Mieux encore, les principes qui le rattachent à ses «alliés » et l'opposent à ses « ennemis » sont ceux-là mêmes qui unissent les êtres physiques, biologiques, chimiques entre eux. Tout concourt à prouver que le lien homme-nature est aussi un lien nature-nature. L'humanité avec ses bras, ses nerfs, ses cerveaux s'amalgame aux puissances qu'elle pénètre. L'homme est donc cheval, gravité, électricité, et réciproquement. Il y a longtemps qu'Antiphon a énoncé cette vérité : "Par notre habileté nous conquérons la maîtrise sur les choses dans lesquelles nous sommes conquis par la nature. » Ce ne sont donc point des termes extérieurs l'un à l'autre. La mythologie de leur mutuelle violence, reprisée et répétée à satiété, s'avère tissu de notions vagues, dénuées de signification, impropre à jeter une lumière quelconque sur les faits historiques concrets. Le dilemme qu'elle rend plausible - entre l'homme dissocié de la matière et l'homme enchaîné à la matière, spectateur et acteur dont le seul recours serait la domination comme envers de son impuissance - perd rapidement son pouvoir évocateur et ses vertus mobilisatrices. Et en particulier à une époque où il est plutôt question de défendre la nature contre l'homme que de défendre l'homme contre la nature.

L'apparence de clivage entre ce qui est produit par l'homme et ce qui est produit sans l'homme s'estompe chaque jour davantage. Les machines et les outils conçus en vue de prolonger directement le corps humain, dépendant pour leur fonctionnement de ses forces musculaires, ont été, pendant longtemps, les supports illustratifs de cette apparence. Les systèmes automatiques modernes jouissent d'une autonomie, d'une faculté d'autorégulation, voire d'autoproduction telles que 
les spécialistes les apparentent aux systèmes dits naturels. Personne ne voit d'obstacle à ce rapprochement, du fait qu'ils procèdent de l'intelligence, de l'ingéniosité et de l'effort humains. Les recherches physiques, chimiques, mathématiques, de leur côté, débouchent sur la production d' "espèces » physiques ou chimiques sans équivalent dans la " nature » et ne se distinguant en rien des espèces qui se sont formées hors de ces recherches. Le lecteur du tableau de Mendéléeff les retrouve chacune à leur place, qu'elles soient nées dans l'univers ou au laboratoire. On abuserait du langage en les qualifiant d'artefacts, étant donné que ces " espèces » scientifiques ne reproduisent aucune structure matérielle préexistante, ni ne se substituent à une telle structure. Dans le nombre des "espèces " découvertes, il faudrait inclure la nôtre, nos qualités biologiques, nos facultés intellectuelles, nos organes et leurs fonctions devant être comptés, je l'ai dit, parmi les résultats de nos pratiques. Les savoirs et les phénomènes qu'engendrent l'art et les sciences (les exemples sont innombrables) vont de pair avec une conversion de nos capacités et des facteurs du milieu auxquels elles correspondent. Leur aboutissement n'est pas un état antinaturel ou artificiel, mais un "progrès de la nature, en tant que l'espèce humaine en a mis à profit, pour ses besoins et ses désirs, les diverses manifestations ${ }^{5}$.

L'homme joint à la matière, voilà la définition concrète, le contenu véritable de notre état de nature. Persister à qualifier d'artificiel le rapport qui s'y manifeste, cela revient à soutenir que notre espèce n'a jamais existé et n'existera jamais que dans une nature à laquelle elle ne devrait pour ainsi dire rien. Ce qui est assurément absurde et sans fondement. La singularité du rapport en question a trait uniquement à ses modalités et éventuellement à un de ses termes. Ses points d'application, comme pour tout rapport analogue, touchent également à la biologie et à l'écologie. Certes, il diffère des relations correspondantes qu'entretiennent la plupart des espèces ; mais ces relations ne sont pas non plus identiques à ce que l'on observe dans l'interaction des forces chimiques ou physiques. En dernière analyse, la qualification semble reposer surtout sur la confusion des échanges naturels avec la manière d'agir et d'évoluer qui a cours dans le monde animal ;

5 L. BrunsChVICG : L’expérience humaine et la causalité physique, Paris, 1949, p. 591. 
on en déduit le caractère non naturel de tous les autres échanges. On commet, en l'occurrence, une erreur analogue à celle des philosophes pour qui, la société étant bâtie sur la propriété et la propriété assimilée à la propriété privée, tout ce qui l'avait précédée était état de nature, de non-propriété, et non pas forme différente de propriété. Ou encore c'est raisonner comme les socialistes pour lesquels la suppression de la propriété privée signifiait la suppression de toute propriété, et non pas l'avènement d'une de ses formes historiques, la propriété collective. Somme toute, le rabattement de la réalité sur une de ses figures explique pourquoi l'effacement d'un mode d'existence naturelle qui fut en partie celui de l'espèce à ses débuts a été considéré comme la subversion de toute existence naturelle et non pas comme un renouvellement de celle-ci. Rien ne nous oblige à prolonger la confusion ; tout nous incite à mettre fin à la vision d'une nature non humaine et d'un homme non naturel. Aucune partie de l'humanité, à vrai dire, ne saurait être jugée plus proche ou plus éloignée que les autres d'un état épuré, de nature, lui-même en mouvement, ni dans le passé préhominien ou sauvage, ni dans le présent évolué. Ce qui a eu lieu une fois se recrée continuellement, les modalités seules changent. Au poète il n’a pas échappé que

«Au-dessus de cet art

«Qui, dites-vous, ajoute à la nature, il est un art

«Que fait la nature » ${ }^{6}$.

Arts que nous avons repris ensemble, combinés, substituant à ce qui aurait pu être, à ce que l'on aperçoit avoir été une histoire naturelle améliorée de l'homme, une histoire humaine de la nature. L'éclosion d'un trait critique signalant l'irruption de notre espèce, la distanciant des autres espèces n'indique pas une prétendue sortie de la nature : cette rupture n'a jamais eu lieu. Dans le passage, tant recherché, de l'animal à l'homme, elle marque la transition de la première histoire commune où celui-ci apparaît en tant que produit, à la seconde histoire, la sienne propre, où il se produit en tant que principe actif. Le problème des origines s'estompe ainsi, puisque rien ne s'est ajouté qui n'était déjà présent, derrière le problème des transforma-

6 W. SHAKESPEARE : A Winter's Tale, IV, IV, 90-92. 
tions dans l'échange avec le monde matériel. La direction nouvelle que prend l'évolution à cette occasion en est le témoignage.

$\underline{\text { Retour à la Table des Matières }}$

III.

La réalité et le concept de cet état de nature représentent, par bien des côtés, quelque chose de neuf à quoi il faut s'habituer si l'on veut y voir le lieu d'un devenir dont nous sommes un facteur constitutif, nécessaire, et non pas le lieu des obstacles suscités par les puissances matérielles, nos partenaires convenus à la suite d'un accident biologique initial. Non moins important : l'état de société coexiste avec lui, aux divers niveaux du règne animal. Il est désormais difficile d'y voir seulement l'expression d'un art de vivre de notre espèce, subversion de l'art de survivre qui suffit aux autres espèces pour se nourrir et procréer. Ceci nous oblige à prendre une vue différente du rapport qui délimite ces deux états. Le discours courant de notre culture et de notre science le définit, nous le savons, comme un rapport d'exclusion. Afin de l'étayer, il reprend le thème de la démarcation entre société et nature sur trois registres : technique, génétique et politique. Je vais les décrire succinctement.

On note d'abord un fait qui paraît d'observation : la nature est un donné immédiat. Elle comprend les milieux où les individus se sentent à l'unisson avec les créatures qui les entourent, où les rythmes d'activité et la dépense d'énergie expriment le fonctionnement spontané des sens, les normes immémoriales et le lent écoulement du temps. Elle est peuplée d'êtres familiers qui vivent au sein d'une terre maternelle, en suivant leur impulsion. Là le chien connaît son maître, la cigale la fourmi ; les fleurs et les arbres couvrent abondamment la surface du sol, l'homme possède instinctivement les gestes requis pour atteindre un but, accomplir une tâche. La curiosité se nourrit de la récolte apportée par la vue, le toucher, l’odorat ; les choses sont à la mesure de l'individu, prêtes à se laisser découvrir sans résistance et sans contrainte. Partout règne l'harmonie préétablie entre l'organisme et le milieu ambiant ; la vie authentique est ponctuée par la naissance, la maturité et la mort, dans la continuité visible des générations. Cette nature naturelle est libre, aisée, positive, individuelle, et stable. Elle 
correspond étroitement à notre complexion biologique, rendant justice à nos facultés primordiales, établissant des cycles spontanés d'échange entre nous et le monde. Nous sommes en elle et avec elle, dans une double relation d'être et d'appartenance.

Sur son pourtour se dresse l'édifice d'une autre nature, contrariée — " vexée ", disait Francis Bacon - lointaine, difficile à appréhender, objective, universelle, en perpétuelle agitation. Dans le milieu qu'elle représente, nous instaurons une relation de faire, de conquête. Sa masse inerte et froide est l'affaire du savant ou du technicien qui l'enferme dans un système de lois et la reproduit dans son laboratoire ou son atelier. Ayant été forcés d'entrer en contact avec elle, les hommes la connaissent sans la percevoir, la manipulent sans s'y intégrer. Les ressources leur faisant défaut, ils sont allés les chercher là où elles se cachaient. Les moyens dont ils disposent ne sont évidemment pas ceux d'une espèce ordinaire. Les animaux supérieurs, par exemple, sont parfaitement adaptés à leur écologie, grâce à un équipement biologique qui leur permet de résister aux intempéries, de se nourrir et de préserver la vie de leur progéniture. L'espèce humaine, fragile, dépourvue d'un grand nombre de ces avantages a dû dès le début combler ses lacunes. Les sciences, les arts, les techniques sont nés de cet effort : prothèses s'ajoutant au corps et au cerveau, pour lui apporter ce dont les autres espèces disposent normalement. Dans ce processus, sa tâche essentielle consiste à vaincre les obstacles internes et externes, a soumettre le monde à ses exigences, afin d'obtenir les matériaux nécessaires à la vie. Les forces matérielles qui se sont opposées et s'opposent encore à ses entreprises viennent peu à peu à résipiscence. La lutte contre la nature, contre les éléments qui la composent est sans merci. C'est dans l'intention de les vaincre que les individus s'associent, que la société se forme. Après tant de succès, rarement remis en cause, l'humanité s'est persuadée que la victoire finale lui revenait de droit. A chaque étape, elle s'est émancipée de son milieu en exerçant sa domination sur une nouvelle puissance physique l'eau, le feu, l'électricité, etc. - et en acquérant un savoir qui rend ce milieu un peu plus artificiel. L'abondance des ressources, l'emprise totale sur l'univers s'annoncent au terme de cette longue marche. De l'état naturel, de ses mystères et de son opacité, il ne restera plus qu'un souvenir ou une image floue, réfractée par un monde humanisé, une nature technique. 
La nature est double, scindée : originelle par son fondement, artificielle par les circonstances. Le développement historique de l'individu et de la société témoigne de l'arrachement au cadre primitif et à la tyrannie de la matière, origine d'une dénaturation croissante qui a commencé il y a plusieurs millions d'années. A la lumière de cette stricte dichotomie et du mouvement qui l'a produite, les triomphes de la raison et de la science sont comptés pour autant de défaites d'une humanité qui voit écarteler la réalité dont elle procède et ne sait plus bien quel est son habitat véritable. L'action par laquelle elle étend un de ses empires dégrade l'autre ; à la voix qui célèbre son ascension hors des déterminismes universels vers la sur-nature qu'elle érige, répond en écho une autre voix qui l'instruit sur la déchéance dans laquelle est tombé le terroir végétal et animal initial, ravalé à l'état de sous-nature. Pourquoi s'en étonner? Le travail de la connaissance et de l'art, ingrédients de la culture, a pour condition pareille rupture et pareille évolution ; il est dans l'ordre des choses qu'une fois commencé il continue sur sa lancée, indéfiniment.

Malgré tout, l'homme participe du monde animal. Les lois de l'hérédité et de la sélection naturelle ont présidé à la transformation de ses organes, de la main et du cerveau, et l'ont préparé à s'adapter au milieu. Dans ce cadre génétique, l’identité entre les hommes est profonde ; leur distinction d'avec les autres animaux supérieurs, les primates notamment, n'est pas significative. Par le canal de cette parenté, individus et collectivités ressentent l'emprise de la nature qui subsiste en eux et les soumet aux rigueurs de la structure bio-psychique. La pression des instincts, des pulsions sexuelles et agressives, le désir de satisfaction immédiate des besoins élémentaires, la faim, la soif, traduisent la présence du fonds biologique, lien universel de tout ce qui est vivant. Il suffirait donc de peu pour rappeler à la surface et à la vie les comportements, les postures archaïques.

Jusqu'à un certain point, la société est née et se conserve pour élever dans chacun d'entre nous une défense contre l'incessante menace de la nature: menace de l'animalité contre l'humanité, de l'individualité biologique contre la collectivité policée, du présent qui ne sait pas attendre contre le passé et le futur qui mettent les choses à leur place. Le prix que l'humanité paie pour construire son univers 
propre est souvent la guerre, la maladie, la folie, à côté de bien des malformations de l'esprit et du corps. C'est pourtant de ce pénible travail de répression, et afin de l'achever, que naissent les arts, les sciences, la littérature, les mythes ou les religions ; incarnations d'un élan naturel renversé, œuvres domestiquées canalisant une énergie qui se serait, sans cela, perdue dans les ténèbres des temps sauvages. Leur domaine d'élection est ce qu'on a appelé d'un terme fort évocateur le «supra-organique ». Il synthétise un ensemble d'interactions et de comportements appris qui ont rendu les individus plus aptes à affronter la versatilité et l’hétérogénéité du milieu physique, à le maîtriser. Contrôle et plasticité caractérisent les facultés humaines, s'opposant à la dispersion et à la rigidité des capacités animales. Leur développement sous l'égide de la culture est, on l'a relevé, plus accéléré ; il est aussi plus efficace que le développement naturel. On comprend que, l'ayant à sa disposition, l'homme ait renoncé à l'adaptation archaïque, par voie biologique, au bénéfice d'une adaptation éminemment sociale. Du coup on saisit pourquoi les formes qu'ont prises les sociétés sont si dissemblables. Étant donné que, pour l'homme, les transformations organiques sont exclues, ou dépréciées, ce sont les institutions et les instruments techniques qui se réajustent et se remodèlent lorsqu'il s'agit de s'adapter à des conditions nouvelles et des entourages multiples. Les savants ont haussé à la dignité de principe méthodologique cette explication qui, jusqu'à plus ample informé, serait empiriquement fondée. Lorsque, dans le cadre de leurs travaux psychologiques, anthropologiques, ils isolent un trait ou une régularité présents dans toutes les collectivités, ils les déclarent naturels, génétiques, et les attribuent à des causes innées. En revanche, les traits ou les régularités n’ayant pas la même constance sont déclarés sociaux, et on en rend compte par des causes secondaires ou acquises. L'équation de l'espèce humaine pose, du côté biologique, la similitude et l'universel, et, du côté social, la variété et la particularité à l'intérieur et vis-à-vis du milieu extérieur.

Sur la carte du monde tel qu'il fut et tel qu'il est, le plein de la société correspond au creux de la nature, la percée et les dimensions positives de la première sont symétriques du recul et des dimensions négatives de la seconde. Ayant rompu avec les pouvoirs infus, organiques, et les ayant détournés, les hommes ont écarté les obstacles élevés de longue date devant la progression du règne animal. Parce qu'ils 
sont les seuls à avoir réussi, l'ordre social, dont l'architecture matérielle et spirituelle est unique, est considéré comme leur élément naturel. Quant à l'ordre naturel proprement dit, ses limites atteintes, il n'est plus que le vestige dissimulé et contingent d'une association autrefois nécessaire. Il revient sporadiquement à la surface, en profitant d'une faille dans la surveillance de la culture, dans le dressage des individus, ou d'une tolérance inaccoutumée envers les désirs par lesquels ceux-ci sont sollicités. La parenté de l'homme avec le reste de l'univers vivant se dévoile l'espace d'un éclair. Mais dès que l'on retourne à la réalité présente, les ponts sont coupés: alors cet ordre paraît déplacé parmi nous et artificiel.

L'état de nature vient donc du passé. L'état de société témoigne du mouvement d'inclusion de l'individu dans le réseau des obligations collectives, dans une organisation déterminée de celles-ci. L'hypothèse d'un état naturel de l'homme a été proposée dès le XVII siècle pour désigner les conditions primordiales à partir desquelles s'est formée la société avec ses conventions forcément arbitraires, ses rapports de propriété et son pouvoir politique. On peut dire que cet état symbolisait, en fait, une société parfaite où régnait l'égalité entre individus, ayant un libre accès aux richesses et un statut personnel indifférencié. Bodin décrit ainsi la naissance du lien social ${ }^{7}$ : «Alors la pleine et entière liberté que chacun avait de vivre à son plaisir, sans être commandé par personne, fut tournée en pure servitude et du tout ôtée au vaincu... Ainsi les mots de seigneur et de serviteur, de Prince et de sujet, auparavant inconnus, furent mis en usage. " Locke ${ }^{8}$ a poursuivi cette réflexion, présentant le pouvoir politique comme le gauchissement d'une situation où chacun fait ce que lui dicte son bon plaisir, dispose de sa personne et de ses biens à sa guise, sans demander l'autorisation de quiconque ni dépendre d'une volonté étrangère à la sienne, les seules bornes connues et reconnues étant celles des lois de la nature. La juridiction est réciproque, et le pouvoir équitablement distribué ne prend pas la figure d'une violence légitimée, " car il est très évident que des créatures d'une même espèce et d'un même ordre, qui sont nées sans distinction, qui ont part aux mêmes avantages de la nature, qui ont les mêmes facultés, doivent pareillement être égales

7 J. Bodin : Les six livres de la République, Paris, 1579, p. 47 et p. 48.

8 J. Locke : Essai sur le gouvernement civil, Amsterdam, 1691, Chap. I, p. 1. 
entre elles ». Lorsque se sont introduites l'inégalité, la rapine, la servitude et la différenciation des classes, cette « nature » a été changée en « société ». La comparaison des communautés politiques européennes avec les communautés, plus simples, d'Afrique et d'Amérique, connues par les récits des voyageurs, jugées proches de l'état de nature, étoffait ces analyses et rendait leurs conclusions vraisemblables. Jean-Jacques Rousseau les a synthétisées de manière éclatante ${ }^{9}$. Aux origines, conjecture-t-il, les individus pourvoyaient paisiblement à leurs besoins physiques et intellectuels, jouissaient de ressources indépendantes, sans se préoccuper de ce qui est à soi et de ce qui est à autrui. Ils se sentaient suffisamment outillés pour décider seuls de ce qui leur convenait ou ne leur convenait pas sans rechercher constamment l'approbation de leurs congénères. Des mécanismes spontanés veillaient à l'harmonie de leurs relations. L'état de nature, dans lequel ils vivaient, connaît l'aisance et non pas la contrainte, le partage et non pas l'échange, l'accord et non pas l'opposition des intérêts particuliers aux intérêts généraux, la confiance qui naît de la sécurité et non pas la peur qui répond à la menace. Cependant le désir de conservation l'emportant sur les résistances rencontrées pour maintenir cet état, et la limitation des forces que les individus peuvent employer pour se défendre, les ont incités à rechercher un arrangement contractuel collectif, à faire les concessions mutuelles indispensables pour $\mathrm{y}$ arriver. Les hommes sont passés à l'état de société en renonçant à une liberté précaire, dangereuse, au profit d'un joug salutaire. Leurs instincts, dûment épurés, se sont soumis aux exigences d'un ordre où chacun se voit assigner une place, un espace de vie circonscrit. La loi y distingue les droits des forts et les devoirs des faibles, tempère les abus des premiers, amène à l'obéissance les seconds, tient la balance égale entre les prestations qu'impose le groupe et les protections que l'individu réclame. Son ombre s'étend constamment et avec elle la propriété privée, l'autorité politique, pénétrant chaque parcelle de l'existence humaine, l'arrachant à la nature. La société est un mal nécessaire, le philosophe ou le savant motivent la nécessité de ce mal.

Claude Lévi-Strauss a ajouté une dimension anthropologique au problème de la démarcation des liens sociaux. Son point de départ est relativement simple. La nature biologique est le domaine du spontané 
et de l'universel chez les hommes et chez les animaux. La promiscuité qui se manifeste dans le choix des partenaires sexuels et les rencontres propres aux groupes biologiques, notamment les primates, donne la preuve d'une grande versatilité, d'une absence de normes susceptibles de guider sélectivement le comportement. A l'opposé les processus culturels laissent voir, en permanence, l'action des règles, étayées par le langage et par les structures de l'esprit, qui impriment une trajectoire précise aux relations entre les membres d'une collectivité et entre collectivités. Le contraste de la nature à la culture est coextensif au contraste des rapports sexuels promiscus et des rapports sexuels codifiés. La prohibition de l'inceste, du commerce sexuel avec ses géniteurs, ses frères et ses sœurs, leur sert de support et en garde les traces. Elle est universelle, a un champ d'application instinctuel, comme tout phénomène naturel, et inaugure une classe de règles particulières à l'homme, comme tout phénomène culturel ${ }^{10}$. Sa portée n'est pas négative. Car sa signification ne réside pas dans l'interdit d'épouser sa sœur ou sa fille, mais bien dans l'obligation de donner sa sœur ou sa fille à autrui. Les individus qui circulent, sous son empire, assurent l'alliance de leur groupe avec un autre groupe, la communication des biens à l'intérieur du système social et l'équilibre de ses capacités productives. Les règles de mariage prescrivent avec qui une communauté préfère échanger richesses, prestations, personnes, ou avec qui elle est tenue de les échanger. Parce que la famille est l'unité constitutive dans toutes les sociétés, la prohibition de l'inceste, partout et toujours, accomplit les mêmes fonctions: empêcher leurs membres de retomber sous l'emprise de l'instinct, témoigner du dépassement de la nature grâce à la prééminence du collectif sur l’individuel, intégrer les organisations plus simples de la vie animale aux organisations plus complexes de la vie humaine. Mais la règle présuppose, perpétue, une subordination des femmes aux hommes. Le mariage, en tant qu'échange, a lieu entre deux groupes d'hommes; la femme est

10 «La prohibition de l'inceste est le processus par lequel la Nature se dépasse elle-même ; elle allume l'étincelle sous l'action de laquelle une structure d'un nouveau type et plus complexe se forme et se superpose, en les intégrant, aux structures plus simples de la vie psychique, comme ces dernières se superposent, en se les intégrant, aux structures, plus simples qu'elles-mêmes, de la vie animale. Elle opère, et par elle-même constitue, l'avènement d'un ordre nouveau. »C. LÉVI-STRAuss : Les structures élémentaires de la parenté, Paris, 1949, p. 31. 
l'objet à échanger, l'indice physique et symbolique médiant la relation qui s'établit ou se renouvelle à cette occasion. Le jeu social comprend uniquement des acteurs masculins, la donnée féminine offrant les matériaux dont il a besoin.

Nous ne sommés pas très loin des théories de Jean-Jacques Rousseau et des penseurs politiques qui l'ont précédé. Aux yeux de ceuxci, l'état de société met fin à la discontinuité, à l'absence de discrimination, à la libre décision des individus et à leur union accidentelle. La fortune, le rang, le savoir, instruments sociaux, créent, à la place, la continuité, la différenciation, la conduite orientée par la pesanteur des traditions et les exigences collectives. Claude Lévi-Strauss, de son côté, pose, en s'appuyant sur des observations, que la femme est un objet, vivant, certes, appropriable comme une ressource rare. Les donneurs et les récepteurs de femmes les incluent dans leurs transactions, soit à la place d'autres biens, soit mêlées à eux. La prohibition de l'inceste fixe les titres des échangeurs (on ne se marie pas dans sa famille) et fournit sur le "marché » les produits requis. La circulation de l'élément féminin dans les veines du corps social, à chacune de ses stations, contribue fortement à faire respecter l'ascendant du groupe des hommes sur la collectivité. Les règles de mariage sont des règles de répartition de la propriété et du pouvoir selon un critère sexuel. Le sauvage comme le civilisé souscrivent, sous des formes variées, à la formule de James Boswell : "La chasteté des femmes a une importance primordiale, comme toute propriété en dépend.» Ainsi s'ébauche déjà le prototype — du moins si l'on entend ce que la théorie veut dire - de la longue chaîne de maîtres et d'esclaves. Le signe qui les pose et les sépare se confond avec le signe qui pose et sépare l'état de société de l'état de nature. Plus exactement, les hommes qui se réservent le premier et les hommes qui sont identifiés au second sont inconciliables et soudés comme la force et la faiblesse, la richesse et le dénuement, l'élément mâle et l'élément femelle.

Je n'ai pas fait ces rapprochements à cause de leurs résonances éthiques. J'ai voulu souligner la permanence d'un courant de pensée pour lequel la réalité ultime de la vie naturelle est l'égalité, et la réalité ultime de la vie sociale - à travers l'universalité de la propriété et du pouvoir, de la prohibition de l’inceste - l'inégalité. Sans celle-ci, il n’y a ni famille, ni classe, ni statut « de seigneur et de serviteur », si- 
tuation qui signifierait la fin de la culture et le retour à la nature. C'est probablement afin de se prémunir contre une telle éventualité que l'humanité s'est ingéniée à accumuler règles restrictives, interdits et différences, au lieu d'en alléger la charge et d'en diminuer le nombre. Le reste étant utopie, animalité ou archaïsme.

Quel que soit le registre - technique, génétique, politique — auquel elles ont recours, ces conceptions suivent un programme logique commun. Elles atteignent l'exclusion qu'elles visent en combinant une complémentarité et une négation. L’ordre social s'inscrit dans l'espace où sourd le désordre naturel — rareté des ressources, poussée des instincts, promiscuité sexuelle. Le principal est de garantir la stabilité en réintroduisant les uniformités. C'est le rôle de l'ordre social, affirmant ses droits face au monde biologique, matériel, qui les reconnaît pour siens, mais a cessé d'agir, ayant perdu son autonomie à un certain niveau de déséquilibre ou d'évolution. D'autre part, on découvre, et c'est le plus important, les lignes de forces suivant lesquelles est pensée, pour l’homme, la société (ou la culture).

Par le faire, travail ou connaissance, elle complète son équipement organique, le distingue des pouvoirs matériels, lui donne le moyen de les soumettre. Par les systèmes symboliques — langages, rituels - et l'apprentissage, elle le prémunit contre les dangers que lui font courir son fonds animal et les lenteurs du développement biologique, et elle introduit la diversité dans l'identité brute des êtres vivants. Par l'institution - loi ou règle - elle met un frein aux mouvements incontrôlés des individus et enchâsse ceux-ci dans un réseau de droits et de devoirs collectifs.

La coupure provoquée par la société avec ce qui est réputé demeurer hors de l'homme, elle la reproduit en lui. Ainsi le dédoublement de la nature qui lui est donnée et de celle qu'il se donne ; la division de l'individu en ce qui est contraint, interdit, civilisé, et ce qui correspond à la spontanéité, à la jouissance, à la force indomptée de ses pulsions affectives ; la division, encore, mais entre les classes d'hommes, les unes étant les piliers de l'alliance communautaire — les mâles, les maîtres, les peuples d'en haut sur l'échelle historique - les autres les femmes, les esclaves, les peuples d'en bas sur l'échelle historique - évoquant la menace d'un désordre et d'une indifférenciation possi- 
bles. L'opposition du monde social au monde naturel est alors opposition de l'homme à la matière animée ou inanimée, de l'individu à soimême, être de culture et être bio-psychique, d'une fraction de la collectivité entre les mains de laquelle sont déposées les clés de la parenté, de la propriété et de l'État à une seconde fraction des mains de laquelle on les a enlevées par un contrat fondateur. Ce qui est dans l'opposition se forme comme opposé. En se donnant l'état de société, l'humanité s'est donné le moyen d'engendrer le milieu d'artifices qui lui convient. Elle y a aussi trouvé un substitut à la nature qui se parachevait: la communication symbolique à la place de l'hérédité, l'adaptation culturelle à la place de l'adaptation biologique. Mais surtout elle a imaginé, construit cet état à l'instar d'un artifice, où tout ce qui était sauvage est domestiqué. De chacune de ses composantes, on peut écrire ce que Claude Lévi-Strauss écrit au sujet de l'instinct sexuel et de la famille ${ }^{11}$ : « Si la société a eu un commencement, celui-ci n'a pu être que dans l'interdit de l'inceste, puisque l'interdit de l'inceste est en fait une sorte de remodelage des conditions biologiques de l'accouplement et de la procréation (qui ne connaissent pas de règle, ainsi qu'il ressort de l'observation de la vie animale) les forçant à devenir perpétuelles seulement dans le cadre artificiel des tabous et des obligations ". La vie de l'homme est ainsi tout entière contenue dans son artifice suprême.

En définitive, par quelque bout qu'on la prenne - indice de différenciation d'avec le monde animal et matériel, instance intériorisée par les individus, terme d'une opposition ou œuvre d'art — la société est radicalement une contre-nature. Je résume dans cette proposition la quintessence des opinions qui ont été émises et ré-émises maintes fois et qui sont devenues progressivement les catégories stables de notre entendement, de notre éducation et de notre action. Les philosophies, les sciences psychologiques, économiques, anthropologiques ou naturelles, les ont incorporées à leurs théories et leur ont ajouté des preuves empiriques. Elles ont toutes coopéré afin de métamorphoser une croyance très ancienne en un fait d'observation. A savoir que l'espèce humaine est le terme absolu où s'arrête la nature et son couronnement,

11 C. LÉVI-Strauss : The family, in H.L. ShAPIRo (ed.) : Man, Culture and Society, New York, 1956, p. 278. 
la forme supérieure de toute existence présente, passée ou à venir dans l'univers.

Aux attendus qui justifient cet événement et cette consécration s'opposent des constatations troublantes. Je n'insisterai guère sur toutes les raisons qui les rendent telles et me bornerai à indiquer les plus remarquables du point de vue théorique.

La foi dans l'existence d'une seconde nature, culturelle, surajoutée au substrat intact d'une première nature, biologique, est des plus tenaces. On figure, en l'occurrence, une substance organique, structurée par des impulsions autonomes et stéréotypées, sur laquelle est apposée, au cours de l'éducation, la matrice d'activités réglées, de normes rationnelles, de mouvements rythmés par les outils ou les machines. Enlevée, la matrice laisse voir la substance dans son état originel. Toutefois, à y regarder de plus près, ce qui est supposé primitif, purement biogénétique, demeure à jamais inaccessible. Les analyses poussées et les comparaisons approfondies que l'on a faites avec les enfants et les préhominiens nous permettent uniquement d'identifier des adaptations à un milieu, physique, social, devenu intérieur par rapport au milieu encore extérieur; adaptations impliquant des élaborations déjà secondaires. Les réflexes auxquels nous conditionnent les outils ou le raisonnement ne sont que des modifications de réflexes antérieurs, établis à d'autres fins. Aussi loin que nous puissions remonter la chaîne des filiations, nous ne reconnaissons que des secondes natures succédant les unes aux autres, sans aboutir à une nature vraiment première. L'homme sans art, sans technique gestuelle et mentale, nous est inconnu et inconnaissable. Les enfants nouveau-nés ou dits sauvages ne font pas exception. Certes, une organisation biologique préexiste partout ; elle n’est pas directement améliorée ou remplacée en tant que telle. On agit continuellement sur ses qualités transformées qui sont obligatoirement un produit. La seule étape authentiquement « naturelle » serait celle de l'homme-animal ou de l'animal-pasencore-humain. Les spécialistes se demandent encore, par habitude, où se trouve la limite entre le dernier primate et le premier hominien. On a cru fermement à son existence. Les découvertes des dernières décennies dissipent tout espoir de l'identifier. Elles témoignent de la grande ancienneté, trois millions d'années, de notre branchement évolutif. Plusieurs espèces d'hommes, ayant des traits anatomo- 
physiologiques distincts, se sont succédé dans les mêmes sites. Leur mode de vie et leurs occupations, les artefacts inclus, sont voisins. Des créatures analogues à l'homme d'aujourd'hui, sachant courir mais non pas marcher sur deux pattes, et au cerveau aussi gros, ou aussi petit, que celui des simiidés actuels, communiquaient peut-être au moyen d'un langage élémentaire et se livraient à la prédation armées d'outils qu'elles avaient confectionnés. Il en découle que ces formes simples de connaissance, de signalisation et d'opération ont modelé notre corps et nos sens du point de vue somatique, et qu'elles ont provoqué des métamorphoses biologiques visibles. Pour qu'elles aient eu de telles répercussions, il n'est pas indispensable que les caractères acquis aient été héréditaires. Dès l'instant où elles produisaient une différenciation du milieu, influaient sur la capacité de reproduction des populations, elles avantageaient ou désavantageaient la transmission de certaines combinaisons génétiques. Ainsi, à l'intérieur du genre humain, l'homo sapiens par rapport à l'homo erectus, l'homo erectus par rapport à l'australopithèque, etc., apparaissent nature première ou nature seconde, suivant le terme auquel on les compare. Chacune recèle à la fois une composante biologique et une composante culturelle, la superstructure technique d'une phase de l'évolution se manifestant dans l'infrastructure biologique de la phase ultérieure. Ceci enlève toute vraisemblance à l'opinion si répandue d'un développement organique complet des individus, auquel sont venus s'ajouter, résultats d'une invention brusque, les instruments, les artefacts, les savoirs et bien d'autres prothèses. Corrélativement, l'axiome d'une uniformité naturelle des hommes - au cours de l'histoire naturelle — contrastant avec leur variété sociale ne résiste pas davantage à l'examen des faits. Leurs origines sont multiples et se sont renouvelées à plusieurs reprises. Chaque espèce signale moins une naissance qu'une évolution, non pas l'éclipse de la nature et son débordement par la société, mais leur transformation conjointe. Les différences qui se sont dessinées successivement ne furent jamais du ressort de l'une sans être du ressort de l'autre. Le paléontologue et l'anthropologue de nos jours sont conduits à reconnaître la simultanéité des distinctions biologiques et culturelles par une comparaison de leurs séries d'observations dans le temps. Lorsqu'ils regardent autour d'eux, à moins d'être racistes, ils constatent, comme le veut la conception courante, l'homogénéité psychologique, physiologique et anatomique des individus et l’hétérogénéité de leurs comportements sociaux. Le paléontologue et 
l'anthropologue d'il y a six cent mille ans (il en existait sans qu'ils fussent professionnels) pouvaient comparer leurs séries dans l'espace et proposer leurs théories, pour expliquer le sens de l'hétérogénéité qui existait sur les deux plans, puisque coexistaient alors plusieurs espèces d'hommes et plusieurs types de sociétés. Les conceptions les plus actuelles renouent donc avec les plus anciennes et contestent celles qui les ont précédées immédiatement, parce que celles-ci se sont contentées de mettre en paroles ce que chacun croyait voir de ses yeux.

Alors la société est une nature seconde lorsqu'elle écarte l'humanité du règne animal, et représente son signe distinctif. En dessous, elle laisserait subsister une communauté biologique, instinctuelle, avec les espèces composant ce règne. Si l'art est l'homme ajouté à la nature, l'homme est la culture ajoutée au primate. Malgré les apparences, les hommes, qui ont fait des progrès impressionnants pour ce qui est de quelques-unes de leurs techniques ou sciences, ne seraient que des singes prédateurs quant aux conduites essentielles qui sont demeurées, depuis ces temps reculés, sous contrôle génétique. Ces assertions - les ouvrages qui les illustrent, dus à la plume de savants éminents, surabondent - ont le clinquant de l'indigence. Elles envisagent des qualités et des traits séparés sans rapport avec la structure qui les englobe au moment où elles émergent. Or il est évident qu'un élément même ancien dans un ensemble nouveau ne reste pas identique à lui-même, pas plus que ne le reste, par ses effets, un élément chimique dans les diverses combinaisons où il entre. Les sciences biologiques et anthropologiques sont des sciences de l'organisation ; leurs praticiens ne pensent pourtant pas souvent en termes d'organisation. Sinon ils se seraient abstenus de conjecturer des changements de parties qui n'affectent pas le tout ou vice versa. Pris dans le réseau de ses échanges, de son développement, l'homme ne descend pas du singe ainsi que le veut le fameux aphorisme, ni ne s'en sépare uniquement par la culture. Voici ce que nous savons à ce sujet. La lignée hominienne s'est dissociée de celle des anthropoïdes il y a environ vingt millions d'années. Ses caractères génétiques distinctifs, résultat d'une évolution parallèle ${ }^{12}$, peuvent être attribués à des

12 «Un grand nombre des arguments tirés de l'anthropologie se rapportant à la différenciation de l'homme et des primates non humains sont centrés presque 
facteurs sélectifs qui ont permis l'adaptation et la survie. Les primates contemporains qui descendent d'une autre lignée sont aussi éloignés de leur souche «naturelle » que nous le sommes de la nôtre. Pour atteindre le niveau qui est le sien, l'homme n'avait pas à vaincre ou à transformer ses pulsions, sa structure anatomo-physiologique de primate ; il lui a probablement suffi de développer la sienne qui était devenue différente. Les circonstances sociales ont joué, mais il faut croire que les mutations successives sont intervenues, elles aussi. Aucune des espèces passées qui ont lancé le mouvement ayant abouti à nous n’a été ni plus « animale » — c'est-à-dire biologiquement identique aux espèces de la branche voisine - ni significativement moins humaine que l'espèce qui règne à présent. La parenté étroite avec le singe est une parenté de plaisanterie. L'écart qui sépare les hommes du reste des anthropoïdes a, en définitive, autant de racines dans l'ordre organique que dans l'ordre social. Impossible de faire un choix à cet égard, de décider lequel est le plus important : il faut les garder tous deux, au même titre.

Amalgamés, l'individu, l'animal, l'instinct, d'un côté, le collectif, l'homme, la raison ou la loi, de l'autre côté, rendent manifeste la cloison étanche qui sépare la fonction biologique de la fonction sociale. Distinctes, antagonistes, exprimant des tendances divergentes, il semblerait à première vue que ces fonctions ne puissent coexister ni dans un même temps, ni dans un même être, ni dans une même évolution. Présente dans un ordre de réalité, chaque fonction manquerait dans l'ordre de réalité complémentaire ou y serait refoulée. La fragilité de ce partage commence à nous apparaître. Tant qu'on s'intéressait aux mécanismes physiologiques, aux appareils sensoriels, aux squelettes, en prenant l'individu pour unité d'analyse, chez l'homme comme chez l'animal, les groupements établis par ce dernier étaient tenus pour curieux et ponctuels. Les ruches d'abeilles et les colonies de fourmis ont davantage servi de thème à des discours moraux que de matière à des conclusions scientifiques. Néanmoins, les informations affluent,

exclusivement sur la différenciation de la «nature » et de la «culture » et la « substitution » de la culture à la nature. Mais l'homme ne se différencie pas seulement des autres primates par le comportement cumulatif traditionnel appris (et même cette différence est de degré, non d'espèce) ; il constitue un genre différent. » R. Fox: In the Beginning : Aspects of Hominid behavioural Evolution, Man. 1967, 2, p. 417. 
engrangées et classées avec beaucoup de soin par de très nombreux chercheurs. La recension des associations stables à bénéfice réciproque, dans de nombreuses espèces, a montré la corrélation entre les exigences du milieu et les régularités d'un comportement éminemment social. Bref, la société existe partout où existe la matière vivante relativement organisée : elle n’a pas commencé avec notre espèce et rien ne laisse supposer qu'elle disparaîtra avec nous. Ces études ont également fait voir que les créatures non humaines sont capables d'accomplir des tâches que l'on croyait exclusivement humaines, notamment apprendre et inventer. Primates, dauphins, oiseaux même possèdent des facultés d'apprentissage et de création de conduites nouvelles, et en dépendent pour leur nourriture et leur reproduction. Contrairement au cliché d'une maturation biologique individuelle, les animaux, à l'instar des enfants, à l'état sauvage, c'est-à-dire seuls, isolés, ne se développent pas normalement, et le contact avec la mère et les congénères leur est indispensable. Il y a environ un siècle on recherchait de tels enfants-loups afin de prouver que, sans la société, l'individu retombe dans l'animalité, incapable de parler, de penser ainsi qu’il le devrait. Des expériences bien menées ont démontré qu’il en était de même pour les singes et les autres espèces. Sans l'appui du groupe et les soins de sa mère, l'individu jeune voit s'atrophier ses capacités de maîtriser les émotions, de se déplacer, d'interagir. Il rechute dans son animalité, comme l'homme était censé rechuter dans la sienne. Aussi bas et aussi loin que l'on descende sur l'échelle de l'évolution, on n'arrive pas à déceler l'existence d'un individu biologique, totalement non social. On note en revanche, chez les mammifères supérieurs surtout, des comportements et des rôles traditionnels transmis d'une génération à l'autre par une initiation individuelle et collective. La reproduction sexuelle des populations prolonge leur reproduction sociale ou est prolongée par elle. Phénomène capital, sur lequel je reviendrai, les structures des sociétés de primates varient à l'intérieur d'une espèce particulière, témoignant d'une indépendance possible envers le substrat génétique.

De grands efforts intellectuels ont été dépensés pour trouver les racines de la société, système exclusivement humain, dans la nature : on l'imaginait comme un ordre triomphant du désordre, celui-ci animal, s'entend. Les observations auxquelles je fais allusion nous informent que l'homme, s’il est arrivé autrement que la majorité des êtres vi- 
vants à instaurer un tel système, n'a pas pour autant accompli un acte exceptionnel ; il a suivi une tendance commune à tous. La plupart des espèces se donnent une organisation collective afin de régler le volume de la population, la transmission de certains caractères spécifiques, ou de pallier les déséquilibres éventuels avec le milieu favorable à la survie. Cette organisation est un facteur nécessaire et non pas une simple extension extrasomatique, un appendice artificiel surimposé aux mécanismes génétiques. Sa capacité de canaliser les interactions des individus pèse sur le sens des adaptations, la reproduction des groupes, l'emploi des ressources. Pour les primates et les hominiens, nous en sommes certains. De la sorte, ce qui se passe dans le domaine social a des répercussions sur ce qui se passe dans le domaine naturel. On a conçu le premier dans la dépendance stricte du second. Nous observons que le second dépend aussi du premier pour son évolution et sa structure. Longtemps jugés incompatibles et non communicants, nous constatons qu'ils sont compatibles et communiquent, par leurs éléments et par leurs effets. La société est vécue et pensée comme défense contre l'impétuosité du vivant, le dos tourné à la nature. Voici quelle se découvre appui de la nature, partie indispensable à son fonctionnement, appelée à préparer et à provoquer ce qui advient dans le cours ordinaire des choses. On est en droit d'y apercevoir « une option biologique fondamentale au même titre que dans la symétrie bilatérale par opposition à la spécialisation du membre antérieur pour la préhension ${ }^{13}$. Le constat entraîne une conséquence qui mérite mention. On a prétendu expliquer notre singularité et notre genèse par un coup d'éclat extraordinaire, nous arrachant à la nature pour nous enfermer complètement dans la société, qui est aussi sa contrefaçon. Dès l'instant où celle-ci n'est pas apparue avec nous, où elle se retrouve sur toute l'échelle des êtres vivants, le lien de succession postulé, la justification de la césure qui aurait eu lieu, à notre propos, à une époque, déterminée, perdent leur raison d’être, scientifiquement parlant.

Enfin, les bons et les mauvais sauvages d'Afrique, d'Amérique ou d'Australie disparaissent. Les collectivités longtemps figées se mettent à bouger. Dans le tableau de l'humanité, dessiné à grands traits, ces hommes occupaient une place à part, symbolisaient son état naturel. Hors du courant historique significatif, on les décrivait menant

13 A. Leroi-Gourhan : Le geste et la parole, Paris, 1964, p. 205. 
une existence originelle et exotique, sans histoire. L’État, la propriété, du moins ceux qu'ils auraient dû avoir pour être semblables à leur découvreurs, leur faisaient défaut. Les individus composant ces communautés pensaient autrement que ne le stipulent la logique et la philosophie. Leurs institutions morales échappaient aux normes et aux lois puisque le code judéo-chrétien ne s'y appliquait guère. A tous égards ils paraissaient dominés par une pré-pensée sauvage, un ordre social réduit à sa plus simple expression, et soumis aux aléas de l'affectivité et de l'instinct. La distance les séparant de nous les fixait dans le statut d'objets d'un développement où leurs protecteurs, colons, scientifiques ou administrateurs, jouaient le rôle de sujets. omparés au cadre de référence civilisé, ils manifestaient une fraîcheur, une absence étonnante de disciplines et de valeurs indispensables à un être humain évolué. En les rencontrant, les missionnaires et les voyageurs d'abord, les anthropologues ensuite eurent l'impression de toucher là à un état primitif, proche de celui de l'animal ou de l'enfant, vis-à-vis duquel les nations du continent européen figuraient l'état social ou culturel, dans son éclatante maturité.

L'inassouvie intolérance à l'altérité, passion nourricière de notre pensée, nous a poussés à voir un néant dans ce qui ne nous reflète pas, à restituer le différent comme lacunaire. Démarches parfaitement justifiées à partir de l'erreur initiale commise en identifiant les collectivités aborigènes, par exemple, à une ébauche barbare du système social à son point de jonction avec le système naturel, quand tout nous montre qu'elles ont suivi une évolution remarquable, distincte de la nôtre. Cette dernière, comparée à la leur, se révèle en fin de compte moins résistante au temps qui l'engendre et qu'elle engendre. Couverte par cette erreur, l'œuvre de la culture a pu être œuvre de destruction, parce que ses protagonistes se sont toujours donné le droit d'annihiler les débris d'un passé qu'elle estimait avoir mandat d'assimiler et d'effacer. Par ce moyen l'état de société s'est installé partout où existait l'homme blanc.

La majorité des peuples qui habitent le globe se retrouve aujourd'hui interdépendante, convergeant vers un cycle commun d'échanges. Ceux qui étaient parlés, pensés et étudiés parlent, pensent et étudient. Ils abordent ce qui était réservé à une partie de l'humanité en tant que possibilité de vie, d'organisation sociale à venir. La fonc- 
tion de l'espace (et de l'espèce) concédée à la primitivité sort de la claustration, les acteurs qui avaient pour rôle d'illustrer la nature cessent de le faire. On croyait tenir solidement les deux bouts de la chaîne, matérialisant les deux pôles de toute existence. On se retrouve avec un seul, le pôle social, mais différencié. Le contraste des deux mondes humains, auparavant hétérogènes, se vide de son contenu, tandis que l'histoire s'universalise. A la faveur de ce rapprochement, l'autre de la société nous instruit qu'il est une société autre. L'échafaudage que nous avons élevé sur des bases différentes est désormais inutile et inutilisable.

L'homme est un primate différent, et non pas une variante domestiquée de la biologie des primates; les écarts entre hommes et ceux qui les séparent des autres animaux sont sociaux, mais aussi génétiques ; l'antinomie de l'artificiel et du naturel parait s'atténuer, et son caractère illusoire s'affirme ; la fonction sociale est générale et inhérente au règne animal ; le renouvellement du contexte historique nous oblige à reconnaître combien est passagère et particulière à notre culture occidentale l'opposition tranchée de la société à la nature ; telles sont les constatations troublantes auxquelles j'ai fait allusion. Les sciences qui ont découvert les unes, et les événements historiques qui ont provoqué les autres, n’ont pas, pour l'instant, touché la terre ferme. Des incertitudes subsistent, il faudra du temps avant que les controverses s'apaisent. Les évidences d'hier ont toutefois perdu leur consistance et commencent à rejeter les théories et les concepts, sinon le langage, qui, de par sa vocation, résistera plus longtemps. Je n’en veux pour preuve qu'une coïncidence qui m’a frappé. Lors de la publication, en volume ${ }^{14}$, environ quarante ans de distance, de son article sur le Supra-organique, Alfred Kroeber a fait machine arrière et a avoué son doute quant à la valeur d'une séparation entre social et organique et des arguments qui la justifiaient. De même, Claude LéviStrauss, à l'occasion de la réédition, près de vingt ans après sa parution, de son ouvrage Les structures élémentaires de la parenté ${ }^{15}$, note qu'il est malaisé de démarquer la culture de la nature. Il pense devoir ajouter que l'opposition des deux termes ne serait probablement ni

\footnotetext{
14 A. KROEBER : The Nature of Culture, Chicago, 1952.

15 C. LÉVI-STRAuSs : Les structures élémentaires de la parenté, $2^{\mathrm{e}}$ édition, La Haye-Paris, 1967.
} 
une donnée originelle, ni une propriété inhérente à l'ordre du monde, mais une création « artificielle » des hommes.

Que deux savants de cette importance aient eu à revenir sur des conclusions qu'ils avaient formulées avec vigueur et regardées comme fondamentales pour leur science ne saurait être attribué à quelque manque d'information ou à une erreur de déduction décelée par la suite. Les prémisses sur lesquelles ils se sont appuyés semblent être seules en cause et avoir été mises en question par l'expérience et les connaissances qui sont les nôtres en ce moment. Parmi celles-ci, l'existence antérieure supposée d'individus ou de groupes purement biologiques, devenus brusquement - avec le langage, les institutions politiques, la prohibition de l'inceste, etc. - des groupes culturels, sociaux, est des plus touchées. Ces individus et ces groupes ont toujours eu une vie collectivement policée, réglée. On l'a décrite informe, chaotique. Ne nous étonnons pas : tout ordre est désordre aux yeux des tenants d'un ordre différent, humain, en l'occurrence. L'opinion suivant laquelle notre espèce a connu un état bionaturel identique à celui des animaux pour s'installer dans son état social — d'où leur opposition résulte d'un effet d'optique. S'il y a eu, indubitablement, une rupture, celle-ci porte les traces d'un bouleversement de comportements déjà sociaux, propres aux anthropoïdes. Là encore il convient d'être circonspect. La société dite humaine n'a pas débuté avec l'homme, ni vice-versa. Nous pouvons soutenir, sans risquer d'être contredit, que les premiers hominiens ont eu une organisation collective semblable à celle des primates supérieurs et qu'ils ont survécu, progressé, grâce à elle. Dans la perspective d'un développement général, le lieu de naissance de notre société est une autre société. Nous tenons là un de ces constats dont j'ai déjà fait mention. On a trop longtemps hésité à l'admettre, sinon dans les travaux concrets, du moins dans la perspective de la révision de concepts qu'il appelle. Le prenant pour acquis, notre enquête doit porter sur le devenir humain du social et non pas sur le devenir social de l'humain, vers quoi on était conduit d'habitude lorsqu'on voulait trouver, dans la nature, le lieu de naissance de la société. 
IV.

Nature et société ne s'excluent pas mutuellement. La première nous comprend, résultant de notre intervention. La seconde existe partout : elle n'est pas née avec l'homme, et rien ne laisse supposer qu'elle mourra avec nous. L'homme se situe à la confluence de leur structure et de leur mouvement : biologique parce que social, social parce que biologique, il n'est pas le produit spécifique de l'une ou de l'autre. Dégagée du problème de ses origines, de l'opposition de ses deux ordres de réalité, la discontinuité postulée à leur propos se déplace du plan horizontal au plan vertical. Elle n'est plus entre nature et société, elle est à la fois dans celle-ci et dans celle-là, conséquence des changements que nous y avons introduits. Événements, faits et phénomènes se disposent d'une manière différente sur la grille ainsi déplacée. La transition assidûment recherchée de la première à la seconde se découvre, en fait, transition parallèle, solidaire, d'un état naturel commun à un état naturel propre à l'homme, d'un état social commun à un état qui lui est particulier. Ces conséquences s’imposent dès l'instant où l'on reconnaît le caractère concomitant, historique, du lien que l'on envisageait séquentiel, statique, qui unit les processus sociaux et les processus bionaturels. Mais les matériaux sur lesquelles elles s’appuient offrent un intérêt supplémentaire : ils contiennent les éléments d'une solution aux questions soulevées par la transformation, que je viens d'indiquer, de ces processus. Mon travail est consacré à l'élaboration de cette solution. Le lecteur qui m'a suivi jusqu'ici a cependant le droit de savoir où je veux en venir. D’abord, en ce qui concerne le passage d'une nature qui nous a faits à une nature que nous faisons, je montrerai la substitution, au cours de l'évolution,

- d'une division naturelle des hommes, suivant les ressources et les savoir-faire qu'ils engendrent par leurs activités, à la sélection naturelle dont elle reprend les fonctions ;

- de mécanismes de croissance aux mécanismes d'adaptation de la population et des facultés, en relation avec le milieu matériel. 
Sur les traces de cette substitution a eu lieu ce qu'on appelle l'hominisation: éclosion des propriétés anatomo-physiologiques qui nous sont propres à partir des propriétés anatomo-physiologiques anthropoïdes. On l'attribue d'ordinaire à des mutations génétiques ou à une modification dramatique, externe, de l'environnement, en lui assignant des causes précises dans la structure de l'organisme ou du milieu. Dans l'interprétation qui prend corps ici, nous la verrons résulter d'un développement du potentiel prédateur et fabricateur d'outils des primates. Développement dû aux tensions provoquées dans leur organisation sociale par la présence de sous-groupes de mâles surnuméraires non-reproducteurs. La découverte de ces phénomènes a provoqué ces dernières années la floraison d'une littérature qui tend à abuser des analogies partielles entre les groupements animaux et les groupements humains, jusqu'à assimiler les processus sociaux aux processus biologiques. Les rapports entre hommes et femmes, entre générations et entre sociétés, le contrat social, les pratiques cynégétiques, la guerre ou le mariage sont décrits comme des effets de la sélection naturelle, qui passe pour être le principe explicatif de tout ce qui arrive là où il $\mathrm{y}$ a des êtres vivants. Le zoomorphisme remplace l'anthromorphisme comme cadre de pensée. Un examen attentif de ces phénomènes bouleverse au contraire notre vision du biologique et nous amène à accorder une importance plus grande au dynamisme social dans l'interaction avec le milieu, mettant notamment en lumière ce que l'hominisation présente de particulier. Le fait capital, j'y insisterai, n'est pas la spéciation des primates, l'homme descendu du singe, mais la séparation à l'intérieur d'une population vivant de la cueillette, l'éruption hors d'un groupe de collecteurs d'un groupe de prédateurschasseurs ayant son mode propre d'échange avec les forces matérielles. Les limites d'application de la sélection naturelle deviendront claires, la signification de ce qui lui est subrogé aussi.

Ensuite, pour ce qui est de la société humaine succédant à la société des primates, reprise et remodelée à l'intérieur de ce nouveau mode d'échange, je proposerai des éclaircissements basés sur la modification des rapports entre générations et entre sexes : l'individuation de la fonction paternelle, l'avènement de la famille en tant qu'unité constitutive de l'organisation sociale traduisent cette modification des formes d'association des adultes et des jeunes, des mâles en particulier. 
La prohibition de l'inceste règle la position respective des hommes et des femmes, devenus groupes distincts quant à leurs activités, leurs savoirs, leurs ressources. Elle n'a rien d'un interdit contrôlant le débordement des instincts, mettant fin à une promiscuité dont l'existence dans le monde animal est douteuse ; elle est, comme la pensée, l'outil, le cerveau, le langage, etc., une invention que l'homme a élaborée pour articuler la société dans laquelle il vit avec la nature qu'il se donne. L'individuation de la paternité, l'avènement de la famille, la prohibition de l'inceste sont les facettes d'un même passage des sociétés d'affiliation propres aux primates et aux hominiens à la société de parenté, la première que nous ayons conçue sur les débris de celles qui l’ont précédée.

L'analyse du jeu de l'état de nature et de l'état de société, le premier reconnu à son espèce, le second accordé aux autres espèces, ouvre une brèche dans leur concept. Cela n'a rien d'étonnant ; plus étonnant est le fait que l'on y ait prêté aussi peu d'attention et moins encore cherché à y porter remède. De même qu'une notion physique est remise sur le chantier à l'occasion d'un nouveau problème qui oblige à réordonner les connaissances - à cet égard, l'histoire de l'atome est exemplaire - de même le concept en question appelle une refonte analogue. Tout ce qu'il n'est possible de faire ici est de tenter de définir, de situer les deux ordres fondamentaux de réalité, d'en préciser la signification. J'anticipe : dans la délimitation de leurs domaines respectifs, ce que l'on a cru qui obéissait au rapport de négation et de complémentarité s'avère être le reflet partiel d'un rapport de réaffirmation et de réciprocité. A l'étage au-dessus, contemplant un horizon plus vaste, l'idée largement répandue, résumée dans le titre, apparaît renversée : la société n'est pas hors nature et contre nature, elle est dans la nature et par la nature. Et tout le livre s'emploie à démentir son titre, dialogue qu'un contenu engage avec la vision, la chaîne de catégories dont il est à la fois le prisonnier et le gardien.

Pour donner corps à ces conjectures, il me faudra recourir à des informations et à des théories appartenant à plusieurs branches de la science. J'en userai avec discrétion, ces informations et ces théories tirant leur solidité et leur valeur essentiellement de leur contexte d'origine. Hors de ce contexte, ce que j'avancerai et ce qu'on peut 
avancer a un caractère approximatif et spéculatif. Les renseignements relevant de l'évolution des primates, de la civilisation non occidentale, n'ont ainsi qu'une valeur potentielle ; les déductions que j'en tire ne prétendent rendre compte ni de l'évolution, ni de ces civilisations, mais simplement des parentés directes impliquées par les conjectures que je développe. Il ne saurait en être autrement lorsqu'on désire mettre à contribution plusieurs ordres de connaissance pour les faire coopérer à la solution de problèmes communs. Les spécialistes seraient mal venus de s'élever contre pareille tentative, puisque, les premiers, ils se sont aventurés au-delà du domaine de leur compétence, avec raison. On peut regretter qu'ils l'aient fait dans la précipitation, sans la prudence dont ils témoignent en présence de juges plus sévères, et ce pour recueillir l'approbation d'un public dont ils ont plutôt fortifié qu'ébranlé les préjugés. Des travaux révolutionnaires de paléontologie, d'anthropologie ou d'éthologie ont ainsi manqué de produire l'effet escompté.

Je veux espérer que, entre les opinions que je formulerai, un petit nombre pourra être considéré comme vrai et représentant un point de vue nouveau. Je présenterai, dans chaque cas, les raisons qui me font adopter une conception et m'éloignent des autres. Je ne pense pas que ces raisons soient les seules ou qu'elles soient définitives. Le scepticisme serait, après tout, une réaction saine, car il est normal de mettre à l'épreuve avec sérieux ce qui risque de ne pas avoir une consistance suffisante, et de réserver son jugement devant ce qui n’est qu'ébauché. Ce scepticisme serait toutefois regrettable s'il devait jaillir de la rencontre avec le non-familier, être motivé par la non-conformité de ce que je propose et de la réalité immédiate. Le seul reproche que l'on soit en droit de faire à une construction théorique, ce n'est pas de s'éloigner trop du réel mais de ne pas s'en éloigner assez; ce n’est pas d'abuser du pouvoir de l'imaginaire et du langage mais de ne pas en user pleinement. A mon grand regret, j'estime ne pas l'avoir fait, par respect pour les idées et les données empruntées aux divers champs du savoir que je sais être dans un état de transition. Cette situation n'encourage pas une combinatoire très poussée permettant de reculer aussi loin que possible les limites de nos conceptions habituelles et de surprendre les aspects les plus saisissants des phénomènes qui nous entourent. Ce sera un jour la tâche d'une science qui se proposera d'analyser l'altérité et non pas de justifier l'opposition de l'aspect so- 
cial à l'aspect naturel des groupes humains, qui, mettant en pleine lumière ce qu'elle a étouffé hâtivement sous l'appellation, ancillaire, de civilisation matérielle, rendra la place qui leur revient aux informations et aux processus par lesquels un groupe humain se constitue et constitue sa nature. Elle renoncera alors à se concentrer uniquement sur les origines de la religion, de la famille et de l'État. Donc ce sera la tâche d'une telle science d'être rigoureuse là où je ne suis que spéculatif, de pousser à fond le travail de création théorique, là où je m'efforce surtout de faire converger quelques vues sur l'évolution du comportement humain et ses relations avec le monde qui l'environne. 


\section{Première partie : Évolution et histoire}

Pour comprendre l'essence de la culture, il faudrait donc remonter vers sa source et contrarier son élan, renouer les fils rompus en cherchant leur extrémité libre dans d'autres familles animales et même végétale. Finalement, on doit considérer peut-être que l'articulation de la nature et de la culture ne revêt pas l'apparence intéressée d'un règne hiérarchiquement superposé à un autre qui lui serait irréductible, mais plutôt d'une reprise synthétique permise par l'émergence de certaines structures cérébrales qui relèvent elles-mêmes de la culture, de mécanismes déjà montés mais que la vie animale n’illustre que sous la forme disjointe et qu'elle alloue en ordre dispersé.

Claude LÉVI-STRAUSS, les Structures élémentaires de la parenté, $2^{\mathrm{e}}$ éd. Paris-La Haye, 1967, p. XVII. 


\section{Chapitre $\mathrm{I}^{\mathrm{er}}$. \\ Les premiers primates, promoteurs de l'histoire}

\section{Mutation, adaptation et évolution : rappel succinct de leurs mécanismes essentiels.}

\section{$\underline{\text { Retour à la Table des Matières }}$}

J'ai déjà eu l'occasion d'en avertir le lecteur : je me vois obligé, dans cette première partie, d'ouvrir une parenthèse, afin de poser un certain nombre de faits et de notions dont l'intérêt apparaîtra plus tard. J'estime que sans cela il serait impossible d'avancer, et que leur exposé constitue un solide point de départ à la réflexion sur les processus spécifiquement humains. J'ajoute que faits et notions ne sont pas l'expression exacte ni la reconstitution scrupuleuse d'une réalité qui aurait été, dans le passé, celle de notre espèce (note 1). Pour l'instant nous ne disposons cependant pas d'autres moyens ni d'autres informations nous permettant de peindre, avec plus de rigueur, de richesse et de verve un tableau qui doit demeurer incomplet à jamais.

Les organismes se sont développés dans le temps, ils ont changé et continuent à changer. La mutation et la sélection déterminent ce phénomène biologique universel, la première en assurant la transmission des caractères particuliers d'un être vivant, la seconde en infléchissant ses relations avec le milieu ambiant. Les mutations représentent, on le sait, des variations brusques et héréditaires de l'organisme au cours des générations successives. Pour les comprendre, il faut se rappeler que l'héritage biologique d'un individu est constitué par des gènes qu'il a reçus de ses parents. L'ensemble des gènes qui se trouvent dans les cellules sexuelles forme un "génotype ». 
Le gène est une quantité de matière susceptible de se séparer du chromosome qui le renferme pour être remplacée par une fraction correspondante, et par aucune autre, du chromosome analogue des cellules sexuelles. Les chromosomes de tous les organismes, des plus simples aux plus complexes, ont une composition similaire. Par ailleurs les divers gènes d'un même organisme et les gènes d'organismes différents comprennent des substances chimiques faisant partie de la même classe : les nucléo-protéines. Leur partie acide nucléique se compose essentiellement d'une substance très remarquable, l'acide désoxyribonucléique (ADN). Les qualités de celui-ci lui permettent de faire une copie exacte et détaillée de la structure moléculaire, à partir des matériaux offerts par la cellule et le milieu ambiant. L'autoreproduction, il ne faut pas l'oublier, est la fonction fondamentale d'un gène. Il a donc pour activité essentielle de confectionner une sorte de calque de lui-même dans l'intervalle séparant deux divisions de la cellule qui le porte. Sans cette opération, les cellules d'un organisme ne seraient pas munies d'un équipement génétique complet. La structure moléculaire de l'ADN expliquerait comment a lieu cette duplication. Les molécules de l'acide sont des doubles hélices de chaînes polynucléotides. Chaque nucléotide est formé d'un phosphate, d'un acide spécial du sucre (désoxyribose) et d'une base purine ou pyrimidine. Ces bases sont, pour les purines, l'adénine et la guanine, et pour les pyrimidines la cytosine et la thymine. Les deux chaînes de l'hélice sont tenues ensemble par des liaisons hydrogènes qui joignent les bases ; les études expérimentales ont montré que la base adénine d'une chaîne est associée à la thymine de l'autre chaîne, et que la guanine est associée à la cytosine. Les deux chaînes se complètent donc parfaitement. Lorsque la double hélice se sépare en deux fils isolés, chacun est capable de reconstituer une copie identique de la structure double initiale, en appariant les quatre bases de manière appropriée. A l'aide de ces quatre bases, on spécifie, par permutation, les différences existant entre d'innombrables gènes, de même que les vingt-six lettres de l'alphabet, en se combinant, permettent de constituer une variété immense de mots, de phrases et des textes. Le code génétique, à l'instar du code linguistique, est en mesure de fabriquer un grand nombre de " textes ». Il ne produit pas, dans tous les cas, une succession de bases ayant pour résultat un gène fonctionnel, pas plus que le code linguistique ne produit uniquement des mots ou des phrases ayant un sens. 
Concrètement, quand même la plupart des permutations théoriquement concevables seraient extravagantes, il n'en reste pas moins qu'une infinité de structures de gènes deviennent effectives, si le gène est une section de l'hélice comprenant des milliers de nucléotides.

On voit comment les molécules d'acide désoxyribonucléique fonctionnent pour communiquer, d'un organisme à l'autre, le matériel héréditaire. Le processus exposé est conservateur, il assure le succès de l'hérédité, qui est principalement une autoreproduction des gènes. L’évolution serait impossible si le processus n'était contrecarré de temps en temps par un raté : le gène produit une copie imparfaite. La mutation correspond à un défaut de fonctionnement. Elle aurait pour cause, suivant l'hypothèse de la double hélice, des substitutions, des suppressions ou des réarrangements portant sur une ou plusieurs paires de nucléoprotides composant les chaînes d'ADN des chromosomes $\mathrm{A}$ côté de ces mutations dues à une erreur de décodage, on rencontre des mutations structurelles consécutives à la multiplication, à l'élimination et à la réorganisation de séries de chromosomes, de parties de chromosomes ou de chromosomes entiers. Une cellule sexuelle humaine contiendrait plusieurs dizaines de milliers de gènes. Ceux-ci mutent de nombreuses façons, mais même s'ils ne mutaient que d'une seule façon, on voit que des dizaines de milliers de mutations seraient possibles. Les mutations sont des événements rares, car les gènes s'autoreproduisent exactement. Toutefois, étant donné le nombre de ceux-ci, il y a constamment en présence des gènes mutants qui n'existaient pas dans la souche qui les a produits et qui finalement se manifestent par une mutation. Celle-ci est souvent délétère et se traduit par des malformations ou maladies congénitales, mais ce n'est pas toujours le cas. Les mutations représentent la source dernière des transformations qui préparent les étapes de l'évolution organique, du fait qu'elles créent des alternatives à partir desquelles s'opèrent les choix décisifs qui assurent la survie d'une espèce.

Le mécanisme d'autoreproduction - la mutation notamment est, on le remarque, un mécanisme aléatoire ou non dirigé. La raison de cet état de choses est claire. Les copies et les erreurs de copiage obéissent à des principes inhérents. Les mutations ont lieu en fonction d'une structure matérielle donnée, indépendamment de leur utilité pour l'organisme, dans les circonstances où il se trouve. Lorsque, dans 
une population, la petite stature est un avantage biologique, les mutations inhibant la croissance de la taille ne seront ni plus ni moins fréquentes que dans une population qui aurait intérêt à avoir une grande stature. De même, si une population émigre dans une zone plus chaude que celle où vivaient ses ancêtres, les mutations conférant une résistance au froid ne seront pas moins fréquentes qu'auparavant. Le caractère aléatoire du mécanisme de transmission signifie, somme toute, que ses résultats ne sont pas déterminés par une fin qui leur est extérieure ; il ne veut pas dire que tous les résultats possibles sont également, probables. Les modifications chimiques qui ont leur siège dans un gène dépendent de la composition établie du génotype. C'est leur impact sur la faculté de l'organisme à s’adapter qui est aléatoire. Dans ce sens, on peut dire que l'hérédité crée une population d'individus éventuels entre lesquels elle ne fait pas de tri préalable ou définitif.

La sélection naturelle introduit une direction dans les changements organiques. Les êtres vivants ont une tendance à l'expansion. Ils procréent plus de descendants qu'il n'en survit pour procréer à leur tour la génération suivante. Ils se propagent et colonisent les territoires et les milieux disponibles. Leur réussite est fonction de leur rapport au milieu ambiant. Parmi les individus qui naissent dans une population particulière, seule une fraction possède les attributs exigés pour pouvoir se nourrir, exercer l'activité qui est indispensable à la défense contre l'agression, à la protection des congénères, etc. Une population n’a pas d'emblée toutes ces qualités. Les facteurs du milieu ambiant, et ils ne sont pas les seuls, exercent une pression sur elle qui aboutit à un changement de la composition génétique. Les individus survivants sont ceux qui possèdent un arrangement de gènes porteurs de propriétés conformes aux exigences. Ce changement peut entraîner des mutations ou des combinaisons de gènes ayant des conséquences favorables au sein de la population envisagée. Il est bien entendu que les gènes ne sont pas détruits ni ne disparaissent complètement : seule leur répartition est affectée. A cet égard il y a sélection. La constitution réelle d'un groupe, d'une espèce, le sens dans lequel ils se modifient et ce qu'ils conservent, reflètent, tant du point de vue du nombre que des caractères, leur adaptabilité. Celle-ci s’améliore de génération en génération, le groupe ou l'espèce arrivant peu à peu à réduire les écarts avec le milieu ambiant et à y vivre dans de meilleurs condi- 
tions. Les individus qui sont trop aberrants ou qui ne possèdent pas l'équipement nécessaire sont éliminés et leurs chances de se multiplier diminuent en même temps. Le degré d'adaptabilité s'exprime par la correspondance entre les impératifs de l'existence dans le milieu défini, la niche écologique, et l'organisation génétique à laquelle est parvenue la population considérée. A cela s'ajoute une dimension temporelle, car il faut qu'il subsiste un certain rapport entre le rythme des transformations de la population et la transformation inhérente au milieu ambiant. Le déséquilibre entre les deux séries de transformations entraîne la disparition, le remodelage ou la division de l'espèce. A moins que les individus en surplus ne quittent l'habitat ancien pour aller en coloniser un nouveau. Ce mouvement dit de préadaptation suppose que le modèle de comportement, créé à cette occasion, assume une nouvelle fonction dans le nouveau contexte, sans interférer avec la fonction originelle ni faire obstacle à son développement.

La sélection naturelle exprime donc la relation entre le dynamisme interne de la structure génétique et celui des forces matérielles, le rapport entre les propriétés génétiques et le milieu ambiant. C'est à celuici que revient, en dernière analyse, le rôle déterminant, en raison de ses changements incessants et de sa diversité ; il contraint les êtres vivants à " accepter » ou à "refuser » les mutations, à se regrouper dans des entourages différents, et, finalement à se filtrer afin de pouvoir " croître et se multiplier ». C'est lui aussi qui les oblige à diverger, se diversifier, former des espèces, sans qu'il faille toutefois surestimer son importance. Supposons une source donnée de mutation dans une espèce qui s'étend depuis la forêt jusqu'à la savane. Le mécanisme qui rend l'adaptation possible produira une fréquence de certains gènes chez les individus vivant dans la forêt différente de leur fréquence d'apparition chez les individus vivant dans la savane; il y aura cependant des types intermédiaires et l'écoulement des gènes se fera régulièrement d'une extrémité à l'autre. Maintenant, il suffit d'un événement géologique ou climatique pour dresser une barrière qui coupe une fraction de l'espèce du reste. Les mutations, faute de circulation des gènes, donc de croisements, produiront des écarts par rapport au type commun aux deux régions, écarts qui seront d'autant plus marqués que le milieu lui-même aura changé. Le taux de modification dans une petite population sera mathématiquement plus élevé que dans une grande. Au cas où la barrière disparaît et si l'écart n'est pas 
trop accentué, on assistera à une réunification de l'espèce, accompagnée d'une flexibilité accrue d'adaptation au milieu ambiant, due à la diversification des gènes. Il arrive aussi que les deux groupes, exploitant mieux leurs ressources respectives, même s’ils peuvent reprendre des relations, aient avantage à se spécialiser chacun dans sa direction propre, augmentant ainsi leur isolement reproductif. Ceci bien entendu à condition que les deux fractions de l'espèce n'aient pas atteint le point de non-retour ; dans ce dernier cas en effet, elles ne sont plus aptes à s'accoupler entre elles, celle qui est la mieux adaptée survit seule et l'autre disparaît : Transposé à l'ensemble du monde organique, ce schéma de déroulement des événements nous montre que ce qui a été, pour l'évolution, une seule unité, se scinde, laisse la place à de nombreuses unités devenues indépendantes. La diversité biologique est accrue, l'univers se peuple d'organismes disparates. Par ailleurs, une espèce prise seule, pour se modeler sur le milieu ambiant, se reforme progressivement sous l'angle génétique. Elle garde, à nos yeux, son unité évolutive. Pourtant les transformations qui ont lieu sont telles que les populations sont classées en tant qu'espèces ou genres différents, apparus au cours du temps.

La sélection a ces trois conséquences : elle optimise la structure des populations, améliore leurs rapports au milieu ambiant, et les diversifie dans l'espace et dans le temps. Elle travaille par ajustements comme l'hérédité travaille par décalque. Les disparités du milieu ont des conséquences analogues aux erreurs de copiage des processus physicochimiques. La sélection n'a pas trait à l'individu mais à la population dans son ensemble. En effet, ce qui évolue ce n’est pas l'individu - il n’y a pas hérédité des caractères phénotypiques, les gènes mutants sont répartis sur un grand nombre d'individus - mais le groupe au sein duquel se produisent les mutations adaptatives et les croisements qui les stabilisent. Dans une population, la sélection conditionne le seul processus génétique qui, d'après ce que l'on sait, ne soit pas aléatoire : la reproduction. L'augmentation de fréquence de facteurs génétiques propres à la survie d'une génération à l'autre est une fonction directe de la reproduction différentielle, de la corrélation entre les facteurs génétiques chez les parents et leur capacité plus grande à laisser des descendants. L'évolution sera orientée vers les traits les plus adaptatifs, les plus reproductibles. Pendant longtemps on a embrassé une conception " élitaire » : le vainqueur dans la lutte 
pour la vie est l'individu qui survit. Suivant la conception « démocratique » qui prévaut actuellement, la sélection naturelle ne se rapporte pas à la survivance des membres « les plus forts » du groupe, mais à la capacité d'avoir des descendants. Le vainqueur est celui qui subsiste à travers l'abondance de sa progéniture, non pas celui qui vit mais celui qui se reproduit.

$\underline{\text { Retour à la Table des Matières }}$ 


\section{La société adaptative.}

\section{$\underline{\text { Retour à la Table des Matières }}$}

Les êtres cherchent à se conserver ; ils débouchent sur le changement. La double inconstance du monde qu'ils enferment et de celui qui les renferme provoque des événements rares et décisifs. Ce qui leur paraît important et qu'ils poursuivent sans discontinuer n'est pas essentiel ; ce qui est essentiel, opère à leur insu parce que mal repérable ou peu important. L'évolution banalise, apure les contretemps, elle efface les dispositions stables et régulières. Les espèces s'autoproduisent comme matière vivante et se reproduisent comme organismes afin de se garantir contre les aléas qu'elles finissent par produire. Ainsi c'est la vie qui juge la vie et non pas la mort. Reliant la routine nécessaire et les résultats arbitraires des phénomènes, la sélection naturelle tire le meilleur parti des dispositions d'une population, d'un groupement d'individus dans la parcelle d'univers qui est la leur. Elle commande un système d'échanges de l'information génétique susceptible de convenir à un certain agencement du milieu. Mais de cette manière on l'envisage uniquement en ce qu'elle met en rapport une structure déterminée des êtres vivants, la population génétique, et un ordre défini des forces matérielles avec leur distribution dans l'espace et dans le temps. Ce qui est insuffisant. Les individus d'une espèce ont, certes, un fonds héréditaire commun. Ils ne sont pourtant pas identiques ni plongés dans un agrégat informe. La collectivité qui les comprend est toujours plus ou moins réglée. C’est à la fois une combinaison variée d'éléments discrets et une organisation de relations. La reproduction est leur plus grand dénominateur commun. Celle-ci, on l'observe fréquemment, n'est pas une activité sans frein et le travail adaptatif des populations, pour survivre, a trait à leur nombre autant qu'à leurs qualités individuelles. Le volume et l'étendue d'une espèce dépend du volume et de l'étendue de ses ressources. Elle ne saurait se multiplier au-delà de certaines limites sans mettre en danger l'existence de chaque individu et le fonctionnement de l'ensemble. Savoir qui survit et combien d'êtres survivront, ajuster le modèle biologique et « calculer » sa multiplication, ces opérations sont liées. La reproduction différentielle qui favorise un génotype au détriment de l'autre double d'une reproduction discriminative qui contrôle, dans le 
génotype dominant, la procréation des individus. - Elle agit soit en exerçant une influence sur le nombre de jeunes que chaque couple de parents peut procréer, soit en instituant une proportion entre le nombre d'adultes qui entrent dans le cycle reproducteur et ceux qui en sont exclus. Le malthusianisme serait une nécessité permanente dans les populations animales insérées dans un milieu donné et conditionnées par une structure génétique. Suivant une hypothèse séduisante ${ }^{16}$ et conformément à des observations répétées, le long de l'échelle des êtres vivants, toute une série de comportements sociaux — parade, agression, déploiement de caractères secondaires attirants ou répulsifs - semble être consacrée à la régulation du nombre d'individus qui s'accouplent, naissent ou meurent. La limitation rituelle ou saisonnière du commerce sexuel, les territoires dans lesquels les individus interagissent, la hiérarchie qui ouvre à une partie du groupe l'accès aux ressources et aux partenaires sexuels, le type de compétition qui accompagne un degré de densité optimale, sont des moyens employés dans tout le règne animal pour obtenir un taux défini de fécondité, distinguer les individus indispensables des individus surnuméraires, ou pour lutter contre la perte de ressources due à une mortalité excessive. Quel que soit le groupe, les animaux n'interagissent pas au hasard, ni sur le plan de la sexualité, ni sur le plan de la nourriture ou des soins qu'ils reçoivent de leurs congénères. Le réseau des liens collectifs affecte par conséquent la répartition du matériel génétique. L'utilité d'un comportement pour la communauté prévaut sur l'utilité de ce comportement pour un de ses membres. Le bon fonctionnement de l'ensemble en dépend et permet à la survie de tous de prendre le pas sur la survie de chacun. On donne, à ce propos, l'exemple frappant du comportement territorial des oiseaux mâles, qui les rend plus visibles et plus exposés à la prédation. Il a pour effet un partage des territoires d'accouplement qui garantit une nourriture plus abondante aux nouveau-nés. Conjointement, en attirant, par leurs atours, les prédateurs, les mâles les écartent des femelles et des jeunes oiseaux.

Les formations sociales de la plupart des espèces animales interfèrent avec les mécanismes sélectifs, l'adaptation concerne les individus par le truchement de la société (note 2). Paradoxalement, la révolution

16 V.C. WYNE-EDWARdS : Animal Dispersion in relation to Social Behaviour, New York, 1962. 
mendeléenne qui a amené la biologie à penser en termes de populations et non pas en termes d'individus, a simultanément donné un relief extraordinaire aux dynamismes physico-chimiques internucléaires et aux dynamismes collectifs trans-individuels. Quant aux individus eux-mêmes, ils apparaissent de plus en plus comme le produit et non pas comme la donnée dont tout dérive et dans laquelle s'opèrent les transformations significatives. L'étude des sociétés animales, de leur fonction et de leur fonctionnement, est encore dans l'enfance. Nous sommes plus riches en conjectures qu'en expériences ou observations destinées à les vérifier. En voici encore une rendue possible autant par notre ignorance que par notre connaissance. Il serait préférable que les recherches ultérieures, loin de la confirmer, la démentissent. Pour l'instant nous pouvons supputer que le poids relatif des facteurs qui infléchissent le comportement d'une espèce, sa reproduction - le mécanisme directionnel par excellence - varie avec sa situation du point de vue évolutif. Dans l'ensemble, chez les animaux, les composantes biogénétiques dominent les composantes biosociales ; le milieu étant décisif, un surplus d'individus ou une déviation des caractères adaptatifs a pour conséquence la destruction ou la spéciation. Ce qui est avantageux ou désavantageux, génétiquement, se traduit immédiatement par une pression sur le réseau des relations qui enserrent les individus. Une espèce biologique est capable d'instituer une organisation sociale et une seule. Du reste aucune autre ne lui est indispensable.

Dans l'évolution, le substrat génétique est une cause nécessaire, le milieu ambiant une cause suffisante, et la société une cause occasionnelle. Les simiens ébrèchent cet ordre. Les éthologues l'ont relevé lorsqu'ils ont renoncé à observer des animaux captifs dans les jardins zoologiques, en les traitant comme du matériel comparatif, porteur de fonctions humaines diminuées, pour commencer à entrer en contact avec eux dans leur habitat d'origine, là où ils mènent une existence pleine, méritant d'être considérée en elle-même. La progression des connaissances étant toujours proportionnelle au respect de l'autre, fûtce un animal ou un caillou, et au détachement vis-à-vis de soi, fût-on un homme civilisé, on devait s'attendre que les résultats obtenus soient d'un intérêt extrême. Jugeons-en immédiatement. Les collectivités de primates — je parle ici uniquement de simiens — ont pour 
unité constitutive le groupe basal se composant, en général, de femelles, de jeunes des deux sexes et d'au moins un mâle adulte. L'activité sociale et procréatrice a lieu dans son sein, et il témoigne d'une stabilité et d'une cohérence relatives. A côté, suivant les circonstances, on rencontre des groupes appendiculaires, dont le rôle sera bientôt défini, comprenant uniquement des mâles. Les sociétés sont une combinatoire de ces groupes. Je ne décrirai que deux types purs. Le premier type de société est la société de clique. Kummer et Kurt ont donné à leur groupe le nom de "groupe à un seul mâle ». La dénomination n'est pas heureuse, parce qu'elle se réfère au caractère sexuel des individus et non pas à leur fonction, et aussi parce que souvent le mâle dominant est accompagné d'un ayant-droit qui lui succède et le remplace dans ses tâches reproductives. Cependant, effectivement, dans ces sociétés, l'unité constitutive est formée d'un mâle adulte entouré d'un "harem » de femelles avec leurs nourrissons et de jeunes. Ces unités sont associées par des liens extérieurs plutôt lâches. Chez les geladas, elles s'agrègent temporairement afin d'exploiter un territoire riche en nourriture. Chez les hamadryas, grands singes cynocéphales, remarquables par leur crinière, animaux sacrés de l'Égypte antique, les divers groupes se réunissent très régulièrement et leurs relations sont ordonnées avec assez de rigueur. Ni chez les geladas ni chez les hamadryas les territoires ne sont strictement partagés. En marge, il y a fréquemment des groupes appendiculaires, monosexuels, formés surtout de mâles sub-adultes. A l'intérieur du groupe basal, la régulation des comportements est très stricte. Le mâle dominant, maître de céans, contrôle l'étendue des déplacements de ses femelles et réprime les conduites attentatoires à son autorité et à l'intégrité du groupe, quel que soit celui qui les manifeste, jeune ou adulte. Les jeunes singes, en le rencontrant, font les chattemites, pour détourner son agressivité, entrer dans ses bonnes grâces. Pourtant ce mâle n'agit pas en chef, tant il est vrai que les femelles, qui sont seules à prendre soin de la progéniture, s'arrangent entre elles dans le cours ordinaire de la vie. Son rôle est celui d'un gardien et d'un tuteur, ayant pour mission de veiller au bon fonctionnement de la petite collectivité à sa charge. L'expérience acquise, la force, le respect qu'il commande, sont indispensables en cas de menace ou de migration. Le dosage de punitions et de cajoleries auquel il procède renforce les liens d'inclusion et d'exclusion qui l'unissent aux autres membres de la collectivité. Aux yeux de l'observateur, le groupe basal apparaît fermé sur lui-même 
(dans ce sens il est une clique), avec un haut degré de solidarité ; et il s'articule avec d'autres groupes, en tant que tel.

Le second type de société, la société de compagnonnage, comprend des animaux qui partagent occasionnellement ou habituellement des activités communes. Elle présente le spectacle d'une congrégation de plusieurs mâles adultes, de femelles, plus nombreuses, avec leurs jeunes, et aussi d'adolescents. Les barrières qui séparent les différents groupes constitutifs sont plus lâches ; on constate une circulation des mâles d'un groupe à l'autre. Mais l'ensemble, vivant à la lisière de la forêt, sur un terrain boisé ouvert ou dans la savane, se déplace à des distances relativement grandes, suivant un ordre assez bien défini. Quelques espèces défendent leur territoire, néanmoins les chevauchements sont tolérés. Les disputes ont lieu dans les zones frontières : habituellement groupes ou bandes s'évitent et coexistent en s'ignorant. A l'intérieur de chaque société, on relève la présence de sous-systèmes sociaux, avec regroupement temporaire des classes d'âge ou de sexe dans les sites de couchage ou de nourriture, par exemple. Les sous-systèmes ne sont pas clos et les individus passent de l'un à l'autre lorsque les circonstances l'exigent ou le permettent. L'étude des macaques et des babouins de la savane ou des terrains boisés ouverts en Afrique et en Asie a montré une grande régularité en ce qui concerne leurs occupations journalières et l'interdépendance qui règne parmi eux. Ainsi les individus ne vont pas boire les uns sans les autres et se déplacent toujours ensemble. La crainte des prédateurs semble être la raison essentielle de l'impossibilité de s’isoler ou de chercher sa nourriture loin du groupe. La cohésion sociale est maintenue aussi par une hiérarchie complexe entre les mâles qui détermine leur accès aux ressources et à la vie sexuelle. Les individus dominants ont à tous égards la priorité. Les femelles des babouins et des macaques rhésus sont servies par des animaux subordonnés, généralement jeunes, au début de l'œstrus. Toutefois, au point culminant, elles s'accouplent avec les animaux occupant une place élevée dans la hiérarchie. La promiscuité porte donc une marque sociale. Le groupe des mâles de rang supérieur commande à l'ensemble de la société, dirige ses activités et définit la place qui revient à chacun de ses membres. 


\section{L'ancien et le nouvel art de survivre.}

\section{Retour à la Table des Matières}

Les sociétés de clique et les sociétés de compagnonnage sont des types extrêmes entre lesquels on trouve des variantes intermédiaires (note 3). Le fait important n'est cependant pas l'existence de cette diversité, mais son caractère intra-spécifique: une même espèce connaît plusieurs formes d'organisation sociale. Les recherches faites sur les macaques et les babouins, les plus extensives, l'ont amplement prouvé. Ceci soulève trois questions : Par quoi est déterminée la vie collective des primates? Quelles sont les causes de la variation des rapports sociaux dans un groupe ou entre les groupes ? Que signifient, du point de vue évolutif, les différences sociales ? La plupart des théoriciens ont supposé que l'instinct sexuel était responsable de la tendance des individus (à vrai dire, primates ou non) à demeurer dans le groupe, à être attirés les uns par les autres. Leurs conclusions étaient fondées sur l'étude du comportement d'animaux prisonniers, arrachés à leur habitat, dispensés de pourvoir à leur subsistance. Des partis pris extérieurs ont induit, de surcroît, une sélection particulière, mettant l'accent sur le commerce sexuel, en vue de la procréation. Les observations d'animaux à l'état sauvage ont situé les comportements sexuels et agressifs dans un entourage plus large et leur ont attribué une position plus subordonnée dans la constellation globale des comportements. Au-delà de ce poids relatif, le schéma conceptuel luimême est en cause. En effet, si la satisfaction de instincts, notamment des instincts sexuels, explique la sociabilité des animaux, il faut en tirer trois conclusions. La relation au milieu ambiant est secondaire, dépendant exclusivement de la structure génétique; la capacité de procréer, de répondre aux exigences pulsionnelles, conditionne le contenu des relations sociales ; enfin, la reproduction est un acte et non pas un processus, c'est-à-dire qu'elle médie les rapports des individus dans le groupe mais ne médie pas l'adaptation du groupe à son milieu normal. Bref, c'est la société qui est sexuelle, et non pas la sexualité qui est sociale.

On pouvait s'en tenir là, tant que l'on prenait l'individu pour unité d'analyse et que l'on envisageait la société animale hors de 
l'évolution, en se désintéressant des mécanismes généraux qui règlent le volume d'une population (la séparation des individus reproducteurs et non-reproducteurs étant un accident du point de vue instinctuel), et tant que l'on ne connaissait pas l'existence d'une différence sociale intra-spécifique. La remise en question s'est imposée sous la pression des observations et devant la nécessité d'insérer la fonction sociale dans l'évolution. A cet égard l'évidence empirique n'est pas sans défauts. Elle nous laisse cependant voir une correspondance entre les sociétés de primates et le milieu où ils vivent. Dans un travail théorique qui appelle de sérieuses retouches, mais qui n'en est pas moins en tous points remarquable, par sa démarche, John Crook et Stephen Gartlan ont essayé de ranger les systèmes sociaux en cinq classes, le système de clique et le système de compagnonnage étant aux deux extrémités. Ils ont dressé un tableau de corrélations entre la taille du groupe, le type d'unité constitutive, l'organisation des activités diurnes, les préférences alimentaires, la dispersion de la population et l'habitat avec ses propriétés biotiques. Les cinq classes sont rattachées respectivement à la forêt, à la lisière de la forêt, à la savane et aux divers terrains arides. La similitude de système social chez des primates appartenant à plusieurs espèces mais vivant dans des milieux similaires, le parallélisme de ces systèmes et des milieux, témoignent d'un phénomène d'adaptation. La cause de l'hétérogénéité sociale ne réside pas dans l'équipement génétique ou instinctuel de l'animal mais dans le rapport population-milieu ambiant. Comme partout où s'exerce la pression sélective, la survie est associée à la reproduction du groupe, et non à la procréation des individus. Sur ce fondement, il a été possible de formuler quelques hypothèses, encore fragiles - comment en serait-il autrement dans un domaine nouveau ? - afin de rendre compte de l'adéquation d'une organisation sociale particulière à un environnement. Les sociétés de clique se trouvent surtout dans les régions arides, où les risques de prédation sont moindres et la disponibilité en nourriture relativement réduite. La densité de la population est faible, comparée à celle des régions plus riches. Ce fait qui ne surprend guère a été confirmé par plusieurs observateurs. Un tel milieu décourage la formation de congrégations de grande taille; les animaux vivant dans des collectivités trop nombreuses par rapport aux possibilités de s'alimenter réussissent moins bien à se reproduire. Les groupes constituant ce type de société sont effectivement plus petits. On peut se demander pourquoi ces groupes ne comportent qu'un seul 
mâle. Un mâle peut féconder plusieurs femelles. Dans le cas où la population connaît une disette chronique, il devient avantageux de réduire le nombre d'individus masculins reproducteurs, d'atténuer la concurrence pour les ressources alimentaires rares. Au cours du temps, les sociétés qui ont pu survivre sont celles qui ont réalisé ce schéma, optimisant la taille du groupe et la proportion numérique de mâles et de femelles. La structure sociale préserve ces attributs en éliminant, en rendant non-reproducteurs les jeunes mâles qui se réunissent dans les groupes monosexuels appendiculaires. Cette conjecture n’a pas été démentie par les données empiriques. La proportion d'individus de chaque sexe chez les primates hamadryas, geladas, patas, vivant dans des zones arides, montre la disparité de nombre que l'on attendait entre les mâles et les femelles. En revanche, les sociétés de compagnonnage se répandent dans les savanes, à la lisière des forêts, où les variations climatiques sont moins sévères et les ressources plus abondantes. Les mâles de grande taille et dominateurs peuvent y subsister avec les femelles plus petites et leur progéniture. Les communautés plus nombreuses peuvent atteindre plusieurs centaines d'individus. Elles se déplacent dans un espace plus vaste sans que la rivalité entre leurs membres, leurs unités constitutives, dépasse un seuil au-delà duquel la collectivité souffrirait. La discrimination entre mâles reproducteurs et non-reproducteurs est moindre, et il n'est pas rare qu'un individu passe d'un groupe à l'autre. Les risques de prédation étant assez grands, la taille du groupe et la coordination obligatoire entre ses membres constituent une défense efficace. Selon les divers témoignages, les animaux sont plus adroits, ont une faculté de communication vocale et faciale plus complexe que leurs congénères des régions arides et pauvres.

Ces explications ont beaucoup de force et entraînent notre adhésion par leur familiarité. Malheureusement elles se bornent à sérier des corrélations ayant trait au phénomène de variabilité sociale, sans fournir un corps d'hypothèses où causes et effets soient rigoureusement définis. Cette carence nous empêche d'affirmer que nous tenons là une réponse aux problèmes soulevés. Nous n'en sommes pas moins sûrs que l'organisation sociale des primates non humains agit au niveau des génotypes. Chaque individu a son rôle, un programme d'interaction qui l'accompagne, un certain champ d'expériences auquel il est censé faire face et un espace qui lui est alloué. Sa capacité 
d'accomplir ce qui est requis de lui décide de ses chances de se reproduire ou non, donc de laisser une descendance. Ce qui est vrai au niveau de l'individu l'est aussi au niveau du groupement. La conformité aux exigences sociales - et par là écologiques - conditionne le degré d'adaptation et la perpétuation des propriétés anatomophysiologiques appropriées. Dans la mesure où leur réussite est assurée, les différentes sociétés cherchent à se maintenir, à stabiliser les relations entre leurs membres, à préserver les comportements qui les renforcent ainsi que la fréquence et le type d'interaction sociale : Les modèles et les normes appris sont légués d'une génération à l'autre, imités des compagnons et imposés par les adultes aux jeunes. De la socialisation dépend la possibilité du groupement de se renouveler et celle des individus de se procurer les ressources qui leur sont indispensables en occupant une certaine place dans la hiérarchie, de se développer ou de rester confinés dans une position subalterne. Paradoxalement, les nécessités de la vie en commun ont eu pour effet de forger des individualités, de rendre les animaux dissemblables, du point de vue phénotypique; elles n'ont pas conduit à l'uniformité, à l'effacement de la personnalité. Le sexe, l'âge, le statut fixent des limites très larges : le destin de chacun n'en est pas pour autant prédéterminé. Quand on examine par le détail la vie d'une communauté de primates (les comptes rendus minutieux des éthologues sont révélateurs) on remarque une grande diversité de moyens, de comportements, qui permettent d'arriver aux mêmes fins. Les changements écologiques ont, certes, imprimé à la plupart des espèces coexistant dans un milieu semblable des schémas d'organisation, d'interaction voisins. Les écarts qui conditionnent les degrés de liberté des individus et des groupes nous font supposer que la dynamique sociale sousjacente diffère. Le poids de celle-ci doit être très grand. A telle enseigne que l'on peut se demander si le milieu est seul à imprimer sa marque, si les types sociaux décrits se sont toujours formés par réaction aux facteurs externes. On est en droit d'imaginer que, parfois, l'inertie du système oblige les animaux à chercher le milieu qui contribue à leur conservation, à éviter les conséquences délétères qui résultent d'une modification de celui-ci. Le jeu des adaptations du système social au milieu ambiant et du milieu ambiant au système social justifie l'apparition de solutions de compromis, de structures collectives intermédiaires, courantes parmi les primates. La correspondance des deux termes n'est pas en cause : leur corrélation, Ronald Hall l'a sou- 
ligné, n'est pas très grande. Les macaques rhésus et les babouins vivent aussi bien dans les arbres des forêts galeries qu'en terrain découvert dans la savane. Leurs groupements très organisés, agressifs, leur versatilité sont ce que l'on attend d'une espèce bien adaptée à son milieu. En revanche, l'entelle, espèce plus paisible, subsiste dans un milieu analogue et aussi varié. Les chimpanzés dominent indiscutablement leur habitat, dans la forêt, avec une efficacité comparable à celle des babouins et des macaques rhésus. Cependant leurs sociétés de compagnonnage témoignent d'un faible degré d'intégration des individus, d'une grande labilité et d'une relative indifférence au contrôle du territoire où elles se déplacent. L'écologie détermine ainsi la forme globale d'une collectivité, elle ne module cependant pas entièrement les normes et les comportements particuliers. De là, déjà chez les primates, une certaine autonomie de la fonction sociale et des structures qu'elle revêt. Leurs rapports naturels s'en ressentent qui soumettent le substrat génétique à l'emprise du monde social, intervenant à côté du monde physique ou interférant avec lui. Les changements dans l'un comme dans l'autre se répercutent de concert sur le plan des propriétés adaptatives de l'espèce et sur le plan des ressources matérielles nécessaires à la survie. Les limites, quant à ce dernier, sont étroites, puisque les activités et les moyens des primates supérieurs non humains sont grossièrement identiques. Relativement à la plupart des espèces animales, elles sont suffisamment larges — et la flexibilité de l'organisation sociale s'y ajoute - pour réduire la tendance à la spéciation, c'est-à-dire à l'isolement reproducteur des populations et à la circulation de 1 ' «information » génétique. La gamme des combinaisons de traits biologiques possibles, les chances de survie sont accrues, les espèces se propageant dans des milieux qui, aux yeux de l'observateur, sont dissemblables. En réalité l'écologie des primates a une dimension dynamique, une complexité qui les mettent en contact avec des espèces et des conditions de vie offrant une grande variété. Ils exploitent donc plus largement leur potentiel biogénétique et biosocial. Les babouins, les macaques rhésus, les chimpanzés, nous l'avons constaté à plusieurs reprises, utilisent diverses niches écologiques, en forêt et en terrain découvert, au sol, sur les arbres et les buissons. Les remaniements de la taille, de la densité, des comportements et des interactions de la population à l'intérieur du groupe, de la répartition des individus reproducteurs et non-reproducteurs, se substituent à la spéciation avec le succès que l'on connaît. Ils ont dû faciliter la 
préadaptation, l'insertion des individus surnuméraires dans un milieu nouveau, modifiant plutôt leur mode d'interaction que leur génotype qui a pu ainsi se conserver ou varier dans de faibles limites. Dans ce sens, la sélection naturelle a atteint ses limites. Chez les primates, la pression directe du facteur écologique sur le facteur génétique, la dépendance où les relations existant dans une population se trouvent par rapport au milieu, facteurs propres à l'évolution, cessent d'opérer seules, d'avoir une valeur adaptative. La dynamogénie largement autonome de la reproduction sociale, des populations ordonnées et spécifiées par des rôles, des hiérarchies et des subdivisions en classes, impose elle aussi ses exigences quant aux facultés et à l'art de vivre de l'espèce. Par un renversement de priorités, les traditions et les liens qui prédominent à l'intérieur du groupement animal quant à la procréation, à la nourriture, à la délimitation des déplacements et au genre d'activités réservées à chacun, viennent à imprimer partiellement leur marque sur la quantité et la disparité des échanges avec le monde matériel. Le processus historique se greffe de la sorte sur le processus évolutif. Les primates contemporains nous permettent d'entrevoir ce qu'avaient dû être ses modestes débuts. Mais guère davantage. 


\section{Chapitre II.}

\section{Des sociétés sans paroles}

\section{Les hiérarchies bien tempérées.}

\section{$\underline{\text { Retour à la Table des Matières }}$}

Les primates marquent dans le développement des relations avec le monde matériel un tournant que les hommes ont prolongé et définitivement affirmé. Ayant fixé dans le chapitre précédent les conditions préliminaires, je reviendrai sur les circonstances qui ont amené ce passage. Je m'arrête pourtant encore sur l'organisation sociale des singes cynocéphales et anthropoïdes pour mieux tracer les contours de la réalité qui lui a probablement correspondu il y a plusieurs millions d'années. Les descriptions que l'on donne de cette organisation sont saisissantes. Les interactions, les comportements utilitaires et symboliques ont un air de famille avec les comportements et les interactions observés dans certaines sociétés humaines. La tentation est très forte de dire qu'il ne manque aux acteurs que la parole. Afin de tirer tout le fruit de cette similitude, il est souhaitable de combattre le penchant répandu à envisager ces organisations comme des ébauches primitives d'une structure plus achevée, la nôtre. Une telle attitude nous amènerait à négliger l'originalité de leurs mécanismes, à minorer le fait que nous avons affaire à des ordres sociaux tardifs, établis au cours d'un long processus évolutif. Leur particularité ne réside pas dans l'identité biologique de leurs membres, primates non humains, mais il semble que les premiers hominiens aient partagé un mode de vie analogue. De plus, nous ne sommes pas très avancés, théoriquement, lorsque nous qualifions une société d'humaine ou d'animale, tant sont grandes les disparités des institutions ou des configurations sociales que les hommes ou les animaux connaissent ou ont connues. Dans un souci de 
clarté conceptuelle, définissons les sociétés en question comme des sociétés d'affiliation, au vu de leurs caractères communs. Les individus y recherchent constamment la présence d'autrui et le contact avec lui, afin de se rassurer quant à leur position et à leurs possibilités d'agir ou de réagir. Leur association, directe, ne passe pas par l'intermédiaire d'organismes sociaux spécialisés. Les conduites manifestes réduisent l'incertitude concernant la situation respective des groupes et des individus, le partage des ressources et les échanges hiérarchiques. Les rôles existants, prescrits par un système d'incitations, sont clairs. Ils ne sont ni rigides ni constamment attachés à un seul individu. Ainsi un babouin hamadryas peut être le coryphée d'un groupe, passer dans un groupe appendiculaire non reproductif et retrouver, éventuellement, son statut antérieur. A chaque étape de sa vie, l'animal remplit une fonction précise, avec un répertoire de comportements spécifiques, dans un cadre cohérent lui indiquant comment il doit s'approcher d'une partie de ses congénères et en éviter d'autres. L'isolement est dangereux, privant l'individu de la protection et de la stimulation de l'environnement social. La nécessité de s'affilier est un facteur plus prégnant et plus général de congrégation chez les diverses espèces étudiées que la pression écologique ou territoriale. Mais elle a un sens déterminé.

Dans un ouvrage remarquable, Social Groups of Monkeys, Apes and Men ${ }^{17}$, Michaël Chance et Clifford Jolly décrivent la structure de souche (stem structure) des sociétés d'affiliation. Ils y distinguent trois sous-groupes caractérisés. Le premier se compose des femelles et de leurs progéniture, réunies en assemblées de femelles, qui comprennent aussi les sans progéniture. On les observe surtout quand la troupe est au repos, n'est pas occupée à se nourrir ou fuir un assaillant. Elles donnent l'occasion aux jeunes et aux femelles sans descendance de s'occuper des enfants, de les surveiller, de les épouiller, de jouer avec eux. Ces assemblées constituent le trait saillant des collectivités de primates, une modalité de groupement spécifique, bien que leur organisation soit moins rigide que celles des mâles. Elles reproduisent aussi la hiérarchie qui règne dans ces dernières et qui se traduit par le désir de fuite des inférieurs devant les supérieurs. Ce trait social est da-

17 M. Chance et C. Jolly : Social Groups of Monkeys, Apes and Men, Londres, 1970. 
vantage marqué lorsque les mâles n’interviennent pas dans les affaires des femelles ; il s'atténue et disparaît pour les espèces où les rapports entre sexes sont plus accentués, ainsi chez les rhésus qui forment quelquefois des couples, ou chez les gibbons dont les couples sont permanents. Chez les hamadryas, on ne peut parler d'assemblées de femelles, puisque celles-ci se groupent autour d'un seul mâle, et de même chez les macaques à bonnet, pour qui l'épouillage ne traduit pas de relations de préférence.

Les bandes de jeunes regroupent plusieurs classes d'âge, unies dans la recherche de nourriture et les jeux ; les plus jeunes se font porter par les aînés, attitude qui signale la peur devant un membre influent lorsqu'ils sont menacés. Leur grande mobilité sociale amène parfois les jeunes issus de deux groupes à un seul mâle dominant chez les babouins geladas ou les hamadryas - à se rencontrer. Certaines sociétés rejettent les jeunes à la périphérie, d’autres ne s'opposent pas à ce qu'ils jouent près des adultes à condition de ne pas les déranger. Chez les patas, on note toutefois que les bandes de jeunes s'agglutinent aux assemblées de femelles, tandis que, chez les gorilles, les jeunes peuvent aussi bien aller et venir parmi les adultes que se grouper en bandes pour leurs jeux.

Les mâles adultes, eux, forment une cohorte ordonnée par rang dont les membres se séparent rarement. Elle inclut les mâles de la hiérarchie reproductive chez les macaques, les gorilles ainsi que dans les groupes hétérosexuels des babouins vivant dans la savane. On est moins bien renseigné en ce qui concerne les mâles périphériques " célibataires " ; tout ce qu'on sait, c'est que, même lorsqu'ils se rapprochent des mâles reproducteurs, leur groupe ne se disloque pas. A la vérité, on a fort peu étudié ces bandes de célibataires considérées comme un élément accessoire. On n’a pas non plus examiné avec assez d'attention les conditions qui entraînent la dislocation de la cohorte et son regroupement en fractions bisexuées, chez les chimpanzés, par exemple. Suivant les espèces, les cohortes de mâles s'intègrent plus ou moins à la société. Chez le pata et l'entelle, la cohorte ne fraie pas avec le groupe constitué par un animal coryphée et ses femelles, alors que chez le babouin gelada il y a un rapprochement sans fusion. Chez les chimpanzés, l'isolement de la cohorte peut durer plusieurs jours, tandis que, chez les gorilles, la fusion est complète 
entre la cohorte des mâles et le groupe hétérosexuel. Pour d'autres espèces, le degré d’intégration est plus ou moins marqué.

La lecture du plan de nombre de villes ou de villages nous renseigne rapidement sur les groupes qui en composent la population et sur la manière dont ils se combinent. L'observation des groupements spatiaux des primates nous donne de même une idée de la physionomie des rapports qui unissent leurs parties composantes : un ordre déterminé entre les individus et les sous-classes y est omniprésent. Quelques exemples sont suffisamment évocateurs.

Le comportement des singes japonais est délimité par une série de cercles concentriques idéaux. Les animaux n'occupent pas, dans cet espace, n’importe quel emplacement. Au centre sont les « coryphées » - comme je propose d'appeler les mâles dominants, de préférence à « chefs » ou « leaders » - les femelles adultes et les nourrissons, surtout femelles. Dans le second cercle, autour de ce foyer sont dispersés les seconds et les jeunes mâles. Plus loin à la périphérie, demeurent les mâles adultes solitaires.

La stabilité de cette disposition est remarquable. A Takasakiyama, où cette étude a été faite, le nombre des singes a pratiquement triplé en cinq ans. Le coryphée-alpha, le mâle principal, est resté tout ce temps dans sa fonction et dans sa position. Les mâles adultes subordonnés ou seconds ainsi que les jeunes mâles sont demeurés au même échelon social. Les premiers n’ont pas eu accès au foyer du groupe, les derniers ne sont pas devenus les seconds des animaux coryphées. Les barrières sociales paraissent être à la fois élevées et efficaces. En échange, deux des cinq mâles coryphées, occupant un emplacement au centre de la hiérarchie, de même que cinq sur huit des anciens jeunes mâles, se sont retirés de la congrégation pour s’agglutiner aux mâles solitaires. Quelques femelles avec leurs nourrissons ont aussi quitté le foyer pour aller vers la périphérie, devenant ainsi plus autonomes.

Les convois à escorte des babouins, comprenant de 12 à 87 membres, sont peu ordonnés à première vue. On se détrompe immédiatement lorsqu'on observe leur façon de se déplacer en terrain découvert. Les mâles adultes subalternes avec un ou deux adolescents ouvrent la 
marche. En seconde ligne viennent les femelles et la majorité des adolescents. Au centre se trouvent les mâles coryphées, les femelles ayant des nourrissons et les jeunes. L'arrière-garde est symétrique de l'avant-garde : femelles et adolescents, puis, tout à la queue, les mâles subalternes. Spontanément le cortège s'organise de telle sorte que les femelles, les nourrissons et les mâles coryphées soient protégés de toute attaque de la part des prédateurs. Réciproquement, l'ensemble des membres de la collectivité est sous la surveillance des animaux qui ont pour fonction de les guider et de les entraîner, et à qui revient la décision en cas d'hésitation ou de danger.

Les cantonnements de chimpanzés correspondent à leur organisation sociale plus floue et plus composite. Les groupes sont instables et les individus les quittent assez facilement, les échanges étant fréquents. Dans la forêt de Budoryo les observations ont relevé l'existence de congrégations comprenant uniquement : a) des mâles adultes ; b) des adultes mâles et femelles et, parfois, des adolescents ; c) des mères et leurs jeunes enfants ; d) des mères et leurs enfants, d'autres femelles, des adolescents et des adultes mâles. Dans d'autres endroits on a constaté que les troupes de mâles vont tantôt ensemble, tantôt se séparent. Par moments elles se joignent aux jeunes et aux femelles. Sous cette apparence de variété et d'anarchie, on retrouve un peu partout la domination du sous-groupe des mâles sur les autres membres de la société.

La distribution dans l'espace d'une telle organisation attire l'attention à plus d'un titre, mais fait essentiellement ressortir l'existence d'une hiérarchie. Toutes les collectivités de primates la mettent en évidence, mais elle ne fonctionne pas partout dans les mêmes conditions ni en vue des mêmes fins. Il est difficile de savoir en quoi elle consiste exactement, d'autant plus qu'elle revêt des formes très diverses et synthétise trois fonctions : le contrôle, la domination, l'influence. Les écarts de rang qui contribuent au maintien du contrôle social affectent les relations entre mâles et femelles avec leur progéniture. Il est avéré que, dans toutes les espèces étudiées, la prééminence revient aux animaux de sexe masculin ; on ne relève aucune concurrence entre les sexes à ce propos. La position des mâles n'étant pas contestée, ils sont pour leurs compagnes à la fois un point de rallie- 
ment et un point de repère qui détermine leur orientation et leurs liens et un agent qui les oblige à se tenir à leurs rôles respectifs.

Les mouvements d'affiliation se cristallisent à partir d'eux et leur comportement concentre, à chaque instant, les lignes de force et de communication dans le champ collectif. La manière dont se diffusent les innovations illustre bien la direction de ces communications dans un groupe de primates. Les savants japonais ont d'abord observé que les jeunes singes de l'île de Koshima, à l'initiative d'une femelle de 18 mois, avaient pris l'habitude de laver les patates douces avant de les manger. Cette pratique ne fut jamais adoptée par les mâles plus âgés, normalement sans contact avec les jeunes. D’où l'idée, afin d'être certains de leur fait, de proposer une nourriture aux jeunes : des caramels. Peu à peu les jeunes y prirent goût, suivis par les mères et les plus petits, les mâles s'abstenant. Par contre, lorsque du blé fut offert au mâle-coryphée, il en mangea aussitôt, et fut imité par la femelle du rang le plus élevé, puis par les autres membres de la bande ; ceci en l'espace de quelques heures; il avait fallu dix-huit mois pour que la moitié du groupe s'habitue à manger des caramels. L'empreinte de la hiérarchie sur l'inhibition et la facilitation des comportements semble donc être très forte. Ceux qui sont au sommet dessinent un gradient de participation ou de non-participation à la vie du groupe et veillent au maintien d'une dépendance qui est gage de stabilité. Chez les geladas, le mâle coryphée laisse une certaine liberté de déplacement à ses femelles. Toutefois, elles se réunissent régulièrement autour de lui, qu'il les ait ou non invitées à le faire. Dès qu'il manifeste l'intention de changer de lieu, les membres du groupe qui ne cessent de le surveiller se rapprochent de lui pour le suivre. Avec la même constance il circule parmi les femelles afin de prendre note de leur position. Lorsqu'il est séparé d'elles, on le voit prendre une attitude imposante, la tête levée, la queue dressée, scrutant la compagnie rassemblée avec une expression sévère sinon menaçante. Si quelque chose dans l'attitude d'une femelle lui paraît incongru, il va vers elle, et lorsqu'il est tout proche elle s'accroupit et pousse un cri perçant en le regardant. Alors il s’assied et la femelle se met à l'épouiller.

Chez les babouins hamadryas, les femelles suivent le coryphée tout le temps, sans s'en éloigner à plus de quelques mètres. Elles ne connaissent qu'un seul protecteur, et si, par hasard, elles s'en écartent, 
il les amène à résipiscence par une morsure au cou. Dans ces groupes, le souci du mâle adulte est de préserver l'unité et de s'assurer de la loyauté des membres, surtout femelles, envers lui. Ses rapports avec une femelle - ou avec un jeune, surtout de sexe féminin - définissent son degré de participation, le contrôle qu'il exerce en tant que mâle. Les conflits entre femelles et leurs diverses interactions sont dominées par leurs relations avec le coryphée. En courant pour arriver la première auprès de lui, une de ses compagnes vient à occuper un lieu qui lui confère une sécurité évidente. Elle se permet alors d'attaquer ses rivales ou de les écarter de lui. Il n'intervient pas activement dans ces querelles. Toutefois, s'il devait le faire, c'est l'animal le moins proche qui serait sanctionné par une morsure. Dans les diverses espèces, l'ordre du rang corrélatif au contrôle est plus ou moins strict : il contribue partout à rendre cohérent le comportement réciproque des femelles vis-à-vis des mâles et entre elles. La hiérarchie de domination voit s'opposer et se séparer les mâles adultes. Les privilèges des animaux-coryphées leur garantissent des avantages enviables par rapport au reste de la société, sources de tentation pour les animaux qui, ayant atteint un certain âge, en sont privés. Les jeunes adultes ressentent aussi les inconvénients de cet état de choses.

Les geladas et les hamadryas vivent en société de clique ; chez eux le problème est plus simple ; les mâles défavorisés sont repoussés vers les groupes appendiculaires monosexuels. Quelques-uns d'entre eux échappent à cette rigueur en formant équipe avec un coryphée vieillissant. L'animal plus jeune, subordonné, lors du déplacement du groupe prend la tête du mouvement et rapporte à son aîné des informations sur la situation d'ensemble. Celui-ci, à la lumière de son expérience, décide de l'étendue ou de la direction du déplacement projeté. Les différences hiérarchiques sont encore plus visibles chez les babouins. Elles s'expriment très directement à l'occasion des rencontres. Lorsqu'un animal dominant s'approche d'un animal qui ne l'est pas, celuici s'écarte. Dans la vie ordinaire, le coryphée a aussi une liberté de choix relativement plus grande en ce qui concerne les sites de couchage. L’hégémonie, dans une société de compagnonnage comme celle des babouins, revient à une " chefferie », à plusieurs mâles coryphées associés. Le statut de chacun ne dépend donc pas exclusivement de ses aptitudes physiques, force, agilité, mais aussi du réseau d'alliances qu'il a nouées. Comme, d'habitude, ces animaux sortent 
ensemble, ils sont en mesure de s'entraider en cas de menace et de vaincre un individu qui, venant du dehors, essayerait de déloger l'un d'eux de la position qu'il occupe. On attribue la constitution de ce commandement collectif - par opposition au commandement individuel des hamadryas et geladas - au danger accru de prédation. Il est patent qu'il permet de maintenir une discipline assez stricte. Les combats sont rares et les chercheurs japonais n'en ont relevé que dans $15 \%$ des collectivités étudiées. Lorsque des querelles éclatent, les animaux composant le noyau dominant accourent pour y mettre fin. La permanence de la hiérarchie de domination est remarquable, probablement parce qu'elle représente l'action collective d'une sousclasse de la communauté et non pas la force d'un seul individu.

La différenciation sociale afférente à l'influence est plus malaisée à cerner et caractérise surtout les rapports entre mâles adultes et jeunes. Le problème est ici d'assurer de la part de ces derniers une conformité aux règles implicites du groupe et de les amener à respecter les cadres de vie. A l'encontre des mâles adultes surbordonnés, ces jeunes mâles représentent un facteur de perturbation potentielle et non pas actuelle. Paradoxalement, les interactions entre ces classes d'animaux dans une société n’ont pas été analysées, observées de manière très approfondie. Si la présence de jeunes mâles est un facteur de tension, c'est que chaque société, une fois instituée, tend à garder ses traits et ses rapports constants, à résister aux efforts qui pourraient les changer prématurément. Or l'éclosion d'un sous-groupe important de mâles qui demande sa part de ressources et de participation à la vie sociale met en question l'ordre existant. En particulier lorsque fait défaut à celuici un mécanisme ostensible de renouvellement. Une chose est certaine : dans la plupart des sociétés d'affiliation, les jeunes femelles sont gardées dans le groupe basal et les jeunes mâles risquent généralement d'en être chassés. Les premières n'ont pas une très grande liberté de mouvements, les seconds sont nécessairement des migrants. De cette façon ils font l'apprentissage de la rupture et de l'isolement par rapport à leur unité d'origine. Des chercheurs japonais ont signalé la fréquence de ce phénomène chez les macaques. Ceci suppose qu'ils sortent de l'orbite des animaux-coryphées et que leur interférence avec le reste de la collectivité, leur capacité de l'infléchir est diminuée, sinon nulle. Mais le lecteur pourra se demander pourquoi je parle d'une hiérarchie d'influence alors qu'on a l'impression qu'il 
s'agit d'une interaction parent-enfant. Nous n'avons aucune raison de transposer à une société d'affiliation des catégories décrivant ce qui a lieu dans une société où la famille existe. L'organisation collective des primates est fondée sur l'existence d'une distance entre les mâles et le reste du groupe, et ipso facto entre eux et la progéniture en tant que leur progéniture. Elle ne reconnaît du mâle à ses descendants masculins d'autre lien que celui qui s'établit au moment de modeler le comportement de ceux-ci, lien destiné à tempérer leurs initiatives et à réduire les risques de leur intrusion dans la routine des relations sociales quotidiennes. Il est par conséquent impossible, étant donné cette distance et ces rapports, " de retrouver les liens du sang entre père et enfants ; non seulement il en est ainsi objectivement, mais aussi, de la part de l'enfant, il n’y a aucune connaissance de son « père » ${ }^{18}$.

Les différentes formes de hiérarchie n’ont rien de gratuit, et les conséquences qu'elles entraînent pour ceux qui en bénéficient sont certaines. Les animaux qui occupent une place élevée sont aussi ceux qui ont une chance de survie, au sens strict comme au sens sélectif, puisqu'ils peuvent avoir des liens hétérosexuels et se reproduire. Par contre, les individus subordonnés, les jeunes mâles adultes ou subadultes en particulier, sont forcés de quitter le foyer du groupe. Sur eux s'exerce une pression à l'homosexualité, au sens littéral aussi bien que dans le sens d'une sorte d'initiation à une vie qui se déroule surtout avec leurs congénères du même sexe. Leur réunion avec des femelles est difficile et implique pour eux un risque d'agression. Même quand cette possibilité leur est offerte, la surveillance des animaux hégémoniques ne se relâche guère et il est hors de question de former un couple normal. L'accès à la nourriture obéit à un modèle analogue. Les coryphées, les études le montrent, ont une priorité incontestable sur ce plan. Malgré le caractère plausible de cette proposition ou observation, j'avoue ne pas avoir eu connaissance de données empiriques incontestables. Si, elle se confirmait, il faudrait en conclure que la poussée des animaux vers la périphérie de la société et surtout vers les groupes monosexuels se traduit par une mortalité plus grande que celle constatée chez les animaux de rang supérieur demeurant dans les groupes bisexuels. Jusqu'à présent rien ne nous autorise à l'affirmer.

18 J. ITANi : Paternal Care in the Wild Japanese Monkey, Macaca Fuscata, Journal of Primatology, 1962, 2, p. 86. 
Mais tous les chercheurs sont d'accord sur un autre indice de hiérarchisation des membres d'un groupe, à savoir l'épouillage. Cette opération permet de débarrasser la fourrure des saletés et des parasites, sa fonction biologique est patente. On a observé que de nombreux animaux des régions explorées étaient infestés de tiques; en revanche les babouins en sont exempts, car ils consacrent beaucoup de temps à s'épouiller. A travers l'activité à finalité hygiénique, contribuant au bien-être de l'individu, se tissent des liens sociaux plus subtils et plus complexes. Dans une collectivité de primates, tous les animaux reçoivent et effectuent l'épouillage. La quantité d'épouillage reçue, elle, dépend du rang : un mâle de rang élevé en reçoit plus qu'un mâle de rang inférieur. Le réseau des animaux qui l'effectuent envers un animal donné décrit la carte de sa clientèle et de ses affiliés.

La réalité, il s'en faut, ne coïncide pas avec les descriptions que l'on vient de lire. Les structures hiérarchiques d'un groupe de capucins, d'une congrégation de chimpanzés ou d'entelles aux Indes sont très peu accusées. Souvent l'animal-coryphée a une fonction essentiellement de garde : il disperse les individus engagés dans un combat ou réagit à un danger externe, singes étrangers ou hommes.

L'articulation des trois fonctions autour desquelles se constitue la hiérarchie —contrôle, domination, influence — n'est pas uniforme à travers les espèces ou les types de société. Les sociétés de clique, comparées aux sociétés de compagnonnage, les intègrent mieux en les subordonnant les unes aux autres à l'intérieur d'un schéma cohérent. Je ne saurais cependant en dire davantage, car les recherches dont j'ai pu prendre connaissance ont jusqu'à ce jour soulevé plus de questions qu'elles n'en ont résolu, sur ce plan comme sur d'autres. 


\section{Les prescriptions de la vie en commun.}

\section{Retour à la Table des Matières}

La vie en commun ne connaît pas de règles clairement énoncées : elle n'en obéit pas moins à un ensemble d'instructions rigoureuses qu'un membre doit respecter s'il tient à rester dans le groupe, à y occuper une position particulière. Ce cadre est bâti suivant deux dimensions principales : le sexe et l'âge. Plus que par leurs caractères anatomo-physiologiques, c'est par une série d'indices et de comportements sociaux que les individus sont inclus dans l'une de ces classes. La classe des femelles est, par définition, dans une situation de soumission. La signification de cette relation est subtile : d'un côté elle s'encastre dans l'ordre hiérarchique, d'autre part elle connote plutôt une non-domination, un non-contrôle du système social qu'une véritable subordination. L'association d'une ou de plusieurs femelles avec un mâle les fait participer d'un certain statut inférieur ou supérieur. Mais ce n'est pas toujours le cas. Chez les singes rhésus on a constaté que le rang des femelles adultes conditionnait le rang du groupe par rapport aux groupes voisins. Les babouins voient se former une hiérarchie lâche, indépendante de celle des mâles. En revanche, chez les patas, les interactions entre femelles traduisent leurs différences hiérarchiques, fortement marquées, et qui s’imposent à l'ensemble de la collectivité, y compris le mâle. Lorsque celui-ci menace l'enfant d'une femelle de haut rang, elle réagit promptement, appuyée par ses compagnes. L'éthologue anglais Ronald Hall estime que le rôle du mâle-coryphée se borne à contrôler le groupe, à l'entraîner et à le défendre contre les prédateurs, mais que ce sont les femelles, déjà organisées et ordonnées entre elles, qui le choisissent. L'articulation des deux classes manifeste l'autonomie relative de chacune et l'existence d'un certain degré de liberté. Elle correspond à un clivage ou à une distinction des fonctions. Dans l'ensemble, la classe des mâles représente la partie la plus mobile et la classe des femelles, adultes s'entend, la partie la plus stable du système social. Les études faites sur les singes rhésus ont montré que, dans le développement d'une collectivité, les femelles se réunissaient en une unité plus facilement que les mâles. Elles forment le noyau auquel s'ajoute le mâle, la congrégation n'étant possible qu'une fois le groupe basal institué. Si 
le comportement des animaux de sexe féminin crée la possibilité d'une permanence de la vie sociale, la présence d'un animal-coryphée mâle est indispensable à la survie de l'unité sociale dans son ensemble. Les animaux qui ne trouvent pas de place dans la trame collective ainsi dessinée restent solitaires ou s'affilient à une bande monosexuelle masculine. Chez les Papio anubis, les vervets et dans la plupart des sociétés de compagnonnage, les mâles vont facilement d'un groupe à l'autre, tandis que les femelles, liées entre elles, semblent être la fraction la plus constante de la communauté. On observe un phénomène analogue chez les babouins hamadryas. Le coryphée les surveille et les attaque si elles s'éloignent. Les mâles sub-adultes, par contre, peuvent rejoindre ou quitter le groupe à leur convenance, sans grand risque, s'ils n'attentent pas aux statuts et privilèges existants. La dissymétrie des deux classes se retrouve dans les actes essentiels de la vie. Les mâles sont pratiquement obligés de vivre dans le groupe appendiculaire, non reproducteur, du moins lorsqu'ils sont jeunes. Ils passent donc d'une série de relations homosexuelles à une série de relations hétérosexuelles et vice versa. Chaque fois ils doivent faire la preuve de leur capacité à adhérer ou à participer à un groupe dont la structure et la finalité sont particulières. Le changement qui leur est imposé ou qu'ils recherchent affecte à le fois leur statut et leur mode de vie. Le conflit entre l'appartenance à un cycle d'interactions homosexuelles et la participation à des relations hétérosexuelles, les tensions engendrées par l'agression qui marque les rapports de domination, expliquent l'état anachorétique choisi par de nombreux mâles. Les femelles demeurent pendant toute leur vie dans un groupe basal, hétérosexuel, donc reproductif. Pour des raisons diverses, elles cherchent parfois à se retirer à la périphérie de la collectivité, mais n’en sortent jamais. Les mâles adultes s'opposent à une telle migration. Les femelles sont cependant des individus-charnières dans ces société. Elles participent à deux couples : le couple nucléaire, formé par la mère et sa progéniture, et le couple reproducteur, formé par la femelle et le mâle. Ces deux couples sont distincts, parce que fondamentalement les enfants sont les enfants de leur mère jusqu'à un certain âge et qu'elle fournit le schéma d'affiliation des individus jeunes dans l'organisation sociale. Le lien de la mère et du nourrisson dure au moins dix mois. Au cours de cette association, elle lui fournit la sécurité affective dont il a besoin, lui apprend à explorer le milieu ambiant pour se nourrir et aussi comment réagir au comportement des adultes. 
Les mères-babouins ou macaques résistent habituellement à la séparation d'avec leurs enfants, tandis que les mères-entelles enrôlent d'autres femelles pour les aider à élever leurs rejetons après la naissance.

Assurément l'éloignement des jeunes mâles est la règle et ce sont eux qui sortent le plus rapidement de l'orbite des soins maternels, du couple nucléaire. Le temps que celui-ci a duré semble suffisant pour créer des liens. Des observations détaillées des singes rhésus à Cayo Santiago (Porto Rico) ont mis en évidence que les jeunes entretiennent une relation avec leur mère même lorsqu'ils atteignent la maturité physique. Les enfants appartenant à une lignée ont des rapports préférentiels entre eux. Ainsi ils s'épouillent, en grande partie, les uns les autres. Par ailleurs, si un jeune mâle s'approche d'un groupe monosexuel périphérique, il est attaqué, à moins d'être protégé par un mâle qui en fait partie. Les mâles qui les appuient paraissent être des membres du même groupe que le "protégé ». Le mâle adulte du couple reproductif ne s’occupe qu'épisodiquement des jeunes. Les études faites jusqu'à ce jour prouvent qu'il est difficile de parler d'un comportement « paternel » dans les sociétés d'affiliation. Il serait plus exact de dire que les mâles-coryphées s'occupent individuellement plutôt des mères que des enfants. Le savant japonais Itani a constaté des soins que l'on pourrait qualifier de paternels dans 3 des 18 groupements étudiés. Les singes rhésus adultes n'accordent aucune attention aux enfants, et s'ils ne font pas preuve d'hostilité, ils s'en désintéressent lorsque l'un d'eux s'approche. Ceci ne veut point dire que la collectivité, dans sa totalité, néglige le bien-être et la survie de la progéniture. Au contraire on remarque fréquemment des individus qui s'y consacrent en qualité d' " oncles » et de "tantes ». Il n'en reste pas moins que les mâles adultes contrôlent plus longtemps les jeunes femelles que les jeunes mâles ; ceux-ci sont poussés vers la périphérie de la collectivité au fur et à mesure qu'ils approchent de la maturité et de l’indépendance.

La continuité du couple reproducteur et la continuité du couple nucléaire s'opposent donc. L'absence de lien particulier entre le mâle et sa progéniture en est cause. Le couple reproducteur jalonne clairement l'aire de protection, de contrôle, du mâle, il démarque les prestations de service auxquelles il a droit ainsi que le réseau des choix sexuels 
qui sont limités aussi bien que leur régularité. Il s’affirme élément essentiel de l'organisation sociale ; participant à la fois du statut du mâle et de celui de la femelle, discriminant entre le centre de la collectivité et sa périphérie. Le couple nucléaire a une existence plus cachée, plus implicite, parce qu'il est tout entier inclus dans l'univers des femelles. Sa vocation est de tisser des liens interindividuels perdurables en partant principalement d'une femelle ou d'un groupe de femelles. Pour cette raison il n'est ni passager ni purement biologique. On a pu voir qu'il produisait de véritables lignées qui se retrouvent hors du groupe où elles ont pris naissance. Bien entendu, on ne peut pas être très affirmatif à ce sujet; il est cependant logique que les choses se passent ainsi.

Le passage d'un couple à l'autre est assuré, socialement, par la mère qui est le foyer affiliatif en même temps qu'un agent par l'intermédiaire duquel se transmet le rang du mâle à ses descendants. Il faut ajouter, à son insu. Les recherches sur les macaques ont établi que les mâles adolescents sont forcés de passer du centre à la périphérie, accédant ainsi à un rang social inférieur. Les enfants de femelles ayant un rang élevé font exception, ils restent au centre et peuvent occuper à leur tour un rang élevé, sans être astreints à un séjour au " purgatoire », séjour qui peut durer de 3 à 5 ans pour les autres. La dispense de stage à la périphérie de la collectivité a plusieurs conséquences qui assurent la transmission d'avantages du point de vue de la domination. Les enfants des mères hégémoniques ont acquis des attitudes plus agressives, plus assurées et se conduisent en accord avec ce qui leur a été enseigné comme étant naturel et nécessaire. Les enfants des femelles subordonnées ont acquis, pour la plupart, des attitudes de soumission, de peur. Au cours de leurs futures rencontres, les premiers auront nécessairement le dessus sur les seconds. Même avant, probablement, les jeunes étant soignés et protégés par leur mère pendant trois ans; ils sont perçus, lorsqu'ils demeurent auprès de l'animal-coryphée et de sa femelle, comme faisant partie de la zone centrale de la société. A ce titre, ils suscitent des comportements conformes de la part des autres qui, pour leur part, s'habituent à manifester et continuent à manifester, à l'âge adulte, les mêmes postures de soumission. Enfin, les animaux subordonnés ayant intérêt à prendre soin de tout ce qui touche à l'autorité, la sollicitude et la protection du groupe accroissent le bien-être physique, améliorent les facultés in- 
dispensables à la survie. Ceci démontre que, dans ces sociétés, les positions sociales, en général, ne reviennent pas à des individus de façon aléatoire, discontinue ou seulement biogénétique. Il y a un processus de reproduction sociale, reproduction du nombre aussi bien que des attributs requis par les divers rôles qui attendent un individu désigné à l'avance par sa naissance, et un aménagement des situations qui a pour effet de placer l'animal dans une niche de l'organisation globale proche de la niche de ses géniteurs. La commutation des générations a pour opérateur la mère qui, là encore, assure la stabilité de l'ensemble en transmettant aux jeunes l'héritage des adultes. Les mécanismes mis en jeu garantissent la communication des différences statutaires et avec elles la solidité de l’édifice social.

La société d'affiliation, dans sa totalité, est structurée à partir des diverses modalités de hiérarchie des mâles. Descendant au niveau du groupe basal - nous voyons maintenant qu'il est impropre de parler, comme on le fait, de "famille » — on retrouve une répartition assez rigoureuse des fonctions de chaque individu et des normes qui définissent leurs comportements réciproques. Les transgressions sont parfois très sévèrement punies. Le comportement sexuel n’échappe certainement pas à pareille régulation. A vrai dire, ce problème n'a pas été suffisamment étudié pour que l'on puisse dégager un code précis concernant l'accouplement. Il ressort cependant des études faites que les relations sexuelles chez les primates non humains n'ont pas un caractère général de promiscuité. En tenant compte des circonstances démographiques, on constate dans certains cas, notamment dans les sociétés de clique, un quasi-exclusivisme des rapports entre un mâle et ses femelles, avec des aménagements dus à l'âge ou à l'état de l'œstrus de la femelle. Les chimpanzés mâles, comme tous les autres mâles vivant dans des sociétés de compagnonnage, sont plus tolérants envers les autres individus adultes subordonnés ou envers les jeunes. Les cas de promiscuité ne manquent pas. Un éthologue a cru bon de décrire la scène suivante dont il a été témoin : sept chimpanzés mâles (dont un adolescent) ont copulé successivement avec une femelle sans manifester d'agressivité. Mais ce fait divers est bénin, comparé à ce que l'on lit dans les journaux ou dans les annales criminologiques. Les sociétés de primates, comme les sociétés humaines, diffèrent par le degré de promiscuité qu'elles tolèrent. Si l'on comparait le comportement sexuel des cynocéphales et des anthropoïdes avec le compor- 
tement humain réel, et non pas avec son travestissement juridique, religieux ou moral, on serait enclin à opposer leur vie presque vertueuse à la nôtre qui apparaîtrait dissolue et versatile. De même qu'il y a peu de promiscuité, il n’y a pas non plus de rapports sexuels fréquents dans le couple nucléaire, c'est-à-dire entre la mère et ses fils. Ces rapports sont pratiquement impossibles, car le mâle-coryphée chasse les jeunes avant leur maturité. Sade a noté l'absence ou l'évitement des liens incestueux quels qu'ils soient. Il ne saurait en être autrement : l'appariement hétérosexuel est le canal de la distinction et de l'individualisation sociale. Le laisser au hasard plongerait les animaux dans la grisaille de l'indifférenciation des fonctions, des comportements et des liens. Ainsi, sur ce plan comme sur d'autres, la société animale est bien une société.

$\underline{\text { Retour à la Table des Matières }}$ 


\section{Comment réussir à combler le fossé}

qui sépare les générations.

Les collectivités sans conflits ne sont pas utopiques : elles sont impossibles. Les éthologues ou les biologistes qui réduisent ces conflits à l'agressivité ou se bornent à compter les actes hostiles, quand il s'agit d'animaux — et par extension d'hommes — aplatissent un phénomène qu'il faut saisir par référence à un système ayant des répercussions individuelles et inter-individuelles. Ils étudient la société comme un individu à l'intérieur duquel ils projettent la totalité de l'appareil social. Principe d'ordre, la hiérarchie est aussi principe d'antagonisme, créant des conditions auxquelles il est difficile de se résigner, ou des privilèges dont on ne peut jouir indéfiniment. Dans les sociétés formées par les primates non humains, la statique est fournie par les rapports entre les classes sexuelles et la dynamique par les classes d'âge. Il y a une contradiction flagrante entre le fait que les mâles sont dominants en tant que mâles et dominés en tant que jeunes, la jeunesse étant, pour la plupart d'entre eux, cause d'une situation subordonnée. Mous avons vu que les individus occupant une place élevée dans la hiérarchie sont aussi ceux qui bénéficient de l’appui du groupe et ont une chance de survie, au sens strict comme au sens sélectif, car ils peuvent procréer et se reproduire. Par contre, les individus subordonnés, les jeunes mâles subadultes en particulier, sont expulsés du centre du groupe. Leur accès aux ressources et aux femelles est difficile et comporte pour eux des risques. Même s'ils ont accès à ces femelles ou à une partie plus favorable du territoire, ils ne sont autorisés à le faire qu'à certains moments. Donc, de ce point de vue aussi, les relations entre mâles adultes et sub-adultes sont tendues.

Les mâles-coryphées sont constamment préoccupés de leurs prérogatives. Ils savent que l'on cherche à les leur ravir et ils sont, l'objet de nombreuses attaques. Dans une étude sur les entelles aux Indes, on a remarqué que les individus appartenant à des groupes monosexuels attaquaient sans cesse les groupes bisexuels. Au cours d'un de ces combats, un assaillant a ravi presque toutes les femelles de l'animal- 
coryphée. D’autre part, une bande de sept mâles a attaqué un grand groupe pendant dix jours, réussissant à éliminer le mâle dominant et plusieurs jeunes. Quelques jours plus tard, les six mâles surnuméraires furent éliminés, l'animal victorieux gardant les femelles avec lui. Des cas semblables ont été observés plusieurs fois. Il convient d'ajouter que la reprise en main du groupe s'accompagne d'infanticide, les mâles et les jeunes défiant le conquérant. La mise à mort des jeunes et la procréation d'autres enfants permettent aux femelles comme au reste de la collectivité d'accepter sexuellement et socialement le nouvel animal-coryphée. Le changement du couple reproducteur, par cette voie, a des effets délétères pour le couple nucléaire, le renouvellement du premier impliquant la reconstitution presque totale du second. L'existence d'une fraction reproductrice et d'une fraction non reproductrice est source de conflits; les combats qui naissent entre elles représentent un des modes d'ascension dans la hiérarchie sociale, de transformation du statu quo. La recherche de faveurs en est une autre. Les animaux subordonnés tentent de gagner les bonnes grâces des mâles dominants, soit pour accéder au pouvoir, soit pour atténuer les rigueurs de la domination ou du contrôle. Le comportement de « tante » ou d' " oncle » correspond fréquemment à une telle intention. Hinde et Spencer-Booth décrivent la façon dont les mères rhésus acceptent les services d'une femelle à qui elles confient la garde de leur nourrisson. Elles la choisissent généralement parmi les compagnes d'autrefois pour la surveillance et l'épouillage. Et autant que possible de statut social inférieur. Les mères de haut rang commandent les interactions avec le nourrisson par des menaces voilées, tandis que les mères de rang moins élevé " sourient » ou " se présentent » aux " tantes » potentielles de rang plus élevé qu'elles. L’intérêt d'une femelle « inférieure " sans enfant est clair : elle peut participer par ce moyen du statut de la mère de rang plus élevé, ou être protégée par elle. Les macaques japonais mâles adultes s'approchent des jeunes animaux lorsque ceux-ci ont un an. Ils étreignent l'enfant, l'épouillent, l'accompagnent dans ses déplacements et le défendent. Ce comportement est développé chez les animaux-seconds des bandes fortement organisées. De cette façon ils sont tolérés par les animaux-coryphées, et les femelles qui leur sont associées, et à titre d' " oncles » ils peuvent s'élever socialement. On retrouve une stratégie analogue chez les babouins hamadryas. Un mâle plus jeune entre dans le groupe en adoptant un comportement non agressif, soumis, et sans prétendre à s'accoupler 
avec les femelles. L'animal-coryphée l'accepte et l'associe à ses différentes tâches. A mesure que l'adulte vieillit, le jeune se voit autorisé à accéder à la vie sexuelle, tandis que son compagnon garde la direction des déplacements et des activités quotidiennes.

A côté de ces comportements utilitaires, on observe des comportements cérémoniels qui visent à atténuer l'antagonisme des générations, à différer l'hostilité des adultes envers les jeunes. Être toléré plutôt qu'attaqué par les animaux de rang élevé ouvre la possibilité d'une ascension ultérieure, du maintien dans le cercle privilégié de la société. Le modèle de comportement symbolique par lequel on y parvient est celui de la féminisation du jeune ou du subordonné. Toute la cérémonie a pour objet d'établir une relation de non-agression où le dominant traite le dominé comme une femelle. L'animal de rang inférieur, ce qu'est le jeune par définition, découvre son arrière-train, adopte la posture sexuelle féminine, croupe tendue et se présente pour être monté. L'animal de rang supérieur s’apaise et parfois fait le simulacre de l'accouplement, manifestant par là sa bienveillance et son consentement au lien qu'on lui demande d'établir. L'épouillage est un autre moyen employé en vue de la même fin. L'animal subordonné ou jeune fait comprendre à l'adulte son désir de le toiletter en émettant un claquement des lèvres, perçu comme un signal : ce bruit accompagne en effet l'épouillage, dans lequel l'animal porte à sa bouche les divers détritus et les peaux mortes qu'il enlève. L'agresseur potentiel renonce à ses intentions belliqueuses et accepte l'offre de service, au bout d'un temps plus ou moins long, ou bien tolère la présence de celui qui cherche ainsi à l'amadouer.

Tous ces subterfuges - comportement d' " oncle » ou de « tante ", ou de "successeur ", cérémonies de soumission — ouvrent la voie à la montée dans la hiérarchie pour les uns, évitent aux autres d'être rejetés vers la périphérie. La mobilité sociale a lieu dans le cadre existant, les tensions étant diminuées par des concessions réciproques et des atermoiements qui préparent une cohabitation et une succession sans que les contradictions viennent à la surface. La fission du groupe ou le recrutement de femelles sont également possibles, donnant aux individus subordonnés l'occasion de reconstituer le groupe basal dont ils ont besoin et de s'affirmer au sein de l'organisation sociale élargie. L'étude de Takasakiyama déjà mentionnée, met en évidence, avec 
l'accroissement de la population, la formation d'un groupement d'animaux se détachant de l'ancienne unité. Les animaux dominants de la nouvelle unité sont des mâles jeunes, de 12 à 13 ans, qui ont occupé auparavant le second, le troisième et le cinquième rang dans leur classe d'âge. Les autres sont devenus anachorètes avant la scission. Les femelles qui les ont rejoints ont vécu à la périphérie de la collectivité, et la moitié d'entre elles ont fini par s'intégrer au nouveau groupement tandis que les autres oscillaient entre celui-ci et le groupement d'origine. Une fois différenciée, la collectivité des jeunes s'est organisée sur le patron de la collectivité-mère, avec son centre, sa périphérie, etc. A l'encontre de la fission, le recrutement s'apparente au vol. Les mâles sub-adultes prennent les jeunes femelles à leur mère et leur prodiguent des soins de type maternel. Ils les surveillent et les empêchent de s'éloigner. La jeune femelle accepte rapidement ce traitement et épouille son ravisseur. Les rapports, à cette phase, n’ont rien de sexuel et conduisent surtout à préparer une future affiliation plus stable. Suivant un plan analogue, les jeunes mâles choisissent pour protégés des jeunes des deux sexes. Rapidement ils délaissent les mâles pour s'occuper uniquement des femelles et jeter les bases du groupe bisexuel qu'ils contrôleront par la suite. Qu'il s'agisse de combat direct, de recherche de faveurs, de fission ou de reconstitution, la contradiction qui est au fondement de l'organisation sociale n'est jamais entièrement résorbée ni la vie complètement pacifiée. Il reste toujours une partie de la population mâle — on aimerait cependant avoir des renseignements plus précis sur ce point - qui se retire ou qui est obligée de subsister en marge, parfois en cherchant à échapper à sa condition et parfois en s'y résignant. Elle synthétise et rend visible la menace qui pèse constamment sur la collectivité : celle d'une remise en question des positions acquises et d'une chute sans rémission.

Les études portant sur les sociétés de simiens sont fascinantes par les perspectives qu'elles nous ouvrent et les similitudes qu'elles nous font pressentir. Qui, à leur lecture, n'est pas tenté de rapprocher tel comportement anthropoïde de tel comportement humain ? Les cérémonies de soumission avec leurs connotations homosexuelles nous rappellent que la souveraineté, en paroles ou en actes, est souvent associée à un coït anal symbolique. Le vol des femmes, le mariage avec des filles impubères ou de très jeunes gens sont d'une pratique cou- 
rante dans certaines sociétés, tandis que la polygamie s'accommode fort bien d'une cohorte de célibataires auxquels est refusé l'exercice de leur pouvoir viril. Pour ne rien dire de la signification de la hiérarchie et des relations entre les femelles et les mâles qui la sous-tendent. Mais ces associations sont surtout suggestives, et l'on aurait tort de suivre l'exemple des auteurs qui inclinent à identifier complètement ce qui se passe dans les sociétés dites primitives et ce qui a lieu dans les nôtres. Les distinctions sont notables. Si la vie des primates non hominiens est socialisée, cela est surtout vrai pour le domaine des liens interindividuels, de la reproduction du groupe et de ses conditions de survie. L'existence demeure complètement individuelle en ce qui concerne les ressources, la nutrition. A partir du sevrage, chaque individu cherche seul les aliments dont il a besoin, sans s'associer ou se coordonner avec ses congénères pour se les procurer. La consommation de la nourriture est aussi affaire individuelle, le groupe n'intervenant pas pour le partage. Les animaux sont retenus dans les zones riches ou rejetés vers les zones pauvres suivant qu'ils sont ou non reproducteurs : l'intervention collective ne va pas au-delà. De même, il est évident que l'abus d'un langage métaphorique qui fait parler de "famille », de " leadership » ou de " harem » concourt à obscurcir les concepts et les réalités. La parenté, au sens strict, est absente. Certes, on relève des échanges entre mâles, femelles et jeunes ; des éléments identiques ne forment pas nécessairement une structure identique. Or la " famille » en tant que telle est une unité constitutive dans bon nombre de sociétés créées par les hommes, elle ne l'est pas dans celles des primates non humains. On oscille d'habitude entre l’identité complète et l'hétérogénéité complète, les sociétés animales servant soit de prototype des sociétés humaines ultérieures, soit offrant des variantes du substrat biogénétique sans point de comparaison possible. Pour ma part je constate seulement des différences qui les séparent en tant qu'elles combinent autrement les comportements, les liens propres aux individus et aux classes, et qui les rapprochent en tant que sociétés, ayant des exigences souvent communes. Par ce dernier biais elles participent d'une série historique qui transforme les unes dans le devenir, hypothétique, des autres. Et si, me pliant à la coutume, je fais précéder les sociétés sans écriture par des sociétés sans parole, c'est pour souligner l'unité que motive pareil devenir, et non pas le hiatus que constituerait la sortie de la nature. 
Accepter un tel hiatus, Georges Bataille nous en a avisés il n’y a guère, "c'est aller d'une vue abstraite à l'autre, c'est exclure le moment où la totalité de l'être est engagée dans un changement. Il me semble difficile de saisir cette totalité dans un état, ou des états énumérés l'un après l'autre, et le changement donné dans la venue de l'homme ne peut être isolé du devenir de l'être en général, de ce qui est en jeu si l'homme et l'animalité s'opposent en un déchirement exposant la totalité de l'être déchiré. Nous ne pouvons en d'autres termes saisir l'être que dans l'histoire : dans des changements, des passages d'un état à l'autre, non dans des états successifs envisagés isolément ${ }^{19}$.

J'ai essayé de dégager les traits de la transition qu'opèrent, dans la nature, les primates, entre l'évolution et l'histoire, et ceux qui déterminent l'existence de ce qu'il faut bien reconnaître comme représentant les premières sociétés historiques: les sociétés d’affiliation. Après ce détour indispensable, on sera mieux préparé à aborder les questions soulevées initialement et à juger de la valeur des réponses proposées. Ce qui signifie concrètement insérer l'homme dans un mouvement déjà en marche dans le règne animal, découvrir l'instant où il reprend ce contre quoi il a dû se constituer. Nous savons maintenant quel héritage il y a eu à reprendre et contre quoi il s’est constitué.

$\underline{\text { Retour à la Table des Matières }}$

19 G. Bataille : L'érotisme, Paris, 1957, p. 236. 
Retour à la Table des Matières

\section{Notes de la première partie}

\section{$\underline{\text { Retour au texte }}$}

Note 1: Il faut attirer l'attention sur la valeur des sources utilisées et l'incertitude des preuves avancées qui, sans préjuger des résultats, affectent d'un doute fondamental toute démonstration et tout débat dans ce domaine. Les conclusions auxquelles on aboutit au sujet des primates non humains actuels sont basées sur des études en plein développement qui, à la longue, jetteront une lumière tout à fait nouvelle sur l'histoire des espèces. Malheureusement ces primates sont à la fois les proches parents de l'homme et aux antipodes de lui. Leurs conditions de vie présentes diffèrent notablement de celles qui prévalaient à l'époque où les hominiens se sont formés en tant que genre biologique indépendant. En effet, ces diverses familles de simiens ne sont pas nos ancêtres : elles résultent d'une évolution entièrement différente et qui prend son point de départ dans d'autres espèces. N'oublions pas, par ailleurs, que les observations faites sur les sociétés de primates les appréhendent dans un milieu qui, outre ses écarts climatiques et géomorphologiques par rapport à cette période reculée, est marqué par la présence de l’homme. Pour ne pas insister sur la découverte des variations intraspécifiques de leur organisation sociale, qui suppose que la saisie de celle-ci en des points particuliers de l'espace et du temps devrait tempérer toute généralisation prétendant retrouver l'organisation sociale d'une espèce tout entière partout et toujours. Force nous est de nous appuyer sur une hypothèse d'universalité et de nous référer aux observations sur les primates actuels à titre d'analogie. Elles sont le support d'un modèle possible de notre passé, et non pas un témoignage sur celui-ci.

Ce que je viens de noter pour une première source d'information est partiellement vrai pour la seconde, à savoir les témoignages archéologiques, fossiles et géologiques qui aboutissent à reconstituer les formes de vie, l'environnement, les caractères anatomiques des espèces et surtout des hommes. Les moyens de datation moderne nous fournissent, certes, les données chronologiques les plus précises. Les indices que nous employons sont, en revanche, entachés d'ambiguïté. Il est difficile de conclure, avec assurance, lorsqu'il s'agit de décider si certains types organiques expriment la spécificité d'une espèce humaine particulière ou d'un nouveau genre, par exemple. La connaissance du volume d'un crâne ne nous donne pas le droit d'en inférer quoi que ce soit sur le degré d’intelligence, l'aptitude au langage, de l'individu auquel il appartenait. Chacun reste libre de bâtir l'interprétation qui lui convient — ce qui explique que les ro- 
mans sur les origines ne soient pas rares, même s’ils reprennent les uns des autres une trame usée de thèmes identiques. Il y a peu de chances qu'il arrive pour les spéculations sur les espèces hominiennes anciennes ce qui est arrivé à celles sur la biosphère des planètes qui, sur le plan géomorphologique, représentent une forme de notre biosphère. Pour l'instant, les fusées ne voyagent pas suffisamment loin dans l'espace et dans le temps pour aborder un corps céleste où se trouverait logée une espèce qui nous reconnaisse pour son avenir et à qui nous fassions rendre gorge pour figurer notre passé. Quoi qu’il en soit de ces hésitations, il est évident que les témoignages connus nous obligent à envisager l'histoire de l'homme comme histoire de plusieurs espèces, l'homo sapiens étant l'une d'entre elles, et à remonter au-delà de quelques dizaines ou quelques centaines de milliers d'années. Et, malgré les controverses, je m’en tiendrai par la suite aux datations ou aux descriptions qui sont les plus conventionnelles.

La technologie constitue la troisième source. Elle classe les outils des diverses époques, les date, étudie les différences entre eux et leur distribution, etc. Là nous nous trouvons en présence d'un squelette sans corps et surtout d'un squelette incomplet. Tout ce qui était fait de bambou, de bois, matériaux largement employés, a disparu. On ne saurait apprécier le degré d'élaboration d'un outillage uniquement à partir des matériaux qui se sont conservés (pierre, os). Nous ne pouvons donc pas juger de l'habileté des premiers hommes en nous basant sur les seuls artefacts qui ont résisté à l'assaut du temps, quand nous savons qu'ils employaient surtout des substances périssables. La situation est aggravée par le manque d'information sur les activités connexes. Qui nous dira quelle a été la place exacte de ces divers outils dans le système général de production? Nous ne savons même pas quelle était leur fonction précise. Lorsque nous trouvons des pierres non écaillées, nous pouvons émettre deux hypothèses : (a) leurs possesseurs ne savaient pas fabriquer des outils ; (b) ils ne savaient pas fabriquer des outils en pierre (car les pierres non écaillées peuvent parfaitement bien servir à fabriquer des outils de bois ou de bambou). Il nous est impossible de décider entre ces deux hypothèses. Mais il y a plus grave. L'étude de la technologie préhistorique, comme du reste l'étude de la technologie historique, se limitant à l'artefact, à l'inventaire de la « civilisation matérielle », est sommaire tant qu'elle ignore l'habileté, les savoirs, les gestes corporels qui l'ont accompagnée. Cependant l'habileté, les savoirs, les gestes corporels ne doivent pas être considérés en eux-mêmes : ils sont unis aux moyens intellectuels et physiques dans et par l'organisation du travail. Pour l'instant la technologie préhistorique, à de très rares exceptions près, est de l'antiquariat et a donné lieu à peu de généralisations vraiment théoriques. Une nouvelle analyse de l'infrastructure productive ouvrira la voie à la compréhension des collectivités qui ont su découvrir pour nous le langage des pierres, du bois ; des espèces végétales et animales, le langage de la nature, eu même temps que le langage de l'homme. Jusqu’à plus ample informé, les réserves les plus grandes s’imposent. 
La quatrième source d’information, ce sont les sociétés qui ont résisté le plus longtemps à l'érosion du temps et qui semblent suffisamment différentes ou éloignées des sociétés qui prédominent aujourd'hui pour pouvoir être considérées comme des objets. Ces sociétés, que l'on qualifie par un écart ou par un manque — sans histoire, sans écriture, sauvages, élémentaires, naturelles, etc. - et qu'il conviendrait plutôt de percevoir par les propriétés de leur système, sont des sociétés de parenté. De manière fort compliquée, elles entretiennent une relation avec les premières sociétés qui furent spécifiquement humaines quant à leurs structures, codes, occupations, hiérarchies et volume. Avec quelque complaisance, nous pouvons supposer trouver sous nos yeux une réalité qui était pleinement épanouie avant que notre «culture » isolât ou oblitérât ces échantillons de la "nature ». Nous commettrions une erreur à y voir des collectivités primitives ou des collectivités à l'état primitif : elles ont effectué une longue marche historique avant d'atteindre la forme que nous leur connaissons et avant que nous les rendions méconnaissables à elles-mêmes comme à nous. Car, il faut bien l'avouer, ce que nous connaissons, ce sont des systèmes sociaux qui ont perdu leur équilibre et leur dynamisme, qui ont évolué, à notre contact, pour devenir l'ombre de ce qu'ils ont été, puisque nous avons voulu les rendre naturels en leur ôtant la culture, le mode de vie qui leur étaient propres. Après les avoir mutilés comme la soldatesque, les barbares ou le fanatisme religieux ont mutilé monuments et villes, nous leur avons envoyé les anthropologues, comme nous déléguons les historiens, les archéologues, non pour les sauver mais pour sauver leurs témoignages et reconstruire avec peine ce qui avait été saccagé sans retenue. On s’est beaucoup intéressé à leurs mythes, leurs religions et leurs mariages, beaucoup moins au tissu essentiel de leur existence ; et peut-être ces sociétés se sontelles adaptées à nous en exagérant ces aspects de la vie collective, en se rendant plus exotiques que nécessaire, pour venir au-devant de notre désir. Ne voyons-nous pas aujourd'hui des régions entières devenir exclusivement « artisanales » et " agricoles » pour répondre au rêve d'une civilisation urbaine de se donner une réserve naturelle et un passé d'innocence manuelle ? Il est probable que ces peuples conquis se sont adaptés de la même façon ; pour cette raison ils sont, en grande partie, nos contemporains et notre produit. Rien ne nous garantit, sans plus, qu'ils se trouvent sur la même lignée historique que nous, que nous sommes leur avenir obligatoire ou qu'ils sont notre passé. Seul un pari nous incite à les décaler dans le temps et à juger légitime une comparaison à trois termes entre ces sociétés de parenté et les sociétés de classe, d'un côté, les sociétés de parenté et les sociétés d'affiliation des primates non humains, de l'autre. Un tel rapprochement, quels que soient les artifices qu'il met en œuvre, nous donne l'impression, qui répond à un besoin impérieux, que nous sommes en mesure de parcourir, étape par étape, les transformations qui ont conduit du monde animal au monde humain.

Les primates d'aujourd'hui pour l'analogie, les fossiles et les témoi- 
gnages archéologiques dûment reconstitués, les sociétés de parenté à des fins de comparaison, et la technologie intégrée au tissu des habiletés, sont autant des réalités donnant à penser que les substrates d'une pensée sur la réalité. Ils nous entraînent à proximité des facteurs qui ont probablement participé, au cours de l'évolution, à un développement qui connaît les hommes pour sa matière première et qui prend ses acteurs dans la société et dans la nature. Nous n'en savons pas davantage, et il se peut que par la suite nous en prenions une connaissance meilleure mais non pas plus ample. Vu l'impossibilité de décider, parfois, faute de matériaux sûrs, entre les diverses hypothèses, ce que j'expose dans ce livre se fonde sur le libre jeu qui en résulte. Je voudrais insister sur le fait que, malgré les manques et les insuffisances inhérents aux sources utilisées, les propositions que j’avance ne sont pas entièrement hypothétiques. Elles ont été formulées de manière à y inclure, même si ce n'est pas toujours signalé, les paramètres de sécurité que fournit une meilleure connaissance des accidents et des détours du terrain sur lequel on progresse. Retour au texte

\section{$\underline{\text { Retour au texte }}$}

Note 2 : S.A. Altman : A Field Study of the Sociobiology of Rhesus Monkeys, Macaca Mulatta, Annals of New York Academy of Science, 1962, 102, 2, 238-435. - C. R. CARPENTER (ed.) : Naturalistic Behaviour of Nonhuman Primates, Philadelphie, 1964. - M.R.A. CHANCE : Social Structure of a Colony of Macaca Mulatta, British Journal of Animal Behaviour, 1956, 4, 1-13. - J.H. CROOK : Evolutionary Change in Primate Societies, Science Journal, 1967, 3, 6, 66-72. — J.H. CROOK (ed.) : Social Behaviour in Birds and Mammals, Londres, New York, 1970. - J.H. CROOK et P. ALDRICH-BK-LACK : Ecological and Behavioural Contrasts between sympatic ground-dwelling Primates in Ethiopia, Folia primatologica, 1968, 8, 192-227. - J.H. CROOK et J.S. GARTLAN : Evolution of Primate Societies, Nature, 1966, 210, 1200-1203. — I. DE VorE (ed.) : Primate Behaviour : Field Studies of Monkeys and Apes, New York, 1965. - Th. Dobzhansky : Cultural Direction of Human Evolution, Human Biology, 1963, 35, 311-316. - J.S. GARTLAN : Structure and Function in Primate Society, Folia Primatologica, 1968, 8, 89-120. — K.R.L. HALL : plusieurs articles in I. DE Vore (1965) et P. JAY (1968). — P. JAY (ed.) : Primates : Studies in Adaptation and Variability, New York, 1968. - H. KuMMER : Social Organisation of Hamadryas Baboons, Bibliotheca Primatologica, 1968, 6, 1-189. - H. KUMMER : Two Variations in the Social Organisation of Baboons, in P. JAY, op. cit. - H. KUMMER et F. KURT : Social Units of a free-living Population of Hamadryas Baboons, Folia Primatologica, 1963, 1, 4-19. - W.A. MASON : Sociability and Social Organisation in Monkeys and Apes, Advances in Experimental Social Psychology, 1964, 1, 277-305. - J.J. PETTER : Recherches sur l'écologie et l'éthologie des Lémuriens malgaches, Mémoires du Musée national 
d'Histoire Naturelle, 1962, Série A, 27, 1-46. — T.E. RowELL : Variability in the social Organisation of Primates, in Primate Ethology (D. MORRIS, ed.), Londres, 1967.

Ces articles et livres forment le principal des études empiriques et théoriques que j'ai analysées et exposées dans cette partie de mon travail. Bien que j'en diffère sur quelques points de terminologie (très lâche et trop descriptive par ailleurs, dans ce domaine) et d'interprétation, je partage pour l'essentiel les opinions de Crook (1970) et Hall (1968). Je crois qu'elles devront être profondément remaniées, non seulement à cause de leur désaccord avec les faits mais aussi à cause de leur grande simplicité, et, paradoxalement, de leur congruence avec le bon sens scientifique. Pourtant on y trouve une formulation claire des problèmes, une exigence conceptuelle et une liberté par rapport aux notions établies qui sont de bon augure. Retour au texte

\section{$\underline{\text { Retour au texte }}$}

Note 3 : Outre les ouvrages et articles mentionnés en 3, j’ai puisé toute une série d'informations dans les articles suivants. Leurs auteurs font preuve de beaucoup de doigté quant au choix des matériaux empiriques. A de rares exceptions près, ils manquent cependant d'une formation propice à encourager une analyse très poussée des systèmes sociaux.

M.R.A. CHANCE : Attention Structure as the Basis of Primate Rank Order, Man, 1967, 2, 503-518. - W. ETKIN : Social Behavioral Facts in the Emergence of Man, Human Biology, 1963, 65, 299-310. — R.A. HindE et Y. Spencer-Booth : The Behaviour of socially living Rhesus Monkeys, in their first two-and-a-half-years, Animal Behaviour, 1967, 15, 169-196. - K. IMANISHI : Social Organisation of Subhuman Primates in their natural Habitat, Current Anthropology, 1960, 1, 393-407. — J. ITANI : Paternal Care in the wild Japanese Monkey Macaca Fuscata, Primates, 1957, 2, 61-93. - G.B. KofORD : Rank of Mothers and Sons in Bands of Rhesus Monkeys, Science, 1963, 141, 356-357. — G.D. MitchelL : Paternalistic Behaviour in Primates, Psychological Bulletin, 1969, 7, 399-417. - V. REYNOLDS : Kinship and the Family in Monkeys, Apes and Man, Man, 1968, 3, 209-223. - V. REYNOLDS : Open Groups in Hominid Evolution, Man, 1966, 1, 441-452. - T.E. HowELL : Hierarchy in the Organisation of a captive Babbon Group, Animal Behaviour, 1966, 14, 420-443. - D.S. SADE : Some Aspects of Parent-Offspring and Sibling Relations in a Group of Rhesus Monkeys, with a Discussion of Grooming, American Journal of Physical Anthropology, 1965, 23, 1-18. - VAndenBergh : The Development of Social Structure in Free-ranging Monkeys, Behaviour, 1967, 29, 179-95. Retour au texte 


\section{Deuxième partie : \\ La nature de I'homme}

$\underline{\text { Retour à la Table des Matières }}$

Examen de la question de savoir si la nature ne s'est pas profondément transformée à mesure que croissait la culture.

Novalis, Werke, t. III p. 221. 


\section{Chapitre III.}

\section{Le nouveau monde animal}

\section{Dans le no man's land : hominisation ou cynégétisation.}

\section{Retour à la Table des Matières}

L'art de la restauration du passé est le plus ancien et le plus audacieux (note 4). Avec peu d'éléments, il arrive à peupler tout un univers, à l'animer, bien que rien ou presque ne subsiste des acteurs qui ont effectivement occupé la scène. Deux ou trois dizaines de groupements étudiés sont la base de toutes nos connaissances sur les sociétés de primates. Si on leur ajoute quelques centaines de fragments de crânes et de squelettes, un millier de tonnes de pierres et d'ossements, une centaine de sites explorés, on a dressé l'inventaire à peu près complet des témoignages dont nous disposons pour parler d'un accident aussi capital à nos yeux que la venue à l'être de l'homme. Il a eu lieu dans une zone incertaine du temps et de l'espace, sur laquelle nous pouvons faire des conjectures à loisir, et où plusieurs espèces ont tenté, selon toute vraisemblance, une aventure analogue. Notre espèce y a parcouru la distance à la fois courte et infinie qui la sépare des créatures avec lesquelles elle avait et continue à avoir tant de choses en commun. De quelle façon décrit-on le mouvement propre à une grande partie des anthropoïdes alors vivants qui les a détachés du monde animal, la frontière invisible qu'il a instituée ? Le changement du climat, de la faune et de la flore, poussant une partie de nos ancêtres simiens à quitter l'habitat riche et protecteur de la forêt pour les zones moins clémentes de la savane paraît avoir joué le rôle de cause première. Lentement, ils ont dû rompre avec leurs congénères et garder par devers eux uniquement les facultés propices à la poursuite d'une existence plus diverse et plus dangereuse. Le processus de sé- 
lection naturelle y a conduit par le moyen ordinaire des mutations génétiques favorables et des adaptations écologiques heureuses. Seul l'aboutissement, qu'il s'agisse du pouvoir cérébral, technique ou intellectuel, s'est avéré exceptionnel. Autour d'une de ses facultés, lorsqu'elle eut atteint sa pleine maturité, se sont individualisés les hominiens, s'est formé un ensemble organique original. Les savants s'efforcent avec patience de définir celle qui a été déterminante et a marqué, par son unicité, la transition vers une nouvelle classe d'êtres vivants. Ils ont successivement proposé : le volume du cerveau, la station debout, le langage, la capacité de fabriquer des outils. La créature l'ayant manifestée en premier fut vraiment humaine. Depuis, en l'élaborant, les hommes se sont éloignés à grands pas du règne animal et ont enrichi leur équipement anatomo-physiologique d'un équipement culturel : " adaptation biologique, dont les modes de transmission ne sont pas génétiques, et qui complète dans une grande mesure l'évolution somatique ${ }^{20}$.

Chacune de ces facultés a ses partisans. Les faits réunis et les arguments théoriques que l'on expose afin de montrer que l'une d'elles prédomine sont censés prouver son absence dans la plupart des espèces et sa présence dans la nôtre, donc la discontinuité radicale ainsi introduite. Lorsque les contrastes l'emportent sur les analogies avec les primates actuels ou disparus, on est sûr de tenir une démonstration définitive en faveur de celle pour laquelle on a pris une option. Un pelvis rectifié pour marcher, une pierre cassée à côté d'un fémur, une voûte crânienne suffisamment grande donnant a penser que son possesseur a pu prononcer une parole, font déclarer : voici l'homme ! Sa spécificité - bipède, pensant, faiseur d'outils, langagier, etc. — est du coup assurée. L'hominisation coïncide, dans cette optique, avec l'émergence, au sein de la vie animale, d'un organe ou d'un trait signalant la cristallisation d'une catégorie biologique supplémentaire se superposant, à la place la plus haute - mais là nous sommes juge et partie — sur l'échelle des créatures aux catégories qui existent déjà. Nous apprenons ainsi sur quelle dimension une quantité additionnelle a fait pencher la balance vers une animalité, vers un anthropoïde qui ne sont plus quelconques. Par des retouches successives, continuelles,

20 J.N. SPUHLER : The Evolution of Man's Capacity for Culture, Detroit, 1959, p. 1. 
le travail de l'évolution, comme le travail du peintre qui, à travers de multiples esquisses, dégage les contours de ses personnages, a eu cet effet, en modifiant un à un les caractères biologiques jusqu'à les rendre humains. Du singe-homme à l'homme-singe et de celui-ci à l'homme-homme, les essais ont été nombreux et quasi indiscernables. A partir d'un seuil, d'un endroit, d'un instant, le Rubicon, suivant l'expression césarienne des anthropologues, a été franchi sans espoir de retour vers l'horizon simien, et notre espèce s'est trouvée achevée.

Malgré l'ingéniosité des reconstitutions et la variété des détails, cette vue achoppe sur des obstacles et paraît occulter des phénomènes essentiels. Est-ce dû à l'impression persistante qu'un choix arbitraire préside à la description d'un déroulement nécessaire ? Car, à bien y réfléchir, le caractère différentiel motivant ce choix que l'on cherche à isoler n'est qu'une possibilité parmi d'autres, celles-ci probablement équivalentes. Ou bien est-ce parce que la théorie enseigne en quoi et par quoi la marche vers l'homme est une terminaison, un dépassement, et non pas en quoi et par quoi elle est un commencement ? De Darwin nous avons hérité la tâche de comprendre comment notre espèce descend du singe. Une fois cette tâche accomplie, nous n’apprenons pas nécessairement comment a lieu la «montée » — si les expressions monter, descendre ont un sens - vers l'homme, puisque toutes les espèces qui en sont descendues, tel l'australopithèque robuste, n’y sont pas parvenues. La réalité visée par le concept d'hominisation, de son côté, a perdu de sa cohérence et s'est diversifiée. Son contenu, se référant aux propriétés organiques individuelles, cantonné dans l'ordre biologique, impliquait une évolution autonome de celui-ci comme condition préalable à toute transformation technique et culturelle. La croissance du cortex cérébral au-delà du niveau anthropoïde devait précéder la station debout, l'invention de l'outil, de la même façon que l'aptitude à marcher droit devait précéder l'aptitude à courir. Les archives géologiques et paléontologiques ont laissé voir depuis peu que les événements se sont déroulés dans le sens inverse de celui que l'on attendait. Certes, du point de vue fonctionnel, la taille et l'organisation du cerveau et des parties du corps sont toujours les facteurs déterminant les structures techniques ou culturelles qui nous sont propres. Du point de vue historique, toutefois, ce sont ces structures qui ont façonné le cerveau et les parties du corps. La cause s’est muée en effet et réciproquement. D’autre part, 
l'interférence des phénomènes sociaux avec l'ordre biologique le dote d'une dimension méconnue ou systématiquement ignorée. Ils sont reconnus à la fois exceptionnels, producteurs d'un être d'exception, l'homme, et confinés dans un rôle dérisoire de doublure des mécanismes génétiques ayant pour seule conséquence un meilleur rendement adaptatif. Envisagée de manière aussi parcellaire, leur mission se réduit à figurer la chiquenaude initiale, lorsque le besoin s'est fait sentir d'aiguiller le train organique sur une autre voie. La possibilité qu'ils aient introduit un principe différent dans le développement d'ensemble n'a été ni mise à l'épreuve ni exclue. Devant le renversement de la chaîne des causalités, l'irruption massive de preuves techniques et sociales dans le phénomène d'hominisation où elles n’avaient aucune place, la démarche normale aurait été, est une remise en question des attitudes et des prémisses conceptuelles initiales. Au lieu de cela, pour atténuer le choc provoqué par les divers remaniements de la carte du réel, on a proposé un mélange éclectique subtil d'interprétations en fonction de la sélection naturelle, et, là où elles s'arrêtaient, de justifications anthropologiques : le véritable réexamen s’est trouvé retardé d'autant.

Et d'abord la convergence des faits réputés têtus, impatients des vérités sans souffle et des erreurs partagées, le rendent inévitable. Les modifications écologiques portent, dans les explications usuelles, la responsabilité majeure de ce qui nous est advenu. Assurément des corrélations existent entre un environnement et la configuration biologique et sociale qu'il abrite. Cependant leur portée dépend étroitement du niveau d'évolution auquel on se trouve. La simple taille physique de l'animal intervient déjà : plus cette taille est grande, moins l'animal subit le contrecoup des variations qui ont le milieu pour théâtre. Au cours de la diffusion d'une espèce, les fluctuations géomorphologiques sont un danger pour une espèce de stature réduite et peuvent rester sans effet sur une autre, plus massive. Le caractère catastrophique des transformations ou des pressions écologiques n'est donc pas uniforme : les dimensions de l'organisme affecté déterminent leur part de répercussion sur le potentiel reproductif. En ce qui concerne les premiers hominiens, ces transformations et ces pressions ne sont pas contraignantes, pour un motif qui nous est déjà connu. Des simiens ayant des propriétés anatomo-physiologiques opposées subsistent parfaitement dans des environnements identiques et vice versa. Les réor- 
ganisations sociales suffisent parfois, en l'absence de réorganisation génétique, à réaliser les adaptations requises. Constat qui a incité certains savants, notamment Ronald Hall, à nous mettre en garde contre la propension à vouloir rendre compte d'un comportement des espèces humaines disparues à partir de l'information maigre et schématique dont nous disposons sur leur écologie. A fortiori contre la prétention d'en déduire le caractère indispensable de tel ou tel organe ou capacité et de lui faire porter le poids d'une rupture radicale avec le reste du monde animé.

Le temps qu'a pris la naissance de l'homme est beaucoup plus considérable qu'on ne l'a pensé. Le point de référence obligé demeure théoriquement l'animal non humain. Historiquement, nous le trouvons être un autre animal humain. Notre espèce procède, en effet, d'une lignée d'évolutions, très nombreuses, étrangère à la lignée des primates contemporains. La différenciation des anthropoïdes se situe à la période oligocène, il y a environ quarante millions d'années. Au miocène, vingt millions d'années plus tard, le proconsul était encore l'ancêtre que nous avions en commun avec les chimpanzés et les gorilles. Depuis, ces derniers, à travers le dryopithèque, ont débouché sur le mode moderne d'existence dans la forêt. Parallèlement, avec le ramapithèque, les hominoïdes se sont diffusés, ont pris leur essor. Ils serrent de près le cycle évolutif qui, il y a environ deux ou trois millions d'années, s'est concrétisé en deux espèces d'australopithèques, dont l'une au moins était omnivore et habile. L'homme de Pékin et de Java (homo erectus) leur a succédé, cinq ou six cents millénaires avant notre ère. L'homme moderne n'a pas plus de cinquante ou cent mille ans. Les incertitudes ne manquent pas sur la validité de cette chronologie, sur la coexistence des divers embranchements, sur les raisons de leur disparition. A proprement parler, nous ne savons pas quel est l'ancêtre " vrai » du seul héritier du genre, l'homo qui s'est proclamé lui-même sapiens. Il s'est différencié à l'intérieur d'un mouvement qui avait déjà sa spécificité. L'hominisation a dû se produire à plusieurs reprises et de diverses manières suivant les espèces et suivant la période où elles se sont affirmées.

L'usucapion d'un trait unique n'a pas pu garantir la perdurabilité et le détachement d'avec le règne animal des groupements humains qui ont éclos et se sont éteints au cours de ces millions d'années. Il s'est 
inscrit dans un développement d'activités plus complexes et plus profondes.

Par ailleurs, toutes les fois que l'on a cru tenir un tel trait, il a fallu reculer. A peine venait-il d'être choisi, délimité, proposé pour unique, qu'un examen méticuleux en montrait le caractère précaire et conventionnel. De cette manière, on a renoncé aux critères différentiels anatomiques : la station debout — bien que ce ne soit pas le plus mauvais - le volume du cerveau, la main. Les facultés du langage ou de la fabrication des outils, a-t-on estimé, avaient des chances de s'imposer souverainement. Là aussi le désenchantement est venu. Le langage, malgré son importance capitale, est un indice incertain. Suivant la définition adoptée, il peut être jugé du ressort exclusif de l'homme, ou diffus parmi les primates, à titre de système de signes indispensables à la communication. Conjointement une observation poussée du comportement animal, des témoignages paléontologiques et éthologiques ont révélé l'existence d'un savoir-faire instrumental chez plusieurs espèces vivantes ou disparues. Le savant britannique Oakley a suggéré, implicitement, de renoncer à fonder notre originalité sur un trait différentiel tellement imparfait. D’ailleurs, qu'est-ce qui nous autorise à choisir un trait pour marquer la transition du monde animal au monde humain ? Puisque, à première vue, cette transition a dû et pu se produire à plusieurs reprises et de diverses manières selon les espèces, rien ne nous empêche d'imaginer que leur organisation collective et leurs rapports avec le milieu ont accentué ici le poids d'un trait et là le poids d'un autre trait, ici la station debout, là le langage, etc. Bref, nous n'avons pas de raisons très fortes, ni de les rendre singuliers, ni de les faire exclusifs les uns des autres, sinon la conviction d'une vérité douteuse et qui, de toute manière, appelle une refonte, la croyance en une genèse de l'humanité à point de départ et d'arrivée unique sur tous les plans.

L'anthologie de ces indices anatomiques, techniques ou intellectuels exprime une tendance à projeter rétrospectivement une vision de l'homme formulée après coup, à la justifier en réduisant la diversité des solutions - les unes réelles, les autres de simples possibilités, qui sont en même temps un passé et beaucoup plus - à une solution unique, à enfouir sa particularité historique sous l'universalité organique. Comme si, ayant gagné la bataille sur le signe des origines, on déte- 
nait la clé d'une détermination essentielle qui, depuis, n’a plus été remise en question, redéterminée. Comme si l'important était de reconnaître l'identité des éléments et non pas la structure où ils s'inscrivent, le processus qui les engendre. Comme si, étant en mesure de dire ce qu'est l'homme en un point de l'espace et du temps à partir d'une de ses propriétés, on définissait ce qu'il est partout, pour toujours. A cet égard, nous suivons, c'est la sagesse même, une maxime qui nous convie à comprendre l'inférieur par le supérieur, à éclairer l'anatomie du singe par l'anatomie de l'homme ; on ne saurait contester que cette règle mérite d'être appliquée. Néanmoins, dans le domaine des origines, où les apparences scientifiques enveloppent un noyau très compact de valeurs, la compréhension de l'inférieur par le supérieur devient une compréhension de l'inférieur pour le supérieur. L’homme langagier, cérébral est l’homme blanc, civilisé, résultat de la division, inconnue il y a cinq mille ans, du travail manuel et du travail mental, de l'écart entre l'homo faber et l'homo sapiens, qu'une tradition humaniste et religieuse s'emploie vigoureusement à maintenir. La fixation sur l'outil et l'homme technique, gloire de l'industrie, prolonge la conception un peu courte résumée par Benjamin Franklin à l'aube de l'ère mécanique, dont on ne sait si elle constitue le dernier hommage rendu à l'artisan ou la première reconnaissance du pouvoir de l'ingénieur. Partant, l'apparition de notre espèce n'aurait pu avoir d'autre cause qu'une révolution industrielle : « Le premier pas a constitué l'équivalent de la première révolution industrielle ; car il impliquait non seulement que l'on invente la fabrication des outils, mais encore qu'elle se transmette et se perpétue en tant que tradition industrielle $^{21}$. " On imagine dès lors que chaque hominien a vécu et concentré ses opérations habiles autour de la pierre, sa brisure, son polissage, comme chaque ouvrier moderne se confond avec sa machine. Il en résulte une distorsion de la réalité, une attention disproportionnée accordée aux artefacts dans un contexte historique où leur fonction effective était relativement subordonnée. Mais on commence à être prévenu au sujet de ces incongruences : "Pendant une grande partie de ce siècle, écrit un anthropologue ${ }^{22}$, les historiens de la préhistoire ont limité leurs recherches à l'étude des outils de pierre ; les faits que renferme la préhistoire de l'Ancien Monde au pléistocène en

21 A. Montagu : The Human Revolution, Cleveland, 1965, p. 41.

22 C.L. ISAAC, in LEE et DE VORE : Man the Hunter, op. cit., p. 253. 
sont venus à être exprimés exclusivement sur le mode technique en fonction de la morphologie des outils de pierre. L'examen des activités autres que la fabrication des outils a tendance à devenir un domaine secondaire qui ne mérite pas de loin le respect dû à l'interprétation d'artefacts choisis. Visiblement les résultats d'études fort techniques des artefacts ne peuvent apporter qu'une contribution limitée à notre intelligence de l'évolution du comportement humain dans son ensemble. »

La totalité se révèle si l'on emprunte la direction opposée à celle qui a été suivie, en renouvelant le sens des questions posées. Les diverses caractéristiques ou facultés associées à l'hominisation sont interdépendantes, liées quant à leur évolution et, pratiquement, redondantes. Ce sont des chaînons d'un développement unique : le bipédisme facilite l'individuation de la main, la main humaine porte la marque des artefacts et de leur prolifération, l'accroissement volumétrique du cortex entraîne l'émergence d'un langage articulé qui est à son tour conséquence de l'invention d'instruments et de techniques servant à attaquer et se défendre. Il y a aussi simultanéité et répétition des genèses. Le bipédisme de la course, la main nue qui cueille ou attrape les animaux sont contemporains ; ils précèdent le bipédisme de la marche, la main instrumentée capable de tuer une grande proie, de construire un piège ou un abri. Parler de la station debout ou de la différenciation des membres supérieurs et inférieurs comme d'événements uniques, signalant un commencement absolu, n'offre guère d'intérêt, si nous ne savons pas de quelle station debout et de quelle différenciation d'organes, envisagées à un stade précis, il s'agit ; nous comptons des éléments hors d'un ensemble, une succession séparée des interactions qui la rendent possible. La manifestation simultanée et la cohérence évolutive des capacités organiques et techniques laissent entrevoir, à l'arrière-plan, un système d'actions susceptible de les avoir provoquées, orientées, assujetties à l'environnement. Afin de survivre, de se conserver à côté des autres espèces, l'animal humain a bricolé le bois et la pierre, accumulé les ruses, essayé de mieux communiquer avec ses congénères. Au-dessus de tout, il a pris, en quelque sorte, pour matière première et outil principal, son propre corps, lui imprimant le savoir-faire constitué de gestes, de coordinations rythmiques et de schèmes perceptifs. La coopération sociale a accompagné la spécialisation anatomo-physiologique, 
facilitant une combinaison inédite des appareils sensoriels tactiles, visuels et auditifs. Si l'on voulait chercher une formule, elle est là, toute prête : l'homme est un faiseur d'homme, au sens réel, concret, puisqu'il se reconnaît et se saisit en tant que son propre produit, son propre objet. Il est vraisemblable que, dans la période de transition considérée, les techniques essentielles furent, selon l'expression de Marcel Mauss, les techniques du corps, du corps individuel et social. Je suis certain qu'une étude minutieuse confirmerait, jusque dans les sociétés sans écriture d'aujourd’hui, la place capitale occupée par ces techniques comparées aux techniques instrumentales.

Dire cependant que l'homme est un faiseur d'homme, c'est succomber, une fois de plus, à la fascination d'un aphorisme à prétention universelle. Il suffit de le presser un peu pour se convaincre de la faible part de vérité qu'il enferme. Il n'est pourtant pas complètement inutile si on le replace dans le temps où, au sens strict, matériel, il a été valide. Alors les hommes se sont faits hommes en se préparant à leur tâche de chasseurs. En d'autres termes, ils ont essayé d'acquérir des aptitudes, des méthodes aux contours précis, d'entrer en contact avec une partie déterminée du milieu, entreprise qui les a entièrement transformés génétiquement, socialement, technologiquement. Ils se sont trouvés dans l'obligation de faire une nouvelle unité entre eux, et une nouvelle unité avec le monde matériel, qui est naturelle, servant à promouvoir les fonctions de toute espèce : se reproduire, se propager, utiliser et renouveler les ressources. Elle s'impose à tous les éléments composants, a une présence historiquement située, dépasse le domaine des instruments, de la subsistance, touche aux mythes, au rituel, aux échanges émotionnels et intellectuels entre individus. Unité naturelle, aussi, parce qu'elle recouvre les hommes - prédateurs et chasseurs — s'étendant à plusieurs espèces coexistantes (l'australopithèque robuste et l'homo habilis) ou successives (l'homo erectus et l'homo sapiens). Son affermissement amène l'homme à s'opposer aux primates et à s'en éloigner. Mais c'est en tant qu'ils sont liés au monde végétal et en dépendent, et non en tant qu'ils sont primates.

Pendant plusieurs millions d'années, l'homme s'est forgé un corps, s'est répandu à la surface de la terre, s'est affermi et reconnu en qualité de chasseur. Le reste lui est venu autant par nécessité que par surcroît. Cette constatation a pour effet d'allonger l'échelle et d'élargir la 
grille à l'aide desquelles on envisage son passé. Nous avons pris l'habitude de nous limiter à un laps de temps trop bref, à une expérience trop proche de la nôtre. Ainsi nous avons projeté moins d'un dixième d'une longue histoire sur les neuf dixièmes restants. Il ne serait pas moins instructif de procéder à l'exercice inverse, car il est presque absurde d'imaginer les traces d'un tel parcours effacées au point que notre station présente leur soit irrémédiablement étrangère.

Il s'agit de chasse, du monde animal dont nous rejetons l'affinité, qui nous répugne non domestiqué ; mais les premiers hominiens n'avaient pas à s'en dissocier et ne le voulaient pas. Au contraire, leur grande affaire était de multiplier et d'enrichir les liens tissés avec ce monde, de s'y établir solidement. De même que nous pénétrons les arcanes des réactions chimiques, des fissions nucléaires, il leur importait de pénétrer les secrets du bison, du cheval ou du daim. En abandonnant la forêt pour la savane, ils n’ont pas quitté une aire géographique pour une autre, comme l'ont fait bien des anthropoïdes : ils sont allés vers ce monde animal qui les attirait. Au lieu de se situer audessus de lui, ils l'ont marqué, créant un rapport distinct de lui à eux. A terme, les espèces avec lesquelles on cohabitait dans l'indifférence mutuelle - c'est le cas aujourd'hui encore pour les babouins avec les lions ou les antilopes - se sont transformées en partenaires actifs, composantes d'un milieu ambiant, tenants d'une ressource qu'il fallait conserver et faire fructifier.

S'ils sont parvenus à leurs fins, nous savons que l'impulsion ne leur est pas venue de l'extérieur, ne s'est pas imposée à une espèce dépourvue d'initiative. L'avantage initial ne peut non plus être résumé en disant que cette espèce était plus grande, plus intelligente ou plus bavarde que les espèces qui sont devenues ses proies ou ses agresseurs. Seulement son effort se déploie à l'intérieur d'un mouvement déjà commencé, s’écartant du mouvement général, pendant lequel les rudiments de l'histoire s'infiltrent dans l'évolution, dotent les comportements appropriés d'une résonance qu'ils n'avaient pas auparavant. Dans cette conjoncture, nous savons qu'à l'instar de toutes les espèces supérieures elle est capable d'exercer une pression sur le milieu, non seulement par ses qualités biogénétiques mais aussi grâce à son organisation biosociale. Les deux termes du développement - organisme et niche écologique — se façonnent et répondent à des forces, des in- 
citations qui naissent de la distribution fonctionnelle des membres de l'espèce, de leurs rapports collectifs. La brèche a été ouverte par quelques anthropoïdes : elle traduit le nouveau rôle du comportement social dans le cycle naturel. Les groupes hominiens qui ont raccordé les formes d'action ébauchées dans ces circonstances les ont extraites du contexte d'origine, synthétisées dans la prédation systématique ; les incluant dans leur corps et leur cerveau, bâtissant un modèle de vie et de réalité, ils ont poursuivi cette ouverture jusqu'au bout. De la sorte ils n’ont pas seulement acquis des qualités distinctes de celles des autres animaux, ils les ont surtout acquises autrement.

La portée de ce qui s'est accompli à cette occasion réside moins dans la séparation biologique réussie à l'intérieur d'un processus existant que dans le retournement de celui-ci ; elle est moins dans le contenu spécifique des propriétés organiques que dans le principe qui en assure la genèse. Si, au niveau élémentaire de la substance vivante, les organismes n'obéissent pas encore aux règles de sélection et d'adaptation, au niveau qui nous occupe ici ils ne leur obéissent plus qu'accessoirement. Ce qui arrive dans l'évolution est manifestement subordonné à ce qui arrive à l'évolution. Le rameau du genre humain fait donc surgir simultanément une classe biologique et un mouvement singulier dans la nature.

Le phénomène est assez remarquable pour mobiliser notre entière attention. Il suggère deux conduites. La première est de substituer à l'analyse d'une caractéristique anatomique, technique ou intellectuelle privilégiée, l'analyse d'un ensemble organisé, finalisé, la chasse ; de remplacer la recherche d'une manière d'essence universelle de l'homme par l'étude d'une de ses formes particularisée dans l'espace et dans le temps ${ }^{23}$.

« Une histoire de la chasse, on l'a dit avec beaucoup d'à propos ${ }^{24}$, lève la pénible ambiguïté qui entache tant de discussions courantes sur

23 Des travaux, peu nombreux, il est vrai, commencent à s’orienter dans ce sens (le volume publié par De Vore en témoigne). Ils restent cependant tous fidèles au cadre conceptuel ancien, au mélange éclectique de théories darwiniennes et de modèles anthropologiques. La description y trouve son compte mais non pas l'analyse théorique.

24 W.S. LAUGhlin, in LeE et DE VORE, op. cit., p. 304. 
l'importance des outils — ou, ajouterais-je, sur le langage ou la pensée - la question de savoir si l'emploi des outils veut dire que les outils emploient des humains ou que les humains emploient des outils. » La deuxième conduite est de viser au-delà d'un horizon théorique préoccupé uniquement de la place réservée à notre espèce sur l'échelle des êtres, des détails de sa définition et de son écart différentiel. S’en tenir là, à mi-chemin, cédant à l'esprit typologique, revient à estimer connu ce qui ne l'est point : la dynamique de son engendrement. Elle est spécifique, n'en doutons pas, disjoint ses acteurs, les hominiens en devenir, de la plupart des espèces. A travers ce qui la représente - la cynégétisation et non pas l'hominisation des primates, le devenir homme du chasseur et non pas le devenir chasseur de l'homme - je la considère comme étant au centre de mon enquête. Après tout, peutêtre n'est-il pas moins nécessaire de nous comprendre et de nous resituer dans une nature que nous avons faite que dans une nature qui nous a faits. 


\section{Populations, ressources et pressions sur l'environnement.}

$\underline{\text { Retour à la Table des Matières }}$

\section{Description liminaire de deux états stationnaires.}

Le point de départ obligé de l'étude est la description des relations entre les collectivités, humaines et pré-humaines, et leur milieu ambiant. A cette fin il est indispensable de fixer les traits d'une conjoncture idéale qui ne se réalise que rarement ou de façon temporaire, grâce à deux états d'équilibre ou d'harmonie.

Le premier de ces états suppose une correspondance exacte entre le nombre d'individus qui doivent exercer une fonction productive, sociale, et le nombre d'individus qui peuvent le faire, étant données la quantité de richesses exploitées et l'organisation établie de la société. Un groupement de cinquante ou cinq cents personnes, ayant une hiérarchie déterminée, un mode de distribution des fonctions et des biens, s'efforce de se renouveler en préservant le même taux de répartition, les mêmes dimensions. Il recherche l'assimilation, soit par naissance, soit par recrutement, dans le temps, d'individus capables d'assurer sa continuité. Dans le cas le plus simple - la chasse est un tel cas - la densité ou rapport quantitatif de la population au territoire occupé est un indice direct de son potentiel démographique. Les oscillations autour d'un certain volume ou d'une certaine densité sont monnaie courante. Les sociétés s'efforcent de maintenir une constance (note 5) des facteurs démographiques et matériels, interviennent dans l'intention d'assurer un recouvrement satisfaisant de leur structure et du fondement naturel de celle-ci ${ }^{25}$. Ce qui entraîne de leur part une réduction des écarts, soit par un appel à l'immigration et à des ressources inhabituelles lorsqu'il y a manque, soit par un interdit jeté sur tout apport de population, de ressources ou de savoir-faire nouveaux ou supplémentaires lorsqu'il y a excès.

25 J. HELM : The Ecological Approach in Anthropology, American Journal of Sociology, 1962, 67, 630-639. - A.I. HALLOWELL : The Size of Algonkian Hunting Territories, a Function of Ecological Adjustment, American Anthropologist, 1949, 51, 35-45. - G. BARTHOLOMEW et J. BIRDSELL : Ecology and the Protohominids, American Anthropologist, 1953, 55, 481-498. 
Le deuxième état d'équilibre traduit la correspondance des facultés, des savoir-faire qu'une espèce ou une collectivité possède, distribue entre ses membres, avec les forces matérielles, les ressources différenciées du milieu, de sorte qu'il n'y ait rien dans le contenu des premiers qui n'ait de contrepartie parmi les secondes et réciproquement, aussi bien en quantité qu'en qualité. Cette correspondance assure la stabilité des échanges avec le monde objectif, l'articulation des comportements ou des instruments reproduits avec les êtres animés ou inanimés qu'ils concernent. L’équilibre démographique est envisagé, à juste titre, sous l'angle des richesses en nourriture ou d'autres biens économiques permettant à une population de vivre dans une niche écologique. Mais l'abondance ou la rareté en soi ne sont pas seules à conditionner la reproduction. Ceci semble être généralement vrai pour les animaux et a été prouvé, par exemple, pour les rats. Une expérience a montré qu'en créant un environnement riche, fournissant de la nourriture en excès à une population de rongeurs, on ne voyait pas celle-ci se multiplier jusqu'à épuiser les ressources offertes par le milieu. On observe au contraire un plafonnement qui est fonction de l'espace où elle doit se mouvoir et du répertoire de conduites dont elle dispose. A la disponibilité s'ajoute un élément qu'on a tendance à négliger : la capacité à reconnaître, sélectionner, produire (ce dernier terme lorsqu'il s'agit d'hommes) ces richesses, les actions qui sont en rapport avec l'organisation du territoire, la défense, l'entraide des membres du groupe pour affronter un ennemi, un prédateur, par exemple, ou pour exploiter en commun un site. Le degré d'excès ou de manque en dépendent étroitement. On voit donc qu'il ne suffit pas de poser globalement une équation en mettant d'un côté l'organisation ou la population, et de l'autre côté le milieu ou les biens. La mise en relation se rapporte d'abord à la nature des comportements possibles et à la structure du champ ouvert à ces comportements. La conclusion est qu'il faut définir les ressources plus largement et plus exactement qu'on ne fait d'habitude.

Constatons d'abord qu'elles se composent de forces matérielles eau, végétaux, substances chimiques, etc. — d'un côté, de savoir-faire - habileté à marcher, à communiquer, à combiner des mouvements, artefacts, etc. - donc de systèmes de comportements, de l'autre côté. Ensuite, du point de vue de leur importance, de leur place dans l'effort 
collectif, certaines ressources sont principales, forment le noyau autour duquel s'ordonne l'activité physique et intellectuelle de la collectivité ; d'autres sont accidentelles ou complémentaires, car on y a seulement recours de façon occasionnelle ou accessoire, lorsque les circonstances se présentent ou commandent d'en profiter. Le cours ordinaire de la vie se déroule sans qu'on leur accorde de l'attention ou qu'on leur consacre un effort soutenu. L'équilibre à cet égard concerne les dimensions de la société, de l'espèce, quant à ses savoirfaire, le répertoire des comportements qu'elle détient et le maintien de ces dimensions par une focalisation exclusive sur les ressources principales. Ainsi, si son existence dépend du fourragement, de la cueillette de plantes ou de fruits particuliers, les seuls systèmes de comportements, les seules attitudes neuromusculaires ou les seules connaissances impartis et transmis méthodiquement, consciemment, auront trait au fourragement et à la cueillette. Les zones du territoire, les rythmes nocturnes ou diurnes d'activités se concentrent autour de ce qui est transmis de la sorte et périodiquement renforcé. De là vient que, bien souvent, une espèce vit dans une niche délimitée, où elle remplit une fonction qui lui est propre, en ignorant une fraction de la flore et de la faune ; la fraction qui, pour une espèce ou une collectivité différente réalité pleine, vitale, est aux yeux de la première réalité inexistante, néant. De là vient aussi qu'un potentiel écologique, technique, demeure inexploité ; les facultés qui pourraient le mettre en œuvre ne sont pas entretenues et les individus qui seraient capables de s'y consacrer manquent ou ne sont pas poussés dans cette direction. L'éventualité d'un tel débordement du cadre naturel établi est toujours présente à l'horizon et toujours contournée, par des mesures appropriées, puisque l'on reproduit uniquement les propriétés organiques et non organiques qui ont réussi à s’imposer. Tout ce qui déborde ce cadre apparaît contre nature et représente une menace pour la survie de l'ensemble.

Les deux états d'équilibre de la société et de sa base matérielle, des échanges que requiert la correspondance des propriétés intellectuelles et physiques de l'organisme avec les forces composant son milieu, sont rarement atteints (du moins en ce qui concerne certains anthropoïdes et les humains). On ne connaît que des déséquilibres atténués, aux fluctuations plus ou moins grandes. Comment ces déséquilibres, le dernier surtout, prennent-ils naissance ? A quelles conséquences 
conduisent-ils lorsque l'obligation surgit de les réduire, du moins au niveau historique qui nous concerne ? Un des facteurs est la constitution d'une surpopulation, d'une quantité d'individus surnuméraires eu égard aux capacités élaborées, aux pouvoirs matériels reconnus et renouvelés dans un cadre donné. Dans toutes les sociétés ou classes biologiques se produit un décalage entre la tendance de leurs membres à se multiplier et les possibilités dont dispose la collectivité de maintenir constamment son volume à un niveau prédéterminé. Ce décalage provient du fait qu'un mode de reproduction, un rythme de naissance ou de recrutement, une durée moyenne de vie, s'établissent à un moment en rapport avec une organisation collective, relativement à une quantité et à une qualité des ressources. Avec le temps, la " productivité » peut s'accroître et susciter, parallèlement, une fécondité supérieure. Par un jeu de causes variables, interférence avec une autre espèce, limites géographiques ou géologiques, etc., et sauf changement profond, les limites de cette productivité sont rapidement atteintes. Dans l'agriculture extensive sur brûlis, il faut respecter un rapport entre la surface des terres cultivées et la surface des terres cultivables, si l'on veut obtenir une certaine fertilité du sol permettant une culture efficace. Lorsqu'en s'étendant les premières populations agraires ont accru démesurément les terres cultivées, les sols se sont épuisés ou ont été envahis par des plantes parasites, qui les ont dégradés et en ont diminué la fertilité. De même, le perfectionnement de l'art du chasseur a eu pour conséquence la disparition trop rapide de quelques espèces sauvages - par exemple le cheval en Amérique du Nord mettant une partie du groupe dans l'impossibilité d'exercer son art ou l'obligeant à le modifier. La propension à encourager la fécondité individuelle, entraînant la recherche du nécessaire dans un environnement donné, avec, à l'occasion, le déclin de la fertilité de celui-ci, est cause de surpopulation. Ce n'est pas la seule cause, mais peut-être la plus remarquée. Elle est d'autant plus persistante que le changement du modèle et du volume de procréation est un processus très lent, comparé à celui qui affecte les ressources, et ne saurait parfois se réaliser sans bouleverser la structure sociale dans sa totalité, ce qui engendre des résistances supplémentaires sur son chemin. Dans cet interstice, les groupes d'individus en surnombre peuvent devenir une partie inhérente, stable, du système social et reproductif, du moins une partie qu'il est difficile de réduire au taux qui aurait été acceptable antérieurement. Parallèlement à ces individus démographiquement 
superflus se trouvent aussi des ressources et des savoir-faire complémentaires. En exerçant une activité spécifique, toute collectivité, notamment humaine, dépasse le donné, les frontières qui lui sont coutumières ; elle conçoit des procédés originaux, découvre des forces matérielles qui subsistaient auparavant dans son univers soit comme éléments autonomes, soit comme déchets de ses propres activités ou de celles d'autres espèces. Cette création peut être encouragée ou découragée, rencontrer des circonstances favorables ou non : elle n'est jamais totalement arrêtée ou absente. Par là, l'espèce s'approprie, de manière accidentelle ou expérimentale, les éléments d'un milieu différent qu'elle associe au sien. Les théoriciens attribuent à la rareté des ressources, à la concurrence, un rôle moteur dans la diffusion des espèces et leur recherche de nouveaux moyens d'existence. Pour ma part j'estime que ce rôle est rempli par la formation d'une abondance latente qui permet à une espèce, et, disons-le, à l'homme, de pénétrer dans les régions marginales disponibles ou dans les niches écologiques exploitées de façon insuffisante ou moins efficace par les espèces qui les occupent. L’antagonisme se déclare à une étape ultérieure, comme un effet de la coprésence, de la concurrence pour les mêmes richesses, et non pas comme sa cause.

La rencontre d'une surpopulation permanente et de ressources complémentaires, accessoires, assure à la première un champ d'action et tend à stabiliser les secondes, à les rendre moins occasionnelles ou accidentelles. Le cycle des occupations, des dextérités acquises et des facteurs matériels prévalents révèle ses limites et voit se former, à ses côtés, un cycle distinct qui, sans être principal, prouve son utilité, incite une communauté d'individus à s'y attacher et à le développer. L'unité ancienne de l'espèce et du milieu est remise en question par l'unité naissante et la convergence des déséquilibres ne trouve sa solution qu'au terme d'un mouvement réorganisant de fond en comble les conditions dans lesquelles ils ont pris naissance. La prédation témoigne, dans l'évolution humaine, d'une telle rencontre et représente le commencement d'un tel mouvement, avec les répercussions que nous connaissons. 


\section{Les mâles surnuméraires et le petit monde menacé de la forêt.}

Malgré les hésitations que l'on peut avoir devant toute projection rétrospective qui nous aide à découvrir la genèse de la prédation humaine, le spectacle des primates aujourd'hui vivants est encore la meilleure représentation que nous ayons de ce qui a dû se passer autrefois. Leur nomadisme, celui des mâles notamment, est frappant. Les grands singes, chimpanzés, orang-outangs, se déplacent constamment vers les zones où la nourriture est disponible, suivant les lignes de leur répartition. Réunis en bandes de deux à cinq individus, ils parcourent rapidement les vastes étendues de la forêt, éclaireurs ou pionniers de leur collectivité d'appartenance. Une fois découverts les arbres pleins de fruits, on les voit tambouriner avec frénésie, pour annoncer le succès de leur entreprise et signaler l'emplacement des richesses. La tendance à la migration, à l'exploration d'une région parait incoercible. Les femelles, surtout lorsqu'elles sont requises par les tâches maternelles, restent plus attachées à un territoire, à ses routines. Le nomadisme mâle a aussi ses limites dans la permanence des relations sociales. Un individu est intégré à un groupe : en cette qualité, à son retour, il y est immédiatement accepté. S’il prenait l'initiative d'entrer dans un groupe étranger, il devrait combattre et serait combattu. La réunion après séparation temporaire des membres d'une collectivité est l'occasion de salutations affectueuses, on l'observe chez les chimpanzés. Les enfants ou adolescents engagés dans des activités variées avec les mâles adultes migrateurs retournent périodiquement au couple nucléaire qu'ils forment avec leur mère. La vie sociale est nécessaire à tous. Elle facilite la recherche de la subsistance, l'épouillage et protège les individus mieux avertis et mieux défendus contre les prédateurs. L'élasticité de ce système d'échanges et de relations chez les grands singes est remarquable. Elle convient à une population dont la partie masculine est entraînée vers des zones plus variées et plus exposées aux dangers de l'agression, laissant à la partie féminine le domaine où la sécurité de la vie et de l'approvisionnement est plus grande. L'ensemble de la société y trouve son compte. La dissymétrie entre les sexes a encore une autre cause que le relâchement de la concurrence pour les ressources végétales ou la différence de responsabilité reproductive des individus. Dans les sociétés d'affiliation des primates, on note l'existence de mâles solitaires ou rassemblés en groupes appendiculaires dans la forêt et de troupes 
comprenant plusieurs groupes de ce type, exclusivement mâles, en terrain découvert. J'ai déjà décrit plus longuement cette population, en principe non reproductive, surnuméraire par rapport aux richesses disponibles et constituant une solution au déséquilibre qui en résulte. Elle vit en marge de la collectivité, exclue d'un grand nombre des transactions régulières de celle-ci et, bien entendu, de la procréation. Le mécanisme de sa formation et de son isolement participe du fonctionnement social normal. Nous le voyons opérer avec constance à travers la hiérarchie sociale, les comportements réels ou symboliques de rejet et de soumission, la délimitation de l'espace alloué à chaque sous-classe de la collectivité. Les jeunes adultes sont, à de rares exceptions près, automatiquement poussés vers le pourtour du groupe, vivent sous la menace des animaux coryphées. Les adultes subordonnés partagent un sort identique : l'accès aux femelles, aux ressources communes, leur est interdit ou permis dans des limites étroites. La périphérialisation signale un travail d'exclusion, de création d'un surplus eu égard à la population nécessaire qui seule a les chances les plus sérieuses de vivre et de laisser une descendance. Pendant une certaine période de leur vie, ou pendant toute leur vie, les individus concernés sont poussés vers les secteurs les plus déshérités - déshérités quant à la nourriture, la ressource principale du groupe ou de l'espèce, s'entend. Le bénéfice de l'entraide mutuelle, des jouissances sociales et sexuelles procurées par le couple reproductif, leur est mesuré. Les menaces du monde extérieur pèsent plus lourdement sur eux; comme tous les animaux obligés de quitter les sites habituels, ils sont plus exposés à rencontrer des prédateurs, à devenir proies. La recherche de moyens de subsistance et de satisfaction substitutifs est leur tâche constante, rendue plus ardue lorsque la disette survient.

Ce partage du corps social s'accompagne d'une double pression. Le sous-groupe des animaux reproducteurs est incité à s’intégrer fortement dans le cycle courant des interactions et des pratiques. La spécialisation des rôles, des comportements, le mode de distribution des ressources communes, lient chacun à un cadre qui lui assure un développement normal, absorbe complètement ses énergies et dessine les limites de son horizon. La préparation d'une petite partie des jeunes mâles à leur mission hiérarchique, les soins prodigués par les " oncles » et les " tantes », quelles qu'en soient les motivations, leur nonexpulsion vers la périphérie, contribuent au modelage psychologique 
de ceux qui sont destinés à rester dans le noyau durable de la société. Les femelles, par définition, sont conditionnées de manière à demeurer fortement attachées au mode de vie prédominant. Les mâles adultes avec lesquels elles forment un couple ressentent par là même les diverses influences stabilisatrices, sont prêts à les subir et à les exercer à leur tour. Situés au centre de l'attention de tous, consacrés à leur fonction de surveillance et de contrôle, dépositaires d'une expérience et d'un savoir indispensables à la survie, protégés par la solidarité de leurs pairs et la sollicitude de leurs subordonnés, ils sont enfermés dans le moule de la tradition, et leurs actions sont caractérisées par le souci de la continuité. En revanche, le sous-groupe d'individus «superflus », non reproducteurs, subit la pression à ne pas s'intégrer aux activités prédominantes et, sinon à éviter les ressources essentielles de la collectivité, du moins à n'en profiter que de façon précaire. Dans l'intention de pallier les inconvénients de leur position, les êtres qui le composent recourent à des subterfuges - recherche de faveurs, soumission symbolique, etc. — ou, plus rarement, à l'agression directe. Ils ne sont cependant pas bien outillés pour réussir dans ces entreprises puisque, d'emblée, l'apprentissage qu'ils ont fait tend à inhiber ou négliger les facultés correspondantes. Les ethnologues ont parfois relevé le contraste qui sépare la liberté d’allures, l'agressivité, l'agilité des animaux vivant au cœur de la communauté avec le caractère renfermé et craintif, l'aspect contraint des animaux périphériques. Si ces derniers ne peuvent se tourner vers la société pour échapper aux servitudes de leur situation, il leur reste pour seule issue l'exploration intensive du milieu, la création d'une existence possible avec les congénères qui obéissent aux mêmes impératifs. Leur moindre degré de spécialisation quant au mode d'agir prévalent est, sous cet angle, un avantage. Comme il s'agit généralement de jeunes individus, leurs capacités pour l'exploration ne sont pas négligeables. Les études faites à propos des macaques sont, sur ce point, concluantes. Lorsque les circonstances s'y prêtent ou lorsque les conditions changent, on a noté à plusieurs reprises l'apparition de conduites inventives et leur diffusion ultérieure. On observe la locomotion bipède et la natation sur de courtes distances quand les animaux sont requis par des tâches de transport. D'abord accidentels, ces comportements se sont intensifiés jusqu'à devenir habituels. Le lavage des patates douces avant consommation est encore une invention spectaculaire des primates japonais. Dans l'ensemble, la découverte de nouveaux aliments est 
due aux animaux jeunes. Ceux-ci semblent moins enclins à éviter les formes, les matériaux non familiers, ce qui les amène à s'en approcher, à les saisir avec la bouche, et, de temps en temps, à les utiliser. Les adultes constamment engagés dans les rapports sociaux, les routines de leur espace et de leur programme quotidiens, demeurent indifférents aux formes ou aux matériaux nouveaux, tant qu'ils ne présentent pas un danger pour eux ou pour le groupe. La transmission des innovations parcourt un cycle relativement régulier. Le comportement nouveau, élaboré par un individu jeune, à titre de jeu, se diffuse pour commencer chez tous les ,jeunes. Par le canal du couple nucléaire, il est adopté par les mères, et c'est seulement après qu'il apparaît chez les autres adultes mâles. Le changement de comportement ou d'objet du comportement affecte, avec un décalage, les animaux centraux de la collectivité et se généralise au fur et à mesure que les adolescents vieillissent. A l'instar des habitudes motrices ou alimentaires, les modalités d'interaction sociale des jeunes ont pu ou pourraient également se propager dans une population, empruntant un cheminement analogue. Rien ne s'oppose à ce que des méthodes inédites d'explorer et d'utiliser les ressources virtuelles, de s'organiser socialement à cette fin, soient conçues, pratiquées, associées à une tradition, se modifiant progressivement, sans que le milieu géographique, climatique, soit profondément bouleversé. Ainsi tout se passe dans les sociétés d'affiliation comme si le mécanisme d'exclusion était simultanément un mécanisme créateur d'un potentiel transformateur du cycle d’échanges avec le monde matériel.

La première pression, on doit le conjecturer, entraîne une adaptation étroite à une configuration donnée du milieu, le désir d'y rester, tandis que la seconde provoque, à la longue, le besoin de le quitter, en suscitant la possibilité de modifier les rapports avec les éléments qui le composent. La force respective de ces deux pressions dépend, bien entendu, des circonstances. Quand la nourriture végétale est suffisante, la proportion numérique entre mâles et femelles favorable, l'intégration a le dessus et le conflit latent entre mâles est atténué. Par contre, lorsque l'accès à la ressource principale devient difficile et, la reproduction incertaine, une grande partie de la collectivité, sinon la totalité, se muant en surplus, doit émigrer, trouver des solutions nouvelles ou disparaître. L’état réel, envisagé pendant une longue durée, est un état intermédiaire, gravé dans le corps social, engendrant une 
différenciation qui désigne clairement la partie devant se consacrer à l'exploration pour survivre, se soumettre aux aléas de la concurrence avec les autres espèces, et la partie qui est destinée à procréer, à se maintenir dans les conditions inhérentes à l'espèce. On attribue couramment la plasticité des primates et des hommes à leurs qualités physiologiques et intellectuelles. Je crois qu'on serait plus près de la vérité en cherchant sa raison d'être dans le dynamisme d'une organisation sociale susceptible à la fois d'enraciner la plupart de ses membres dans un espace de vie déterminé et d'obliger le restant à le dépasser. Assurément ce dynamisme s'avère opératoire au niveau biologique, où la mort n'est pas la seule réponse au manque relatif de moyens indispensables à la perpétuation du groupe, et où l'association des individus dure suffisamment pour que les aptitudes accumulées soient diffusées et conservées.

Nous avons tous les motifs de supposer, sans avoir les moyens de le prouver entièrement, que ce surplus, concrétisé aujourd'hui par les bandes de jeunes célibataires, les troupes exclusivement mâles, a été un facteur décisif, induisant les espèces-souches, dont sont issus les hominiens, à délaisser l'existence arboréale à laquelle elles étaient accoutumées. Certes, le bouleversement climatique, affectant l'équilibre de la flore, en réduisant l'étendue des zones nécessaires à la nourriture et à la reproduction normales des collectivités animales, a précipité ce mouvement. Mais celles-ci étaient probablement déjà prêtes à partir à la recherche de ces terrains herbeux du myocène, couverts de nouvelles formes de végétation, remplaçant celles de l'éocène et de l'oligocène. Ils offraient des possibilités de préadaptation à une grande variété de mammifères, y compris les primates, habitant une forêt qui avait tendance à reculer. Dans l'Ancien Monde, à la différence du Nouveau, leurs essais pour s'installer au sol sont patents. Les babouins, les singes patas, les vervets, les macaques y ont réussi. Les ancêtres des hominiens aussi. Ils ont commencé à émigrer vers la savane boisée, intermédiaire entre la haute futaie et le terrain découvert. Les chances de devenir proie, surtout en s'éloignant de l'habitat, s'étaient notablement accrues par la même occasion. Cependant les groupes composés de plusieurs mâles étaient mieux préparés à affronter ce risque, leur coopération forcée leur apportant divers avantages. Nous devons imaginer, par ailleurs, qu'ils étaient déjà prédateurs et partiellement carnivores. Cette tendance se manifeste aujourd'hui de 
façon accidentelle chez les primates : elle a dû être plus forte, il y a des millions d'années, avant que leur environnement soit transformé par l'homme. La plupart des simiens pillent les nids pour dérober les œufs ou les oisillons. Les babouins mâles tuent et consomment parfois des animaux, généralement les jeunes antilopes venant de naître. Les chimpanzés font de même avec certaines espèces, plus petites, de singes. A l'encontre des primates qui, malgré tout, ont cherché une richesse végétale de remplacement, c'est la richesse animale, d'abord accessoire, qui a attiré les préhominiens. Les perturbations survenues parmi les mammifères lors des changements du climat et de la flore les ayant dépouillés en partie de leurs ressources usuelles, ont créé un vide que des animaux plus entreprenants avaient le loisir d'occuper. Les remaniements écologiques n'ont pas joué un rôle causal et n'ont pas mis les espèces supérieures en position d'affronter une situation totalement différente. Ils ont simplement aggravé le phénomène de surpopulation, les tensions internes des collectivités anthropoïdes et hominoïdes, les amenant à explorer intensivement les possibilités existant dans les territoires adjacents. Les plus aptes à entreprendre des efforts dans ce sens et à en subir le contrecoup, les mieux préadaptées, à vrai dire, furent les espèces qui avaient un surplus important, relativement déspécialisé, constitué d'individus habitués à s’aventurer ensemble dans des régions nouvelles et à se coordonner effectivement. Et, nous l'avons observé, disposés à s'approcher des formes et des objets inédits, à élaborer des comportements nouveaux, à les diffuser, étant donné leur âge et leur situation périphérique. L'expérience déjà acquise au cours des prédations intermittentes antérieures a pu ainsi se développer. Durant la période proto-hominienne, de transition, une ressource longtemps accidentelle, supplémentaire, commença à devenir régulière, à prendre de la consistance. Paradoxalement, le déficit en végétaux, l'obligation de les concéder à la fraction reproductrice de l'espèce, libéra les animaux non reproducteurs des liens préexistants, les mit plus à l'aise pour acquérir le goût de la viande, de la capture des proies, et les aida à déployer les savoir-faire et l'agressivité correspondants. «Ce changement, écrit John Crook ${ }^{26}$, peut fort bien avoir été associé au développement de groupes entièrement mâles (que l'on connaît pour les chimpanzés et les babouins geladas, par exemple) se transformant en groupes de chasseurs, et à l'institution de

26 J.H. CROOK : Cooperation in Primates, The Eugenics Review, 1966, 58, p. 68. 
lieux de campement jadis abandonnés pendant les expéditions diurnes des animaux. » En effet, on ne voit guère quelle autre fraction de la population aurait été mieux désignée, incitée socialement et biologiquement à aller vers des milieux inconnus pour y instaurer des relations différentes avec les êtres qui les habitent et profiter de leur généralisation. Convertir l'accessoire en nécessaire est le seul moyen laissé aux « superflus » de forcer les portes de la vie.

Retour à la Table des Matières 


\section{La cueillette des animaux.}

\section{Retour à la Table des Matières}

L'univers préhominien et même l'univers hominien à ses débuts sont des univers mixtes. L'espèce savait déjà se tenir debout et avait l'habitude de se suspendre aux branches des arbres ; elle se mouvait à l'aise dans la savane comme dans la forêt. Le jour, les bandes de jeunes mâles parcouraient les diverses zones de la plaine et de la broussaille à la lisière de la forêt. Le soir venu, le retour vers les arbres, au sommet desquels la communauté se rassemblait pour dormir, s'imposait. Chacun s'y sentait à l'abri des grands chasseurs de nuit. Les végétaux continuaient à représenter une proportion importante du régime alimentaire, et les individus pratiquaient couramment la cueillette. La prédation était toutefois déjà présente, fournissant son contingent de vivres, occupant de manière importante la partie masculine du groupe. Son endroit et son envers ne coïncident guère. A l'endroit, elle symbolise une activité particulière, supplémentaire, qui se réalise aux dépens des petits animaux recherchés, pourchassés, attrapés et tués. A l'envers, il ne s'agit que d'un fourragement de carnivore, suivant la trace des autres carnassiers pour profiter des déchets de leur chasse, consommant, en bon nécrophage, les carcasses, les viandes qu'ils n'ont pas consommées. Des deux côtés, il faut apprendre à suivre à la piste les animaux soit pour les guetter, soit pour les attaquer, mieux connaître leurs particularités et leur habitat, ainsi que la topographie des lieux. Les déplacements couvrent des régions plus vastes et se font plus fréquents. Ils sont aussi très différenciés par rapport à ceux des primates. Aller loin, aller vite est une faculté indispensable mais assez simple. En revanche, explorer des sites en vue de trouver des fruits et des proies dispersés dans l'espace implique sa diversification et son amplification sur le plan individuel, organique, autant que sur le plan social. Les efforts exigés sont plus importants : la flore de la savane est pauvre, les arbres plus rares offrent moins de refuges, il faut couvrir une longue distance pour l'atteindre et pour la quitter à la tombée du jour. De plus, il faut transporter la nourriture et réunir les moyens artefactuels, à vrai dire peu nombreux, tels que pierres ou bouts de bois. Un individu isolé ne saurait accomplir ces tâches et serait, par ailleurs, une proie toute désignée. Sa force ou les instru- 
ments qu'il possède, bâtons et pierres, ne lui seraient d'aucun secours au cas où un félin s'acharnerait contre lui. La seule arme efficace consiste à se grouper avec les autres membres de la collectivité. Cette coopération est accessoire dans l'attaque, les jeunes et les animaux de petite taille se prêtant à la capture individuelle. La défense et les explorations en revanche exigent un partage des informations, la signalisation des emplacements des charniers ou des futures victimes; un rassemblement, par sa masse, éloigne les prédateurs les plus audacieux. La coordination sociale, celle des mâles notamment, prend un sens technique.

Devenues habituelles, diffusées, les activités prédatrices et leurs prolongements ont fait naître, comme on le voit, trois séries de tâches :

- courir et couvrir de longues distances pour explorer le terrain, trouver les proies ou les déchets des tueries des grands carnassiers, et revenir régulièrement à son point de départ ;

— saisir les animaux, les faire sortir de leurs abris, les tuer et les dépecer une fois morts ;

- signaler les lieux, les adversaires, ainsi que ses propres mouvements et ses propres intentions aux individus avec lesquels on coopère.

Pour accomplir ces tâches, tirer parti des ressources complémentaires en nourriture carnée, les primates supérieurs disposent de ressources complémentaires en comportements et savoir-faire. Les artefacts, j'y reviendrai dans le chapitre suivant, étaient connus des primates non humains. La station debout, lorsque besoin est, n'est pas exceptionnelle chez eux, ni la différenciation entre l'activité des membres inférieurs et celle des membres supérieurs. Les gibbons marchent et courent facilement sur deux pattes. Les chimpanzés et les gorilles n'ont aucune peine à se déplacer dans cette position, où le poids du corps vient naturellement se porter sur un axe vertical, perpendiculaire aux pieds. Les orang-outangs captifs se dressent parfois sur leurs pattes de derrière, mais c'est sans aisance qu'ils pratiquent la locomotion bipède. Quant aux babouins, ils demeurent résolument quadrupèdes, 
mais chez eux la spécialisation des fonctions est déjà ébauchée, et les terrains découverts rocailleux sont leur habitat de prédilection. De toute façon, on relève entre leurs mains et leurs pieds une différence assez grande pour qu'on puisse la dire inscrite dans l'espèce de longue date. On observe, par ailleurs, l'emploi de systèmes de communication simples : les gorilles pratiquent le tambourinement sur la poitrine, et les chimpanzés frappent rythmiquement le sol ou les troncs des arbres. L'installation dans un habitat mixte et l'exercice systématique de la prédation ont entraîné l'usage régulier de ces facultés et, consécutivement, des transformations anatomo-physiologiques propres à notre lignée d'espèces.

Surveiller le territoire commun est une activité qui exige la généralisation de la station debout. L'hominoïde devait pouvoir discerner rapidement prédateurs et proies. Les yeux de ses ancêtres arboricoles s'étaient déjà agrandis et placés de front, lui conférant une vision stéréoscopique. Ce développement des organes et du sens optiques a eu pour corollaire la régression de la région nasale. Le sens olfactif, qui permet aux animaux vivant au sol de flairer l'ennemi ou la victime, devient moins efficace ; celui de la vue y remédie. Qu'il s'agisse d'accomplir des reconnaissances ou d'élargir le champ de vision, il est indispensable de se tenir debout. Le bipédisme, dont nous avons vu l'ébauche chez les anthropoïdes, l'a permis grâce à des mutations nouvelles favorables. Le transport des fardeaux de toute sorte a multiplié les occasions de renforcer cette tendance et de consolider les appareils neuro-musculaires afférents. L'étude des macaques demisauvages de Koshima, au Japon, fournit une preuve à ce sujet. Elle montre comment la locomotion bipède, aptitude potentielle chez ces singes, s'est intégrée dans un schéma de comportements conçu à l'occasion d'un changement de régime alimentaire. Entre 1952 et 1962, ces macaques ont inventé le lavage des tubercules, invention qui les a obligés à quitter la forêt épaisse pour le terrain découvert et à fréquenter la plage de l'île où ils habitaient. Là, une possibilité leur fut offerte de se nourrir avec du blé, et quelques-uns d'entre eux inventèrent une technique de ramassage des grains en les séparant du sable auquel ils se trouvaient mêlés. Le lavage des tubercules, la sélection par lavage des grains conduisirent les singes à porter tubercule ou sable jusqu'à l'eau, dans leurs mains. On les vit alors marcher sur les pattes postérieures. Dans d'autres circonstances, on observa qu'ils 
gardaient une posture analogue pendant un laps de temps relativement long. A la fin de la période indiquée, $71 \%$ des membres de la communauté savaient marcher dans la mer, recueillir des mollusques et diverses nourritures marines. Dans des conditions similaires, il y a plusieurs millions d'années, les proto-hominiens ont dû arriver au même résultat, et ce résultat s'est transmis de génération en génération au sein d'un milieu qui nécessitait sa conversion.

Cependant le prédateur n'a pas seulement besoin de surveiller ou de porter : courir est vital pour lui. Son bipédisme est lié à la course plutôt qu'à la marche, à un déplacement rapide plutôt qu'à un déplacement très long. Les restes du pelvis des hominiens confirment cette hypothèse. Ils appartiennent sans conteste à des créatures bipèdes. La comparaison du pelvis de l'anthropoïde, de l'hominien et de l'homme moderne permet de noter que la partie supérieure est moins large et plus longue chez le premier que chez ces derniers. La longueur du pelvis supérieur du singe anthropoïde est un trait commun à la plupart des quadrupèdes. L'os court et large de l'ischium, facilitant la locomotion à l'aide des membres inférieurs est propre à l'homme. Il permet l'extension de la jambe jusqu'à un point situé en arrière de l'axe vertical de la colonne vertébrale. De plus, les muscles fessiers petit et moyen, articulés avec la hanche, stabilisent le pelvis au cours de chaque grande enjambée spécifique de la marche. Chez les premiers hominiens, les dimensions de l'ischium du pelvis se rapprochent de celles de l'ischium du pelvis des anthropoïdes et l'évolution des muscles fessiers n'est pas suffisante pour leur permettre de remplir leur rôle stabilisateur, autoriser l'extension normale de la jambe. Ceci fait qu'ils ne se déplacent qu'au petit trot rapide, hanches et genoux légèrement fléchis. Il en résulte une dépense d'énergie telle que cette locomotion ne peut se prolonger longtemps et leur interdit de parcourir de grandes distances d'une seule traite.

La station debout, impliquée par la surveillance statique du terrain et la locomotion bipède réclamée par les opérations dynamiques liées à l'attaque, à la défense et au transport, n’ont pas été des phénomènes isolés. Elles ont entraîné des modifications morphologiques secondaires et rendu possible l'individuation d'organes qui étaient auparavant associés à l'exercice de fonctions identiques. Notamment celle des membres antérieurs, devenus par la suite nos bras et nos mains. Leur 
intervention agonistique, les efforts et les habiletés qu'on leur demande pour casser les branches, charrier les cadavres ou les pierres, ne dépassent pas ce dont sont capables les mains préhensiles des primates. Elles sont différenciées dans le contexte des activités prédatrices mais non pas spécialisées ; elles sont dégagées en tant que parties anatomiques d'un corps redressé, elles ne sont pas encore particularisées en tant qu'organes humains. La dissymétrie évolutive des pieds et des mains est frappante. L'examen du squelette de l'australopithèque montre que la ressemblance avec celui de l'homme moderne est plus marquée pour les os des pieds que pour ceux des mains. Ces derniers mettent en évidence que l'espèce vivant au sol n'était pas brachiante. Ils sont de constitution plus robuste, et le corps des phalanges présente une courbe dorsale plus accentuée.

D'autres indices nous permettent de tirer des conclusions analogues. De fortes raisons militent en faveur d'un parallélisme entre la spécialisation de la main et l'augmentation du poids du cerveau. Le changement de régime alimentaire qui influe sur la dentition, la station debout et la modification de la forme du visage remodèlent la boîte crânienne. Le volume demeure du même ordre que pour les simiens, préservant le même rapport au poids du corps. En revanche la mâchoire s'est développée, et le crâne est plus haut que celui des anthropoïdes; le tore occipital et l'inion bas, la place en avant des condyles occipitaux sont autant de traits qui annoncent l'espèce humaine. Bien entendu, faute de pouvoir étudier la structure neurophysiologique du cortex, qui nous demeure à jamais inaccessible, les conjectures fondées sur l'indice quantitatif seul — de 435 à $680 \mathrm{~cm}^{3}$, chiffre voisin de celui des primates — restent des plus contestables.

La main différenciée est muette. Le cerveau des premiers hominiens, si l'on met à part la morphologie crânienne, ne semble pas avoir subi une transformation égalant en profondeur la transformation des autres parties du corps. Pourtant l'animal humain, dans les conditions qui sont devenues les siennes, était amené à créer un système de communication plus adéquat que celui des primates. La mimique et la lecture des signes raciaux, auxquelles les singes excellent, sont inopérantes dans l'obscurité. L'emploi de la vision pour émettre et recevoir des messages à des distances moyennes est d'une efficacité douteuse. Le décodage auditif de signaux acoustiques perd de sa précision, ces 
signaux étant noyés dans le « bruit » des diverses espèces vocalisant en même temps. S'y ajoute la nécessité d'imiter les cris des animaux afin de les attirer ou de les éloigner, bref, en un sens, de communiquer avec eux. La coordination des initiatives individuelles réclame de son côté le recours à des sons particuliers et, peut-être, un accompagnement rythmique de gestes et de mouvements. Les schémas vocaux jouent un grand rôle chez les singes. Ils commandent le mouvement des groupes, règlent les écarts territoriaux entre les troupes, marquent les positions hiérarchiques à l'intérieur des collectivités, etc. Sur une base matérielle analogue, mis dans l'impossibilité d'employer un système d'indices visuels et tactiles, les premiers hominiens, disposant d'un organisme et d'un milieu remodelés, pouvaient aller plus loin sur la voie de la communication vocale et la remanier par une invention capitale : le langage. La taille du cortex plaiderait contre la spécificité d'une faculté linguistique chez les deux espèces d'australopithèques. Dans la mesure où nous ne savons rien sur l'allure de ses circonvolutions, sur le nombre de ganglions des centres nerveux et sur leurs connexions ou le degré de spécialisation des cellules, il est difficile de sous-estimer l'obstacle présenté par la capacité volumétrique. Toutefois il est bon de rappeler que l'enfant de l'homme moderne commence à parler vers l'âge de deux ans, avec un cerveau de $650 \mathrm{~cm}^{3}$. Pourquoi n'aurait-on pas pu parler il y a deux ou trois millions d'années avec un cerveau de dimensions identiques ? La locomotion bipède, la diversification de l'utilisation de la main ont rendu la bouche qui servait auparavant à happer et porter disponible pour les modes d'agir qui étaient les siens : menacer, crier, imiter des sons, etc. Ils trouvaient devant eux un espace libre pour leur extension et leur perfectionnement. L'articulation des syncinésies corporelles et des schémas vocaux appelait de son côté un rajustement consécutif au remaniement des appareils sensoriels et moteurs. La spécialisation et l'insertion des organes phonatoires dans cet ensemble anatomophysiologique a certainement influé sur la nature des éléments phoniques produits et sur leur organisation en vue de l'émission et de la réception des commandements, des informations ou des signaux requis. Si la faculté langagière a existé de façon indubitable, il est souhaitable d'apporter des précisions sur la nature de ce langage dont l'invention correspond à l'apparition. 
Le langage a, comme on le sait, deux fonctions : une fonction communicative, instrumentale, associée surtout au comportement concret et à la signalisation, et une fonction de codage des renseignements tirés du monde physique et social en vue de leur stockage mnémonique et de leur réutilisation intellectuelle. La fonction communicative recourant de manière simultanée ou substitutive à des noyaux d'indices verbaux et non verbaux a été probablement la première à se transformer et à se détacher sur le fond des systèmes symboliques préexistants. Les mots, incrustés dans une chaîne vocale, ont agglutiné, stabilisé des familles de sons, reproduisant analogiquement d'autres sons. Agissant sur le comportement du sujet émetteur et de ses congénères, en localisant des sous-groupes ou des animaux, ils ont, par leur particularité qui les détache du « bruit » environnant, envahi progressivement le champ de leurs équivalents non lexicaux. Le langage de ces hominiens est, pour reprendre l'expression du philosophe anglais John Langshaw Austin, performatif. Parler est alors faire, aménager pratiquement les relations communautaires, les échanges avec le milieu et les espèces qui l'habitent. L'énonciation linguistique s'entre-tisse aux actes techniques, biologiques et y adhère, elle participe de la puissance de l'individu sur les forces qui l'entourent, et confère enfin au silence une signification. La fonction de codification, le poids modeste du cortex nous oblige à l'admettre, est encore remplie suivant la méthode commune aux anthropoïdes sans affecter le langage ni être affectée par lui. Cette architecture du système de communication, des pouvoirs intellectuels, se conforme à ce que demandait une organisation sociale proche de celle des primates modernes, une activité grâce à laquelle l'homme se sentait sur un pied d'égalité avec n'importe quel animal, étant bien sûr proie mais aussi prédateur ${ }^{27}$.

La station debout, la locomotion bipède, la main différenciée, le langage performatif, la banalisation des artefacts, l'alimentation omnivore consacrent la présence biologique du genre humain. L'australopithèque robuste et l'australopithèque africain ( $\mathrm{y}$ compris l'homme habile découvert par Leakey) sont les deux espèces qui correspondent à la période, très reculée, où ces innovations organiques et techniques ont eu lieu. Elles résultent toutes de la généralisation 
d'aptitudes et de comportements qui étaient et sont répandus chez les primates et les singes anthropoïdes. De rares, ils deviennent fréquents ; de sporadiques ou occasionnels, ils deviennent systématiques. Ce qui était destiné à périr ou pourrir, les restes de la prédation animale, entre dans le circuit de la subsistance humaine à côté des fruits, noix, bourgeons, pousses, tubercules, reptiles, crustacés, insectes, œufs, petits mammifères jusques et y compris les babouins. Les reliefs d'antilopes et de girafes trouvés dans les sites préhistoriques, provenant probablement de la prédation des lions, prouvent que les cadavres ont été exploités avant d'être produits. Les ressources accidentelles en savoir-faire et nourriture perdant leur caractère fortuit s'ajoutent pour les compléter aux ressources principales de l'espèce. Les changements génétiques accompagnent et expriment ce mouvement d'habitude et de synthèse dans la prédation des moyens instrumentaux et des habiletés anatomiques et intellectuelles diffuses, marginales, dans les espèces supérieures. Toutefois si les termes du rapport naturel, l’organisme, le milieu se transforment, évoluent, ce rapport, lui demeure le même. Certes son rayon d'action s'étend, les modalités d'échange qui lui sont propres sont transférées d'un objet à un autre — du végétal à l'animal — d'une région à un autre — de la forêt à la savane. Pourtant nous restons dans le contexte prédominant du fourragement, de la cueillette. Concrètement aussi bien que métaphoriquement, l'activité prédatrice est une façon de cueillir les animaux, surtout lorsqu'ils sont petits ou morts, de les cueillir à la main pour ainsi dire, et la cueillette est une sorte de prédation des végétaux. La combinaison des deux formes de cueillette, suivant l'action, ou de prédation, suivant l'objet, concourt à réaliser un meilleur équilibre écologique, une autonomie relative vis-à-vis du milieu. Exploitant moins intensivement chacune des espèces végétales ou animales, on les laisse se reconstituer et on instaure des relations plus stables avec elles. De plus, quand l'une d'entre elles devient rare pour des raisons qui peuvent être indépendantes de l'intervention humaine, on a la possibilité de se rabattre sur celle qui est plus commune ou à laquelle on a permis de l'être. D'autre part, l'espèce humaine a le loisir de se propager, comme elle est en mesure de survivre dans des conditions où les éléments de son habitat se combinent selon des proportions variées et non plus rigides. Les australopithèques ont ainsi pu se répandre rapidement dans la plupart des régions tropicales et subtropicales, insti- 
tuant partout leur genre de vie particulier et interférant avec le genre de vie des autres animaux en le bouleversant.

En même temps se consolide la différenciation initiale. L’impulsion en a été donnée par les individus surnuméraires, la surpopulation apparue dans le monde des primates, exclue de la plupart des privilèges du groupe, maintenue par le fonctionnement des sociétés d'affiliation. La hiérarchie et la répartition sociales qui retiennent une fraction de la population et excluent la fraction des jeunes et des mâles subordonnés les obligent à diversifier leurs contacts et leur approvisionnement au-delà de ce qui constitue la niche écologique spécifique, à s'intéresser à ses alentours. La migration sans spéciation et la socialisation de la migration ont pour effet de les écarter de la solution commune sur l'échelle animale. Condamnés à vivre, ils sont obligés de reprendre et de banaliser ce qui se trouve à leur disposition, c'est-à-dire des éléments secondaires par rapport à ceux qui ont une valeur vitale, adaptative, pour l'ensemble du groupe. Les oppositions internes sont d'ailleurs moins fortes envers ce qui fait partie du domaine marginal, peu défendu et peu soumis à la concurrence de la part des congénères. La poussée vers la prédation, donc sur une ligne de moindre résistance, est une poussée vers la seule solution de rechange accessible aux mâles non reproducteurs pour survivre. Elle convertit la différenciation biosociale en différenciation des activités. Les zones vers lesquelles on émigre désormais se situent dans la savane, offrant des espaces où la biomasse des mammifères est telle qu'un prédateur, un nécrophage capable y trouve un champ favorable à ses entreprises. Les protosémitiques, à l'instar des primates, ont coexisté avec les autres espèces animales, se disputant et se partageant les ressources végétales d'un environnement commun. Transformant ces espèces en ressources, les hominiens ajoutent une dimension supplémentaire à cet environnement et se l'approprient, différencié. Dans l'évolution, la variété des configurations du monde extérieur a déterminé le sens qu'a pris la diversification organique des populations et de leur démographie. Avec l'embranchement des espèces humaines, ce sont au contraire la démographie et les formations sociales qui donnent au monde extérieur une figure nouvelle. 


\section{Chapitre IV}

\section{Les deux naissances de l'homme}

\section{De la prédation à la chasse.}

\section{Retour à la Table des Matières}

\section{La barrière des ressources principales.}

Reprenant les ressources secondaires des primates, les hommes se sont faits prédateurs. L'équilibre atteint est cependant précaire. Les causes qui ont fait surgir une activité importante à côté de la cueillette continuent à agir et à en presser le développement. Certes, dans le circuit normal des préoccupations, des comportements encouragés et reproduits, les procédés neufs et les aliments nouveaux demeurent subordonnés à ceux qui prédominent, sans qu’il y ait là une résistance déraisonnable ou volontaire de la part des divers groupes. Ayant pour vocation de se perpétuer, ils s'y efforcent en évitant les initiatives trop hardies. Du reste, la tradition sociale, l'équipement génétique sont engagés sur une certaine voie. Faute de mécanisme régulateur particulier, la difficulté serait trop grande de suivre une seconde voie réclamant des qualités opposées aux qualités normales imparties à l'espèce. La prédation devait tôt ou tard toucher aux limites de son essor dans le cadre de la cueillette. Séparées par des tensions et même des incompatibilités, elles ne mettent pas en œuvre les mêmes dextérités ; elles supposent un rapport différent aux ressources et ne s'exercent pas non plus dans le même entourage matériel.

Et d'abord la cueillette, le fourragement sont des activités passives. L'habileté nécessaire pour s'y adonner se réduit souvent à la reconnaissance des plantes et des fruits, aux gestes permettant de les arra- 
cher en vue de la consommation immédiate. Chaque individu, quel que soit son âge, son sexe, est capable de les pratiquer dès l'instant où il est biologiquement indépendant. Il n'a besoin ni d'un instrument particulier, ni de la collaboration constante de ses congénères. La flore est immobile, se renouvelle dans un rayon borné ; aux mêmes endroits, et ne résiste guère à celui qui l'explore ou la détruit. La prédation, elle, est active, suppose un répertoire de savoirs plus étendu, portant sur la localisation variée des proies, des prédateurs dont on consomme les dépouilles, ainsi que sur les moyens indispensables à la capture et à la mise à mort des petits animaux. Ceux-ci, à l'encontre des plantes, sont mobiles et migrants, parcourent rapidement de grandes distances, passent d'un habitat à l'autre suivant la saison. Dérangés ou attaqués, ils ripostent individuellement, mais le plus souvent collectivement, à leurs assaillants. Les prédateurs humains sont constamment obligés, connaissant ces réactions, de prévoir une suite d'actions complexes, à l'issue incertaine. Ils ne sauraient entretenir avec les espèces animales les relations que les cueilleurs entretiennent d'habitude. A savoir, se déplacer parmi elles, partager les mêmes aires d'approvisionnement et les mêmes points d'eau paisiblement, en les ignorant. Comme il se doit, un groupe de prédateurs voit dans chaque animal une proie possible, un adversaire ou un concurrent : le butin d'un lion, par exemple, attire immédiatement plusieurs espèces nécrophages, et celles-ci sont autant de ressources en puissance pour le prédateur, objet d'un partage différentiel, en fonction de l'adresse dont fait montre chacun. D'où une attitude à la fois plus agressive et plus défensive. La coexistence dans l'habitat mixte, solution de transition et solution de compromis, prolongée au-delà d'un certain degré d'efficacité des entreprises cynégétiques, révèle le plus clairement les oppositions fondamentales. Le va-et-vient constant d'une zone propice à l'approvisionnement végétal à une zone propice à l'approvisionnement animal, du terrain ouvert diurne qui prolonge la savane boisée à l'abri nocturne, implique une dépense considérable d'énergie. C'est l'implantation des arbres et non la richesse en gibier qui détermine en l'occurrence l'aire de recherche et d'exploration. On observe chez les babouins l'importance de cette contrainte. On a ainsi noté, dans un secteur près d'un marécage, à Amboise, riche en eau et en nourriture, une importante population de lions et trois troupes nombreuses de babouins qui y faisaient des incursions diurnes ; mais, les arbres étant rares, ils se retiraient pour dormir à un kilomètre de là, 
ce qui leur permettait de subsister sans danger. Les hominiens ont dû connaître une situation analogue. En tant que prédateurs, ils étaient désavantagés d'un autre point de vue encore, à savoir la répartition géographique des ressources. Les populations ont tendance à rechercher les espèces dans les régions où elles abondent et à s'y fixer, ce qui les oblige à renoncer à d'autres espèces plus rares dans le secteur défini. Celles qui se consacrent à la cueillette suivront les lignes de force de la flore au détriment de celles de la faune, car les plantes comestibles et les animaux de grande taille ne coïncident ni dans le temps ni dans l'espace.

Les collectivités ou les individus qui s'attachent à la fois aux deux sortes de ressources sont condamnés à la médiocrité, pris entre la nécessité de choisir entre l'activité principale qui leur est refusée et l'activité secondaire au caractère aléatoire. C'est pourquoi les hommes obligés de se rabattre sur les ressources complémentaires en nourriture et savoir-faire n'ont eu d'autre recours que de se séparer de leurs congénères pour former des groupements distincts. En se rendant indépendants du milieu de la cueillette qui ne leur était plus favorable, ils ont cherché à mieux s’intégrer à celui de la prédation qui leur offrait des possibilités plus grandes, à condition d'être meilleurs prédateurs que les meilleurs. La migration des populations vers les zones où ils s'éloignaient des tropiques, zones où la végétation habituelle fait défaut tandis que le gibier y abonde, a facilité cette tâche en la rendant urgente. Plus tard, dans les régions subarctiques, le régime carné servant à procurer des moyens de subsistance pour l'hiver est devenu presque exclusif. La dissociation qui s'était d'abord inscrite dans le temps a pris le caractère d'un partage de l'espace, dédoublement de l'entourage physique parallèle à celui des facultés qui lui avait donné naissance. Les cueilleurs demeurent dans les régions chaudes, les prédateurs s'avancent dans les régions froides et s’y installent irrévocablement. Cette division a imprimé une direction très précise à l'évolution des hommes et ses traces profondes sont encore visibles. Pareil résultat, on s'en doute, n’a pas été recherché consciemment. Le redéploiement de l'espèce, la répartition de son surplus, la redéfinition de ses caractères organiques et inorganiques n’ont pas été motivés par le désir impérieux d'innovation ou d'aventure ; il s'agissait plutôt de continuer ce qui existait, de poursuivre les gestes auxquels on était accoutumé. Les proto-hominiens ont mangé des animaux dans l'espoir 
de rester dans le monde des végétaux, tout comme, plus tard, les hommes ont employé les métaux dans l'intention d'élargir la gamme des pierres. Ce qu'il faut nommer l'effet Colomb, chercher les Indes et découvrir l'Amérique, a de la sorte marqué de nombreuses tentatives aux répercussions historiques incalculables.

\section{$\underline{\text { Retour à la Table des Matières }}$}

\section{Une séparation maîtresse.}

L'élan visant à perfectionner les méthodes de prédation et les dextérités des prédateurs, à vaincre les obstacles dressés par le cadre matériel et mental de la cueillette, l'impossibilité de se cantonner dans un rôle second et de répondre pleinement aux besoins qui ont suscité les nouvelles activités arrachent les dernières amarres. Les hommes qui, pendant des millénaires, se sont évertués à être des prédateurs supérieurs aux espèces avec lesquelles ils se mesuraient, devenant supérieurs à eux-mêmes se font prédateurs autrement que ces espèces : ils se transforment et se découvrent chasseurs. L'indice majeur, apparent, de ce tournant est la production des cadavres que l'on avait l'habitude de consommer. La prédation a appris aux hommes à tuer les petits animaux et à se nourrir des dépouilles des grands. Les deux facettes de leur activité fusionnent, grâce au transfert, du second groupe au premier, des techniques inventées en vue de la capture et de la mise à mort. Jusque-là, en effet, on savait seulement dépecer les carcasses de grande taille, en prélever la peau, en transporter la chair. La proie change de dimension, les opérations d'échelle. Les troupeaux affrontés, poursuivis, se composent désormais d'individus de taille imposante, nettement plus agiles, parcourant des régions très variées, dispersés sur un espace plus vaste. Le butin n'est pas consommable immédiatement ou en entier. Il faut découper l'animal, en emporter une partie. D'où la naissance d'une série d'opérations annexes concernant le dépeçage, la conservation, l'usage différentiel de la viande, de la peau et des os, avec les habiletés et l'outillage appropriés. Le champ de la coordination sociale s'étend dans deux directions. Durant la prédation, elle est motivée par la défense contre les grands prédateurs, la signalisation et la consommation sur place des reliefs des carnassiers, analogues à la signalisation et à la consommation sur place des fruits. La chasse — bien qu'il ne faille pas exclure la possibilité de captures 
individuelles - fait appel à la coopération depuis l'organisation de l'expédition jusqu'à la capture du gibier, sans omettre la répartition des tâches avant et après la prise. D'autre part, le travail de l'individu dépend, à chaque instant, de celui de ses compagnons. Ceux-ci à leur tour veillent à ce qu'il soit capable de remplir parfaitement sa fonction. La socialisation générale des actions, leur articulation dans un ensemble, s'accompagnent d'un soin soutenu accordé aux qualités intellectuelles et physiques des participants, d'une uniformisation de leurs capacités, ayant pour enjeu le succès et la survie de tous. L'homme débute ainsi dans sa carrière de faiseur d'hommes. Les savoir-faire et les instruments matériels et sociaux lui permettent de donner un caractère substantiel aux ressources animales qui pourvoient à toute une série de besoins courants, à commencer par la nourriture. Elles commencent à se substituer avantageusement aux ressources végétales. Les collectivités humaines peuvent dès lors se diriger, sans mettre en péril leur existence, vers les régions où celles-ci sont plus pauvres, renoncer définitivement à l'habitat protégé qu'elles leur offraient, s’installer dans un habitat différent, tout aussi protégé et aussi viable.

Les groupements - et on peut dire les espèces - de chasseurs s’écartent des groupements de prédateurs ; à l'habileté relativement diffuse, aux pratiques individuelles de ceux-ci, ils opposent leur habileté spécifique et leurs pratiques socialisées. Cette évolution a pris une forme plus radicale et plus reconnaissable. La redistribution stricte du champ opératoire des mâles et des femelles suit de près la sortie de la prédation de l'horizon de la cueillette, sa conversion en chasse. Nous avons là une des conditions nécessaires de cette sortie. Les raisons en sont apparentes. Un même individu ne pouvait guère mener de front deux activités ayant des exigences si contradictoires, faisant appel à des aptitudes si divergentes. Ce qui était frein d'un côté était moteur de l'autre et vice versa. Les talents nécessaires à la prédation n'ont pu se développer que jusqu'à un certain niveau - une analogie s'offre avec la poterie, pratiquée d'abord par la cultivatrice comme une activité d'appoint. Pour que ce niveau soit dépassé, il faut qu'une fraction du groupe prenne entièrement l'activité en charge, la rende autonome et principale - l'artisan, dans le cas de la poterie. Pour ce qui est de la chasse, la fraction masculine était toute désignée. Ses qualités physiques de force ou d'endurance n'y sont pour rien, ou presque. Si les 
chasseurs sont des mâles, c'est parce que les sous-groupes non reproducteurs, pionniers, objets de discrimination sociale, tenus de se vouer au règne animal, étaient formés de mâles. Pendant ce temps, les femmes ont gardé intact le fonds grâce auquel l'espèce continuait à survivre, elles ont persévéré, poussées par les circonstances, sur la voie autrefois commune. Au fur et à mesure que la chasse s'individualise, elles ajoutent ou conservent parmi leurs occupations ordinaires, complétant l'arrachement, le fourragement des plantes et la " cueillette » des petits animaux, l'utilisation des débris de la prédation. Il ne s'agit pas là, comme on l'affirme souvent, d'une simple division du travail, où les hommes s'occuperaient des tubercules et des grands animaux, tandis que les femmes se réserveraient les fruits et les petits animaux, chaque sexe entretenant un rapport unique avec le milieu en se spécialisant dans l'exploration et le travail d'objets différents faisant partie d'une seule ressource globale. Nous observons, au contraire, que les deux modalités d'insertion dans le cycle naturel s'écartent, comme ce serait le cas pour deux espèces parentes dont l'une vivrait sous l'eau tandis que l'autre se serait donné les moyens organiques de respirer hors de l'eau. L'aspect ponctuel, individuel, et pour ainsi dire préhumain de la cueillette est frappant. Un observateur anglais note que « pendant la période qui débute il y a 350000 ans pour s'achever il y a 11000 ans, les hommes avaient deux occupations principales, la cueillette de nourriture, comme les grands singes anthropoïdes (souligné par moi) ou comme certains primitifs contemporains, et la chasse, comme les Pygmées et les Eskimos " ${ }^{28}$. La chasse embrasse, nous venons de le voir, une chaîne complexe d'actions préparées, organisées, collectives, un équipement intellectuel et technique demandant une formation préalable des individus. Sous cet angle, la distance qui sépare le chasseur de sa compagne qui cueille, fourrage, est comparable à la distance qui sépare une espèce humaine d'une espèce protohumaine ou non humaine. La séparation des sexes autour de leurs ressources et de leurs facultés respectives suggère l'écart entre deux codes de la réalité, deux langues telles que le français et l'allemand par exemple, plutôt que la variation d'accent, d'idiotismes ou de style que pratiquent les personnes parlant la même langue mais appartenant à des classes sociales différentes, où l'on pourrait voir une analogie avec la division du travail. Elle n'établit pas seulement une communi-

28 A. COMFort : The Nature of Human Nature, New York, 1968, p. 68. 
cation difficile mais une incommunicabilité durable ; elle permet à une catégorie d'activités et de comportements sans équivalent dans le monde animal de se consolider, de lutter contre l'érosion du temps, et d'affirmer ce qui y distingue les hommes de façon durable.

Retour à la Table des Matières

\section{Les arts de la ruse et de la mort.}

La grammaire met à notre disposition un temps admirable, le présent historique. Il semble rapprocher le passé de nous, tout en le maintenant dans l'indéfini temporel. A plus forte raison lorsque ce passé est lui-même indéfini, et qu'il est malaisé d'en démêler les divers moments à travers des traces aussi incertaines dans leur existence que dans leur signification. Réticents à avouer notre ignorance et notre impuissance devant une déperdition si considérable d'événements, de travaux, et de sociétés, bouleversés par l'idée de nous trouver là sans savoir comment nous y sommes parvenus et d'être sans savoir comment nous sommes devenus, force nous est d'emprunter, à droite et à gauche, afin de tenter de reconstituer ce qui est irrémédiablement disparu. Les mémoires d'archives et les fausses réminiscences semblent préférables à l'amnésie, à l'histoire vidée de ses faits, destructrice involontaire de sa propre substance. Par la force des choses, notre récit se déroule toujours au conditionnel, s'alourdit d'artifices de langage et de controverses sur des vestiges que nous déchiffrons à la seule condition de leur retirer ce caractère d'épaves pour les assimiler à ce qui continue à vivre. Parlant des chasseurs d'hier, dont nous ne savons presque rien, nous nous résignons à les voir semblables aux chasseurs d'aujourd'hui, en leur attribuant la réalité que nous espérons avoir été la leur.

Reprenons le fil de l'exposé un instant interrompu par la nécessité de mettre le lecteur au courant de ce mélange inévitable des temps. Déterminés par les contraintes de la cueillette, les prédateurs humains sont incités à exploiter en nécrophages les espèces animales disponibles. Ils ne se montrent pas plus sélectifs qu'ils ne l'étaient dans le choix de la nourriture végétale. Le régime alimentaire des babouins, qui fournissent un terme de comparaison, ne comporte pas moins de cinquante espèces de plantes dont ils consomment les fruits, les bour- 
geons ou les pousses. L'alimentation carnée des hominiens semble avoir été tout aussi variée. Dans deux sites d'Afrique, datant d'il y a cinq cent mille ans - époque de transition à plusieurs égards - le butin inventorié comprend trois espèces de simiens, deux de carnivores, trois de moutons, trois de girafes, des buffles et un grand nombre de restes d'antilopes, de rongeurs, d'oiseaux et de tortues. Les proies ou les cadavres accumulés appartenaient donc à un large éventail de la faune, des mammifères notamment. L'abandon du contexte de la cueillette, l'évolution de la chasse vers l'autonomie, se signalent par une spécialisation poussée ${ }^{29}$. Les peuples de chasseurs se cantonnent dans la poursuite d'un nombre restreint d'espèces animales, accroissant leur expérience et concentrant leurs efforts afin d'obtenir du gibier plus abondant. Les vestiges trouvés près de Pékin, dans le célèbre site de Chou Kou Tien appartiennent à des mammifères carnivores et ongulés, deux espèces de cerfs seulement ayant fourni près des deux tiers des restes exhumés. En Espagne, près de Torralba, on ne chassait que l'éléphant, le bœuf sauvage, le cheval. En Croatie, une caverne d'époque aurignacienne contenait pour quatre-vingt-dix pour cent d'ossements d'ours. Dans le Sud de la Russie et en Europe centrale, les mammouths formaient le gibier principal. A Solutré, en Dordogne, on a découvert les restes de cent mille chevaux, tandis qu'à une époque plus tardive, il semble qu'on ait chassé de préférence le renne. Ces indications n'ont pas une valeur absolue ; il n'en reste pas moins que la chasse a tendance à se subdiviser, à devenir, préférentielle : les collectivités récentes, celles de l'homo sapiens, s'intéressent systématiquement à une seule espèce, en vue de satisfaire leurs besoins de tout ordre. Le règne animal est ainsi lui-même subdivisé, partagé, différencié.

La spécialisation des ressources va de pair avec la spécialisation des habiletés ; la continuité de leur emploi, dans un même cadre, à propos des mêmes espèces, conduit à un approfondissement et à une organisation stricte des opérations impliquées. Les chasseurs les cultivent méthodiquement, les condensent en un ensemble compact qui renferme un grand nombre de connaissances, un répertoire cohérent de comportements. On a dit avec raison que « la chasse pour l'homme

29 L.R. BINFORD, S.R. BINFORD : The Predatory Revolution, American Anthropologist, 1966, 68, 508-512. 
est faite par les outils mais est beaucoup plus qu'une technique ou même une variété de techniques ${ }^{30}$. Le piège en est le cœur. Art suprême, dans la chasse, son champ d'expérimentation est très vaste. Visant constamment un nombre restreint de communautés animales, il les a marquées, comme il a marqué les individus qui l'ont façonné. Son noyau essentiel est la capture, dont l'efficacité surpasse celle de l'attaque directe et met en œuvre des moyens complexes, étant données la sensibilité et la mobilité des futures victimes. Pour piéger, il faut concevoir et connaître l'usage des filets, des nasses, de fosses, des trappes, des piquets, susceptibles d'immobiliser l'animal, de l'emprisonner ; il faut prévoir le moment opportun pour l'assommer ou l'empoisonner aux moindres risques. Les Eskimos jusqu'à une date récente chassaient l'ours brun sur l'île de Kodiak par une méthode simple. Le chasseur fichait son javelot en terre, le maintenant à l'aide du pied, et l'ours s'empalait sur la pointe. Parfois on fixait une traverse au javelot pour tenir l'animal à distance du chasseur une fois qu'il s'était empalé. Cet ours, le plus grand carnivore actuel, atteint 800 kilos, tandis que le poids de l'Eskimo ne dépasse pas 65 kilos. Outre l'efficacité, ce qui compte pour le chasseur, dans cette méthode, c'est sa sûreté.

Le piégeage, et les techniques annexes incluent l'attaque et la défense dans une seule action. Ceux qui les pratiquent doivent connaître les habitudes, les pistes suivies par le gibier, repérer ce qui l'attire ou le fait fuir, savoir la distribution des individus d'une bande suivant l’âge, le sexe, le degré de coordination, la place dans la hiérarchie et le seuil de discrimination du leurre. Une telle somme de connaissances est l'œuvre de générations, matérialisée dans des langages, des mythes et des rites, reprise par une science orale constamment enrichie. Le contenu technique et intellectuel du piégeage porte témoignage du fait que la chasse est d'abord maîtrise de soi, résistance, mais surtout ruse. Ce dernier trait est capital dans la psychologie des peuples qui s'y adonnent ; il transforme une position de faiblesse en une position de force et ajoute à l'apparent, au donné, la dimension du simulé et du construit.

30 S.L. WASHBURN, C.S. LANCASTER : The Evolution of Hunting, in N. KorN et F. THOMSON, op. cit., p. 68. 
En tant qu'agression directe, la chasse requiert adresse et précision. Déclencher une avalanche de pierres, lancer des flèches ou des objets lithiques effilés, comporte un effort disproportionné au résultat. L'important est de bien viser la cible mouvante ou fixe, d'avoir une perception nette de l'anatomie de l'animal, de discerner ses parties vulnérables et de savoir comment il réagira une fois atteint. Les notions ayant trait à la vitesse, à la direction du déplacement, aux blessures causées par tel ou tel instrument, sont indispensables pour éviter d'être attaqué par l'animal rendu furieux. Les chasseurs d'aujourd'hui ont, semble-t-il, recours à l'observation et à la dissection, à des fin médicales et hygiéniques, notamment en vue d'entraîner leur corps. Ils recueillent ainsi des connaissances étendues, qu'ils projettent sur les objets inanimés. Aux îles Aléoutiennes, les différentes parties de la planche de jet ont reçu des noms congruant avec ceux des parties du corps qu'elles reproduisent : ainsi la petite cheville d'ivoire servant à fixer le javelot se nomme le «ziphisternum », et l'on a de même le front, la bosse, la paume ; quant à la planche, peinte en noir d'un côté et en rouge de l'autre, elle symbolise la fourrure de l'animal et son sang. Nous procédons de manière analogue quand nous reconstituons les fondements de l'univers, la structure des objets, le fonctionnement de l'intelligence ou du corps à partir de la force matérielle prédominante, les faisant mécaniques avec les forces mécaniques, chimiques avec les forces chimiques, etc.

Piégées, attaquées, empoisonnées, massacrées inconsidérément, les espèces animales seraient menacées de disparition. Les peuples de chasseurs spécialisés, conscients de cette menace, "cultivent » le gibier, lui permettent de se reproduire et de survivre. C'est là une innovation par rapport à la prédation et à la cueillette. Assurer le renouvellement de la population animale sans cesser de la chasser, tuer sans exterminer, appelle un sens de la prévision et du contrôle très poussé, une théorie sous-jacente aux actions et au commerce avec les forces du milieu ambiant. Ce sens, que l'on observe aujourd'hui encore, a dû naître il y a très longtemps. Il interdit de s'attaquer aux animaux trop jeunes, ou en période de fécondation, ou à n'importe quel moment de l'année. Les tabous ont souvent eu la fonction heureuse de conserver les ressources vives des sociétés humaines. En voici un exemple frappant sinon concluant. Lors de la dernière glaciation, les groupes de chasseurs auraient découvert une nouvelle méthode de capture desti- 
née à leur procurer un butin massif. En guidant les troupeaux d'animaux, ils les poussaient jusqu'au bord d'une falaise, d'où ils les faisaient tomber dans un précipice pour les anéantir. Les tribus de Tasmanie connaissaient une méthode analogue. Elles encerclaient une vaste zone à l'intérieur de laquelle le gibier, rabattu vers le centre, était massacré. Les résultats de telles pratiques, on s'en rendit compte, risquaient d'être catastrophiques à la longue. Un tabou limita la fréquence de son emploi, préservant de la destruction une source d'approvisionnement irremplaçable. On ne saura probablement jamais si, à la dernière glaciation, il y a plus d'un demi-million d'années, on était arrivé à une semblable régulation des liens entre les collectivités humaines et animales; il n'y a pas de raison de croire qu'elle ait fait entièrement défaut.

De tout temps la chasse a incité les individus à respecter les habitudes des diverses espèces animales, à préserver leur habitat. Les Toungouses et les Aléoutiens, et ils ne sont pas les seuls, reconnaissent à l'animal la propriété d'un certain territoire et essaient de l'apaiser lorsqu'ils en franchissent les limites en usant d'un langage que l'animal est censé comprendre ; ou bien ils cherchent à le tenir en respect en le terrifiant. Ces conduites sont fondées sur l'étude de l'animal, l'observation systématique de ses comportements, choses très courantes. Un groupe de Toungouses ayant cru observer qu'un certain oiseau disparaissait dans un trou de la glace et émergeait en un autre point par un trou différent eut l'idée de fixer un fil à l'animal pour suivre son parcours sous-marin. Puis ils le tuèrent et en firent la dissection, examinant soigneusement sa peau pour voir si elle recelait des insectes. Il leur arrive fréquemment de garder des animaux jeunes pour les observer et mieux les connaître, tout en prétendant qu'il ne s'agit là que de jouets destinés à amuser leurs enfants. En fait l'examen attentif auquel se livrent les adultes ne trompe pas.

On peut donc soutenir à juste titre que «la connaissance acquise par l'homme de la morphologie, de la physiologie et du comportement de l'animal pourrait devancer de beaucoup l'adaptation technique. En fait il semblerait des plus vraisemblables que des outils furent confectionnés dans des buts spécifiques tels que ceux-ci, au nombre desquels 
figurait la recherche de nourriture ${ }^{31}$. La chasse a également entraîné les hommes dans la voie de la coopération sociale globale. A cette fin, il faut que les gestes, les renseignements, les signaux soient conventionnalisés, exprimés de façon à être compris et échangés facilement. Les postures, le rythme des mouvements prennent un aspect collectif et l'organisme de chacun est envisagé dans la perspective de la tâche commune. Cette domestication du corps et de l'intelligence commence dès que l'enfant mâle sait à peine marcher, et se poursuit jusqu'à l'âge où il est pleinement intégré à son groupe. Le chasseur affirme sa particularité en étant possesseur d'une faculté, d'un pouvoir auquel il s'identifie et qui le distingue. Ce n'est plus uniquement un prédateur spécialisé, un homme détenteur d'une habileté définie, formée au cours des millénaires : c'est un homme à part, qui se conçoit comme tel, éloigné du reste des hommes. Et notamment de la femme.

$\underline{\text { Retour à la Table des Matières }}$

31 W.S. LaUghLin : Acquisition of anatomical Knowledge by Ancient Man, in S.L. WASHBURN : Social Life of Early Man, Chicago, 1961, p. 169. 


\section{L’homme dénaturé.}

Retour à la Table des Matières

\section{A l'école des anthropoïdes artificiers.}

Jusqu'ici j'ai omis de parler des outils, à dessein. Ils jalonnent la séparation décrite, symbolisent aux yeux de tous, acteurs d'hier, observateurs d'aujourd'hui, la relation au monde qui en résulte. Je tenais à définir le cadre de leur apparition avant d'en préciser le sens. Mais là je me heurte à quelques notions diffuses sur lesquelles il est nécessaire de s'arrêter.

Extraits de l'ensemble où ils s'encastrent si parfaitement, la chasse, les outils sont censés nous avoir arrachés à la poursuite animale, aux déterminations communes de la nature, en nous préservant dans cette grave solitude qui nous rend si fiers pour le passé et si inquiets pour le présent. Péremptoirement on déclare qu'ils concrétisent le trait distinctif de l'homme : «L'emploi des outils semble être le caractère biologique principal de l'homme, car, considérés dans leur fonction, ce sont des prolongements détachables de ses membres antérieurs ${ }^{32}$. " D’emblée, on semble considérer en tant qu'outils les seuls instruments lithiques, en laissant de côté nasses, filets, pièges, poisons, feu, tous artefacts qui ne prolongent pas une partie visible du corps. L'association exclusive de l'outil à la main qui, rappelons-le en passant, en est suivant l'évolution le prolongement organique, et non l'inverse, projette dans le passé, en le tronquant, une réalité artisanale. Mieux encore, se limiter aux objets obtenus à partir de la pierre d'où les divers "âges de la pierre " - revient à choisir entre toutes les matières premières employées à cette époque la seule qui ait échappé à la destruction, indépendamment de son importance effective dans l'ensemble des matières premières utilisées ${ }^{33}$. Pourtant, elle ne caractérise pas plus exactement l'état des savoir-faire et de l'homme d'alors que les métaphores l' « âge atomique » ou 1' « âge du

32 K.P. OAKLEY : Man the Tool-Maker, op. cit., p. 1.

33 Pour une critique analogue, voir V.G. CHILDE, Social Evolution, Londres, 1951. 
plastique » ne caractérisent notre état actuel. Adopter ce point de vue, c'est se condamner à n'envisager qu'un secteur restreint de l'activité humaine, que l'on analyse uniquement sur le mode technologique, en faisant abstraction des connaissances qui l'ont engendré, de l'organisation du travail qui l'a requis, et des besoins qui l'ont suscité en son temps. La comparaison avec les machines d'aujourd'hui, dont il constituerait la première ébauche, semblerait ainsi être plus pertinente que la comparaison avec la série des instruments et des pratiques à laquelle il s'intégrait. Mais un outil envisagé indépendamment de ses fins spécifiques n'est pas un outil du tout. Retrouvons donc ces fins au plus tôt.

La position du prédateur s'écartant de ses congénères humains et anthropoïdes attachés au fourragement des végétaux lui a facilité la saisie de la plupart des moyens employés comme autant d'éléments objectifs qu'il s'agissait d'essayer et de développer. Parmi ces moyens, à titre de ressources complémentaires ou accessoires, il faut compter les artefacts. A l'encontre d'une croyance largement répandue, ceux-ci ne sont pas apparus avec l'homme. Leur invention remonte à l'évolution préhumaine. Nous les rencontrons chez les espèces dites inférieures et chez les primates. Les jeunes babouins portent dans leurs mains des bâtons et des branches. Ils sont tous capables de casser un rameau lorsqu'ils cherchent une nourriture, larves ou fourmis. A l'occasion, on les voit tripoter, manier, examiner des objets peu familiers, déplacer une dalle de rocher pour satisfaire leur appétit insectivore. Les orang-outangs préparent soigneusement des bâtonnets qu'ils enfoncent dans un nid d'insectes pour les en retirer enduits d'une nourriture qui semble leur plaire. Les chimpanzés, à leur tour, confectionnent des artefacts dans le même but. Pour déloger et manger les insectes cachés dans les fentes des arbres, ils fabriquent des bâtons ayant les dimensions voulues. Parfois ils enfoncent de longues brindilles dans une ruche et les en retirent enrobées de miel. En regardant faire les adultes et en les imitant les jeunes chimpanzés apprennent à se procurer des termites. Leurs congénères de Liberia Creek savent ouvrir les noix de coco en les cassant à coups de pierre. D'autres se font des gobelets ou des éponges de feuilles pour aspirer l'eau et boire. Une équipe d'ethnologues a pu filmer le comportement d'une bande de chimpanzés mis subitement en présence d'un léopard empaillé. Le premier instant de stupeur passé, une explosion de cris 
aigus ou rauques salue la découverte de l'animal. Le gros de la troupe se précipite en avant, bientôt rejoint par les quelques fuyards qui avaient pris peur, et se lance à l'assaut en projetant des armes improvisées, gourdins ou arbres arrachés, accompagnant l'attaque de piétinements et de martèlements. Ils fustigent la proie au moyen d'arbres encore enracinés qu'ils inclinent dans la direction voulue. Dans presque tous les cas, les chimpanzés se tiennent debout, se servant de leurs membres antérieurs pour manier et lancer les projectiles. Alors que, dans les rapports sociaux habituels, les gestes de violence se bornent à des menaces fictives, ici ils sont nettement dirigés contre le léopard et visent un but précis, sa tête, autour de laquelle ils décrivent à peu près un demi-cercle. Les chimpanzés vivant dans la savane sont généralement plus adroits et se font de meilleures massues, en dépouillant de leurs feuilles des branches d'un à deux mètres de long, que leurs congénères de la forêt. Chez tous les primates ou anthropoïdes où l'on relève une conduite en relation avec la prédation et l'alimentation carnée, on note la présence d'instruments. Les aptitudes, si rudimentaires soient-elles, à les employer et à les confectionner, sont malgré tout assez répandues. L'outil n'est donc nullement notre apanage exclusif.

Devant ce constat, décevant, il faut le croire, on a voulu déplacer d'un cran la différence décisive recherchée, en soutenant qu'elle réside dans la capacité humaine de fabriquer des outils, concédant aux animaux celle de les utiliser. L'ordre de succession a son intérêt, puisqu'il exprimerait une supériorité intellectuelle : «La fabrication des outils exige un degré d'intelligence plus élevé que leur emploi ${ }^{34}$. » La distinction paraît subtile mais sa réalité est contestable. Elle naît d'une illusion rétrospective tendant à maintenir une division fonctionnelle propre à notre industrie dans un cadre où elle manque de fondement. En effet, prendre une pierre placée devant soi ou casser une branche d'arbre dans l'intention de les lancer contre un prédateur ne signifie pas utiliser un outil de la même façon que nous en utilisons un quand nous prenons un marteau ou une pince. La pierre ou la branche n’étaient pas des outils avant d'être arrachées, saisies, cassées. Le geste qui les dissocie des autres pierres ou des autres branches, qui les jette ou les manipule, leur donne une forme ou une fin, les classe en

34 K.P. OAKLEY : On Man's Fire, with comments on Tool-Making and Hunting, in S.L. WASHBURN, op. cit., p. 187. 
même temps parmi les instruments. Elles prolongent alors le bras ou le mouvement de projection, au lieu de prolonger des rameaux d'arbre ou de rester accolées à un tas de gravier. Elles se manifestent par des propriétés différentes : solidité, poids, souplesse, tranchant, efficacité. A tous les stades, aussi élémentaires soient-ils, les artefacts, comme leur nom l'indique, sont faits, ils sont le produit d'un effort et la transformation d'un matériau brut. Comment qualifier la pierre non travaillée, maniée pour façonner un morceau de bois ? L’individu qui exécute ce travail est-il utilisateur d'outils au niveau de la pierre et faiseur d'outils au niveau du bois?

La question a un sens précis. L’identification des objets lithiques préhistoriques est difficile. Les morceaux de pierre fracturée qu'étudie l'archéologue pourraient être aussi bien l'œuvre de l'homme que le résultat d'un accident quelconque indépendant de son intervention. Seule leur accumulation à côté de vestiges humains autorise à les considérer comme le produit d'un travail délibéré et non pas comme un phénomène matériel spontané. On est moins frappé, en les voyant, par la forme de chaque morceau que par le fait de les trouver en grande quantité dans un lieu insolite. Ainsi, dans le site de Sterkfontein, en Afrique du Sud, nombre de ces outils sont de simples galets fluviaux n'ayant subi aucune transformation. Il a cependant fallu un motif précis pour qu'une communauté humaine les transportât depuis les graviers situés à plusieurs kilomètres de là. Leur entassement pur et simple avait peut-être un sens instrumental que nous ignorons. A supposer que ce ne soit pas le cas, on ne saurait en conclure que ces communautés, capables de dépenser tant d'énergie pour accomplir une tâche précise, ne faisaient qu'utiliser des outils. Elles auraient été en mesure de confectionner une gamme étendue d'instruments en bois, ou en os. Les branches ou les bâtons sont normalement peu commodes, trop grands ou trop petits et souvent pourris. Leur emploi courant n'est possible qu'une fois qu'ils ont été façonnés. Les hommes préhistoriques étaient certainement capables de les couper à la dimension voulue, de leur donner une forme pointue ou spatulée. Plusieurs sites en Europe attestent, à une époque relativement tardive, il est vrai, l'existence d'une telle capacité. La combinaison de la pierre et du bois, l'un servant d'outil à l'autre, se retrouve chez les tribus australiennes qui coupent parfois des arbres et se font des instruments de bois en prenant pour outils des pierres à l'arête coupante. L'os sert 
de matière première à de nombreux artefacts obtenus par brisure et clivage, présents en plusieurs sites. On peut du reste se demander pourquoi casser une pierre serait un acte technique, alors que casser un os ou une branche ne l'est pas. Contrairement aux matériaux lithiques, les témoignages qui nous sont parvenus par ces matériaux, très périssables, sont fort maigres. Leur absence dans nos archives n'implique cependant pas qu'ils aient été peu importants ou aient fait complètement défaut dans la vie de ceux qui pouvaient difficilement s'en passer. L'image de l'homme faiseur d'outils, à la suite d'un éclair de la pensée conceptuelle convertissant une créature sous-humaine en créature pleinement humaine, couronnant le processus d'hominisation, cette image a trop vécu. La ligne qui sépare l'artefact-produit de l'artefact naturellement donné s'évanouit dès qu'on essaie de la dessiner rigoureusement. Les postulats sur lesquels on s'appuie sont fragiles, et son existence, notamment du point de vue archéologique, est sujette à caution.

Envisagée historiquement, notre habileté instrumentale est l'extension quantitative d'une habileté analogue, manifeste chez beaucoup de primates supérieurs ou anthropoïdes. Si ceux-ci ne l'ont pas poussée plus loin, c'est qu'elle ne présentait pas pour eux un intérêt évident. Les chimpanzés sont, instrumentalement parlant, fort avancés. Leur organisation sociale et leur adaptation physique apparaissent pourtant, aux yeux de l'observateur, moins réussis que l'organisation sociale et l'adaptation physique des macaques. Les outils n'ont pas en eux-mêmes, pour toute espèce et à tout moment, le privilège d'une fonction supérieure. Il n'est pas opportun de leur conférer un tel privilège, sans tenir compte des comportements et des ressources qui les requièrent. Il est inexact de les envisager, de les définir comme des appendices ou prolongements directs du corps, à l'instar des cisailles ou des burins prolongeant une machine, la machine humaine en l'occurrence. A l'encontre d'une opinion répandue, ce ne sont pas des «adjonctions au corps qui complètent les mains et les dents » ${ }^{35}$. Seule la prédation, que l'homme a menée à son terme, leur assure une fonction constante, les articule avec une activité stable et cohérente, leur imprime une forme et les complète anatomiquement. Au cours de ce long développement, tout dépend d'elle, et non pas de la décou-

35

Idem, p. 167. 
verte d'un savoir-faire industriel, à part, qu'aucune espèce animale n’aurait possédé : « Les êtres humains ne sont pas devenus humains, écrit un anthropologue américain ${ }^{36}$, pour apprendre ensuite à chasser d'autres animaux ; ils étaient déjà chasseurs et ont tout simplement modifié leurs méthodes de chasse en liaison avec l'acquisition d'idées qui permettaient l'emploi des outils, et, plus tard, leur fabrication. » La conception et l'emploi des artefacts - les objets lithiques inclus — sont des conséquences et non pas des facteurs indépendants ou des causes dans le processus d'assimilation à un ensemble de pratiques et de connaissances ayant une organisation particulière. A l'intérieur de cet ensemble, le rôle des outils est limité, voire modeste. Ils consomment une fraction minime du temps disponible. Les instruments et les armes improvisés sont suffisants pour accomplir la plupart des tâches courantes. Les conséquences de leur adoption sont cependant telles qu'on les a qualifiées: immenses. Une fois inclus dans l'ensemble auquel ils participent, ils concourent, en tant qu'éléments de cet ensemble, au remodelage des parties du corps rendu apte à exécuter les tâches qui sont les siennes. Il ne s'agit pas seulement de nourrir et de soigner ce corps, de satisfaire ses divers besoins instinctuels, physiologiques, subjectifs, afin de le maintenir en vie dans l'habitat matériel et social coutumier. Il faut encore développer sa vigueur et son adresse, qualités indispensables lorsqu'il affronte les autres espèces, en le prenant en quelque sorte pour objet, pour matériau consciemment élaboré. Il est dépositaire de la force individuelle ou collective opposée aux forces du milieu ambiant, non pas en tant que donnée mais en tant que produit minutieusement travaillé. Il émerge du fonds indifférencié, terme de référence, pôle d'une relation où il a le milieu pour contrepartie. C'est sur ce point que l'homme a surpassé ses maîtres ès artifices, les anthropoïdes chez lesquels il a tout pris et tout appris.

Retour à la Table des Matières

(2) La naturalisation des artifices.

Les outils enlevés à l'ordre de succession qui conduit à la machine et replacés dans celui qui a eu pour aboutissement la chasse, leur évo-

36 W.S. LAUGHLIN : art. cit., p. 151. 
lution s'est vraisemblablement déroulée en deux temps. La première étape est celle de la généralisation de leur usage et de leur fabrication. Mais de quels outils s'agit-il ? Après avoir fait leur inventaire dans le règne animal, Ronald Hall a eu l'heureuse idée de les classer en outils domestiques, prolongeant l'action du corps afin d'obtenir une nourriture qu'il est impossible d'atteindre autrement, et outils agonistiques nécessaires à l'attaque et à la défense contre les prédateurs. Les premiers sont repérés un peu partout sur l'échelle des espèces. Les seconds sont l'apanage des primates et des anthropoïdes. Les hominiens, si l'on pense à leurs conditions de vie, les trouvant à leur disposition, produits d'une longue évolution, les ont repris et perfectionnés. Sur ce point la station debout leur a été un atout considérable. Un quadrupède est à même de manier, en posture assise, un instrument domestique quand il s'agit de découper sa viande, d'écraser des os, et, au besoin, de déloger un petit animal. Pour manipuler efficacement un instrument agonistique, par contre, il faut que l'individu puisse courir facilement, se tenir droit, viser loin. La libération des mains, la locomotion bipède correspondent à ces fins et les favorisent. Les plus anciens des sites explorés nous montrent que ces instruments se sont diffusés sous formes d'éolithes — silex aux bords naturellement taillés — de haches à main à peine modifiées par cassure d'os brisés, de cailloux et de petites dalles de pierre effilées. Les pierres arrondies ou coupantes, accumulées autour des lieux d'habitation ou des campements provisoires, constituaient vraisemblablement des réserves de projectiles, là où l'on n'avait pas d'arbres sous la main pour en arracher les branches et les jeter contre l'intrus. Cet arsenal devait comprendre des massues défoliées d'une bonne longueur. Les connaissances afférentes, en vue de la protection ou de l'agression, s'organisent autour de ces outils. L’uniformité des objets choisis et partiellement ouvrés en, témoigne. La présence de l'homme sur la terre est attestée par la première fois, sans doute possible, par l'existence d'instruments grossiers, que l'on a groupés sous le nom de "culture lithique ». Ils sont formés d'une matière première conçue et travaillée en vue d'atteindre une fin particulière. Dans le bassin du Haut Kalifa, à 80 km au nord d'Elisabethville, on a recueilli des quartzites portant la trace de coups violents sur un côté, parfois une des faces du caillou a été travaillée. Selon l'Abbé Breuil, ces pierres à l'arête tranchante devaient servir d'outils pour le travail du bois. Ce qui frappe est leur uniformité, leur adaptation à un but précis, la façon dont elles s’intègrent en permanence aux occupa- 
tions courantes. La pierre se range désormais au nombre des matériaux familiers.

Ensuite, lorsque la chasse devient une occupation pleine et un domaine à part, elle s'accompagne d'une différenciation des instruments. C'est peut-être en cela, beaucoup plus que dans leur fabrication, que réside leur caractère proprement humain. Ou plutôt, pour être précis, leur caractère masculin, puisqu'ils sont l'œuvre des confréries cynégétiques mâles. L'éventail instrumental s'élargit au fur et à mesure que les opérations se multiplient, les sous-produits de l'animal étant exploités systématiquement. La pierre passe de l'usage agonistique, de l'état de matériel lourd, à lancer, à l'état de matériel solide, à manier. L'outil lithique a des propriétés dont on a dû vite prendre conscience. Avec un fragment écaillé, on peut couper facilement la chair et l'os. Tenu dans la main, il sert à concasser, gratter ou creuser. Plus que pour la mise à mort, c'est pour dépecer et écorcher l'animal que les communautés de chasseurs conçoivent les pierres écaillées. Grâce à elles, ils peuvent opérer sur place le démembrement des carcasses, le découpage de la viande, évitant d'avoir à les transporter ou de laisser pourrir une quantité considérable d'aliments. Pointes, grattoirs, couteaux, lissoirs sont inventés successivement et adaptés à une tâche particulière. Les burins - lame dont les côtés amincis obliquement se joignent en une fine pointe de ciseau - permettent le travail de la pierre tendre, de l'os, de l'andouiller et du bois. Les merlins et les javelots, les couperets en Asie, s'ajoutent à cette gamme ; les spécimens trouvés près des vestiges de l'homme de Pékin montrent ces artefacts dans leurs tout premiers stades. A partir de là, un faisceau de talents se manifeste dans chaque site où l'on a relevé des traces d'existence humaine. Les méthodes et les styles portent la marque d'une population, d'une région, de l'animal que l'on chasse habituellement. En les spécialisant et en les rattachant à un emploi précis, les chasseurs font de ces éléments accessoires des instruments nécessaires, moyens d'action et moyens de dévoilement de leurs propriétés ainsi que des propriétés de leur milieu. Ils représentent moins les prothèses d'un organisme modifié, qu'un savoir-faire rigoureux, établi, moins un système technique séparé qu'une fraction d'une chaîne de comportements intellectuels et sociaux. 
La structure anatomo-physiologique analogue à celle des anthropoïdes redressée, aménagée, atteint le seuil supérieur de son adéquation à cette chaîne de comportements et à l'entourage naissant. Il faut à présent qu'interviennent des modifications permettant d'inclure les composantes intellectuelles, perceptives et instrumentales de l'art du chasseur à sa biologie. La réussite a été si remarquable que l'on pense souvent que celui-là a été rendu nécessaire par celle-ci : l'art a imité la nature, même là où il a été imité. La paléontologie a rafraîchi notre mémoire, réparé un oubli : la marche, la spécialisation de la main, l'expansion relative du cortex renforcent neurologiquement, musculairement des facultés non biologiques. Elles font partie intégrante de la nature première des hommes actuels, mais non de celle des hommes ayant vécu il y a un million d'années. Celle-ci les a produites : l'art d'un homme devient toujours la nature d'un autre homme.

Les mains de tous les primates anthropoïdes sont préhensiles. Sauf chez l'homme, elles servent aussi d'organes locomoteurs. Leur mode de préhension se manifeste par la position respective des doigts, de la paume, du bras pour tenir un objet ou par leur manière de se tendre pour le saisir. La main doit pouvoir tenir l'objet de façon sûre et le maintenir dans une position fixe. Les opérations manuelles combinent une prise de force et une prise de précision. La prise de force stabilise l'objet en l'enserrant dans un crampon formé par la flexion partielle des doigts; le pouce renforce la saisie en exerçant une pression en sens contraire. La prise de précision consiste à saisir l'objet entre les phalanges terminales respectivement du pouce et des doigts qui interviennent tous plus ou moins selon la finesse de l'objet. Dans les diverses espèces, les schémas moteurs servant à la prise de force sont plus ou moins coordonnés avec les schémas moteurs sous-tendant la prise de précision. Le babouin utilise l'index et le pouce pour extraire le dard d'un scorpion, et le chimpanzé de même extirpe une épine qui s'est logée dans sa peau avec une adresse dont peu d'entre nous seraient capables. Mais la faiblesse relative de leur pouce les empêche d'exercer une prise de force, sans compter que sa longueur est bien inférieure à celle des autres doigts. Le raffinement et l'extension du domaine d'application des dextérités manuelles ont provoqué des modifications des os, des liaisons sensorielles et musculaires destinées à fonctionner lorsqu'il s'agit de saisir un animal, un instrument, un aliment, avec la maîtrise nécessaire. En particulier, le développement, 
chez l'homme, d'un pouce complètement opposable aux autres doigts lorsqu'ils sont fléchis, a certainement répondu au besoin de minutie, minutie nécessaire pour préparer un leurre, travailler un artefact ou dépecer les parties d'un animal. La main est désormais capable de se commander et de commander, la gamme de ses possibilités est déterminée pour longtemps. La mise en place du pelvis humain, avec les grandes enjambées qu'il autorise et le parcours des grandes distances qu'il tolère est, en comparaison, un changement mineur.

Le cerveau humain est le fils de la main humaine. Il est surtout le fils de la chasse ${ }^{37}$. La chasse provoque la rupture avec une évolution qui, même pendant la prédation, maintenait le cerveau proche de celui des primates et des singes anthropoïdes. Cette rupture a d'emblée un aspect quantitatif. La capacité crânienne de l'homme moderne est de 1200 à $1500 \mathrm{~cm}^{3}$. L'homo erectus d'il y a environ cinq cent mille ans avait déjà une capacité de 710 à $1100 \mathrm{~cm}^{3}$. La forme de son crâne était certes différente de celle de l'homme moderne. La calotte était longue et plate, l'os frontal, analogue à l'os frontal des chimpanzés et des gibbons, dessinait une arête continue au-dessus des yeux. Malgré ces similitudes morphologiques, le cortex ainsi abrité atteignait un volume qu'il n'est pas exceptionnel de rencontrer aujourd'hui, même chez des individus d'une très haute intelligence. Les hommes de Java et de Pékin étaient donc, par quelque côté, nos contemporains. Il est malaisé d'expliquer la croissance volumétrique du cortex. Elle est due, en grande partie, à l'accroissement de la taille du corps. La propension au contrôle strict des mouvements, à la discipline des organes d'agression et d'expression a eu, de son côté, une influence sur l'agrandissement des amygdales et parallèlement sur les parties « domestiques » du cortex qui leur sont rattachées. Mais le cerveau de l'homme n'est pas tant un cerveau plus grand qu'un cerveau différent. Depuis l'australopithèque, le nombre de cellules a augmenté de façon

37 « Le mode de vie du chasseur utilisant des outils et vivant au sol a créé le grand cerveau, au lieu qu'un homme à grand cerveau ait découvert certains modes de vie. Les auteurs croient que cette conclusion est de celles qui s'accompagnent d'implications ayant une vaste portée (implications que les auteurs n’ont cependant pas dégagées, S.M.) pour l'interprétation du comportement humain et pour les origines de celui-ci. " S.L. WASHBURN et F.C. Howell : Human Evolution and Culture, in Sol TAW (ed.), Evolution after Darwin, Chicago, 1960, t. 1, p. 49. 
significative. Cette évolution ne s'est pas faite au hasard. Ainsi chez le singe la zone du cortex qui correspond à la main est à peu près de la même taille que celle qui correspond au pied. A partir de l'homo erectus, les zones de projection du pouce, de la main, se développent de manière prédominante. Les aires sensori-motrices destinées à recevoir les informations tactiles provenant des doigts, à commander leur flexion et leur extension, se spécialisent et entrent dans une combinaison originale. Quand une région du cortex prend de l'ampleur et se modifie, les régions voisines suivent le mouvement, rendant « exploitables » de nouveaux territoires neuro-physiologiques. Chez les singes, les fonctions sensorielles et motrices occupent la plupart des surfaces disponibles. Leur essor, chez l'homme, a entraîné l'essor des segments adjacents du cortex, facilitant leur association avec l'habileté, la mémoire et, forcément, le langage.

La coupure des deux hémisphères, la dissymétrie de la gauche et de la droite, la subordination des liaisons nerveuses périphériques à un système central de coordination, inaugurent une économie mentale dont nous n'avons pas encore débrouillé les écheveaux. Elle s'est mise en place parallèlement à la mise en place des habiletés instrumentales cynégétiques et porte, assurément plus que nous ne le pensons, leur empreinte. La séparation qui les a accompagnées dans la recherche d'un développement indépendant s'est infiltrée profondément. Le dualisme logique persistant l'exprime, dans notre intelligence, dans le mode de construire nos codes de communication et d'organiser les éléments de l'espace et du temps. Si elle n'est pas une donnée génétique, dans le sens de propriété spontanée de la manière vivante, elle en est devenue une dans la mesure où notre cerveau, notre corps, la distinction des membres supérieurs et inférieurs, de la main droite habile et de la main gauche auxiliaire, l'ont résorbée et stabilisée. Dans le flux continuel des informations et des interactions, la trame perpétuée à travers les générations, se poursuit le travail de découpage des séquences significatives, introduisant non pas la discontinuité mais une discontinuité propre à l'exploration, à la connaissance et à la survie dans un ordre naturel que nous avons produit en nous produisant. L'aire linguistique, projetée de manière autonome sur le cortex, s'est agrandie en se renouvelant. La description des situations, des relations, la classification des animaux avec leurs traits et leurs habitudes, la spécification des outils, des matériaux et de leurs 
propriétés, sont des nécessités impérieuses, quotidiennes dans la vie d'une collectivité de chasseurs. A la fonction de communication se joint une fonction de codification ; au langage performatif déjà existant se superpose, en l'assimilant, le langage constatif, susceptible d'opérer par les moyens que nous lui connaissons. Il sous-tend les échanges complexes, répond au besoin d'ordonner et de transmettre d'un groupe à l'autre une grande variété de renseignements. La fusion des expériences accumulées est assurée, la coordination des tâches techniques et sociales facilitée, l'homme se sait parlant. Le langage, canal où circulent les savoir-faire avec les savoir-dire, engendre une temporalité, une substance historique du groupe restituant la voix des absents, incorporant le passé au présent et encadrant le présent dans le passé. Tournant décisif, puisque, dans le monde animal, la discontinuité est la règle, les générations s'effritent, les groupes tombent en poussière dès que le soutien écologique leur est retiré. La pensée conceptuelle, objet de tant de louanges, figure, en regard, une matière ou un pouvoir translucides, se glissant dans les interstices du jeu ininterrompu de l'acte et du mot. D'où la toute-puissance que tant de peuples lui ont généreusement reconnue. Il a fallu deux spéciations l'homo erectus et l'homo sapiens - pour l'amarrer définitivement et rendre naturels les deux organes les plus artificiels, la main et le cerveau. 


\section{Remarques finales : l'élément humain et la structure humaine.}

Pendant la plus grande partie de leur histoire, les sociétés humaines ont pratiqué la cueillette et la chasse. La genèse de cette dernière peut être décrite comme une suite d'adjonctions et de transformations qui affectent les facteurs organiques et physiques préexistants. En surface, son déroulement est subordonné à un combat direct avec les adversités climatiques, géologiques, de l'entourage matériel, scandé par des inventions qui, à chaque étape indécise, résolvent un problème, améliorent la technologie, engendrent des ressources là où elles faisaient défaut. Progressivement, l'aptitude à utiliser l'outil est complétée par l'aptitude à le fabriquer ; la richesse végétale ordinaire est augmentée par l'apport de la richesse animale supplémentaire. Examinée de près, cette genèse est surtout un mouvement de scission, d'écartèlement. Parti d'un démarquage hiérarchique des groupes reproducteurs et non reproducteurs chez les anthropoïdes puis les proto-hominiens, passant par la différenciation fonctionnelle correspondant à la prédation, il instaure, en même temps que la chasse et avec la chasse pour but, une véritable division structurelle, ayant sa contrepartie dans l'organisation de l'univers physique immédiat. L'impulsion initiale donnée par la surpopulation, sous les auspices d'un aménagement, inégalitaire, des rapports sociaux, débouche sur leur conversion en tant que rapports objectifs, sur une forme d'insertion naturelle distincte de la forme d'insertion initiale. Les bandes de chasseurs mâles, lancées sur cette voie indépendante, ont pu découvrir une somme de savoirs et de techniques considérable, aboutir à une modalité de reproduction des talents humains finalisée, disciplinée et communicable. La distance qu'ils ont ainsi instaurée entre eux et la cueillette s'exprime dans le partage, aux conséquences si vastes, des forces matérielles, de la flore et de la faune, des collectivités humaines, les femmes et les hommes, associées, identifiées à leurs tâches écologiques respectives. Le feu aidant, on a su faire sortir le gibier d'un endroit clos : un leurre de plus. Mais on a surtout réussi à rendre possible l'occupation des cavernes et des rochers. Les hommes quittant la 
forêt quittent aussi l'habitat " végétal » : ils s'installent partout où vivent les animaux, à leur place. Et de même que les ressources autrefois complémentaires acquièrent de l'ampleur, deviennent principales, indispensables, de même l'ancienne population superflue perd son caractère surnuméraire ; disparaissant comme superflue, elle reparaît comme nécessaire. A mesure qu'elles se propagent des tropiques vers les pôles, ces ressources façonnent l'environnement à l'intérieur duquel les sociétés émigrent, se concentrent, en assimilant pour subsister les éléments du milieu. Leur existence, reconstituée dans ses fondements, n'a pas été médiocre. Les tribus de chasseurs d'aujourd’hui montrent qu'en travaillant de deux à quatre heures par jour elles peuvent mener une vie décente, sans souci excessif du lendemain. Nous n'avons aucune raison d'imaginer que la situation ait été beaucoup plus critique il y a une dizaine ou une centaine de milliers d'années.

Au niveau désormais atteint, l'équilibre entre le volume des populations, les richesses disponibles et l'espace indispensable à leurs mouvements et à leurs entreprises est rétabli, et pour longtemps. Si les observations contemporaines ont une valeur indicative pour le passé, elles nous permettent de connaître ce niveau. L'étude de la densité démographique et de la distribution géomorphologique de 123 territoires appartenant aux tribus australiennes a mis en évidence une corrélation entre les dimensions des groupes et les ressources alimentaires du secteur habité. Chaque tribu occupe en effet un territoire d'autant plus vaste que la rareté des pluies y fait croître une végétation moins abondante et y diminue la population animale, donc le gibier. Cette recherche d'équilibre apparaît de façon particulièrement nette pour les tribus des zones dépourvues de points d'eau et de rivages. Un examen détaillé des données fait ressortir une dimension constante pour ces tribus, qui sont de 500 personnes environ, tandis que les unités qui les composent dépassent rarement 40 à 50 personnes. Pendant très longtemps, les collectivités de chasseurs ont ainsi maintenu leur volume stationnaire, en apprenant à vivre de ce que leur offrait l'environnement ; de la sorte elles ont réussi à se perpétuer. A l'arrière-plan de cet équilibre se profile la séparation préalable du groupe qui chasse et du groupe qui fourrage, du monde animal et du monde végétal. Elle concerne moins ces termes que leurs relations respectives avec le milieu, les capacités organiques et inorganiques qui les traduisent. Ainsi est née la faculté d'être homme et de faire des hommes: les animaux eux- 
mêmes, comme le recommande la spécialisation de la chasse et du gibier, sortent de leur indifférenciation pour devenir des espèces.

Je ne prétends instruire personne en relatant des faits qui sont tous connus. On les convoque régulièrement au tribunal de la science pour les faire témoigner de notre origine, nous assurer de son caractère d'événement. La poésie d'une telle recherche des commencements lointains est d'un attrait indéniable. Pourtant baliser les lieux où s'est opéré le passage éventuel du primate à l'homme demeure une entreprise troublante. C'est vouloir toucher du doigt, suivre à la surface de l'eau une ligne d'horizon s'éloignant dès qu'on s'en approche. Il manque, il manquera toujours un chaînon dans la succession qui va du singe-homme à l'homme moderne en passant par l'homme-singe. Lorsqu'on détache les caractères biologiques, intellectuels, techniques, de la totalité dont ils participent, les filiations se font linéaires, leur décalage apparaît arbitraire, et les discontinuités radicales provoquées par les pratiques qui ont suscité ces caractères se trouvent ramenées à des encoches sur une échelle continue. L'impression subsiste cependant - elle se fait jour à travers les controverses scientifiques - qu'il y a quelque chose de plus dans cette interférence des hominiens qui sont déjà hommes sans l'être entièrement et des espèces humaines n'ayant plus rien de l'anthropoïde sans s'en être dégagées complètement. Les essais tentés pour la dissiper : estimer la portée d'un instrument, d'une capacité mentale, d'une rectification anatomique, ou bien apprendre à parler à un chimpanzé, lui faire résoudre un problème dans l'intention d'évaluer avec exactitude notre proximité ou notre éloignement par rapport aux simiens, réussissent rarement. A vrai dire, l'impression est fondée. Les hominiens sont des hommes par leurs qualités discrètes dans un ensemble naturel anthropoïde, tandis que les hommes conservent des qualités anthropoïdes dans un ensemble naturel qui, lui, ne l'est plus du tout. Le retournement n'a rien d'étonnant. Les grands événements, la Renaissance, la Réforme, la Révolution française ou la Révolution bolchévique ont lieu plusieurs fois. Ils reçoivent cependant une date, ils se condensent dans une signification unique, pour les commodités de la mémoire et des célébrations collectives. De même, notre espèce n'a pas une seule naissance, mais deux. Les indices en sont visibles à plusieurs niveaux. Au niveau neurophysiologique se singularisent d'abord les centres locaux de la sensibilité sensori-motrice, du comportement verbal, ensuite le cortex 
avec ses centres spécialisés de coordination. Au niveau de la communication linguistique, le langage performatif insérant ses unités phonétiques dans un système de signaux non verbaux précède le langage constatif lequel intègre les éléments non lexiques dans le système de règles et de codage qui lui est propre. Enfin, au niveau de l'outil, on a commencé par uniformiser son emploi, sa fabrication, réalisant une extension quantitative du donné, avant de le différencier, ce qui a transformé qualitativement ses fonctions et son effet.

La première naissance est, dans la mouvance des hominidés, celle de l'animal particulier, se préoccupant de rendre commun ce qui était exceptionnel et de concentrer ce qui était diffus. Les facultés éparses dans l'univers anthropoïde, entretenues ou vivifiées de façon occasionnelle - poursuivre une proie, s'aider d'un artefact, se déplacer debout, explorer et inventer, transmettre et reconnaître des symboles - sont exercées régulièrement et de concert par un même groupe d'individus. Du coup, elles convergent dans un élément unitaire, fortement accentué, prenant du relief dans cet univers à côté des éléments qui sont depuis longtemps entrés dans sa définition. La prédation tisse la toile de fond sur laquelle comportements et échanges se croisent, se renforcent mutuellement, augmentant la fréquence, élargissant l'éventail des mutations génétiques qui, sans cela, seraient restées enfermées dans les replis de la matière vivante. Vu de loin, rien ou presque n'est vraiment neuf ; vu de près, tout a changé. Les espèces australopithèques résument cette condition dans leur corps et leurs ressources différenciés, incarnent la modalité particulière d'action qui la fonde. L'humanité, tout entière consacrée à la résolution du problème posé à la surpopulation par son milieu, est encore complémentaire de l'animalité. Le mouvement auquel elle a donné une si grande impulsion est encore réversible, le monde des primates lui reste ouvert.

A sa seconde naissance, l'homme, faisant fond sur les virtualités, les procédés, les organes empruntés aux autres espèces, développe les virtualités, les procédés, les organes qu’il a synthétisés, produits. L'homo erectus et l'homo sapiens cessent d'être à la fois proie et prédateur, de vivre dans la forêt et dans la savane, de confondre ce qui a trait au végétal et ce qui a trait à l'animal. Ce dernier, passant de l'état de congénère ou de concurrent à celui de force naturelle et de ressource, est, certes, précieux pour la nourriture carnée qu’il apporte. 
Mais, bien plus, lorsque la chasse mobilise les énergies masculines de manière exclusive, toute l'existence en dépend, est imprégnée par lui. Dans la réalité quotidienne, l'animal est aliment, habillement, matériau artistique, calendrier, ce dont on parle, ce à quoi on pense, le foyer autour duquel sont ordonnées les fêtes, les migrations saisonnières, les étendues. Les générations, les sexes, les groupes se situent dans ce cadre, tout comme, de nos jours, les classes sociales, les nations, les professions se définissent eu égard aux forces mécaniques, chimiques ou nucléaires. La possibilité biologique de consommer de la viande, celle de capturer, de manger des animaux réels ou imaginaires, a permis aux hommes de se manger et de se capturer entre eux comme animaux et comme surplus ${ }^{38}$. La «nature » anthropoïde est rarement agressive ou féroce. L’agressivité spécifique ou la férocité atteignent un seuil vraiment élevé avec le chasseur. Le cannibalisme, dont les traces sont nombreuses dans les sites préhistoriques, prolonge la chasse ; d'un certain point de vue, il en constitue le sommet. On cherche souvent le secret de la disparition des diverses espèces hominiennes et de l'unicité des deux dernières dans les modifications du climat, de la faune et de la flore. Ce secret est vraisemblablement enfoui dans la dynamique de leurs relations. Les hommes possédant des habiletés supérieures ont été en mesure de chasser ceux dont ils se démarquaient, demeurés à l'état de simples prédateurs, disposant de moyens techniques et sociaux de défense et d'attaque relativement modestes. Ou bien, dans une perspective analogue, en piégeant et tuant avec beaucoup plus de succès les animaux qui leur étaient communs, ils ont rendu la nécrophagie onéreuse, privant nombre de collectivités d'une de leurs sources d'approvisionnement ; ils ont modifié de façon radicale le milieu coutumier et fini par provoquer la disparition de l'espèce en question. Les séquelles des glaciations, avec le flux et le reflux des populations, ont complété ce travail millénaire. Que ce soit par voie de cannibalisme, ou à la suite de modifications de la faune dans un sens favorable à la chasse, l'héritage anatomophysiologique, technique, social, des primates non humains, conservé par les hominiens, s'est effacé, a été défait et retravaillé à l'intérieur de l'héritage anatomo-physiologique, technique, social, des hommes non primates. Avec des conséquences, comme on le sait, irréversibles.

38 E. MAYER : Taxonomic Categories in fossil Hominids, Quart. Biology, 1950, 15, 109-118. 
Sous nos yeux se sont déroulées les phases d'un mouvement de séparation, qui a duré plus d'un million d'années, le seul important, comparé aux effets cumulatifs ou de sélection sur lesquels on insiste tant. Ce mouvement est à la fois le point de départ d'une série d'incompatibilités et le résultat de leur résolution. Les grandes ruptures, les grandes divisions ont effacé les origines et imposé des recommencements. Elles sont l'œuvre d'hommes appliquant leur énergie et leur intelligence à convertir le superflu en indispensable, à maintenir ou transformer ce qui était condamné à disparaître. Attirer à soi les êtres animés et inanimés, uniformiser et démultiplier les gestes ou les instruments que des circonstances exceptionnelles amènent à la surface, régulariser le hasard et l'accidentel pour les intégrer dans le cours naturel des choses, est une entreprise parfois désespérée, toujours créatrice. Ces qualités qui sont nôtres, les milieux où nous vivons, nos ancêtres hominiens les ont activement recherchés : à la lettre, ils les ont produits. Il serait faux de dire qu'ils en ont eu la révélation, ou encore que les seuls changements géophysiques les ont contraints à quitter la forêt. On croit souvent que notre espèce a pour destin, depuis toujours, de lutter contre la nature, de maîtriser les forces externes, les éléments, la flore, la faune. Cette lutte est en réalité provoquée par l'homme. Les chasseurs se sont efforcés de maîtriser les proies, mais ces proies revêtaient pour eux une importance vitale ; elles avaient une portée humaine, puisque toute l'architecture biologique, psychologique, sociale dépendait d'elles. Constante, la lutte n'est pas toujours dirigée contre les mêmes adversaires. Elle se déroule chaque fois qu'une partie de l'humanité doit édifier sa réalité propre, dépasser le donné présent ; se dégager du passé, s’affirmer en prenant une option sur l'avenir. La survie est à ce prix. 


\section{Chapitre V.}

\section{De la sélection naturelle à la division naturelle}

\section{Sur l'inversion des rapports entre population et milieu.}

\section{$\underline{\text { Retour à la Table des Matières }}$}

La séparation de la chasse et de la cueillette est le premier exemple d'un phénomène dont il convient maintenant de formuler les traits généraux. On sait que les espèces sont assujetties à un destin qui les a choisies en alliant l'aléatoire des mutations au déterminisme de l'hérédité, reversant l'arbitraire cumulé des processus physicochimiques dans le cours nécessaire des processus organiques. Avant d'exister pleinement, toute espèce est réversible, ensuite chacune est indispensable. Le plan de son développement biologique tracé par son passé est reconstitué en permanence par son avenir ; sa substance est formée par l'addition des accidents repris en charge et stabilisés ; sa direction lui est conférée par la rectification du poids des termes au sein d'un système qui garde, dans l'ensemble, son invariance. La reproduction génétique assure partout les opérations qui modèlent sa substance ; le travail sélectif, en répercutant une partie des pressions de l'environnement, infléchit la direction qu'elle prend. La diffusion de la matière organique, phénomène constant, son évolution rendue ainsi impérative, nous montrent que ce que nous appelons survie est production ininterrompue de la vie. Les grands embranchements du règne animal correspondent à une migration en extension et en profondeur, traduisent les dispositions inhérentes aux espèces à envahir des espaces écologiquement nouveaux et à s'y installer. La reproduction différentielle des qualités spécifiques détermine quels sont les individus capables ou non de se prolonger dans leur progéniture ; elle ponctue cette évolution, en permettant aux êtres animés de s'adapter à 
un entourage matériel, de s'y maintenir et de faire s'épanouir une tendance qui leur est provisoirement propre : vivre dans l'obscurité ou sous l'eau, fourrager ou se nourrir de proies. En même temps, on enregistre les effets concordants d'une reproduction discriminante sociale en vérité - qui manifeste moins la structure génétique que le comportement des organismes constitués.

Quoiqu'il affecte des individus génétiquement similaires, le jeu des mécanismes internes, sociaux, de l'espèce, introduit un coefficient supplémentaire quant à leurs chances de survivre et de procréer. Expression la plus simple de ce mécanisme, une hiérarchie s'institue de ce fait entre eux qui modifie leur rapport à l'entourage tel qu'il résulterait des différenciations au sein d'une population inséparables de leurs qualités génétiques. Les objets, les stimuli physiques et sexuels, les aires de déplacement sont dotés d'une signification additionnelle qui varie avec chaque sous-groupe : ce qui paraît à l'un attrayant ou permis devient menaçant, interdit aux yeux des autres. Les relations ainsi marquées s'imposent à l'individu sa vie durant, et l'on peut dire que la population se compose de quelques catégories statutaires ayant des réactions communes. Ces catégories peuvent être le produit d'une transmission institutionnalisée de tâches et de comportements, ce qui expliquerait que, dans de nombreuses espèces, de mammifères en particulier, on rencontre des associations d'individus, des écarts de rang qui ne reposent pas seulement sur le sexe et sur l'âge. Le mode de reproduction décrit, introduisant dans chaque espèce une hétérogénéité sociale à partir d'une relative uniformité génétique et une homogénéité des fonctions attribuées à des individus à travers les espèces, malgré la diversité génétique, a accru le pouvoir organique général et déterminé ses performances. Il a rempli, à cet égard, pendant l'évolution, un rôle d'équilibration surtout quantitative. Chez les primates, les anthropoïdes, les hominiens, son influence comparée à la reproduction différentielle, sexuelle, est devenue prépondérante. Une seule espèce est désormais capable d'engendrer et de perpétuer plusieurs systèmes de rapports collectifs auparavant réalisés par des espèces séparées ayant un système unique ; et un processus ayant produit successivement les éléments et les combinaisons d'éléments sociaux mûrit au point de les refaire, de les employer ou de les articuler simultanément. A l'échelle de l'univers, tout se passe comme si un procédé ancien s'était instruit et enrichi grâce à la confluence de maints procédés 
conçus dans différentes régions de l'espace et du temps, par des créatures biologiquement distinctes. Les idées d'indépendance envers le milieu, de suspension du travail sélectif, notamment en ce qui concerne l'homme, ont traduit habituellement la portée de ce changement de rapport entre le social et le génétique. " Il est évident, écrit Jacques Monod ${ }^{39}$, que la part des performances téléonomiques dans l'orientation de la sélection devient de plus en plus grande à mesure que s'élève le niveau d'organisation, donc d'autonomie de l'organisme à l'égard du milieu. Et cela au point qu'on peut sans doute considérer cette part comme décisive chez les organismes supérieurs, dont la survie et la reproduction dépendent avant tout de leur comportement. » Le même auteur affirme aussi que, dans ces sociétés, la sélection a été supprimée. Du moins n’a-t-elle plus rien de «naturel » au sens darwinien du terme ${ }^{40}$.

Nous nous sommes en effet détachés d'une partie de la biosphère, celle qui est commune à la plupart des espèces animales. En revanche, nous avons établi des liens de dépendance avec ses parties non communes, plus négligées ou plus cachées, restructurant différemment notre entourage physique direct. Prendre cette transformation pour un signe d'autonomie, c'est n'en retenir que l'aspect négatif, souligner le contraste avec un état antérieur. L'aspect positif nous intéresse davantage : il s'agit du parachèvement d'une évolution qui situe le comportement en tant que véritable médiateur entre l'organisme et le milieu. Les facteurs qui y ont contribué sont connus. Tout d'abord, l'allongement de la durée de la vie au-delà de la période de procréation sexuelle. Les intervalles entre générations augmentent, le rythme de croissance des populations, des modifications génétiques se ralentit probablement, facilitant la stabilisation des groupes spécifiques et des rapports entre leurs membres. La prolongation du développement préet post-natal, ayant pour conséquence de faire naître l'individu avec un système neuro-physiologique inachevé, est le second facteur. Il semble avoir pour cause l'augmentation du volume du cerveau. Elle suppose un cerveau plus grand chez le fœtus, donc un pelvis plus grand chez la mère. Paradoxalement, la locomotion bipède a eu pour effet de rétrécir le canal osseux par lequel a lieu l'accouchement. La

39 J. Monod : Le Hasard et la Nécessité, Paris, 1970, p. 142.
40
Idem, p. 179. 
solution de ce problème d'obstétrique est inscrite dans la constitution féminine : l'enfant est mis au monde à un stade de maturation moins avancé et appelle des soins relativement plus nombreux avant de compléter sa croissance normale. Cette tendance se dessine nettement chez les primates et chez les singes anthropoïdes, mais le cerveau et le corps atteignent pour ces espèces une taille et une proportion définitives à un âge plus précoce que chez l'homme. La capacité crânienne du bébé humain est environ 25 \% de la capacité adulte, tandis qu'elle est de 35 à $60 \%$ chez les simiens. L'existence d'un tel décalage donne toute sa signification à l'immaturation de notre espèce, qui n'est que l'accentuation d'une tendance générale. Il ne s'agit cependant pas d'un phénomène biologique à part, qui a eu lieu d'une manière indépendante, et devant lequel les collectivités humaines se sont vu obligées de réagir, instituant une famille ou une société destinée à lui faire face. Il est plus conforme à la réalité d'y voir une conséquence des nouvelles activités, de la chasse, principalement responsable de l'évolution quantitative et qualitative du cortex, donc aussi de l'immaturation fœtale décrite, avec pour contrepartie la longue dépendance du jeune envers l'adulte. La disponibilité et la lenteur organiques sont devenues à la longue un avantage en même temps qu'une charge, les hommes plus que les autres espèces, étant mis dans l'obligation de compléter le processus biopsychique, d'interférer avec lui. La plasticité, la non-spécialisation des nouveau-nés les aide à assimiler les comportements sensori-moteurs proposés et imposés. L'initiation aux formes de vie courante, au milieu particulier, ressemble en l'occurrence à l'imprégnation, telle que l'étudient les éthologues, de l'organisme par les postures, les mouvements, les modèles de stimulation visuelle, sonore, etc. L'apprentissage proprement dit s'y superpose ultérieurement. Du reste, jusqu'à une date très récente, il est bon de le rappeler, l'introduction aux pratiques et aux techniques s'effectuait très tôt par l'observation, l'exemple et la communication orale. Ainsi ce qui appartient à l'univers des adultes - instruments, ruses, attitudes ou gestes relatifs aux animaux et végétaux - s'entretisse avec les appareils neuro-physiologiques avant leur mise en place définitive. La capacité innée à assimiler les éléments extérieurs, à les intégrer aux structures opératoires existantes comprend un secteur dans lequel s'articulent les comportements disponibles; elle dépasse le niveau de ce qui est donné par la reproduction génétique pour ramener celle-ci à un segment de la reproduction naturelle des hommes. 
D'un autre côté s'opère une inversion dans le rapport entre la population et le milieu. De longue date, mais surtout depuis Malthus, on a considéré la population comme étant entièrement fonction des disponibilités du monde matériel ; en termes techniques, elle est définie comme une variable dépendante fluctuant au gré de la variable indépendante exogène représentée par les ressources. La biologie darwinienne a généralisé à l'ensemble du monde vivant cette conception qui est devenue une loi d'airain pour les espèces ; elle a aussi cherché à démontrer que tout ce qui les affecte génétiquement, du point de vue de leur volume et de leurs caractères, traduit l'organisation et l'étendue de leur environnement. La capacité d'adaptation équivaut à un équilibre quantitatif et qualitatif établi dans ces circonstances, facilitant la survie des individus et leur reproduction. Assurément, il faut garder l'hypothèse d'une constance démographique; sa réalité, on doit en convenir, peut être contestée, et l'a été : « Une des généralisations les plus importantes que l'on puisse faire au sujet des populations d'animaux sauvages, écrit un spécialiste de l'écologie animale ${ }^{41}$, est que leur nombre est sujet à d'importantes fluctuations. Les naturalistes du XIX ${ }^{\mathrm{e}}$ siècle ont repris sans la modifier l'idée d'un équilibre de la vie, c'est-à-dire d'une population constante. Les conceptions religieuses antérieures incluaient l'idée que le monde avait été créé de façon ordonnée et attribuaient les perturbations de cet ordre à l'action de Dieu punissant l'homme de sa présomption à bouleverser cet ordre ou peut-être à faire quoi que ce fût. Cette idée générale a trouvé tout naturellement place dans les théories biologiques ultérieures de l'adaptation chez les animaux, puisque l'on supposait (à juste titre) que les animaux étaient étroitement adaptés à leur entourage et (à tort) que cette adaptation conduisait à un état d'équilibre stable entre les membres des différentes espèces. "

A supposer que ce soit le cas, nous ignorons pour l'instant quel est le véritable facteur limitatif de la fécondité animale. On a cru pouvoir affirmer que les variations démographiques annuelles étaient liées à la quantité de nourriture disponible, indice de la productivité et de l'adéquation au milieu. Mais on n’en a pas donné d'exemple probant, pas plus qu’on n’a décelé le mécanisme de cette prétendue limitation

41 C. ELton : The Ecology of Animals, Londres, 1968, p. 61. 
de la population en fonction de la quantité et de la nature des aliments. Des cas troublants pour la théorie ont été décrits. Ainsi les populations de lémurs, à Madagascar, ne consomment pas toute la nourriture qui est à leur portée. Les crabes décimés par l'explosion atomique sur l'atoll d'Eniwetok l'ont repeuplé par la suite et ont retrouvé leur densité initiale. Faute d'algues en quantité suffisante, ils se sont rabattus sur les fibres extérieures des tiges des plantes. Ces exemples tendraient à prouver l'existence d'une densité donnée pour une population et la tendance des individus composant la population à préserver cette densité en face de ressources variables et variées. Force est de renoncer à l'idée d'un rapport rigoureux entre le nombre d'individus et la quantité de nourriture : "L'idée malthusienne, si populaire, que le nombre d'individus qui survit chaque année est déterminé par la quantité de nourriture disponible, la population excédentaire mourant de faim, n’est plus défendue par aucun de ceux qui étudient la population naturelle ${ }^{42}$. » Toutefois, tant de faits s'appuient sur ses autres aspects que l'on doit, malgré tout, prendre cette théorie en considération. Elle présuppose une invariance qui subsiste à travers les fluctuations démographiques annuelles et déduit que l'apparition d'un "superflu » en mutations ou en individus a pour effets négatifs l'exploitation excessive des ressources, la propagation de maladies pernicieuses et, à la longue, la destruction d'une partie de l'espèce. Le retour à une situation normale se produit si, entre temps, l'espèce n'a pas été dangereusement diminuée ou n’a pas donné naissance à d'autres espèces, les barrières écologiques ayant interféré avec la circulation des gènes.

A partir des primates supérieurs peut-être, à partir des hommes sûrement, ce modèle et ses conséquences ne s'appliquent plus. La population semble jouer le rôle d'une variable indépendante, d'un élément moteur qui soumet l'univers matériel à sa pression. Le surplus démographique intervient de manière constante, entraînant la différenciation et la recombinaison des ressources en nourriture, savoir-faire, talents, incitant les collectivités à s'étendre, à inclure ces ressources dans leur cadre de vie au lieu de se soumettre à la configuration préexistante. La propension des groupes humains à respecter une certaine

42 D.H. StotT : Cultural and Natural Checks on Population Growth, in A.P. VAYDA : Environmental and Cultural Behavior, New York, 1969, p. 191. 
densité, à proportionner leur nombre à l'espace et aux richesses qu'ils possèdent, est très ancienne et constitue un effort nécessaire de conservation. Elle traduit le souci de rétablir l'équilibre dans un mouvement qui tôt ou tard l'emporte, provoquant des migrations puissantes ou la mobilisation de moyens destinés à créer les matières premières et inventer les connaissances propres à fixer la population au plafond qu'elle a atteint. Que ce dépassement, l'existence d'individus en surnombre, soit responsable d'une redistribution des pouvoirs matériels, des organismes et des instruments appropriés, nous venons de le voir au sujet de la chasse, et on l'a montré récemment pour l'agriculture ${ }^{43}$. Loin d'être un facteur négatif, et uniquement destructeur d'harmonies qui se sont longuement, péniblement établies, ainsi qu'il apparaît dans l'évolution, la surpopulation remplit une fonction positive, puisqu'elle ouvre la voie au renouvellement des propriétés et des relations de l'espèce et de la nature. Elle «crée, écrivait Élie Faure en observateur attentif, des besoins nouveaux, les besoins nouveaux des idées vivantes, les idées vivantes des ressources insoupçonnées auparavant ${ }^{44}$. De ce fait, nous devons l'envisager comme une cause, une force motrice ayant son autonomie, et non pas comme un effet des oscillations purement extérieures du milieu ambiant. Là encore Karl Marx a été près de la vérité en constatant que « l'accroissement de la population... résume à lui tout seul le développement des forces productives $»{ }^{45}$. Dans l'inventaire de celles-ci figurent, à côté des techniques, toutes les dispositions biologiques, physiques et intellectuelles de l'espèce ${ }^{46}$.

La formation d'un important potentiel de reproduction génétique et non génétique, la tendance de la population à déborder les frontières écologiques, en diminuant la pression sur les mutations génétiques, maintenues à titre virtuel dans des conditions plus complexes et plus variées, expliquent que les relations entre l'organisme et le milieu ne soient plus naturelles au sens darwinien du terme. Conjointement, quand une partie d'un groupement animal est forcée de quitter son

43

44

45

46

E. BOSERUP : Evolution agraire et pression démographique, Paris, 1970.

E. FAURE : Regards sur la Terre promise, Paris, 1970, p. 249.

K. MARX : Les fondements de la critique de l'économie politique, Paris, 1967, p. 184.

S. Moscovici : Le marxisme et la question naturelle, L'Homme et la Société, 1968, 13, p. 59-109. 
habitat (allant de la prairie vers le désert, par exemple) ou que cet habitat change (la prairie devenant un désert), elle affronte directement un autre climat, une autre végétation. La discontinuité géographique détermine la mortalité des individus, leur génotype et la conversion des propriétés spécifiques héritées ou acquises. En revanche, lorsque les sous-populations ayant atteint le stade évolutif qui nous intéresse ici sont amenées à explorer l'inconnu au cours de leurs déplacements et entrent en contact avec une ressource inexploitée, elles le font, le mécanisme de séparation sociale le permet, à titre d'activité complémentaire. La possibilité leur est donnée d'éprouver, de créer les richesses matérielles et intellectuelles nouvelles sans être obligées de renoncer complètement aux fonctions et aux richesses anciennes, sans rompre irrémédiablement avec leur environnement. Il en a été ainsi, nous l'avons constaté, de la prédation. En outre, les échanges des sous-populations humaines ou proto-hominiennes sont médiés par une autre sous-population : les chasseurs ont abordé la savane après avoir assimilé les moyens biopsychiques des prédateurs, anthropoïdes ou hominiens. L'institution d'un rapport, en l'occurrence de l'homme à la matière, passe continuellement par un autre homme qui a déjà intégré de façon provisoire certains de ses aspects dans son corps et son cerveau. On pourrait dire que la sélection naturelle rencontre là les frontières de son application.

Fréquemment un processus fondamental de par son travail engendre ses propres limites, débouche sur un processus réel différent, de même qu'un système solaire obéissant scrupuleusement à ses lois explose, s'éteint ou entre dans l'orbite d'un autre système solaire. L'universalité, la constance sont des états provisoires, difficiles à atteindre et à maintenir. Que la sélection naturelle ait esquissé il y a environ vingt millions d'années ou il y a quatre millions d'années les prémisses d'une combinaison distincte de l'organisme et du milieu, les conditions d'une existence naturelle différente, n'a donc rien d'extraordinaire. De même qu'elle avait été rendue possible par un passage aux conséquences analogues, elle a simplement rendu possible le passage à un principe de vie distinct. Je veux dire l'éclosion d'une dynamique inédite des forces objectives agissant dans le monde matériel. L'autonomie de cette dynamique se dégage, a pour résultats solidaires la fissure ouverte dans l'univers des anthropoïdes et de la cueillette et l'avènement des espèces humaines et de la chasse. L'écart 
qui sépare la partie masculine des sociétés de leur partie féminine, et dans l'environnement le potentiel animal du potentiel végétal, a valeur de signe : "Par contraste avec les carnivores, la chasse humaine est le fait des mâles ; fondée sur la division du travail, c'est une adaptation sociale et technique qui diffère foncièrement de celle des autres mammifères ${ }^{47}$. "

La notation est pertinente. Mais il faut clarifier immédiatement un point important. Cette division désigne un écart, une séparation relatifs aux rapports avec le monde matériel, aux propriétés du champ d'action du travail et des groupes humains qui l'accomplissent. Ses effets se font sentir au niveau de la création des facultés de l'espèce et de ses ressources, du bouleversement de ces rapports. Je veux dire par là qu'elle ne se ramène pas à la spécialisation des tâches exécutées par des sous-groupes particuliers, à la division ou plutôt subdivision du travail. Qu'un groupe chasse l'antilope et l'autre l'éléphant, qu'une partie de la population fourrage à proximité du campement tandis que l'autre s'en éloigne, cette répartition assure un meilleur emploi des facultés, une mise en œuvre plus raffinée des ressources. La définition des opérations et de l'objet améliore les dextérités, ouvre la voie à une organisation sociale plus efficace de la production. Elle ne traduit pas une différence de liens avec le monde matériel, ni un processus de transformation de ces rapports. Manifestement, la subdivision qui a lieu entre chasseurs, entre collecteurs - et de même entre agriculteurs, entre artisans, etc. - , est une chose, la division entre chasseurs et collecteurs (ou entre agriculteurs et artisans, etc.) en est une autre. La première exprime la diversification d'un savoir-faire, de moyens matériels communs existants ; la seconde signifie la formation de savoir-faire, de moyens matériels différents, voire opposés. Depuis l'émergence des hominiens et des hommes, cette dernière division a donné au problème de la reproduction des populations, de leur insertion dans le milieu une autre solution que celle que lui avait donnée la sélection. Je l'ai décrite à l'œuvre dans le cas particulier du chasseur, et j’ai démontré ailleurs ${ }^{48}$ qu'elle traduit un principe général déterminant la genèse des qualités d'une fraction de l'humanité et leur transfert à une autre fraction, tandis que sont réorganisés les rap-

47 S.L. WASHBURN et C.S. LANCASTER in LEE et DE VORE, op. cit., p. 293.
48 S. MosCOVICI : Essai sur l'histoire humaine de la nature, op. cit. 
ports entre elle et le monde matériel. Les conditions d'un tel renouvellement de la composition des populations et des forces biologiques, physiques, sont, chaque fois, l'existence d'un déséquilibre dans cette composition, la rencontre de ressources secondaires disponibles en substances utilisables, instruments et dextérités avec des groupes d'individus en surnombre. Pareille rencontre actualise et unit les propriétés potentielles des deux termes, rend possible un travail continu visant à les inventer et reproduire de manière intensive et adéquate. Ainsi surgit, à chaque époque, une classe d'hommes - chasseurs, agriculteurs, artisans, ingénieurs, etc. - dotés de savoir-faire, de traits biosociaux et de pouvoirs matériels spécifiques, aptes à forger des liens nouveaux associant l'organisme au milieu, et qui finissent par se substituer aux liens existants. La distance qui sépare ces classes entraîne une redéfinition des éléments, les replaçant dans un cadre matériel distinct. Pour les collectivités originelles vivant de la cueillette, les arbres de la forêt offrent l'abri et la subsistance, la pierre est un objet quelconque à déloger ou un obstacle. Le prédateur ne voit dans la forêt qu'un lieu de refuge, tandis que la pierre devient, entre ses mains, un instrument agonistique ou une matière première. Mais cette distance a encore une autre signification. Les rapports entre groupes humains la reprennent et la retrouvent comme rapport entre les entités non humaines. Le chasseur se pose devant l'agriculteur comme le règne animal devant le règne végétal ; l’ingénieur représente le pouvoir mécanique en face de l'artisan qui incarne le pouvoir manuel, organique. La diversité créée à l'intérieur de l'humanité a de la sorte, pour contrepartie, la diversité éclose, au cours du temps, à l'intérieur de l'architecture cosmique. Le moment venu, chacune de ces classes donne une figure particulière à la nature et conçoit ses fondements ultimes en accord avec la configuration de la matière animée ou inanimée corrélative. Le mouvement qui embrasse ces ordres naturels est visible et l'impulsion que l'homme lui imprime est dirigée par ce principe et s'explique par son action. 


\section{Faire des femmes, faire des hommes.}

\section{Retour à la Table des Matières}

Au seuil du passage de l'évolution à l'histoire, d'une humanité qu'elle découvre à une humanité qui la découvre, la division naturelle prend le pas sur la sélection naturelle. Il en découle pour le processus de reproduction deux séries de conséquences. D’abord, il se dédouble, facilitant la pression sur le milieu, permettant, au besoin, de remanier les données du réel, de convertir les surplus ou les déchets, hommes ou ressources, en nécessaire, de les retenir au sens propre du terme dans la vie de l'univers. Le processus d'invention qui en résulte et se détache répond à cette exigence et acquiert peu à peu un rôle prépondérant. En commandant la genèse des propriétés somatiques et extrasomatiques, il concrétise la capacité ébauchée chez les primates, mûrie chez les hommes, d'atténuer les répercussions de la sélection grâce à la diffusion de comportements atypiques. Ensuite la reproduction que nous avons vu présider partout à la spéciation et à l'évolution des êtres organisés perd son rôle prédominant. Elle se transforme pour assurer les relations entre groupes humains distincts, s'ajoutant aux relations avec l'environnement qui, cette fois à titre de produit et non plus de donné, filtre les mutations avantageuses et désavantageuses, imprime aux facteurs anatomiques ou physiologiques une structure qui assure leur coordination avec lui et les rend aptes à subsister dans son sein. On obtient ainsi l'apparence d'une adaptation, d'une transmission héréditaire et quasi héréditaire des facultés humaines progressivement élaborées. La. réalité n'a d'exceptionnel que l'interpolation des mécanismes biologiques ordinaires dans un réseau soumis au mécanisme de transmission sociale et de division des facultés appropriées, dont ils constituent une des boucles.

\section{$\underline{\text { Retour à la Table des Matières }}$}

\section{(1) Invention et croissance.}

Toute mythologie mise à part, l'invention est ordinaire. Elle se glisse dans tous les efforts d'exploration, de résolution de problèmes, grands ou petits, dans chaque combinaison de deux opérations ou de 
deux matériaux servant à accomplir une tâche quelconque. L'inspiration et le hasard, le génie des individus, y contribuent ; ils ne sont pas essentiels. Dans l'invention, les habiletés, les connaissances, les réflexes constitués sont composés ou décomposés, réorganisés, débouchant sur des habiletés, des connaissances, des réflexes nouveaux. Parallèlement les phénomènes physiques ou biologiques sont réordonnés, rendus plus rares ou plus fréquents, se substituent les uns aux autres, donnent naissance à des effets inconnus auparavant. Ainsi surgissent des qualités des éléments humains et non humains dans un univers commun. L'animal capturé dans un piège est une invention, comme l'outil de bois taillé au moyen d'une pierre, ou la station debout pour manier un projectile. Continuellement les propriétés de la matière se changent, à ce propos, en lois et propriétés de l'organisme et réciproquement. Ce que nous appelons savoir-faire, ressources, est créé par cette voie, représentant une ouverture et une fusion de formes et de domaines d'existence. Leur séparation est un indice du développement des échanges entre les collectivités et les pouvoirs matériels, élargissant l'éventail des possibilités que présente une configuration stable et prédéterminée. A l'intérieur de celle-ci, les diverses parties semblent concorder et s'imbriquer complètement. L'espèce primate ou hominienne qui s'adonne à la cueillette ne dépend pas de n'importe laquelle des plantes qui subsistent dans son espace habituel. Elle n'a pas pour milieu véritable, l'air, le soleil ou les montagnes lointaines, mais tel secteur de la flore, telle zone où elle se déplace, telle texture géologique, envers laquelle elle remplit une fonction particulière. Les comportements, les appareils sensoriels et intellectuels auxquels elle a recours ou qu'elle mobilise régulièrement répondent à la nature des stimulations, à la distribution spatiale propres à ces êtres inanimés qui délimitent et peuplent son univers. Ce qui se constitue ainsi, c'est un système "homme-matière » - la faune et la flore font partie évidemment de cette dernière - patiemment rodé, que l'on peut isoler, décrire, analyser, et qui a eu longtemps une existence indépendante effective. Lorsque la prédation commence à y prendre de l'importance, ce système est à la fois étendu, renforcé et enrichi. Le phénomène inventif fonctionne d'abord comme reproduction du contenu d'une région ancienne transposé à une région nouvelle. Les habitudes et les opérations propres à la cueillette des végétaux sont transférées à la « cueillette » des animaux attrapés ou trouvés morts ; le massacre de petits animaux, auparavant mesure de défense, est seu- 
lement multiplié et rendu banal. Les frontières du territoire sont repoussées et retracées de manière à y inclure les points où l'on recherche le butin.

A partir d'un certain moment, les événements prennent une direction différente : la reproduction devient une invention. La diffusion des pratiques, la conviction partagée que chacune d'elles est indispensable à l'existence commune et aux besoins courants, ouvre la chaîne des substitutions. L'abri de la forêt est remplacé par un abri dans la savane ; l'espace favorable à la cueillette fait place à un espace favorable à la chasse, le campement centré sur le fourrage est abandonné pour un campement centré sur le piégeage et la capture du gibier : ce sont là quelques-unes des solutions nouvelles adoptées. L'ingéniosité des hommes y a pourvu et leur a ajouté de nombreuses découvertes surprenantes. La répercussion la plus certaine est cependant que la couche de savoir-faire, de modes de vie, de niches écologiques se trouve doublée. Un système "homme-matière " nouveau s'arrache à l'ancien, il se glisse également à ses côtés et le dépasse. L'espèce réussit, de cette façon, à vivre dans deux milieux distincts que prolongent les deux " systèmes » naturels respectifs; mais on peut aussi dire qu'elle subsiste dans un seul milieu particulier après l'avoir déplié. L'unité biosociale, enjambant cette discontinuité, voire cette disconnexion, autorise la simultanéité d'une mise en série des aires, d'une dislocation des espaces - ici l'on cueille et là on chasse - et de leur superposition, car là où l'on chasse on arrive à fourrager, cueillir, et vice versa. Dans un premier moment, tout s'étend et se diversifie ; dans un second moment, tout se concentre, se découpant sur une ligne pour ainsi dire verticale. Le mouvement centrifuge vers les ressources animales éloignées et le mouvement centripète qui consiste à redécouvrir sur place les ressources connues se croisent constamment, et nous leur devons l'état actuel de la faune et de la flore de la plupart des continents. Ils créent une hétérogénéité de l'environnement, l'espace nécessaire à une population plus nombreuse ou ayant des besoins plus importants. Vivre de la chasse et de la cueillette, séparément, dans une région plus vaste, relâche la tension provoquée par une densité démographique alarmante ; vivre dans cette même région, un peu partout, de chasse et de cueillette pratiquées de concert, élargit le territoire et accroît le rendement des efforts collectifs. Le rythme et le poids de la spéciation diminuent, non pas tant parce que le genre hu- 
main est capable de vivre dans des conditions géologiques, climatiques et alimentaires variées, mais surtout parce qu'il a réussi à diversifier le milieu où règnent ces conditions. L’impulsion écologique remplace l'impulsion génétique ; la création d'une diversité dans la biosphère suspend l'urgence d'une sélection des organismes. Ceci explique en grande partie le changement qui a eu lieu il y a environ cinq cent mille ans. Auparavant plusieurs espèces hominiennes coexistaient, comme coexistent les autres espèces. Leurs migrations et des obstacles géomorphologiques les ont partagées biologiquement. Après l'homo erectus - fait sans équivalent dans l'évolution - on ne rencontre plus qu'une seule espèce humaine. L'humanité semble composer une seule population mendélienne au fond génétique commun. Entre temps, la chasse - et ses inventions - ayant affiné l'art d'engendrer plusieurs mondes pour une seule espèce, il n'était plus nécessaire d'engendrer plusieurs espèces pour un seul monde. La variété des circonstances externes encourage les mutations génétiques : l'unité de l'espèce leur permet de se propager rapidement, d'entrer sans délai dans la composition du génotype humain.

La pression répétée de la surpopulation, les répercussions qu'elle a eu ont longtemps fait surgir talents, substances, organes anatomophysiologiques, au cours de cette période inaugurale, comme un résultat spontané. Par la suite, des sociétés en quête d'un univers disponible, réceptif à leurs visées d'expansion, ont engendré systématiquement cette pression et la surpopulation qui lui correspond. Dans ce contexte, l'excédent n'est plus un " superflu » que l'on tolère et maintient tant bien que mal, mais une nécessité à laquelle on cherche à répondre. Que les séparations successives aient été dues à des écarts démographiques spontanés ou délibérés, elles n’ont fait qu'enraciner plus profondément le phénomène d'invention. Il exprime et sert la capacité des collectivités d'augmenter leur volume, les savoir-faire relatifs aux puissances matérielles auxquelles elles confèrent une structure et un statut. En surface, le nombre d'individus est conservé ou accru. En profondeur, la population dans son ensemble est réorganisée. Le passage de la cueillette à la chasse ne signifie pas seulement ou essentiellement que 5000 individus peuvent désormais se nourrir là où se nourrissaient 1000 individus. Ces individus doivent aussi être engendrés avec d'autres facultés, en faisant d'autres investissements répartis proportionnellement à l'efficacité prospective de leur travail. Chaque 
trait biologique ou non biologique s’intègre à l'activité correspondante et y demeure tant qu'il trouve sa justification. Du point de vue de la croissance, des groupes qui ont été contraints et prêts à l'amener à un degré plus élevé, la survie a été garantie au plus faible et non pas au plus fort, à celui qui avait été empêché de se reproduire et non pas à celui qui s'est le mieux reproduit. Mais ce n'est là qu'une apparence, si l'on considère que survie signifie conservation de l'espèce et non pas de l'individu, capacité de laisser une descendance. Tel est bien le but de la division naturelle, puisqu'elle assure la survie du groupe avec de nouvelles facultés, sur une base transformée. Et telle est bien la signification de l'invention ; l'inventeur ne découvre pas un objet, une matière, mais la façon de l'utiliser, le savoir-faire qui le rend accessible à tous et permet de le multiplier. Le bénéfice de son invention, est collectif et son premier souci est de la diffuser.

Compte tenu de ces faits et de leurs répercussions, peut-on continuer, comme l'habitude en a été prise, à voir dans la capacité d'adaptation le critère et la caractéristique des espèces humaines aussi bien que de toute autre espèce ? Est-on en droit, par exemple, de soutenir que la chasse représente une meilleure adaptation à l'environnement, ou que les qualités sélectionnées sont celles qui sont le plus adaptatives ? Bien entendu, en disant, comme on l'a fait, que les hommes qui chassaient isolés avaient moins de chances de survivre que ceux qui chassaient groupés, ou que les outils, le langage, la station debout leur ont permis de s'ajuster à des conditions externes précaires, on n'affirme rien de faux. Pourtant ce qu'on avance est inutile, puisqu'il n'y a point de chasseur isolé, et que la station debout, le langage et les outils se sont spécifiés parallèlement aux conjonctures physiques et aux interactions sociales sur lesquelles ils s'appuyaient. Dire que le chasseur ou l'agriculteur est adapté à son milieu est une tautologie, puisqu'il l'a formé à cette fin ; dire que l'un est mieux adapté que l'autre est un non-sens, puisque leurs environnements respectifs diffèrent. En revanche, nous sommes parfaitement à l'aise quand nous comparons leurs capacités de croissance; capacités d'élargir et non pas d'ajuster la niche écologique, de rayonner à partir d'elle et non pas d'y rester, de subdiviser les habiletés disponibles et les individus qui les acquièrent, d'étendre le territoire habitable proportionnellement au nombre de ceux qui l'habitent à ses divers étages. Et nous avons noté jusqu'à quel point l'apparition de chacun de ces 
étages est une œuvre de longue haleine, un jeu complexe de déplacements et de substitutions dans les systèmes objectifs où l'homme et les puissances matérielles sont les pôles observables. A une extrémité de la chaîne se profilent le manque de ressources et l'abondance du surnuméraire et du déchet. L’alerte donnée, une première répartition unit le périssable au périssable, le complémentaire au complémentaire, l’incertain à l'incertain.

Les hommes, comme toutes les espèces, ne fuient pas la mort : ils ne peuvent éviter la vie lorsqu'elle est déjà là. Aussi emploient-ils au mieux ce qui leur est alloué, étendant la gamme des talents et des richesses avant que ceux dont ils disposent ne s'épuisent et n'amènent la destruction de l'ensemble. La séparation des grands groupes, à l'autre extrémité de la chaîne, élimine les antagonismes, enlève à ce qui était en trop son caractère précaire et exceptionnel, lui assigne une place dans l'humanité et découpe sa part de réalité dans le monde. Que le fait de la croissance doive passer par la division n'est pas étonnant, puisque le donné se conserve provisoirement et se multiplie durablement. La réciproque est plus surprenante, aussi longtemps que l'on exclut la tentative faite par une population de se donner sa propre terre, de s'inventer une existence qui lui est refusée. Détachée du cadre principal, elle accepte sa condition et force sa chance. Ayant découvert son propre mode de subsistance, elle provoque l'éclatement du cadre de vie général et sa reconstitution sur une base élargie ${ }^{49}$.

Retour à la Table des Matières

49 L'expression fatiguée d'un phénomène plus profond fait l'unanimité à ce propos: au lieu de s'adapter au milieu, l'homme l'adapte à lui-même. Le contraste, souligné par cette idée, avec le reste des animaux permute les termes sans toucher à la relation qui, elle, est tenue pour universelle. L'homme y paraît figé une fois pour toutes, les éléments extérieurs se conformant à ses besoins, perpétuant son adaptation initiale. L'analyse du devenir cynégétique nous a enseigné que rien de tout cela n'est vrai et qu'une relation nouvelle, exprimée par la notion de croissance, est responsable de la constitution des termes. 


\section{(2) Transmettre et conserver.}

Ce renouvellement a marqué le processus de reproduction. Celui-ci agit dans l'espèce biologique modale par le jeu des mutations et des adaptations sélectives. L'individu naît pratiquement avec les qualités requises, atteint rapidement la maturité. Des mécanismes génétiques règlent, en grande partie, la taille de la population, le partage des fonctions, empêchant une procréation excessive, un recrutement intempestif de nouveaux membres ; dans le cas d'une défaillance de ces mécanismes, l'environnement décide de la vie et de la mort de chacun. Les sociétés animales proches des nôtres perpétuent leur organisation en consacrant une partie des énergies, du temps disponibles à inculquer aux plus jeunes de leurs membres les comportements dont elles ont la garde. Les primates supérieurs sont, jusqu'à plus ample informé, les premiers à avoir soustrait aux déterminations génétiques strictes et poursuivi intensément la communication et le façonnage des qualités biopsychiques des individus suivant le rôle - animal-coryphée, mère, mâle subordonné, etc. — qu'ils devront remplir dans la collectivité eu égard à leur organisation particulière. La reproduction sociale répond ainsi à des exigences qui lui sont inhérentes en s'éloignant de la reproduction naturelle. Celle-ci, qui nous concerne maintenant au premier chef, a trait à une communication et à un façonnage du même genre, touchant aux habiletés d'un individu, à la coordination des actions de plusieurs individus, aux appareils sensori-moteurs mobilisés pour s'articuler avec les éléments matériels définissant le milieu. Par son truchement, l'espèce poursuit ses échanges internes et externes avec le monde physique, répète sa composition du point de vue de l'éventail de ses qualités et de leur efficacité à un moment donné. Elle se conserve non pas dans mais à travers les individus, cherchant à vivifier ses facultés, ses rapports naturels, les organes qui les concrétisent. La reproduction naturelle de toutes les espèces - y compris probablement les primates supérieurs - est, à tout prendre, génétique. Ceci n'est plus vrai pour les hominiens, chez qui elle devient un domaine autonome, ayant, ses fins propres, ses pratiques spécifiques. La durée accrue de la vie, le développement incomplet du système neuromusculaire du nouveau-né lui laissent le champ libre, après la naissance, fournissent la base naturelle indispensable à son action. Ils ne définissent cependant pas son originalité historique. A savoir, d'être une reproduction des divisions entre groupes humains, une reproduc- 
tion séparatrice. Généralement les individus appartenant à une espèce, même s'ils exécutent des tâches spécialisées, sont inclus dans un cycle identique d'échanges ou d'opérations. Les espèces humaines répartissent les individus entre des secteurs différenciés à l'extrême, dans des cycles d'échanges et d'opérations radicalement distincts, voire opposés. Les conditions d'exercice et de création des savoir-faire, d'emploi des ressources, expliquent pourquoi cela est nécessaire.

L’organisation des dextérités spécifiques, leur association à des organes humains empêchent qu'un même groupe d'hommes puisse pratiquer des genres de travail requérant des qualités antagonistes. Pensons aux relations que chasseur et pasteur entretiennent avec l'animal : le premier guette, traque, poursuit, piège l'animal pour le tuer immédiatement ; le second le soigne et s'efforce de le maintenir en vie, suit sa croissance et s'en occupe quotidiennement. Ou que l'on compare, encore, l'ingénieur à l'artisan. L'un multiplie ses habiletés, ses connaissances, surmonte les difficultés rencontrées grâce aux instruments de mesure et à l'invention de mécanismes ; l'autre poursuit ces mêmes fins en améliorant son agilité manuelle, la coordination de ses gestes, sa sensibilité aux qualités perceptibles des matières premières, etc. Ces exemples montrent que chaque groupe, afin de conserver et de compléter le spectre de ses dons particuliers, est obligé de les reproduire séparément. La composante organique, subjective, de toute ressource intellectuelle ou physique amène ceux oui détiennent cette ressource à se dissocier de la chaîne des pratiques ou des comportements qui contreviendraient à la logique interne de leur développement.

La séparation de la cueillette et de la chasse a obéi aux mêmes impératifs. Là où elle a eu lieu, les individus ont été, pour ainsi dire dès le berceau pré-parés, destinés à une initiation, ou bien à une simple accoutumance à leur univers, comme chez les primates supérieurs ou proto-hominiens. Et tout d'abord, dès leur naissance, on décidait s'ils avaient ou non droit à l'existence. L'infanticide, sans que l'on veuille exagérer son importance ou sa généralité, a été la méthode sinon la plus courante, du moins la plus sûre pour canaliser le courant reproductif. Les données que l'on possède sur les peuplades australiennes ou les Eskimos d'aujourd'hui aussi bien que les vestiges du pléistocène attestent une pratique systématique de la mise à mort des nou- 
veau-nés, avoisinant un taux variant entre 15 et $50 \%$. S'agissant de la chasse et du chasseur, on ne s'étonnera point d'observer que les bébés sacrifiés sont du sexe féminin. La situation semble donc s'être renversée : dans les sociétés de primates, le « superflu » potentiel est masculin, tandis qu'il est féminin dans les sociétés humaines.

La tendance à la formation différentielle se poursuit pour les individus laissés en vie. La cueillette et le fourragement restant très longtemps proche des pratiques pré-hominiennes, comme nous l'avons noté, ils appellent une initiation des plus rudimentaires. Il s'agit pour le jeune enfant de s'accoutumer au milieu à l'aide des sens et des mouvements du corps. Le temps nécessaire à cette mise en route ne dépasse pas de beaucoup la maturation biologique proprement dite. Les filles s'intègrent très rapidement à leur domaine d'activité, sans subir de préparation intensive spécifique préalable. La prédation et la chasse exigent davantage. Dès le jeune âge, le garçon est amené à se familiariser avec la capture et le piégeage, à savoir discriminer les espèces animales, à reconnaître odeurs et cris, à discipliner ses gestes et son rythme corporel, à les accorder aux gestes et aux rythmes de ses futurs compagnons. Les recettes, les lieux, les distances se fixent dans la mémoire individuelle et collective, les instruments et les matériaux dans le système des réflexes. Conjointement le corps est soumis à des exercices réguliers, afin d'accentuer certaines de ses virtualités comme l'illustre l'allongement du tendon pour la chasse en kayak. Dès qu'il commence à marcher, on installe le jeune garçon sur un siège ou un lieu plan, les talons appuyés sur un autre siège. Un homme de sa famille (père, oncle, etc.) se place derrière lui et lui saisit le bras servant à lancer pour le tirer en l'élevant vers l'arrière. Cet exercice d'assouplissement de l'épaule s'accompagne souvent d'un chant rythmé qui le grave dans la mémoire. Le bras acquiert ainsi une plus grande mobilité, et pourra lancer le javelot ou le harpon plus loin, fonctionnant comme un levier allongé, dans toutes les positions qui sont celles du chasseur en kayak. Un autre exercice consiste à faire asseoir l'enfant jambes étendues et à le pousser en avant en lui appliquant la main dans le dos, dont les muscles se trouvent fortifiés. Un troisième, pour prévenir la fatigue des jambes qui doivent demeurer longtemps dans la même position, porte sur les tendons des jambes que l'on allonge par une flexion répétée des genoux, toujours en position assise. 
Chez un grand nombre de peuples de chasseurs, on rencontre des programmes analogues d'exercices corporels, de complexité variable. Leur stricte application préside au déroulement d'une existence enfantine, sculpte, au sens exact du terme, le matériau anatomophysiologique humain, scande les étapes de l'entrée dans le groupe adulte. Bien entendu, seuls les jeunes mâles sont soumis à ces rigueurs. Le répertoire des conduites et des savoirs ayant trait à l'emploi des arcs, des lances, des bateaux, des harpons, etc., leur est aussi exclusivement réservé. Communiquer des facultés physiques et intellectuelles, ce n'est pas seulement enseigner ou faire apprendre, assurer le remplacement des individus et des groupes : est garanti du même coup le renouvellement des liens avec un milieu particulier. Vu sous un certain angle, celui-ci est un agencement externe d'objets, le lieu des ressources ; sous un autre angle, il est un livre, un ouvrage composé au cours de nombreuses générations. Elles y ont imprimé les directions de mouvement, distribué les espèces constituant la flore et la faune, dessiné les trajectoires par rapport aux habitations, au soleil, aux rivières ou aux montagnes; et elles ont aussi donné une signification aux bruits et aux couleurs, classé les événements ponctuant le jour ou la nuit et la succession des saisons. Pendant ses années d'initiation, le jeune déchiffre cet ouvrage, s’en pénètre, se l’incorpore.

C'est pourquoi en reproduisant les individus on reproduit le milieu ambiant lui-même. Que l'une de ces facultés ne se renouvelle point, et l'on voit l'ordre, les forces animées ou inanimées qui lui correspondent, disparaître ou se dissoudre dans un monde distinct. Que l'homme cesse de chasser une espèce, ou que la chasse dans son ensemble devienne inutile, la distribution des espèces s'en ressent, les lignes de force de l'espace et les rapports entre ses éléments perdent de leur signification; ils ne sont plus reconnus et progressivement s'évanouissent, se vident de toute réalité objective et subjective. Le travail qui préserve et rajeunit ces facultés se concrétise dans des moyens, des biens, dans le volume de la population ; c'est également un travail qui préserve et rajeunit le monde matériel où la population est installée et son organisation caractéristique. Dans l'univers humain, il engendre un écart, une dénivellation. La reproduction des individus munis de leur savoir-faire, de leurs artefacts éventuels, est reproduction du rapport qui existe entre deux groupes, entre l'homme et 
la femme, entre ceux qui chassent et ceux qui cueillent, chacun étant situé à l'intérieur du système cohérent qu'il forme avec les pouvoirs matériels respectifs, eu égard à la constitution et au dynamisme ayant provoqué leur réunion distincte. A aucun moment ils ne sont maintenus en vie et formés en tant que membres d'une espèce : ils le sont en tant que membres de ce que j'ai appelé une catégorie naturelle, c'està-dire un ensemble d'individus identiques du point de vue biologique et social mais qui se distinguent des autres sous l'angle des capacités associées à leur cerveau, à leur corps et sous l'angle de leur symbiose avec les puissances du milieu ambiant. Chaque fois qu'ils ont créé ou se sont partagés des ressources externes et internes, les hommes l'ont fait, jusqu'à ce jour, au titre de la catégorie à laquelle ils appartenaient, et ils se sont trouvés les uns en face des autres éloignés de la distance même qui sépare ces catégories.

Toutes ces raisons conjointes font que cette reproduction est naturelle. Elle accompagne et prolonge la maturation biologique, la rétention des mutations indispensables à l'espèce dans l'assimilation des régularités et des lois de l'univers ambiant. Son action constante vise au renforcement et au développement, comme partout chez les êtres vivants, d'un rapport entre une population déterminée et son fond matériel, d'une interaction qui accentue les qualités de la substance organique et les propriétés de la substance inorganique. Par ailleurs elle adapte, en le différenciant, le flux croissant de la matière vivante humaine aux configurations écologiques dans lesquelles elle se diffuse. A cette nuance près qu'une telle adaptation est une réaction à une configuration qui n'a pas été pré-établie, donnée aux hommes, mais engendrée par eux. Ce faisant ils créent une nouvelle interdépendance entre les éléments, la faune et la flore, parallèle à celle qui existe entre les catégories naturelles, les facteurs biologiques ou géomorphologiques. La complexion dans laquelle ils s’inscrivent, la proximité ou l'interférence des espèces, la pression sur le nombre ou les barrières qui séparent les populations animales et végétales, les circonstances physiques, climatiques auxquelles elles font face, tout ceci a été et est suscité par notre truchement. Les êtres animés ou inanimés participent ainsi d'une nouvelle histoire parcourue avec nous, faisant suite à une évolution qu'ils ont parcourue sans nous. C'est cette dernière que nous avons recommencée, reproduite ; mais ses traces se font rares et finissent par s'effacer jusqu’à devenir inexistantes. 
Retour à la Table des Matières 


\section{Le processus de division est naturel.}

\section{Retour à la Table des Matières}

Cette description serre de près les faits, notamment le fait essentiel auquel nous avons accordé le plus d'attention : la cynégétisation. Sa théorie tient compte des phénomènes les plus importants révélés par l'étude du règne animal et des vestiges préhistoriques : la généralité de la fonction sociale dans l'évolution organique, son interférence avec les mécanismes génétiques, d'un côté ; la présence d’outils aux divers degrés de l'échelle animale, leur usage intermittent, l'antériorité des dextérités prédatrices par rapport aux changements anatomophysiologiques de notre espèce, de l'autre. Partant, au lieu de raisonner sur des caractères psychologiques ou physiologiques restreints à une fraction de l'échelle des êtres vivants, il est devenu souhaitable de raisonner sur des organisations collectives prises sous l'angle du dynamisme de leur totalité. Du reste, les analogies entre les comportements des primates et ceux des hommes nous y obligent, car nous ne sommes pas en mesure d'en saisir la valeur, pris isolément. Les correspondances ou les différences entre leurs significations ne peuvent conduire qu'à une réflexion sur les ensembles dans lesquels ils se situent. La chaîne de ces ensembles distincts n’est pas nécessairement continue et on ne saurait expliquer rigoureusement un maillon par un autre. Là encore, nous sommes amenés à appréhender tout ce que cette chaîne contient, pour l'instant, afin de préciser, dans la mesure du possible, les articulations qui importent. Il n'en découle pas que le mouvement dessiné, à l'aide des matériaux ainsi réunis et ordonnés, se soit déroulé uniformément et qu'il se soit matérialisé partout et toujours de façon identique. Les conditions de départ ont été différentes, les combinaisons de parcours ont certes varié suivant les entourages concrets. Dans ses grands traits le processus a toutefois été commun.

Même si l'exposé qui précède ne soulève pas d'objection, il demeure dans son fond incongru et choquant, puisqu'il fait état d'une division naturelle et attribue ce caractère à l'histoire à laquelle nous participons. Suivant ce qui est devenu convention courante et sagesse commune, l'arrêt de l'évolution organique, de l'action du principe de 
sélection, permet à l'évolution culturelle commençante de se donner libre cours : la nature s'efface devant la société. Et ce à la faveur d'un armistice que l'hominisation aurait conclu dans la lutte universelle pour la vie. Pour limpides que soient une telle disposition des réalités et un tel partage des notions, ils n'éclairent pas grand chose ni ne favorisent la déduction, puisqu'on s'abstient de formuler la loi de la seconde évolution, la notre, en laissant dans l'ombre les conditions précises qui l'ont rendue possible et nécessaire. D'autre part, la justification empirique de la séquence postulée est définitivement ébranlée : «On a cru parfois pouvoir soutenir que, dans l'évolution humaine, l'adaptation suivant des procédés génétiques avait été remplacée par l'adaptation suivant des procédés culturels. Dans cette optique, on considère en fait que l'évolution de la morphologie et de la biologie humaines s'est achevée avant que ne débute l'évolution culturelle qui en a pris la relève. Mais cette affirmation semble démentie par les connaissances que nous possédons : la fabrication des outils indique qu'un certain degré de culture avait été atteint avant le terme de l'évolution qui devait donner à l'homme tous ses caractères, notamment la taille actuelle du cerveau. Mais c'est là encore raisonner de façon trop simple à notre avis. Dès que les activités culturelles ont débuté, elles ont dû avoir une action en retour sur les activités biologiques, comme l'existence de la culture avait le pouvoir de changer considérablement la valeur adaptative des gènes ${ }^{50}$. "

Il n'y a pas, ou, à vrai dire, il n'y a plus de contradiction entre la détection d'une influence du facteur culturel humain, et le caractère profondément naturel d'un développement historique. Les poids respectifs, les rapports du génétique et du social peuvent être et sont différents chez l'animal et chez l'homme : rien n'autorise à en conclure que là ils sont de la nature et qu'ici ils n'en sont pas. Tout au plus le sont-ils autrement ; là ils visent à la sélection et à l'adaptation, ici à la division et à la croissance. Et nous venons d'expliquer les raisons d'une telle substitution et les conditions objectives dans lesquelles elle se déroule. Mais les arguments les plus solides poussent sur le sol de la réalité. La division naturelle se dessine à l'arrière-plan de la métamorphose et de la structure des ressources humaines et non humaines,

50 E.W. CASPARI: Some genetic Implications of Human Evolution, in S.L. WASHBURN : Social Life of Early Man, op. cit., p. 274. 
de ce qui a été chaque lois vécu et conçu comme ordre naturel. Constamment sont levés les obstacles devant les démarches particulières et antagonistes servant à engendrer les capacités inorganiques et les facultés organiques. L'agriculture ou la chasse s'éloignent de la cueillette moins par leur objet que par la manière de le traiter, d'observer, de signaler les végétaux ou les animaux, ainsi que par la nécessité imposée à ceux qui les exercent de subir une initiation plus ou moins longue, d'assimiler systématiquement les réflexes physiques et intellectuels indispensables. L'apparition d'un de ces faisceaux de savoirfaire provoque le vieillissement ou la disparition des précédents, le changement des circuits établis entre l'homme et le milieu matériel. La refonte de l'espèce, de sa composition, de son étendue, de ses apports avec les forces objectives, en est la conséquence. Certes, nul n'ignore que les habiletés, les moyens et les sources d'énergie s'accumulent et s'ajoutent à la somme des habiletés, des moyens et des sources d'énergie qui ont été inventés, reproduits au cours de millions d'années. Ce développement s'éclaire pourtant si l'on observe que, régulièrement, un ensemble de phénomènes, un groupe d'êtres animés ou inanimés accèdent à la suprématie : à côté de leur rôle de ressource principale, ils ont vocation de foyer de l'ordre cosmique. Tour à tour, le règne animal et le chasseur, la puissance sensorielle, musculaire, et l'artisan, la force mécanique et l'ingénieur, ont imprimé une figure particulière de la nature, couple zoomorphe avec le premier, organique avec le second, mécanique avec le troisième. La succession qui embrasse ces états naturels, "définitifs » aux yeux des contemporains, a des fondements visibles. Elle enregistre l'avènement et la hiérarchie des secteurs de l'univers, chaque fois que l'un de ceux-ci s'impose avec la formation d'une catégorie d'hommes susceptibles d'assurer son équilibre. La correspondance est immédiate. Elle traduit les circonstances qui incitent à recombiner les éléments du monde physique auquel nous faisons face et à redistribuer les facultés, les arts entre lesquels nous nous divisions.

Les liens continuellement noués des puissances matérielles avec l'humanité (qui fait aussi partie d'elles) est réciproque. Le pied qui marche, l'œil qui épie, la main qui saisit s'approprient des instruments, des dextérités ; mais le pied, l'œil, la main participent de tout agencement matériel, écologique, et lui sont homogènes. Sans cette participation, une fois qu'elle a débuté, aucune puissance non hu- 
maine ne peut s'exercer ni aucune espèce exister. Tout animal appelle notre intelligence, notre effort animal ; tout pouvoir mécanique réclame notre force physique et nos appareils sensoriels. Aucune de ces forces, de ces espèces, ne saurait être dite externe, indépendante, ni agir sans nous, dans l'enchaînement des cycles vitaux d'une biosphère dont nous sommes un des facteurs organisateurs. Par la différenciation de ses attributs, l'homme se renouvelle de manière aussi bien génétique que naturelle, au sens qui est le sien. Suivant une opinion courante, l'ère des changements anatomiques et physiologiques est close : seule la superstructure des artifices, des connaissances, de l'acquis continue à se modifier. Cette conclusion semble prématurée. Des transformations biologiques subtiles afférentes à la longévité, à la fréquence des maladies, aux connexions corticales possibles, sont susceptibles de prendre un jour un caractère plus massif. Et puis, l'homme moderne, la dernière en date des espèces humaines, n'est relativement parlant qu'au milieu de sa course. Les pouvoirs objectifs sont devenus des parties de son être, comme ils ont fait partie des espèces qui l'ont précédé. L'organisme humain est un grand livre sur lequel s'inscrivent les lois des éléments cosmiques. Les sens, les membres, le cortex, les muscles de l'homme remplissent la double tâche de reproduire des forces quelconques - animal avec les animaux, pouvoir mécanique avec les mécanismes - et de contenir leur essence : les règles et les principes qui convertissent ces forces en parties de lui-même. Le savoir-faire et les instruments, la coordination sociale des actions et la procréation des individus se combinent afin d'intégrer l'entourage matériel à notre constitution, et, inversement, d'articuler notre constitution avec l'entourage matériel. Les séparations successives ont permis d'aboutir à ces résultats, de les fixer dans notre mémoire et dans notre environnement, de les retrouver dans la diversité de nos activités. Parce qu'elles ont eu l'homme pour objet et pour sujet, on les ignore, sur la foi de la conception prévalente, qui a pour fondement le plus sûr sa répétition ; ou bien l'on est enclin à leur dénier la qualité naturelle que l'on reconnaît aux sélections adaptatives. Elles ont pourtant eu un impact décisif sur notre spéciation, sur l'apparition de la plupart de nos propriétés biologiques. Celles-ci ne sont pas plus artificielles que ne le sont les propriétés équivalentes des primates ou des requins, ni les mutations intervenues dans bien des espèces animales ou végétales dites sauvages, dont nous avons bouleversé le mode de vie. 
L’interférence de l'homme avec les phénomènes organiques, évolutifs, comme son interférence avec les phénomènes physicochimiques, ne rend pas le processus de division non naturel, pas plus qu'elle ne rend non naturel celui de sélection. Du moins sont-ils naturels de la même façon : « Naturelle » dans la « sélection naturelle », écrit le biologiste Dobzhansky ${ }^{51}$, ne veut pas dire l'état des choses précédant ou excluant les changements faits par l'homme. » En acceptant cette idée, on écarte l'incompatibilité qui existe entre le fait de reconnaître à l'homme une place dans la nature en tant qu'être génétique et psychique et la tendance à l'exclure de la nature en raison de ce qu'il accomplit individuellement et collectivement. Nous savons qu'il participe à un mouvement aboutissant chaque fois à l'institution d'un ordre naturel et non d'une nature humanisée. Cette dernière serait une réalité modelée par le travail, un substrat naturel subsistant inchangé, revêtant une forme adéquate aux impulsions d'un agent qui, au demeurant, lui serait complètement extérieur. L'acte transformateur est cependant un acte constitutif ; il aboutit à une organisation historique particulière de la matière et des facultés humaines. Il est impossible de trouver un critère assez net qui permette de dire que l'homme appartient à la nature sous son aspect premier et s'en écarte sous son aspect second. Ces derniers développements prouvent bien que le devenir homme de l'homme fait partie de notre histoire. Les débuts de cette histoire ont été tâtonnants ; la sélection sexuelle et naturelle a été mise en place peu à peu, les directions prises l'ont été sans plan ni volonté consciente, le patron général se dégageant lentement à partir de ses propres effets, à l'instar des rivières coulant vers un fleuve qui n'existe pas encore, d'un fleuve creusant son lit pour se jeter dans une mer inconnue. A chaque étape, les faux recommencements mêlés aux vrais, débouchant sur une impasse ou sur une voie plus ouverte, ont nécessité une remise en route des énergies ou des mécanismes bloqués. Les espèces disparues ont emporté avec elles le secret de ces instants exceptionnels, prouvant combien l'équilibre, la conservation sont choses difficiles, fragiles, rares, comparés au flux normal, permanent, des changements. Ceux-ci, dûment inventoriés, ont balayé

51 Th. Dobzhansky : The present Evolution of Man, Scientific American, September 1960, p. 3. 
l'illusion de l'homme naturel, venu à être tel par une autre histoire que la leur.

$\underline{\text { Retour à la Table des Matières }}$ 
Retour à la Table des Matières

\section{Notes de la deuxième partie}

\section{$\underline{\text { Retour au texte }}$}

Note 4: La littérature sur le sujet traité dans cette deuxième partie est immense et son contenu est tombé dans le domaine commun. Quelques articles et ouvrages m’ont cependant aidé de manière particulière à clarifier les idées que j'expose.

L.R. BinforD, S.R. BinforD : A preliminary Analysis of Functional Variability in the Mousterian of Levallois Facies, American Anthropologist, 1966, 68, 239-294. - M. DETIENNE, J.-P. VERnANT : La métis du renard et du poulpe, Revue d'Études grecques, 1969, 82, 291-317. — K.R.L. HALL : Tool-using Performances as Indicators of Behavioral Adaptability, Current Anthropology, 1963, 4, 479-494. — K.J. HAYES et C. HAYEs: The Cultural Capacity of Chimpanzees, Human Biology, 1954, 26, 288-303. G.H. HewES : Hominid Bipedalism : Independent Evidence for the FoodCarrying Theory, Science, 1964, 146, 416-418. - H.J. JERISON : Interpreting the Evolution of the Brain, Human Biology, 1963, 35, 263-291. R.B. LeE, I. DE Vore: Man the Hunter, Chicago, 1968. - A. LeROIGOURHAN : Le geste et la parole, éd. cit. — P.R. MARLER : Animal Communication Signals : function and structure, Science, 1967, 157, 769-774. - J. NAPIER: The Evolution of the Hand, Scientific American, 1962, 207, p. 157. - K.P. OAKLEY: Man the Toolmaker, Londres, 1961. — Th.A. SEBEOK (ed.): Animal Communication, Bloomington, 1968. - C.H. SoutHwick: Primate Social Behaviour, Princeton, 1963. - I. VINE: Communication by Facial Visual Signals in J.H. CROOK (ed.), op. cit., pp. 279-354. - S.L. WASHBURN : Australopithecines: the Hunters or the Hunted, American Anthropologist, 1957, 59, 612-614. — S.L. WASHBURN : Classification and Human Evolution, Londres, 1964. — S.L. WASHBURN : Speculations on the Interrelations of the History of Tools and, Biological Evolution, Human Biology, 1959, 31, 21-31. - S.L. WASHBURN et C.S. LANCASTER : The Evolution of Hunting, in N. KorN et F. Thomson (eds.) : Human Evolution, New York, 1967, p. 68. - S. ZuCKERMAN: The Social Life of Monkeys and Apes, Londres, 1932. Retour au texte

\section{$\underline{\text { Retour au texte }}$}

Note 5 : On a attribué cet effort de constance à un objectif précis : la maîtrise de la fécondité, processus purement biologique. Le raisonnement usuel est 
le suivant : les êtres vivants ont une tendance inhérente à procréer. Laissée libre, cette tendance conduirait à un accroissement indéfini de la population. Face aux risques d'une fécondité explosive, le corps social se constitue de manière à la maîtriser par des mécanismes régulateurs — avortements, contrôle des actes sexuels, hiérarchie sociale, etc. — qui ont pour effet de la tempérer, d'atteindre un état optimal. La société serait donc en quelque sorte au service de la reproduction, les contraintes qu'elle exerce étant extérieures au processus biologique indépendant et s'appliquant à ce processus, dans un sens qui est toujours restrictif. Dans la description que je propose, il ne s'agit pas de la recherche d'un optimum mais de celle d'un état stationnaire, la reproduction étant, au contraire, au service de la société. Celle-ci cherche, d'une part, à obtenir par naissance, recrutement, etc., les individus qui lui sont indispensables, vu son organisation, son mode d'échange avec le monde matériel. D’autre part elle instaure des limites, crée des obstacles à l'expansion démographique, quelle qu'en soit l'origine - la fécondité en étant un facteur — pour conserver ses capacités d'intégration, l'ordre qui lui est particulier. Les deux mouvements, d'incitation et de limitation, tous deux internes, sont également sociaux. Leur conflit est constant et les oscillations avérées. Pourtant, pendant des périodes plus ou moins longues, une sorte de bilan tolérable s’établit entre les deux et l'on peut alors parler d'état stationnaire ou d'équilibre. Par ailleurs remarquons que la théorie qui attribue à la fécondité ce rôle de menace permanente méconnaît le fait qu'aucune population animale ou humaine ne manifeste une tendance à procréer indéfiniment de façon désordonnée. Retour au texte 


\section{Troisième partie : Société animale et société humaine}

$\underline{\text { Retour à la Table des Matières }}$

L'histoire est elle-même une partie réelle de l'histoire naturelle, de la transformation de la nature en homme. Mais les sciences naturelles engloberont par la suite la science de l'homme, tout comme la science de l'homme englobera les sciences naturelles : il n'y aura plus qu'une science.

Karl MARX, Euvres Philosophiques, t. VI, p. 36. 


\section{Chapitre VI.}

\section{Les sociétés qui viennent de nulle part}

\section{La nature prise en défaut.}

\section{$\underline{\text { Retour à la Table des Matières }}$}

Une transformation profonde est en cours. Soudainement et de plusieurs côtés, les notions d'individu, d'instinct, le contenu de ce que nous désignons par société ou nature humaine, subissent l'érosion du temps accéléré, l'assaut de phénomènes récemment mis à jour. Le terme de société s’applique maintenant plus pleinement aux relations entre animaux ; quant à la nature, j'espère l'avoir montré avec suffisamment de clarté, elle inclut les échanges avec les forces de l'environnement déclenchées par nous. Dans un sens comme dans l'autre, le concept vient à inclure ce qui en était exclu auparavant. La trajectoire que retracent les deux parties précédentes souligne une conjoncture générale : la dimension sociale du réel, au lieu de se disjoindre ou de se substituer progressivement à sa dimension naturelle, semble au contraire s'en rapprocher, y jouer un rôle de plus en plus actif. La logique des manifestations évolutives le dégage avec une netteté croissante.

J’ai déjà indiqué les répercussions qu'entraîne cette situation scientifique du point de vue de la nature, de son histoire humaine. Maintenant — renouant avec le second thème de ce travail — je vais en analyser les implications du point de vue de la société, notamment à propos de la genèse des premières organisations sociales humaines. A l'arrière-plan se profilent une convention - la société sort de la nature - une question - comment la société surmonte-t-elle le désordre inhérent à la nature ? — une définition — la société est une réalité 
seconde. Convention, question, définition qui, selon le poids qu'on leur donne, orientent tous les essais théoriques.

Pour l'homme, l'état de nature est son passé, l'état de société son présent et son futur. La discontinuité est complète : toute tentative de rétablir un lien présumé rompu est qualifiée de retour. Elle prend nécessairement figure de situation exceptionnelle, signifie un danger : " Et lorsque les idéaux et les institutions de la culture échouent à satisfaire les besoins humains universels, toujours s'élève le cri de retour à la nature ${ }^{52}$. " $\mathrm{A}$ aucun instant les efforts sociaux ne se diluent à se répercuter sur le domaine proprement organique ou inorganique, à contribuer aux échanges courants des forces matérielles en les façonnant. Ils sont entièrement consacrés aux relations politiques, religieuses, économiques : " On admet, écrit Algirdas-Julien Greimas ${ }^{53}$, selon la description de C. Lévi-Strauss, que les sociétés humaines divisent leur univers sémantique en deux dimensions, la culture et la nature, la première définie par les contenus qu'elles assument et où elles s'investissent, la seconde par ceux qu'elles rejettent. " Lapidaire, parce qu'elle délustre une pensée autrement plus subtile, cette formule n'en traduit pas moins un sentiment commun. Les hommes, organisés en collectivité, retranchés pour ainsi dire dans la biosphère, exploitent ses ressources, lui appliquent leurs savoirs par le truchement de leurs artifices. Ils n’ont ni le projet ni la possibilité de la conditionner ou d'interférer avec ses processus essentiels. Grâce à d'autres artifices, ils imposent silence aux passions qui les habitent, réfrènent les tentations de transgresser les limites imposées. Maîtres de la terre comme d'eux-mêmes, leur être est d'exception.

Le passage de la forme de vie animale la plus élevée à la forme la plus élémentaire de vie humaine est investi d'une aura sacrée. Le moment primitif aurait le privilège de la transparence ; sa particularité serait de révéler d'un seul coup l'universel, les matériaux bruts que nous n'avons cessé de travailler depuis. Partant des commencements difficiles, lointains mais authentiques, on cherche à découvrir les fondations permanentes des institutions et à extraire, des alluvions dépo-

52 D. BIDNEY : Theoretical Anthropology, New York, 1970, p. XXXIII.

53 A.-J. Greimas : Du sens, Paris, 1970, p. 142. 
sées ultérieurement, l'essence de la sociabilité. Les collectivités de primates et celles des premiers hommes en représentent le degré zéro. Leur reconstitution, que l'on croyait conforme à la réalité, à des fins de comparaison, en tant qu'états culturels dont se sont dégagés les états sociaux, s'est opérée suivant deux modèles complémentaires : l'un ramenait leurs propriétés à celles des organismes individuels qui les composent, l'autre les assimilait à un agrégat instable d'individus. Dans l'optique du premier modèle, la société animale, à la différence de l'individu biologique, n'a rien de spécifique ; dans l'optique du second, la spécificité de la société animale vis-à-vis de la société humaine est d'être biologique.

Sur ces bases, le sens de l'évolution se laisse décrire aisément. Initialement, la nature interne se confond avec l'individu, unité psychobiologique constitutive, les instincts ou forces primaires de l'organisme agissant partout où existent des êtres animés; la nature externe comprend l'ensemble des milieux matériels. L'individu humain, mû par une énergie intrinsèque, sans liens préférentiels, a, dans cette hypothèse, une existence rigoureusement déterminée et indépendante, forgée au cours de l'évolution, lui donnant les moyens de s'adapter partiellement à l'entourage physique qui lui est dévolu. Étant relativement autonome, rien ne l'empêcherait de rester à l'écart, de satisfaire seul ses besoins, n’étaient les imperfections inhérentes à l'espèce et les exigences de la procréation :

«Il se peut, écrit David Bidney ${ }^{54}$, que l'individu satisfasse sa faim, son besoin d'abri et son besoin de repos sans l'aide de la société. D'autres besoins, tels que la reproduction, de toute évidence exigent des institutions sociales. " Les instincts, montages génétiques, directement liés à l'organisation anatomo-physiologique, transmis héréditairement, de par leur spontanéité, leur uniformité, sont les noyaux irréductibles de tout comportement. Le règne animal les connaît à l'état pur. Soumis aux injonctions des appétits antagonistes, réagissant automatiquement à des stimulations stéréotypées, les animaux offrent le spectacle d'un monde désordonné ne connaissant ni retenue ni délai et manquant des moyens de commander aux pulsions qui les mobili- 
sent. Les enfants - et, pourquoi pas, les sauvages - en sont proches, animés par des impulsions, surtout sexuelles et agressives, sans égard envers autrui ou les intérêts supérieurs de la communauté. L'anarchie et la promiscuité régneraient parmi les animaux, primates inclus ; elles sont potentielles parmi les hommes, manifestant leur côté naturel.

A l'opposé, la société est un système dérivé, hiérarchiquement supérieur, intégrant la somme des sujets humains dispersés en familles, classes, collectivités régionales, dotées de normes, de religions et de pouvoirs, comme une langue superpose à la multitude des phonèmes une syntaxe les obligeant à s'accorder, à se ranger dans des mots, des phrases et des messages. Elle a aussi la vocation d'imposer des restrictions aux instincts, détournant les énergies organiques vers des objets compatibles avec l'établissement de rapports inter-individuels stables et continus. L'ascèse et la contrainte sont les armes, la parenté le premier barrage, suivi d'autres, que dresse l'humanité devant le déluge impétueux de l'animalité. Involontairement, sous la tyrannie des pulsions, les hommes seraient pareils à cette race des Barbares, dont les mœurs indécentes suscitent la vertueuse colère d'Hermione: "Le père s'y unit à la fille, le fils à la mère, la sœur au frère ; les amis les plus chers s'entr'égorgent, et la loi n'interdit rien de tout cela ${ }^{55}$. » Ces accouplements, engendrant conflits et rivalités, feraient obstacle à la coopération nécessaire, à l'établissement de relations durables. Dans ces conditions, personne ne consentirait à des sacrifices, imposant de surseoir à la satisfaction de ses tendances égoïstes, immédiates, afin d'aider son congénère ou l'enfant, encore impuissant, à devenir adulte. L'équilibre d'ensemble menacé, la continuité du lien entre générations compromise, l'individu lui-même court à sa destruction ou amène la destruction de ses proches. D’où le recours à des mécanismes éliminant les tensions délétères, l'invention de la loi comme instrument propre à domestiquer les hommes et mettre un terme à la violence originelle : " La fonction fondamentale de la loi, affirme Bronislaw Malinowski ${ }^{56}$, est de freiner certaines tendances naturelles, de limiter et de dominer les instincts humains, et d'imposer un comportement obligatoire non spontané ; en d'autres termes, d'assurer un genre de coopération basé sur les sacrifices et les concessions récipro- 
ques en vue d'une fin commune. Pour accomplir cette tâche, il faut que soit présente une force nouvelle, différant des dons innés, spontanés. »

La plupart des formations culturelles sont nées au cours de ce travail dépensé à contrecarrer les penchants agressifs ou érotiques, à écarter la menace qu'ils représentent pour la coexistence d'un grand nombre de sujets dans un espace déterminé. Sous la surface unie des alliances, des valeurs, des rituels pédagogiques, des créations artistiques ou scientifiques, sourd le mouvement incessant des désirs subjectifs illimités, le souvenir ou la réalité de la haine, du tumulte instinctuel, de la guerre de tous contre tous. Que la croûte se craquèle, que la barrière des multiples garde-fous cède, on assistera «à la confusion des âges, au mélange des générations, à la désorganisation des sentiments, à un renversement brutal de tous les rôles " ${ }^{57}$. La société s'est formée, dans des temps immémoriaux, afin de prévenir cette catastrophe. Elle est gage du triomphe remporté sur la spontanéité naturelle qui la précède et l'entoure, sur l'individu hanté par des tentations obscures, adhérences résiduelles au monde animal. La répression est le visage que prend l'adaptation lorsque l'évolution touche l'homme.

Elle est aussi, le second modèle le suggère, le commencement d'un ordre à part, transformant les associations instables à l'intérieur de l'agrégat biologique en liaisons stables du système social exclusif à notre espèce. Avant nous, les congrégations d'individus obéissent aux cycles hormonaux, à la périodicité de l'œstrus, à la distribution des naissances, autant de paramètres indépendants ou presque, dont les effets combinés sont imprévisibles. Les rencontres de mâles et de femelles en vue de la procréation ont lieu au hasard. L'attitude d'un animal envers un autre animal est versatile ${ }^{58}$ : il n'y a pas de distinction entre le jeune et l'adulte, le membre du groupe biologique et l'étranger. A l'instar de la main invisible commandant les transactions des échangistes sur le marché, une main invisible dirige dans la nature les mouvements des sujets isolés. Les préférences des partenaires sont antagonistes: seule la force du milieu, des besoins fluctuants, les

Idem.

58 C. LÉvi-Strauss : Les Structures élémentaires de la parenté, op. cit., éd. 1949, p. 6. 
oblige à converger vers une cohabitation fragile, sans cesse remise en question. L'homme a arrêté ce mouvement brownien en séparant, dans la nuit naturelle, le domaine du permis et celui de l'interdit, discriminant soi et autrui, imposant les règles de leurs déplacements sur l'échiquier collectif dans le mariage, la production et les communications. Véritable démon de Maxwell, guidé par les lois de son esprit, il a institué, dans le grand jour de la culture, des écarts là où il n’y en avait point, redressé le cours d'une histoire qui allait vers un désordre croissant. Le grand physicien anglais, on s'en souvient, avait imaginé un démon posté au seuil par où devait nécessairement passer un gaz quelconque en provenance de deux enceintes différentes où régnait la même température initiale. Agissant sur une vanne imaginaire, il pouvait autoriser ou empêcher la circulation des molécules sans aucune dépense d'énergie, plus exactement laisser passer dans un sens les molécules lentes à faible énergie et dans le sens inverse les molécules rapides à haute énergie. Au bout d'un certain temps, l'une des enceintes était réchauffée, l'autre refroidie, et ceci sans perte ni transformation d'énergie.

Les codes matrimoniaux, linguistiques, économiques que les sociétés ont édictés ont eu des conséquences "démoniques " analogues. Le déroulement de chaque existence individuelle suit une direction précise, elles se combinent harmonieusement, les groupes biologiques reçoivent une place définie dans le tableau des alliances perpétuées par le système total qui les comprend. Comme dans les enceintes manœuvrées par le démon de Maxwell, la mesure subroge la démesure et l'altérité, l'identité. Le processus de sélection rend possible ce démarquage qui a pour résultat historique l'opposition de la culture à la nature.

Selon cette vision théorique, la société humaine naît, pour s’y opposer, de son contraire organique, individuel, animal, comme d'un néant ou d'une nébuleuse, après une période d’hésitation chez les peuples sauvages «naturels ». La primitivité, fait et idée, atteste la genèse des différences et de l'antagonisme fondateurs de notre réalité spécifique. Elle indique moins l'instant d'un commencement que celui d'une rupture, moins les attributs d'un état des communautés humaines que la génération, la création de ces communautés à partir de ce qui pourrait être tenu pour un accident ou un rien. Du préhumain à 
l'humain on suit une progression indistincte sur une échelle de valeurs et de complications. Nos sociétés, surtout les plus récentes, sont décrites comme à la fois les plus accomplies et les plus complexes. A chaque étape du développement, elles contiendraient moins de naturel et plus de culturel, supprimant toujours plus le premier pour favoriser le second. Leur qualité d'exception et d'unicité invite à chercher une cause exceptionnelle, à les lier à un événement unique. La supériorité de la société devient ainsi le principe explicatif et le phénomène à expliquer. C'est pourquoi on cherche toujours à rendre compte de la formation du lien humain par des raisonnements qui légitiment en même temps sa perdurabilité et justifient ses avantages. Nous l'avons constaté. L’émergence de la société — la causalité s'avère dès lors exceptionnelle - est associée à la nécessité de surmonter un passé animal, d'écarter l'imminence d'une rechute au cas où les « trous » de la nature ne seraient pas bouchés, ses imperfections rabotées, ses manifestations combattues. Elle y parvient en imposant sa discipline aux individus, en se rendant indépendante d'eux. La turbulence des instances organiques, la promiscuité des rapports, mais aussi la rareté des ressources dans le monde, la fertilité envahissante des populations, ou l'immensité des puissances matérielles, sont citées parmi les manques ou les menaces qui ont appelé et continuent à appeler de notre part une réponse collective vigoureuse afin de combler les vides ou de réduire les excès. Les coutumes, les institutions, les prohibitions que les hommes se dictent mutuellement sont dirigées contre le fonds biologique qui demeure en eux, contre l'incertitude de leurs comportements individuels. Si tout dans l'univers et en nous-mêmes avait fonctionné harmonieusement, le phénomène social n'aurait aucune raison de se singulariser. Il est là, nous l'avons produit, péniblement, dans l'intention de corriger, quand le besoin s'en fait sentir, l'économie défectueuse de l'univers ou de l'organisme, bricolage hâtif d'une espèce aux prises avec les rigueurs d'un environnement inclément et d'une force psychobiologique débordante. L'imposition d'un contrôle, d'une régulation contractuelle des interactions, est à la fois la solution apportée à un problème général qui n’avait pas été résolu à l'échelle animale, et le moyen prothétique imaginé pour faire face à nos adversaires. Contrôle et régulation définissent la société qui s’appuie sur eux comme une négation de tout ce qui est inclination psychique spontanée, différence des sujets agissants, donnée naturelle en nous et hors de nous. Ils la situent aussi comme une réalité secondaire quant à 
sa genèse, son importance dans la vie de tous les êtres, l'homme excepté. Aux yeux de celui-ci, elle assure, devant les tendances contradictoires, chaotiques, devant la tyrannie des besoins et des congénères, l'équilibre et l'harmonie ; elle incarne la loi morale au-dedans de soi, la solidarité avec les proches hors de soi. Mais, même dans ces cas, elle demeure une réalité d'emprunt.

$\underline{\text { Retour à la Table des Matières }}$ 


\section{Du désordre biologique et animal.}

Ensemble ou seuls, cela ne fait aucune différence, les animaux demeurent dans la même condition, abandonnés aux vicissitudes de leur biologie. Ensemble les hommes sont dans la société, seuls ils sont soumis aux impératifs de la nature : environnement contraignant, hérédité inflexible. A mi-chemin, l'homme policé intériorise le réel dans l'individuel, ramène la diversité des pulsions à l'uniformité des règles, subsume la variété naturelle sous l'identité culturelle. La route qui nous a éloignés de la plupart des espèces a été longue. La vigilance constante face à ce qui nous reste de cette évolution est la condition primordiale de notre originalité et de notre survie. Ceci explique pourquoi notre vie commune, afin de rester humaine, est, doit être une combinaison d'oppressions et de renoncements, de déguisements et de satisfactions continuellement remises. De cette manière, nous évitons le désordre qui rôde autour de nous dans le règne animal, en nousmêmes, anciens animaux. Une dualité intrinsèque caractérise notre espèce et nous subsistons dans les deux cadres irrémédiablement hétérogènes de l'inné et de l'acquis, de l'individuel et du social. «Il y a donc là deux grands ordres de faits, déclarait Claude Lévi-Strauss ${ }^{59}$, l'un grâce auquel nous tenons à l'animalité par tout ce que nous sommes, du fait même de notre naissance et des caractéristiques que nous ont léguées nos parents et nos ancêtres, lesquelles relèvent de la biologie, de la psychologie quelquefois ; et d'autre part tout cet univers artificiel qui est celui dans lequel nous vivons en tant que membres d'une société. » Toute instance jugée supérieure, spécifique à cet « univers artificiel» — la raison, la science, l'art, la civilisation, la communication symbolique - vise la suppression d'un besoin impérieux, d'un processus organique autonome, universel ; nous sommes implicitement invités à la conserver si nous ne voulons pas retomber dans la confusion de nos prédécesseurs non humains, ni rester sous la tyrannie de l'héritage ancestral, privés de la solidarité collectivement organisée. Le choix nous est d'autre part refusé. Le départ de l'état naturel a eu lieu à une époque très reculée, et que cela plaise ou non,

59 G. Charbonnier : Entretiens avec Claude Lévi-Strauss, Paris, 1959, p. 154. 
la société contient, encastrées dans sa structure, les conditions - la prohibition de l'inceste, la langue, les techniques - qui ont rendu ce départ définitif. Réduite à sa plus simple expression, voilà la trame de la conception qui justifie bon nombre de nos institutions, sous-tend nos théories biologiques et sociales, et, à l'occasion, inspire l’interprétation de notre expérience quotidienne.

On est cependant amené à soupçonner de plus en plus que la fascination exercée par un ordre humain opposé au désordre animal, la phobie éprouvée devant ce qui est spontané, organique, instinctuel, le génie dépensé à dresser des barrières sur notre pourtour ont conduit à une vue inexacte de nous-mêmes, de l'univers animé et inanimé en général. Les récits portant sur les communautés animales (ou primitives), sur les pulsions "bestiales », s'exerçant sans frein et sans mesure, si entremêlés de terreurs affectives, de lieux communs, doivent leur consistance à la complicité du récitant et du public. La lutte pour la vie confondue avec la lutte pour la nourriture, pour la possession d'un mâle ou d'une femelle, totalement aveugles à leur identité réciproque et à leurs positions sociales respectives, participait sans doute possible de ces fictions qu'il est licite d'inventer lorsqu'on sait ne pas trop presser l'expérience. Ce qui a été fait en considérant la tempérance, cultivée, des désirs agressifs ou sexuels, la redistribution des énergies biologiques innées, la fin de la promiscuité animale, comme des événements motivés objectivement, ayant effectivement eu lieu dans l'histoire. Je ne nie pas l'existence de ces conflits, de ces dissipations, attisés par les tendances les plus diverses. Ils ne sont cependant pas des conséquences aussi inéluctables qu'on veut bien le dire de la réalisation de ces tendances.Dans de nombreuses sociétés, le père, le fils ou les frères partagent paisiblement la même épouse, la mère, les filles et les sœurs partagent, sans rivalités outrancières, le même époux. Les rapports incestueux ne sont pas absents parmi les hommes, ni nécessairement abhorrés, ni condamnés toujours. L'antagonisme, dans tous ces cas, cède le pas à la cohabitation paisible, et les disputes éventuelles sont parfaitement maîtrisées, comme l'atteste la diffusion de la polygamie et de la polyandrie. D'ailleurs, nous sommes mal avisés de voir dans la société humaine l'avènement d'une organisation harmonieuse à la place d'une vie naturelle, foncièrement déréglée ou défectueuse, de la tenir pour une réaction adéquate aux forces menaçant la coexistence stable des individus des autres espèces. Au lieu de 
céder à un sentiment de triomphe, il vaudrait mieux se rappeler que, s’il y a peu de vertus que les cultures ont négligé de prôner, il y a encore moins de crimes devant lesquels elles ont reculé. Si les lois, les prohibitions et les coutumes; la famille ou la hiérarchie ont été conçues, comme on le prétend, afin de mettre un terme à la violence disruptive des pouvoirs organiques auxquels sont soumis les autres espèces ou les individus isolés, jugées à leurs résultats elles semblent avoir échoué, entraînant des conséquences contraires à celles que l'on escomptait. Nous pourrions, au vu de leurs effets - l'observation comparative nous l'assure - revenir sans crainte aux lois inconstantes de la jungle qui continuent, paraît-il, à agir en nous et hors de nous. Le bilan que le zoologue dresse de la vie des animaux est rose en regard de celui dressé par l'historien ou, plus modestement, par le profane ayant atteint l'âge mûr vers le milieu de ce siècle. L'expérience collective, l'aération des peurs et des préjugés, le peu d'attrait d'une humanité déçue pour une animalité ou sa propre animalité inutilement humiliée, inclinent vers une plus grande lucidité. On admet que ce qui a été attribué à la nature, la séparation que l'on s'efforce de mettre en lumière, est une somme de projections, d'émotions, de situations internes à la société et à l'homme, dans une réalité méconnue, plutôt qu'une représentation exacte de celle-ci. La confrontation de ces projections à la réalité les infirme immanquablement. Sur deux points en particulier, le démenti de ce que l'on avait cru fermement est évident :

1. La notion d'individu donné, irréductible à une autre unité d'analyse, s’apparente à celle d'atome insécable dans les théories mécanistes, élément de construction à partir duquel se constitue la matière, contenant de ses propriétés essentielles. C'est lui qui est censé être le porteur de l'hérédité et détenir les comportements innés. Ses qualités proviennent de ce qu'il affronte directement le milieu ambiant : la lutte pour la vie serait en grande partie une lutte assurant la survie du plus apte ou du plus fort. A cet égard, chaque individu représente toute l'espèce, il est biologiquement complet, fermé. Les structures complexes, notamment sociales, seraient des assemblages d'éléments simples, se conservant tels quels, comme les éléments chimiques purs dans les combinaisons. La notion, à l'instar de celle d'atome physique indivisible, subit une éclipse. Nous savons que l'individu exprime une configuration transitoire, prête à assurer la 
transmission d'une substance héréditaire, contenue dans ses chromosomes, mutant à son insu. Le métabolisme des cellules, les mécanismes de croissance sont sous la commande d'un code contenu dans les acides nucléiques de la double hélice fondamentale, communiqué à l'aide de messages chimiques appropriés. Les facteurs déterminant les attributs organiques, la naissance et la mort, travaillent suivant un programme protégé autant que faire se peut contre les accidents ; ils sont précontraints, prédisposés, agencés de manière à répéter indéfiniment leur action. Ensemble ils reproduisent l'architecture régulière de l'espèce et non pas celle d'un sujet isolé. Celui-ci se définit sur une grille de distribution des divers complexes génétiques : ce qu'il est ne représente qu'une version de ce qu'il est susceptible d'être. Virtuellement d'autres sujets possibles sont esquissés, capables de se réaliser lorsque les circonstances se prêtent à l'éclosion de l'imprévisible. L'évolution l'envisage en tant qu'élément d'une population où les organismes, loin d'être les exemplaires identiques d'une série itérative, offrent de nombreuses variantes. L'espèce correspondant à une telle population est définie en tenant compte de la gamme entière : un individu ou une classe d'individus, pris séparément, en donnent une image particulière et vraisemblablement biaisée. Le collectif est dans l'individuel et l'individuel dans le collectif même du point de vue du biologique, de l'inné. Cette constatation déplace le centre de gravité de l'organisme singulier vers les groupements, de la clôture des êtres vivants à l'intérieur d'une hiérarchie classificatrice vers l'interdépendance réclamée par la combinatoire générale des agents génétiques.

Parallèlement, le libre et illimité déploiement des instincts afférents à un sujet indépendant de ses congénères ou au règne animal, conjurés ou dissimulés dans l'interaction avec autrui, dans le règne humain, est une image en train de jaunir. Il était trop commode de les réduire à des mobilisations énergétiques, se déchargeant aveuglément, sans mesure et sans objectif. Il était contraire à la logique de leur attribuer pour seul effet, redoutable, la dispersion des animaux, obligés de s’éviter, afin d'esquiver les tensions provoquées par une rencontre où l'un des antagonistes était certain de l'emporter, impitoyablement. Seul l'animal économique poursuit son intérêt exclusif ; l'animal biologique est mieux avisé. Certes, les actes instinctuels jouissent d'une grande autonomie, et leur déroulement implique uniformité et automa- 
tisme. Pourvu que la stimulation soit suffisante, leur déclenchement est assuré de manière relativement simple ${ }^{\mathbf{6 0}}$. Toutefois, observés rigoureusement, les comportements corrélatifs, surtout les comportements sexuels et agressifs, les plus étudiés, n'ont rien d'outrancier, de purement brutal, quant aux buts. Des conventions qui ne doivent rien à l'anatomo-physiologie ou au milieu habituel confèrent un sens aux mouvements de stimulation et de réponse, sens reconnu uniquement par les membres de l'espèce. Le battement de la queue est un indice de paix chez les chiens et un indice de menace chez les félins. Le rapport qui unit la capacité à mettre en action le schéma inné de l'espèce et la fonction est arbitraire. Sous l'angle de la fonction, le lien du mouvement caudal avec la paix ou la menace aurait pu être changé, l'effet n'en aurait pas été affecté. Le geste qui déclenche une approche ou un évitement doit sa force à la convention et à aucun autre facteur. Dans un grand nombre d'espèces, les combats sont soumis à une étiquette, au respect des relations collectives, attestant une ritualisation remarquable. Un code existe, partiellement inscrit dans le matériel génétique, partiellement appris, et chacun le suit. La virulence est réglée, les conséquences destructives sont bloquées grâce à des dispositifs appropriés. On sait que les membres d'une espèce évitent en général de se heurter directement, et que ce comportement s'observe encore plus rarement chez les animaux en liberté que chez les captifs. Parfois les sujets cherchent uniquement à réduire au minimum les occasions de conflit. Les félins en liberté marquent leur passage par des traces d'odeur, supprimant ainsi le désagrément de rencontres inopinées avec les affrontements qu'elles entraîneraient ; chacun peut détecter l'autre à la trace, connaître sa position, l'approcher s'il le désire. Les rapports hiérarchiques servent aussi à diminuer l'agressivité et à modérer les combats, comme on le remarque dans la plupart des sociétés d'affiliation. Une autre façon de freiner la violence des luttes consiste à les ritualiser, en totalité ou en partie. Les comportements cérémoniels sont destinés à régler les disputes sans faire appel à la brutalité, ou à mettre un terme au corps-à-corps avant qu'il n'entraîne des dommages irréparables. Dans les conditions qui règnent naturellement - ceci vaut pour les loups comme pour les simiens - le vain$\mathrm{cu}$ a la ressource de s'enfuir, ou de faire la preuve de sa soumission en 
prenant une posture destinée à apaiser son adversaire, bien souvent en lui présentant une partie vulnérable de son corps.

Les instincts sexuels suivent un modèle de régulation similaire. Les rencontres antagonistes ou celles d'accouplement sont minutieusement ordonnées, assurent conjointement la survie et la satisfaction des besoins des partenaires. Les conduites instinctuelles ne sont pas inertes : elles consolident les liens intra-spécifiques et les renouvellent au gré des circonstances. Loin de disloquer les groupements animaux, ces conduites sont un ferment d'attirance, de rassemblement, stimulent la co-présence spatiale et temporelle des congénères désirés. L'individu subordonné recherche l'individu supra-ordonné, l'agresseur l'agressé qu'il épargne, pour ne rien dire des manœuvres " sexuelles » au cours de la quête d'un mâle ou d'une femelle par ailleurs désignés grâce à des indices sociaux ou physiologiques. L'inné n'est donc pas nécessairement source de désorganisation, contraire à la vie commune, à une finalité qui l'englobe.

Ainsi l'organique ne se confond pas avec l'individuel, ni l'instinct avec l'autonomie, la spontanéité d'une manifestation défiant un contrôle interne à résonance collective. Le sujet plongé dans une situation d'interaction est aussi biologique ou biologiquement aussi pur qu'un sujet isolé. On pourrait même soutenir le contraire. Chez les animaux captifs, on a relevé l'inachèvement des actes instinctifs par diminution de l'intensité des réactions intimes ou par perte de leur portée biologique au cours de leur déroulement, vu la modification des conditions externes usuelles. Quant à l'homme — ou aux espèces qui s'en approchent - il est moins " naturel » seul qu'associé à ses congénères. Les preuves et les hypothèse avancées en anthropologie, en psychologie, à propos des enfants sauvages, des sauvages enfants, d'un résidu animal décelable dans chacun avant qu'il n'entame le commerce avec un autrui adulte, civilisé, ou s'il évite tout contact avec la société ou la culture, perdent leur valeur heuristique. Je veux dire par là que tout individu demeurant séparé ne représente pas notre fondement organique : il manifeste plutôt une carence neurologique, physiologique, psychique. Les appareils sensoriels, instinctuels, intellectuels, à l'instar de ceux de n'importe quelle espèce relativement évoluée, sont prédisposés et structurés épigénétiquement en vue d’une interdépendance, d'une maturation des capacités à communiquer, à 
agir dans le cadre d'un groupement spécifique. « Les anthropologues en sont venus à comprendre, déclare-t-on ${ }^{61}$, que l'évolution du comportement de l'homme, en particulier son comportement social, a joué un rôle que l'on ne peut dissocier de son évolution biologique. » Les moyens ou la justification nous manquent de comparer, en observant les animaux ou même les enfants, un développement normal, indépendant, d'une pulsion ou d'une faculté que l'on serait en droit de qualifier rigoureusement d'individuel, avec un développement défini, par contraste, comme collectif. Le laboratoire parvient à les créer, la réalité ignore la distinction.

Généralement, cependant, hors des jardins zoologiques et autres endroits de réclusion ou de captivité, les propriétés organiques d'un être animé sont parachevées - et non pas déviées ou contraintes par le côté social. Compte tenu du caractère hautement coordonné, maîtrisé des comportements, considérer ce côté comme antinaturel, comme un système surajouté de contrôles a posteriori, paraît inutile et artificiel. A vrai dire, les interactions collectives et les phénomènes biologiques convergent d'emblée, se modulent à chaque étape importante de leur expression. De manière concertée, ils se remplacent ou se relaient afin de contribuer à un résultat, à une structure qu'ils partagent. Les premiers ne sont pas tout ordre, harmonie, acquis, ni les seconds tout désordre, mécanismes platement itératifs, inné.

Les conduites d'exhibition et de parade, de substitution, d'arrêt d'une séquence de mouvements spontanés, abondent dans le règne animal. Les " cérémonies » y sont aussi importantes et plus fréquentes que le passage à l'acte. Elles révèlent la puissance et la retenue des pulsions inhérentes à l'espèce, leur faculté subtile d'automotivation et de rétroaction, le fait que, dans le calcul des comportements associés, le lien à autrui est présent dès le départ. L'absence de ce calcul, souvent postulée, est plutôt le signe d'un fonctionnement pathologique que d'un fonctionnement normal. Les autres espèces, confrontées à des problèmes analogues aux nôtres posés par les instincts de tous ordres et l'interaction avec le milieu, ne les ont pas laissés sans réponse en attendant l'émergence de l'homme : ils ont imaginé des solutions

61 S.L. WAshburn, I. De Vore : The Social Life of Baboons, Scientific American, 1961, 204, 62-71. 
distinctes des nôtres pour s'adapter aux exigences de l'interdépendance, de la survie commune. Si, par hasard, il nous arrivait d'oublier ou de perdre nos règles sociales, nous ne tomberions pas dans une situation d'anomie ou de non-société : nous retrouverions autour de nous, chez les autres animaux, un capital de normes, le modèle de différents ordres sociaux. Il est superflu de croire que les arrangements culturels auxquels nous avons procédé diminuent les pulsions sexuelles ou agressives du singe ou du loup rémanentes en nous ; leurs formations sociales pourvoient à leurs pulsions, nos formations sociales à celles qui nous sont propres. Nous n'avons pas à nous préoccuper outre mesure du prétendu passé naturel résidant en permanence dans notre biologie, ce passé s'est tiré d'affaire tout seul. Si, ainsi que le soutient Konrad Lorenz, les instincts sont assimilables à des organes, s'ils évoluent, nous pouvons supposer que les nôtres nous sont spécifiques et qu'ils correspondent à une organisation collective particulière : "L'évolution de l'acte instinctif dans le système zoologique, écrit-il ${ }^{62}$, nous montre d'une manière pénétrante combien il est insensé de vouloir parler de "l'instinct»; nos constatations ne pourront jamais s'appliquer qu'à des mouvements innés, qu’à des actes instinctifs pour une fraction plus ou moins grande du système zoologique. »

Quelles que soient les raisons pour lesquelles on a établi deux compartiments hétérogènes du réel, de son analyse ${ }^{63}$, en y plaçant sans qu'ils se touchent, en hiérarchie ou en opposition, les processus biologiques et les processus sociaux, la pertinence de l'opération commence à nous échapper. Ces derniers ne sont pas plaqués en quelque sorte de l'extérieur sur les premiers, la partie individuelle ne préexiste pas, intacte, complète, au groupement spécifique la comprenant. Au contraire, nous les voyons entraînés dans un mouvement commun, entrelacés au niveau de l’organisation et de l'évolution.

2. Comment l'ordre social naît-il de son antagoniste naturel ? Sur quoi se fonde la spécificité de chacun ? Quelle est la genèse des différences que nous sommes censés apercevoir entre l'un et l'autre ? Ces questions dérivent toutes de l'hypothèse d'une discontinuité, repérée

62 K. LORENZ : op. cit., p. 251.
63 H. CALLAN : Ethology and Society, Oxford, 1970. 
dans le temps, de notre société vis-à-vis de la nature, de l'histoire de celle-là en tant qu'abandon progressif de celle-ci. Le monde non social est toujours décrit comme étant soumis aux aléas des mécanismes physiologiques, des automatismes non appris, de l'élan individuel incontrôlé, de la violence sourde des besoins, manquant d'autre part d'institutions stables et fermement disciplinées. La frontière de cet état a d'abord été fixée chez les peuples sauvages, fluctuants dans leurs conduites, débordés par les désirs, fragiles et démunis devant les forces de l'univers. Dans l'esprit d'Hegel, le Noir représente l'homme « naturel », qui « manque de contrainte. », ne connaît pas « Dieu et la loi ». La culture commençait au-delà, avec la famille, la religion, l'éducation et la raison. Dès qu'on cessa de se fier aux impressions des voyageurs, aux spéculations des philosophes, et qu'on connut mieux ces peuples grâce à la diligence des anthropologues, on constata qu'aucun des traits exclusifs de la culture - la domination del'adulte sur le jeune, du mâle sur la femelle, l'exclusion de l'étranger, l'infanticide, la coopération, l'échange, les croyances irrationnelles, l'interdiction des jouissances fondamentales, etc. - ne leur manquait, et que leur nature était profondément sociale. Ce que, à sa manière, Sigmund Freud tenait encore à apprendre à ses lecteurs en écrivant : « Nous ne nous attendrions certainement pas que la vie sexuelle de ces pauvres cannibales nus soit normale, au sens que nous donnons à ce terme, ou que leurs instincts sexuels soient soumis à un grand degré de restriction. Nous trouvons pourtant qu'ils se proposent avec le soin le plus scrupuleux et la plus grande sévérité d'éviter les rapports incestueux ${ }^{64}$. $»$

La frontières se trouva ipso facto reculée vers les espèces non humaines. Les descriptions et les attributions de l'état non social restaient les mêmes : seuls le contexte et le sujet furent changés. Mais les notions d'individu et d'instinct qui les étayent sont, nous l'avons vu rapidement, en instance de rectification. Les ethnologues, comme les anthropologues avant eux, sont allés étudier de près l'existence des populations «sauvages». Leur promiscuité animale, la réalité des groupes exclusivement biologiques "naturels », bref, pré- ou antisociaux, ne résistent guère à l'examen. Nous savons maintenant, de science certaine, que les primates, notre référence parmi les animaux, 
le totem de notre espèce, ont une organisation sociale stricte, ce qui veut dire une activité sexuelle, des modes de communication, de rencontre, etc., exprimant une certaine régularité, des normes permanentes, différentes des nôtres, mais enfin des régularités et des normes conservées solidairement et transmises soigneusement. A la limite, on serait en droit de dire que la promiscuité est notre crainte et notre problème ; ce n'est pas une calamité prête à s’abattre sur les groupements de simiens. Là où elle apparaît, il s'agit d'un accident : " La promiscuité aléatoire est rare et résulte en général de quelque rupture sociale ${ }^{65}$. " On a donc pris un cas exceptionnel pour définir leur état naturel et notre état social pour ce qui leur est coutumier. Il semble que certains aient de la peine à admettre cette continuité. Malgré la qualité des observations, beaucoup hésitent encore à renoncer à des concepts élimés et cherchent à reconnaître les faits tout en essayant d'en minimiser la portée : «Un groupe biologique « naturel » est amorphe, écrit un anthropologue américain ${ }^{66}$, sauf en ce qui concerne la domination, les couples sexuels, s'il y en a, et les dyades mèreenfant. » D’après quel critère les groupes biologiques sont-ils jugés amorphes ? A partir de quel instant une hiérarchie, les couples reproducteurs ou nucléaires sont-ils acceptés comme sociaux? Cela ne nous est pas dit explicitement. Le sentiment de cet anthropologue contraste avec celui des zoologues et des ethnologues dont j'ai rendu compte à plusieurs reprises.

Si la valeur des phénomènes de hiérarchie et d'association chez les primates est réduite à si peu de chose, je présume que l'unique raison en est la nécessité de garder intacte une vision qui, présupposant la carence du dynamisme social en ce qui les concerne, situe là l'écart du règne animal au règne humain. Pourtant, il n'y a pas plus de " horde », de communauté uniquement organique, instinctuelle, chez eux, qu'il n'y en a et qu'il n’y en a eu chez nous. « On ne saurait imaginer plus grand contraste que celui qui existe entre l'image naissante d'une société ordonnée, note $\mathrm{S}$. Washburn ${ }^{67}$, fondée de façon prépondérante sur des actions sociales obéissant à des motifs affectifs ou

\footnotetext{
65 R. Fox : Kinship and Marriage, Baltimore, 1967, p. 29.

66 F.R. SERVICE : Primitive Social Organisation, New York, 1968, p. 69.

67 S.L. WASHBURN, P.C. JAY : Perspectives on Human Evolution, New York, 1968.
} 
coopératifs, et structurée par des rapports de domination stables, et l'ancienne idée d'une bande indisciplinée de singes dominés par un tyran. Les tenants de l'évolution, au $\mathrm{XIX}^{\mathrm{e}}$ siècle, attribuaient aux sociétés des hommes primitifs beaucoup moins d'ordre qu'il n'en existe, nous le savons à présent, dans les sociétés de singes et d'anthropoïdes vivant de nos jours. » La nouvelle frontière ne s'avère pas plus résistante ou mieux placée que l'ancienne. Bien entendu, entre les sociétés humaines et celles des primates, il y a de nombreuses différences manifestes quant aux qualités des individus, au rapport de l'inné à l'acquis, aux cycles d'échange avec le milieu, etc., sur lesquelles il est inutile d'insister. Le démon de Maxwell préposé à ces étranges manœuvres les a élaborées patiemment, au cours de millions d'années. Mais il y est arrivé en modifiant les coordonnées d'un mouvement générateur d'organisations équivalentes, et non pas en réglant l'agitation de molécules biologiques ponctuelles. Les essais théoriques successifs de séparer la société de la nature ou de poser la nature visà-vis de la société comme son passé ou son double hétérogène ont régulièrement abouti à la découverte d'une société autre, de l'essence d'une société distincte, celle du sauvage, celle de l'animal.

En résumé, la société apparaît comme une réalité positive et primaire analogue à la matière ou à la vie. Elle a sa propre économie née de l'économie de la nature et articulée avec celle-ci. Son existence a été redécouverte, éprouvée des centaines de fois par des espèces différentes. Sa continuité réside dans la capacité de se reformer, de se reproduire et de provoquer des changements lorsque les circonstances l'exigent. Nous n'expliquons pas la formation des systèmes biologiques par une anomalie de l'univers chimico-physique : nous n'avons pas davantage à expliquer celle de la société par une anomalie semblable, à y voir un pis-aller, la réponse à un dysfonctionnement extérieur. Des événements où le hasard a eu sa part l'ont suscitée avant que les nécessités de la survie, des adaptations successives l'amalgament à un grand nombre de processus objectifs. De même que la symétrie bilatérale est préférée à la symétrie radiale, la reproduction sexuelle à la division cellulaire, de même la sociabilité est une option fondamentale diffusée partout parmi les êtres vivants ${ }^{68}$. Elle remplit dans l'évolution une fonction décisive, et l'on peut se deman- 
der si l'on a le droit de parler à ce propos d'évolution biologique, au sens strict du terme, sans faire violence à la réalité. Loin d'avoir créé la société, notre espèce lui a simplement imprimé une structure particulière. La nature de l'homme est sociale, disait-on, afin de l'opposer aux autres espèces. La nature d'un très grand nombre d'espèces est sociale, constate-t-on, et l'opposition ne tient plus sous ce rapport.

Retour à la Table des Matières 


\section{Les sociétés « avec » et les sociétés « sans ».}

\section{Retour à la Table des Matières}

Rien n'autorise donc à fonder la supériorité et la permanence des institutions sur une différence radicale entre deux ordres de réalité, à y voir des réponses accumulées, au cours de l'évolution, à l'instinct et au désordre primordial, remèdes à une situation qui n'en avait pas auparavant. Les exemples étudiés nous montrent une propension constante à définir le passé naturel ou sauvage par l'absence d'un caractère qui ne se trouve que dans la civilisation. On oppose, à un étage quelconque de la vie humaine ou animale, les sociétés transitives sans parenté, sans règle, sans histoire, sans écriture, sans classe, aux sociétés intransitives avec parenté, avec règle, avec histoire, avec écriture, avec classe. Seules ces dernières apparaissent véritablement sociales, alors que le doute plane sur les premières en raison de leurs manques. La notion de passage devient dès lors essentielle, signalant un développement orienté qui prend par la main les collectivités déficitaires pour les acheminer vers ce qui fait la supériorité des nôtres. Le saut d'un tel état présocial ou non social à un état pleinement social ou non naturel comporte l'introduction de l'élément absent dans une structure préparée à cet effet et qui le réclame. Retracer la voie suivie pour arriver à ce terme et à cette fin équivaut à décrire la genèse de la culture ou de l'humanité. La différence comblée s'explique par le caractère de l'élément choisi - la règle, la parenté, etc. — au lieu que ce soit la différence constitutive qui rende compte de la manière dont l'élément a été engendré. Ne devons-nous pas considérer, non seulement les points de départ de l'évolution dans la perspective des points d'arrivée, mais aussi les points d'arrivée dans la perspective des points de départ?

Quelle que soit l'espèce considérée, les sociétés qui ont précédé les nôtres ont leurs règles, leurs hiérarchies, leurs moyens de communication, leur mouvement historique, assurément distincts des nôtres mais équivalents. Leurs pierres ne sont pas des pierres d'attente mais des pierres d'assise, fondations d'une construction complète. Leur devenir suit toujours plusieurs trajectoires possibles, subit des arrêts et des involutions, débouche sur plusieurs solutions, toutes réelles. Son tissu 
est composé des transformations des systèmes d'ensemble, c'est-àdire qu'elles subissent l'épreuve de la discontinuité et de l'opposition, reconstituent la continuité et la convergence, opèrent la substitution de facteurs qui se remplacent mutuellement. Les additions et les soustractions auxquelles on attache tant d'importance se ramènent à des conséquences secondaires, et les divers passages ne sont que le reflet formel du mouvement réel. A l'intérieur de celui-ci, ce qui advient sur l'échelle humaine a sa contrepartie sur l'échelle animale, qu'il s'agisse de la régulation des instincts, de la coexistence des individus ou des comportements concertés en vue d'une fin. Les interdits sexuels humains, entre autres, ne sont pas provoqués par leur objet. A aucun moment il n'est apparu qu'ils visaient des comportements débridés. Ils ont été suscités, pour des motifs propres, par les sujets qui les ont repris, raffinés et transmis.

Le passé d'une société n'est pas la nature interne ou externe : c'est une société autre. Nous étant accoutumés à l'idée que notre physiologie, notre anatomie "descendent» de celles des primates, nous devons encore nous faire à l'idée qu'il en est de même de notre corps social. De quelque côté qu'on regarde, vers le monde matériel ou vers le monde biologique, sa continuité, son autonomie sont certaines. Ses instances sont " conventionnelles »; d'autres instances, chez d'autres espèces, sont susceptibles de produire des effets analogues quant aux relations entre milieu et organisme et, s'étant élaborées au cours d'une période suffisamment longue, d'acquérir une inertie qui les rend indispensables. De sorte qu'une fois les événements ou les actes socialisés, ils conditionnent ceux qui les suivent, s'intègrent dans les systèmes d'interaction successifs. La place respective des sexes, des générations, plus tard des catégories sociologiques, a été transposée, impartie itérativement sur la carte des comportements, des normes, avec leurs compléments logiques, dans la chaîne des collectivités qui se sont succédé jusqu'à ce jour. A chaque étape, une cohérence interne nouvelle a été constituée, une congruence nouvelle avec les facteurs objectifs visés. Dans cet enchaînement, aucune société n’est «plus » société qu'une autre, ni celle des hommes vis-à-vis des primates, ni celle des peuples d'aujourd'hui vis-à-vis des peuples sauvages, de même qu'un atome électrique n'est pas "plus » atome qu'un atome chimique. Ces comparaisons ont le même fondement que la célèbre constitution d'Animal Farm, de George Orwell : «Article I ${ }^{\mathrm{er}}$ : tous les 
animaux sont égaux. Article II : certains animaux sont plus égaux que les autres. » Il y a eu vraisemblablement, et il y a eu sûrement, même si nous en connaissons peu ou si nous les connaissons mal, des sociétés meilleures ou préférables. On soupçonne que les nôtres sont préférables ou meilleures que celles des primates tandis que, aux yeux de certains, les sociétés sauvages paraissent meilleures ou préférables aux sociétés civilisées. L'inclination à les classer du côté de la nature ou du côté de la culture procède de la conviction que nous sommes en mesure de calculer une échelle de perfection dont nous connaîtrions le degré zéro et le degré cent. Et qu'elle aurait de plus une réalité tangible, puisque l'ethnologue, quittant l'Europe, la prend pour guide : « Il s'agissait d'échapper, écrit Jacques Monod à propos d'un départ pour une étude sur le terrain ${ }^{69}$, au cercle d'Occident, par une épreuve de distanciation qui ne portât plus seulement sur le langage... mais sur tout ce qui me reliait au monde et aux autres, par « immersion totale » dans une culture que je croyais "plus proche de la nature " que la mienne. » Conviction sans fondement, avouons-le, tant elle est basée sur des indices fluctuants et arbitraires. Leur valeur, quant à traduire un état de perfection, est relative à l'observateur, influencée par l'esprit du temps. Des distances et des hétérogénéités entre sociétés sont monnaie courante: les analyser va de soi. Il ne nous incombe toutefois pas d'établir une hiérarchie et d'apprécier leurs qualités, inspirés par les jugements des contemporains ou de la postérité, pas plus que nous ne décidons de l'excellence d'une langue parce que nous la parlons bien ou du fait qu'elle possède un vocabulaire plus fourni. Du point de vue de la méthode, si toutes les sociétés sont également sociétés quant à leurs fonctions, leurs structures et leur correspondance aux circonstances qui les suscitent, mieux vaut alors saisir la nôtre dans une succession qui a débuté très tôt sur l'arbre de la vie, relever celle des primates. En d'autres termes, leurs sociétés d'affiliation font partie de notre histoire. Hypothèse qui nous dispense de bâtir le roman de l'existence d'un conglomérat rudimentaire d'animaux ou d'une horde primitive d'individus instables que le texte suivant illustre: " Elle (la société) émerge de la nature dès l’instant à jamais indéchiffrable où, sans doute par l'intermédiaire du langage, ces hordes préhominiennes découvrent des Nous en face d'autres Nous, des femmes

69 J. Monod : Un riche cannibale, Les Temps Modernes, déc.-jan. 1970-71, p. 1065. 
et des aliments que Nous possédons, d'une part, des femmes et des aliments qu'ils possèdent, d'autre part, fussent-ils rigoureusement équivalents " ${ }^{70}$. Les mots « indéchiffrable », « sans doute » et « rigoureusement équivalents » donnent du vernis à cette image d’Épinal, qui pourrait s'appliquer à n'importe quelle espèce, langagière ou non ; ils ne la sauvent pas d'une caducité théorique que seule la connivence du sens commun retarde. Mais, outre cet aspect de sobriété intellectuelle, l'hypothèse a pour elle la vraisemblance: les sociétés d'affiliation - est-il nécessaire de le rappeler ? — ayant précédé celles de parenté, ayant été adoptées et à coup sûr achevées par les hominiens, elles sont un point de départ et de comparaison effectif.

Ensuite, ceci nous amène à traiter l'avènement de nos premières conditions de vie collective comme s'il s'agissait de la succession et non, pas du passage - du féodalisme au capitalisme, sans plus. Le changement d'un tel système social, à moins que ce ne soit un miracle ou le résultat d'une génération spontanée, est un mouvement de formation et de transformation qui, parallèlement à des facteurs novateurs, conserve, dans un contexte modifié, des traits du système auquel il se substitue. D'une certaine manière, l'esclavage antique persiste à travers la féodalité et ne disparaît pas complètement avec le capitalisme : De même, les éléments de la société de parenté ne sont pas obligatoirement tous sa création : quelques-uns, provenant des sociétés de compagnonnage et de clique ont été repris dans une combinaison nouvelle au moment où des éléments inédits s'inséraient dans une combinaison ancienne, préhominienne. Nous le verrons à propos de l'exogamie et de la prohibition de l'inceste. Le changement qui a eu lieu fut néanmoins un changement de la société animale en société humaine, d'une première société humaine non spécifique en une seconde, spécifique. Le fait que ce soit un double changement une organisation sociale commune aux deux espèces, les primates et les hommes, devenant une organisation sociale appartenant exclusivement à ces derniers - lui confère une caractère extraordinaire. Extraordinaire, en vérité, par l'ampleur du bouleversement, mais non parce qu'il s'agirait d'un commencement absolu.

70 L. De Heusch : Pourquoi l'épouser ? Paris, 1971, p. 97. 
Dans ces conditions, la primitivité où l'on a l'habitude de voir un commencement, une rupture, l'instauration d'un ordre dans un système lacunaire par réduction du chaos initial et comblement des manques, est en réalité, si on la compare à son propre passé et non plus à notre présent, un état tardif et évolué. Les écarts de la société à la nature, censés être les jalons de la distance qui sépare celle-là de son origine dans celle-ci, se révèlent être des écarts de la société à ellemême, aux diverses étapes de son développement, les indices de transformations multiples. Bref, il n'y a pas de nature avant la culture, il n'y a pas d'homme avant l'homme, puisqu'on ne le trouve pas préexistant à lui-même sur le mode biologique avant de se manifester sur le mode non biologique. Concrètement il a toujours été dans la nature comme un être social et dans la société comme un être naturel: " L'ordre naturel, écrit Robert Jaulin ${ }^{71}$, est celui du milieu en lequel, contre lequel et par lequel les espèces vivent et non pas une négation de la culture ou une culture inverse, pas plus que la culture ne constitue une négation de la nature ou une nature inverse. D'un point de vue biologique, la nature de chaque espèce, dont l'humaine, est son mode particulier d'existence, son être dans le monde, sa culture. » A la vérité, le mouvement de la société a changé, le mouvement de la nature aussi. Envisagées dans leur évolution globale, dans leur histoire humaine, la discontinuité absolue et l'extériorité postulée s'estompent, et l'on voit la société sortir continuellement de la nature pour y retourner ; on pourrait comparer l'induction des effets réciproques qui sont les leurs à l'induction oscillante qui s'exerce entre un champ magnétique et un champ électrique. A chaque époque, l'homme ressuscite leur antagonisme, leur assigne de nouvelles frontières, donne un poids différent à l'une ou à l'autre, institue des modalités différentes d'échange, sans pour autant quitter la nature ou intégrer la société, parce qu'il ne peut faire qu'il n'appartienne à l'une et l'autre à la fois. Dire qu'une fraction de l'humanité est plus proche ou plus éloignée qu'une autre de l'état de nature n'a pas davantage de sens pour le passé initial que pour le présent évolué. Il est donc vain de vouloir prouver à tout prix que l'homme est un être unique, puisque - et ce fait n'a rien de dégradant - la société et la nature qui lui sont propres se placent dans la suite des créations que suscitent dans l'univers des es-

71 R. JAUlin : La distribution des femmes et des biens chez les Mara, Cahiers d'Études Africaines, 1966, 6, p. 461. 
pèces dont la nôtre est peut-être la plus récente mais ne sera pas forcément la dernière.

$\underline{\text { Retour à la Table des Matières }}$ 


\section{Chapitre VII.}

\section{La chasse et la parenté : premières constatations}

\section{Les trois dimensions de l'entreprise cynégétique.}

\section{$\underline{\text { Retour à la Table des Matières }}$}

Système de rapports sociaux fondés sur le mariage et la cellule familiale, la parenté forme l'ossature des collectivités archaïques. Elle s'est instituée en même temps que s'élaborait le savoir-faire et que s’organisaient les groupes spécialisés en vue de la chasse. Comparée au mode antérieur d'exploitation des ressources et d'interaction avec le monde matériel, la chasse entraîne des changements d'importance.

La collectivisation, d'abord, porte aussi bien sur ce qui constitue une ébauche de la production que sur la consommation. Les singes et les anthropoïdes se procurent chacun seul leur subsistance. Une fois sevré l'enfant ne dépend plus des adultes pour sa nourriture. Sauf cas exceptionnel, mâles et femelles, animaux jeunes ou vieux sont déliés de toute responsabilité réciproque à cet égard. On observe une certaine coordination en ce qui concerne le repérage de nouveaux sites ou la défense contre les prédateurs : son impact sur la vie interne du groupe demeure limité. La diffusion de la chasse et sa séparation d'avec la cueillette transforment les conditions d'existence. Hommes et femmes se consacrent à obtenir des ressources différentes et complémentaires. L'alimentation régulière des individus appelle une mise en commun de ces ressources et la possibilité d'y avoir accès lorsqu'elles sont présentes. Les liens entre générations en sont aussi affectés ${ }^{72}$. Puisqu'il faut plus de temps aux enfants pour atteindre la matu-

72 V. REYNOLDS : Kinship and the family in Monkeys, Apes and Man, art. cit. 
rité biologique, ils exigent des soins plus nombreux et prolongés. D'autre part, et ceci est surtout vrai pour les garçons, ils ne sont pas aptes avant un certain âge à prendre part aux activités des adultes. Les parents voient leurs charges accrues d'autant. Les femmes sèvrent les nourrissons beaucoup plus tard — si l'on se réfère à l'exemple des Eskimos ou des Australiens, l'allaitement se prolonge jusqu'à trois ans au moins, en raison notamment du manque ou de la précarité des autres nourritures et continuent à assurer par la suite leur alimentation et leur transport. De leur côté, les hommes doivent enseigner aux adolescents les disciplines physiques et intellectuelles de la chasse. En revanche, les jeunes ont l'obligation, parfois révocable, de pourvoir aux besoins des vieux, de leur réserver une partie de leur butin. L'interdépendance des classes d'âge et de sexe, au niveau de la consommation, devient générale.

La cueillette et le fourragement, l'observation des primates l'atteste, sont des occupations individuelles; la chasse, surtout aux grands animaux, suppose la coopération d'un nombre assez élevé d'individus, leur intégration en vue de l'accomplissement de tâches voisines, pour un but qui les concerne tous. Je n'insiste pas ici sur son aspect technique. Je me réfère à l'articulation des groupes, à la confiance qu'ils doivent se faire à long terme pour se retrouver périodiquement dans les lieux repérés et désignés, au mutuel secours qu’ils ont à se porter en cas de danger, blessure, etc., à l'assurance qu'ils se donnent d'exécuter chacun la mission qui lui est impartie au cours des expéditions cynégétiques. La solidarité des mâles, leur fraternité, répondent aux circonstances dans lesquelles se déroulent leurs activités ; le passage de la cueillette à la chasse - on peut le comparer au passage de la production artisanale à la production manufacturière - représente une collectivisation des moyens et des fins, une subordination réglée des parties au tout. On est fondé à dire qu'il s'agit à la fois d'une extension de la reproduction, dont le domaine élargi ne comprend plus seulement la sexualité mais aussi les savoirs, les instruments, les échanges avec le milieu matériel - la production en tant que telle ne devenant autonome que plus tard, grâce à l'accumulation et à la conservation des biens - et d'une collectivisation de l'ensemble de cette reproduction naturelle, au sens humain. Cette prééminence et cet élargissement de l'intervention sociale, dans des aspects de la vie dont les groupements de primates ne se mêlaient pas, 
instaurent le partage des ressources, des occupations et des devoirs, en tant que principe de relation et d'organisation. Dans les tribus australiennes de nos jours —si l'on admet l'illustration — l'appartenance à un clan ou à une bande obéit à des règles claires ; elle n'entraîne nullement l'exclusion d'autres clans ou bandes du territoire en question. Maintes fois - c'est le cas des Boschimans et des Eskimos - cette appartenance aussi bien que l'espace réservé à la cueillette ou à la chasse varient suivant les saisons, de manière à faciliter l'existence de tous. A plusieurs titres, la dépendance réciproque directe colore liens et comportements. Aucune fraction de la collectivité ne monopolise définitivement les richesses, ne se ferme aux individus amenés à participer à ses entreprises, ne mesure son aide à ceux qui la sollicitent en temps de disette ou en cas de maladie. "Refuser de donner, négliger d'inviter, comme refuser de prendre, équivaut à déclarer la guerre ; c'est refuser l'alliance et la communion. Ensuite, on donne parce qu'on y est forcé, parce que le donataire a une sorte de droit de propriété sur tout ce qui appartient au donateur ${ }^{73}$. " Donner et recevoir, tenir à la disposition d'autrui ce qui est à soi sont les impératifs d'une convention qui garantit à chaque homme d'être le partenaire d'un autre homme. Faute de quoi il n’y a ni bien-être, ni survie.

Ensuite le chasseur est constamment à la poursuite de sa proie. A des époques déterminées de l'année, il lui faut quitter son campement habituel, rencontrer d'autres chasseurs pour explorer avec eux les terrains giboyeux. Le gibier qui comprend plusieurs espèces ne se trouve pas toujours à des endroits précis, son importance numérique varie. De son côté, la recherche des tubercules et des fruits implique des déplacements moins étendus mais non négligeables, compte tenu de ce que le groupe doit transporter les enfants et quelquefois les objets indispensables pour camper. Le nomadisme est un style de vie caractéristique pour qui examine ces sociétés à proprement parler volantes : « Comme les chasseurs-collecteurs, écrit Desmond Clarke ${ }^{74}$, dépendent entièrement des ressources naturelles de leur milieu, leur survie est liée à la présence d’une réserve de nourriture appropriée, toujours disponible. Ils ne peuvent donc s'établir dans des demeures permanentes mais doivent être sans cesse en route. Pendant la saison des pluies,

73 M. MAUSS : Sociologie et anthropologie, Paris, 1965, p. 162.

74 J.D. Clark : The Prehistory of southern Africa, Londres, 1959, p. 39. 
ils se disséminent pour récolter les nombreux fruits sauvages et autres ressources que l'on trouve à cette époque, et pendant la saison sèche, lorsque la nourriture végétale se fait rare, ils se concentrent plus autour des points d'eau permanents (et, voudrait-on suggérer, font appel à une nourriture carnée). Ainsi le groupe se déplace sans cesse autour de sa réserve de chasse. On a beaucoup parlé des habitudes carnivores de l'homme préhistorique, mais il est certain que les nourritures végétales ont dû fournir une source de provende tout aussi importante pour ses besoins et, pour commencer, étaient probablement de beaucoup la plus importante. Un groupe de chasseurs parcourait probablement un territoire de plusieurs centaines de kilomètres carrés en l'espace d'un an, allant d'un endroit à l'autre, se nourrissant de fruits et de racines sauvages à mesure qu'ils mûrissaient ou qu'ils étaient de saison. Pour s'assurer une nourriture carnée, le groupe suivait aussi le gibier qui, surtout les troupeaux d'herbivores, se disséminait loin des points d'eau permanents pendant la saison des pluies et, à mesure que les sources temporaires tarissaient, se rabattait sur les rivières et autres points d'eau permanents à la saison sèche. Ce mouvement saisonnier est une règle de vie que suivent en fait rigidement tous les groupes primitifs de chasseurs-collecteurs et qui offre des ressemblances avec les habitudes des mammifères supérieurs. »

Cette continuelle mouvance n'est pas, on le voit, pur désordre. Elle est déterminée par des facteurs constants et a des effets sociaux patents. Les femmes et les hommes suivent des trajectoires indépendantes, dans certaines limites, pour s'approvisionner et mettre à la disposition de la famille ou du clan les biens qu'ils sont censés leur fournir. Les territoires sont répartis entre eux, particuliers à chaque groupe. L'espace occupé, les espèces sont ordonnées en fonction de cette ligne de clivage et de cette alternance des périodes où l'un ou l'autre sexe récupère sa position privilégiée. L’étendue de la collectivité se soumet à ces données. Les groupes doivent rester relativement petits sous peine d'épuiser les réserves végétales d'une région. D’autre part, la chasse exige, pour être menée à bien, la concentration de plusieurs familles, clans ou bandes. La coordination dans le temps introduit une dimension rythmique dans la société, des périodes de dispersion et des périodes d'agrégation corrélatives à une contraction et à une dilatation 
du groupe. «Le milieu, observait Marcel Mauss ${ }^{75}$, agit, non sur l'individu, mais sur le groupe dans son ensemble. » Des exemples contemporains en fournissent une image.

Daryle Forde ${ }^{76}$ nous a donné une description instructive des Blackfoot, tribus vivant de part et d'autre de la frontière canadienne. L'animal chassé est le buffle, et c'est lui qui commande, par ses déplacements, les migrations saisonnières. A la fin du printemps et au début de l'été, les immenses troupeaux de buffles paissent l'herbe des gras pâturages abondants dans les plaines du nord-ouest. A partir de l'automne, le déclin de la végétation disperse les animaux en troupes beaucoup moins nombreuses et plus mobiles. Les chasseurs à leur tour suivent ces règles de peuplement. En été, ils se réunissent en groupes importants, bien encadrés, pour aborder les troupeaux concentrés dans les plaines ; en hiver, par contre, la population se dissémine, en petites unités, à travers le territoire, afin de rechercher séparément des ressources devenues moins abondantes. Ces unités sociales qui se forment pour des raisons objectives évidentes ne perdent pas leur identité en se fondant dans des groupements plus vastes. Chacune dispose d'une portion de territoire, séparée des autres par des limites naturelles, cours d'eau ou accidents de terrain. Elles ont pour centre des hommes apparentés d'une même famille, bien que parfois d'autres familles ou des individus isolés s'y joignent. Les actes de la vie collective, rencontres et fêtes, obéissent à ces modèles de regroupement et de dispersion au rythme des saisons. En été a lieu l'assemblée tribale qui se réunit dans un camp circulaire de près de cinq cents mètres de rayon, où les tentes sont disposées sur trois ou quatre rangs de profondeur. Chaque unité y retrouve sa place traditionnelle d'une année à l'autre. La chasse ayant été fructueuse, son succès est fêté par des festins et des cérémonies. Des charmes, des objets magiques accompagnés des formules rituelles permettant de s'en servir s'échangent contre des peaux de bêtes et des chevaux. Les associations d'hommes se livrent à des danses rituelles. Le moment venu, des groupes effectuent les transactions et négociations prolongées, sous des tentes construites spécialement à cet effet, au cours desquelles certains individus

75 M. MAUSs : Essai sur les variations saisonnières des sociétés eskimos, Année sociologique, 1905, 9 ; p. 65.

76 C.D. FordE : Habitat, Economy and Society, New York, 1963. 
passent d'un groupe à l'autre. Le rassemblement se termine par la cérémonie annuelle de la Danse du Soleil, qui peut durer plus d'une semaine. L'astre est censé conférer prospérité et pouvoir à ceux qui l'adorent. La fête précède de peu la dislocation du camp, chaque groupe prenant ses quartiers d'hiver dans le territoire de l'ouest, plus vallonné et moins fertile, et retrouvant son indépendance relative visà-vis des chefs et du conseil de tribu.

L'exemple des Eskimos Netsilik est aussi révélateur. Habitant le nord et l'ouest de la baie d'Hudson, ils se dispersent en juin, le groupe étant la famille élargie. Ils vivent sur le rivage où ils ont dressé leurs tentes, puis à l'intérieur des terres, pêchant au harpon et chassant le caribou avec l'arc et les flèches. En août débute la pêche au saumon ; les poissons sont pris au trident en grand nombre, les rivières ayant été obstruées par des barrages. En septembre on chasse le caribou poursuivi en kayak; en octobre on pêche la truite saumonée, à travers la mince couche de glace des fleuves. Puis les groupes se rejoignent pour la chasse aux phoques. Ces animaux respirant à travers les trous de la glace, il est avantageux de poster un chasseur à chaque trou, prêt à intervenir avec son harpon. Les communautés qui peuvent dépasser soixante personnes vivent dans des igloos sur la mer gelée. L'hiver est la saison de la vie sociale, des pratiques magiques et d'autres cérémonies. A propos de l'ensemble du monde eskimo, Marcel Mauss a analysé finement ce caractère cyclique des rapports collectifs, la phase du rassemblement hivernal étant aussi celle de la communication, de l'élaboration des mythes et des contes transmis de génération en génération, d'une longue fête où la collectivité célèbre et se célèbre. La construction même des habitations favorise la fusion des familles; l'existence d'une maison d'hiver, lieu d'assemblée et demeure des hommes, où les adultes mariés et célibataires couchent à part des femmes et des enfants, manifeste l'unité de la communauté. La dispersion des groupes en été amorce une nouvelle phase ; sur la terre ferme, la vie familiale reprend ses droits, ce qui diminue, de toute évidence, l'intensité des échanges collectifs et atténue l'emprise de la société. L’alternance obligatoire de la réunion et de la séparation, du contrôle et de l'autonomie, enserre l'individu dans un double lien : il appartient à la fois à une collectivité privée permanente et à une collectivité publique périodique. 
Mais un autre facteur infléchit cette alternance. Les ressources végétales et animales fluctuent suivant les années, d'une région à l'autre, et ce sont elles, en définitive, qui commandent l'organisation sociale, lui imposent sa flexibilité, motivent les déplacements de la population. Les cérémonies, les rituels et le règlement des affaires communes sont reportés, dans ces conditions, aux époques, aux années où le gibier abonde ${ }^{77}$. «La population, notait Marcel Mauss ${ }^{78}$, se condense ou se dissémine comme le gibier. Le mouvement dont est animée la société est synchronique à ceux de la vie ambiante. » Les lois de l'hospitalité assurent la soudure, entent la continuité sur la discontinuité, cimentent les liens entre les groupes, chacun se faisant un devoir d'inviter à son tour celui qui l'a reçu.

Le nomadisme brise avec la co-présence, le contact visuel tactile en tant que moyens de reconnaissance et de délimitation du champ social. Il effectue le brassage et la redistribution continuels des individus ou des familles, empêchant la formation de hiérarchies stables, de conduites d'appropriation rigides. En contrepartie, l'interdépendance des groupes et leur dépendance commune, manifestes, par rapport à des espèces végétales et animales individualisées dans l'espace et dans le temps, la possession d'un savoir unique partagé, la nécessité de se retrouver afin de mettre en œuvre ce savoir et d'agir sur ces espèces, situent le tout au-dessus des parties, supra-ordonnent la société aux bandes, clans ou familles qui la composent.

Enfin, la division naturelle de la chasse et de la cueillette transforme, tout en s'y appuyant, la distinction des sexes. Celle-ci, de purement biosociale, comme chez les primates, devient différence vis-àvis du monde matériel. Dans les sociétés archaïques, elle est essentielle et engendre deux champs d'activité nettement séparés. On a observé, par exemple, chez les Ainu, population subarctique, que les activités de l'homme et de la femme étaient très différenciées et ne se recouvraient pour ainsi dire pas. La femme ne sort guère, pour son ramassage et sa collecte, d'un espace étroitement circonscrit près du camp, alors que la chasse amène l'homme à parcourir un terrain beaucoup plus vaste. Même lorsque les femmes chassent, les disparités

77 J.H. Steward: Theory of Cultural Change, Urbana (III).

78 M. MAUSS : art. cit. p. 65. 
subsistent. Les femmes capturent les petits animaux et ne disposent pour cela d'aucune arme spécialisée ; elles ont recours à des bâtons, armes improvisées ou se servent de leurs mains nues. Il peut leur arriver, en de rares occasions, d'emprunter les harpons ou autres objets de chasse masculins. Ordinairement il leur est interdit de s'en servir. Dans les chasses collectives, le rôle des femmes se limite au rabattage, et elles ne s'attaquent aux mammifères de grande taille que de façon exceptionnelle. Chez les Semang, la différenciation des activités des deux sexes est beaucoup moins marquée, s'agissant d'une population qui ne pratique la chasse que de manière épisodique et dépend beaucoup plus étroitement des ressources végétales. Chez les Kuchin, peuple de grands chasseurs, au contraire, la séparation est nette et s'observe déjà lorsqu'on lève le camp. La femme, lourdement chargée, se déplace lentement en ligue droite vers le nouveau camp; l'homme, ne portant que son arme, explore le territoire adjacent et parvient à destination après maint détour. Pour les Lele d'Afrique, la forêt où l'on chasse est un domaine exclusivement masculin, tandis que les femmes règnent sur la savane où elles cultivent l'arachide. La division est si générale qu'il faudrait plusieurs volumes pour en consigner les modalités par le menu. « Il semble certain que la différenciation du champ d'activité selon le sexe soit un phénomène universel parmi les modernes collecteurs de nourriture. Il se peut que ce soit un caractère écologique de l'homme. Par suite de cette différenciation, la structure de l'habitat de l'homme diffère de celle des primates non humains ${ }^{79}$. »

Cette différenciation connote une complémentarité et une séparation. La complémentarité est objective, aucune fraction du groupe ne pouvant subsister indépendamment de l'autre. La séparation, elle, est beaucoup plus importante et significative, puisqu'elle demande à être étayée, déterminant la plupart des institutions sociales et rituelles. La dissymétrie des sexes en découle comme une conséquence remarquable, dont la reproduction naturelle est un aspect décisif. Afin d'obtenir des ressources équilibrées, de répondre aux besoins collectifs, il est nécessaire de développer les caractères spécifiques des hommes et des

79 H. WATANABE : Subsistence and Ecology of Northern food gatherers with special reference to the Ainu, in R.B. LEE, I. DE Vore, Man the Hunter, op. cit., p. 76. 
femmes, et de disposer en quantité suffisante d'individus ayant ces caractères. La complexion sexuelle de ce qu'il faut nommer, déjà, la force de travail, prend un sens nouveau et s'avère déterminante. La contribution d'un groupe aux résultats de la chasse dépend du nombre des hommes, de leurs qualités physiques et de leur habileté. Ainsi une carence prononcée d'éléments masculins en état de chasser entraîne une perturbation et met le groupe en état d'infériorité. Par suite, la transmission des facultés, des modèles de coordination sensorielle et intellectuelle, l'imprégnation psychologique par les propriétés du milieu doivent être distinctes pour les filles et pour les garçons. Le laps de temps nécessaire pour y parvenir n'est pas non plus le même. Non seulement l'initiation s'adresse à des individus de sexe différent, elle est l'œuvre d'adultes de sexe différent, qui participent séparément à la chasse et à la cueillette. Chacun des deux univers est ainsi perpétué à part, sous-tendu par des pratiques qui le renouvellent de génération en génération.

La chasse est une occupation collective, tandis que la cueillette demeure largement individuelle. Les liens entre hommes ont tendance à se resserrer et à s'institutionnaliser, les liens entre femmes restent lâches et épisodiques. La réunion périodique à l'occasion des chasses collectives est surtout un rassemblement d'hommes, consacré par les activités de ceux-ci, qui occupent le centre de la vie sociale aussi bien que matérielle. Les dissolutions successives renvoient à l'univers particulier de la cueillette, à la prédation individuelle, à la dispersion propre au monde féminin. Les oscillations régulières de la société ressuscitée et de la société mise en sommeil, de la vie cérémonielle successivement retrouvée et suspendue, le retour périodique des mois d'abondance et des mois de disette, des "vaches grasses » et des "vaches maigres », portent la marque de la séparation, chaque fraction étant associée de manière symbolique, avec prédilection, à l'un des sexes.

Au-delà de cette dissymétrie du facteur masculin et du facteur féminin, c'est à une sorte de renversement que l'on assiste si l'on compare les collectivités humaines aux collectivités des primates ou des premiers hominiens. Dans ces dernières, les jeunes mâles représentaient un surplus, rejeté vers la périphérie du groupe, condamné à disparaître en priorité. La cynégétisation les valorise parce qu’ils sont 
devenus indispensables et leur donne un pouvoir de négociation avec les adultes inestimable. "Mais les jeunes hommes, observe Luc de Heusch ${ }^{80}$ à propos des sociétés de parenté actuelles — et ceci a dû être vrai des sociétés passées - détiennent un atout appréciable dans le secteur cynégétique : leur force de travail. Ils entrent dès lors dans un double rapport de réciprocité avec les hommes âgés : au sein du clan matrilinéaire, ils recevront une épouse d’un oncle maternel âgé ; au sein de leur groupe d'âge, ils sont unis à la génération alterne qui mettra à leur disposition une épouse polyandrique, une " épouse de village ». » Ceci vient de ce que leur sous-groupe négatif se transforme en sous-groupe positif, pour la production et la reproduction en général. Le mâle adulte humain, loin de les ignorer, souhaite s'ouvrir, avoir accès aux enfants. Il doit rester en contact suffisamment prolongé avec eux pour assurer leur formation, les entraîner dans le monde où ont cours les relations avec l'animal, qui exercent une si grande fascination. A l'inverse des fourrageurs-collecteurs, voire des prédateurs, il a également intérêt à retenir les adolescents dont le départ serait une perte sèche pour la collectivité. Il est probable que les efforts que représente l’initiation des jeunes seraient découragés si les initiés n'avaient pas, d'une manière ou d'une autre, un rapport plus stable avec les initiateurs, et si ceux-ci ne disposaient d'aucun moyen de les garder parmi eux. Ce à quoi la société s'applique. L'intérêt commun exige que les jeunes mâles, enclins à explorer, vagabonder, ne s’éloignent pas définitivement pour aller grossir les rangs d'un groupe avec lequel leur groupe d'origine ne chasse pas. Dans un sens, et par symétrie, ce sont les enfants de sexe féminin qui deviennent un surplus, une population surnuméraire, une charge aux yeux des familles. "Les parents, disait un informateur indigène à un anthropologue, considèrent souvent qu'ils ne peuvent se permettre de gaspiller plusieurs années à élever une fille. Nous vieillissons si vite qu'il nous faut nous dépêcher d'avoir un fils. » La position nouvelle du jeune mâle stabilise les relations des parents avec leurs enfants, en particulier la relation du géniteur mâle à ses fils, et leur confère un caractère de nécessité qu'elles n’avaient pas auparavant. C'est là une innovation proprement humaine, une mutation radicale induite par la division naturelle par rapport à tout ce que l'on sait de l'organisation collective des simiidés. 
Ces divers changements sont solidaires, provenant de la même racine. Collectivisation du processus reproductif, nomadisation des sociétés humaines, division naturelle des ressources humaines et non humaines traduisent un mouvement unique qui modifie les données du milieu et les propriétés de l'organisme, avec pour résultat un renouvellement du mode de fonctionnement social. Les conditions nouvelles dans lesquelles se fait l'exploration du monde ont en effet entraîné, contrepartie évidente, une refonte de l'unité constitutive, de la régulation reproductive, de la hiérarchie des sociétés d'affiliation. La diversité de celles-ci a eu pour répercussion certaine une diversité des solutions adoptées à cette occasion. Nous n'avons aucune raison de postuler, à tout prix, une uniformité initiale. La pluralité observée dans les collectivités archaïques s'explique, en partie, par la pluralité des matériaux sociaux qu'il leur a fallu reprendre et retravailler. La parenté les a cependant rapprochées dans un mouvement unique. Ses traits correspondent et se comprennent — je ne parle pas de leur détermination qui soulèverait de tout autres problèmes (note 6) — en tenant compte des savoirs et des pratiques que ces collectivités ont été les premières à singulariser et à développer. Ceux-ci tournent, on le sait, autour de trois pivots ; la paternité, l'impératif du mariage et la prohibition de l'inceste. Le premier est la grande innovation des sociétés de parenté, le second représente une nouvelle manière d'élargir d'anciens rapports, le troisième prolonge une subordination qui existait déjà dans les sociétés de primates. Aucun de ces pivots n'appelle l'existence de l'autre, et chacun pourrait, à la rigueur, exister séparément. Mais c'est ensemble, liés au soubassement naturel commun, qu'ils soutiennent le dessin basal des rapports de pouvoir et d'alliance devenus proprement humains. 


\section{Le problème du mâle.}

$\underline{\text { Retour à la Table des Matières }}$

\section{(1) Découverte de la paternité.}

Des sociétés d'affiliation, qui ont servi de moule et de conducteur à ces pivots, on a déjà relevé les constances ${ }^{81}$. La distance qui sépare les groupes mâles, non reproducteurs, des groupes hétérosexuels en est une. Inclus dans l'organisation commune, ces prétendus célibataires forment un groupe appendiculaire, occupent une place à part, reconnue et nécessaire, du système de relations sociales ; ils sont soumis aux autres mâles. Femelles et mâles constituent les groupes de base, s’agrégeant séparément dans les diverses occasions qui ont été mentionnées. Leur réunion, sauf chez les gibbons, est le plus souvent polygyne. Elle est marquée par l'existence d'une hiérarchie de contrôle : les mâles occupent une position de direction, les femelles une position de suivantes. A l'intérieur de ces groupes de base, on relève le clivage du couple nucléaire et du couple reproductif, formés, l'un, de la femelle et de sa progéniture, l'autre, d'un mâle et d'une ou plusieurs femelles. Le premier couple, comparé au second, est faiblement hiérarchisé ; seul est perceptible, pendant une période relativement brève, l'attachement réciproque des mères et de leurs enfants. Ces couples nucléaires se transforment rarement en couples reproductifs. Les savants japonais ont étudié avec soin le choix des partenaires sexuels chez les primates et en ont conclu que, lors de la formation des couples au printemps, il y a très peu de couples constitués par une mère et son fils. Des mâles adultes aux jeunes, on constate une sorte de coupure, une quasi-absence de liens positifs analogues à ceux qu'établissent les femelles. Le mâle adulte considère les jeunes mâles, surtout à partir d'un certain âge, comme appartenant à une catégorie subordonnée, avec laquelle il n’a que des rapports de domination.

La prééminence des mâles reproducteurs, qui entraîne l'isolement et le maintien de groupes, aux frontières fluctuantes, de mâles " célibataires » non reproducteurs, la spécificité des couples nucléaires et

81

Chap. II. 
des couples reproductifs, paraissent, jusqu'à plus ample informé, définir les sociétés d'affiliation décrites chez les primates. Ce sont, notons-le, des sociétés directes. A l'encontre de nos sociétés plus récentes, que l'on pourrait qualifier de différées, elles existent uniquement à travers les faisceaux de rôles, de tâches, que remplit chaque individu ou chaque fraction du groupe. Les actions accomplies ou exigées sont entreprises et contrôlées spontanément par ceux qui les exécutent, aucun organe extérieur ou spécialisé n’ayant l'autorité ou les moyens de le faire. L'ensemble est soudé par des forces internes, à défaut de forces sociales externes ou d'une structure qui l'encadre et lui impose la cohésion. Les ressources sont celles des différents membres, aucune provision en individus, biens ou territoires n'étant réservée à la communauté en tant que telle. $\mathrm{Nu}$ en quelque sorte dans l'univers qui l'entoure, le corps social se tient fermement grâce à la coordination et au contrôle constants de ses membres.

La chasse y introduit et requiert un certain degré de collectivisation. Elle amène les deux classes de couples propres aux sociétés d'affiliation à se fondre dans une unité à la fois nucléaire et reproductive, comme elle associe étroitement la reproduction et la production du groupe en son entier. Les individus des deux sexes sont indispensables pour assurer la subsistance, et les générations engagées solidairement dans l'œuvre de survie du groupe ; les jeunes vis-à-vis des adultes et réciproquement. Les enfants sont devenus nécessaires à la fraction masculine de la population, amenée à recruter des compagnons parmi les jeunes garçons. Quoique l'usage terminologique soit répandu même en ce qui concerne les primates, la famille n’a pu apparaître avant la formation des collectivités de chasseurs-collecteurs : « Bien qu'une famille nucléaire - unité minimale composée du père, de la mère et des enfants - serve de base à toutes les études ethnographiques, observe un anthropologue américain ${ }^{82}$, il se peut qu'elle n'ait pas existé aux époques primitives. Chez les chasseurs et collecteurs, la famille est fondée sur une forte complémentarité des sexes ; l'homme est surtout chasseur, la femme porte le bois et l'eau, fait la collecte des graines, garde le camp et élève les enfants. »

82 J.H. STEwARD : Causal Factors and Process in the Evolution of Pre-farming Societies, in R. LEE, I. DE VORE, op. cit., p. 330. 
Nous venons de voir les motifs qui l'ont amenée à se constituer. On a attribué son apparition à l'impuissance biologique de l'enfant, au fait que le prolongement de la période d'immaturation aurait exigé la collaboration d'un père et d'une mère. Cette impuissance n'est pas une pure donnée biologique : elle résulte du développement social et naturel de l'espèce. Si les rôles éducatifs des hommes et des femmes ne s'étaient pas complétés, surtout en ce qui concerne la formation des garçons - et je crois que c'est principalement à cause d'eux que le lien conjugal s'est établi — s’il n’y avait pas eu un rapport préalable fondé sur une autre base, l'allongement de la période de dépendance vis-à-vis de la mère aurait simplement abouti à un renforcement des attaches avec elle, à une consolidation du couple nucléaire. L'insertion du mâle dans ce couple a dû être un des premiers problèmes à résoudre. Insertion créatrice de tensions, certes. L'agressivité masculine, la tendance à tuer les jeunes au cours des accouplements - on l'a observé chez les babouins et chez les rhésus - ou lors d'un changement de partenaires, ne pouvaient avoir que des effets délétères. La rivalité, ou l'habitude d'essayer de briser les relations des mères avec leur progéniture, allaient aussi à l'encontre d'une stabilisation des rapports avec cette dernière. Cette stabilisation impliquait qu'entre l'homme et le jeune garçon s'établisse un analogue du couple nucléaire à l'intérieur et sous l'égide du couple reproductif. Or ce dernier n’est pas hiérarchisé, tandis que les relations des deux générations mâles étaient probablement hiérarchisées, comme dans toutes les sociétés que nous avons passées en revue. S'y ajoute la dissymétrie de l'enfant ou de l'adolescent vis-à-vis des deux groupes: situation d'appartenance et différenciée du côté féminin, de soumission et indifférenciée du côté masculin. Par un paradoxe qui n'en est un qu'en apparence, c'est dans l'interaction homosexuelle que la dimension sexuelle prend un relief social, et non pas dans l'interaction hétérosexuelle. Les cérémonies de soumission entre mâles par la présentation des organes génitaux l'expriment clairement. La coexistence prolongée imposait la cessation de ces pratiques. La solution a consisté à rendre hiérarchiques les rapports nucléaires de la mère à ses enfants, et nucléaires les rapports hiérarchiques du géniteur à sa progéniture, c'est-à-dire que l'on a introduit une distance là où il n'y en avait pas et que l'on a réduit la distance là où elle existait. La paternité représente la diffusion de l'autorité des mâles dans un domaine qui restait auparavant du ressort exclusif du sexe féminin, la rétention des jeunes gar- 
çons, à l'instar des jeunes filles, dans le groupe reproductif, avec pour conséquence que le père s'arroge un droit particulier sur eux. Une relation de consanguinité s'installe là où elle était méconnue. Les formes prises par la cellule conjugale varient considérablement suivant les sociétés.

Dans bien des cas, le père, la mère et les enfants vivent ensemble. On relève toutefois l'existence de groupes de mères avec des enfants auxquels s'ajoutent des pères. Parfois les hommes demeurent à part, s'unissant périodiquement avec les femmes. Plus rarement, les femmes ont plusieurs compagnons sans former avec aucun une famille proprement dite. Le caractère du père en tant que mâle s'ajoutant au couple nucléaire mère-enfants n'a pas complètement disparu. Aux îles Trobriand, le père est un étranger, un outsider vis-à-vis des autres membres de la famille. La polygynie étant la règle plutôt que l'exception accentue le caractère intrusif du personnage paternel. Personnage tantôt individuel - et les enfants se rattachent à lui tantôt collectif, comme chez les Lele du Kasar dont les enfants ont en quelque sorte pour père un groupe d'hommes. Sans trop insister, on ne peut s'empêcher de rapprocher cet état de choses de celui qui règne dans les sociétés d'affiliation, où l'on observe une disjonction similaire entre un groupe de mâles associé à une communauté de femelles et, subsidiairement, d'enfants, et un mâle associé à plusieurs femelles et par dérivation à sa progéniture. Tout se passe comme si les animaux-coryphées s'étaient transformés, collectivement ou individuellement, en pères. Dans la mesure où les liens - toujours plus directs avec les filles, médiés par la mère avec les garçons — dont ils forment le centre et la source sont devenus permanents et définissent la place de chacun dans l'espace domestique, l'affiliation se convertit en parenté. A la lignée de la mère s'ajoute ou se substitue celle du père, et la société de parenté se dévoile immédiatement société de paternité. C'est ce trait qui oppose le plus fortement le système social des primates au système social humain, la société humaine qui a précédé la chasse à celle qui lui a succédé. Partout la paternité a force de loi, sur le plan juridique comme sur le plan moral ; le mariage doit nécessairement précéder la conception des enfants et l'adoption supplée, le cas échéant, à l'absence de progéniture. A défaut, les enfants sont déchus de leurs droits ; la société les flétrit du nom significatif d'enfants naturels. Le père fait les enfants, et sans père il n’y a pas d'enfants, socia- 
lement parlant. Réciproquement, ce sont eux qui donnent une signification au couple, lui garantissant la finalité qui lui a été assignée ; faute de quoi l'union scellée s'avère inutile. Le couple sans enfants est à peine considéré comme faisant partie de la société humaine. Certaines populations rejettent celui ou celle qui n'a pas procréé en ne lui accordant qu'un statut mitigé, de même qu'ils sont exclus de cette société supérieure qui regroupe les ancêtres, honorés par leurs descendants. D'où l'importance extrême que les collectivités archaïques attachent à la fécondité individuelle, même là où l'infanticide est chose courante. Mais être le rejeton d'une mère et d'un père, entrer dans la vie par l'une et dans la société par l'autre, se lier au père par la mère et s'associer au premier en rompant avec la seconde, éprouver sans cesse un des parents comme un vis-à-vis et comme un tiers, terme d'une relation et médiateur, vivre enfin cette relation sous le signe de la violence et la violence comme le prix de sa sauvegarde, voilà ce qui change dans la famille, une fois qu'elle s'est établie, le problème du mâle en problème de l'enfant.

\section{Retour à la Table des Matières}

\section{(2) Du célibat : le mariage et l'égalité des hommes.}

« Sans cesse, dans l'histoire du monde, les tribus sauvages ont dû avoir clairement présente à l'esprit cette simple alternative pratique : se marier au dehors ou être exterminées. » De toute évidence, ce choix que Tylor formule en termes dramatiques n'en est pas un. Il illustre pourtant avec force le fait que le mariage est d'abord un troc, où l'on reçoit la vie contre la mort, et en second lieu seulement une alliance. Dans un monde où le nomadisme est la règle, pouvoir séjourner chez autrui comme chez soi et réciproquement, s'unir à un individu pris hors de son groupe est le seul moyen d'ajouter à la vie donnée une vie obtenue qui prolonge la première. La norme d'exogamie répond d'emblée à cette dure nécessité. En contrepoint, le déni de l'endogamie — la prohibition de l'inceste s'y associe — freine l'autarcie de la famille, du clan, qui tendraient à s'isoler virtuellement de la société. L'obligation de l'alliance et la stérilité de la clôture reconnaissent l'une et l'autre le préalable de la famille, l'impératif du mariage comme état ordinaire de l'être humain. Cet impératif dépend de deux conditions qui le précisent. 
La première exclut le célibat comme second terme de l'alternative. Dans les sociétés dites primitives, l'individu non marié mène une existence matérielle précaire et est en butte au mépris général. Il reste marginal, n'accède jamais à un statut social acceptable, ne participe pas pleinement aux échanges collectifs. Le vocable qui le désigne s'emploie aussi comme injure grossière, et il n'est pas rare de voir assimiler le célibataire à l'infirme ou au sorcier malfaisant, d'en faire, comme pour ceux-ci, un être maudit. Il expie la faute d'avoir cru pouvoir recevoir la vie sans la donner. "Plus frappant encore, écrit Claude Lévi-Strauss ${ }^{83}$, est le véritable sentiment de répulsion qu’ont la plupart des sociétés envers le célibat. Généralement parlant, on peut dire que, parmi les tribus dites primitives, il n'y a pas de célibataires, pour la simple raison qu'ils ne pourraient pas survivre. » Et il ajoute, rapportant un souvenir personnel, l'impression que lui a faite la rencontre, chez les Bororo du Brésil, d'un homme dont le délabrement physique et l'air d'abandon manifeste ne provenaient pas, selon ses interlocuteurs, d'une maladie, mais tout simplement de son état de célibataire.

La deuxième condition est l'obligation pour chaque groupe de contracter une alliance durable, épargnant à ses membres la déchéance du célibat. De là ces promesses d'union formulées par les parents pour leurs enfants en bas âge, ou ces mariages entre un adulte et un jeune enfant de l'autre sexe, dont il s'occupera jusqu'à ce que son conjoint puisse accomplir ses devoirs. L'interdiction pratique et morale du célibat, la prime donnée à l'union hétérosexuelle montrent que le mariage n'allait pas de soi, qu'il a fallu l'action de forces puissantes pour l'imposer : les groupes devaient l'accepter, les adultes le rendre possible. Pourquoi donc ? Nous pensons souvent au mariage en prenant pour évident le désir biologique de l'individu de s'unir à un individu du sexe opposé : nous décrivons alors l'institution comme un mode d'union réglée individuelle, suscité par les groupes qui souhaitent le contrôler ; il se substitue à une union arbitraire, et crée une hétérosexualité socialisée qui rehausse l'hétérosexualité organique. Bref nous y voyons le modèle de la reproduction humaine prenant le relais de la reproduction animale. Mais c'est là une interprétation partielle 
de la réalité, un choix dévalué du point où fixer ce relais. En effet, ce qui frappe dans les sociétés de primates, c'est la présence d'une obligation au célibat, avec le caractère social discriminant, réservé, de l'accouplement sexuel, mais non point sa versatilité ou son désordre. La différenciation des individus reproducteurs et non reproducteurs manifestant une échelle de rangs, liée aux chances de survie, l'opposition des congrégations bisexuées et monosexuées (ces dernières surtout masculines), sont, des composantes fondamentales de la majeure partie des sociétés animales.

L'étude des simiidés a amplement mis en lumière le rôle des cohortes de mâles qui se voient refuser la possibilité de relations régulières avec une femelle, signe de leur infériorité et de leur exclusion. On peut formuler en ces termes le second problème qui se pose aux mâles : le désir d'une partie d'entre eux de se mêler aux groupes mixtes pour y trouver une compagne, mais surtout le besoin de devenir dominants à leur tour, d'avoir part aux ressources principales du groupe, sont constamment contrecarrés. Cette situation fait peser une menace sur la société - l'animal non reproducteur cherchant à détruire un couple reproducteur ou à s'y associer — et engendre un conflit mobilisant les jeunes contre les adultes, les adultes défavorisés contre les adultes privilégiés. Si la distance instituée entre le groupe basal, bisexuel, et le groupe appendiculaire, monosexuel, dans les sociétés d'affiliation facilite la survie de l'ensemble, elle entretient néanmoins des ferments de dissolution. Or cette distance - et l'existence même de ces groupes permanents enferme une double contradiction dans le contexte de la chasse. D'une part, l'interdépendance des hommes s'étant accrue, toute discrimination l'affaiblit. D'autre part, la surveillance étroite exercée par les mâles-coryphées sur leurs compagnes tenues à l'écart des mâles subordonnés - telle qu'on l'observe chez les rhésus ou les babouins - interdit tout déplacement, si l'on ne veut voir disparaître mères et progéniture, signes du pouvoir. Le besoin d'un lien stable et d'un nouvel équilibre entre les générations, masculines surtout, exige l'abolition de la ségrégation en question. La possibilité offerte à chaque membre de la société d'entrer dans le groupe des reproducteurs éloigne le spectre du conflit là où l'on cherche à resserrer les rapports. La non-reproduction conserve le sens d'une infériorité sociale passagère, d'une modalité de régulation démographique ; elle cesse d'être l'opposé de la reproduction pour en devenir une 
simple phase ; le trait caractéristique d'une catégorie d'individus se change en trait lié à la croissance de tous les individus. L’impératif du mariage signale un renversement de la tendance évolutive, efface la discrimination perpétuée dans les sociétés animales. Il représente probablement une conquête des mâles subordonnés, une subversion de l’ordre régnant dans les collectivités hominiennes.

Par antériorité logique, avant d'être un code d'interdits et de prescriptions, dictant avec qui on s'allie et avec qui on ne s'allie pas, le mariage est un droit et un devoir. Droit pour chacun, notamment, d'avoir un partenaire sexuel, de conserver sa progéniture, d'accéder au statut de reproducteur et devoir pour tous de fournir les moyens à cette fin. Le propre des droits est de devenir des devoirs, comme celui des devoirs est de se changer en droits. L'individu est obligé de se marier ; en s’abstenant, il met en question la légitimité de la règle et du comportement général. La collectivité exige de lui qu'il s'acquitte de son obligation ; de cette façon, elle justifie ses efforts, ne lui laissant de choix qu'entre l'obéissance et l'exclusion. Je ne vois pas de meilleure analogie que celle du vote. Le droit en a été conquis de haute lutte contre les classes qui le voulaient réservé ou sélectif. Une fois acquis, le droit se transforme en devoir du citoyen. En s'y soustrayant, celui-ci gauchit le fonctionnement de la démocratie représentative ; dans la mesure où les lois le lui permettent, il enfreint une norme ou subit l'opprobre qui flétrit le mauvais citoyen. Avoir droit de cité dans une société dite primitive, c'est faire valoir ses droits à l'union conjugale. Celle-ci a pour fonction d'abolir à terme un état d'infériorité, d'introduire et de maintenir l'égalité là où régnait l'inégalité. Égalité partielle, à vrai dire, puisqu'elle concerne surtout les hommes, la position des femmes demeurant inchangée. La liberté, l'égalité, la fraternité ou la mort de la révolution bourgeoise avaient trait aux seuls individus propriétaires. La liberté, l'égalité, la fraternité ou la mort de la révolution matrimoniale intéressent les pères, puisque, dans ces sociétés, être homme c'est être père. Lorsque la rivalité se manifeste, elle prend la forme d'un désir de possession qui a pour enjeu l'élément féminin — on y a vu une lutte pour la mère — mais cette possession n'est qu'un moyen de briser le cercle de la soumission, d'empêcher la dimension hiérarchique du rapport nucléaire unissant le fils au père de se figer en rapport de subordination tout court. Le mariage nous révèle ainsi une signification différente de la paternité. Celle-ci se présente 
en effet comme la réalisation d'un contrat, un mode d'association des hommes par le truchement des femmes et des enfants. Envisagée sous cet angle, elle est à la fois la négation absolue du fondement des sociétés d'affiliation, et une manière d'ouvrir l'accès de la majorité à un privilège autrefois réservé à une minorité, symbolisé par le couple reproducteur. De la sorte, ce qui était auparavant l'apanage du petit nombre devient, en généralisant l'échelle des valeurs, le droit de tous.

Retour à la Table des Matières 


\section{Le principe du partage don et réciprocité.}

$\underline{\text { Retour à la Table des Matières }}$

Donne autant que tu prends, tout sera très bien.

Proverbe maori

\section{(1) L'exogamie conjugale.}

Pour fonder une famille, un homme et une femme sont dans l'obligation de se marier. Où trouver cette femme, cet homme ? En dehors de la famille, de la bande, du clan d'origine, répondent la plupart des sociétés. Elles proclament ainsi l'opportunité pour une personne et un groupe de s'allier à une personne ou à un groupe auxquels ils ne sont pas alliés, ou de renouveler l'alliance établie à la génération antérieure. L'incitation à l'exogamie est précise. On peut se demander quelle en est la signification; sa grande ancienneté nous interdit de tenter plus qu'une reconstitution spéculative.

Lorsque se forme une institution ou une catégorie sociale - ici la famille, unité productive et reproductive, ailleurs la manufacture, l'armée, l'université, la classe marchande, etc. - cette formation a lieu dans une société existante. Ceci, bien entendu, à condition de les considérer comme des segments du système social, engendrés par celui-ci, et non pas comme des réseaux de liens entre personnes ou groupes préformés, à l'état de nature. La société dans laquelle la famille a pris corps est, nous l'avons observé, une société directe, ne disposant pas de moyens politiques ou économiques propres, dépourvue de la coquille des institutions capables de s'imposer aux parties qui la composent. Dans une société de ce type, l'apparition de la famille étendue, liée normalement à d'autres familles, représente un risque d'éclatement. En effet, après la fusion en un seul du couple nucléaire et du couple reproductif, celui-ci a tout intérêt à retenir, comme la possibilité lui en est donnée, un nombre relativement élevé d'individus, et il peut, théoriquement, se suffire à lui-même du point de vue matériel. L'atténuation d'une des deux formes de subordination - celle qui a trait aux rapports internes à la fraction masculine consolide le mouvement de socialisation des activités et des échanges, 
élimine une source de tension à l'intérieur du groupe. Pis encore dans la perspective de la société - en s'attachant des jeunes ou des individus marginaux, conjoints disponibles, il arriverait à s'accroître aux dépens des bandes ou des groupements voisins. Par la suite, en se subdivisant, en facilitant la création de nouveaux groupements familiaux, il finirait par sortir du cadre de la société à laquelle il appartient et par reproduire sa propre société. Ce risque d'éclatement fait peser une menace d'autant plus grave que la chasse nécessite la constitution de liens stables. La collaboration indispensable est ainsi entachée d'incertitude, soumise aux aléas que représente le développement d'une structure de groupes capables de se soustraire au contrôle de la collectivité.

De plus, la famille, institution originale et nécessairement subversive à ce titre, éclôt dans une société qui change elle-même, les deux phénomènes étant évidemment liés. La collectivité cesse d'être une organisation compacte, dans l'espace et dans le temps, où les communications sont pour ainsi dire immédiates, la co-présence quasi physique assurée, puisqu'elle se caractérise précisément par la réunion fréquente, régulière, sinon quotidienne, de ses unités et ses membres. Son territoire s'élargit considérablement, le contact au sens réel perd une partie de son efficacité, sa fonction de réaffirmation des rapports sociaux. Les déterminismes écologiques et productifs provoquent une série d'oscillations entre la période de " réunion » et celle de "séparation », entre les époques où la société est "présente » et celle où elle est " absente ", entre son existence concrète et son existence abstraite aux yeux des individus et des fractions qui la composent. Psychologiquement, cet état de choses a dû encourager les processus d'intériorisation des symboles et des événements communs, de manière à rendre vivante, palpable, une totalité périodiquement sujette à des éloignements et à des éclipses. Culturellement, il était nécessaire d'inventer des comportements et des conventions permettant de préserver et de renouer un lien sans cesse défait et destiné à se défaire. A l'inverse, la société se devait d'encourager la dispersion des unités (famille, clan, bande) qui ne pouvaient coexister, compte tenu des nouveaux besoins et des nouvelles habitudes, sur un territoire limité où les ressources venaient tôt ou tard à se raréfier. L'interdépendance suppose ici une autonomie prolongée, elle, qui permet à chacun des partenaires sociaux de subsister de son côté, à des époques fixées 
d'avance ; et l'autonomie s'accompagne d'une interdépendance, de la possibilité de retrouver les partenaires nécessaires lorsque les circonstances imposent le rassemblement, la quête de ressources différentes que les groupes ne sauraient, se procurer séparément. Le nomadisme qui provoque ces bouleversements touche à la nature même de la société ; il les transforme aussi en une menace pour les divers groupes : risque pour l'un d'entre eux de se trouver isolé, ou, au contraire, danger de coalition ou d'association avec d'autres groupes au hasard des déplacements ou des relations de voisinage, entraînant la formation d’une nouvelle société, par coopération ou absorption.

La menace est particulièrement grave pour la famille ou le petit rassemblement de familles. Supposons un tel rassemblement d'environ cinquante personnes, ou neuf couples qui procréent ; étant donné le taux de fécondité, un enfant tous les trois ans par couple, sur les neuf enfants mis au monde en l'espace de trois ans, le groupe n'en gardera que trois, les autres ayant peu de chances de survivre jusqu'à l'âge adulte. Le sexe étant aléatoire, il est probable que les écarts par rapport à la proportion théorique d'un garçon à une fille seront notables. Afin de réduire ces écarts et de se reproduire convenablement, en excluant la solution de la polygynie, il y a tout intérêt pour l'unité en question à se fondre dans une société comptant cinq cents personnes, une centaine de couples environ. Sinon elle ne pourra éviter le déséquilibre démographique.

L’absence de partenaires épousables, un surplus éventuel de jeunes du sexe masculin, deviennent, quand la famille est l'unité de base, un autre facteur d'involution sociale. Dans les sociétés de primates ou d'hominiens, la séparation des individus reproducteurs et non reproducteurs, le refoulement de ces derniers dans un groupe appendiculaire, résout la difficulté. Une collectivité où le rôle du mariage est, à la fois, de fournir à un homme épouse et enfants, et de modifier son statut social, a de fortes chances de voir ses membres s'éloigner, car elle bloque les relations instituées entre sexes et générations. L’union avec, par exemple, la sœur ou la mère, achopperait à des obstacles analogues. En épousant leur mère, les garçons resteraient enfants du père ; le couple formé de la mère et du fils serait par définition stérile, donc de statut inférieur. Un mariage avec la sœur conduirait à maintenir la domination paternelle, ou à entrer en conflit avec elle, le pouvoir 
du mari s'opposant à celui du père. Bref, la ségrégation d'un groupe restreint de familles engendrerait cette promiscuité que l'on attribue aux animaux, bien qu'elle n'ait socialement aucun sens dans les organisations de clique ou de compagnonnage des primates ou des hominiens. Elle a, en revanche, une existence notoire, et aussi un sens, dès l'instant où la famille prend corps et se diffuse dans l'organisation sociale humaine. A tous les niveaux, ces circonstances sociodémographiques expriment un phénomène plus vaste. S'agissant d'une unité productive autant que reproductive, la composition du groupe suppose un nombre équilibré d'hommes et de femmes, un volume suffisant pour pratiquer, en alternance, la chasse et la cueillette, en deçà duquel sa disparition est inéluctable.

La situation se présente en termes clairs. La société directe et nomade doit se prémunir contre la tendance des groupes comprenant plusieurs familles à se soustraire à son contrôle ; simultanément il lui faut préserver l'autonomie de ces groupes, leur mobilité, qui leur permet de vivre éloignés les uns des autres. De leur côté ces groupes à base familiale cherchent évidemment à se renouveler en perpétuant leurs caractères dans un milieu social approprié ; s'ils acceptent la perspective de la séparation, c'est avec l'assurance de pouvoir renouer à terme les liens interrompus. De part et d'autre des garanties sont nécessaires : la société veut être certaine que les transactions ayant lieu à l'intérieur de chaque groupe se font en son nom et ne sont pas contraires à sa cohésion ; la famille, le clan ou la bande ont besoin de savoir que leur isolement dans le temps et dans l'espace ne les fera pas sortir du cadre communautaire, qu'ils y trouveront le moyen de se régénérer biologiquement et de reconstituer leurs réserves matérielles et intellectuelles. En cherchant des alliances au dehors, on est certain de parvenir à ces buts dans le cadre défini. La société, en imposant cette règle à ses membres, tisse les liens de dépendance qui rendent son intervention indispensable. L'exogamie, l'obligation de s'unir à un individu extérieur au groupe - non seulement la famille mais le clan ou la bande - représente la réponse à ces exigences. « L'exogamie, constate Claude Lévi-Strauss ${ }^{84}$, fournit le seul moyen de maintenir le groupe comme groupe, d'éviter le fractionnement et le cloisonnement indéfinis qu'apporterait la pratique des mariages consanguins ; si l'on 
avait recours à eux avec persistance, ou seulement de façon trop fréquente, ceux-ci ne tarderaient pas à faire " éclater » le groupe social en une multitude de familles qui formeraient autant de systèmes clos, de monades sans porte ni fenêtre, et dont aucune harmonie préétablie ne pourrait prévenir la prolifération et l'antagonisme. »

\section{$\underline{\text { Retour à la Table des Matières }}$}

\section{(2) L'endogamie sociale et le pouvoir généalogique.}

Le problème à résoudre était celui de la distribution des individus dans des conditions où l'unité constitutive était nomade et continue, la société discontinue et stable, le facteur spatial lui-même réduisant les interactions entre tribus ${ }^{85}$. L'application de la règle exogamique revient à interdire, à l'intérieur d'un groupe, un certain nombre d'actes : se marier avec un membre du groupe, consommer certains aliments, etc. Ainsi chaque groupe est amené à rendre disponibles des hommes ou des femmes, des territoires ou des ressources, et non seulement à les rendre disponibles mais à les donner, donc à se lier à un groupe différent qui effectue la même opération envers lui et ne saurait se soustraire à ses obligations par le refus ou la thésaurisation. "Car un clan, une maisonnée, une compagnie, un hôte, ne sont pas libres de ne pas demander l'hospitalité, de ne pas recevoir de cadeaux, de ne pas commercer, de ne pas contracter alliance, par les femmes et par le sang ${ }^{86}$. »

Un courant de réciprocité s’instaure qui colore toutes les activités et impose la soudure des diverses parties du corps social pour résorber le surplus et obtenir le nécessaire, participer aux cérémonies ou aux entreprises collectives d'exploration. Par ailleurs, les biens réservés à autrui, rendus disponibles, ne sont pas répartis au gré de la volonté de chaque unité sociale ou de chaque individu, libre de s'unir tantôt avec tel groupe ou telle personne, tantôt avec d'autres, suivant l'abondance ou la pénurie, le caractère avantageux de l'offre et de la demande. Les partenaires sont clairement désignés, les choix fixés d'avance : ce qui soustrait la famille aux aléas déjà mentionnés. Les prescriptions de

85 J.B. BIRDSELL : On Population Structure in Generalized Hunting and Collecting Societies, Evolution, 1958, 12, 189-195.

86

M. MAUSs : Sociologie et Anthropologie, op. cit., p. 161. 
mariage et les prestations requises sont connues d'emblée : le groupe ou la famille $\mathrm{X}$ marie ses fils ou ses filles avec le groupe $\mathrm{Y}$ suivant des règles relativement rigides, le conjoint présentant avec le sujet un rapport de parenté ou un rapport d'appartenance à un clan ou à une classe matrimoniale. Plus exactement, les épousables sont déjà parents entre eux et les alliances nouées sont semblables à celles qui s'étaient formées antérieurement : ce ne sont pas des étrangers qui établissent les liens conjugaux, ni avec des étrangers. Les ascendants proposent à leurs descendants la gamme des unions possibles en spécifiant dans la liste des parents les époux interdits et les époux permis. La succession des générations peut introduire une variation notable, lorsque les règles de la société indiquent que les mariages d'une génération, au lieu de reproduire ceux des parents, se feront comme ceux des grandsparents. L'interdit de s'épouser à l'intérieur d'une catégorie matrimoniale donnée définit l'exogamie du groupe.

L'union préférentielle, prescription d'épouser les individus issus d'une autre catégorie, définit l'endogamie de la société, qui délimite ainsi le choix et le déplacement des individus à l'intérieur d'un circuit de relations préétabli. Ni la logique ni l'histoire ne fournissent de motifs permettant de croire que l'exogamie a pu être précédée par une période d'unions endogamiques au niveau des groupes où la consanguinité prédominait sur d'autres formes d'affinité. De telles unions supposeraient que les épouses soient considérées propriété commune du groupe ; les sociétés d'affiliation n'en offrent guère d'exemples, pas plus que les sociétés archaïques. Par contre, le fait que l'endogamie se situe au niveau du système social traduit, à travers les alliances qui se nouent, l'emprise du système sur ses segments, de la société sur la bande, la famille ou le clan ; c’est la société qui décide du sens à donner à la relation ou aux termes qui la composent. Par suite, la famille ou le rassemblement de familles joue le rôle d'unité au sein d'un réseau plus vaste fondé sur d'autres obligations, en accord avec la résidence et le statut. Il serait erroné de l'opposer aux autres familles ou rassemblements comme une unité détachée, complètement indépendante, ou de l'envisager dans une réunion d'unités semblables et indifférentes ; au contraire, chacun est marqué par des lignées, le réseau orienté qui lui a imposé des associations décidées à l'avance. 
A l'encontre de ce qui se passe sur un marché ou entre échangistes, cet ascendant et cette insertion dans un réseau orienté tiennent compte de l'ensemble des transactions synchroniques et diachroniques ; chaque individu " donné » est nécessairement un "rendu », aussi bien dans l'immédiat que dans la suite des temps, et les unions expriment une interdépendance généralisée qui s'étend aux générations futures tout comme elle a lié les générations passées. Jusqu’à un certain point, on peut dire que, matériellement et spirituellement, les sociétés passées indiquent ce qui est possible et ce qui est impossible aux sociétés présentes, et celles-ci, à leur tour, prescrivent aux sociétés futures ce qui est licite et ce qui ne l'est pas. A l'alliance concrète, effective, se superpose l'alliance abstraite et indicative, impliquant là subordination de la première à la seconde, la mainmise de la totalité sociale intemporelle sur ses réalisations au cours des générations, de même que la société temporelle subordonne les sous-groupes qu'elle inclut et dans lesquels elle s’actualise, concentrée, et se conserve, dispersée.

Mais une telle alliance supra-ordonnée est une forme de pouvoir, le pouvoir généalogique, qui transcende les décisions particulières, les conditions pratiques de l'existence quotidienne, imposant la présence du collectif à travers la plupart des opérations privées. La constellation des mariages conclus offre une image de l'adhésion plus ou moins fidèle de chaque famille, de chaque clan et du rôle qu'ils jouent dans l'ensemble. Les limites des relations généalogiques, revivifiées par des unions matrimoniales, sont celles de la société, puisqu'elles circonscrivent le champ au-delà duquel il n’y a plus ni liens, ni alliance, ni langue, ni dons réciproques. De cette manière, la distribution de cette richesse que sont les individus est stabilisée et se trouve mis en évidence le pouvoir apte à la répartir. Et celui-ci n’a rien d'abstrait. Il incarne la domination du géniteur sur la progéniture, des ancêtres ou de la totalité sur chaque génération particulière. Sous son emprise, le mariage n'est pas seulement le lien direct d'une femme à un homme, ni même d'un groupe à un autre groupe. Il est toujours un lien indirect, ayant pour médiateur et terme le père ou la mère, qui fixent la filiation, certes, mais interviennent aussi de façon plus immédiate ; c'est en effet par leur entremise que l'on obtient un homme ou une femme, quand ce ne sont pas eux qui concèdent un homme ou une femme, qui, par ailleurs, leur appartient. En fait, il s'agit d'une domination différée, d'une coalition où les membres d'une génération se 
prêtent main forte afin de préserver la tutelle des parents sur les enfants. Le contrôle collectif, très dense, combine l'entrecroisement des réciprocités avec l'emboîtement hiérarchique, transformant constamment la parenté en alliance et l'alliance en parenté. La famille est son lieu géométrique, fournissant la base sur laquelle s'établissent les rapports d'autorité et de justice, à travers les relations de personne à personne qui se nouent en accord avec les choix matrimoniaux offerts aussi bien qu'avec les autres rapports généalogiques institués. Telle est la place à laquelle l'a conduite l'évolution que nous avons examinée, et qui l'insère dans la société, tandis que la société, qui se développe sur le modèle de la famille, devient société de parenté.

Retour à la Table des Matières

\section{(3) La généralité du partage exogamique.}

L'exogamie a servi d'opérateur à cette transformation. Ses effets et sa signification ne sont pas limités aux règles de mariage ; exprimant un principe général de répartition particulier aux sociétés archaïques, elle est omniprésente. Quelques exemples le feront mieux voir. La fraternité par le sang, pratique répandue, associant les hommes d'une même génération, obéit à des normes montrant bien qu'elle est respectée ${ }^{87}$. Pour se dire frères, les hommes échangent leur sang. Cette forme d'alliance ne saurait en aucun cas s'établir avec une femme. Elle procure un sentiment de sécurité, soit parce que l'on est sûr de recevoir assistance sur place, soit parce que l'on peut voyager sans danger dans des contrées lointaines. En effet, lorsqu'un homme se déplace dans un territoire voisin du sien, il prend soin d'entrer en contact avec un homme de son clan ou du clan de sa mère qui lui sert de répondant. En pays étranger, ce répondant ne peut être que le frère par le sang. Dans les circonstances ordinaires, avant de conclure une telle alliance, les futur frères doivent consulter leurs proches et obtenir leur assentiment, les obligations envers le frère de sang étant aussi des obligations envers le clan de celui-ci. Semblable en cela aux alliances matrimoniales, ce don réciproque de sang ne peut se faire entre les hommes de la famille élargie ou du clan. Le pacte, une fois ces préalables remplis, se conclut au cours d'une cérémonie ; comme en toute 
occasion, y compris le mariage, où se noue une alliance, il s'accompagne d'un échange de cadeaux. Les partenaires s'engagent à ne pas commettre l'adultère avec la femme de leur " frère » et à donner de préférence leur fille à celui-ci. Lorsque l'un d'eux rend visite à l'autre, ce dernier est tenu de le nourrir, de lui procurer des armes ou de lui offrir les présents qu'il réclame, de l'assister s'il est attaqué, de le défendre, et s'il tue des animaux à la chasse, de lui en réserver une partie.

L'initiation et la circoncision dont les cérémonies et les pratiques sont liées sont soumises, pour certains de leurs aspects, à des déterminations analogues. En Australie, par exemple, l'initiation d'un jeune homme dépasse le groupe territorial auquel il appartient. Y prennent part le frère de sa mère, son cousin croisé du côté du père (qui est souvent son beau-frère), le mari de sa sœur, ainsi qu'un membre du groupe qui lui donnera plus tard une épouse. Chacun des participants représente un groupe territorial différent de celui du jeune homme, tout en étant lié à celui-ci. L'initiation dure deux ou plusieurs années, pendant lesquelles il est soumis à la tutelle d'un homme plus âgé, par exemple le mari de sa sœur, qui lui enseigne à chasser et d'autres techniques pratiquées par les seuls hommes. L'initiateur en retire des avantages puisque le produit de la chasse ou de la cueillette du néophyte lui revient de droit. Le rapport qui s'établit à ce propos offre des analogies évidentes avec le rapport du tuteur à son épouse, la sœur du jeune homme. Les missionnaires ont souvent désigné celui-ci comme un enfant-esclave. A Groote Englandt, le rapt de jeunes épouses était chose fréquente, et tout aussi fréquent le rapt de jeunes garçons constituant une " main-d'œuvre " gratuite — arrachés à leur tuteur et initiateur. Les cérémonies de circoncision se déroulent sur un arrièreplan semblable et font appel aux mêmes acteurs. Chez les Nambuti d'Australie, l'opération est pratiquée par le futur beau-père du garçon, assisté de deux ou trois frères de la mère. La cérémonie est appelée ulkuteta (avec la bouche), ce qui signifie adoption. Elle lie le garçon de façon durable au circonciseur, qui effectue aussi la subcision ; ils communiquent dans un idiome ésotérique et ont entre eux des rapports homosexuels où le garçon joue le rôle de la femme. L'âge venu, le garçon accède au plein statut d'adulte et reçoit pour épouse la fille de son circonciseur. Dans d'autres tribus, le futur beau-père, après 
l'opération, conserve le prépuce un certain temps et finit par le remettre au garçon ${ }^{88}$.

Tous ces modes d'alliance, auxquels on pourrait ajouter les modes de répartition de certaines nourritures, des terrains de chasse ou des ressources, respectent un code de pratiques et de règles identiques. $\mathrm{A}$ juste titre, Émile Durkheim considérait que l'exogamie qui les caractérise avait précédé et englobé les autres institutions des sociétés archaïques. Il supposait cependant un point du temps où ces sociétés disposaient d'un jeu complet d'institutions, intégrées et en quelque sorte statiques. Et c'est bien dans cette optique qu'on les regarde, en cherchant à reconstituer l'ensemble synchronique de règles sociales, éthiques et rituelles, que le rude assaut de la colonisation a démantelé, en les dépouillant de la dynamique qui devait leur être particulière. Dans la perspective de cette dynamique, l'exogamie, régissant la manière de distribuer entre les groupes, dans ces sociétés, personnes, biens, temps et forces de travail, module un principe de partage diffus à travers les pratiques propres à chacune, au même titre que l'impératif du profit et de la productivité parcourt toutes les veines de la société capitaliste. C'est ce principe que nous voyons à l'œuvre dans les comportements quotidiens, aussi bien que dans l'organisation des cérémonies et les divisions du corps social. Dans la chasse, dans la cueillette, dans ses occupations quotidiennes, en procréant ses enfants, l'individu ou le groupe réserve une partie de ses produits, une part de son activité, une parcelle de son territoire, certains de ses enfants à la collectivité, c'est-à-dire aux autres groupes et individus, avec l'assurance que ceux-ci agiront de même, procédure qui permet de renforcer constamment les liens sociaux. C'est la raison pour laquelle les biens concédés et les biens reçus participent de celui qui les concède et les reçoit : « La communion et l'alliance qu'elles établissent, affirme Marcel Mauss ${ }^{89}$, sont relativement indissolubles. En réalité, ce symbole de la vie sociale - la permanence d'influence des choses échangées - ne fait que traduire assez directement la manière dont les sous-groupes de ces sociétés segmentées, de type archaïque,

88 G. RoHeIM : Héros phalliques et symboles maternels dans la mythologie australienne, Paris, 1970.

89 M. MAUSS : op. cit., p. 194. 
sont constamment imbriqués les uns dans les autres, et surtout qu'ils se doivent tout. »

La pratique du don et l'attente de la réciprocité sont les deux faces d'un principe unique : offrir pour demander, apporter afin de recevoir. L'intérêt commun veut que chacun ait le nécessaire, d'où l'obligation de le lui procurer ; il exige aussi que les richesses humaines et non humaines auxquelles ont droit tous les membres de la collectivité soient redistribuées afin de réduire les écarts entre eux. Dans cette mesure, le partage remplace les formes de répartition des sociétés d'affiliation qui sont purement coordinatrices, le groupe intervenant seulement pour tracer les limites à l'intérieur desquelles chacun obtient ce qu'il lui faut ou ce qu'il désire. Quand l'accumulation et la conservation se seront notablement accrues, rendant la production indépendante de la reproduction, l'appropriation des choses n'ira plus de pair avec celle des hommes, l'appropriation des hommes pourra se faire par l'intermédiaire des choses, et le partage deviendra échange, transmission de biens et de personnes dont la valeur se trouve fixée par rapport à un étalon. Dans le partage, ce qui est offert et ce qui est reçu a la signification d'un lien recherché, opération synthétique où chaque transaction est plus que la transmission d'une ressource particulière, don et valeur ; dans l'échange, la tendance se renverse, les deux partenaires retrouvent leur autonomie en rendant ce qu'ils ont reçu et ne se doivent plus rien, opération analytique où chaque transaction n'est que la transmission d'une ressource sous des espèces différentes mais équivalentes, richesse et signe. Partager, c'est introduire la continuité au sein des actes discontinus, obliger autrui à entrer en relation avec soi, viser une restitution équitable à long terme, se créer un droit sur des biens futurs ; échanger, c'est introduire la discontinuité dans la continuité, se libérer de l'obligation d'une relation à autrui, accepter la restitution à court terme, limiter son droit aux biens présents. Là, on accorde le nécessaire, ici, on concède le superflu ; là les biens sont médiateurs de conjonction, ici ils sont facteurs de disjonction. Mais il a probablement fallu que l'humanité, avant de valoriser l'indépendance et le détachement, apprenne la dépendance et l'attachement, et qu'avant de savoir s'associer par contrat elle s'allie par réciprocité. 
Un regard jeté sur l'apparition de la famille et de l'exogamie, dans le contexte du partage précédant l'échange, permet de voir jusqu'à quel point elles ont bouleversé le fondement des sociétés d'affiliation, prédominant chez les primates, en accord avec le mouvement de collectivisation et de nomadisation qui a emporté celles-ci. L'alternance de la dispersion et du rassemblement, de la présence vécue et de la présence conçue succédant à l'absence conçue et à l'absence vécue de la communauté, rompent avec l'emprise de l'immédiat, affaiblissent la vertu du spontané, éloignent les parties de la totalité. Le symbolique pénètre le réel, étant le pouvoir de la collectivité de maintenir là où elle n'est pas, dans la séparation et l'autonomie, la signification de l'union et de la dépendance ; et la réalité prend le caractère du symbolique, la collectivité cherchant à se révéler, là où elle est union et dépendance, comme le signifié de la séparation et de l'autonomie. D’où la charge d'émotion et de tension qui pèse sur les retrouvailles et les séparations, la surabondance des célébrations notées dans les sociétés primitives, comme si chacun voulait surcharger l'autre des paroles qu'il n'a pas prononcées, des signes qu'il n’a pas faits, et emporter avec soi le plus de paroles entendues, de signes perçus, afin de retenir encore, dans le silence retrouvé, l'écho du discours commun, de continuer le dialogue polyphonique avec son interlocuteur invisible, de préparer les répliques qu’il devra lui donner. La mémoire devient ainsi gage, substance de la vie sociale, et la vie sociale gage et substance de la mémoire. Dans cet enrobage, les actes commencent à signifier, à manifester le passé dans le présent, l'autre dans soi, le collectif dans l'individuel, la relation dans l'événement. Après avoir été des sociétés à rappel, comme les sociétés des primates et des hominiens, avant d'être des sociétés à projets, telles que se veulent les nôtres, les collectivités ont été des sociétés à souvenirs ; elles sont allées de la rareté des indices à l'économie des informations en passant par la période très longue d'une production proliférante de signes. Elles sont ainsi passées d'une histoire subie et pratiquée à une histoire assumée et éprouvée, avant de s'engager dans une histoire voulue et figurée. Suspendue dans cette position intermédiaire, traçant une voie nouvelle, la société apprend à équilibrer forces centrifuges et forces centripètes ; elle s'efforce de garder les portes ouvertes à une humanité qui amorce ses grandes migrations ; elle enseigne aux individus et aux groupes à œuvrer les uns pour les autres, à l'intérioriser dans leur esprit et dans leur travail, à recréer constamment les rapports qui la 
représentent, et à agir en son nom. Mais on constate aussi qu'elle a préservé les distances, instaurées entre générations et entre sexes, qui avaient précédé l'émergence de l'homme, et qu'en édifiant les systèmes de parenté, elle a pris précisément ces distances pour base des rapports humains.

Retour à la Table des Matières 


\section{Chapitre VIII.}

\section{Les femmes dans la société des hommes: le problème de l'inceste}

\section{Pourquoi les femmes?}

\section{$\underline{\text { Retour à la Table des Matières }}$}

Le mariage, distribution exogamique des membres d'une société, ne présuppose aucune discrimination statutaire des femmes et des hommes. Pourtant ce sont les femmes qui sont distribuées par les hommes et entre les hommes. La réalité et la signification de cette discrimination sont entièrement contenues dans l'inceste. Un très grand nombre de peuples le condamnent. La plupart des individus répugnent à le commettre. Sa prohibition sert de modèle à toutes les prohibitions que les sociétés ont édictées au cours de l'histoire. L’importance que lui ont accordée les théoriciens se justifie amplement, s'agissant d'un phénomène qui a modelé en profondeur l'existence sociale et le psychisme humain. On a souvent tenté de l'expliquer, beaucoup plus rarement de l'analyser. Dans les Structures élémentaires de la parenté, hymne à la prohibition de l'inceste désigné comme principe fondateur de la culture, Claude Lévi-Strauss donne aux problèmes qu'il soulève leur véritable dimension. Il part de la constatation suivante : depuis les temps primitifs, les hommes ont eu à pallier le manque de nourriture ou de partenaires sexuels, aléas qui contrarient la satisfaction de leurs besoins. Chaque fois que la collectivité se heurte à une insuffisance, à la distribution aléatoire d'une ressource fondamentale, une intervention particulière de la société est nécessaire, afin de mettre un terme aux fluctuations, à la répartition 
désordonnée des biens qui risquerait, à la longue, d'entraîner sa dislocation et son dépérissement.

Dans le domaine essentiel de la reproduction, de l'union sexuelle, c'est à la prohibition de l'inceste qu'est dévolue la tâche d'introduire cette organisation, en soustrayant les hommes à l'incertitude qui découle de la rareté de ce bien que sont les femmes. Les fils, les frères et les pères, renonçant à former des couples consanguins avec leurs mères, leurs sœurs et leurs filles, s’ouvrent la possibilité d'avoir accès à un éventail plus large de partenaires éventuelles : « En somme elle (la prohibition de l'inceste) affirme que ce n'est pas sur la base de leur répartition naturelle que les femmes doivent recevoir un usage social. Reste alors à définir sur quelle base. Pour emprunter une expression familière à la réglementation moderne (mais en quelque sorte, aussi, éternelle) du " produit raréfié », la prohibition de l'inceste a d'abord, logiquement, pour but de "geler » les femmes au sein de la famille, afin que la répartition des femmes ou la compétition pour les femmes se fasse dans le groupe et sous le contrôle du groupe, et non sous un régime privé. C’est le seul aspect que nous ayons jusqu'à présent examiné : mais on voit que c'est là l'aspect primordial, le seul qui soit coextensif à la prohibition tout entière ${ }^{90}$. "

Cette thésaurisation du " produit raréfié » qu'est la femme, valeur d'usage fondamentale, le sacrifice d'une satisfaction présente à une satisfaction future, la subordination du bien-être individuel au bienêtre commun commandent la transaction et lui confèrent sa portée. A savoir, de donner sa fille ou sa sœur à un autre homme, le forçant du même coup à retourner le geste par le truchement d'une fille ou d'une sœur : "Ainsi toutes les stipulations négatives de la prohibition ontelles une contrepartie positive. La défense équivaut à une obligation ; et la renonciation ouvre la voie à une revendication ${ }^{91}$. " Le sacrifice se justifie, car le mariage est un moyen de communiquer des richesses, une prestation de services qui incluent aussi les femmes: «L’inclusion des femmes au nombre des prestations réciproques de

\footnotetext{
90 C. LÉVI-STRAuSs : Les structures élémentaires de la parenté, op. cit., p. 55. 
groupe à groupe, et de tribu à tribu, est une coutume si générale qu'un volume ne suffirait pas à en énumérer les instances ${ }^{92}$. "

La réciprocité ainsi vivifiée, le partage auquel on ne saurait se dérober, entraînent l'alliance qui convertit les étrangers en conjoints, les adversaires potentiels en compagnons. Le transfert des femmes pourrait sembler à première vue une opération vide, aiguillage d'individus dispersés ; il a pour effet une plus-value : le lien social. Les prescriptions exogamiques en sont le chiffre de code ; la société dans son ensemble les a édictées et chacun les respecte. Donner et recevoir des épouses, des cadeaux, est une conduite guidée à la fois par l'intérêt et par un faisceau d'impulsions généreuses, qui obéit à un rite précis, entouré de toute la solennité voulue, conférant au pacte social la charge d'émotion qui le grave dans le souvenir de tous les participants. Cependant la transaction qui lui préexiste, génératrice de toutes ces opérations, est une fonction liée à l'émergence de la pensée symbolique. L'apparition du langage, la coupure avec la nature la préparent. Pénétrant dans le domaine de l'alliance et de la parenté, elle transforme les règles de mariage en règles d'un discours communicable, ayant les femmes pour éléments lexiques originaux : "L'émergence de la pensée symbolique devait exiger que les femmes, comme les paroles, fussent des choses qui s'échangent. C'était en effet, dans ce nouveau cas, le seul moyen de surmonter la contradiction qui, faisait percevoir la même femme sous deux aspects incompatibles : d'une part objet de désir propre, et donc excitant des instincts sexuels et d'appropriation, et en même temps sujet, perçu comme tel, du désir d'autrui, c'est-à-dire moyen de se lier en s'alliant ${ }^{93}$. " Pensée et désir, masculins déjà, décrivent le double visage de la prohibition. Au revers, elle retient et interdit, figeant les femmes dans la région des biens et des ressources ; à l'avers, elle délivre et permet, consacrant les hommes dans la position d'échangistes, auteurs de liens sociaux, qui assurent la perpétuation du groupe à leur charge. Elle précise en même temps ce qui restait indéfini dans le partage exogamique : les termes de la réciprocité, les représentants du groupe qui en énoncent les règles en s’associant.

\footnotetext{
92 Idem, p. 80-81.

93 Idem, p. 616.
} 
Ce sont toujours des hommes. La rareté qui inclut la femme dans le champ des denrées - les Grecs le désignaient par le vocable d'oikomema, une chose - l'exclut du cercle des rapports collectifs essentiels. Sa situation aux îles Trobriand, telle que la décrit Bronislaw Malinowski, n’est pas exceptionnelle : «La femme étant éliminée de l'exercice du pouvoir et de la propriété foncière et étant privée de beaucoup d'autres privilèges, il s'ensuit qu'elle ne peut prendre part aux réunions de la tribu ni faire entendre sa voix dans les délibérations publiques où sont discutées les affaires se rapportant au jardinage, à la chasse, à la pêche, aux expéditions maritimes, aux détails cérémoniels, aux fêtes et aux danses ${ }^{94}$. "Converties en signes, les femmes sont les emblèmes du statut de l'homme, les marques de sa virilité en face des autres hommes. Mais de ce fait elles ne mènent qu'une existence sociale diminuée. La valorisation de leur beauté et de leur grâce en est un indice, figure de rhétorique du langage public, conçu par les hommes pour les hommes. La communication avec la femme reste forcément une communication privée, car elle « ne pouvait jamais devenir signe et rien que cela, puisque dans un monde d'hommes, elle est tout de même une personne et que, dans la mesure où on la définit comme signe, on s'oblige à reconnaître en elle un producteur de signes. Dans le dialogue matrimonial des hommes, la femme n'est jamais, purement, ce dont on parle ; car si les femmes, en général, représentent une certaine catégorie de signes, destinés à un certain type de communication, chaque femme conserve une valeur particulière, qui tient à son talent, avant et après le mariage, à tenir sa partie dans un duo ${ }^{95}$. »

Rendu possible par les règles de l'alliance, mais inopérant sur elles, ce dialogue fait de la femme, à la limite, une émettrice de signes naturels : elle est de la nature. C'est pourquoi on la situe souvent en marge de la culture ou en dehors d'elle. Son dialogue avec le serpent dans la Genèse a inspiré à un rabbin ce commentaire révélateur : " La femme avait l'intelligence et la connaissance de la langue animale », savoir qui semble avoir fait défaut à l'homme. Aucun doute n'est laissé quant à la réalité de cette distance et à la courbure qu'elle imprime

94 B. MALinOwSKi : La vie sexuelle des sauvages du nord-ouest-de la Mélanésie, Paris, 1930, p. 51.

95

C. LÉVI-STRAUSS : Les structures élémentaires de la parenté, p. 616. 
aux liens de parenté : «La relation globale d'échange qui constitue le mariage ne s'établit pas entre un homme et une femme qui, chacun doit, et chacun reçoit quelque chose : elle s'établit entre deux groupes d'hommes et la femme y figure, comme un des objets de l'échange, et non comme un des partenaires entre lesquels il a lieu. Cela reste vrai, même quand les sentiments de la jeune fille sont pris en considération, comme c'est d'ailleurs habituellement le cas. (...) Ce point de vue doit être maintenu dans toute sa rigueur, même en ce qui concerne notre société, où le mariage prend l'apparence d'un contrat entre des personnes ${ }^{96}$. " Le rapport de réciprocité, qui régit si profondément et si généralement le cycle des prestations et des associations entre individus ou groupes, a pour gardien l'élément masculin de la société, le seul auquel il s’applique. L'élément féminin est éliminé de ce rapport. Réfléchissant sur le fait qu'aux îles Trobriand les relations sexuelles sont traitées comme des services rendus par la femme à l'homme, Claude Lévi-Strauss note : «Le manque de réciprocité qui semble les caractériser aux îles Trobriand, comme dans la plupart des sociétés humaines, n'est que la contrepartie d'un fait universel : le lien de réciprocité qui fonde le mariage n'a pas été établi entre des hommes et des femmes, mais entre des hommes au moyen de femmes, qui en sont seulement la principale occasion ${ }^{97}$. $"$ Et il en suggère la raison : « Dans la société humaine, elles n’occupent ni la même place ni le même rang. L'oublier serait méconnaître le fait fondamental que ce sont les hommes qui échangent les femmes, non le contraire ${ }^{98}$. "

Ces constatations éclairent mieux la prohibition de l'inceste. J'ai multiplié à dessein les citations afin de dégager la signification qui me semble la plus profonde. Il est évident que cette prohibition s'exerce au sein d'une intervention qui opère à deux niveaux dont l'un s'appuie sur l'autre. D'une part elle corrige, théoriquement, le déséquilibre résultant de la distribution de la denrée supposée rare que sont les femmes : elle sert d'instrument à une rationalité qui se substitue aux caprices des volontés individuelles. D'autre part elle consacre et traduit la dissymétrie sociale des deux sexes, transformant les écarts quantitatifs dus au nombre en écarts qualitatifs de statut. En effet, si l'on y

Idem, p. 148.

97

Idem, p. 149.

98

Idem, p. 147. 
prend garde, la fille, la sœur ou la mère qui renoncent à leur père, leur frère ou leur fils ne le font pas au même titre que le père qui renonce à sa fille, le frère qui renonce à sa sœur ou le fils qui renonce à sa mère. Une telle renonciation dans le premier cas n'est pas un don appelant un contre-don, l'acte inaugurant une chaîne de prestations réciproques. Par ailleurs, un homme ne peut obtenir une femme que d'un autre homme, jamais d'une autre femme, et il est tout à fait exclu qu'une femme puisse s'associer librement à un homme, en partenaire ou en égale : leur alliance est impossible. L'univers masculin et l'univers féminin se déplacent sur deux orbites distinctes, dans des directions opposées. Les hommes vivent dans un monde de symboles, les femmes dans un monde de valeurs ; ceux-là connaissent le mariage à travers l'alliance, celles-ci l'alliance à travers le mariage ; pour eux la parenté est un moyen, pour elles c'est une fin. Si la prohibition de l'inceste marque le passage de la nature à la culture, elle est passage d'un état où le monde féminin et le monde masculin étaient équivalents à un état où ce dernier a la préséance sur le premier, affectant d'un signe positif tout ce qu'il inclut et d'un signe négatif tout ce qu'il écarte. "La " différence fondamentale » est une différence orientée », nous précise-t-on ${ }^{99}$. Certes, puisque l'intervention collective - et la règle en question - aboutit à un dédoublement des relations : de réciprocité, sur le patron de l'exogamie, pour les hommes ; de subordination, pour les femmes. La seconde est condition de la première.

Nous pouvons alors renverser l'ordre des raisons et retrouver par un autre moyen l'origine de l'asymétrie qui conduit à voir dans la réciprocité des hommes un phénomène de culture et dans la nonréciprocité des femmes, leur subordination, un phénomène de nature. L'exclusion de ces dernières du domaine des alliances autorise à les assimiler aux commodités, afin de les traiter comme telles. Leur absence du contrat social qu'elles ne sont pas invitées à signer les situe automatiquement dans le camp des objets sur lesquels porte ce contrat. Devenues un élément de prestation, elles sont destinées à être partagées, à circuler dans les veines de la société pour répondre aux besoins formulés par ceux qui la gouvernent, les hommes.

$\underline{\text { Retour à la Table des Matières }}$

99 Idem, p. 151. 


\section{Loi naturelle ou règle sociale ?}

\section{Retour à la Table des Matières}

La prohibition de l'inceste définit le rapport entre deux fractions de la société qui ne sont pas mises sur le même plan. Cet aspect n'est généralement pas perçu, car on l'envisage sur le modèle d'une loi physique qui s'applique de manière uniforme à une série de conditions ou d'éléments objectifs, soit les femmes, les hommes, les instincts, les comportements de reproduction sexuelle, les phénomènes génétiques. Ceux qui l'ont édictée n'ont fait que se substituer à la nature, tout en procédant comme elle : «La loi, écrivait James Frazer, reprenant une opinion commune ${ }^{100}$, interdit seulement aux hommes de faire ce que leurs instincts les inclinent à faire ; ce que la nature elle-même prohibe et punit, il serait superflu que la loi l'interdît et le punît. Nous pouvons donc supposer toujours sans risque de nous tromper que les crimes interdits par la loi sont les crimes que la plupart ont une tendance naturelle à commettre. Si cette tendance n'existait pas, il n'y aurait pas de tels crimes, et si ces crimes n'étaient pas commis, quel besoin y aurait-il de les interdire ? Au lieu de supposer, par conséquent, d'après la prohibition de l'inceste par la loi, qu'il y a une aversion naturelle envers l'inceste, nous devons plutôt supposer qu'il y a un instinct naturel en sa faveur, et que si la loi le réprime comme elle réprime d'autres instincts naturels, c'est parce que les hommes civilisés, arrivent à la conclusion que la satisfaction des instincts naturels nuit à l’intérêt général de la société. »

Les relations que la loi met au jour visent des êtres donnés avec leurs qualités et leur dynamisme. Lui préexistant, ils ne lui doivent rien et elle ne leur ajoute que peu de chose : ainsi la rareté des femmes, la capacité des hommes de les échanger, le désir de chaque individu de s'unir à l'individu de sexe opposé qui lui est le plus proche. La direction prise par ces relations résulte de ce que les termes qu'elles assemblent sont différents (êtres de sexe opposé, jouissant de pouvoirs intellectuels et physiques particuliers); la réciprocité ou la non-réciprocité qu'elle institue, l’inclusion dans le circuit social ou

100 J. Frazer : Totemism and Exogamy, Londres, 1910, t. V, p. 97. 
l'exclusion de ce circuit, partielle ou totale, dépendent de la valeur intrinsèque de ces facteurs. Et puisque la loi exprime le rapport entre deux êtres différents, se borne à combiner des termes hétérogènes, elle est la réplique de conditions qu'elle n’a pas créées, de phénomènes objectifs à propos desquels son intervention, sous le seul aspect d'une technique intellectuelle et pratique, fait échapper à l'arbitraire et charge de son poids d'universalité et d'indépendance ceux qui la décrètent, sans tolérer de déviations. On a investi la prohibition de l'inceste du caractère de nécessité que l'on reconnaît à la gravitation universelle ou à la sélection naturelle; on lui a supposé le pouvoir d'introduire dans les rapports entre les sexes la régularité qu'ils n'avaient pas ou qu'ils n'auraient pas eue sans elle; on y a vu l'instrument permettant de détourner leur désir de sa franche satisfaction, de réprimer l'inceste omniprésent. Toutes ces affirmations peuvent être réfutées sans peine.

En première approximation, tant qu'il n’y a pas de parenté, ou bien là où la parenté n'est pas reconnue comme lien social essentiel, on ne saurait parler de prohibition de l'inceste : les hominiens n'ont pas dû la connaître. Rien ne nous permet de supposer que la prohibition ait existé chez eux, donc de la rendre coextensive au genre humain, inséparable des organisations sociales qu'il s'est données ou qu'il se donnera. La rareté invoquée la réclame-t-elle ? La fonction d’une telle rareté mérite d'être examinée avec d'autant plus d'attention qu'elle est, d'emblée, attribuée aux femmes, définies en tant que commodités. Elle semble plausible, à condition toutefois d'assimiler, en anthropologie, la société à la parenté, de même que l'on assimile, en économie, la société au marché. Elles ont pour commun mécanisme l'échange qui suppose, ici et là, une catégorie de biens rares mais parfaitement interchangeables. Ces biens peuvent être très divers pour l'économie, alors que, pour la famille, seule la femme rentre dans cette catégorie. L'unique résultat de la transaction envisagée est l'équilibre obtenu à son terme, qui implique la réciprocité des agents sociaux donnés, les participants à l'échange, propriétaires indépendants de leurs biens. Les lois de la pensée qui ont énoncé les normes du mariage, défini la femme comme parole de la communication dans la société des hommes, ont une grande ressemblance, quant à leur portée, avec les lois de la rationalité qui incitent les possesseurs de marchandises, acheteurs et vendeurs, à optimiser leurs utilités, en surmontant la contradiction en- 
tre l'avantage de l'individu et l'intérêt de la collectivité. L'interdit de l'inceste pour les échanges matrimoniaux, les règles de propriété pour les échanges mercantiles, déterminent qui a le droit de donner et de recevoir, de demander et d'offrir, bref énoncent les règles du jeu et de la communication et nomment les partenaires.

Le statut de la rareté pose dès lors une question théorique et non plus une question de fait. Elle est naturelle si on la prend pour une donnée, en mettant entre parenthèses, à propos de la parenté, de même qu'on l'a fait à propos du marché, les pouvoirs multiples qui interviennent : la structure politique, l'état des arts industriels, les différences de classe, etc. Dès que l'on réintroduit ces pouvoirs et les phénomènes provisoirement isolés dans la totalité sociale - les règles de parenté, écrit Robert Jaulin, « ne pourraient prendre leur signification qu'à l'intérieur de la totalité culturelle, dans le cadre d'une unité de vie ${ }^{101}$ " - la rareté se dévoile sous l'aspect d'un produit, conséquence de l'action des groupes qui la recherchent dans un but précis. C'est un fait d'observation qu'il naît autant d'enfants du sexe féminin que du sexe masculin dans la plupart des sociétés. Le biais affectant leur distribution ultérieure peut avoir pour origine plusieurs facteurs ; les plus notables sont l'infanticide, la polygynie, l'accaparement des femmes par les vieillards. Une comparaison avec les primates nous inciterait à croire que, à l'origine, il y avait abondance de ressources féminines et répartition ordonnée.

En d'autres termes, rien n'interdit d'envisager la rareté des femmes dans les sociétés archaïques comme un résultat artificiel. Si elles ont édicté des règles de mariage à partir d'un manque, tout porte à croire qu'il s'agit d'un manque sciemment entretenu. La pratique du commerce, provoquant la pénurie dans l'intention de faire monter les prix ou de les maintenir à un certain niveau, nous fournit un exemple ad hoc. Commerçants avisés, les hommes n’échangeraient alors pas leurs denrées féminines parce qu'elles sont naturellement rares, mais les raréfieraient socialement afin de les échanger de façon avantageuse. Les conclusions seraient identiques si l'on adoptait une définition différente de l'échange, tablant sur le fait que les collectivités cèdent d'ordinaire ce qu'elles ont en trop, le surplus. Les femmes auraient

101 R. JAULIN : La paix blanche, Paris, 1970, p. 296. 
ainsi une moindre valeur que les hommes : ce sont elles que les groupes auraient un intérêt puissant à troquer contre la richesse du voisin ou d'un demandeur quelconque. Dans les sociétés archaïques, nous connaissons au moins une classe de transactions se conformant à ce schème. Les hommes âgés stockent les femmes et les cèdent aux jeunes contre des prestations réglementées par la tradition ${ }^{102}$.

On ne saurait donc attribuer sans hésiter la prohibition de l'inceste à la fluctuation quantitative du facteur démographique. Celle-ci pourrait à la rigueur découler de la prohibition - par le jeu de la thésaurisation et de la libération de l'objet convoité - et dans ce cas il faudrait chercher la motivation en question ailleurs que dans un état présocial qui aurait créé cette situation. Paradoxalement, si tel est le stratagème, l'inceste devient nécessaire, solution d'attente avant que les femmes soient mises en circulation - et ceci expliquerait qu'on l'autorise tout comme l'épouse polyandrique des jeunes sert de solution provisoire avant qu’on leur accorde à chacun une épouse.

Peut-on dire, alors, que la prohibition sert à déterminer les relations effectives entre les sexes, à éviter les unions consanguines ${ }^{103}$, à canaliser les pulsions menaçant l'existence collective ? La question a été posée : l'inceste n'est pas concerné. Son interdit met en jeu des frères, des sœurs, des fils, des mères socialement définis. Défense est faite à un individu d'épouser un autre individu qui lui est donné comme « mère ", " sœur ", " père ", " frère " par le groupe, mais non par la naissance, par les liens génétiques. Dans ce cas l'interdit ne protège guère contre les dangers eugéniques - malformations physiques, altération des facultés intellectuelles, etc. - des unions endogames. D’une société à l'autre, les degrés de parenté qui rendent le mariage ou le commerce sexuel licite ou au contraire le proscrivent varient grandement. Les prohibitions reposent moins sur le danger de la consanguinité que sur des normes ; ainsi le mariage peut être interdit entre cousins parallèles et permis entre cousins croisés. Et de même il est prohibé entre les enfants qui ont été allaités par la même mère, bien que ceux-ci puissent n'avoir aucun lien de parenté. Les partenai-

102 L.W. Simmons : The role of the aged in primitive societies, s.l., 1970.

103 D.F. Aberle et al. : The Incest Taboo and the Mating Patterns of Animals, American Anthropologist, 1963, 65, 253-265. 
res sexuels défendus sont souvent biologiquement très éloignés et leur progéniture serait à l'abri de toute conséquence supposée néfaste. Au contraire, les Australiens acceptent des unions, celle du grand-oncle avec la petite-nièce, par exemple, qui devraient, en principe, avoir des effets génétiques délétères. La décision de ce qui est incestueux revient toujours à la société. Chez les Iatmul ${ }^{104}$, les mariages endogames avec les «sœurs" classificatoires sont chose courante. Si le groupe devient trop endogame, il décide que la moitié du clan appartient à la proue de la pirogue de guerre et l'autre moitié à la poupe : le tour est joué, ce qui était union incestueuse cesse de l’être.

L'observation montre que l'inceste n'est pas toujours objet de réprobation, n'engendre pas obligatoirement un sentiment d'horreur. Puisque, tout en étant proscrit par le droit et la coutume, il est néanmoins pratiqué - et beaucoup plus souvent, et dans un plus grand nombre de collectivités ${ }^{105}$, qu'une opinion publique liée par une consigne de silence ne voudrait le reconnaître. La prohibition ne se dresse pas en tous lieux comme une barrière élevée contre le désir, destinée, à assujettir la sexualité individuelle à l'intérêt social. Inversement l'accomplissement du désir incestueux n'est pas incompatible avec la prohibition - et le mariage - car le commerce sexuel peut intervenir fréquemment de façon licite entre des individus qui sont dans l'impossibilité de se marier. Pareils faits témoignent d'une certaine autonomie des règles exogamiques vis-à-vis de l'interdit ${ }^{\mathbf{1 0 6}}$. « Il se peut que l'exogamie et la prohibition de l'inceste soient fondées sur les mêmes règles, mais elles ont chacune leurs propres règles ${ }^{107}$. " Quant à supposer que les défenses et les prescriptions de partenaires sexuels servent à mettre un terme ou empêcher le retour à une promiscuité animale, au choix arbitraire des partenaires, nous savons à présent que cette hypothèse ne tient plus.

\footnotetext{
104 G. BATESON : La cérémonie du naven, Paris, 1971.

105 M. MEAD : Sex and Temperament in three Primitive Societies, New York, 1953.

106 A.F. RadCliffe-Brown, D. Forde: African Systems of Kinship and Marriage, New York, 1950.

107 D. SPERBER : Le structuralisme en anthropologie, in Qu'est-ce que te structuralisme ? Paris, 1968, p. 181.
} 
La plupart des conceptions qui se rencontrent autour de cet interdit oscillent entre la tendance à souligner sa fonction adaptative dans l'intérêt de la reproduction, et l'importance de sa fonction répressive face au danger que constitue l'instinct, le désordre d'origine biologique. Ou, pour être plus près de la vérité, elles ont entretenu la confusion au sujet de ces deux fonctions ; dès lors rien ne permet de savoir s’il représente la réponse spécifique des sociétés humaines à un problème universel, celui du nombre, de la dégénérescence des qualités génétiques entraînée par les unions consanguines, ou bien s'il est la réponse universelle des sociétés humaines à une question qui leur serait spécifique, à savoir, assurer le renouvellement d'un lien collectif là où rôdent, porteurs d'incohérence, l'instinct sexuel, la versatilité du désir individuel. Envisagées dans toute leur extension, les unions permanentes et temporelles, virtuelles et réelles, des individus censés contracter des liens de parenté, obéissent à une norme tout en concrétisant un grand nombre de combinaisons imaginables. L'opportunité dicte la conduite, la psychologie et la biologie s'en accommodent. Sur la base de ces combinaisons, chaque société en déclare une partie impossible et une autre partie possible. L’inceste est ainsi créé par la règle ; sans elle, avant elle, il n’a ni sens ni réalité. Sa racine est dans la société qui l'institue, et sa raison d'être au-delà de l'organique et en deçà du lien conjugal, qui n'en épuise pas la signification. 


\section{Le seul inceste vrai : celui de la mère.}

\section{Retour à la Table des Matières}

Cette signification ne ressort guère, nous venons de le voir, des considérations pragmatiques ayant directement trait aux impératifs psychiques, biologiques ou démographiques. Pis encore, la règle perd de sa vigueur, voire de son utilité, si l'on y aperçoit en priorité le moyen de canaliser les rapports sexuels, de réduire les conflits provoques par l'union à l'intérieur des groupes sociaux restreints. La plupart des espèces possèdent de tels moyens et on ne saisit pas pourquoi la nôtre, pour suivre le raisonnement de James Frazer, aurait eu à s'embarrasser d'une loi là où la nature en fournissait déjà une. Les diverses conceptions exposées partent toutes de l'hypothèse, dont nous avons vu sur quelle base fragile elle se fonde, d'un état initial de promiscuité. Elles sont dénuées d'intérêt à partir du moment où il s'avère que cet état n'a jamais existé. Il vaut donc mieux demeurer au niveau de la règle et considérer ses exceptions et ses normes d'application. Nous savons, en effet, que la plupart des sociétés discriminent les relations incestueuses de celles qui ne le sont pas et réservent à l'inceste une place sur leur tableau de valeurs. Nous en déduisons l'universalité de la prohibition. Son absence, les transgressions, les tolérances prennent alors toute leur importance. Loin d'attester une mise en échec, un manque de généralité de l'institution, comme on l'a soutenu parfois, elles la précisent.

Quelles sont ces exceptions ou ces normes d'application ? La série la plus marquante se réfère à l'opération d'une règle distincte : l'obligation de l'inceste. On l'a étudiée moins attentivement en tant que règle et on l'a réduite à désigner une classe d'exemples isolés, puisque son extension est moindre que celle de la prohibition. Ce point de vue paraît insoutenable. L'écriture de droite à gauche, ou le boustrophédon dont les lignes alternent de gauche à droite et de droite à gauche, ne constituent nullement des épiphénomènes alors que l'écriture de gauche à droite serait la règle ; ce sont des manières différentes d'écrire, dont la fréquence a pu varier au cours de l'histoire. De même, l'obligation avouée de commettre l'inceste représente un mode particulier, mais équivalent à la prohibition, de définir les rela- 
tions entre les groupes sociaux, entre les hommes et les femmes, et non pas une chaîne discontinue d'accidents ou de tolérances dans le règne universel de l'interdit. La portée des actes est claire, leur place dans la vie collective précise ; ils ne réalisent pas seulement une inversion arbitraire de la règle ni ne trichent avec elle en vertu du bon plaisir ou d'une perversion, pas plus que l'écriture de droite à gauche n'est l'écriture en miroir d'un individu dyslexique ou la graphie secrète de Léonard de Vinci.

Dans les sociétés évoluées, là où cette obligation a été imposée, elle concerne surtout les classes supérieures. Au Cambodge, les unions incestueuses sont autorisées parmi les membres de l'aristocratie. Les mariages entre père et fille, frère et sœur étaient fréquents en Perse ; les documents ne permettent cependant pas de savoir si cette pratique s'étendait à toute la société ou si elle était restreinte à sa couche dominante. A Madagascar, l'interdit de la mère, de la sœur, du cousin joue pour les gens du commun ; pour les rois et les nobles il n'existe que l'interdit de la mère. Au Pérou, l’Inca épousait sa sœur. En Polynésie, si le premier-né est une fille, afin d'éliminer la rivalité entre lignées à propos de la chefferie, elle est mariée à son frère cadet. Les unions consanguines étaient autorisées en Égypte dans la classe régnante ; les unions entre frère et sœur surtout étaient nombreuses chez les artisans et les petits fonctionnaires. A Samoa, un noble peut épouser sa sœur, non sa mère ou sa fille. A Hawaï, le rang d'un chef était déterminé par l'union incestueuse dont il était issu et le mariage incestueux qu'il contractait. Les honneurs rendus aux chefs devaient être d'autant plus grands que le degré de consanguinité de leur mariage et celui de leurs ascendants étaient plus proches. Le mariage du degré le plus élevé, le plus sacré, était celui d'un frère et d'une sœur ayant même mère et même père, bien entendu, de haut rang. Suivant la doctrine de cette aristocratie, l'enfant né d'un tel mariage était un dieu, vénéré, et " chaud du plus ardent des tabous ». Plus généralement, les rois que James Frazer a qualifiés de « divins » ont tous une chose en commun : ils descendent de familles incestueuses et commettent rituellement l'inceste, notamment l'inceste maternel qui occupe une place à part. Il s'agit là d'une condition préalable et d'un acte nécessaire pour établir leur qualité de souverains. Luc de Heusch a inventorié et analysé très finement l'aspect réel et symbolique de 
cette obligation institutionnelle, si répandue ${ }^{108}$. Bien qu'elle soit ressentie et représente une violation reconnue de la règle, elle est néanmoins, en tant que violation, un signe de sortie du commun, du cadre ordinaire de la vie sociale. Un signe parmi d'autres, car ceux qui sont sollicités ou autorisés à commettre l'inceste sont aussi sollicités ou autorisés à enfreindre les tabous relatifs à la nourriture et parfois à tuer un homme. En brisant les règles, en commettant l'acte défendu, en perpétrant des crimes contre ce qui ailleurs constitue l'armature de la solidarité collective, on acquiert le pouvoir effectif et magique, on trace la frontière entre les groupes. La transgression qui transforme les hommes ordinaires en sous-hommes, en bêtes humaines, et les rejette de la culture dans la nature, transforme certains privilégiés en surhommes, en bêtes divines, les projette de la nature au centre de la culture. Ce qui d'un côté est droit est de l'autre interdit et réciproquement. Ainsi l'aristocratie marque son rang, affirme ses prérogatives, découpe sa place à part dans le système social, refuse le mélange avec d'autres classes qu'entraînerait, dans bien des cas, l'union exogamique. La loi est la même pour tous ; mais son application fait que tous ne sont pas les mêmes suivant le côté où ils se situent.

A l'évidence, l'acte que quiconque se voit défendre de commettre, se marier avec un parent proche, consanguin ou non, sépare deux sphères de la collectivité, détermine un ordre sacré - parfois stérile, il est vrai - superposé à l'ordre profane toujours inférieur. L'inceste obligatoire instaure de façon réelle ou symbolique une réciprocité relative des sexes, une égalité introduite par contraste dans l'élite de la société, et rend à la mère, dont le lustre se trouve rehaussée, son autorité sur les enfants qui ne lui sont plus arrachés sans contrepartie. L’ordre profane est frappé du sceau de la prohibition. L’ordre sacré se détache nettement comme la négation de l'autre, et son adoption a pour fin de substituer, aux pouvoirs issus de la parenté, les pouvoirs propres à une organisation quasi étatique qui le dépasse. Le seul fait de les classer suivant les catégories du sacré et du profane suffit à indiquer qu'il s'agit d'une différence entre le supérieur et l'inférieur, d'une séparation entre le dominant et le dominé, l'élite et le peuple, découpant deux règnes distincts : celui d'en haut, pour lequel le lien

108 L. De Heusch : Essais sur le symbolisme de l'inceste royal en Afrique, Bruxelles, 1958. 
incestueux est non seulement abordable mais requis, et celui d'en bas auquel ce lien est interdit. Le noble qui le désire a toute liberté de suivre les coutumes des gens du commun ; les gens du commun n'ont pas le droit de suivre la coutume des nobles. Le peuple apprend de la sorte quel est son véritable état, et aussi à donner sans rien recevoir en échange, qu'il s'agisse de filles à marier, de biens ou de services. Que le peuple décide à son tour d'adopter la règle d'obligation au lieu de l'interdit qui lui est imposé, et la promiscuité ronge comme la rouille les piliers de la religion, elle détruit, comme la mauvaise herbe, l'ordonnance cultivée du jardin des mœurs. On peut donc dire que l'exception n'en est jamais une. Elle change la prohibition de l'inceste en signe de l'hétérogénéité et du classement des groupes humains; par la même occasion, elle nie l'existence de ce classement au sein des catégories privilégiées, ou, à tout le moins, récuse la différenciation de ces catégories suivant les critères valables pour le commun des mortels.

En outre, la prohibition de l'inceste connaît elle-même des degrés, et n'a pas la même rigueur pour les hommes que pour les femmes. Pour les sociétés des îles Trobriand, "l'inceste avec la mère est considéré comme un acte vraiment horrible, mais aussi bien par le mécanisme à la faveur duquel il fonctionne que par la manière dont il est envisagé, ce tabou diffère essentiellement de celui qui pèse sur le frère ou sur la sœur ». " Il convient d'avoir bien présent à l'esprit le fait que, tout en étant considéré comme mauvais, l'inceste de père à fille n'est pas désigné par le mot suvasova (exogamie de clan ou inceste proprement dit) et qu'il n'est suivi d'aucune maladie ${ }^{109}$. »

Si l'on recensait les diverses manières dont la défense est pratiquement mise en œuvre, les condamnations qui frappent ceux qui la transgressent et les sanctions qu'elles entraînent, on obtiendrait une échelle de valeurs adoptée par la plupart des sociétés humaines. Au sommet, la prohibition touche le plus durement l'union de la mère et du fils ; en bas, fortement atténuée, elle limite l'union du père et de la fille ; entre les deux extrêmes se situent les unions entre frères et sœurs. A telle enseigne qu'on est enclin à voir dans l'interdit de l'inceste avec la mère le seul interdit vraiment universel. Passer outre,

109 B. MALINOWSKI : op. cit., p. 490 et p. 497. 
commettre l'acte réprouvé et défendu entre tous, c'est s'exposer à la vindicte générale, tant chacun au tréfonds de son être le ressent comme un événement impossible et impensable, aux répercussions incalculables sur le plan individuel et social. En regard, le père n'est pas assujetti à une règle aussi rigoureuse ; on tolère de sa part le libre accès à toutes les femmes, ses filles incluses. On inclinerait à croire que c'est en vertu d'une concession faite aux femmes par les hommes pour des raisons de symétrie que l'interdit a été élargi à son cas : « Il y a des raisons de penser que les prohibitions du totem étaient dirigées contre les désirs incestueux du fils », écrit Freud ${ }^{\mathbf{1 1 0}}$ qui ajoute : «Il est intéressant d'observer que les premières restrictions apportées par l'introduction des classes de mariage affectaient la liberté sexuelle des jeunes générations. (c'est-à-dire l'inceste entre frère et sœur et entre fils et mère) alors qu'il fallut une nouvelle extension de l'interdit pour empêcher l'inceste du père et de la fille ${ }^{111}$. "

Sans qu'il s'agisse d'une exception licite, la dérogation suffit à dispenser le père de respecter la norme supposée commune, transformant dans bien des cas en mauvaise ou simplement répréhensible une conduite qui, s'agissant de ses compagnes, est radicalement condamnée. L’inégalité devant la loi que nous ne connaissons que trop, le quod licet jovi non licet bovi romain le dit, l'infraction réprimée chez les uns et passée sous silence chez les autres, est prouvée par cet exemple, montrant pour qui une loi est faite et contre qui, témoignant de toute la distance qui sépare ceux qui la décrètent de ceux qui la subissent. Son énoncé, sous couvert de généralité, ne les distingue pas ; sa pratique, traduisant une norme implicite d'application, les particularise forcément. Nul n'est censé lui désobéir ; les juges savent pourtant parfaitement qui est tenu de la connaître et d'obtempérer, lorsque, appelés à statuer, ils épargnent leurs pairs et leurs auxiliaires. Ainsi l'inégalité devant l'inceste reflète la situation asymétrique de l'homme et de la femme.

Les interdits sont gravés dans la différence. Jusqu'à ce jour, les collectivités n’ont toléré les différences qu'en les transformant en hiérarchies, et les hiérarchies se sont édifiées en creusant profondément

110 S. FREUD : Totem and Taboo, op. cit., p. 5.

111 Idem, p. 121. 
les différences. Aussi nous apprennent-ils beaucoup plus de choses sur leur sujet que sur leur objet. Ils n’ont été énoncés ni par quelqu'un ni par le genre humain dans son ensemble qui n'est pas un sujet, mais édictés au nom de quelqu'un, derrière lequel s'abrite le vrai sujet qui essaie de se poser comme tel et d'accentuer un écart, une absence de réciprocité entre lui et les autres. Ce n'est jamais dans les plis de la nature qu'il faut chercher l'origine d'un interdit, mais toujours dans les écarts de la société, qu'il augmente tout en les masquant. La prohibition de l'inceste ne fait pas exception. Nous la savons orientée. Elle départage avec clarté, aux yeux de tous, la classe d'individus tenus de la respecter avec rigueur de la classe d'individus qui jouissent d'une certaine latitude à son égard, ou auxquels il est fait obligation de le commettre ; ce seul critère suffit à ranger les premiers dans l'ordre inférieur du profane et les seconds dans l'ordre supérieur du sacré. La règle produit une relation de différence entre ces classes; à un pôle l'inceste désigne le pouvoir social, public ; à l'autre pôle la prohibition traduit la soumission domestique, privée. A partir de là, les couches de la société se définissent en fonction de ce qui est requis ou toléré de la part de l'une et de ce qui est imposé à l'autre. Dans les diverses sociétés énumérées, l'interdit a pour rôle de discriminer entre les nobles, les chefs et le peuple, de déterminer leurs privilèges et leurs devoirs respectifs, d'indiquer qui peut recevoir et qui doit donner ; sur une échelle plus réduite, son usage conduit à des obligations analogues dans les sociétés archaïques. En lui conférant une telle portée, les premières sociétés politiques, étatiques, ont magnifié les forces qu'il recelait concentrées et latentes dans les sociétés de parenté. Mais c'est justement parce qu'il avait le pouvoir d'établir une telle distance, de créer les termes ou de maintenir en eux des qualités distinctes, transformant les uns en objets, en matériaux des autres, subordonnant les uns aux autres : les femmes aux hommes, la classe du peuple à la classe aristocratique et assurément les jeunes aux adultes.

Si l'on voit dans la prohibition de l'inceste la répression de l'instinct sexuel, ou du désir individuel de choisir le partenaire de la jouissance, à travers l'instinct ou l'individu, c'est la répression de l'instinct ou du désir de l'autre - le sexe féminin, en l'occurrence c'est leur contrôle qui est sous-entendu, c'est en eux et non pas dans l'inceste ou la pulsion qu'est localisée la menace ; ainsi, dans les sociétés policées, la répression de la violence ou de l'agressivité, présen- 
tée comme ayant trait à l'instinct, est répression de la violence et de l'agressivité d'une fraction de la société, les jeunes, les défavorisés, etc., la violence de l'autre fraction passant pour légitime, légitimée ou légitimable.

Bref, une règle, qu'elle soit inscrite sur les tables de la loi, dans les droits de l'homme et du citoyen, ou dans la mémoire des peuples, dès lors qu'elle est sociale, une fois dépouillée de son déguisement de rapport à l'objet, de rapport à un différent, exprime toujours un rapport à un sujet, un rapport de différence, c'est-à-dire un rapport entre groupes sociaux en fonction desquels sont déterminés à la fois les rôles d'objet et de sujet et les qualités qui rendent les termes distincts. 


\section{Les règles de parenté, règles de domination.}

\section{Retour à la Table des Matières}

La prohibition de l'inceste a donc un caractère discriminatoire et hiérarchique. Les règles de parenté font voir que les femmes, dans cette condition, sont communiquées d'un côté à titre de biens et de services, de l'autre côté circulent à titre de messages ayant la qualité de biens et de services. Mais, qu'il s'agisse de biens humains ou non humains, chaque fois un art, ayant ses règles et ses outils, est indispensable pour transformer les ressources brutes en objets et en signes que l'on puisse donner, émettre, recevoir. Faute de cet art, aucun objet ne peut devenir signe, donné naturel rencontrant un besoin social, ni aucun signe devenir objet, produit social concrétisé par un matériau naturel. L'art de convertir une fraction de la société en commodité, l'autre fraction se posant en créateur de cette commodité, par le truchement d'une série appropriée de discriminations de rang, n'a rien d'exceptionnel. Bien des collectivités humaines ont mis un groupe d'individus au nombre des richesses, des instruments matériels, équivalant à d'autres richesses ou instruments, et susceptible d'être donné, vendu ou partagé. Cet état de choses implique toujours un rapport particulier entre ceux qui ont le pouvoir d'imposer pareil traitement et ceux qui le subissent. L'esclavage est un tel instrument, produit de l'art raffiné de la domination, un bien éminemment précieux, car il conditionne tous les autres, que son propriétaire a le droit de concéder, d'employer, d'acquérir, de détruire suivant les règles de l'art. Signe d'opulence et d'autorité, pouvant engendrer la solidarité de ses possesseurs, s'accumuler au pôle des riches et se diluer au pôle des pauvres, il est dépourvu d'existence civique et n'apparaît nulle part terme d'une relation, sauf de celle qui consacre possession d'autrui ce « bétail humain »: "L'esclave est en quelque sorte une propriété animée... Ce qu'on appelle proprement des outils sont donc des instruments de production, mais l'esclave est instrument d'utilité... Or la vie est usage et non pas production ; voilà pourquoi l'esclave est le ministre des choses qui servent à l'usage. On l'appelle aussi chose possédée, partie, car ce mot exprime non seulement ce qui est partie d'une chose, mais ce qui en dépend entièrement ;... c’est celui qui ne 
s'appartient pas à lui-même, mais qui appartient à un autre et qui pourtant est homme, celui-là est esclave par nature ${ }^{112}$. »

Mieux loti parce que plus libre de ses décisions, le prolétaire apparaît sur le marché à l'instar de n'importe quelle marchandise. Le manque de capital, le fait d'avoir pour tout bien sa force de travail le conduisent à la vendre au plus offrant, à subir les fluctuations des prix, à être un surplus en période de chômage, à se raréfier, cherchant un remède dans la migration. La loi du profit, les relations sociales capitalistes ne cessent de produire cette marchandise particulière, transformant des hommes en salariés, commodité que ceux qui détiennent les leviers du pouvoir et de la richesse manipulent sans relâche, appuyés par l'appareil politique, idéologique et social.

Nous avons notre disposition d'autres éléments de comparaison non moins valables, tirés du domaine des professions ; les sociétés ont en effet tendance à classer les individus qui les exercent sur une échelle indiquant le degré de dignité ou d'indignité qu'elles leur attribuent. Or, dans les sociétés archaïques, les femmes et les hommes pratiquent respectivement, en général, la cueillette et la chasse. L'infériorité des premières, la supériorité des seconds peut, à la rigueur, refléter, à travers normes, institutions et mythes, le choix fait entre deux familles de techniques, les mesures prises afin de maintenir séparés, chacun à sa place respective, les groupes qui exercent l'une ou l'autre. Le moment n'est pas encore venu d'explorer ces analogies. Elles facilitent pour l'instant un rapprochement suggestif : semblables en cela aux femmes, les prolétaires, les esclaves, etc. jouent dans la conscience et la pratique collectives le rôle de biens quelconques ou d'indices mesurant l'échange des richesses. Convergence pleinement saisie, d'ailleurs, par le maître français qui écrit : "Qu'il y a là un aspect absolument fondamental de nos mythes, et qu'ils nous font accéder à un état décisif de la pensée humaine, dont, par le monde, d'innombrables mythes et rites attestent la réalité, le troisième volume de ces Mythologiques achèvera de le montrer. Tout semble se passer comme si, dans une soumission mystique des femmes à leur empire, les hommes avaient perçu, pour la première fois, mais d'une manière symbolique, le principe qui leur permettra un jour de résoudre les pro-

112 Aristote : Politique, Livre I, chap. II. 
blèmes que pose le nombre à la société, comme si en subordonnant un sexe à l'autre, ils avaient tracé l'épure des solutions réelles mais encore inconcevables ou impraticables pour eux, qui consistent, tel l'esclavage, dans l'assujettissement d'hommes à la domination d'autres hommes ${ }^{113}$. »

Quel que soit le contexte dans lequel la femme a été assujettie d'abord, l'identité des effets obtenus au cours du temps et le genre distinct de différence - de classe ou de facultés productives - enlèvent un doute à son propos : ce n'est pas la nature qui la donne pour objet ou signe, c'est la société qui la prépare à pareille fonction. Celleci reflète un lien social ; la substance biologique est secondaire. Reprenant le fil des métaphores, on peut dire que, si les relations conjugales déterminent le sexe féminin à être don, prestation, monnaie d'échange, de même que les relations marchandes déterminent la force de travail à être marchandise, c'est parce que le système collectif auquel appartiennent la femme ou la force de travail les conserve ou les rend telles, objet de partage ou de commerce. La pensée a le pouvoir de sculpter et polir l'architecture du système, enchâssant chaque catégorie dans l'alvéole qui est le sien : la gradation des rangs n'en découle pas, n'étant vraisemblablement pas inscrite dans sa constitution comme l'est celle de la gauche et de la droite. Dans l'architecture de la famille, la prohibition de l'inceste concourt à diviser le groupe social et non pas à l'unir, à transformer les règles de parenté en coordonnées d'une «différence orientée » entre hommes et femmes, au lieu de l'estomper dans les liens du sang. La réciprocité a, ici, pour préalable son contraire ; la cohésion masculine repose sur la soumission féminine ; l'intervention entraîne la hiérarchie des personnes. La constatation n'a rien de surprenant, car l'art de la domination n'est pas inconnu aux sociétés archaïques, et, dit Nietzsche, "celui qui revient aux origines trouvera de nouvelles origines ».

$\underline{\text { Retour à la Table des Matières }}$

113 C. LÉvi-Strauss : Du Miel aux Cendres, Paris, 1967, p. 244. 


\section{Chapitre IX.}

\section{La lutte des sexes}

\section{Deux sociétés en une seule.}

\section{(1) La société du secret.}

Dans une vue globale qui la ramène à l'essentiel, l'organisation des sociétés archaïques s'ordonne sur deux axes principaux : l'axe de la différence, séparant ceux que l'on peut épouser et ceux que l'on ne peut pas épouser, donc les alliés et les parents, et l'axe de la dichotomie sexuelle entre hommes et femmes, qui traverse tous les rapports de l'existence, travail, espace, habitats, objets, nourriture, événements, langage. «Dans les cultures primitives, constate Mary Douglas ${ }^{114}$, la distinction entre les sexes est, presque par définition, la première de toutes les distinctions sociales. De sorte que certaines institutions importantes reposent en permanence sur la distinction entre les sexes. » Le choix entre ce qui est permis et ce qui est interdit met simultanément en jeu les deux dimensions, les combine pour discriminer le semblable du différent, soi et autrui. La terminologie de la parenté détermine les groupes d'individus qui sont autorisés à se marier entre eux ou non en tenant compte de l'identité sexuelle en termes sociaux : les enfants d'un homme peuvent épouser les enfants de sa sœur mais non ceux de son frère, pas plus que ne peuvent se marier ensemble les enfants issus de deux sœurs : Cette règle n’est ni générale ni toujours aussi directe ; elle est cependant suffisamment employée pour attester

114 M. Douglas : De la souillure, Paris, 1971, p. 184. 
l'intersection du principe généalogique d'alliance et du principe social de hiérarchie sexuelle.

Ce dernier ne juxtapose pas seulement deux groupements caractérisés par leur appartenance à un sexe, il les sépare par certains côtés et les discrimine par d'autres, faisant pencher la balance en faveur des hommes. Afin de charger leur plateau de tout le poids nécessaire et de le maintenir aussi incliné que le rapport des forces le permet, ceux-ci ont créé des associations dotées des moyens, du statut et du prestige nécessaires. La solidarité et l'ascendant masculins constituent un trait fondamental du mode de vie archaïque, sur lequel reposent l'idéologie, l'économie, voire la politique de ces groupements. Les variations éventuelles sont de degré, non de nature. La présence, dans ce cadre, de sociétés d'hommes plus ou moins secrètes est un phénomène trop répandu pour ne pas attirer l'attention. La rareté ou la faiblesse de sociétés féminines de ce genre également. Les confréries masculines sont signalées un peu partout: Mélanésie, Afrique noire, Amérique du Nord, Malaisie, Polynésie. Leur mode de recrutement et de fonctionnement mérite d'être décrit brièvement, ne serait-ce qu'à propos d'un cas.

Chez les Blackfoot, on atteste l'existence de plusieurs sociétés qui se livrent à des danses et à d'autres cérémonies et assument la charge de prévoir les campements lors des migrations estivales. Une tribu peut en comporter jusqu'à douze et plus, elles se nomment « tous camarades » et ont aussi chacune un nom particulier. Chacune comprend des hommes sensiblement de même âge, mais qui n'y demeurent que quatre ans environ, après quoi ils passent dans la société de classe d'âge supérieure, en même temps que leur place est prise par des hommes plus jeunes. Bien que ces échanges s'effectuent simultanément, pendant le camp d'été, ce sont des transactions entre individus, monnayées en chevaux, armes ou vêtements qui donnent droit au postulant d'occuper le rang et de revêtir les insignes de l'homme auquel il succède. Ce dernier doit s'assurer une promotion semblable, de sorte que tous les quatre ans environ chacun, sauf les anciens, passe dans une société supérieure, tandis que les jeunes accèdent pour la première fois à la société de dernier rang. Outre les rites, propres à chacune, ces sociétés exercent une fonction d'organisation des camps d'été ; leurs dirigeants proposent aux chefs de tribu des itinéraires pour les migra- 
tions et des terrains de campement; deux ou trois d'entre elles sont agréées et ont pour tâche de surveiller ces migrations et de faire régner l'ordre. Les hommes ainsi désignés plantent leurs tentes au milieu du camp et patrouillent pendant la nuit. Les sociétés ont aussi pour mission d'observer les déplacements des troupeaux de buffles, raison d'être de ces camps, et organisent les chasses. Les chefs de tribu savent qu'ils peuvent faire appel à ces hommes en cas de besoin. Cependant la rotation des membres et la limitation de la durée effective des sociétés, qui tombent en sommeil pendant la plus grande partie de l'année, les empêchent de jouer un rôle prépondérant dans la tribu et de constituer une menace pour ceux qui détiennent le pouvoir.

Ces sociétés, partout où leur présence est relevée, exercent néanmoins un grand ascendant. Elles disposent d'une force non négligeable, et jouissent d'un prestige religieux, car elles sont le siège d'un culte public et de cérémonies d'initiation ${ }^{115}$. Il n'est pas rare que le lien qu'elles établissent entre hommes l'emporte sur le lien clanique entre hommes et femmes ${ }^{116}$; c'est notamment le cas en Australie où les associations tribales groupent les hommes pour des pratiques dont le secret est jalousement réservé aux yeux des femmes et des noninitiés. Prenant l'habitude de rencontrer les hommes des autres clans (ceux de l'Aigle-Vautour, de la Chauve-Souris, de la Corneille et de la Grenouille), les hommes du clan du Casoar, par exemple, se sentent plus proches d'eux que des membres de leur propre clan.

\section{$\underline{\text { Retour à la Table des Matières }}$}

\section{(2) Les discriminations sexuelles.}

La séparation des sexes a une répercussion dans le domaine de l'alimentation. Il est interdit aux hommes d'un clan de consommer la chair de leur totem, et cette défense vaut aussi pour les femmes ; mais les Yualarois font du vautour brun un aliment prohibé pour les femmes de n'importe quel clan. L'appartenance à un sexe l'emporte donc sur l'appartenance au groupe totémique. Aux îles Banks, l'isolement des sexes est porté au maximum dans la vie quotidienne ; les hommes

115 K.L. LiTTLE : The Role of Secret Society in cultural Specialization, American Anthropologist, 1949, 51, 199-212.

116 R. LOWIE : Traité de sociologie primitive, Paris, 1969, p. 248. 
mangent et dorment dans le sukwe (club) et quiconque n'est pas admis à y entrer encourt le mépris de tous. Contraint de prendre ses repas avec les femmes, il n'a d'autre issue, pour réduire ce que R. Lowie appelle la "période d'ignominie » ${ }^{117}$, que de susciter la pitié d'un ami, c'est-à-dire que celui-ci consent, en échange d'une somme importante, à l'y introduire. Ces associations masculines disposent presque toujours d'objets et de masques que les non-initiés n’ont le droit de voir que de loin, à l'occasion d'une procession. Jeter les yeux sur ces objets à d'autres moments serait criminel pour eux, comme pour les femmes, exclues des cérémonies publiques. Ces dernières y sont admises sous certaines conditions, ainsi en Nouvelle-Guinée, chez les Iatmul : " Quand les femmes prennent part à la cérémonie, elles font quelque chose de tout à fait étranger aux normes de leur existence, mais qui est normal pour les hommes ; aussi adoptent-elles en ces occasions particulières des éléments de la culture masculine. Elles se tiennent comme les hommes et portent les ornements qui leur sont normalement réservés ${ }^{118}$. "

Dans les collectivités archaïques, le pouvoir appartient toujours l'existence de ces sociétés secrètes et exclusives l'illustre - aux hommes et non aux femmes. Organisés pour chasser, rassemblés pour accomplir les diverses tâches cérémonielles, ils le sont aussi pour ramener à l'obéissance ceux qui enfreignent les règles collectives, leur fait et leur œuvre. On a quelquefois du mal à apprécier le degré de rigueur avec lequel elles sont appliquées. Du moins l'intention qui préside à l'établissement de telles règles ne fait point de doute : d'une part isoler les femmes, d'autre part les frapper d'un traitement discriminatif. Rappelons quelques faits à ce propos. Dans beaucoup de populations, hommes et femmes sont censés manger à part et consommer des nourritures particulières. La langue ou le vocabulaire, on l'a observé chez les Guyacurus et dans les Caraïbes, peuvent différer pour chaque sexe. Le même critère préside à l'organisation de l'habitat. Dans nombre de tribus, aux îles Mortlock par exemple, le chef et tous les hommes du clan dorment dans une maison collective au centre du village, entourée de demeures plus petites qui abritent les femmes et les jeunes filles. Les femmes ne peuvent résider avec leurs

117 Idem, p. 92.

118 G. BATESON : op. cit., p. 211. 
maris, puisque ceux-ci appartiennent à un clan différent. En Californie, la maison de famille des Hupa est la demeure des femmes, où les hommes viennent pour les repas. Mais le soir venu ils se rendent au sudatorium pour prendre des bains turcs et y passent la nuit. On note cependant une variation saisonnière, une séparation moins stricte en été. La séparation est plus rigoureuse aux îles Marquises, aux îles Salomon, aux îles Banks, cas extrême que je viens de mentionner. Et dans l'Alaska les Eskimos ont une maison dont les femmes sont exclues, tandis que chez les Athapaskan du Nord on sépare les filles des garçons, et les femmes ne peuvent prendre part aux danses. Cette ségrégation des sexes se reflète dans le détail des activités de la tribu, la section masculine ayant, comme l'écrit Geza Roheim, les activités d'un " groupe d'hommes unis dans le culte d'un objet qui est un pénis matérialisé, et excluant les femmes de leur société ${ }^{119}$. »

Les comportements quotidiens sont marqués par une préoccupation analogue. Chez les Samoyèdes, il est interdit aux hommes de toucher un objet qui a servi à une femme, tandis que les Boschimans redoutent d'être atteints dans leur virilité s'ils s'assoient par mégarde du côté réservé aux femmes. L'évitement de tout ce qui a trait au sang menstruel est connu, et je ne reviendrai pas sur sa signification. La séparation des deux sexes qui commence très tôt est presque toujours rigoureuse à partir de l'adolescence. En Corée, en Mélanésie, en NouvelleCalédonie, chez les Indiens de Californie, frères et sœurs cessent de se parler lorsqu'ils ont atteint l'âge de la puberté. Chez les Lethas de Birmanie, garçons et filles qui se croisent sont obligés de détourner leurs regards. Émile Durkheim, quelle que soit la valeur de l'explication qu'il en donne, a qualifié avec pertinence la relation qui distancie et oppose les sexes : «On aperçoit, écrit-il ${ }^{\mathbf{1 2 0}}$, le rapport qu'il y a entre ces interdictions et l'exogamie. Celle-ci consiste également dans la prohibition d'un contact: ce qu'elle défend, c'est le rapprochement sexuel entre hommes et femmes d'un même clan. Les deux sexes doivent mettre à s'éviter le même soin que le profane à fuir le sacré, et le sacré le profane ; et toute infraction à la règle sou-

119 G. RoHeIM : Psychoanalysis of Primitive Cultural Types, Intern. Journal of Psychoanalysis, 1932, 18, p. 152.

120 E. DuRKheIM : La prohibition de l'inceste et ses origines, Année sociologique, 1896, 1, p. 40. 
lève un sentiment d'horreur qui ne diffère pas en nature de celui qui s'attache à toute violation d'un tabou. Comme quand il s'agit de tabous avérés, la sanction de cette défense est une peine qui tantôt est due à une intervention formelle de la société, mais tantôt aussi tombe d'elle-même sur la tête du coupable, par l'effet naturel des forces en jeu. Ce dernier fait surtout suffirait à démontrer la nature religieuse des sentiments qui sont à la base de l'exogamie. Elle doit donc très vraisemblablement dépendre de quelque caractère religieux dont est empreint l'un des sexes et qui le rendant redoutable à l'autre, fait le vide entre eux. Nous allons voir que, effectivement, les femmes sont alors investies par l'opinion d'un pouvoir isolant en quelque sorte, qui tient à distance la population masculine, non seulement pour ce qui concerne les relations sexuelles, mais dans tous les détails de l'existence journalière. »

Tel est bien le rapport que nous voulons faire ressortir. Dans ces collectivités, les femmes, investies d'un pouvoir négatif, constituent une menace ; elles sont parallèlement tenues à l'écart des hommes. Les mesures destinées à consolider l'écart, ces quelques exemples nous ont permis de le voir, sont en vigueur pendant toute la vie adulte des individus. Elles ont trait à tous les aspects de l'organisation sociale, s'étendent à toutes les institutions, et c'est seulement de façon accessoire qu'elles se rapportent au commerce sexuel. Les hommes ne s’identifient pas nécessairement avec le sacré, ni les femmes avec le profane ; l'emploi de ces catégories permet cependant de remarquer, au-delà d’une hétérogénéité préservée à tout prix, la tendance à accentuer entre les deux sexes le contraste qui sépare une condition supérieure d'une condition inférieure, et à éviter le mélange. Dans les faits, assurément, aucune fraction d'une collectivité n'est en mesure de faire prévaloir de manière absolue son autorité au détriment de l'autre fraction avec laquelle elle est en rapport. Tous les groupes masculins s'efforcent d'imposer aux femmes et aux jeunes une discipline, de brimer les tentatives d'autonomie, en superposant les exigences de la société dont ils se sont proclamés les gardiens aux volontés individuelles qui pourraient se faire jour. Comme il arrive en pareil cas, cette action revêt deux formes : brutale et directe lorsque la possibilité en est donnée, idéologique et indirecte si les circonstances l'exigent. 
L'anthropologue Mary Douglas a observé ${ }^{121}$ — et elle n'est pas la seule $^{122}$ — que les différents tabous liés à la pollution ont pour but principal d'obliger les femmes à se tenir à la place qui est la leur dans la vie collective. Dans les sociétés qui respectent strictement la gradation des rôles sexuels, on trouve rarement la sexualité associée à la pollution. L'anthropologue anglaise donne l'exemple des Walbiri d'Australie. La structure sociale est fondée, comme pour tous les peuples australiens, sur les rapports matrimoniaux. La rareté des ressources dans un territoire désertique et la difficulté de survivre exigent de la part des membres une grande solidarité, chaque personne valide contribuant à l'entretien de ceux qui ne peuvent subvenir à leurs besoins. Elle s'accompagne d'une stricte hiérarchie qui met les jeunes sous la domination des vieux et les femmes sous celle des hommes. Éloignée de son père et des frères, la femme mariée ne peut en attendre la protection qu'elle serait en droit d'espérer. Son mari a sur elle pouvoir de vie et de mort ; les plaintes de la femme ne sont pas écoutées, son meurtrier est assuré de l'impunité. Elle ne trouve personne pour la défendre, les hommes, même lorsqu'ils séduisent une épouse, ne prenant pas fait et cause pour elle. Par ailleurs les Walbiri n'ont pas d'interdits concernant le sang menstruel et ne croient pas qu'il puisse souiller.

La plupart des sociétés primitives ne disposent guère des moyens d'appliquer les châtiments qu'elles décrètent contre ceux qui violent les interdits, notamment lorsqu'ils découlent du principe de domination masculine. Il s'agit de sociétés qui par ailleurs accordent aux femmes certains degrés de liberté, ou bien qui protègent les êtres faibles contre la violence des forts. Les règles de pollution sexuelle $\mathrm{y}$ sont plus développées. Les tabous du sang menstruel, fort répandus, permettent aux hommes de tenir les femmes à l'écart. On peut les rapprocher des mesures de ségrégation sociale prises à l'encontre d'une ethnie ou d'une caste traitée en inférieure. Parfois des cultes sont institués, qui exaltent la supériorité masculine dans l'ordre du sacré, en même temps qu’ils défendent les hommes contre les menaces que ren-

121 M. DOUGLAS : op. cit.

122 F.W. Young, A.A. BuCDAyAn : Menstrual Taboos and Social Rigidity, Ethnology, 1965, 4, 225-240. 
ferment les menstrues, et vont jusqu'à célébrer la capacité des hommes de procréer, seuls, des fils ${ }^{123}$.

Ces exemples ne doivent pas nous surprendre. Dans nos sociétés dites avancées et modernes, basées sur le principe de l'égalité et les droits de l'homme, l'inégalité est la règle, la violence, l'instrument, la propriété le stimulant d'une ségrégation tenace et d'une construction collective par emboîtement des classes, des races, des groupes, des nations superposés les uns aux autres. Les innombrables préjugés, auxquels les sciences contribuent parfois à leur insu, concernant les différences d'intelligence, d'ardeur au travail, d'esprit d'entreprise, de prédisposition criminelle, s'ajoutent à ceux qui présentent le contact avec le Noir, le pauvre, le sauvage, l'Arabe, dans les pays occidentaux, comme une souillure. L'odeur, la couleur, la texture de la peau, la forme du visage, le crêpelage des cheveux : autant de signes qui, à l'instar de la pollution par le sang menstruel, indiquent un danger d'impureté ; celui qui les possède ou qui transgresse les normes édictées à leur sujet perd en partie sa qualité d'homme aux yeux de la collectivité d'origine. Le but inavoué de ces préjugés est d'assurer l'intégrité et la domination d'un groupe, de discriminer étroitement le semblable du différent, les hommes avec qui il est licite d'avoir des relations d'appartenance et ceux avec qui il vaut mieux ne pas avoir de relations du tout, ou qu'il convient en tout cas de maintenir dans une position inférieure.

A l'arrière-plan de ces valeurs, de ces attitudes qui façonnent les traits symboliques de la nature humaine, se profile toujours l'armature solide des institutions fortes de leur bras séculier. Ces barrières visibles ou invisibles servent à cloisonner plusieurs sociétés, plusieurs cultures à l'intérieur d'un seul système social. Il n'y a guère eu de peuple où la catégorie la plus basse n'ait été plus ou moins exclue de ce qu'on estimait être la société au sens strict et obligée d'avoir sa propre société. Les attributs soit positifs mais dérogatoires, soit négatifs, qui ont été conférés aux femmes, la position effective qui a été la leur, l'isolement dans lequel on a cherché à les tenir, ont eu des résultats analogues. La société des hommes s’y est efforcée constamment,

123 A. Strathern : The Female and Male Spirit Cults in Mount Hajen, Man, 1970, 5, 571-586. 
et ce par bien des moyens. Le mystère que nous voulons préserver à leur sujet, l'enthousiasme que suscitent leur beauté, leur douceur ou leur abandon, miroirs à facettes multiples que nous leur tendons, continuent à dresser les mêmes barrières, cette fois teintées de poésie, et travaillées avec art. " Mais, rappelait Émile Durkheim, d'un autre côté, nous n'aurions pas connu ces besoins si des raisons depuis longtemps oubliées n'avaient déterminé les sexes à se séparer et à former en quelque sorte deux sociétés dans la société ; car rien, dans la constitution de l'un ni dans celle de l'autre, ne rendait nécessaire une semblable séparation ${ }^{124}$. "

Retour à la Table des Matières

124 E. DURKHEIM : art. cit,, p. 68. 


\section{Les hommes entre eux.}

\section{$\underline{\text { Retour à la Table des Matières }}$}

« Au revers qui paraît l'endroit, au cœur d'une prise sans emprise, au long des heures, à l'orée de l'indéfiniment prolongé de l'espace et du temps, attrape-dehors, attrape-dedans, attrape-nigaud, dis, qu'est-ce que tu fais ? Qu'est-ce que tu es, nuit sombre ou dedans d'une pierre ? »

Henri MICHAUX, Poteaux d'angle.

\section{(1) Devenir homme.}

Les diverses sortes de faits que nous avons rapportés précisent, sans nécessiter grand commentaire, la situation respective des deux sexes. Mais c'est à propos de l'initiation que la non-réciprocité des hommes et des femmes, par le truchement des enfants, prend corps, de même que la réciprocité des hommes appartenant à des groupes distincts prend corps à propos du mariage par le truchement des femmes. Il y a certes quelque outrance à rapprocher l'initiation du mariage, et leur contraste apparaît à maint égard simplificateur. D’autres facteurs interviennent - le clivage des générations, les impératifs de la reproduction des forces de travail, etc. - qui s'inscrivent tous dans le cadre d'une altérité orientée. Dans le discours social, la langue commune des règles de mariage instaurant la communication des hommes à propos des femmes se double d'une langue ésotérique de la noncommunication des hommes et des femmes à propos des enfants, mâles surtout. Les valeurs sont révélées sous l'espèce des signes, et les signes celés sous l'espèce des valeurs : on transforme ainsi en équivalent ce qui ne l'est pas - les femmes - et en non équivalent ce qui l'est - les enfants. Ces diverses opérations aboutissent à consolider et renforcer 1e groupe des hommes, en assurant sa position et sa force numérique.

Il est rare que les phénomènes sociaux se laissent enfermer dans le moule d'une régularité sans bavures. Les phénomènes d'initiation ne font pas exception, cela va sans dire. D’emblée il apparaît qu'ils concernent surtout les hommes et s'adressent à eux. L'initiation des filles, là où elle a lieu, reste un acte social mineur ; sa généralité et son ampleur sont moindres. Examinée dans une certaine perspective, 
l'initiation des garçons signifie assurément l'inclusion dans la communauté cynégétique, la consécration d'une maturité physique et technique et du savoir-faire lentement acquis. Être homme, c'est être chasseur, et à coup sûr l'adolescent aperçoit dans le rituel la réalisation d'un idéal dont il s'est pénétré très tôt. L'absence d'initiation des filles ou le caractère très fruste de leur initiation, comparée à celle des garçons, est inscrite dans les conditions de reproduction naturelle de leurs talents et de leurs échanges respectifs avec le milieu. On ne peut cependant manquer de voir, dans cette différence de traitement, une confirmation de la défaveur qui frappe le sexe féminin : en effet les manifestations de la vie sociale et religieuse étant réservées aux seuls initiés, les femmes en sont nécessairement exclues et se trouvent cantonnées, dès leur jeune âge, dans la sphère des occupations domestiques.

Pour saisir cette conversion et les obstacles qui s'y opposent, examinons les rites d'initiation. Ils comprennent des épreuves et des cérémonies. Les épreuves sont d'ordre physique et moral : le jeune garçon subit des brimades, des châtiments douloureux, parfois des opérations. Il est tenu de faire la preuve de son endurance et de sa virilité. Il n'a pas le droit d'approcher les femmes, et on l'isole en particulier de sa mère et de ses sœurs. Les adultes lui font sentir leur autorité de toutes les façons possibles. Les cérémonies, célébrant la cohésion et la pérennité du groupe, comportent toute une série de festivités et de rituels qui consacrent le passage de l'individu à un nouveau statut social, sa participation à des activités et son accès à un corps de savoirs qui lui étaient jusque là interdits. A la Terre de Feu, la puberté d'une fille donne lieu à un rituel simple ; après un festin, la jeune fille, couverte de peinture, est confinée dans une demeure à l'écart, et les femmes lui enseignent la conduite qui sera désormais la sienne. La puberté d'un garçon est l'occasion d'une cérémonie développée, le klokoten, qui comporte l'initiation à des secrets réservés aux hommes et des épreuves physiques sévères. Les individus des deux sexes sont séparés. Des adultes, des chamans costumés et masqués incarnent, devant les groupes assemblés, des êtres surnaturels qui effraient les femmes et les enfants, ceux-ci étant censés croire qu'ils ont affaire à des esprits. Au contraire ces " esprits » révèlent leur identité aux garçons, tout en leur faisant jurer le secret. Les jeunes gens subissant l'initiation ont auparavant jeûné, on les a obligés à passer vingt-quatre 
heures allongés dans la neige. Ils ont reçu des instructions minutieuses sur leur rôle d'adultes. Enfin, après un festin, le groupe reprend ses activités habituelles. Au Chili, chez les Indiens yaghan, le garçon qui doit être initié est revêtu d'un costume spécial. Il a trois " parrains ». Les épreuves sont sévères : le néophyte est obligé de boire en se servant d'un os d'oiseau, de se gratter au moyen d'un bâton, de se faire tatouer ; il apprend des chants et des préceptes professionnels et moraux. La cérémonie qui comprend des chants et des danses est suivie d'une bataille fictive entre les sexes, et d'un festin. Seuls sont considérés comme adultes ceux qui ont subi l'initiation, et seuls ceux qui l'ont subie deux fois ont accès au mystère de la cérémonie du kina, qui a souvent lieu après les rites d'initiation et rappelle le klo-koten des Ona et le yinchiana des Alcaley, laissant supposer que ces divers groupes ont échangé leurs rituels. Le kina représente une période mythique où les femmes dominaient les hommes en s'incarnant dans des esprits; les hommes découvrent la supercherie cachée derrière cette incarnation et prennent la direction de la société en soumettant les femmes. Ils jouent cette scène en se déguisant et en frappant de terreur les jeunes et les non-initiés.

L'initiation s'accompagne fréquemment de circoncision. Les Wiko de l'Angola bâtissent une maisonnette pour la circoncision à l'extérieur du village. Elle symbolise l'accord conclu entre les hommes et les femmes, permettant aux garçons de devenir des hommes dignes de ce nom. Pendant cette période, les relations sexuelles sont interdites entre les parents ; les mères cèdent les garçons aux pères, avec lesquels ils vont s'identifier. Cette distance des générations est clairement signifiée et représentée dans une danse exécutée par des hommes masqués. Le conflit des sexes est plus puissamment dessiné dans l'opposition de la maisonnette et du village, représentant respectivement les hommes et les femmes, dans le fait que les garçons initiés sont considérés comme étant pris aux femmes. Il n'y a jamais d'initiation pour les filles. Celles-ci sont mises au nombre des femmes, de même que les garçons non initiés. L’initiation détache le garçon de sa mère dont il quitte la hutte; il n'a plus le droit de se tenir près d'elle, et il est défendu à la mère de voir le pénis de son fils. Il est prêt à se marier, a le droit de s'asseoir avec les hommes pour manger, il peut prendre part aux procès et faire la guerre. Dans ce cas aussi, l'objectif principal de la cérémonie est de remplacer la mère par le 
groupe d'hommes, d'intégrer le jeune homme dans le clan du père. Et parfois, au cours du rituel, le père joue le rôle de celui qui sépare l'enfant de la mère. Les acteurs principaux de l'initiation - le groupe des femmes et le groupe des hommes - son but — le transfert des enfants mâles du premier groupe au second — l'atmosphère — celle d'un antagonisme entre les deux sexes — le résultat — la victoire des hommes sur les femmes - sont clairement définis. Dans un beau livre, la Mort Sara ${ }^{125}$ où il raconte sa propre initiation dans une tribu du Tchad, Robert Jaulin a décrit cette institution, de façon très vivante, nous faisant mieux pénétrer sa nature. J'en retiens quelques éléments parmi les plus significatifs.

La cérémonie se déroule dans le plus grand secret, les femmes sont tenues à l'écart de ses préparatifs. Pour effrayer les femmes et les néophytes, les hommes ont recours à toutes sortes de moyens, en particulier au bruit du rhombe qui siffle, vrombit, ulule ou rappelle le hurlement du vent. Cet instrument sert à reproduire les râles et les gémissements des ancêtres morts, dont les adultes imitent aussi la voix en appelant les " petits-fils » à rejoindre le groupe des hommes. (En Australie, on emploie à cet effet le bull-roarer, dalle de pierre que l'on fait tournoyer au bout d'une ficelle). Les garçons qui vont subir l'initiation sont toujours mis dans le secret de la supercherie, qu'il s'agisse du bruit des rhombes, des menaces, etc. La duplicité est un aspect essentiel de la cérémonie, servant à maintenir les femmes dans l'ignorance et à leur inspirer le respect. Paroles, bruits, gestes ne jouent pas un rôle véritable dans l'initiation mais ont pour fonction de préserver cette distance. L'initiation signifie une mise à mort — les esprits des ancêtres engloutissent les enfants - et une renaissance, puisque, délivrés par les hommes, les enfants reparaissent armés des qualités et des savoirs des ancêtres, c'est-à-dire qu'ils sont devenus les enfants de leurs pères. De façon symbolique pour l'initié et littérale pour le profane, l'acte d'initiation revêt souvent l'aspect d'une parturition : un homme s'empare d'un enfant et le fait naître homme, en usant de techniques magiques dérobées aux femmes dans la nuit des temps. Ces techniques perdraient tout pouvoir si les femmes en étaient spectatrices, c'est-à-dire si elles voyaient qu'en fait il n'est pas possible à un homme de mettre au monde un enfant. La légitimité de

125 R. JAULIN : La mort Sara, Paris, 1971. Plon. 10/18. 
l'appropriation est ainsi établie par la négation de la naissance conférée par la femme et par l'affirmation de la naissance conférée par l'homme, négation et affirmation que l'on veut faire accroire aux femmes, tandis que les hommes et les enfants sont justement instruits de la simulation et de la convention.

L'ensemble des rites a pour objet réel de séparer les femmes de leurs enfants, séparation moins physique que sociologique, puisque l'enfant quitte l'entourage féminin pour entrer dans le cercle des hommes. Si le garçon refusait de quitter sa mère, son acte l'empêcherait de devenir un être social et équivaudrait à un inceste, crime commis contre la tribu tout entière. Dans cette éventualité, ce serait les filles qui deviendraient des fils en passant de la mère au père.

Le néophyte entre dans le cercle des initiés, où la vérité des morts est le secret des vivants, en triomphant de nombre d'épreuves qui lèvent toute incertitude au sujet de son sexe puisqu'il s'y est conduit en homme. Il pénètre simultanément dans le monde du discours masculin qui a sa langue — on lui enseigne la langue secrète — ses ruses — il est mis dans le secret des rites - et sa clôture. Il apprend ce qui ne doit jamais franchir les limites du domaine des hommes, ce qui demeure caché aux femmes. Telles sont sans doute les raisons pour lesquelles, au cours des cérémonies, les hommes s'efforcent constamment de dérober leurs gestes et le sens de leurs pratiques aux regards de leurs compagnes et des mères, et déclarent que ce qui est recherché et obtenu dans l'initiation proclame « une conquête de l'homme sur lui-même et sur le monde matériel et domestique : cependant il se mêlait à l'affirmation systématique et bien chantée de la victoire sur les femmes un air de ruse qui semblait porter en lui-même sa contradiction, laissant entendre qu'il s'agissait moins d'une conquête sur le sexe faible que d'une opération qui, battant en brèche l'autorité de celui-ci, l'équilibrait ${ }^{126}$. "

La chimie des rapports sociaux a donc pour effet, grâce à des formules efficaces, de permettre aux pères d'engendrer leurs enfants mâles par parthénogenèse ; elle change les pères en " mères sociales », si

126 Idem, p. 124. 
l'on veut. Elle accomplit aussi une opération bénéfique : en faisant prendre aux mères les pratiques de l'initiation pour des réalités, elle les oblige, après les avoir dépouillées de leurs fils, à payer le prix nécessaire si elles veulent continuer à s'en occuper après la cérémonie, c'est-à-dire si elles veulent les racheter à leurs pères qui, eux, les ont obtenus pour rien.

$\underline{\text { Retour à la Table des Matières }}$

\section{(2) La ruse de la raison.}

Ces rituels témoignent de l'intersection de deux plans. Sur un plan, initier un garçon revient à changer son aspect, à définir son sexe avant, il est parfois censé avoir une nature féminine - à lui imposer un grand nombre de tâches qui le placent ailleurs, effacent les traces de son passé, lui inculquant à cette occasion la supériorité des adultes sur les jeunes. Là où son sexe est déterminé aux yeux de tous, dès la naissance, ces rituels n'ont pas lieu. Ainsi, à Samoa, l'absence de rite d'initiation à l'époque de l'adolescence vient de ce que l'enfant quitte la compagnie des femmes dès le sevrage; il commence, en accord avec les tabous de la nourriture, à prendre tous ses repas avec les hommes, et devient donc homme lui-même ${ }^{127}$. Pour rendre la rupture tangible, pour faire prendre au garçon conscience du monde nouveau dans lequel il pénètre, il arrive fréquemment qu'on l'envoie parcourir de grandes distances, errer de longues semaines dans la brousse, affronter des dangers. Ce n'est qu'en triomphant d'obstacles physiques et en faisant preuve de résistance morale qu'il est admis dans la communauté des hommes.

Le sens des épreuves, des mauvais traitements que l'on fait subir aux garçons n’a pas été correctement interprété. Au cours de l'enfance et avant l'initiation, le jeune adolescent est identifié et probablement s’identifie à un personnage féminin, la sœur ou la mère. C'est en tant que tel qu'il aborde le cérémonial. "Dans une certaine mesure, écrit Gregory Bateson à propos des Iatmul ${ }^{\mathbf{1 2 8}}$ et particulièrement au cours des premières phases de l'initiation, ils jouent le rôle des femmes. »

127 E. NORBECK, D.E. WALKer, M. COHEN: The Interpretation of Data : Puberty Rites, American Anthropologist, 1962, 64, 463-485.

128 G. BATESON : op. cit., p. 142. 
Parce qu'ils symbolisent l'autre sexe, les néophytes sont brimés, maltraités, et les initiateurs qui leur font manipuler leur pénis les désignent comme leurs « femmes ». Le vocabulaire usité en ces occasions pointe vers une analogie des relations entre hommes et femmes et entre initiateurs et initiés. Tout semble se passer comme si à la fois le rituel tendait à faire éprouver concrètement l'antagonisme des sexes, rendu actuel, et à démontrer l'issue inéluctable, en exorcisant la part de féminin qu'enferme le masculin. Il enseigne une loi de la société, en assurant la défaite de l'un et le triomphe de l'autre, en humiliant l'enfant de la femme pour glorifier l'enfant de l'homme, afin de préserver la pureté des membres du groupe qui ne contient plus que des hommes véritables.

Le temps de l'innocence domestique prend alors fin pour le jeune garçon. On lui révèle des secrets, on lui enseigne des interdits, on lui fait don d'objets ayant une valeur symbolique ou un but magique. C'est le véritable commencement de sa jeune existence, moment solennel vécu avec intensité : «L'avidité avec laquelle les jeunes nouvellement initiés entrent dans la vie cérémonielle et assimilent la signification cachée des traditions et usages mythiques de leur tribu est remarquable. Le jeune homme pratique avec assiduité les chants et danses des aînés. Quand il est au loin en train de chasser avec ses compagnons, il se lance dans les aventures les plus audacieuses, sans jamais cependant manquer de revenir en arrière et d'effacer les traces laissées sur le sol, signes révélateurs de sa présence, qu'une femme pourrait apercevoir ou qu'un ancien pourrait remarquer, auquel cas sa négligence lui vaudrait une réprimande ou une punition. Il voyage sans cesse, parcourt de longues distances pour assister aux initiations et autres cérémonies qui se tiennent parfois en des lieux très éloignés de ceux que fréquentent normalement les gens de sa tribu. Son habileté à la chasse, sa connaissance des points d'approvisionnement en eau situés sur toute l'étendue de son pays se développent ainsi de façon extraordinaire, et il voit les lieux des mythes et des légendes dont il absorbe si rapidement le contenu caché ${ }^{129}$. »

La sphère des hommes, dans le sens matériel et social, cessant d'être un domaine lointain, prend consistance pour lui. Mais aussi elle

129 N.N. Tindale, in G. Roheim : Héros phalliques, etc., op. cit., p. 215. 
se referme sur lui. Presque sans transition, il est devenu le détenteur des chiffres d'un code dont il ne doit divulguer la teneur sous aucun prétexte, le porteur d'un pouvoir qu'il lui faut conserver intact en toute occasion. Le sens profond de la connaissance lui est révélé : moins appréhension de l'inconnu que saisie de l'interdit, moins découverte du réel que déchiffrement de ses déguisements, elle est lumière et libération pour celui qui a acquis le droit de l'approcher, à condition de demeurer terreur et obscurité pour celui à qui ce droit est refusé. Ceci traduit la duplicité de la connaissance, inséparable de la dissimulation : la raison du sujet est ruse et par la ruse seule le sujet obtient sa raison d'être ce qu'il est. Le discours et les règles et les institutions sociales sont à double face, leur littéralité et leur sens ne coïncident pas. Tout ce qui est considéré comme vrai doit être masqué par des histoires, des légendes, des actions ad mulierem, afin d'empêcher que sa transparence ne nuise à la vie publique.

Mais dans ce domaine on n'atteint jamais la clarté, et le couple de ceux qui savent et de ceux qui ne savent pas est lié par une incertitude commune : ceux (ou plutôt celles) qui sont censés ignorer ne connaissent-ils pas la vérité ? ceux qui connaissent la vérité n’en ignorent-ils pas l'autre face ? La réalité sociale se profile comme envers d'une cérémonie. Le secret demandé tranche le dilemme sans le résoudre, rend la convention efficace sans pourtant lui assurer un fondement substantiel, puisqu'il est ruse, faux-fuyant, équivoque. La complicité oblige à une loyauté sans faille envers les compagnons. Même si la voie du mariage est désormais ouverte à l'homme, son alliance profonde est avec eux, les hommes, car c'est d'eux que dépendent sa subsistance, son autorité, son existence tout court. Il a surmonté l'épreuve de la mort et du mensonge pour atteindre la vie et la vérité. La page de l'enfance, qui était aussi celle de la non-initiation ou de la féminité classificatoire, est désormais tournée. L'individu s'éloigne vigoureusement de ce qui la représente dans le présent et la réalité, par désir d'accentuer le contraste, de démontrer sa nouvelle fidélité, de combattre ce qui, malgré tout, reste vivace en lui.

Sur un deuxième plan l'initiation se situe nettement dans le cadre de la coupure et de l'antagonisme entre femmes et hommes. Les enfants en constituent l'enjeu qu'il s'agit de dérober aux mères pour que les hommes en prennent possession. Ce passage est inséparable d'une 
certaine violence, en actes et en paroles. Gestes et rites, chants et mimes représentent la lutte des hommes contre les femmes ; les premiers remportent la victoire et exercent désormais la souveraineté : ce qui indique bien la tonalité de l'endoctrinement initiatique. Les cérémonies célèbrent scissions et combats ; sous la menace de la terreur, les femmes sont mises en demeure d'honorer le renouvellement du pacte qui les soumet. La réalité montre l'interruption effective du commerce sexuel, l'évitement réciproque des individus des deux sexes, assurant leurs positions respectives vis-à-vis de cet acte social par excellence qu'est le devenir homme d'un fils. S'il est entouré d'une telle aura, c'est que «le but de tous les rites d'initiation est de séparer les fils d'avec les mères et de les intégrer à la société des pères " ${ }^{130}$. Il ne s'agit pas de la simple promotion d'un adolescent, d'une concession formelle convenue entre le père et la mère d'une famille nucléaire d'aujourd'hui. L'enfant qui aurait pu coopérer avec la mère, devenir un de ses auxiliaires, lui est enlevé sans contrepartie, intégré et soumis au groupe des hommes. C'est probablement parce qu'ils ont conscience de ce dol que les peuples archaïques ont tissé, pour l'envelopper, ces comportements et ces mythes qui font appel aux ancêtres, aux obligations envers les morts, à la vie donnée par le père, créant un droit et le justifiant aux yeux de tous, parents et enfants. Des événements d'une si grande portée exigent qu'on s'y prépare de longue main, afin que la société, comme l'individu, soit en mesure de les supporter sans dommage grave. C'est à quoi servaient les interdits, prévenant l'éventualité d'un affrontement réel que les adultes simulaient. Saisis de terreur, ils en revivaient la possibilité, de même que nous revivons parfois en représentation une catastrophe qui aurait pu nous atteindre, éprouvant avec une intensité particulière la chose qui n'est jamais arrivée et qui suscite notre émotion pour le seul motif que nous l'avons crue possible. En l'occurrence, la catastrophe aurait été que les mères retiennent les enfants mâles, mettant en question l'ascendant des pères. La conquête est célébrée avec une réjouissance d'autant plus vive que le pire a pu être évité, les obstacles ont été franchis sans encombre; et une fois de plus les hommes sont sortis victorieux du combat qui est posé explicitement au cœur de la transmutation des adolescents. Autant que la ségrégation de l'habitat et de la nourriture, l'initiation qui transfère la possession des enfants mâles

130 G. RoHEIM : Héros phalliques, etc., p. 215. 
des mères aux pères est une des facettes du processus d'assujettissement du sexe faible au sexe fort.

Ce processus les saisit et les traite comme deux catégories sociales distinctes et les articule en tant que telles. Là où l'organisation collective et les liens de parenté sont fluides, chez les chasseurs hadza, par exemple, cette relation entre sexes comme relation entre catégories et son caractère premier ressortent avec vigueur : "Où que soient les Hadza, écrit Mary Douglas ${ }^{131}$, et quoi qu’ils fassent, ils sont toujours sous l'emprise de la division entre les sexes. Cette division sépare deux classes hostiles, dont chacune peut à l'occasion s'organiser en vue de la défense ou de l'attaque virulente contre l'autre. Cette conscience extraordinairement intense de la différence sexuelle est le seul niveau permanent d'organisation auquel les Hadza atteignent jamais. »

A la vérité, au-delà de telle ou telle population particulière, cette différence traverse toutes les manifestations sociales ou techniques significatives, de la langue au comportement, de la pensée au rite, de la disposition spatiale des collectivités à la répartition des tâches. Elle n’est pas sans répercussion sur la sexualité ; la frigidité est parfois le seul moyen de proclamer une liberté, une non-reconnaissance, une non-participation, dans sa passivité freinatrice, de la femme réduite à l'état de subordonnée, qui en même temps laisse à l'homme le soin de ranimer une impulsion qu'il a éteinte. De même, la virilité et la puissance masculines sont un reflet inversé qui s'affirme en tant que maîtrise et conquête d'une femme qui n'est là que pour les célébrer, consentante. Que ces relations changent, que la femme échappe à la dépendance, ainsi qu'il est arrivé récemment en Afrique, et l'équilibre sexuel s'en ressent. Robert Le Vine rapporte le cas des Yoruba chez qui il a pu observer une telle perturbation, qui se traduit de deux façons. D'une part, l'impuissance masculine, effective chez certains hommes mariés, redoutée par d'autres, et tenant une large place dans les conversations, comme elle est un souci majeur; d'autre part, le rituel et les coutumes qui amènent souvent les hommes à se déguiser en femmes : ainsi les prêtres revêtent des vêtements et coiffures féminins pour les besoins du culte et les hommes se groupent en un club

131 M. Douglas : Natural Symbols, Londres, 1970, p. 101. 
qui exige de ses membres qu'ils se travestissent en femmes lors de sa fête annuelle. «Ces fragments de preuves disparates, écrit-il ${ }^{132}$, laissent penser que, chez les Yoruba, la modification des rôles traditionnels a atteint le stade où non seulement les hommes éprouvent du ressentiment envers l'indépendance des femmes, mais sentent qu'elle les émascule, et l'envient. »

Pleine d'enseignement pour les problèmes du temps présent — la non-réciprocité et l'hostilité qui habitent les rapports sexuels — une telle réaction projette aussi une lumière sur le passé. Si la femme est apparue comme objet et signe du système de parenté, et, à beaucoup d'égards, elle le reste, c'est au prix d'un travail de longue haleine assumé par ces sociétés et qui ne s'est point relâché. Ces signes et ces objets étant aussi des êtres en chair et en os, une fraction distincte de la collectivité capable de résister à l'autre ou de déchirer le voile de la ruse dont se protégeaient les maîtres, la fragilité des effets et des mécanismes mis en jeu a dû souvent être ressentie à tel point que l'on y a lu une menace contre l'échelle des valeurs, contre le principe masculin dominant, donc contre l'ordre social et humain tout court. Le renouvellement continuel du pacte social, ce à quoi chacun est initié, et la raison partielle de l'initiation, annule la menace et restaure l'ordre. Derrière la relation que l'on veut naturelle, posant l'un des sexes au pôle des ressources à donner et recevoir - épouses, filles et sœurs et l'autre au pôle des donateurs et des donataires - époux, pères, frères - se profile la lutte des sexes, transposée, rationalisée et figurée de nombreuses façons, dont les traces dans notre organisme, notre psychologie, notre histoire sont si vives.

Captée au cœur des sociétés archaïques, cette lutte nous en donne une image moins unie et moins cristalline que celle que nous en avons. Elle atténue, par contre, l'étrangeté de ces sociétés « sans » visà-vis de nos sociétés " avec » en les faisant surgir, sur leur propre registre, plus vivantes, engagées dans un effort d'autocréation, au lieu de se contenter de réagir à une biologie exigeante et à un milieu ingrat, obsédées par leur subsistance. Sous le couvert des règles de parenté, sous la fiction de la séparation de la nature et de la culture -

132 R. LE VINE : Sex Roles and Economic Change in Africa, Ethnology, 1966, 5, p. 191. 
séparation à vrai dire du monde féminin et du monde masculin - à travers les mythes qui en sont le discours, et la mise en place des institutions, la science de ces sociétés la découvre être son vrai objet. Et l'on doit admettre l'existence d'une relation entre l'interdit de l'inceste, colonne vertébrale d'autres interdits, opérateur transformant la dichotomie sexuelle en hiérarchie sociale, et la lutte des sexes. Cette relation ressort de l'homologie entre le sens véritable de l'interdit - que j'ai exposé dans le chapitre précédent et qui doit apparaître plus clairement maintenant - et les rangs respectifs des hommes et des femmes sur l'échelle collective. Mais il faut encore examiner les raisons qui ont rendu nécessaires les liens consignés par la règle et leur teneur (note 7 ).

$\underline{\text { Retour à la Table des Matières }}$ 


\section{Chapitre X.}

\section{La moitié-nature et la moitié-culture}

\section{La différence fondamentale.}

\section{$\underline{\text { Retour à la Table des Matières }}$}

\section{(1) Deux phénomènes universels.}

Deux phénomènes ont le privilège de l'universalité dans les sociétés humaines : la prohibition de l'inceste et la division des activités selon le sexe ${ }^{133}$. Cette coïncidence n'est nullement fortuite : elle fournit un fil conducteur capable de nous guider vers une explication du sens qu'il faut donner à la lutte des sexes et à la règle qui traduit leurs rapports hiérarchiques. L'importance de la division, les motifs pour lesquels on la néglige dans l'analyse des phénomènes sociaux ont déjà été mis au jour il y a un demi-siècle : "La division par sexe est une division fondamentale qui a grevé de son poids les sociétés à un degré que nous ne soupçonnons pas. Notre sociologie sur ce point est très inférieure à ce qu'elle devrait être. On peut dire à nos étudiants, surtout à ceux et à celles qui un jour pourraient faire des observations sur le terrain, que nous n'avons fait que la sociologie des hommes et non pas la sociologie des femmes ou des deux sexes ${ }^{134}$. " Depuis l'époque où Marcel Mauss écrivait ces lignes, rien n’a vraiment changé. Les femmes sont toujours une terre inconnue, un à-côté de notre savoir, présentes dans la réalité mais absentes de la théorie (note 8), tout comme les peuples ou les classes, il y a moins de deux siècles, jouaient dans l'histoire le rôle de simples figurants. Celle-ci ne 
s'occupait guère que des rois et des nobles, les autres états n'étant pas inclus dans la définition de la société. Lorsque, à la faveur des révolutions à travers le problème social, ils firent entendre leur voix, l'histoire commença à les compter parmi les acteurs, et les sciences sociales s'efforcèrent de les connaître. Je reprendrai, bien incomplètement, la suggestion du grand sociologue français : je montrerai que les femmes, objets des rapports collectifs, sont aussi des termes de ces rapports, afin de faire ressortir le sens de la coïncidence signalée. Option qui invite à faire un retour à la division naturelle et un détour par les sociétés d'affiliation.

Pour quelle raison ? La famille archaïque combine une cellule sociale et une unité productive ; elle réunit les individus qui peuvent s'épouser et exclut ceux qui ne le peuvent pas, elle associe un homme et une femme dont chacun apporte des ressources et des savoirs complémentaires. Seule la réunion dans la cellule familiale semblait devoir requérir une explication, la prohibition et l'exogamie en ont fourni une. L'association dans l'unité productive et la différence qui la suscite semblaient aller de soi et n'avoir aucunement besoin d'être justifiées. Pour deux raisons : ce qui a trait à l'activité productive, à la civilisation matérielle, au côté instrumental de la vie collective, est tenu pour accessoire, n'engage pas l'essentiel des institutions, placées à un niveau plus élevé de la culture et n'en rend pas compte. Par ailleurs, l'attribution de la particularité des travaux féminins et masculins à leur spécificité biologique exclut la nécessité de telles institutions. Parmi celles-ci, la prohibition de l'inceste se limite au domaine de la parenté, à la différence entre les femmes que l'on peut épouser et celles qu'il est interdit d'épouser ; elle laisse de côté la différence entre les hommes et les femmes suivant les ressources et les talents dont ils disposent. En d'autres termes, la réciprocité des hommes, concrétisée dans l'alliance matrimoniale, la prend pour règle, se situant dans la société, tandis que l'association avec les femmes, se situant dans la nature, peut se passer d'une telle règle puisque la nature a déjà appliqué la sienne. L’hétérogénéité que je viens d’évoquer a empêché de voir la concordance des deux phénomènes ; c’est pourquoi il importe d'examiner de plus près les arguments avancés, afin de tirer au clair le processus de division en le replaçant dans le cadre qui lui a donné naissance. 
La division des activités selon le sexe des individus est conçue d'emblée en tant que réponse rationnelle à une donnée physiologique objective : les tâches propres à la maternité et à l'éducation des enfants. Les femmes y sont astreintes, les hommes en sont dispensés, ce qui entraîne le partage de la vie sociale, le confinement des premières aux menus travaux du foyer, et l'obligation pour les seconds de prendre en charge les devoirs importants de la collectivité. "Pendant la plus grande partie de l'histoire humaine, écrit l'anthropologue anglais Robin Fox ${ }^{135}$, les femmes s'occupaient de la tâche hautement spécialisée de mettre au monde et d'élever les enfants. C'étaient les hommes qui chassaient le gibier, combattaient les ennemis, et prenaient les décisions. Ceci est enraciné, j'en suis convaincu, dans la nature des primates, et alors que les conditions sociales, au cours d'un passé très récent, dans certaines sociétés avancées ont donné aux femmes l'occasion d'avoir plus souvent voix au chapitre, je continue à penser que la plupart d'entre elles accepteraient ce que j'affirme... mais que les nécessités purement physiques de l'enfantement rendent le rôle de la femme secondaire vis-à-vis de celui de l'homme pour ce qui est de prendre des décisions à tout niveau supérieur à celui des simples affaires domestiques. »

Outre la nécessité d'enfanter, les aptitudes organiques qui auraient empêché les femmes de participer pleinement, sur un pied d'égalité avec les hommes, aux entreprises collectives, la morphologie et la particularité de leurs aptitudes psychologiques et physiques les élimineraient de certains travaux productifs, ou à tout le moins limiteraient la gamme de travaux qu'elles sont en mesure d'exécuter. Voici quelques textes significatifs : "Les femmes sont capables de travail monotone et continu, disposition que ne partagent pas les hommes; les hommes au contraire sont capables de mobiliser de brusques sursauts d'énergie, et connaissent ensuite le besoin de se reposer et de récupérer leurs forces ${ }^{136}$. " " En vertu des différences premières entre sexes, un homme et une femme composent en coopérant une unité exceptionnellement efficace. L'homme doué d'une force physique plus grande est mieux à même d'entreprendre le travail le plus pénible...

135 R. Fox : Kindship and Marriage, op. cit., p. 32.

136 M. MEAD : Male and Female, New York, 1949, p. 164. 
N'étant pas handicapé, comme l'est la femme, par les fardeaux physiologiques de la grossesse et de l'allaitement, il peut s'éloigner davantage pour chasser, pêcher, garder les troupeaux, et faire du commerce. La femme n'est cependant pas défavorisée dans les tâches plus légères qui peuvent être accomplies à la maison ou dans les parages... Toutes les sociétés connues ont développé la spécialisation et la coopération entre les sexes plus ou moins selon la ligne de clivage biologiquement déterminée ${ }^{137}$. » Émile Durkheim voyait dans cette ligne de clivage plutôt la conséquence d'une division qui a conduit le sexe féminin à être doux, faible, à accaparer les fonctions affectives et l'a rendu psychiquement, anatomiquement et neurologiquement distinct du sexe masculin : " Non seulement la taille, le poids, les formes générales, écrivait ${ }^{138}$, sont très dissemblables chez l'homme et chez la femme, mais... avec le progrès de la civilisation le cerveau des deux sexes se différencie de plus en plus. »

Cet état de choses entraîne logiquement l'infériorité sociale des femmes, la supériorité des hommes. La chaîne des facteurs déterminants est visible : le dimorphisme biologique induit une répartition des activités collectives et productives, le degré d'importance de ces activités infléchit à son tour le statut de chaque sexe. Un économiste a calculé que les femmes occupent une position inférieure dans $73 \%$ des communautés agraires et dans $87 \%$ des communautés pastorales. Et de justifier l'écart en disant que la domestication des animaux est œuvre d'hommes, que partout le soin des troupeaux est confié aux hommes, alors que la culture n'est pas fermée aux femmes de la même façon. Ayant une base organique aussi manifeste, transmise par l'intermédiaire des lois génétiques, la division des sexes n'a besoin du soutien d'aucune autre loi artificielle pour être étayée et perpétuée. Cette conclusion n'est pas toujours tirée explicitement : elle va de soi, compte tenu du fait qu'on n'a pas cherché à dégager la loi qui lui correspondrait ni à examiner ses prolongements dans la structure sociale. Les savants ont répété à ce sujet, sur un ton plus retenu et froid, comme découlant de science sûre, ce que le Marquis de Sade avait déclaré avec plus de fougue et de parti pris : " Que vois-je en procédant de sang-froid à cet examen ? Une créature chétive, toujours infé-

137 G.P. Murdock : Social Structure, New York, 1949, p. 47.

138 E. Durkheim : De la division du travail social, Paris, 1960, $7^{\mathrm{e}}$ édition, p. 24. 
rieure à l'homme, infiniment moins belle que lui, moins ingénieuse, moins sage, constituée d'une manière dégoûtante... une créature si perverse enfin, qu'il fut très sérieusement agité dans le concile de Mâcon, pendant plusieurs séances, si cet individu bizarre, aussi distinct de l'homme que l'est de l'homme le singe des bois, pouvait prétendre au titre de créature humaine ${ }^{139}$. "Le concile, apparemment, n’a pas encore terminé ses travaux.

Cependant, en juxtaposant les données ethnographiques et historiques, on aboutit à plusieurs constatations : la spécialisation forcée dans la reproduction - les femmes produisent des hommes, les hommes produisent des biens - est compatible avec une activité productive à plein temps. Pendant la majeure partie de notre histoire, les femmes ont en effet assumé ou reçu des besognes essentielles. C'est seulement dans les familles de purs " consommateurs » des classes aisées (aristocratie, bourgeoisie, etc.) que leur rôle se borne à entretenir la flamme du foyer et à mettre au monde des héritiers. Les nombreuses pratiques anticonceptionnelles - espacement des naissances, infanticide, etc. - peuvent être mises en rapport direct avec la participation des femmes au travail collectif. Réduisant les soins à donner aux enfants, elles dégagent du temps supplémentaire qui est consacré aux occupations productives. Les tâches féminines ne sont pas toutes disposées autour de la maison, elles ne sont pas moins lourdes ou moins chargées de responsabilité dans la collecte, l'agriculture ou le commerce. Le contenu de ce que l'on entend par travail masculin et travail féminin — le fait n'a rien de surprenant — est interprété de manière très variée. En Amérique du Nord, la préparation des peaux est réservée aux femmes; dans le Sud-ouest, ce sont les hommes qui s'en occupent. Dans le nord de l'Arizona, chez les Hopi - en Égypte, rapporte Hérodote - les hommes, filent et tissent, alors que chez leurs voisins, les Navaho - et en Grèce - ces métiers sont féminins. Les Bantou ne permettent pas aux femmes de soigner le bétail, mais les femmes des Hottentots traient les vaches. En tous lieux cependant est attestée la différenciation des activités, et le travail exécuté par les femmes est généralement estimé être de qualité inférieure, il est méprisé ou évité même quand on a affaire à des opérations techniquement voisines. 
Donc le dimorphisme sexuel, les facultés organiques ne constituent pas une raison suffisante pour justifier la distribution rigoureuse des occupations confiées aux uns ou aux autres. D’ailleurs, si cette distribution avait un fondement anatomo-physiologique, si elle répondait spontanément aux aptitudes de chaque sexe, on ne comprendrait pas pourquoi de nombreuses sociétés ont cru bon d'interdire aux femmes de chasser ou de se servir d'instruments à l'usage exclusif des hommes. La galanterie masculine et la sollicitude du futur père vis-à-vis de ses compagnes (généralement plurielles) n’étant pas des explications recevables, quoi qu'en aient pensé leurs auteurs, force est de croire que les mesures édictées étaient destinées à créer une distance, à maintenir intacte une différenciation du savoir-faire, bref à consolider un clivage qui n'avait rien de biologique. On peut ajouter que la hiérarchie n'apparaît pas comme l'effet de la division et de la répartition des occupations productives et reproductives; la biologie étant impuissante à en rendre compte, l'écart social ne renvoyant pas à un écart naturel, on est bien obligé d'admettre que c'est la hiérarchie qui entraîne la valorisation ou la dévalorisation des travaux et leur distribution ultérieure. Elles supposent tension et séparation, concurrence et compétition, coordination et distance ; elles n'ont pas trait à des individus ou à des familles qui décident tranquillement des tâches appropriées à chacun, mais à la société dans son ensemble, et, à travers les diverses sociétés, à l'ensemble de l'humanité. En tout état de cause, on ne peut soutenir l'hétérogénéité de tout ce qui a trait à la parenté, que l'on situerait dans la société et du côté de l'homme, et de tout ce qui concerne la division, que l'on situerait dans la nature et du côté de la femme.

Retour à la Table des Matières

\section{(2) Le sexe avant la classe.}

L'impossibilité, d'attribuer la répartition des activités selon le sexe à des conditions biologiques incite à l'envisager dans le cadre d'un développement plus général : celui des divisions naturelles. Notre étude se borne ici à la phase qui nous importe et qui est aussi la plus longue dans l'histoire de l'humanité; la collecte est devenue l'occupation des femmes, la chasse l'apanage des hommes, au cours 
d'une série de transformations, que nous avons analysées, des ressources, du volume de la population, des facultés organiques et inorganiques, des échanges avec le monde extérieur. En l'absence de ces transformations, il n'y aurait point eu de dichotomie entre les sexes, puisque, ainsi qu’on la noté : « La collecte de nourriture, pour autant que nous sachions, ne crée pas une division sexuelle du travail, car les deux sexes y procèdent de la même façon ${ }^{140}$. » Leur hétérogénéité du point de vue des savoir-faire, des instruments, n'est pas une situation de fait, le partage optimal conscient des qualités et des moyens, ni le résultat de l'intervention d'une planification cachée; elle traduit l'aboutissement d'une suite de changements dans lesquels ils ont été entraînés. L'insertion de chaque sexe dans un domaine distinct n'est pas la réponse à un milieu matériel prédéterminé, aux aptitudes des individus, mais leur œuvre, le contrepoint de leurs relations en tant que groupes, des facultés et des milieux qu'ils ont découverts, conservés et organisés. Comme toutes les divisions semblables ${ }^{141}$, elle se conforme à des exigences générales. La séparation ferme et presque rigide des collecteurs et des chasseurs, dans cet ordre d'idées, est une condition sine qua non. Elle a pour fonction d'orienter les interactions avec les forces matérielles, en évitant toute interférence ; elle détermine les groupes ainsi circonscrits à se consacrer à la transmission et au perfectionnement des talents qui leur sont propres, à renouveler sans cesse le lien avec l'environnement, environnement végétal et animal en l'occurrence, chaque sexe ayant seul accès à un des aspects de la nature. La situation ne comporte pour eux ni alternative ni contrainte : ils sont constitués et se constituent dans l'univers qui est le leur, lequel imprègne toute leur façon de voir et d'agir, ils y sont pour ainsi dire adaptés après l'avoir instauré. Un tel groupe - ou catégorie naturelle - tend à considérer son univers comme unique ; il l'envisage aussi comme général et, par conséquent, cherche à l'élargir en rapportant tout à lui. Toute diminution de cet univers passe pour un amoindrissement du groupe et tout accroissement est salué comme un enrichissement. Son propre art, son savoir, sa réalité sont vécus et traités comme l'art, le savoir, la réalité. Par la force des choses, la scission prend le sens d'une négation, entraîne l'édification de barrières de tous ordres autour du noyau dur qu'une fraction de la société

\footnotetext{
140 V.H. STEWARD : chap. cit., p. 335.

141 S. Moscovici : Essai sur l'histoire humaine de la nature, op. cit.
} 
s'attribue, le refusant à l'autre fraction. Cette dernière, vue sous un certain angle, est identifiée à la matérialité, au non-savoir, puisque lui fait défaut l'art que possède la première fraction. C'est ainsi que le chasseur rejette le collecteur du monde de l'habileté et de la culture ; et plus tard l'agriculteur fait de même pour le chasseur, l'artisan pour l'agriculteur, l'ingénieur pour l'artisan. La quintessence de l'humain change ainsi de figure, de même que le non-humain ; la distance instaurée se trouve justifiée et reçoit force de loi.

Le caractère absolu du contraste, de la distinction qu'il introduit, ne devrait pas surprendre. Dès qu'un groupement humain est amené à se conduire et à se penser comme s'il concentrait dans ses moyens et ses fins le principal de la réalité matérielle et sociale, le reste lui paraît accessoire et dépourvu d'autonomie. Ses pratiques intellectuelles, idéologiques, voire religieuses ressentent le contrecoup de ce besoin d'asseoir son existence, de légitimer ses relations vis-à-vis d'un autre groupement. Ces phénomènes concomitants de la division naturelle n'ont pas fait l'objet d'une étude systématique. On en trouve cependant d'abondants témoignages - le chasseur, le pasteur, l'agriculteur sont des personnages clé, leurs rapports des thèmes récurrents — dans les mythes et les religions, que ceux-ci aient trait à la période qui suit la révolution néolithique ou à celle qui la précède ${ }^{142}$. Les beaux travaux de MM. Vernant, Détienne et Vidal-Naquet achèvent de nous convaincre que la philosophie grecque a continué dans cette voie.

La séparation ne va pas sans antagonisme. Une catégorie qui possède des facultés spécifiques se développe aux dépens des facultés et des ressources d'une autre catégorie qui lui paraît limiter son propre essor. Les chasseurs sont entrés en conflit avec les collecteurs, car les époques ou les régions les plus favorables aux entreprises cynégétiques ne correspondaient guère aux époques et aux régions permettant la cueillette. Plus tard les agriculteurs ont pâti de la concurrence des artisans, dans la mesure où ceux-ci les dépouillaient de la partie domestique de leur savoir-faire en travaillant leurs matières premières de façon plus habile et plus raffinée. La liste pourrait être allongée. Chacune de ces catégories, corrélativement, ayant acquis ou s'efforçant

142 H. FrANKFORT : Before Philosophy, Londres, 1949 ; J.B. PRITCHARD : Ancient Near Eastern Texts relating to the Old Testament, Princeton, 1955. 
d'acquérir un droit à l'existence sociale, sinon la suprématie, en tâchant de devenir une force productive reconnue, sinon dominante, en donnant pour assise aux richesses de la collectivité ses propres ressources matérielles et intellectuelles, intensifie résistances et oppositions. Le récit biblique du meurtre du pasteur Abel par l'agriculteur Caïn les concrétise sous leur forme extrême. L'enjeu est double : intégrité du champ d'activité, position focale dans la vie collective. La catégorie nouvelle, mettant en avant ses talents et ses productions, enlève à celle qui prédominait sa raison d'être, son pouvoir. Ses arts dévalorisés sont rabaissés au second rang ou tombent en désuétude ; elle ne trouve plus exactement sa place dans l'univers matériel changé. Les sociétés qui en subissent le contrecoup prennent nécessairement parti ; entérinant les séquelles de ce processus, elles adoptent les nouveaux arts et les nouvelles productions et se réorganisent à cet effet. Et si l'artisanat ou la chasse prennent de l'ascendant, le collecteur ou l'agriculteur, pour nous en tenir à ces cas, s'effacent, deviennent subalternes. Avant d'atteindre la prépondérance, une catégorie nouvelle demeure quelque temps marginale. Dans certaines tribus africaines, forgerons et travailleurs du bois, tenus à l'écart du village ; privés du droit de cultiver la terre, réduits à quémander des céréales, sont associés à l'hyène ou au renard, animaux figurant la brousse, la nonculture et la mort ${ }^{143}$. Le comportement n'a rien d'exceptionnel. ; à force de pression et d'identification, l'artisan prenait les traits qu'on lui attribuait, se faisait inquiétant, rusé, etc. En Grèce, où il a triomphé, il est intelligence, réflexion ; et les philosophes s'instruisent à son école.

L'antagonisme s'inscrit dans un ordre et ne s'atténue qu'en se convertissant en un ordre. Ses termes sont modifiés dans et par l'histoire: ils sont néanmoins partout et toujours rangés sur une échelle de valeurs, donnant à la disparité son orientation. « En remontant plus haut dans le passé, écrit Gilbert Simondon ${ }^{144}$, on trouverait que telle ou telle civilisation faisait aussi un choix entre les techniques nobles et les techniques non nobles : l'histoire du peuple hébreu accorde un véritable privilège aux techniques pastorales, et considère la

143 G. CAlAme-Griaule et Z. Ligers : L'homme-hyène dans les traditions soudanaises, L'Homme, 1961, I, 89-119.

144 G. Simondon : Du mode d'existence des objets techniques, Paris, 1958, p. 86. 
terre comme maudite. L’Éternel agrée les offrandes d'Abel et non celles de Caïn : le pasteur est supérieur à l'agriculteur. La Bible contient une multitude de schèmes de pensées et de paradigmes tirés de la manière de faire prospérer les troupeaux. Les Évangiles au contraire introduisent les modes de pensée tirés de l'expérience de l'agriculteur. Peut-être pourrait-on, aux origines des mythologies et des religions, trouver un certain parti pris technologique consacrant comme noble une technique et refusant le droit de cité aux autres, même quand elles sont effectivement utilisées : ce choix initial entre une technique majoritaire et une technique minoritaire, entre une technique valorisée et une technique dévalorisée, donne à la culture qui incorpore les schèmes techniques ainsi découverts un aspect de partialité, de nonuniversalité. »

Séparation, antagonisme, hiérarchie, symptômes et moyens, ont partie liée avec la division naturelle, quel que soit le sexe ou la classe sociale auxquels appartiennent les groupes concernés. Toutefois, en tant que catégorie de possesseurs de talents spécifiques et de producteurs, les femmes, comparées aux hommes, ont été rejetées à un rang inférieur, et ce indépendamment de leurs productions et de leurs talents. Friedrich Engels a observé avec pénétration - et nos propres remarques ont tendu à montrer comment et pourquoi — « La division du travail entre les sexes est conditionnée par des raisons autres que la position de la femme dans la société ${ }^{145}$. " A quoi tient cet écart ? En vérité la société amène les divisions successives, leurs moyens et leurs symptômes à épouser sa courbure, à s'exprimer dans son langage : celui de la disparité et de la différence des sexes notamment. Pour en saisir le sens, il ne faut pas oublier que la domination du sexe masculin sur le sexe féminin, sans avoir un caractère génétique - les essais entrepris afin de le prouver étant peu concluants ${ }^{146}$ — plonge ses racines dans les sociétés d'affiliation. Là, elle a trait uniquement aux liens sexuels et statutaires; les liens avec le milieu, les facultés, les activités sont pratiquement identiques pour les deux sexes. Les mâles assurent tout au plus la défense du groupe, le respect des limites territoriales. La spécialisation des chasseurs et des collecteurs étend ces liens au domaine des facultés et des échanges avec le règne animal et

145 F. ENGELS : L'origine de la famille, Paris, 1954, p. 51.

146 L. Tiger : Men in Groups, Londres, 1969. 
végétal. L'ascendant des hommes est limité, du même coup, par une double dépendance vis-à-vis des femmes. D’une part, en devenant chasseurs, c'est-à-dire en formant une classe de producteurs définie, les hommes viennent à établir les femmes en tant que classe de producteurs équivalente et différente ; ils sont contraints de s'assurer leur concours, de veiller à ce qu'elles leur réservent un approvisionnement indispensable ; à ce titre les tâches féminines demeurent compatibles avec les leurs. A l'uniformité initiale se substitue le dédoublement. Par ailleurs, dans les collectivités de primates et celles des premiers hominiens, la scission du sexe masculin en individus reproducteurs et non reproducteurs se répercute sur l'organisation des échanges, sur la délimitation des aires d'exploration des ressources. Sur ce plan - et par voie de conséquence sur le plan social - se font jour des tensions qui traversent toutes les générations, masculines surtout. Il faut supposer qu'avec la division, ces tensions se transportent aux rapports entre les sexes, les différences entre les générations passant à l’arrière-plan.

D'autre part, on se rappelle que les sociétés d'affiliation ont tendance à rejeter les jeunes mâles à la périphérie. Le seul lien, faible et menacé, il est vrai, des bandes d'adolescents est avec l'assemblée des femelles, avec les mères. Les cohortes de mâles s'en désintéressent : l'intégrité du groupe est à ce prix. Or les groupes d'hommes ont intérêt à assurer le recrutement des garçons, à briser leurs attaches avec le groupe des femmes. Dès l'instant où chaque sexe détient des facultés particulières et règne sur son champ d'action spécifique, la progéniture mâle devient un enjeu de taille. La suprématie maintenue dans la vie collective permet aux hommes de s'assurer la disposition de cet enjeu. A côté d'eux, les femmes constituent une entité jouissant d'une autonomie certaine, d'un pouvoir non négligeable de s'opposer ; les hommes sont obligés de composer avec elles, tout en essayant de les plier à leur propre autorité. A cet égard, le contrôle social, dans ces collectivités, s’exerce immédiatement sur les personnes ; la hiérarchie est fondée sur la régulation de leur participation aux actes essentiels de la vie des groupes, et les objets se confondent avec la personne. Au contraire, dans les nôtres où le contrôle social s'exerce sur les objets, la hiérarchie est fondée sur la propriété, et la personne se confond avec l'objet. 
Sans vouloir dépasser les limites d'une spéculation raisonnable, on peut affirmer que la première division de la chasse et de la cueillette a eu lieu et s'est diffusée dans le cadre d'une hiérarchie de participation où les écarts de rang étaient fortement accentués et marqués dans tous les plis de l'organisation collective. Ils ont contribué à accroître la séparation et l'antagonisme au point que leurs manifestations ont pu faire écrire à Bronislaw Malinowski que «la division du travail est enracinée dans la brutalisation du sexe faible par le sexe fort $"{ }^{147}$. Les divisions suivantes ont été prédéterminées; elles ont préservé le même style de répartition des facultés humaines. S'y conformant, les occupations, les ressources, les modalités d'invention et de reproduction des savoirs, les liens singuliers avec le milieu ont été l'œuvre des hommes ou des femmes, portant la marque d'un sexe défini, ont été attribués soit aux hommes soit aux femmes. Arts, instruments ou pouvoirs matériels ont été jugés, à un moment ou à un autre, suivant leur degré de développement ou les aires géographiques - il y a une agriculture ou un artisanat « féminins » de même qu'il y a une agriculture ou un artisanat " masculins » - comme étant l'apanage exclusif de l'un ou l'autre sexe, frappés de son sceau. Contrepartie évidente, on les a ordonnés suivant la ligne de clivage préétablie et de ce chef valorisés ou dévalorisés, estimés principaux ou secondaires, dominants ou subalternes. La hiérarchie sociale, dont on discerne ici les origines lointaines, a interféré avec la hiérarchie des divisions naturelles en lui imprimant sa coloration; Cette combinaison modifie son point d'application et ses propriétés, en l'élargissant à la sphère productive et reproductive qui auparavant n'était pas de son ressort.

On serait en droit de penser que, pendant toute cette période, la double dépendance envers les ressources des femmes et leur progéniture - a gardé sa force intacte, qu'elle s'est manifestée sur tous les plans des comportements collectifs, imposant le maintien des institutions et des normes appropriées. Et ce d'autant plus que, avant que la distance des possédants aux non-possédants ne reflète fidèlement l'état de propriété et que ne s'opère la rupture entre ceux qui produisent et ceux qui vivent du travail d'autrui, les relations entre groupes sociaux sont simultanément des relations entre groupes de produc-

147 B. MALINOWSKI : The Family among the Australian Aborigines ; a sociological Study, Londres, 1913, p. 287. 
teurs. Le rang des individus dans la société est déterminé par le rang de leurs occupations dont l'honneur ou le déshonneur rejaillit sur eux. L'humanité se compose surtout de chasseurs, collecteurs, agriculteurs, pasteurs, etc., chacune de ces subdivisions indiquant aussi leur appartenance à un clan, une tribu, un sexe, une classe d'âge, des pouvoirs biologiques et psychiques particuliers, en un mot le statut social qui est le leur, auquel sont associées des prérogatives ou des servitudes. C'est pourquoi les articulations des groupements humains sont vécues et pensées dans le prolongement de l'articulation des espèces biologiques ou physiques: "Dans l'une et l'autre perspective (des castes et des groupes totémiques), écrit Claude Lévi-Strauss, il faut admettre que le système des fonctions sociales correspond au système des espèces naturelles, le monde des êtres au monde des objets ${ }^{148}$. »

Ainsi les groupements sociaux sont identifiés aux catégories naturelles : la société est une nature, la nature une société. Pour autant que groupes et catégories, avec leur champ d'action respectif, sont assimilés à l'un ou l'autre sexe, le double mouvement de socialisation et de naturalisation contribue à renforcer l'asymétrie, l'opposition qui les sépare dans l'existence et l'évolution collectives. Il les projette également sur le plan cosmique, car tout ce qui apparaît et se dessine sur la terre comme au ciel, tout ce qui se manifeste, croît, réagit, et les régions mêmes de l'espace et du temps, est soit féminin, soit masculin. L’épistémologie est sexuée, la physique aussi, et l'astronomie, la technologie, la biologie. Mythes et religions en ont recueilli l'écho et lui ont donné une forme durable, qu'il serait grave d’interpréter à la lettre, plus grave encore de prendre pour de simples fictions de l'esprit, sans rapport avec la manière dont l'homme construit le réel ${ }^{149}$.

L’éclosion de la société de classes a opéré une transformation radicale. Les circuits économiques et politiques indirects se sont substitués aux circuits directs, la hiérarchie et l'organisation fondées sur l'appropriation ont remplacé la hiérarchie et l'organisation fondées sur la participation des hommes et des femmes. La qualité de possédant ou de non-possédant est décisive et court à travers tout le tissu des au-

148 C. LÉVI-STRAUSS : La pensée sauvage, Paris, 1962, p. 169.

149 C. LÉvi-Strauss : Mythologiques, Paris, 1964, 1967 et 1968. 
tres relations. Au critère du sexe servant à classer et diviser les facultés, les ressources et leurs praticiens - ceux que Marc Bloch nommait les " porteurs d'invention » — succède le critère, également organique, qui oppose la main au cerveau, le travail manuel au travail intellectuel, celui-là jugé indigne d'un être humain — c'est-à-dire du maître - et celui-ci couvert d'honneurs parfois fictifs. Les principes masculin et féminin, ordonnateurs du monde, sont détrônés au profit du principe spirituel et du principe matériel, dichotomie de l'intelligence et de la substance, dans la fabrique et la composition de l'homme et du monde. Leur montage désigne clairement le terme supérieur et le terme inférieur. L'épistémologie est démonique — démon qui peut être forme, idée ou corps - tout comme l'astronomie, la physique, la biologie, la psychologie. Ce bouleversement mis à part, les procédés n’ont pas changé qui traduisent, dans une société, les différences, les oppositions, les disparités des catégories de producteurs et inventeurs d'arts, le hiatus qui sépare les modalités particulières de se rapporter au monde matériel et de le remodeler. Ils semblent dénoter une nécessité du dur combat contre la nature, pour employer une expression courante, combat, en fait, d'un groupe humain contre un autre groupe humain et contre une nature qu'il refuse de reconnaître pour sienne.

La division a sa dynamique propre, génératrice de faisceaux de talents et de réalités, de frontières qui introduisent entre les hommes un classement virtuel. Dans les espaces ainsi délimités, la collectivité, suivant les lois de sa structure, distribue ses membres, les sépare ou les réunit, dirige leur mouvement vers le centre ou la périphérie, les régions supérieures ou inférieures. De nombreux savants ont méconnu cette dynamique, en recherchant ses causes dans les particularités anatomo-physiologiques de l'espèce, en confondant ses effets, notamment les formes qu'a prises la subordination des femmes dans les sociétés archaïques, avec les effets d'un processus génétique. Parler à ce propos d'appropriation des femmes par les hommes, comme l'a fait Friedrich Engels, de rapport de classes ou d'un contrôle identique au contrôle relevé dans les sociétés de primates, explication dont se contentent éthologues et anthropologues, c'est demeurer au niveau des prothèses analogiques. Ces assimilations indues ravalent la répartition des activités, les rapports entre hommes et femmes au niveau de problèmes de pure technique, d'économie ou d’instinct. Les disparités 
sont interprétées à la lumière du dimorphisme, le conflit entre eux est rendu inutile ; de plus les contraintes biologiques en font une impossibilité théorique, à vrai dire, et le rejettent à un plan secondaire. Par contre, si l'on se persuade de la fragilité de ces constructions, l'ampleur de la dynamique décrite rend compte de la lutte des sexes ; elle en donne les raisons et en explique la persistance. Par ailleurs, la lutte matérialise la confluence de la division, conçue selon ses vraies dimensions, et d'une organisation sociale qui s'y manifeste, garantissant aux hommes l'autorité du point de vue institutionnel et productif. De la sorte, la subordination se trouve élucidée, sans qu'il soit besoin de faire appel à une foule de reconstitutions hypothétiques, allant de la promiscuité aux charges de la maternité, de la taille du cerveau à la génétique des primates, de la pénurie des épouses à l'insuffisance des pouvoirs physiques ou intellectuels. Et aussi le fait qu'à la longue les sociétés se soient ralliées à une coutume ou à une loi — la prohibition de l'inceste - qui accompagne le polymorphisme de cette lutte dans les diverses strates du réel qu'elle traverse. 


\section{La place de la prohibition de l'inceste dans le partage exogamique et la division naturelle.}

$\underline{\text { Retour à la Table des Matières }}$

L'interdit s'accorde avec les exigences principales de l'organisation concrète que les hommes se sont donnée au cours de cette longue période: hommes et femmes sont strictement séparés quant aux ressources, aux facultés, et aux instruments. Les interférences sont réduites et les domaines respectifs sauvegardés afin d'assurer la continuité des occupations nécessaires aux collectivités. Chaque fraction de la société se trouve associée à une fraction du réel, par le jeu des barrières dressées et des prohibitions édictées, clôtures de son horizon intellectuel et pratique, garantissant aussi la préservation des pouvoirs relatifs, des fonctions singulières au sein de l'ensemble. Ici l'individuation est au service d'une interdépendance que l'on doit revivifier sans discontinuer : les efforts sont distribués, les déplacements ordonnés, les tâches obéissent au rythme annuel et saisonnier. Dès la naissance, le sexe détermine la place de l'individu dans un secteur ou un autre, le sélectionne en vue de perpétuer les groupes sociaux et producteurs. L'insertion des filles dans le groupe des femmes et le recrutement des garçons par les hommes s'imposent à l'évidence, et tout concours à cette fin. "Il suffit peut-être d'attirer l'attention, écrit un anthropologue démographe au sujet des sociétés primitives ${ }^{150}$ sur deux différences majeures (avec notre société) : celle des relations mère-fils et père-fille. Aussitôt qu'un garçon sort de l'enfance, il ne subit plus que la seule autorité paternelle. Sa mère cesse d'avoir son mot à dire dans son éducation et d'exercer sur lui une réelle influence. D'autre part, la fille vit sous contrôle presque exclusif de sa mère, et, en dehors des accords prénuptiaux, son père s'intéresse peu à ses affaires. "

Pour y parvenir, rien n'étant donné dans la constitution biologique qui incite à cette divergence et à l'acceptation d'une relation préférentielle avec le père ou la mère, les hommes ou les femmes, il est indis-

150 E. Evans-PRITCHARD : La femme dans les sociétés primitives, Paris, 1971, p. 41. 
pensable de préparer les enfants : toute une série de règles écartant les garçons des filles, les soumettant à des disciplines diverses, entraînent l'évitement réciproque et façonnent leur comportement avec pour résultat l'isolement et l'opposition qui suivent obligatoirement la différenciation : «Dans la mesure où les sociétés ont une division sexuelle du travail et une certaine forme de mariage, un rôle sexuel bien défini devient une nécessité fonctionnelle lorsque le garçon s’approche du seuil de participation à ce modèle social ${ }^{151}$. " Sur l'axe des contemporains ou sur celui des générations, dans la sphère de la reproduction des personnes comme dans celle de la production des biens, la ligne de partage qu'il importe de tracer et de respecter est celle de la masculinité et de la féminité.

La séparation de la mère et du fils se présente comme un aspect particulier d'une telle tendance. Les hommes ayant tout intérêt à s'associer les garçons au lieu de les éliminer, la seule façon pour eux de les obtenir consistait à les prendre aux femmes. Pour les garder dans le groupe en les empêchant de se coaliser, de faire cause commune avec celles-ci, il fallait aussi les fixer au père, en interdisant toutes relations avec la mère susceptible d'affaiblir les liens noués. Les sociétés d'affiliation ne connaissent guère ce danger : l'autonomie du couple reproductif et du couple nucléaire, l'absence consécutive des rapports de parenté y rendent l'inceste à la fois inutile et inopérant. Joignant les deux couples dans l'unité constitutive qu'elle représente, la famille fait apparaître la possibilité de l'inceste et son opération est un moyen de réaliser cette unité : l'union de la mère et du fils enlevé au père résorbe le couple reproductif dans le couple nucléaire, tout comme l'union de la fille et du père refusant de la donner à un autre homme identifie le couple nucléaire au couple reproductif ; dans le premier cas, la femme contrarie l'autorité de l'homme, dans le second cas le groupe familial se soustrait à ses liens de dépendance envers la société. Pallier un tel danger, conserver les relations avec les enfants et s'en faire des auxiliaires transforme l'évitement des rapports prolongés, notamment des rapports sexuels avec la mère, en un préalable de l'initiation qui consolide ce mouvement. Lorsque la propriété viendra à dominer, et avec elle l'argent et le pouvoir séculier, le cérémo-

151 F.W. Young : The Function of Male Initiation Ceremonies, American Journal of Sociology, 1962, 68, p. 381. 
nial restera, l'institution deviendra inutile. Le droit de nature parlera à sa place qui « veut que le vainqueur soit le maître et seigneur du vaincu. D'où il s'ensuit que par ce même droit un enfant est sous la domination immédiate de celui qui le premier le tient en puissance. Or, estil que l'enfant qui vient de naître est en puissance de sa mère avant qu'en celle d'aucun autre, de sorte qu'elle le peut élever ou l'exposer, ainsi que bon lui semble et sans qu'elle en soit responsable à personne. Si, par le contrat de mariage, la femme s'oblige à vivre sous la puissance du mari, les enfants communs seront sous la domination paternelle à cause de cette même domination étant déjà sur la mère ${ }^{152}$.

Quelques auteurs ont fait état, se référant à ces temps plus reculés, d'un combat entre père et fils et d'une entente entre eux pour la possession des femmes. Il serait plus exact de dire que toutes les sociétés archaïques sont centrées, au contraire, sur la lutte des pères et des mères pour la possession des enfants, des fils. Bien des rituels servent principalement à cette fin, car « il est également évident que c'est le père qui sépare le fils d'avec la mère, et que c'est le conflit œdipien qui est à l'origine de cette séparation » ${ }^{153}$. A moins qu'il ne soit plus plausible d'affirmer que cette séparation et les causes qui l'ont provoquée sont elles-mêmes à l'origine du conflit œdipien, en tant que structure psychologique élaborée et transmise par les collectivités qui se sont succédé jusqu’à ce jour.

Les différences de rang se diffusent dans toutes les branches de la vie collective. La disparité des hommes et des femmes se heurte théoriquement, dans la famille, à un obstacle. Il serait impossible de la préserver si frères, sœurs, pères, mères, fils consanguins ou classificatoires se mariaient entre eux. Un homme ne pourrait en effet traiter sa sœur, sa fille, etc. comme un objet, une ressource ou une inférieure si des liens d'ordre parental coexistaient avec les liens d'ordre conjugal. Par ailleurs les rapports de filiation qui uniraient la femme à l'homme seraient rejetés dans une position subordonnée : l’homme lui-même en serait affecté. De plus, à supposer que le partage exogamique n'éloigne qu'une partie des femmes, l'autre partie demeurant dans le

152 T. HobBes : De Civitate, chap. IX.
153 G. RoHEIM : op. cit., p. 108. 
clan, les fils circulant aussi bien que les filles, l'obstacle ne serait pas levé : il serait difficile d'appliquer des règles communes de nonréciprocité à un groupe aussi composite.

La prohibition de l'inceste répond à cette double exigence de séparation et de hiérarchie. De manière générale, elle dresse une barrière entre les sexes, convertit la différence en scission ; qu'il s'agisse en effet de mère, de sœur, de fille d'un côté, de père, fils ou frère de l'autre, chaque fois est en question la constitution d'un rapport interne à chaque sexe, l'établissement d'un autre rapport qui les éloigne. Pour se préparer à la vie qui sera la leur, il convient que filles et garçons vivent une rupture, soient aiguillés vers des voies différentes avant qu'une association trop forte ne s'établisse entre eux. On les prépare en même temps à un changement de rang, la fille et la sœur étant destinées à devenir socialement inférieures au père, au frère. La charge exceptionnelle de l'interdit qui pèse sur la mère se comprend dans la mesure où l'on vise non seulement l'éloignement mais aussi la mise en disponibilité des enfants mâles afin de les concéder entièrement aux hommes. De la sorte, en tant que non-reproducteurs, ils peuvent rester dans la cellule clanique ou familiale. Le risque de voir les rôles sociaux remaniés diminue encore lorsque les garçons sont intégrés au groupe des hommes, réengendrés par ceux-ci. On a souvent remarqué que bien des peuples primitifs n'établissaient pas de relation de cause à effet entre les rapports sexuels et la conception. On peut invoquer le manque de connaissances; il est aussi permis de supposer qu'ils avaient de bonnes raisons de ne pas apercevoir cette relation, car le réengendrement des enfants par les hommes, même assistés des morts, n’aurait pas effacé les droits que les femmes auraient fait valoir. Le rituel initiatique scelle cet éloignement au plus profond, comme il scelle toutes les séparations, donnant l'apparence du passage d'une génération à une autre au passage d'un sexe à l'autre, d'un rang à l'autre.

L'ampleur de la prohibition, la signification qu'elle confère à l'individuation des groupements en les réintégrant aux divisions déterminantes des facultés et des activités humaines, vient de ce qu'audelà des êtres particuliers, des hommes et des femmes, elle s'étend aux connaissances, aux forces matérielles, aux aliments, etc., pour atteindre une portée cosmique. Un esprit positiviste y verrait une projec- 
tion fantasmagorique de l'homme vers l'univers, de la réglementation du commerce biologique à celle des événements naturels. Outre que les pratiques s'y plient, mettant en harmonie la réalité sociale, matérielle, et l'imaginaire, rien ne prouve que la direction ait été conforme à celle que l'on décrit, en allant des groupes sociaux vers les objets et le milieu; je croirais volontiers que l'interdiction a été signifiée sur tous ces plans à la fois, puisque, partout, l'univers des femmes est distinct de celui des hommes. Étant solidaires, ce que l'on validait dans l'ordre de la société se prolongeait dans l'ordre de la nature par l'intermédiaire des êtres humains, l'échelle de ceux-ci servant à graduer tout le reste et réciproquement. L'évitement du mélange, le confinement des parties aux lieux qui leur sont assignés, sont les conditions d'unité, de symétrie dans toute architecture de ce type. Par la suite, on a préféré se guider sur la disparité du haut et du bas, du monde céleste et du monde terrestre, de l'esprit et de la matière, de l'être et du devenir, chacun maintenu en sa pureté, et l'on sait que la philosophie des Grecs, aux âges classiques, réfléchit, à sa façon, un intermédiaire humain. Dans son cadre propre, la prohibition a joué le même rôle ordonnateur et différenciateur.

En empêchant, au niveau de la parenté, les membres d'un groupe de s'unir entre eux, elle lève l'obstacle qui s'oppose à l'introduction de la gradation propre à la société dans la famille, dans le clan. Émile Durkheim l'a bien vu qui écrivait : " La cause qui empêche les hommes et femmes d'un même clan de contracter des relations conjugales est aussi celle qui les oblige à réduire au minimum possible leurs relations de toutes sortes ${ }^{154}$. " Le mariage avec les femmes d'un autre groupe souvent rival, est la solution. A la place des sœurs, épouses, non soumises, non discriminables socialement ou seulement au prix de grandes difficultés, on dispose d'épouses, de sœurs soumises et discriminables. Elles ont pour qualité première d'être étrangères, ce qui justifie pleinement le comportement dont on use envers elles. "Pour ce qui a trait à la mentalité du chasseur, lit-on dans un ouvrage qui fait autorité ${ }^{155}$, les Ona (de la Terre de Feu) nous renseignent de façon très explicite. Il y avait un certain nombre d'institutions très ingénieuses qui avaient pour but de réduire au minimum les hostilités

154 E. DuRKHEIM : art. cit., p. 47.
155 R.B. LEE, I. DE VORE : op. cit., p. 94. 
entre les groupes et de canaliser l'agressivité en la dirigeant vers les étrangers et les épouses, elles aussi des étrangères à strictement parler. " Étrangères, on peut les assimiler à des sujettes, les maintenir dans une position subalterne, sans qu'il y ait conflit entre la subordination et la parenté, entre les liens établis à l'intérieur de la famille et ceux qu'on entretient hors d'elle. De plus, en donnant ses femmes, on fait par l'intermédiaire d'un autre groupe ce qu'on ne saurait aisément faire soi-même, à savoir traiter en inférieures ses propres sœurs et filles. Ce dernier cas n'est pas exclu : il y a des limites cependant et cette conduite interfère avec d'autres. Au contraire, le transfert des femmes d'un groupe à l'autre, leur partage, les associations qui se renouent de clan à clan concourent à préserver un accord qui fixe chaque sexe et âge à sa place.

Et l'on arrive, théoriquement, à ce résultat que les femmes d'un clan sont originaires d'un autre clan ; chaque clan — ou bande ou famille étendue - se compose de deux moitiés extérieures l'une à l'autre et situées aux deux extrémités de l'échelle sociale. Pour l'Australie, un anthropologue décrit sobrement la situation des femmes ainsi introduites : " La condition de la femme y est telle qu'elle a peu de chances d'agir sur la langue, les cérémonies, ou les fonctions des hommes dans la culture totale. Ces épouses importées sont des non-entités silencieuses, jusqu'à ce qu'elles aient appris la langue du groupe de leur mari ; et à ce moment-là elles s'intègrent rapidement à la bande ou à la tribu du mari ${ }^{156}$. " Instrument servant à communiquer, à coopérer, dès sa création le langage est simultanément instrument servant à ne pas communiquer, à dissocier. La prolifération des dialectes, des syntaxes, des champs sémantiques, des canaux physiques de transmission, des signes, la superposition des doubles et des triples sens répondent au désir d'être entre soi, d'insérer l'incompréhensible et le différent dans le compréhensible et le commun. Peut-être les hommes ont-ils commencé à parler pour leurrer les animaux, et écrit pour leurrer ceux qui parlent, se donnant ainsi une des armes les plus puissantes d'arrachement et de menace. Que l'on songe à l'éventail des possibilités d'intimidation et de terreur contenues dans une langue que l'on ne possède pas. Suivant ce sillage, à bien examiner les religions, les mythes, les philosophies et les scien-

156 J.B. BIRDSELL : art. cit., p. 196. 
ces, dans les lois du travail qu'ils effectuent pour extraire une signification du chaos, le même effort de rattacher les effets à leurs causes, en un mot, de rendre intelligible l'inintelligible, réinjecte le chaos dans la signification, sépare les effets de leurs causes, double la lumière d'une zone obscure. La pensée désigne en effaçant, construit en dérobant. Pris dans la turbulence des mouvements opposés, celui qui y est entraîné entrevoit le noyau dur autour duquel flottent des figures mobiles, saisit, de ce qui est dit, la séduction, de ce qui est pensé, la découverte. Dans cette étrangeté de la langue, des coutumes, du commerce intellectuel, les nouvelles épouses sont dépossédées de leur monde, font l'apprentissage de la sujétion, objets et signes d'un discours dont elles se sentent exclues et démunies.

Par ce système, compte tenu de l'âge précoce auquel sont conclues les unions, en particulier dans le groupe patrilinéaire, ce dernier contient rarement une femme adulte née à l'intérieur du clan qui puisse revendiquer d'autres droits que ceux de protection découlant de la filiation. De même, les tâches de chaque sexe sont fortement inscrites sur la grille des valeurs, et, par le jeu des échanges, assurées sans défaillance. Le mariage, libératoire pour l'homme qui entre dans le cercle des détenteurs de l'autorité, du pouvoir cérémonial et du savoir supérieur, est sujétion pour la femme qui se voit reléguée à un rang inférieur et enfermée dans la région domestique où elles poursuit la besogne qu'accomplissait sa mère. La mainmise des hommes sur les enfants mâles touche des femmes qui appartiennent, en quelque sorte, à un autre groupe, à un clan différent qui n'a pas la possibilité de se reconstituer, de battre en brèche le clan dominant : elle ne fait que renforcer la soumission. L'alliance des hommes est rongée à cet endroit par une profonde ambiguïté : à l'instant où ils célèbrent réciprocité et communication par le truchement des femmes, de leurs lignées respectives, ils proclament aussi qu'en elles ils se traiteront dans la nonréciprocité et la non-communication en étrangers et en inférieurs, qu'ils ne cesseront pas d'être adversaires. C'est pourquoi Georges Davy constatait que le mariage est imbriqué «à la lutte des sexes et par voie de conséquence aux luttes privées entre familles et entre tribus ${ }^{157}$. Le fait qu'il soit inséparable de la prohibition de l'inceste exprime le but auquel tend celle-ci dans les collectivités.

157 G. DAVY : La foi jurée, Paris, 1922, p. 118. 
Elle est leur œuvre, se rapportant aux liens naturels et sociaux. Sa première mission est de favoriser, en réaffirmant la distance entre les domaines d'activité, les talents appropriés, les relations entretenues avec le milieu, l'autonomie de chaque sexe en tant que sujet doué de valeurs et de responsabilité. Sur le plan des organisations tribales, claniques et familiales, on retrouve, en transparence, la nécessité qui les fait refluer vers le cadre de vie singulier. Dans ces limites, on peut dire que la règle assure la stabilité de l'ordre matériel sur lequel est édifiée la société ; si cette scission n'existait pas, si hommes et femmes ne suivaient pas la voie désignée, la disposition de l'environnement serait compromise. Son application à la production des ressources en individus, savoirs et biens garantit la pérennité du milieu ; les membres de chaque groupe sont d'emblée orientés dans une direction déterminée, en même temps qu'ils prennent en charge un secteur de la réalité objective. Ensuite, en prescrivant cette séparation, elle l'a adaptée à la courbure de la structure sociale, à sa valorisation du domaine masculin, au contrôle exercé par les hommes sur les femmes. Là se dévoile sa véritable raison d'être, car c'est bien dans la sphère des interactions de notre espèce avec le monde - la recherche réglée d'un partenaire n'est qu'un aspect secondaire - que cette prohibition a été indispensable et qu’elle a opéré. Manifestation sociale de la division naturelle - d'où la convergence que j'ai signalée plus haut - elle est l'instance qui, une fois le couple de la chasse et de la collecte formé, a permis aux sociétés de dériver les institutions idoines à la création et au développement de leur nombre et de leurs facultés. Elle a aussi donné à la différence entre les sexes sa signification humaine.

Agissant sur la famille, unité productive, elle la raccorde en tant que cellule sociale aux propriétés d'ensemble du système collectif. Ensuite se généralise le caractère d'opposition et d'asymétrie, l'autonomie devenant dépendance unilatérale. Le partage exogamique, infléchi, est mis en accord avec la hiérarchie de participation avant de s'ajuster à la hiérarchie d'appropriation sous la figure de l'échange le sexe masculin s'arrogeant, là aussi, l'autorité et la préséance sur le sexe féminin. Les règles de parenté tracent le tableau des alliances, entre hommes, la sociologie le précise, pour que les femmes, permutées, se tiennent au rang qui est le leur, celui d'objets et de biens, ta- 
bleau d'alliances dirigées donc contre elles, ce que la sociologie laisse seulement entendre avec discrétion. La circulation des épouses les transforme en possessions et signes, l'interdit servant à la fois d'opérateur de la transformation et de marque de ce qui est transformé.

La loi concernant l'inceste est aussi à double inscription. Associée à la division, elle exprime la fonction de la société tournée vers son fondement naturel la distribution de ses membres entre les divers secteurs du réel, de la connaissance, du travail, le modelage des instincts et l'ancrage des comportements appropriés. Associée à l'exogamie, elle traduit la fonction de la société d'unir entre eux les groupes qui la composent, orientant à cette fin le pouvoir qui s'exerce par le canal de la parenté dans une direction sexuellement définie. Elle est, à cet égard, l'écho d'un ordre ancien, la primauté de l'homme étant respectée, et l'indice d'un ordre nouveau, dès lors qu'ont été changés le répertoire et le théâtre où les deux sexes se rencontrent pour agir. 


\section{Chapitre XI. \\ Éloge de l'ordre}

\section{L'inceste, menace de la culture.}

\section{$\underline{\text { Retour à la Table des Matières }}$}

\section{(1) La Grande Peur.}

« Si la lutte s’établissait entre les hommes et les femmes, ce serait bien autre chose que celle qu'on a vue entre les Grands et les Petits, entre les Blancs et les Noirs. »

\section{NAPOLÉON : Lettre à Gourgaud.}

L'ordre humain est dans sa totalité un hommage à la prohibition de l'inceste. Il s'y reconnaît en son essence d'interdit et de partage. La barrière permanente dressée pour endiguer les pratiques contraires aux intérêts de la collectivité prévient la résurgence d'un état révolu, impose un terme à la tyrannie des instincts. Rompant avec le passé, soumettant les pulsions à la discipline des coutumes, la prohibition apaise les tensions, fournit une armature à la culture, comme en témoigne l'horreur de l'inceste que tous les peuples ressentent et conjurent. « Il y aurait ainsi dans l'horreur de l'inceste, écrit Georges Bataille ${ }^{158}$, un élément qui nous désigne en tant qu'hommes, et le problème qui en découle serait celui de l'homme lui-même en tant qu'il ajoute à l'animalité ce qu'il a d'humain. » Signe du dérèglement, de la dissolution des liens qui unissent les membres du groupe, l'inceste atteste aussi la compétition meurtrière qui oppose le père et le fils, les hommes pour la possession des femmes. A son horizon on voit poindre le resserrement des affinités biologiques au détriment des affinités socia- 
les, la rupture des freins intellectuels et moraux, la retombée dans la nature. Il inspire l'effroi inhérent au mélange de ce qui doit être séparé, à la transgression de la règle, à la menace de sanctions proportionnées. Et voici le nœud du problème. M'appuyant sur les idées que j’ai avancées, à moins de le nier, je dois récuser les motifs invoqués pour expliquer la réprobation dont on entoure l'inceste. En effet, à aucun moment, je ne fais l'hypothèse d'un état d'indifférenciation initiale des sociétés, de l'union sexuelle aléatoire des individus ou de la possibilité d'un comportement incestueux fréquent ayant des répercussions graves. Je ne présuppose pas non plus que l'interdit ait été requis par un désordre existant ou une menace de désordre ; a fortiori je me refuse à rendre compte de sa genèse en invoquant leurs conséquences délétères réelles ou présumées.

L'horreur en question est-elle donc immotivée ? Ne signifie-t-elle pas le retour à la promiscuité, l'abandon de la culture, la revanche de l'animal sur l'homme? Le fait est que, malgré les certitudes affichées quant à ses effets, contestables, le mystère à son sujet demeure épais, ses racines obscures. " Nous ignorons l'origine de l'inceste, constatait Sigmund Freud, et ne savons même pas dans quelle direction chercher ${ }^{159}$. " Le seul fil conducteur dont nous disposions nous est évidemment fourni par la prohibition elle-même. Nous avons vu ses deux lignes, d'intervention : elle sépare les sexes en les plaçant dans leur champ respectif d'action, et isole notamment les enfants mâles des mères en vue de l'initiation et par l'initiation ; elle instaure entre eux un système de relations hiérarchiques. Les actes prohibés doivent donc être envisagés, sous l'angle de leurs répercussions, dans le contexte des rapports entre groupes d'hommes et groupes de femmes. Précision inutile, jugera-t-on. Non pas, étant donné que les théoriciens passés et présents ont généralement étudié les rapports internes aux groupes d'hommes - opposition des fils aux pères, alliance en vue de la possession des femmes, etc. - et du seul point de vue masculin. « Nous retenons, déclarait l'anthropologue Luc de Heusch, seulement la perspective masculine, car il paraît assez établi par l'argumentation de M. Lévi-Strauss que la réglementation du mariage est essentiellement œuvre des hommes ${ }^{160}$. "

159 S. FREUD : Totem and Taboo, op. cit., p. 125.

160 L. De Heusch : Pourquoi l'épouser? op. cit., p. 17. 
Si la prohibition s'affirme, à contre-jour de cette réglementation, comme soutien d'une différence et d'une hiérarchie, la menace que l'on décèle un peu partout dans l'esprit des collectivités a trait à leur avenir et non à leur passé ; ce n'est pas la réapparition du désordre que l'on redoute, mais la disparition de l'ordre existant. L'interdit n'est pas tourné vers un danger qui a été, qui pourrait renaître ; il a pour but d'éloigner un danger qui plane comme une éventualité, la réaction logique à une organisation - la société des hommes - conçue en vue de la domination. Il ne sert pas à régler les unions sexuelles, ni à transformer en groupe social le groupe biologique consanguin. Strictement parlant, il est difficile de cerner les traits d'un groupe qui soit exclusivement biologique. Qui dit groupe, quelle que soit l'espèce, indique un degré de sociabilité forcément supérieur à zéro et la présence d'une norme défendant l'inceste n'est pas indispensable à cet effet. Nulle part, à aucun moment, l'homme n'affronte la biologie dépourvu de culture, la sexualité menant la danse des unions, dans une famille réduite à la consanguinité, sans que le choix des partenaires soit dirigé. Il n'est nul besoin de réprimer la promiscuité qui n’a pas été aux commencements et n'est pas attendue au détour d'une transgression d'allure catastrophique. Le caractère de l'aversion inspirée par l'inceste ressort beaucoup plus clairement lorsqu'on rapproche la prohibition de toutes les conduites discriminatoires ${ }^{161}$, des préjugés, des lois écrites ou non tendant à préserver une distance sociale. Ils ne tablent nullement sur la réalité des comportements redoutés, sur la cohabitation des personnes visées par l'interdit et de celles qui décrètent les mesures d'exception, sur leur mélange spontané ou arbitraire, sur un état d'indistinction ou d'égalité auquel on mettrait un terme par ce moyen. Les préjugés raciaux contre les Noirs en Amérique du Nord ont commencé avec l'esclavage ; les règles de ségrégation ouverte ou cachée à l'encontre des pauvres se fondent sur l'opposition prétendue indéracinable de la richesse et de la pauvreté ; les doctrines rabaissant le travail manuel et rehaussant le travail intellectuel partent d'une distinction faite, entre deux formes de travail et la position respective de ceux qui les exercent. Dans tous ces cas, différence et asymétrie sont données dès le départ, et pour en établir les manifestations on prend

161 R. FIRTH : We, the Tikopia, A Sociological Study of Kinship in Primitive Polynesia, Londres, 1936. 
prétexte d'un signe - couleur, vêtement, quartier, langage, main ou cerveau — qui est ensuite fixé et développé logiquement.

Ce signe sert à ranger les éléments du monde en supra-ordonné et subordonné, juste et injuste, haut et bas, humain et non humain, permis et prohibé, exaltant ce qu'a d'unique la race, la classe, la faculté possédée, tandis que tout ce que l'on juge étranger, décrété inférieur et contraire aux valeurs, est rejeté à la limite de l'humanité véritable. Les règles de ségrégation et de classification sont parfois négligées dans les affaires privées - même en ce qui concerne la prohibition de l'inceste - mais les rapports publics en tiennent nécessairement compte. Partout où la race, la classe, la profession confèrent un statut et des prérogatives, tout ce qui déroge à la règle constitue pour le système un danger qu'il faut éliminer à tout prix. Néanmoins la dichotomie, ciselée par les doctrinaires, inscrite sur les tables de la loi par les législateurs, assimilée par les coutumes de l'ensemble de l'appareil social, acceptée par ceux qu'elle lèse autant que par ceux qu'elle favorise, montrant qu'ils se complètent jusque dans le détail, dévoile précisément la réversibilité des termes, l'antithèse de la thèse : «En conséquence, note Hegel, la vérité de la conscience indépendante est la conscience servile. Sans doute, cette conscience servile apparaît tout d'abord à l'extérieur de soi et comme n'étant pas la vérité de la conscience de soi. Mais de même que la domination montre que son essence est l'inverse de ce qu'elle veut être, de même la servitude deviendra plutôt dans son propre accomplissement le contraire de ce qu'elle est immédiatement ; elle ira en soi-même comme conscience refoulée en soi-même et se transformera, par un renversement, en véritable indépendance ${ }^{162}$. "

Chacun voit clairement - d'où la grande peur de l'éveil — que l'ordre fait de restriction, de sélection, de limitation, fournit les matériaux d'un autre ordre, sa négation, qui ne peut être que libération, ouverture, élargissement. L'histoire a enregistré d'abondance ce phénomène. Les classes d'hommes, les classes de maîtres qui se sont succédé ont vécu dans la hantise constante du jour où les serviteurs refuseraient d'obéir, où les esclaves se révolteraient, où la haine éclaterait au grand jour, où les instruments animés devenus indociles se mouvraient

162 G.W.F. HegEL : La phénoménologie de l'esprit, Paris, 1939, t. I, p. 163. 
vers des fins propres, où la propriété serait arrachée aux individus et restituée à la communauté, où les étrangers submergeraient les nationaux, où les races sauvages, inférieures, cesseraient d'accepter le sort qui leur est réservé. Chaque fois, le mélange, le contact, l'indifférenciation, l'abolition de la loi sont pris pour symbole de la fin du règne humain, et l'endogamie - celle des richesses aussi bien que celle des êtres - se profile comme fossoyeur de la société. La disparition des fonctions et des rôles établis, le partage de la propriété, l'égalité et la confusion des rangs ont été constamment ressentis, décrits comme annonciateurs du retour de la nature enfouie, de l'offensive de la sauvagerie dépassée, de la ruine de la civilisation, anéantissant les efforts dépensés pour les refouler. La hiérarchie passe pour être la colonne vertébrale de tout, et ce qui la brise brise le tout :

"Quels fléaux, quels sinistres présages, quelles discordes,

Quelles tempêtes sur mer, quels tremblements de terre,

Commotion des vents, terreurs, changements, horreurs

Dévient, brisent, déchirent et déracinent

L'unité et la calme harmonie des États

Jusqu'en leurs fondements. Oh ! quand la hiérarchie est ébranlée,

Seule échelle qui accède à tous les hauts desseins,

L'entreprise est bien malade. Comment les sociétés,

L’ordre dans les écoles, les confréries dans les cités,

Le commerce paisible entre rivages séparés,

Les droits de primogéniture et de naissance,

Le privilège de l'âge, des couronnes, des sceptres, des lauriers,

Pourraient-ils, sans la hiérarchie, garder leur place ? » ${ }^{163}$

En contraste avec l'interdit édicté afin de préserver une distance, de faire respecter une échelle de privilèges et d'obligations différant pour chaque sexe, l'inceste a l'aspect d'une force qui ronge et mine cette échelle, détruit l'emboîtement soigné des rangs, sape les bases de l'alliance entre hommes, nie l'éthique qui s’y appuie. Son éventualité sert de révélateur, dénonce la fragilité d'une relation où l'un des termes domine l'autre, la précarité des positions respectives, l'assise extra-rationnelle d'une rationalité qui n'a d'autre but que de garantir à une fraction de la collectivité le contrôle de l'autre fraction. Le tremblement des hommes supérieurs quintessence de l'homme tout court, à l'idée qu'on puisse bouleverser la hiérarchie, en modifier les critères,

${ }^{163}$ W. ShaKesPeARe : Troilus and Cressida, I, 3, 96-108 (trad. A. Digeon). 
n'a d'égal que la crainte de voir se dresser, élevant la voix, ceux qui occupent le bas et fracasser, au cours d'une fête sanglante et joyeuse, dans des massacres et des spoliations, l'architecture parfaite construite avec tant de peine dans les hauts quartiers de l'esprit. Les violations d'un interdit sont autant d'indices qu'il convient de mobiliser tous les moyens de l'intelligence et du pouvoir afin de lui barrer la route. Depuis qu'il existe un ordre social ayant ses maîtres, cette peur panique se manifeste avec suffisamment de constance pour que l'on puisse estimer qu'elle constitue un symptôme manifeste des concomitants idéologiques et psychiques du geste même par lequel ils s'arrogent des droits sur autrui, lui imposent leur loi. A chaque instant la présence de cet autrui soumis ravive la peur, rappelle l'existence de l'acte décrété illicite, fait planer le spectre de l'anarchie, de la « confusion des relations » (ce qu'exprime à la lettre le caractère chinois qui signifie l'inceste ${ }^{164}$ ), en un mot le Babel permanent, confusion des langues. Les contrevenants outrepassent les bornes, qu'on leur a indiquées, de la société, et mainte légende dénonce cette violence, cause du devenir animal, qui change l'homme en singe, rappelant le danger qu'on encourt à succomber à l'acte prohibé, à céder au geste tentateur. Se livrer à l'inceste équivaut à faire disparaître les écarts, à niveler les différences séparant hommes et femmes : c'est bien ce qu'évoquent les métamorphoses en monstres, les promiscuités retrouvées, les animalités de légende. Si ces menaces, cette horreur ont amené certains à déduire que promiscuité, monstruosité, animalité appartiennent au passé de l'espèce humaine, ceux-ci n’ont fait que suivre le schéma des justifications mais non celui des réalités. Dans les réalités chargées d'incertitudes et d'antagonismes comprenant des groupes d'hommes et de femmes en chair et en os, la prohibition apparaît comme une violence qui infléchit leurs rapports et donne à l'inceste une odeur de poudre, libération vis-à-vis d'une loi qui le consacre à tous les étages de la vie collective. Il est l'arme que le sexe féminin, directement visé par la loi, peut brandir, le symbole de la capacité de ce sexe à bouleverser le monde arrangé par les hommes et pour eux, en bloquant le jeu des règles de parenté, en retenant les fils au lieu de les donner, en refusant d'être l'objet qui ouvre la voie à la réciprocité des hommes, le partenaire dupé d'une transaction inégale. Il n'est pas le seul acte à ruiner le pouvoir masculin, à lui faire éprouver le ressentiment sourd,

164 G. LiEnHARDT : Social Anthropology, Londres, 1966. 
amer, mais non impuissant de celles sur lesquelles s'exerce l'autorité - que l'on songe aux fantasmes répandus des épouses harpies, des vagins dentés - mais il est le seul à avoir une résonance collective profonde.

Retour à la Table des Matières

\section{(2) Fdipe et Antigone.}

Rien n'exprime mieux son mystère que la tragédie qui, entre toutes, a pris la place centrale parmi nos mythes, la tragédie d'Edipe. Deux énigmes résolues, aux conséquences diamétralement opposées, en résument le déroulement. La première, obscure quant à sa portée et à ses implications, est posée dans la rencontre avec le Sphinx, qui est rencontre avec la prohibition effective, obstacle sur la route qui mène vers le trône de Thèbes, obstruction au mariage avec la mère. En se soumettant à son épreuve, on lui reconnaît le pouvoir de définir l'homme ; il se distingue de l'animal, il se sépare de l'élément féminin. Du même coup, on voit pourquoi cette créature extraordinaire propose ses énigmes : afin de s'assurer que les conditions préalables à l'acte interdit sont bien remplies, et d'indiquer celui qui peut le commettre. Sous aucun prétexte, il ne saurait être un hybride, mi-homme mi-animal, mi-masculin mi-féminin, un Tirésias par exemple. Le filtrage des acteurs de la tragédie atteste que l'inceste est possible, et n'a de valeur qu'à partir de l'instant où il est le fait d'un homme, inclus dans la société humaine, exclu lorsque règnent l'indifférenciation et la promiscuité animale, sur le versant du monde où le Sphinx n'est qu'une femme dévorante. Fort de cette prouesse, Edipe apporte à la cité dont il devient le roi prospérité et gloire.

La seconde énigme est celle de sa naissance. Les calamités qui s'abattent sur Thèbes obligent à rechercher un coupable. Le filet des présomptions se resserre autour d'Édipe et il est amené à découvrir, dans son passé, qui il est, comment il est devenu un objet de malédiction pour ses sujets. Il apprend ainsi ce que chacun semblait savoir : il a tué son père Laïos et épousé sa mère Jocaste. Les deux crimes, Sigmund Freud l'affirme, ont été provoqués par la rivalité avec le père et le désir incestueux pour la mère. Ces éléments ont uniquement trait aux acteurs masculins : désirs et rivalités n’occupent certainement pas 
le premier plan de la tragédie, ils n'en sont que l'écorce, arbitrairement isolée au détriment de l'ensemble. Le nœud de la tragédie est l'antagonisme, à première vue inéluctable, des parents d'Edipe. Pour combattre le décret du destin - mourir de la main d'un fils qui épouserait sa femme, donc déchoir de la paternité — Laïos a commandé de tuer Edipe. Sa mère s'emploie à lui épargner ce sort. Elle s’oppose à la loi de l'homme dont elle cause la perte. Les visées des parents sont contradictoires, chacun s'efforçant de parvenir à ses fins contre l'autre par le truchement de l'enfant. Laïos sait que la survie d'un fils dont il n'aura pas l'entière disposition donnera le pouvoir à Jocaste, Jocaste sait qu'en sauvant ce fils elle détruira Laïos. Edipe épouse Jocaste : ce qui pour lui est innocence - mariage, procréation, royauté - est, pour ceux qui connaissent les secrets de la parenté, acte incestueux. Et d'abord pour la mère, qui non seulement l'a rendu possible mais s'efforce, lorsque le drame se déchaîne, d'étouffer les témoignages, de dissuader Edipe de rechercher la vérité. Elle le veut aveugle : qu'il accomplisse le destin aveugle par le non-savoir, et qu'il soit l'aveugle du destin une fois qu'il sait. La reine agit en connaissance de cause : ce qui arrive ne concerne pas Edipe mais se joue entre elle et l'homme, le père. De plus, aux yeux de tous, c'est elle qui a déclenché la catastrophe, subverti l'ordre dans la cité, et qui en subira les conséquences les plus graves.

Dans la tragédie, il y a deux moments particulièrement forts. L’un indéchiffré et indéchiffrable, de la mort donnée et non reçue : à signification initiatique évidemment. Là où le père échoue, la mère réussit, car celui qui initie le fils le réengendre à l'aide de la mort, le possède. Par une inversion de la loi, c'est la femme qui gagne là où l'homme a perdu, et qui s'approprie le fils. Comment celui-ci n'aurait-il pas senti passer le souffle du danger, l'hostilité qui l'entoure ? Les rituels où la vie et la mort sont coprésentes raniment l'opposition de l'homme et de la femme, celle-ci parce qu'y est scellée sa soumission, celui-là parce qu'il n'a jamais la certitude entière que son bon droit sera respecté, tandis que l'enfant se sent l'enjeu et le médiateur d'une transaction qui décide de son existence : il les sait coalisés contre lui dans la mesure où chaque parent poursuit son destin propre et voit en lui le signe de l'autre. C'est la froide perspective de la mort, sourdant de la tension qui l'environne, plus que l'attirance ou la concurrence de l'un d'entre eux qui s'infiltre dans sa conscience. Celui qui devait mourir, 
c’était bien Edipe, et par leur volonté : « Laisse-moi bien plutôt habiter les montagnes, ce Cithéron qui est mon lot. Mon père et ma mère, de leur vivant même, l'avaient désigné pour être ma tombe : je mourrai donc ainsi par ceux-là qui voulaient ma mort ${ }^{165}$. "

Le second temps fort est que la mort déjouée de l'enfant, réelle et initiatique, entraîne la ruine des parents, victimes de leur rivalité, en particulier la mère qui s'élève contre le cours prévu par l'homme. Laïos cherche à prévenir l'inceste : sa vie, son autorité en dépendent. Jocaste au contraire en crée l'occasion, c'est elle qui le désire avec tout son cortège. Edipe tue son père, accomplissant l'oracle, le destin que son père avait voulu conjurer : il en est l'instrument mais non l'agent. Il tue sa mère, devenant l'agent du destin qu'elle avait choisi : en épousant son fils, elle savait qu'elle encourait le châtiment suprême. Ayant cherché à renverser l'ordre des choses, la femme se détruit elle-même, unique moyen pour elle de contrecarrer cet ordre des hommes. Edipe seul reste vivant, ayant infligé la mort à ceux qui l'avaient provoqué, le père en lui cherchant querelle, la mère en le prenant pour époux, vengeances réciproques et tentatives d'échapper à l'antagonisme qui les divisait. La responsabilité, ils la partagent : «Le désastre a éclaté, non par sa faute à lui seul, mais par le fait de tous deux à la fois: il est l'œuvre commune de la femme et de l'homme ${ }^{166}$. "

Ce parcours d'un des trajets de la tragédie nous livre la prohibition en tant que séparation. Répondant au Sphinx, Edipe, se détachait du monde de la femme. Remontant vers son passé, il découvre le conflit de ses parents, pour son appropriation et sa destruction - l'initiation. Ce qui est en question n'est pas la possession de la mère par le fils, mais, à l'inverse, la possession du fils par la mère ; en d'autres termes, la prohibition se rapporte à l'éloignement de la mère et du fils.

La seconde trajectoire est celle même de la tragédie, une fois l'inceste commis ; elle met en lumière ses raisons et ses séquelles. Si l'union sexuelle a pu servir de motif, l'inceste apparaît surtout comme une tentative de renverser la hiérarchie qui emprisonne la femme. Les

165 SOPHOCLE : Edipe-Roi, trad. P. Mazon, 1450-1455.

166 Idem, 1275-1280. 
actes de Jocaste sont guidés par ce but et sa mort représente un sacrifice. En sauvant Edipe, en causant la perte de son mari, en se donnant pour mari son fils, et en étant acculée à la mort, elle assure néanmoins la pérennité à sa descendance féminine. Après la transgression de la règle, les positions se trouvent bouleversées, les filles prennent la place des fils, se substituent aux hommes. Ce sont elles qui accompagnent leur père aveugle dans son exil à Colone, où, après une période de malheurs, on lui rend des honneurs quasi divins. Edipe reconnaît la permutation : "Ah ! qu'ils sont donc bien faits, ceux-là, pour les coutumes de l'Égypte, avec pareils instincts et pareille existence! L'homme, là-bas, reste au logis, tissant la toile, tandis que la femme sans cesse est dehors, lui cherchant à manger. De même pour vous, mes enfants. Tandis que ceux à qui un tel soin revenait gardent la maison, tout comme les filles, c'est vous qui, à leur place, portez péniblement tout le malheur de votre pauvre père ${ }^{167}$. " L'insulte est grave : une partie des Grecs considéraient le travail artisanal, tel qu'il s'exerce dans le contexte agraire, comme travail féminin, inférieur, l'agriculture étant seule jugée digne des hommes, et ils n'avaient que mépris pour les hommes accomplissant des besognes domestiques. Voici donc les fils d'Edipe relégués par leur père au rang des femmes. Le mythe originel des Bushong éclaire le sens de la permutation. L'ancêtre mythique Woot s'est enivré, ses fils n'ont que sarcasmes pour sa débauche et son indécence. Au contraire ses filles jettent une couverture sur lui pour dissimuler sa nudité. Lorsque Woot est informé de leur conduite, il chasse ses fils et les condamne à subir les épreuves de l'initiation; il fait de ses filles ses héritières et établit pour elles la descendance matrilinéaire. On peut se demander si Edipe, repoussant ses fils et favorisant ses filles, ne créait pas l'occasion d'une transformation analogue, rendue possible et nécessaire par le non-respect de la prohibition dont il a été l'agent. L'auteur tragique est muet sur ce point.

La vérité de l'inceste - mise en péril de l'autorité des hommes, conversion de la règle par les femmes - se dévoile à travers le personnage d'Antigone. Sa mère Jocaste en représentait le moment négatif, le personnage qui se meut dans la nuit des interdits, ruse avec la loi, connaît ses fins, mais ne choisit rien de ce qui arrive ni ne brave

167 SopHOCLE : Edipe à Colone, trad. P. Mazon, 335-345. 
en face ceux qu'elle combat. Ceux qui voient sont aveugles, ceux qui sont aveugles voient. Dans la tragédie d'Antigone, les yeux sont ouverts, tout est visible : les choix qui s'offrent à chacun, l'objet du conflit, les enjeux ; le masque de l'inceste n'est plus nécessaire pour que la femme, en la personne de la fille d'EEdipe, s'élève contre le décret des hommes. Créon son oncle, devenu roi, le comprend bien ainsi, à qui elle inspire une longue tirade : " Il n'est pas, en revanche, fléau pire que l'anarchie. C'est elle qui perd les États, qui détruit les maisons, qui au jour du combat rompt le front des alliés et provoque les déroutes ; tandis que, chez les vainqueurs, qui donc sauve les vies en masse ? La discipline. Voilà pourquoi il convient de soutenir les mesures qui sont prises en vue de l'ordre, et de ne céder jamais à une femme, à aucun prix. Mieux vaut, si c'est nécessaire, succomber sous le bras d'un homme, de façon qu'on ne dise pas que nous sommes aux ordres des femmes ${ }^{168}$."

On ne saurait dénoncer plus clairement le danger qui couve sous l'anarchie : c'est que la femme puisse discuter la volonté du maître, manifester son pouvoir ou même commander. Antigone est non seulement la femme qui refuse d'obéir à la loi, mais aussi la femme qui tient tête aux hommes. Le contraste avec sa sœur Ismène le souligne davantage. " Rends-toi compte d'abord que nous ne sommes que des femmes, objecte celle-ci : la nature ne nous a pas faites pour lutter contre des hommes ; ensuite que nous sommes soumises à des maîtres, et dès lors contraintes d'observer leurs ordres ${ }^{169}$. " Antigone s'y refuse, tous ses actes le prouvent, qui sont inspirés par le même désir : « Ton choix est fait : la vie, et le mien, c’est la mort », réplique-t-elle un peu plus tard ${ }^{170}$. La vie, c'est-à-dire la soumission de celle qui renonce à lutter. La mort, c'est-à-dire le refus de la soumission et l'éventualité de la défaite. Elle, la révoltée, prétend substituer aux règles de la cité — règles des hommes — d'autres règles, du devoir envers ses proches, qui suscitent l'irritation, la colère, l'opposition de Créon. Le roi va jusqu'à accepter la mort de son fils, fiancé à Antigone. Il dit de celui-ci : « Il me semble que ce garçon se fait le champion de la femme », ou bien : «Ah ! fi ! quelle bassesse ! se mettre

\footnotetext{
168 SopHOCLE : Antigone, trad. P. Mazon, 670-680.

169 Idem, 60-65.

170 Idem, 555.
} 
aux ordres d'une femme ! ${ }^{171}$ » Et de celle-là : « Désormais, ce n’est plus moi, mais c'est elle qui est l'homme, si elle doit jouir ici impunément de son triomphe ${ }^{172}$. " Puis, lorsque le drame culmine : «Eh bien donc, s'il te faut aimer, va-t'en sous terre aimer les morts ! Moi, tant que je vivrai, ce n'est pas une femme qui me fera la loi ${ }^{173}$. » Une loi qui suspendrait la prohibition, puisque Antigone, en prétendant ensevelir le cadavre de son frère Polynice, commettrait un inceste symbolique, ce que Créon ne saurait tolérer à aucun prix.

En s'opposant avec obstination à l'anarchie, en se cantonnant dans son rôle de défenseur de la loi, Créon tue son fils qui a subi l'attraction et la volonté impérative d'une femme; il ferme ainsi le cycle ouvert par Edipe tuant son père, victime des menées souterraines d'une autre femme. Sur le registre du destin présagé, mobilisant les forces cosmiques, c'est à travers la disparité des hommes et des femmes, la délimitation de leur rôle paternel et maternel et la lutte qui les oppose dans l'initiation du fils, achevant de fixer leur sort respectif, que l'inceste s'accomplit : sur le registre de la loi édictée, mobilisant les forces de la société, l'autorité d'un sexe sur l'autre, le droit de l'un à la parole qui formule les normes et l'obligation pour l'autre de se taire et d'obéir, sont au cœur de l'affrontement et rencontrent le scandale de la prohibition ignorée. Alors que la mère veillait à ce que personne n'enfreigne les consignes de silence ni ne déplace les pièces du jeu qu'elle avait disposées avec tant de soin, la fille oblige chacun à s'exprimer à haute voix en dénonçant un arrangement introduit de force.

Leurs actes et leur existence à toutes deux rappellent la présence menaçante de l'inceste, le souffle destructeur qui rôde autour de l'interdit. La règle et la non-réciprocité sur laquelle les hommes, les pères assoient leur autorité une fois mises en question, les femmes revendiqueraient une autre règle, la réciprocité, elles feraient la loi à leurs époux et maîtres - Laïos le craint obscurément, Créon le proclame ouvertement - substituant à la lignée de l'époux celle de l'épouse, ruinant la gradation des rangs. La mère refuserait de donner

\footnotetext{
171 Idem, 740 et 746.

172 Idem, 780.

173 Idem, 524-526.
} 
son enfant, son contre-don, la sœur de se séparer de son frère, et ce qui a lieu dans la rivalité des parents et des sexes, cœur de la tragédie, aurait lieu dans le partage et le respect mutuel. Ce que l'on redoute à l'égal d'une catastrophe n'est donc pas le retour à cette nature aussi dévorante que le Sphinx, ni l'arbitraire, mais un remaniement des relations ayant la femme pour auteur ou partenaire. Tentative désespérée au départ, les forces en lutte étant trop inégales. Antigone est emmurée vivante parmi les morts, comme Jocaste avait couru à sa perte pour avoir bravé les lois qu'elles jugent injustes : l'une en proclamant hautement son dessein, l'autre en falsifiant le jeu des règles que les hommes ont conçu à leur usage. D’une famille vouée à la destruction, seule survit, effacée, Ismène qui a accepté de se soumettre.

Dans son sombre éclat, la tragédie concentre la famille, la cité autour d'un noyau agonistique dont les enfants sont l'enjeu, les groupes masculins et féminins les protagonistes, la prohibition de l'inceste le principe hiérarchique. Inviolée, elle viole, en brisant les liens, en contredisant les penchants spontanés des individus, en leur laissant la vie en échange de la servitude et en la leur ôtant dès qu'ils prétendent y échapper. Formulée par la fraction de la société qui détient l'autorité, elle décrète criminelles les initiatives de la fraction exclue de l'exercice de cette autorité, sanctionne tout acte qui lui résiste ou s'écarte de la norme. Il ne s'agit plus alors de transgression mais d'anarchie et d'horreur, signe évident d'une tentative de renverser le sens des événements et des lois, de bouleverser la position respective des groupes concernés. Dans cette lumière, et les peuples l'ont senti depuis longtemps, ce n'est pas la prohibition qui met un terme au chaos, un frein à la pulsion, c'est au contraire le prétendu chaos qui met un terme à la prohibition fondée sur la distinction initiale des sexes qu'elle a pour mission de renforcer et de prolonger. Elle n’instaure pas l'ordre dans le désordre : elle signale la virtualité permanente du désordre au sein de l'ordre imposé, dans la mesure où elle charrie la violence et la répression et où tout ce qui a caractère de règle est violence. Elle contraint ainsi les groupes fonctionnant en tant qu'objet et signe, dans la soumission, à la nier en tant que valeur et signe pour pouvoir agir et vivre, ce qui est l'essence même du travail et de l'histoire. Au-delà du spectre de la confusion, de la retombée dans la nature, de toutes les frayeurs soulevées par l'insubordination, on entrevoit non pas le vide mais un autre ordre, l'ordre de ceux qui 
étaient privés de la jouissance et du pouvoir, avec lesquels n'avait lieu ni partage ni échange, à qui était refusée l'humanité et la culture. L’inceste est précisément l'indice d'une telle fin possible, suspension abolie au profit d'une règle différente, amorce d'un autre ordre, et c'est dans ce sens que j'ai souligné son adoption par certaines classes qui en faisaient la marque de leur supériorité. Sur ce plan, il est associé à l'état envisagé dans les mythes, qui sont œuvre des hommes, comme le "règne des femmes », opposé au leur : « Leur insubordination sociale (des femmes), souvent évoquée par les mythes, offre l'image anticipée, sous la forme du "règne des femmes ", d’un péril qui serait infiniment plus grave : celui de leur insubordination physiologique. Aussi faut-il que les femmes soient soumises à des règles. Et celles que l'éducation leur inculque, comme celles qu'un ordre social voulu et conçu par les hommes leur impose, fût-ce au prix de leur asservissement, fournissent le gage et le symbole d'autres règles, dont la nature physiologique atteste la solidarité qui unit les rythmes sociaux et cosmiques ${ }^{174}$. " L'atmosphère de terreur que l'éventualité de ce règne inspire est bien la preuve la plus éclatante que son interdit plonge ses racines dans un rapport de domination prolongé, que sa fonction n'est pas d'établir la réciprocité mais de consolider la nonréciprocité ; qu'il sert moins à préserver une rupture consommée avec la nature qu'à prévenir la transformation des relations dans la société, que loin d'avoir pour but d'éliminer la violence au moyen de la loi, il sert à défendre la loi par le moyen de la violence. Rejetant le postulat de la promiscuité originelle, la théorie que j'ai exposée met en relief la lutte des sexes, en fonction d'un principe de séparation et de hiérarchie. L'essence de l'ordre humain n'est pas la loi que menaceraient de possibles transgressions - les animaux se montrent plus scrupuleux sur ce chapitre. Il a pour condition la tension engendrée par la hantise perpétuelle d'un soulèvement de ceux qu'il soumet et le remaniement consécutif à sa mise en échec.

L'émergence de l'ordre, en un point du temps, n'est pas à elle seule aiguillon et marque de la culture : ce que la loi prohibe, projetant audelà de son monde les contours d'un monde distinct, participe également de cette culture. Les interdits séparent le connu, le transmissible, le transparent de ce qui doit demeurer inconnu, intransmissible, opa-

174 C. LÉVI-Strauss : L'origine des manières de table, Paris, 1968, p. 182. 
que. Leur abolition convertit l'inconnu en connu, l'intransmissible en transmissible, l'opaque en transparent; en reculant de la sorte les frontières, on découvre l'arbitraire et le particulier dans ce qui se donne pour nécessaire et universel. Dans ce mouvement, le savoir naît de l'ignorance, les esclaves d'hier préfigurent les maîtres de demain, le désordre dénoncé comme retour au passé est commencement d'un ordre, les écarts accumulés ébauchent une nouvelle règle. La prohibition, intervention des hommes, rencontre dans l'inceste, menace des femmes, au lieu d'une matrice organique, création de la nature, symbole de l'animalité, sa négation que la société engendre ; à la place d'une destruction inévitable, une tension dirigée vers la redéfinition virtuelle de la réalité, des règles du jeu humain. 


\section{L'éternel présent.}

\section{$\underline{\text { Retour à la Table des Matières }}$}

Dans une lettre du 30 avril 1932 adressée à Marie Bonaparte, Sigmund Freud portait le jugement suivant : " Il est assez étrange — mais peut-être aisément compréhensible — que les interdictions les plus puissantes de l'humanité soient les plus difficiles à justifier. Ceci est dû au fait que les justifications sont préhistoriques et ont leurs racines dans le passé de l'homme. La situation pour l'inceste est exactement parallèle à celle du cannibalisme. Il y a naturellement de bonnes raisons pour que dans la vie moderne on ne tue pas un homme pour le dévorer, mais aucune raison quelle qu'elle soit pour ne pas manger de chair humaine au lieu de viande. Pourtant la plupart d'entre nous trouveraient cela tout à fait impossible. L'inceste n'est pas aussi éloigné et ne se produit en fait que trop souvent. Nous pouvons finalement nous rendre compte que s’il était pratiqué sur une large échelle, il serait socialement aussi nuisible aujourd'hui qu'il l'était dans le passé. C'est ce mal social qui constitue le noyau de ce qui, paré d'un tabou, est devenu une telle affaire ${ }^{175}$. »

En vérité, il est difficile, aucun peuple n'ayant pratiqué l'inceste sur une grande échelle, de mesurer son degré de nocivité et, s’agissant d'un phénomène d'un telle ancienneté, de proposer une théorie qui puisse rendre compte avec exactitude de sa généralité, des formes qu'il revêt. A condition d'être non contradictoire en face de la question qu'elle pose, chaque théorie aboutit à une combinaison de faits qui la confirment. Loin d'être un aveu d'éclectisme, c'est la reconnaissance d'une limite, lorsqu'on a affaire à une réalité dont l'essentiel appartient au passé et dont on ignore l'évolution. La prohibition de l'inceste est toujours associée pour nous au cadre dans lequel se perpétuent la distance et la hiérarchie des sexes et des générations. Sa rémanence n'a rien de mystérieux. Les anciennes divisions des activités ont été reprises et ont reçu un contenu différent dans les systèmes collectifs postérieurs à l'époque archaïque. Les sociétés renoncent difficilement à des institutions qui leur conviennent, elles bâtis-

175 E. Jones : La vie et l'œuvre de Sigmund Freud, Paris, 1969, t. 3, p. 511. 
sent dessus et alentour, comme les villes modernes se construisent à partir des cités anciennes, dont elles bouchent ou irriguent les artères, s’approprient le plan, les édifices, les matériaux.

Il serait inexact de parler de survivance, de décalage, de continuité aveugle, d'inertie. Au contraire, le neuf absorbe l'ancien, en modifie l'emploi, lui donne une vie nouvelle. Rajustés, raffinés, traduits, les conditionnements psychiques, les mythes, les langages, les attitudes sont assimilés aux conditionnements, aux mythes - voire aux philosophies ou aux sciences - aux langages qui prédominent dans d'autres secteurs, et deviennent intelligibles aux hommes vivant à un autre moment, dans une société différente. Sigmund Freud a parfaitement raison : ce que nous trouvons impossible n'est plus nécessairement justifié, mais l'inceste continue à être une menace. Les hommes et les femmes se définissent toujours par rapport à elle (note 9). Son poids entretient la disparité qui permet aux premiers et nous fait condamner chez les secondes, en pratique sinon en théorie, la polygamie licite ou illicite, la participation pleine à la vie sociale et politique, l'union sexuelle ou conjugale avec les individus jeunes. Dans la discipline que les adultes exigent des jeunes, dans la rivalité des parents au sujet des enfants, nous perpétuons l'antagonisme des sexes. Le mariage et la vie familiale sont soumis à l'impératif de l'interdit.

Cependant il nous faut considérer que la prohibition a acquis un sens et une fonction différents de ceux qu'elle avait dans la société qui lui a donné naissance. Ce changement a commencé par l'isoler, il l'a ensuite centrée sur les relations sexuelles, puis il l'a située dans l'opposition de la famille et de la société. Dans son cadre d'origine, elle unissait un aspect positif à un aspect négatif, l'alliance à l'exclusion. Le rétrécissement du champ de la parenté, la famille, de diffuse, devenue restreinte, simple différenciation du système collectif, subordonnée aux autres grands secteurs sociaux : classes, castes, régions, divisions politiques ou religieuses, tout le côté positif est, pour ainsi dire, tombé en désuétude. La fortune, le pouvoir, la profession, la résidence ont accédé au rang de critères prescriptifs, parfois cachés, sur lesquels se règle l'union des hommes et des femmes. Tout ce qui était soumis au partage, tout ce qui exprimait la solidarité de l'affrontement direct, l'authenticité souvent brutale des rapports, a été rendu abstrait ou incertain, le lien conjugal figurant un point de cris- 
tallisation dans un mouvement dominé par la loi des grands nombres, laissant à une promiscuité tempérée par les mécanismes économiques et idéologiques le soin de le reconstituer là où il est indispensable, tel qu'il est. Chacun, frère ou père, sait à qui il doit renoncer et ce qu'il ne peut faire, ignorant les partenaires éventuels de la transaction en faveur desquels il renonce à sa sœur ou à sa fille. Nous ne vivons plus ni sous l'emprise du don, ni, à proprement parler, en exogamie, puisque rien ne nous indique exactement avec qui nous marier, puisque nous épousons des individus semblables et non plus différents. Lorsqu'une indication existe, elle correspond à des facteurs extérieurs à la parenté ou à la généalogie. L'exogamie et l'endogamie sont désormais remplacées par l'état d’agamie.

Là, justement, l'inceste est possible: non seulement les dangers biologiques sont inexistants, la mobilité et la taille des populations y veillent, mais aussi la transgression n'entraverait en rien la bonne marche d'une société qui obéit aux principes de la propriété et du pouvoir étatique. La société archaïque voyait, dans la famille imbriquée au réseau des alliances parentales, l'unité constitutive, son modèle et son moteur. La vie sociale, sous l'angle productif et reproductif, était affaire de mariage, et le mariage affaire de la vie sociale. L'interdit du comportement incestueux avait trait à un acte associant un groupe à un groupe ; et non un homme à une femme, un individu à un autre individu. L'écart mettait en cause l'ensemble des rapports collectifs, les échanges avec le monde matériel, et, à un moindre degré, l'équilibre individuel. Notre société actuelle sera affectée, disparaîtra, non pas le jour où parents et enfants, sœurs et frères auront des relations sexuelles, voire conjugales, licites, où les sexes jouiront de droits égaux, où l'homme aura perdu l'autorité sur la femme et ses enfants, mais le jour où les empiétements de la propriété privée rendront impossibles les transactions sur le marché, le jour où le commerce, l'industrie, la police ne seront plus aptes à échanger, produire, défendre le profit ou l’État.

Ce que nous décrétons universel, règle des règles, n'est qu'une restriction, l'envers d'un échange entre les groupes, le négatif d'une association du sexe masculin avec le sexe féminin, sans aucune emprise sur les rapports sociaux effectifs. Il n'est donc pas vrai de dire que la société et la culture sont inséparables de l'interdit ; il serait plus vrai 
de dire que, compte tenu du type de société et de culture qui est le nôtre, nous ne pouvons nous passer d'interdit, et ce uniquement dans la sphère domestique qui sert de refuge aux liens d'affinité, de sécurité, limitant l'autonomie du groupe restreint et des individus proches. Bref, il se rapporte davantage aux relations interindividuelles qu'aux relations collectives ${ }^{176}$. Dans une société archaïque, comme dans une société animale, distinguer le couple biologique, l'individu biologique, l'amorphisme naturel n'a aucun sens. Dans les sociétés évoluées, au contraire, vu leurs dimensions leur organisation et le niveau auquel elles se perçoivent, couples, individus, amorphisme des échanges entre les sexes peuvent passer pour biologiques. Là se manifeste la coupure du privé et du public, de l'organique et du social, de l'individu et du collectif. Et si la prohibition de l'inceste a cette importance, si elle exerce cette fascination, c'est parce qu'on l'a placée au centre du monde privé en l'opposant au monde public dont la structure n'est nullement ébranlée du fait que l'acte prohibé ait lieu ou non. Elle est devenue l'indice d'une sociabilité scindée, servant à effectuer la régulation des pulsions, proportionner la satisfaction des instincts et restreindre à un cercle étroit la rencontre des sexes, sans plus.

Sans être immuable, la prohibition n'est cependant pas universelle, au sens strict. Toutes les classes de la société ne l'ont pas respectée ni toutes les espèces humaines connues. Le fait est d'importance pour la vérification des théories. Celles-ci sont impuissantes à reconstituer le passé, à décider entre les différentes interprétations que l'on peut en donner, et entre les phénomènes destinés à être inclus dans ce qui fera l'objet d'une interprétation. Leur espoir réside dans une expérience qui pourrait trancher et que seule l'histoire est en mesure de mener à bien, une expérience sociale et psychologique passionnante. Sa teneur ne fait point de doute. Les relations entre les sexes et les générations sont actuellement en pleine effervescence. Nous les saisissons uniquement sous l'angle de l'économie et des transformations globales de la société. L’essence de tout ce qui les affecte, scission, subordina-

176 On a soutenu que les névroses avaient pour motif la nature des rapports familiaux ; on peut se demander si ce motif ne doit pas plutôt être cherché dans la manière dont ils se perpétuent, leur écart aux rapports sociaux dans leur ensemble. En d'autres termes, ce n'est pas la névrose qui découle du devenir familial, mais la famille qui est devenue névrotique. 
tion, non-réciprocité, se trouve dans la parenté. Un système social qui ne touche pas à celle-ci — la famille, disait Émile Durkheim, est le " nerf de toute la discipline collective " - ne modifie en rien la base même des relations entre hommes et femmes, jeunes et vieux, si, par ailleurs, il leur assure l'égalité civique et économique. Mais la famille bouge. Au fur et à mesure que la population s'accroît, la mobilité sociale, l'urbanisation arrachent les êtres aux liens rigides, créant une situation de promiscuité au vrai sens du terme ; elles libèrent la société du danger qui résulte de la consanguinité, de la monopolisation des femmes dans la cellule parentale, en leur ôtant tout caractère de contrainte, si jamais elles en ont eu un. Les pressions diverses rendent impérative la restructuration de l'unité sociale de base. L'exode rural, la concentration de la population dans les villes, ont disséminé les membres de la famille qui vivaient autrefois dans la demeure commune (ascendants, collatéraux, etc.) aboutissant à la dispersion et à l'isolement de noyaux familiaux qui comprennent uniquement le père, la mère et les enfants. Le rôle des parents s'est accru dès l'instant où ils ne pouvaient plus compter sur l'aide des autres personnes de la famille pour partager avec eux les tâches de surveillance, d'instruction des enfants dont ils doivent assumer seuls l'entretien, la protection, la socialisation. Ces fonctions mêmes sont désormais diluées : éducation, intégration productive, socialisation, une fois transférées, se répartissent entre l'école, l'industrie, les corps politiques ou administratifs. En même temps que l'importance de la famille dans le cycle productif diminuait, l'autorité parentale a légalement décru ; puissance paternelle et puissance maternelle ont vu leur différence s’amenuiser.

S'il est vrai que la prohibition de l'inceste est le nœud de la hiérarchie qui oppose les sexes et les générations, si c'est bien elle que sert à définir les individus sous l'action d'une division qui fait de la société une société d'hommes dont les femmes sont exclues, alors toute tentative de reconstituer ou de remplacer la famille, comportant un autre type de rapports entre sexes et générations, tel que la différence qui les sépare ne soit pas obligatoirement une inégalité, mais se fonde sur la réciprocité, a pour condition première la disparition de la prohibition.

Cela ne signifie pas que l'inceste s'y commette — dans une société ayant les dimensions de la nôtre, sa probabilité est bien moindre que 
dans une société stable, et l'endogamie a peu de chances de se produire - mais que son interdit aura été aboli ; qu'il cesse d'être le principe servant à régler les rapports qui se nouent entre individus, et que sa figure négative ne constitue plus une dimension de la vie sociale. Au lieu de représenter le signe d'une catastrophe, l'exception qui sépare les formes de vie humaine des formes de vie animale, il sera l'indice d'un ordre supérieur où les individus seront libérés de toutes les entraves psychologiques et sociales qui l'accompagnent. Il en serait de l'inceste comme du cannibalisme : personne ne le commet, sans qu'il soit besoin d'aucune règle pour l'interdire. En somme, il ne serait plus nécessaire, pas plus nécessaire que le cannibalisme. Il se peut que, dans l'évolution de l'humanité, de telles lois jugées impératives ne soient en réalité que des étapes dans le façonnement d'un comportement qui finit par disparaître. A la longue, on en vient à juger celui-ci impossible, parce qu'improbable, et non plus défendu parce que possible. Ou bien on ne lui accorderait pas plus d'importance qu'à d'autres liens que les individus contractent entre eux.

Si, par contre, il devait être avéré qu'aucune société ne saurait vivre sans imposer l'asymétrie des sexes — l'humanité ne le désirant pas ou n'étant pas en mesure de l'atteindre — ou si, même après la disparition de cette asymétrie, la prohibition devait se conserver, en ce cas les escapades spéculatives auxquelles je me suis livré resteraient de pures escapades ; car nous pouvons faire que les choses soient ce qu'elles sont, mais nous ne pouvons faire qu'elles soient ce qu'elles ne sont pas. Faute d'une telle expérience, les constructions logiques les plus cohérentes demeurent des constructions, et rien ne saurait trancher entre elles, pour la raison énoncée par Freud.

Au-delà de la prohibition de l'inceste, ce qui est en jeu dans une telle expérience, c'est la question de savoir si la société peut se fonder sur autre chose qu'un interdit. 


\section{Conclusion.}

\section{Retour à la Table des Matières}

La paternité, le partage exogamique, la prohibition de l'inceste sont les pivots de la société de parenté archaïque. Pour l'essentiel, aucune de ces institutions ne semble avoir précédé l'autre dans le temps ; aucune ne semble être la conséquence de l'autre. Au cours de l'histoire, elles se sont modelées réciproquement sans que l'on puisse démêler une cause ou un commencement unique. Envisagées séparément, elles projettent un éclairage sur un grand nombre de systèmes sociaux dans lesquels elles se sont combinées suivant des formules différentes. Les solutions auxquelles ont abouti les hommes, dès le début, n’ont été ni identiques ni remarquables, par leur stabilité. Des contradictions se sont logées dans les édifices qu'ils ont bâtis, que seule l'illusion due à la distance nous empêche d'apercevoir. Ce qui explique que de grands pans de ces institutions soient tombés, dont les uns ont fourni des matériaux utilisables et utilisés ultérieurement, tandis que les autres s'effritaient en poussière.

Mais plutôt que d'examiner ces métamorphoses, je veux rappeler l'évolution au cours de laquelle les sociétés de primates se sont transformées en société humaine. Avec leurs clivages physiques et les hiérarchies distinguant le sexe et l'âge, elles lui ont fourni à la fois un modèle et une matière première. La collectivisation des activités, le nomadisme et la division de la chasse et de la cueillette avec la répartition correspondante des habiletés et des parties du milieu ambiant, les ont en quelque sorte transportées dans un nouveau monde, leur ont conféré un contenu intellectuel et organique différent, aboutissant à la nécessité d'une redéfinition du réel, à des formes nouvelles de celuici. La famille a intégré le couple reproductif et le couple nucléaire, les a soudés dans une institution complexe ; elle a introduit le mâle dans le rapport de la femelle à sa progéniture, et cette dernière dans le rapport des sexes entre eux ; elle a rendu possible leur coexistence prolongée, en modifiant le caractère de leur coopération et de leur antagonisme, en les étendant au domaine productif, à la reproduction des habiletés indispensables aux échanges avec les puissances matérielles. Quel que soit le type de mariage qui la concrétise, elle est l'unité ba- 
sale vers laquelle la collectivité dirige ses membres, reconnus uniquement pour autant qu'ils y sont inclus. De plus, la société est dans l'obligation de s'organiser sur un espace non seulement plus vaste mais surtout différencié quant aux opérations exigées, à l'alternance des groupes et de leurs activités. Le rythme des saisons s'identifie au rythme des chasses et des cueillettes, de la réunion et de la séparation des communautés. Le réseau des interdépendances se ramifie, les plans de diversification se multiplient, accroissant le cycle des prestations et des obligations réciproques, provoquant une ouverture capable de survivre à la coprésence des partenaires individuels ou collectifs. Le partage devient le mode de distribution prévalent, que l'on retrouve dans tous les secteurs de la vie, des personnes aux biens, des instruments aux territoires, l'exogamie étant la norme dans la parenté, les ressources et les savoir-faire. En mettant ses fractions dans l'obligation d'avoir besoin les unes des autres, en les amenant à prendre conscience de leurs insuffisances dans quelques circonstances qu'elles se trouvent, la société a pu assimiler le nomadisme et rendre le nomadisme social. La hiérarchie où l'élément masculin domine l'élément féminin a dû recevoir une nouvelle expression, rien n'étant venu la mettre en question. L’hétérogénéité des sexes a pris plus d'ampleur, en se rapportant à leur emprise respective sur le monde matériel.

La reproduction sexuelle était en cause, non pas au niveau biologique mais au niveau naturel, c'est-à-dire pour ce qui était de préserver et de renouveler l'ensemble formé par les hommes et les forces objectives qui leur sont associées, moyens, organes techniques et talents assurant la cohésion de l'ensemble. L'union d'une femme et d'un homme dans la famille, du groupe des femmes et du groupe des hommes dans la société, opérait la soudure de deux fragments de l'univers réel, du monde végétal et du monde animal, de deux régions spatialement disjointes. La sexualité, dépassant sa définition organique, s’y affirme naturelle ; la nature, dépassant sa définition matérielle, se découvre sexuée. Ici la division est une nécessité qui régit l'ordre cosmique qu'il s'agit de maintenir à tout prix ; imposer la division, c'est garantir la permanence et le réengendrement de cet ordre. Cependant ceux qui sont divisés sont inégaux, l’un est subordonné à l'autre. Pour pouvoir à la fois respecter la division nouvelle et garder la hiérarchie ancienne, pour séparer d'emblée les individus qui de- 
vront assumer leurs devoirs d'hommes et de femmes sans que soit menacée l'autorité des premiers sur les secondes, dans chaque groupe et dans la population, il fallait que, dans le partage qui s'opère par le mariage, les femmes quittent en priorité leur famille ou leur clan, et que d'autres femmes viennent prendre leur place dans la famille ou dans le clan : ce qui permettait de les traiter en subalternes, de les assujettir à des règles de discrimination parce que étrangères, d'en user comme avec des étrangères parce que vouées à la subordination et à la ségrégation.

La prohibition de l'inceste, qui concourt à la séparation des sexes et à la transformation de l'un en objet cessible, de l'autre, traduit à la fois la signification élargie de leurs rapports et transpose dans cette régulation la hiérarchie propre à ces rapports. Certes, on peut toujours récuser les reconstitutions, soit que l'on dénie aux congrégations animales la qualité de société ; soit que, tout en leur reconnaissant cette qualité, on donne à la société humaine pour ombre la culture, en prétendant que cette ombre n'appartient qu'à elle. Laissant ces controverses de côté, si l'on suit la chaîne évolutive, la genèse des institutions pivots de la société conduit bien, par voie chimique, d’un état social instauré par les primates à un état social instauré par les hommes - et non point, par voie alchimique, d'un état naturel à un état social. Dès ses premiers pas, notre espèce a choisi le chemin le plus difficile, non sans se consoler de temps en temps, devant les aléas et les déboires d'une aventure dont elle n'avait pas prévu les développements, en prétendant y être parvenue par miracle. 


\section{Chapitre XII.}

\section{Rétrospective}

\section{Le paradigme en question.}

\section{$\underline{\text { Retour à la Table des Matières }}$}

Le rapport de l'homme à la nature, de la société à la nature, est redevenu, sous différentes formes, scientifiques, pratiques, voire politiques, un problème. On ne peut l'aborder ou le résoudre si l'on ne quitte le niveau auquel on s'est arrêté longtemps, celui des théories établies à propos de chacun des termes, pour le transposer à celui des paradigmes de la société et de la nature, en précisant ce que l'on entend par l'une et par l'autre. Mais quelle signification faut-il accorder au fait que l'on situe le débat à un niveau différent ? Un paradigme admet, en général, plus d'une théorie. Ainsi les astronomes, adoptant le modèle héliocentrique qui place le soleil au centre du système planétaire, dont les divers éléments sont tenus ensemble par la force de gravitation, ont conçu la gravité de plusieurs façons - il suffit de mentionner les noms de Newton et d'Einstein — ont attribué à la trajectoire des planètes une forme tantôt circulaire - ce fut la position de Copernic et de Galilée — tantôt elliptique, avec Kepler.

Dans le cas qui nous occupe, si l'on donne à la société un ancrage biologique et pour fonction de réprimer les instincts, il est possible de mettre l'accent soit sur l'instinct sexuel, soit sur l'instinct agressif, et d'accumuler les observations et les hypothèses qui font ressortir la prépondérance de l'un au détriment de l'autre et vice versa. Les résultats sont cependant rapportés à un cadre unique, même si, guidés par des présupposés supplémentaires - l'homme est-il bon ? est-il mauvais ? - leurs divergences les situent à des pôles opposés. Lorsque, 
par ailleurs, on conçoit ce paradigme historique à deux étages dont l'un, l'infrastructure, enferme les facteurs économiques, techniques, tandis que l'autre, la superstructure, contient les facteurs psychiques ou idéologiques, on peut bâtir deux théories contraires. Pour la première, les facteurs économiques sont déterminants et les facteurs psychiques ou idéologiques déterminés ; pour la seconde, le rôle moteur revient aux facteurs psychiques ou idéologiques, les facteurs économiques ne jouant qu'un rôle subalterne. Les controverses modernes de l'histoire ont été motivées, comme chacun sait, par le besoin de mettre ces théories qui s'excluent mutuellement à l'épreuve des faits recueillis.

On peut pousser plus loin l'analyse comparative. Les techniques et la conscience sociale, pour Lewis Morgan, la propriété et la production, pour Friedrich Engels, la pensée symbolique et la prohibition de l'inceste, pour Claude Lévi-Strauss, représentent à la fois l'essence de la culture humaine et la voie par laquelle elle s'éloigne de la nature. Toutes n'en reposent pas moins sur le postulat de la promiscuité sexuelle entre les membres du groupe biologique originel, postulat qui, malgré leurs divergences, les oblige à chercher leurs réponses dans une direction unique.

Certes, les diverses théories du système planétaire ou de la société, pour m'en tenir aux exemples donnés, ne se valent pas; le choix que l'on fait entre elles n'est pas arbitraire, le rôle décisif revenant à la cohérence et au pouvoir démonstratif appliqués aux données objectives. Toutefois, et c'est une observation élémentaire, les familles de théories se conforment à un patron commun, sorte de schéma organisateur contenant le réel dans ses limites et système d'équations général reliant cause et effets, variables et paramètres, sur lequel elles se guident afin de rendre intelligibles les phénomènes qu'il permet d'appréhender et d'exclure ceux qui apparaissent négligeables dans son cadre.

Ce patron n’a rien de permanent ; à des moments précis, la nécessité commande de le remplacer, lorsque des points de référence, des intérêts nouveaux se font jour, à côté de faits récemment découverts, Le modèle héliocentrique a pris la relève du modèle géocentrique qui mettait la terre au centre du système planétaire, moins parce que les 
modifications successivement apportées à celui-ci s’étaient révélées infructueuses que parce que les mouvements des corps célestes et les liens qui les unissent furent imaginés à la façon de ceux d'un mécanisme d'horlogerie. Au-delà des lois et des expériences particulières, des explications locales, s’imposa le choix - les écrits de Galilée et de Descartes en témoignent — entre deux systèmes du monde.

Nous nous trouvons dans une situation de crise analogue en ce qui concerne notre conception de la société et de la nature. La signification que nous donnons à l'une et à l'autre, aussi bien qu'à la relation que nous supposons entre elles, nous en avons pris le modèle chez les Grecs. Il s'est d'abord rapporté à la sphère politique, à l'existence de l'État, au corps humain en tant que lieu privilégié du connaître et de l'agir. Par la suite, l'importance croissante de la production industrielle, l'éclatement des limites domestiques dans lesquelles avait été enfermée l'économie, l'individualisme et la lutte des classes, la récurrence des révolutions, le triomphe de la mécanique, lui ont ajouté une dimension historique, parachevant la figure que nous connaissons. La raison et le contrat sont les moyens, la conquête de l'univers matériel et social la fin satisfaisant aux besoins physiques et spirituels des agents économiques et épistémologiques individuels ; la séparation de l'ordre naturel et de l'ordre social passent pour être l'origine et le couronnement de la condition humaine.

Les contours de ce paradigme sont débordés par le mouvement réel. Si, jusqu'à une date récente, on pouvait tenir l'amélioration des facultés humaines, les découvertes scientifiques et la diversification des forces matérielles pour des phénomènes aussi incontrôlables ou spontanés que les changements de saison ou les marées, sans rapport direct à la vie collective, on en vient à reconnaître que leur orientation, leur rythme dépendent, au contraire, entièrement de nous. En amont et en aval de la production, l'invention et la reproduction des savoirs, des techniques, la rattachent directement, mais de manière subordonnée, aux échanges avec les forces matérielles. Tout ce qui a trait à la communication avec le monde objectif — découverte de matières premières, création d' " espèces " physiques, chimiques ou biologiques, aménagement du milieu, promotion des sciences, etc. — résulte d'une intervention continue de l'homme, est le fruit d'un effort systématique de sa part. Le bouleversement dont nous sommes auteurs et 
témoins, aussi bien par l'effet de masse de l'espèce que par son activité dans la biosphère tout entière, donne une ampleur inconnue auparavant et une physionomie nouvelle à la connaissance associée au travail et qui se mue en travail, à la relation à l'intérieur de laquelle nous modelons notre univers. Obligés d'y faire face, le corps social et les institutions politiques voient leurs fonctions et leurs organes changer, se diversifier, s'étendre à des domaines qu'ils négligeaient auparavant, et finiront par se retrouver modifiés de fond en comble en présence d'une réalité qui, elle aussi, aura été redéfinie. Ni fondement inerte, ni réservoir de forces, ni dépotoir de déchets, le milieu naturel se montrera, à l'évidence, comme le lieu, le prétexte et l'expression de l'action des hommes associés à lui.

Toutes ces circonstances, jointes au grand nombre de constats que l'on a faits à propos de la biologie et de la sociologie de l'homme et de l'animal — ceux mêmes que j'ai exposés dans ce livre - invitent à la recherche d'un paradigme de la société et de la nature qui leur corresponde. Le principal de mon travail se situe dans la ligne d'une telle recherche. L'examen des concepts et des faits particuliers auquel j'ai procédé lui est subordonné ; il représente, si l'on veut, un essai de mise en pratique. Les éléments ou les ensembles déjà prouvés sont évidemment conservés dans un contexte remanié, comme il arrive pour tous les ordres de connaissance, mais leur champ de validité est reconsidéré. La réussite, dont je ne suis pas juge, importe moins, en l'occurrence, que la conviction que la tâche est entamée et doit être menée à son terme. 


\section{Le thème de la rupture et de la conquête}

$\underline{\text { Retour à la Table des Matières }}$

\section{(1) L'artifice social.}

Les traits d'un paradigme sont familiers. La nature est un assemblage autonome de forces et de créatures qui existent de tout temps, suivant un développement propre, harmonieux. Ces forces et ces créatures dont chacun peut disposer immédiatement représentent des « dons gratuits », n'exigeant de la part de celui qui veut les utiliser, se les approprier temporairement, aucun effort particulier autre que celui qui est inscrit dans le cours des choses. La lumière, l'air, l'eau, les végétaux, les animaux symbolisent une telle disponibilité. L'homme est parmi eux le fruit d'une évolution tardive qui lui a assigné une place, des propriétés uniformes, repérables dans chaque individu de l'espèce. Son équipement physique et intellectuel et son environnement sont des données autoconstituées, préalables logiques et matériels d'un devenir ultérieur, marqué par la rencontre d'une limite. Soit que le milieu ou l'organisme présente une déficience ou que ce dernier se voie doté de caractères originaux - capacité crânienne, station debout, langage, etc. - l'équilibre initial a été rompu. La nécessité s'est fait jour de le rétablir grâce à une organisation collective, à une activité finalisée. Associés pour échapper à la nature et contraindre à la soumission ses forces et ses créatures, désormais étrangères, les hommes ont bâti un milieu différent, celui des artifices, et un artifice unique qui leur sert de milieu propre, la société. Elle témoigne du pouvoir d'intervention de l'homme, de son emprise sur la nature interne biologique en faisant de l'individu isolé et complet une partie d'un ensemble qui le dépasse et qui veille à satisfaire ses besoins en distribuant les richesses; règle, morale, culture collectives étoffent le mode d'existence précaire qui serait le sien sans elles. Dans la conscience de l'être qui, en accédant au jour humain, a conservé intactes les traces enfouies de la nuit animale, la foi et la raison face à l'instinct et aux passions du cœur, la pensée et le langage face au corps et au travail assument la fonction de discipline du tout sur les parties. La société est aussi un moyen, l'instrument de la lutte contre les forces matérielles auxquelles les individus réunis veulent se frayer un libre accès, 
contre la nature qui s’y manifeste et leur résiste. De diverses façons, les connaissances, les arts, la production naissent de ce combat. Ce que l'homme atteint par leur intermédiaire cesse d'appartenir aux circuits naturels, à la manière de ces objets ready made, insignifiants, quelconques, délaissés, que l'artiste, du seul fait qu'il les distingue et sépare de leur entourage pour les incorporer à son œuvre, change en objets d'art. Le contraste du donné et du produit exprime moins l'acte que l'acteur, à la présence ou à l'absence duquel sont rapportés les efforts. Tout ce qui renvoie à une immixtion, à une ingérence humaines, brise avec la nature et éloigne d'elle.

Plus généralement encore, le changement, parce qu'il porte l'empreinte d'un effort et d'une intention, est irrévocablement déclaré antinaturel, et ne saurait avoir lieu que dirigé contre la nature. Conquérir celle-ci est une tâche quotidienne. Tout ce qui est arraché à son domaine est transvasé dans celui de la société. L'accumulation continue de savoirs, de biens, d'institutions, de lois, de monuments, de techniques, de formes d'énergie, d'espèces animales et végétales domestiquées, de villes, de savants, signale un enlèvement régulier, ajoutant d'un côté ce qui est soustrait de l'autre. On peut mesurer le degré de culture à la quantité de ces éléments réunis : elle regarde de plus loin et de plus haut vers son passé animal, se sent d'autant mieux abritée du monde matériel qu'elle se sait assise sur un amoncellement plus imposant et plus élevé. L’histoire serait essentiellement ce processus d'enlèvement et de thésaurisation qui, à travers la succession des triomphes, consolide l'entreprise de contrôle de l'univers, synonyme de son exploitation. Comblement du manque initial, substitution de l'aisance à la rareté : c'est ainsi que l'on représente le terme de son mouvement, libérant le système social des amarres qui le rattachent encore au système naturel. D'où la préoccupation constante d'opposer ces systèmes, de planter les jalons de leur éloignement et de chercher la preuve d'une séparation définitive dans les couples de contraires : le collectif et l'individuel, l'urbain et le rural (qui traduit aussi la fameuse opposition du social et du communautaire), le civilisé et le primitif, le domestique et le sauvage. La négation signifie ici distance et rupture. La communication, le travail, la pensée, la prohibition de l'inceste sont considérés non comme les transformations d'éléments antérieurs mais comme des mutations sans précédent, les actes qui 
font prendre pied au genre humain dans la nature sans qu'il soit de la nature.

$\underline{\text { Retour à la Table des Matières }}$

\section{(2) La pollution par l'humain.}

La société est une modalité d'oubli de la nature. Elle a une réalité seconde, d'emprunt. Son développement jouit d'une liberté surveillée, engagée dans la stricte application d'un contrat : la restauration locale d'un ordre dans le désordre, l'administration d'un remède là où il y a déficience. Aussitôt qu'emportés par un élan irrépressible, poussant à son point extrême la logique de la négation, ses actes, ses savoirs, ses institutions imaginés à cette fin prennent un caractère usurpateur, s'abandonnent à la tendance interne des artifices, déferlent hors du domaine qui leur a été concédé à l'origine, le mouvement déclenché n'est plus maîtrisé, l'ancien lien au milieu est trahi, les symptômes de démesure prolifèrent. Mais qu'appelle-t-on nature dans cette vision ? A intervalles réguliers, on nous somme impérativement de revenir à elle, de faire machine arrière, d'annuler les changements, de travailler à rétablir l'équilibre, de retourner à son cadre, notre passé, seule issue pratique. Il n'est nul besoin de recourir à des textes savants pour dégager ses traits, le modèle de ses rapports à la société ; ils ressortent des nombreuses déclarations et des cris d'alarme qui parviennent d'un peu partout.

D'une part, on paraît s'aviser du danger qui pèse sur la nature interne, organique : la survie de l'espèce est en jeu. Le décalage entre le contrôle obtenu sur les forces matérielles, entre l'exploitation réussie et illimitée de l'univers, et l'incapacité de régler les affaires humaines, de les hausser au niveau de ce contrôle et de cette exploitation, inquiète et désole. Le but si longtemps poursuivi, la conquête, a été atteint. Cependant, pour avoir dépassé les limites inhérentes à notre constitution, le mouvement dégénère en mépris et indifférence envers celle-ci. L'homme croyait avoir accumulé suffisamment de puissance pour se libérer de son être biologique, mais l'artifice social tend à détruire cet héritage. La violence collective a faussé ou brisé les mécanismes qui règlent le combat entre animaux des autres espèces. La densité urbaine atteint un seuil critique. L’agressivité s’accroît cons- 
tamment dans les villes où bientôt les hommes, comme les rats emprisonnés dans une enceinte trop étroite, se massacreront sans pitié. La tension que la surabondance des stimulations - bruit, rapidité des déplacements, etc. - les rapports entre générations et nations font peser sur le corps social aura raison des institutions avant que la famine survienne. Elle n'est pas compensée par l'amélioration de la coordination des fonctions du cerveau, et les risques d'explosion affective vont croissant. Verra-t-on l'humanité retomber au niveau de l'animalité, au lieu de préserver une sage mesure entre ses actions et ses capacités psychiques, physiologiques et instinctuelles ? Le risque en paraît d'autant plus grand qu'elle menace d'abattre les barrières écologiques qui sont les siennes pour empiéter sur le domaine des autres espèces et modifier l'environnement jusqu'à le rendre impropre à la vie. Le contact serait rompu entre les données de l'organisme et celles de la biosphère. Le "mal éthologique » ronge nos sociétés, dont les causes sont « les péchés mortels envers la biologie et l'écologie de l'humanité, que perpètrent sans cesse tous les gouvernements, l'exploitation, la pollution, la destruction définitive de la biosphère, dans laquelle et de laquelle nous vivons ${ }^{177}$. » L'homme n'a d'autre recours, s’il veut survivre, que de se réconcilier avec sa nature animale, d'en respecter les exigences génétiques permanentes, et de modifier en ce sens les choix qu'il fait dans la société.

Les rapports qu'il entretient, d'autre part, avec la nature externe ne paraissent pas meilleurs. Jusqu'ici l'humanité s'est montrée discrète, de sorte que l'équilibre fondamental a été préservé à chaque instant. Mais le pacte a été brusquement rompu. Les diverses pollutions s'ajoutent et se généralisent. La surpopulation en premier. Au cours des vingt dernières années, le nombre d'êtres humains s'est accru de $50 \%$, exerçant une pression extraordinaire sur le milieu. La faim, la sous-alimentation, le conflit des générations, la menace de guerre, la détérioration de la flore et de la faune en découlent. La plupart des espèces freinent leur expansion démographique, préservent une proportion optimale entre leur volume et les ressources de leur environnement. La nôtre semble avoir oublié ces règles de prudence et il convient de les appliquer d'urgence : «Si la surpopulation, écrit-on,

177 K. LORENZ : The Enmity between Generations and its probable Ethological Cause, The Psycho-analytic Review, 1970, 57, p. 365. 
est en effet un des facteurs fondamentaux de la conservation de la nature au $\mathrm{XX}^{\mathrm{e}}$ siècle, il ne fait aucun doute au biologiste qu'il est tout aussi important à prendre en considération pour la survie de l'homme sur terre ${ }^{178}$. »

Le diagnostic porte en lui-même la solution. Le contrôle des naissances par des moyens contraceptifs et l'autorisation de l'avortement, ramenant le taux de natalité à un niveau tolérable, serait en accord avec le mécanisme évoqué. Les besoins quantitatifs en aliments et en produits industriels seraient tempérés, la violence et l'agressivité résultant de l'entassement sur un territoire exigu se relâcheraient, la paix du monde se rétablirait, ou presque. Une fois que la pression sur l'environnement aurait décru, il se régénérerait plus aisément.

Ces arguments s'enchaînent suivant une logique peu convaincante, dont les failles permettent d'escamoter l'inapplicabilité des notions aux faits. En ce qui concerne la surpopulation, les choses sont relativement nettes. Lorsque les savants agitent l'épouvantail de l'explosion démographique, ils ne tiennent pas compte de la dynamique propre aux populations humaines. Ils négligent le fait que, dans notre histoire, l'augmentation du nombre est requise par la croissance, la transformation de l'ensemble homme - pouvoirs matériels, l'assimilation des ressources complémentaires en savoirs, individus et richesses. A moins d'un changement radical, on ne peut guère supposer que l'on parvienne jamais à éliminer le phénomène de surpopulation, facteur décisif dans la mécanique subtile de nos relations objectives, dont dépend en dernier ressort l'allure de ces relations. Sans population surnuméraire, nous l'avons constaté en son lieu, il n'y aurait point eu de division naturelle et point d'homme sous sa forme biologique et sociale actuelle. Par ailleurs, on n'aura pas l'indiscrétion de demander à ces mêmes savants de nous livrer les chiffres d'une population normale et les critères qui leur permettent de les calculer. Tenons-nous en à quelques observations. Lorsque, sur un ton pathétique, on dénonce l'excès de population qui désorganise nos sociétés et le cortège de conflits, de violence et de famine qu'elle entraîne, on se réfère à l'accroissement général du nombre d'hommes au-delà du volume censé être raisonnable aussi bien qu'à l'entassement en milieu

178 J. DoRst : La nature dénaturée, Paris, 1970, p. 130. 
urbain. Il s'agit là de deux phénomènes distincts. Même à population constante, l'afflux dans les villes est inscrit dans le mouvement des sociétés. Certes, il bouleverse des modes de vie, des organisations sociales, des comportements. Les concomitants économiques et politiques d'un tel bouleversement expliquent suffisamment la turbulence déplorée. Lier l'entassement à la rivalité, faire de la tension qui l'accompagne un simple effet de la densité analogue à celui que l'on constate dans les sociétés animales est contestable ; et l'on ne voit pas pourquoi, dans ce domaine, on serait autorisé à extrapoler de l'animal à l'homme avec moins de retenue que dans d'autres.

Le lien qui unit l'explosion démographique à la menace écologique demeure malgré tout très tenu. Cette explosion a lieu dans les pays pauvres d'Asie ou d'Amérique latine, et ne se fait pas sentir dans les pays riches d'Europe ou d'Amérique du Nord. Inversement, c'est dans ces derniers que la menace écologique se dessine, terrifiante, les premiers n'en souffrant guère. Les peuples qui se multiplient ont beaucoup d'air pour respirer et beaucoup de réserves matérielles, dont les nations opulentes usent largement; les populations dont la densité s'accroît modérément manquent d'oxygène ou de réserves matérielles. De sorte qu'il est difficile de saisir, du moins dans les termes où elle $\mathrm{a}$ été énoncée, le sens d'une relation qui fait de la dégradation du milieu une conséquence de l'augmentation du nombre d'organismes. La solution que l'on a préconisée est en tout cas naïve ou vaine. Le contrôle de la population par des moyens contraceptifs et par l'avortement peut difficilement passer pour une mesure révolutionnaire. D'une manière ou d'une autre, en y joignant l'infanticide, on le pratique depuis toujours. Croit-on vraiment qu'une fois l'expansion démographique freinée les peuples sortiront de l'état de dénutrition et de dénuement qui est le leur ? Si l'on voulait avancer une suggestion concrète, il faudrait se rappeler que la diminution du nombre des naissances est souvent un résultat, et non pas une cause, de l'aisance ; solution de riches, elle a été adoptée par les nations et les classes favorisées. Que les mêmes voix recommandent donc aux pays qui connaissent le fléau de la surpopulation de s'enrichir, d'élever leur niveau de vie, de se donner l'organisation sociale adéquate, et nous aurons atteint le but recherché. 
Le progrès scientifique et technique est un autre facteur de pollution. L'emploi des produits chimiques, la circulation des véhicules, la radioactivité propagée dans le monde par les retombées des explosions nucléaires ont des conséquences délétères. Le climat de la terre change ou changera ; nous sommes les agents de ces modifications, en raison des énormes quantités de gaz carbonique répandues dans l'atmosphère. L'eau, de son côté, est empoisonnée par les énormes quantités de déchets que la civilisation industrielle y déverse. La nature est devenue un dépotoir, gémit-on. L'équilibre qu'une technologie et une science tempérées réussissaient à maintenir est détruit par une technologie et une science à haut rendement, le changement se révélant irréversible à la longue. Leurs effets sont stériles et stérilisants. Certes, toute œuvre humaine, on s'accorde sur ce point, nie la nature, ou sert de médiation aux échanges de la nature et de l'homme. Mais la technique fruit de l'invention scientifique et du labeur industriel ne transforme pas la nature : elle l'élimine. Le ralentissement de l'activité scientifique et technique, en diminuant la pollution atmosphérique, alimentaire, que le développement de 1 ' «industrie de la connaissance » a si dangereusement accrue, contribuerait à rétablir des conditions de vie saines, à l'échelle des besoins nouveaux.

En quoi consisterait une telle contraception de l'esprit et du faire humains, on ne nous l'apprend guère, à vrai dire. Pas plus qu'on ne nous indique ce qu'il faut entendre par cette fameuse balance of nature, cette nature en équilibre. Les hommes, comme les autres espèces, n’ont pu évoluer qu'en la perturbant, les innombrables catastrophes dont nous voyons l'exemple dans les diverses parties du cosmos ayant forcé la recherche de solutions neuves. Mais il y a plus. Lorsqu'on dénonce la nuisance associée à la technique et à la science, on a l'air de s'en tenir au point de vue de l'Homme, avec une majuscule, c'est-à-dire que l'on prétend envisager les choses d'un point de vue universel. Or c'est là pure imagination, projection de la situation particulière de collectivités qui se figurent avoir atteint un sommet et pouvoir s’offrir un répit. Les autres collectivités, qui sont la majorité, ont au contraire intérêt non seulement à appliquer ces techniques et ces sciences, mais aussi à les promouvoir afin de pouvoir surmonter les difficultés qui leur sont propres. A moins de les persuader et de nous persuader que nous nous représentons sérieusement leur présent ou leur passé comme notre avenir, elles verront, à juste titre, dans ce 
choix de l'ignorance, dans cette mort de l'invention, une supercherie de plus, destinée à préserver les écarts existants au nom des intérêts suprêmes de l'espèce. Cette attitude trahit la profonde ambiguïté dont on fait preuve depuis longtemps envers la connaissance et le travail, considérés comme des activités auxquelles on s’adonne par nécessité, des contraintes auxquelles les communautés doivent se soumettre, des occupations subordonnées à leurs besoins, appendices et non parties intégrantes, d'une humanité statique. Certes, à des instants ou dans des circonstances d'exception, on les célèbre en tant que triomphes et forces de l'homme en général ; mais aussitôt que l'on constate qu'ils affectent le réel, entraînent des changements profonds, créent le devenir humain, la réaction est brutale. Tout est condamné en bloc : on se livre à l'éloge du non-savoir et de la spontanéité, on recherche la vie brute et la fraîcheur supposée de l'instinct, on glorifie le monde donné sans effort et sans pensée ; le reste, c'est-à-dire la discipline de l'art et de la connaissance, est flétri du nom de concret borné, troublant ou interrompant la fête naturelle, purgatoire qui risque de mener, si l'on n’y prend garde, du paradis passé à l'enfer à venir.

L'exploitation abusive des ressources, enfin, sous l'impulsion de la productivité et de la surpopulation combinées, conduit à leur épuisement sans qu'il soit possible de les reconstituer. Ayant atteint l'apogée de ce qu'on croyait être le progrès, on se découvre tout à coup en pleine sauvagerie, à la veille de la pénurie, les fonctions physiologiques élémentaires elles-mêmes — respirer, manger, etc. — ne pouvant plus s'accomplir. L'humanité est mise dans l'obligation de faire face à des problèmes analogues à ceux qui assaillent toute espèce animale au sein d'un milieu dangereux — dans notre cas rendu tel par nos œuvres - avec un héritage génétique et collectif diminué, les normes d'une saine adaptation perturbées. «L'homme, écrit le grand biologiste Konrad Lorenz, est en train de détruire peu à peu la nature, de ravager le biotope dans lequel et duquel il vit ${ }^{179}$. » La protection de la nature complète la panoplie des solutions, mobilise les bureaucrates, crée la possibilité d'emplois politiques et l'occasion de grandes conférences internationales. Pratiquement, on propose de sauvegarder les sites, d'arrêter le massacre des espèces animales, de restituer au paysage végétal son intégrité et sa splendeur. «Il faut, affirme un académi- 
cien ${ }^{180}$, que l'exploitation nécessaire des ressources naturelles préserve les beaux paysages, lieux où repose l'esprit de l'homme autant que dans les œuvres d'art. » De son côté, le président de la République avec une haute autorité déclare : «La France ne doit pas devenir une poussière d'agglomérations urbaines, dans un désert même verdoyant. Il faut sauver la nature, premier besoin de l'homme moderne, la nature cultivée et habitée. Une autre nature est une nature funèbre ${ }^{181}$. " Ce sauvetage offre en plus l'avantage de l'économie, le commentaire le laisse voir : «Et même si on se place au point de vue de la comptabilité économique, cette solution serait la meilleure : garder certains paysans à la terre, quitte à les aider à rester, coûterait moins cher que de faire entretenir des réserves par des fonctionnaires. » D'autant plus que ces paysans sont aussi des électeurs qui garderont avec la même vigilance le paysage politique et le paysage naturel.

La recommandation la plus fervente vise à constituer des réserves de nature vierge, une sorte de chaîne de musées de la nature, tout comme on a créé des réserves de populations primitives, mis l'art dans des vitrines, et enfermé les animaux dans des jardins zoologiques. Ces lieux seraient interdits d'accès et leur aspect devrait être gardé intact : « La première et la plus importante mesure est, aux yeux des naturalistes, écrit Jean Dorst ${ }^{182}$, la constitution de réserves naturelles intégrales, placées sous le contrôle public et dans lesquelles tout acte humain tendant à modifier les habitats ou à apporter des perturbations quelconques à la faune ou à la flore se trouve strictement prohibé. La nature y est donc abandonnée à elle-même, tout se passant au moins en théorie comme si l'homme n'existait pas. » La seule utilisation reconnue à ces réserves serait d'être des laboratoires naturels pour le biologiste (et bien entendu des lieux d'excursion pour le touriste) de même que - le rapprochement s'impose - les populations primitives en sont ou auraient pu en être pour l'anthropologue. Ces territoires protégés formeraient un point zéro du milieu ; l'intervention humaine y demeurant minimale et réfléchie, on pourrait les comparer utilement à d'autres milieux, à commencer par celui où nous vivons : " Ces étu-

\footnotetext{
180 Le Monde, 29 mai 1971, p. 11.

181 Le Monde, 29 juin 1971, p. 8.

182 J. DORST : op. cit., p. 170.
} 
des d'intérêt capital en ce qui concerne la recherche pure sont également fondamentales quant aux sciences appliquées, le milieu naturel ainsi conservé dans les réserves servant de terme de comparaison avec les milieux transformés par l'homme ${ }^{183}$. »

La doctrine de la protection de la nature que l'on désire isoler et embellir dans les parcs, comme le baroque l'a recréée dans ses châteaux, repose sur un paradoxe subtil.On décide que tout ce qui n'est pas végétal ou animal est artificiel, qu'il n’y a pas d'autres équilibres dans la biosphère que ceux de la plante et de l'organisme. Ce qui n'empêche pas de les décrire en facteurs quantitatifs d'oxygène, de gaz carbonique, d'énergie, c'est-à-dire en termes non organiques, chimiques ou physiques. Nous sommes invités à retourner vers le cadre qui était prétendument le nôtre, à regagner le monde des montagnes, des prairies, des eaux pures, notre état naturel, sans liaison avec l'état technique dont le bilan s'établit en machines, lois abstraites, chiffres, laboratoires, etc. Le chemin suggéré contournerait une partie de notre réalité, dont seraient expulsés travail et connaissance. Repeuplant les villages rendus inutiles par la machine économique et sociale, désertés par leurs habitants et tombant en ruines, la végétation redevenue brousse, les animaux errant, les hommes regagneraient l'innocence perdue, fêteraient leurs retrouvailles avec la nature: paysans et artisans d'opérette qui retournent à la terre non pour la féconder mais pour y faire retraite. Rien d'étonnant que l'on envisage la nature en la coupant de tout savoir et de toute activité, puisqu'on lui assigne une fonction purement végétative, de dépaysement, et non pas une fonction active, d'enracinement. La relation établie est foncièrement artificielle. Elle fractionne la nature en l'émiettant sous forme de parcs disséminés dans un environnement mécanisé, elle en fait un objet quelconque, à l'instar des objets techniques ou scientifiques soumis à l'observation et au contrôle des instruments de mesure et de comparaison. Loin d'aboutir à former un complexe d'éléments originaux libre de toute intervention humaine, celle-ci a lieu de la façon qui est habituellement la sienne dans les sciences et les techniques. La proposition de créer des "réserves » végétales et animales, où seuls pénétreraient les savants, représentant le degré zéro d'intervention humaine, ne diffère en rien, dans son esprit, de la proposition de cons-

183 Idem, p. 173. 
truire un accélérateur linéaire très puissant ou d'éviter toute contamination de l'air lunaire. Son seul effet serait de changer le théâtre de la nature, sujet de curiosité qui suscite l'invention, en une nature de théâtre, décor en trompe-l'œil plaqué sur un immense appareil mécanique et chimique. La protection ainsi conférée semble bien douteuse ; elle ne ramène pas à un état stable, abrité des transformations, mais débouche sur une gigantesque illusion, ayant ses côtés opératoires, qui se borne à retirer une partie des espèces du circuit qui était le leur pour les brancher, sur d'autres circuits qui finiront, à la longue, par les altérer, soit que les échanges qui étaient malgré tout stabilisés avec nous et nos savoir-faire amènent à péricliter nombre de ces espèces animales ou végétales, soit que l'on obtienne un milieu plus nature que nature : un parc.

Ainsi, pour remédier au mal du siècle, ne suggère-t-on d'autre remède que le retour à l'état antérieur, la naturalisation de la société. La politique à suivre ne diffère en rien de celle qui est préconisée par ailleurs. On cherche à insuffler une nouvelle âme à ce qui est censé ne plus en avoir, on veut maintenir en vie au lieu de changer la vie. Dans beaucoup de domaines, saignés de leur principe vital, ce principe est ensuite offert à titre de thérapeutique et de médication. Le travail n’apporte ni joie ni intérêt : adonnez-vous au loisir compensateur. Les villes se dégradent : évadez-vous à la campagne. Les aliments industrialisés sont nocifs : remplacez les substances qui leur font défaut par des équivalents chimiques. Et, dans le cas qui nous occupe, lorsqu'on envisage de conserver les parcs et les forêts, de purifier l'air et les rivières, on tolère, moyennant quelques aménagements, que les forces de production continuent à se développer suivant les modalités connues, conduisant aux effets dénoncés. La conservation et la contraception ne sont pas une réponse vraie à ces problèmes, pas plus que la philanthropie ne remédie à la pauvreté. Certes, il s'agit de manifestations graves, et il faut leur consacrer beaucoup d'énergie pour comprendre leurs raisons, les définir correctement et saisir leur évolution. Mais ni la voie prise ni les concepts employés ne vont au fond des choses. Pas plus que l'on n'essaie de transformer le travail, de construire autrement les villes en tenant compte du sens actuel du phénomène urbain, d'œuvrer dans la biosphère suivant d'autres méthodes, on ne met objectivement en question les principes d'une technique et d'une culture qui se sont développées chaotiquement, se 
comportant en prédatrices envers les ressources matérielles décrétées par la théorie libres et gratuites afin qu'elles servent plus facilement de proie aux « maîtres et possesseurs » individuels. Le retour à la nature est une panacée du même ordre, capable, croit-on, de réparer les dommages que nous lui avons infligés et que nous commençons, nous aussi, à subir, pour avoir oublié le respect que nous lui devons, pour avoir accumulé des artifices trop savants, en renonçant aux pouvoirs de la sensibilité, au contact direct avec les êtres, au plaisir de posséder un jardin comme à celui de respirer la senteur de la terre après la pluie.

La nature, dans cette pensée, qu'elle soit consignée dans les écrits des théoriciens ou qu'elle coure à travers le discours social, est un ordre relativement fixe, correspondant à la complexion anatomophysiologique des hommes. Elle est commune à toutes les espèces donc uniforme - de par leur caractère organique, si on envisage son essence elle-même. Quelle que soit la structure sociale d'une population, celle-ci est toujours décrite en termes biologiques, sa relation au milieu apparaît comme celle d'un organisme individuel, cohabitant avec d'autres êtres animés. Bref, on appelle nature l'ensemble de la flore et de la faune, sans en excepter l'homme qui y occupe toutefois une place à part. Les échanges avec les forces matérielles inanimées sont jugés sortir de son cadre. On aboutit à dédoubler la nature, ainsi que je l'ai exposé au début de ce livre. La solution préconisée consisterait donc à découper une partie de notre réalité, où n'interviendraient pas le travail et la connaissance, une partie qui demeurerait naturelle, tandis que l'autre partie les admettrait, s'affirmerait technique; la première serait un système au repos, l'autre un système en action. Nature et société apparaissent comme deux ensembles clos, emboîtés l'un dans l'autre, qui fonctionnent de façon autonome, la plupart du temps, à condition que les règles du jeu soient respectées. Elles sont dans une relation d'inclusion : la plus grande, la nature (environnement ou biosphère), enferme la plus petite, la société, comme les orbes solides de la cosmologie aristotélicienne entouraient les planètes sublunaires. Les interactions ou les ouvertures ne se produisent que de façon exceptionnelle. Le manque dans la nature provoque l'existence de la société ; l'excès de la société conduit à réintégrer la nature. Leur unité demeure négative, externe. Non seulement parce que les circonstances dans lesquelles elles communiquent et se nécessitent récipro- 
quement sont des moments de crise, mais aussi parce que chacune est, dans l'autre, une absence. L'homme ne peut vivre dans les deux états : il est soit dans la société, soit dans la nature. La rupture avec la première le rejette automatiquement dans la seconde. C'est pourquoi les chemins rebroussés vers la sauvagerie, la revendication des anciens modes de vie, la protestation contre le savoir et le faire revêtent la signification de réactions radicales contre toute société et toute culture, et non pas contre une société ou une culture. L'impression prévaut que l'on peut annuler la division intervenue, regagner l'état d'indifférenciation, décaper le vernis de l'acquis pour restituer les formes de vie immuables et authentiques. Il faudrait pour cela des révolutions topiques faisant revivre des états qui ont déjà existé - opposées en cela aux révolutions utopiques qui inventent des états n’ayant jamais existé — tant est grand le poids de la réalité présente.

La nature doit donc rétrograder, pour que la société puisse s'épanouir pleinement; la société doit mesurer ses effets ou s'évanouir, pour que l'on retourne à la nature. Pendant de longs interrègnes, elles réussissent à se maintenir dans une condition d'extériorité et de tolérance. L'histoire apparaît comme un mouvement de navette entre le point où la nature, en se corrigeant, rend possible l'éclosion de la culture, et le point où la culture cherche à retrouver la nature, c'est-à-dire à se corriger à l'aide de celle-ci. L’alternance des progressions et des régressions, la réversibilité impliquée donnent l'illusion d'un devenir, phénomène de surface qui laisse inchangées les structures latentes dans les profondeurs. Le véritable lieu de permanence de ces structures est l'individu organique, avec ses composantes génétiques et psychiques. Tout part de lui et tout y aboutit. Le lien au fondement naturel est essentiellement un lien à la biologie ; il convient de la maîtriser, de l'adapter, de la conserver. Le social est l'envers et la contrainte de l'individuel — Louis Dumont insiste à juste titre sur l'atomisme individuel de la pensée occidentale — et de l'organique, ce qui explique sa variabilité et sa fragilité. A un extrême ses œuvres, à l'autre extrême son animalité définissent dans des combinaisons diverses, pour l'homme, le cours du monde. Compris de cette façon, le paradigme de l'ordre naturel et social, de leurs relations, succinctement résumées ici, discutées à plusieurs reprises ailleurs, est biocentrique. 
Retour à la Table des Matières 


\section{Le thème du changement et de la création.}

$\underline{\text { Retour à la Table des Matières }}$

\section{(1) La nature historique.}

A priori il semble difficile de résoudre le problème des rapports de l'homme et de la société à la nature quand on pose l'homme et la société en dehors de la nature, contre elle. Cette conception est née de l'idée de l'universalité — entendons par là identité et uniformité — de la nature et de la particularité de la société. Le contraste entre l'unicité de la première et la diversité de la seconde fait partie de ce lot restreint de certitudes sur le bien-fondé desquelles, malgré les saines habitudes de l'esprit scientifique porté à tout réexaminer, on ne s’interroge guère.

Des témoignages ont été rassemblés dans le cours de ce travail, montrant la particularité, organique ou non, de la nature et l'universalité de la société ; il convient à présent de les reprendre dans un cadre plus précis, celui d'une nature engendrée, redéfinie par l'action de l'homme, et d'en tirer les conséquences.

La notion de nature uniforme, rappelons-le, suggère aussitôt l'existence d'une configuration stable de forces matérielles dans l'espace et dans le temps, de ressources déterminées communes à tous les organismes et correspondant à leur structure interne. Elle a un caractère prescriptif quant à l'adéquation des comportements des êtres qui la composent, normatif quant au contenu qui la caractérise. Elle signifie, à sa manière, que l'ordre naturel admet tout au plus un mode d'interférence que partagent toutes les espèces quand elles y aménagent leur niche écologique ; toute autre action, celle de l'homme en particulier, le contrarie, c'est pourquoi il convient de l'y soustraire. Mais cette notion n'a guère de fondement. En quoi l'acte d'intervenir dans le cours et l'architecture des systèmes matériels serait-il exceptionnel, pourquoi faudrait-il le décourager à tout prix ? Il est, au contraire, des plus ordinaires, et chaque espèce, selon ses facultés, l'effectue journellement, transformant substances et énergies. En revanche, ce serait plutôt l'obstruction, le non-déploiement des facultés, 
la non-ingérence dans les cycles propres au milieu, qui constituerait l'exception, et l'artifice. Certes, l'argument est dirigé aujourd'hui comme autrefois - contre la technique contemporaine et tolère d'autres formes d'action passées qui reposent néanmoins sur une technique nécessairement différente. L'homme sans art, sans technique n'existe pas, n'a jamais existé. Chacun de ses contacts avec les pouvoirs de l'univers ne peut se faire que par le truchement de savoirs organisés, auxquels sont associés et intégrés les appareils sensoriels et intellectuel : une autre forme de contact est abstraction pure. Une puissance matérielle à laquelle nous n’aurions pas accès de cette façon n'est rien pour nous : pour entrer dans notre monde, il faut qu'elle s'articule avec nos capacités, à la fois biologiques et instrumentales, et se relie aux puissances et aux substances qui s’y trouvent déjà.

Même ce milieu protégé, que l'on s'apprête à parquer et mettre en réserve, en le voulant pur de tout artifice, livré à des rythmes spontanés, parangon de la non-intervention humaine, lui aussi est notre création, notre fait. Les Anglais s'indignent parfois que l'on veuille tailler les haies, geste contre-nature guidé par la science de l'agriculteur moderne. Ils oublient que ces haies ont elles-mêmes été plantées et cultivées. On peut en dire autant de maint paysage " naturel » qui résulte d'abord d'un labeur et d'un soin considérables. Les destructions de nature dont nous sommes journellement témoins sont avant tout destruction d'un certain travail, fait qui paraît échapper à ceux qui déplorent ces disparitions. Les vallées verdoyantes, les étendues dites sauvages n'ont pas toujours existé ni ne se sont formées au hasard. L'emploi du feu pour effrayer le gibier ou défricher les terrains a profondément modifié l'aspect des continents. Ici un excellent sol arable, là, comme en Amérique du Nord, la prairie entretenue par les troupeaux qui y paissaient. A Madagascar, la terre ravagée par les feux de brousse, ravinée par les pluies est devenue sur les hauts plateaux impropre à toute culture, et on l'a comparée à la brique dont elle a la couleur.

L'Afrique offre peut-être le tableau des plus grands bouleversements. Son territoire ne présente plus aucune ressemblance avec le paysage qu'a pu y connaître l'espèce humaine à ses débuts. La savane elle aussi est le produit du feu, et à son tour elle a permis aux espèces qui se nourrissaient de l'herbe et broutaient les plantes de se multi- 
plier. Le développement extraordinaire des mammifères a commencé là. La pollution de la vie et par la vie vient de très loin, les sites naturels sont son œuvre. Allons jusqu'au bout. On a coutume de partager les animaux en sauvages et domestiques, les premiers étant censés s’être formés et avoir évolué à l'écart de toute interférence de notre part. En réalité, cette interférence a seulement eu un caractère différent, les a affectés d'une autre façon. Aucune espèce n'est, à vrai dire, demeurée dans cet hypothétique état primitif : certaines ont disparu à notre contact, par exemple les ongulés, atteints par les mêmes parasites que nous ; d'autres, tels les chats, les chiens, les micro-organismes ont prospéré dans notre voisinage. En épuisant les terres, l'agriculture a privé de leur habitat et de leurs ressources des animaux qui ont été obligés de modifier leur façon de vivre, pour échapper au dépérissement certain, tandis que la prédation - activité « naturelle » par excellence - faisait des hécatombes contre lesquelles aucune espèce n’avait de moyens de défense efficaces. La chasse à son tour a décimé les prédateurs. Il est difficile de mesurer exactement le degré d'influence en chaque cas, si l'on prend en considération non seulement l'action immédiate mais aussi les effets les moins manifestes ; vis-à-vis de presque toutes les espèces, la nôtre a joué le rôle de force matérielle, transformant les données de la biologie ${ }^{184}$. Voyant des macaques dans une région déboisée, des savants en ont conclu qu'ils avaient affaire à une espèce vivant au sol, analogue aux babouins d'Afrique. En vérité, l'Est de l'Asie et le littoral du bassin méditerranéen ont été profondément modifiés par l'agriculture ; les macaques en question étaient à l'origine, vraisemblablement, une espèce arboricole, tout comme leurs congénères du Japon et du Sud de l'Asie où la forêt est demeurée intacte. Il ne serait pas faux de dire que les animaux sauvages sont ceux qui se sont adaptés à l'homme, aux conditions qu'il a créées, alors que les animaux domestiques sont ceux auxquels l'homme s'est adapté, qui sont entrés dans le circuit de ses échanges avec le milieu.

Ce n'est pas, comme on le prétend, avec la technique envahissante que le processus a débuté, mais bien plus tôt, avant même l'apparition de l'homo sapiens, et depuis il se poursuit sans discontinuer. On invoque souvent, à ce propos, les dangers que court notre biologie. Où se-

${ }^{184}$ M. Chance et C. Jolly : op. cit. 
rions-nous si elle ne les avait pas courus ? La locomotion bipède, le changement volumétrique du cerveau - le langage et la pensée à cette occasion - l'alimentation carnée, les pratiques prédatrices ont été, chacun en son temps, un danger de cet ordre, atteignant le matériel génétique et l'équipement instinctuel. On hésite à mettre sous les yeux du lecteur des faits aussi indubitables, quoique si rarement mentionnés. A leur propos, la croyance en un état dégagé de toute intervention humaine - ce degré zéro ardemment souhaité par certains biologistes — révèle ses limites et ses illusions : il s'agit là d'un état relatif à un certain mode d'intervention, cynégétique, agricole, artisanale, scientifique ; et c'est bien à tort que l'on fait abstraction des autres modes, chacun ayant joué son rôle dans le monde animal ou végétal qu'il a contribué à façonner. La nature, telle que la perçoit une espèce animale, est l'art de l'homme, et la nature que l'homme s'efforce de préserver intacte et de protéger de toute interférence est l'art d'un autre homme, ou encore l'art de l'animal, auprès duquel les Grecs nous conseillaient de nous instruire. Le milieu naturel normatif, initial, échappe à toute saisie : il n'est pas. L'agriculteur le situait dans la forêt et la savane broussailleuse, pour le cultivateur de maïs il était représenté par la chasse et l'animal, et les végétaux lui apparaissaient comme des produits de la culture. Plus près de nous, à l'âge mécanique, les arts manuels qui prolongent le corps et s'appliquent à des matières premières végétales ont constitué une autre sorte de milieu naturel. Bientôt nous le verrons retiré dans les mines et les hauts fourneaux, à mesure que ces établissements disparaîtront de notre vie quotidienne. A chaque époque, pour chaque groupe de possesseurs de facultés spécifiques, ce milieu naturel authentique, où l'humanité est en rapport direct avec les choses qui satisfont ses besoins immédiats, est perçu comme un " ailleurs ». On a déjà compris que les frontières tout comme le contenu de la nature première et de la technique cultivée sont, pour le moins, variables, que plusieurs configurations différentes peuvent mériter également le qualificatif de naturelles; aucune ne jouit des prérogatives du commencement absolu et de la norme.

Toutes ces observations nous obligent à renoncer à la notion de nature uniforme, nous amènent à abandonner l'idée que nous ne participons pas activement à son organisation. Au contraire, la nature n’a de sens et de forme que par rapport à ce que nous en faisons. Parmi les pouvoirs matériels qui jouent un rôle déterminant dans sa création, il 
convient d'inclure l'homme avec autant de vigueur qu'on en a mis autrefois à l'exclure. A défaut, il ne reste plus qu'à la qualifier, en reprenant les termes employés par Sigmund Freud, d' " abstraction vide, dépourvue d'intérêt pratique ${ }^{185}$. ». Dans ces conditions, le retour à la nature est une double impossibilité : sur le plan de la réalité, ce que l'on savait déjà, et aussi sur le plan de l'hypothèse, ce que l'on s'efforce d'ignorer. Ni concrètement ni en pensée on ne peut en faire l'expérience.

Ces remarques invitent à sortir une fois pour toutes le substrat naturel du domaine des entités passives, à accepter l'ingérence de l'homme dans le cours ordinaire d'une nature qui n'est pas un réceptacle inerte de forces matérielles, et la transformation de celle-ci, sous l'impulsion humaine, dans l'espace et dans le temps. L'environnement autonome, pur contenant où subsistent des êtres biologiques et sociaux qui ne lui doivent rien, est un mythe, ruiné par l'évidence de la succession évolutive : nous dépendons de notre milieu, car nous l'avons fait autant qu'il nous a faits. Lorsque apparaît une substance ou un processus matériel nouveaux, ils entraînent forcément une refonte de tout l'agencement naturel, ils font virer son histoire dans une direction différente ; ainsi l'émergence de la vie a eu des répercussions sur la constitution des hydrocarbures, une réaction biogénétique prenant la place d'une réaction physico-chimique. Dans toute la biosphère, systèmes organiques et inorganiques s'influencent réciproquement. La division cellulaire, puis la reproduction sexuelle ont donné une impulsion décisive à l'évolution organique, créant ce milieu que nous déclarons avec emphase vouloir protéger, après avoir si longtemps ressenti le besoin de nous protéger contre lui. Les composantes et les contours de la réalité naturelle changent d'une galaxie à une autre, d'une époque à une autre. Concevoir un agencement naturel unique, c'est ignorer les différences rapportées aux divers points de l'espace et du temps pour ne retenir que les forces ou les relations appartenant à la plupart des combinaisons inventoriées jusqu'à ce jour. Une telle réduction reviendrait à inscrire dans le tableau cosmique les seules forces physiques, à accepter uniquement les lois qui les régissent, bref, à tracer une épure basée sur le plus grand diviseur commun des configurations connues dans l'histoire du monde.

185 S. FREUD : The Future f an Illusion, Works, t. XXI, Londres, 1968, p 56. 
Il faut cependant définir la nature comme une constellation de matières organisées en séries simultanées ou successives. Les phénomènes biologiques au-delà du stade cellulaire, la sélection naturelle, on vient de le signaler, lui ont ajouté une dimension, y ont introduit un développement singulier, dont l'origine est datée. De même, avec notre espèce, les forces sociales diffuses ont pris de la consistance, ont commencé à infléchir de leur poids spécifique le processus général. Ce que l'on présente d'habitude comme éloignement de l'univers naturel, le passage des primates à l'homme, est en réalité une transformation et une expansion, non pas une sortie mais une autre orientation du mouvement préexistant. La reproduction de l'espèce humaine, en formant des individus et des groupes d'un type inédit, perpétue une série de comportements, de savoirs, de conventions, qui tous sont répartis selon une loi objective, et qui représentent une modalité de relation avec les échanges matériels. Les talents, les dextérités, à l'instar de certains instincts aux divers degrés de l'évolution, sont en corrélation avec les appareils anatomo-physiologiques, augmentent la puissance ou l'acuité sensorielle, ajoutent de l'intelligence ou des sens qui faisaient défaut - mesure, rapidité, etc. - donc sont relatifs aux organes de reproduction qui en assurent la conservation et l'exercice. Les instruments mis en œuvre sont des moyens de reproduction. Et l'on peut ranger parmi eux la coordination sociale, y voir un de ces outils que les espèces utilisent avec des fortunes diverses.

Le rôle décisif dans la naissance de l'homme revient non pas à une mutation heureuse mais à la présence d'un surplus de ressources complémentaires en individus, les bandes de mâles, et en techniques station debout, locomotion bipède occasionnelle, utilisation du bois et de la pierre en guise d'artefacts - qui s'est combiné, les conditions génétiques et sociales ayant atteint un niveau favorable, avec les ressources complémentaires en nourriture, petits animaux et cadavres laissés par les grands fauves. La conversion en prédatrice d'une espèce qui figurait parmi les proies a banalisé des conduites, des savoirs, des outils, des virtualités biologiques, et projeté les animaux parmi les éléments du milieu hominien, essentiellement végétal. Cette modification qualitative s'est accompagnée d'une modification quantitative, l'aire de déplacement s'est accrue, l'espèce sylvicole a annexé la savane. A la longue, la vie dans un univers mixte n’a plus été possible. 
Les facultés nouvelles exigeaient d'être cultivées avec un soin particulier, le choix s'imposait entre la chasse et la cueillette qui se déroulent dans des lieux et des temps différents. Tout portait les chasseurs à briser avec les activités et les échanges qui contrariaient le développement normal, l'épanouissement de leurs propres échanges et activités. En prenant une distance par rapport aux groupes de collecteurs, en s'opposant à eux, ils s'écartaient aussi des autres animaux, des primates notamment, se tournaient contre eux et en venaient à les considérer comme des objets, des parties du milieu. Ils les percevaient sous la forme d répertoires d'habiletés et de ressources qu'ils s'efforçaient de s'approprier et de reproduire à leur façon. Les organisations sociales, dont les liens étaient déjà assouplis vis-à-vis de leurs constituantes biologiques, servaient à renforcer ou précipiter le mouvement. La première ébauche d'une nature vraiment humaine était en place.

La séparation de la chasse et de la cueillette coïncide avec cet écart que marque l'homme entre son univers et celui de l'animal. Elle favorise la croissance numérique et l'apparition d'espèces hétérogènes. Les ressources secondaires deviennent principales, le surplus démographique se transforme en une partie intégrante de la population qui se définit à un autre niveau. Sous les dehors d'une interaction avec le monde extérieur s'accomplit une métamorphose des organismes. Ils conservent la station debout et la locomotion bipède, ce qui entraîne des changements anatomiques et neurophysiologiques indispensables. A cette occasion, les espèces hominiennes ont bouleversé les modes de communication et de relation, insérant le langage et la technique dans la complexion biologique, les transactions collectives et dans le contact avec les autres créatures. La pensée symbolique, qui les résume, inscrit dans les appareils sensoriels et les circuits neuroniques son acquis devenu génétique, mode d'ordination qui contribue à intégrer les comportements, à distribuer les informations circulant autour de l'homme, à infléchir les rapports entre individus et groupes. L'horizon de l'animal est complètement assimilé et transformé. On ne peut parler, à ce propos, de barrière, de différence absolue localisée à un instant précis du passé, de comblement d'une déficience par le moyen d'une instance telle que la pensée, le langage, la technique. Ce sont les prémisses d'un développement de la nature qui est propre à nos espèces ou à notre espèce. L'homme a désormais le pouvoir de susciter, de combiner les forces matérielles en fonction de l'impératif 
de la collectivité, compte tenu de son étendue et de sa structure; la croissance du volume de la population va de pair avec la croissance et la diversification des capacités qui permettent de multiplier, de réorganiser ces forces, en jouant d'un éventail plus large d'aptitudes physiques et intellectuelles. La division naturelle des groupes humains qui se distinguent par leurs facultés leur donne la possibilité de vivre sur un même territoire en s'attachant respectivement à des forces matérielles distinctes, à l'imitation des espèces biologiques séparées qui habitent une aire commune en exploitant des ressources spécifiques. Cette multiplication évolutive qui se produit dans le temps signifie, en l'occurrence, qu'une force matérielle se substitue à une autre, que celle-ci est subordonnée ou abandonnée au profit de celle-là, ou qu'elles finissent par s'articuler dans un système naturel correspondant.

La division de la chasse et de la cueillette est la première séparation dans la ligne du développement propre indiqué. L'homme en est le produit et non pas le donné. Depuis, à plusieurs reprises, d'autres « humanités » se sont détachées, avec le cortège des forces matérielles auxquelles elles s'associaient : les agriculteurs, les artisans, les ingénieurs, les scientifiques. Les différents groupes ont mis en avant les propriétés respectives des végétaux et des animaux, du corps humain, des forces mécaniques, des phénomènes chimiques et électriques qui caractérisent les divisions ultérieures. Autour d'eux, autour de leurs disciplines - arts, philosophies, techniques, sciences — se sont déployés les états naturels qui les prolongeaient. Seuls ces états ont une réalité, seuls ils représentent la nature sous ses aspects successifs, dynamiques, dont chacun est une totalité originale de matières organisées. La pluralité des ordres naturels implique évidemment leur devenir. Certains ont précédé l'arrivée de l'homme ou ignorent sa présence, ou bien lui survivront. L’ignorance est réciproque, et ces ordres n'ont aucune importance pour nous. Parmi ceux qui nous comprennent, quelques-uns suscitent en vain le regret d'une nature antérieure, ni plus ni moins « naturelle » que la nature qui la remplace. Nous n'en connaissons pas d'autre. Nos sciences, nos arts y jouent un rôle constitutif. Ici, opposer l'effet technique au phénomène matériel, le milieu technique au milieu naturel, n’a pas de sens : tous deux sont inséparables et se font valoir réciproquement. Le technique découpe le naturel qu'il met en œuvre. Artifices et savoirs sont les médiateurs concrets 
entre l'humain et le non-humain ; plutôt que l'anti-naturel, il faudrait voir en eux le pré-naturel, élément indispensable du processus qui engendre notre nature familière. Historiquement, la métamorphose d'une totalité artificielle en totalité naturelle suit un déroulement continu. Il a fallu trois siècles pour voir dans le mouvement des planètes la transposition du mouvement mécanique de l'horloge, et un laps de temps équivalent s'est écoulé avant que l'on reconnaisse dans l'électricité, que l'on croyait être un effet instrumental, un phénomène qui ne requérait pas l'action de l'homme et avait lieu dans tout l'univers. Tout ce que nous posons comme donné est aussi, par bien des aspects, notre produit, et l'on peut dire de l'homme en général ce que Paul Klee disait de l'artiste - mais l'homme n'est-il pas toujours créateur? qu'avec son faire "plus s'imprime en lui, au lieu d'une image de la nature, celle-là seule qui importe - de la création comme genèse ${ }^{186}$ ". C'est pourquoi il n'y a de nature que là où il y a travail et connaissance ; et partout où l'on aperçoit la nature, on peut découvrir le travail et la connaissance qui la sous-tendent. La nature de nulle part et d'aucun temps, la nature sans sujet, est à notre égard un pur néant.

Ce n'est pas tout. Sous plusieurs prétextes, on entretient l'idée d'une accumulation des techniques, d'un gonflement de leur milieu, boule de neige qui roule depuis les origines au détriment de notre milieu naturel ; on leur suppose une marche uniforme et continue, un savoir et un monde matériel unis par un rapport de contrôle s'enrichissant constamment, les dimensions physiques ne cessant d'augmenter. Ce n'est là qu'une apparence. En profondeur, ces accroissements se sont accompagnés de destructions incessantes sans lesquelles aucune invention, aucun renouvellement n'est possible. Les deux effets contradictoires sont indissolublement liés. Que les agriculteurs se multiplient, qu'ils cherchent à mieux faire valoir leurs terres et à encourager la reproduction de certaines espèces végétales, ils n’y parviennent qu'en éliminant ou repoussant aux confins de leur domaine des espèces, pour eux sauvages, en défrichant la forêt ou en limitant l'étendue de celle-ci, donc en réduisant à néant ce que, pendant des centaines d'années, collecteurs et chasseurs avaient conservé. Et de même le mécanicien ou le pasteur, l'artisan ou le scientifique,

186 P. KLEE : Théorie de l'art moderne, Paris, 1964, p. 28. 
chacun à son tour, pour assurer l'essor de son propre ordre naturel des choses, a fait dépérir, a détruit ou transformé des substances, des réflexes moteurs, des tournures d'esprit, des qualités longtemps reconnues aux êtres animés et inanimés. L'enchaînement des découvertes et des arts, leur disparition et leur retrait dessinent, de façon générale, les bouleversements d'une association des forces matérielles, incluant l'homme, qui se change en une gamme nouvelle de forces satisfaisant de nouveaux besoins organiques à travers la modification des facultés intellectuelles et sensorielles. Ce qui est remis en cause à cette occasion, ce qui surgit au terme de la transformation, c'est un état naturel. La distance qui nous sépare des communautés paléolithiques ou néolithiques ne se mesure pas en quantités d'énergie, de pouvoirs matériels annexés, ou par la meilleure emprise que nous aurions sur l'univers, et il serait également faux de dire que les difficultés que nous avons rencontrées dans les derniers millénaires ont été plus grandes que celles qu'elles ont eu à surmonter au cours de centaines de milliers d'années. Cette différence se trouve essentiellement dans le caractère que nous entretenons avec les matières et les énergies, créant un autre état des rapports entre l'homme et le milieu matériel : organique pour les Grecs, mécanique à l'époque classique, on peut aujourd'hui le qualifier de cybernétique. Le monde naturel n'est pas devenu un monde technique : il a simplement évolué. Le clivage entre ces états, ces natures si souvent décrétées ultimes, voilà le résultat frappant de ce qu'il faut nommer l'histoire humaine de la nature, qui se différencie des autres histoires repérées çà et là. Les comparaisons terme à terme auxquelles on se livre fébrilement de nos jours en essayant de replacer notre espèce parmi les autres, de substituer le zoomorphisme à l'anthropomorphisme, en faisant régner la «terreur biologique », ne se justifient guère, puisqu'elles méconnaissent l’hétérogénéité fondamentale.

Il faut donc renoncer à l'hypothèse d'un équilibre de la nature qui se serait établi spontanément, à quelque époque que ce soit, en présence de l'espèce humaine, sans que celle-ci soit intervenue de tout son poids pour l'instaurer. Il n'y a pas davantage de signification à opposer une période ou une région où cette ingérence aurait été discrète, à une période ou région où l'harmonie aurait été gravement perturbée par l'action massive de l'homme. Croire que l'on puisse retrouver l'état antérieur d'équilibre est encore plus dénué de fonde- 
ment : à aucun moment les forces naturelles ne se contrebalancent sans heurt, et il ne peut s'agir en aucun cas d'une situation permanente, donnée une fois pour toutes. Celle-ci exclurait toute évolution, notamment l'apparition et la disparition d'étoiles et de planètes que nous observons, entre autres. L'espoir mis en une stabilité fondamentale est pernicieux, et l'on ne voit guère pourquoi elle jouirait d'un statut privilégié dans les actions et les pensées. Les espèces qui ont été le plus fidèles à leur condition première, qui s'y cramponnent sans changer depuis des dizaines et des centaines de milliers d'années, ne sont pas plus glorieuses que les espèces qui ont été emportées dans le flot du changement et ont su y nager, jusqu'à atteindre la pointe fragile et oscillante de l'évolution. Le seul équilibre auquel on puisse raisonnablement prétendre est celui de la mise en commun des facultés et des forces matérielles en présence - flore et faune, mais aussi substances chimiques et énergies nucléaires - compte tenu de leurs rapports mutuels et de la situation du savoir, du système solidaire qu'elles peuvent former. A condition d'y inclure l'homme, non seulement son organisme mais surtout sa société. Cet équilibre, en tout cas, ne saurait être que momentané, marquant une configuration évolutive. Il n'est pas la remémoration ou la restauration de ce qui a été, mais l'aboutissement du changement de l'état existant, l'avènement d'un nouvel état qui nous est aussi naturel que celui que nous avons mis en danger. Autant dire qu'il faut, à chaque fois, réinventer l'équilibre.

On ne saurait rien espérer d'autre : nous n’atteignons jamais la nature, pas plus que la société, toutes deux étant historiques. Notre effort ne peut porter que sur des sociétés, des natures ; nous en définissons les contours et l'organisation, recensons les êtres qu'elles incluent et les qualités qui leur sont nécessaires pour coexister. La tension extraordinaire qui en résulte incite à chercher une issue qui abolirait le devenir, suspendrait le mouvement, instaurerait la paix des temps révolus. On voudrait contrecarrer le futur en s'aidant du passé, promouvoir et revitaliser le passé comme futur, et par là figer le présent dans le statu quo. Cette abstraction est renvoyée à une réalité qui a élaboré ses propres solutions et qu'il faut aborder non pas à reculons mais de face, jusqu'à ce qu'elles finissent par prendre corps. Dans cette découverte, la tension se résout enfin, les hommes comprenant, chaque fois, que vivre c’est vérifier les lois de la nature. 


\section{(2) La société positive et négative.}

Le rôle que jouent les hommes dans la formation des états de nature matérialise la présence et l'action de la société humaine. Il manifeste aussi la diffusion du social sur l'arbre de la vie. A mesure que nous étudions mieux les espèces animales, nous découvrons que, à quelques exceptions près - le léopard, le blaireau, la martre ou le vison, par exemple - toutes se rattachent à une forme de vie collective ; elles ont des mécanismes qui assurent leur diversification lorsqu'elles ont à remplir des fonctions spécifiques, d'autres qui servent à satisfaire le besoin de réunion des individus en couples et en groupes. Il y a quelque cinq cent millions d'années que la division cellulaire a fait place à la reproduction sexuelle qui substitue à la bipartition d'un être l'union de deux êtres distincts. Ce moyen offrait des possibilités de différenciation si nombreuses, il accroissait tellement les chances de survie qu'il est devenu la règle pour une très grande partie des êtres vivants. Certains bouleversements du milieu peuvent avoir des conséquences dramatiques sur la vie d'une espèce ou sur les rapports entre plusieurs espèces, allant jusqu'à entraîner la disparition d'une famille entière d'individus semblables. Cependant, lorsque les individus présentent des caractères différents, ils sont beaucoup moins vulnérables à ce genre de catastrophes qui ne les atteint pas tous également; ils s'adaptent aussi plus aisément à un milieu diversifié dont ils peuvent reconnaître et aménager les éléments au gré de leurs besoins. Plus on avance dans l'évolution, plus sont nombreuses les occasions d'association à des fins autres que reproductives : explorer le territoire, partager les ressources, éviter les prédateurs, éduquer les jeunes. Les nécessités de la reproduction imposent la dissolution momentanée des groupes et la formation de couples, mais on voit en même temps des animaux lutter et périr pour assurer la défense de la bande.

Les qualités inhérentes au groupe se transmettent d'une génération à l'autre : traits anatomo-physiologiques, certes, mais de plus mécanismes de coordination qui articulent l'individu avec la collectivité, l'amenant à servir l'intérêt général plutôt que son intérêt personnel, dans toutes les situations où la vie sociale l'exige. L'adaptation et la 
sélection agissent au niveau de la concurrence entre individus dans l'existence quotidienne. Elles se manifestent encore pour maintenir la cohésion du groupe contre les aléas de l'environnement. Un double courant se dessine ainsi. Il distribue les potentialités organiques et tient ensemble les subdivisions instituées en donnant la possibilité d'induire une pluralité de relations avec le milieu, et en filtrant les effets du milieu sur les divers organismes. Il reproduit le génotype et renouvelle les phénotypes de manière à préserver les individualités à partir d'une multitude de types, la collectivité produit les individus qui lui sont indispensables - et à mettre en place des mécanismes d'intervention interne parallèles aux mécanismes d'action externe. Ce qui se crée ainsi, c’est la société. Soulignant sa généralité et sa nécessité, le biologiste W.C. Allen a pu écrire que « la sociabilité n’est pas un accident apparaissant sporadiquement chez quelques animaux hautement évolués, mais un phénomène normal et fondamental ».

La société humaine est évidemment un chaînon de cette séquence puisqu'elle résulte, nous l'avons vu, d'une transformation profonde des sociétés de primates. Celles-ci, avec leurs types d'organisation et leurs rituels, avec leurs clivages et leurs hiérarchies distinguant âges, sexes, fonctions, lui ont fourni un modèle et une matière première. La collectivisation des activités productives et reproductives, le nomadisme, la division de la chasse et de la cueillette ont transporté la matière première et le modèle sociaux dans un autre univers naturel, leur ont donné un contenu biologique et mental adéquat, pour aboutir à l'obligation de redécouper et d'élargir la définition du domaine collectif et d'y créer des structures nouvelles - tout comme l'introduction d'un moteur, dans une calèche a incité les constructeurs à redessiner l'objet entier conçu sur des nouveaux principes pour en faire l'automobile, avec son profil et son aérodynamique propres.

Compte tenu de son appartenance à l'ensemble des sociétés et de l'action qu'à leur instar elle exerce sur l'organisme et le milieu, la société humaine apparaît sous deux aspects, opérateur de la nature et organisation autonome, qui redoublent ses contraintes. D'un côté elle est société positive, puissance matérielle qui regarde vers les autres puissances matérielles, forme que prennent les rapports des hommes qui se groupent pour créer leur environnement. En font partie les moyens qu'ils ont de conserver et d'étendre leurs facultés organiques 
et psychiques, de reproduire les ressources humaines et non humaines. Reproduire, c'est-à-dire répéter et réinventer, conserver et renouveler, se donner les instruments d'une continuité qui modifie l'organisme ou le milieu, indépendamment des exigences immédiates de l'organisation collective ou physique.

Le façonnage d'une partie des instincts, des appareils neurosensoriels, s'intègre à ce cadre ; il serait faux de leur attribuer une existence à part : "Il est d'une grande importance, avertit Niko Tinbergen ${ }^{187}$, pour l'intelligence du comportement instinctif dans son ensemble, de nous rendre compte que les divers instincts ne sont pas indépendants les uns des autres. " Ils s'articulent constamment avec une activité spécifique qui précise et conditionne leur combinaison, leur poids, leurs liens de complémentarité ou d'incompatibilité. Dans leur nombre figurent les tendances ayant pour motif la faim, la soif, l'agression, la procréation. Il faudrait y ajouter, pour l'étudier davantage et dans toutes ses ramifications, l'instinct épistémique. Moins visible et moins fixé à un montage neurophysiologique individuel, il représente, sans conteste, une énergie mobilisatrice qui investit, enveloppe l'organisme ou le déplace, tantôt à la recherche d'une stimulation plus forte et plus neuve, tantôt en quête d'une stimulation plus atténuée et plus ancienne. Nous avons remarqué que les anthropoïdes ont un vif penchant à l'exploration du milieu, un intérêt pour les objets inconnus, voire dangereux. Dans de nombreux cas, l'examen des diverses parties du corps, l'inspection du territoire, les modes de rencontre hostile ou amoureuse, l'épouillage ou le toilettage servent, de manière détournée, à satisfaire le penchant épistémique. Le couple de la connaissance et de la reconnaissance du monde, la forte envie d'éprouver celui-ci et de s'éprouver, d'engendrer des tensions et de les apaiser, de vérifier que l'on est vivant, que l'on a des aversions et de préférences, dissolvent la grisaille de l'uniforme, favorisent l'imprégnation par le divers. Les hommes partagent avec une grande partie des êtres animés le désir de créer de l'information qui les pousse à provoquer l'événement, faire des essais et des expériences, quitter l'entourage commun, s'attaquer aux problèmes par leur côté incongru, contourner les schèmes stables qui exercent souvent des contraintes dévitalisantes. Le nomadisme des chasseurs, les déplace-

187 N. TinBERGEN : op. cit., p. 158. 
ments de populations ont certainement un lien avec cette propension. Les structures collectives intègrent la totalité des dispositions organiques, les proportionnent et les distribuent entre les différentes catégories d'individus. La chasse ou l'agriculture, la mécanique ou la cueillette réalisent de telles combinaisons, suivant les patrons qui sont particuliers à chacune. Ces structures qui ont des répercussions sur toutes les opérations et font sentir leur poids dans toutes les disciplines étayent les biosphères successivement créées par la découverte et l'utilisation de nouvelles ressources. Elles trouvent leur prolongement dans l'architecture de ces biosphères, les mythes ou la philosophie d'Aristote, entre autres, nous le rappellent.

Parler à ce propos, en termes morcelés et privatifs, uniquement de civilisation matérielle ou de technique, de contrôle de l'énergie ${ }^{188}$, de volume démographique, de biens et de services, de recettes de cuisine et de remèdes thérapeutiques, de catalogues de plantes et de classes d'animaux, c'est manquer le principal ; c'est laisser croire que ces choses s'engendrent toutes seules, puisées en quelque lieu étrange et lointain pour répondre à un besoin préexistant des individus, tribut payé par le monde non humain concourant à l'édification du monde humain. On s'en tient à une vue mécanique des échanges qui relient la collectivité à son entourage objectif, réduisant celui-ci au rôle de réceptacle, important mais externe, faisant de celle-là un reflet, complet et plat, d'un jeu de forces autonomes. Cependant, à travers ces efforts envisagés en ordre dispersé et subordonnés, ce qui est à l'œuvre, ce sont les propriétés organiques et inorganiques de l'espèce, la tendance générale des êtres vivants à se propager et à coordonner leur action dans une écologie qui leur convient. De cette façon, la société se donne un fondement naturel et le renouvelle sans cesse, non point parce qu'un individu fabrique un instrument ou accomplit une opération - chasser, cueillir, cultiver - mais parce que les propriétés d'une force matérielle se sont transformées en qualités physiques et psychiques. Les arts, les techniques, les disciplines poïetiques ou mythiques synthétisent les tentatives faites au cours de l'histoire pour insérer les hommes coalisés dans le mouvement des phénomènes cosmiques et les phénomènes cosmiques dans le mouvement des

188 L.A. WHITE : Energy and the Evolution of Culture, American Anthropologist, 1943, 45, 335-356. 
hommes, en allant au-delà des limites marquées, les fictions ou les ébauches d'un moment devenant les réalités et la plénitude du moment d'après, tout comme les tâtonnements et les déchets d'un groupe ou d'une espèce se muent en certitudes et ressources, en nature pleine d'un autre groupe ou d'une autre espèce qui a su en faire sa création.

La séparation des individus reproducteurs et non reproducteurs dans les sociétés d'affiliation inaugure une nouvelle répartition des espaces, des dons d'exploration, des savoir-faire, et suscite, finalement, l'alliance avec le règne végétal et le règne animal différenciés. La coupure ultérieure de la chasse et de la cueillette a repris en charge les tentatives multiples et aléatoires. Exigeant une solidarité accrue des mâles, convertissant le corps social en instrument de défense et d'attaque, elle a entraîné la révision des relations entre les sexes en leur permettant d'aborder le milieu divisé et de tirer parti de ses ressources mobiles et immobiles. La transmission des facultés, leur application stricte, le souci de maintenir un certain rapport quantitatif entre le volume de la population et les moyens dont elle dispose, le choix et la fixation des propriétés neuro-physiologiques indispensables à la pléiade correspondante de comportements, sont autant de tâches figurant dans le cahier des charges des organes collectifs naissants. La réussite et l'échec intéressent toutes les espèces, dans la mesure où ils affectent les adaptations réciproques. Dans la conception actuelle, l'individu représente l'humanité tournée vers le monde matériel et biologique, ce qui est, nous l'avons constaté, une abstraction. Du point de vue logique et réel, au contraire, c'est la dimension sociale qui occupe la place laissée vacante par l'individu et le manifeste en tant que pôle de la nature.

De l'autre côté, telle que nous l'avons vue, la société est négative, tournée vers elle-même, et elle a son propre dynamisme qui s'est établi évolutivement, historiquement. Elle concentre une série d'intérêts, d'entreprises, d'échanges organisés autour du pouvoir, de la richesse, des hiérarchies qui séparent et ordonnent les classes, les sexes et les régions. Le renouvellement de ces configurations mobilise les énergies, engendre un langage et des modes d'interaction congruents. La distribution des biens, des services, des symboles de prestige, en départageant les groupes, oblige à énoncer des lois ou des interdits, crée des inégalités ou des différences qui règlent la vie et l'organisation 
collectives. L'uniformité est obtenue au détriment de la déviance et de la singularité en soumettant les facultés individuelles au moule commun des modèles de penser, de sentir, d'agir normalisés. Construire des barrières qui isolent les groupes en les empêchant d'échapper à la dépendance est aussi de son ressort, la cohésion étant la fin recherchée. Les cérémonies, les rituels, les dons symboliques de biens et de personnes accompagnés de sanctions et de récompenses sont indispensables à la réaffirmation du groupe, à la légitimation des règles, au déroulement de l'existence individuelle, au fonctionnement efficace des procédés qui rendent la routine de l'existence quotidienne supportable, la perspective de la maladie, du sacrifice et de la mort tolérable. Ils préservent simultanément la stabilité du système, renouant les liens de solidarité, créant, sous un certain angle, l'impression d’homogénéité dans la réciprocité des partenaires sociaux, rappelant, sous un angle distinct, leurs distances respectives, la non-réciprocité de leur condition. De la sorte, chacun peut à la fois éprouver la communion avec les autres, l'ouverture de l'ensemble, et accepter ce qui le discrimine d'eux, la fermeture de sa sphère particulière. Par le jeu d'une transmission qui soumet la génération montante à celles qui l'ont précédée, la collectivité se reconstitue, identique et exclusive, en masquant les germes de sa propre différenciation dans le temps. Les soins donnés aux enfants, l'élaboration de techniques appropriées, la communication des affects et des traditions qui insèrent chaque personne et chaque groupe dans un ensemble plus vaste, servent aussi à prévenir la menace du discontinu. Constamment, la collectivité doit se prémunir contre ses tensions et ses contradictions, la contradiction majeure étant pour elle l'obligation absolue de se diversifier, de produire les classes d'hommes aptes à assurer les fonctions variées, et en même temps de maîtriser les tendances des individus ainsi séparés.

L'obéissance aux lois, le respect des coutumes, qui les maintiennent dans une société exerçant l'autorité suprême, exigent de courber les volontés particulières, en formant chacun pour le rôle qui sera le sien et en l'amenant à accepter les normes de conduite qui le guideront en toutes circonstances. La discipline, la répression et l'inhibition sont les moyens appliqués en vue de ces fins. Mais la société remplit des fonctions plus nombreuses ; les êtres qui la composent ne lui préexistent pas, isolés dans la nature, et rassemblés par un contrat d'association. C'est elle, au contraire, qui leur préexiste et qui a pour 
mission de convertir les énergies biologiques, les ressources et les facultés en une diversité économique, psychique, politique ; il n’est pas question pour elle de les égaliser ou de limer les traits qui les distinguent. Nous ne la voyons pas s'édifier dans les interstices de la matière biologique, à partir d'individus qui se combinent spontanément, ou par effacement de leurs besoins : les catégories d'individus, la disposition de ces interstices, l'intensité et l'orientation de ces besoins sont son œuvre. Tenir en éveil les désirs, les instincts, les échanges mutuels est une tâche constante à laquelle se livre chaque espèce, chaque communauté pour son propre compte, suivant des modalités adéquates. Afin de se reproduire, la société, humaine ou non, biologise un individu social, lui assure une épaisseur organique, instinctuelle, mentale - elle ne socialise pas un individu biologique en lui ôtant une fraction de ses impulsions originelles, de ses capacités qu'elle détournerait de leur objet authentique. Si le vécu subjectif éprouve la mainmise visible de la société objective comme une contrainte, ce n'est pas nécessairement, on peut en faire l'hypothèse, parce qu'elle opprime en lui une réserve cachée, intrinsèquement libre et spontanément naturelle, mais parce qu'elle le fait par une méthode oppressive.

La société est une instance qui inhibe ce qu'elle stimule, tempère et excite les tendances agressives, épistémiques, sexuelles, accroît et diminue la fréquence de leur satisfaction suivant ses subdivisions, imagine les interdits et prépare les voies de leur transgression. Conçue, en définitive, du moins jusqu'à ce jour, dans le seul but de se conserver, elle combat par la règle et l'institution le devenir ; à l'intérieur elle s'efforce d'imposer la collusion des forces antagonistes suscitées, à l'extérieur elle tâche de rejeter la possibilité d'une alternative ou d'une pluralité. La collectivité y parvient en agissant comme si son ordre était unique, retenant seulement les qualités qui sont les siennes ou celles dont la diversité se présente comme ébauche des siennes dans le temps et dans l'espace. Les notions de progression, de linéarité de l'histoire servent à la définir, la fixant comme but exclusif et suprême. L’ostracisme qui a frappé, depuis les temps les plus reculés, les étrangers, les peuples primitifs, les barbares, voire les animaux, illustre sa propension à dénier l'existence pleine à tout ce qui contredit sa singularité. Les collectivités susceptibles de le faire sont dissoutes dans l'obscurité, assimilées à la nature. En déclarant que la société ou la culture est artificielle, on semble parler le langage de la modestie. 
Toutefois, puisque l'artifice est signe humain, on sous-entend en réalité que sa société, sa culture est la seule humaine, la seule qui mérite l'appellation d'effort et d'œuvre, et dont on valorise les règles et les institutions - les autres cultures s'étant contentées d'enregistrer les pressions de l'environnement et les besoins de l'organisme. La distance qui nous sépare d'elles est distance à l'univers naturel, la négation de celui-ci est leur négation : l'identité se maintient ainsi par exclusion de l’altérité.

Les conceptions modernes de la société ont insisté sur l'opposition de l'homme à la nature ; elles ont mis l'accent sur la prééminence de la règle, de l'économique ou du politique, et sous-estimé l'importance de la régulation que nous opérons envers les forces matérielles aussi bien qu'elles ont négligé la création des facultés productives et scientifiques.

La société, cependant, se dévoile à l'examen forme et fond, rapport à soi et rapport à l'univers, système autonome et partie d'un système cosmique plus vaste, totalité dotée d'une existence spécifique incluse dans la totalité des ordres naturels et sociaux. Réalité première, elle est force et action collectives, comprenant le faire, le savoir et les ressources ; réalité seconde, dérivée, elle transforme le faire, le savoir, les ressources en richesse, pouvoir et idéologie.

$\mathrm{Au}$ lieu de laisser le premier de ces deux aspects dans l'ombre en faisant converger toute la lumière sur le second, il faut la saisir des deux façons à la fois, dans un rapport positif à la nature, comme pôle et facteur historique de celle-ci : ainsi peut-on résumer l'essentiel de ce paradigme qui, à l'encontre du précédent, est sociocentrique. 


\section{Le retour dans la nature.}

\section{Retour à la Table des Matières}

Les sociétés sont des organismes menacés. Aperçues de loin, vêtues de l'habit de lumière des temps passés et de la gloire des temps futurs, le présent mis entre parenthèses, on y fait loger l'espace de la perfection ou de l'étrange. Sous leur enveloppe lisse, cependant, sourdent les forces de désordre qu'elles provoquent, bouillonnent les passions qu'elles enferment, pressent les injustices qu'elles étouffent. Leur ordre couvre des blessures, tient soigneusement à l'écart ceux qui seraient enclins à en dénoncer les failles, oblige à rétracter les pseudopodes qui, franchissant les limites, crèveraient la surface unie. En lutte perpétuelle contre les courants violents prêts à les emporter et à les disloquer, elles y puisent l'énergie requise pour tromper l'angoisse, pour combattre le précaire et le provisoire, et effacer les traces de l'effort et du travail dépensé à atteindre l'harmonie, afin de paraître sur scène, comme l'acteur, tout calme et tout sourire. A cet instant là, baignées de spontanéité et d'innocence, les choses ont le visage de ce qu'elles doivent être, de ce qu'elles ne sauraient pas ne pas être, allégées du poids de l'arbitraire, couronnées par l'évidence de la nécessité. L'art se fond dans l'être, le produit dans le donné. Mais la mémoire, invitée à oublier ce tour de passe-passe, ne peut faire qu'elle ne se souvienne, et les sociétés, fortes de l'endroit solide de leurs œuvres, ne cessent d'être fascinées par leur envers, trame provisoire que le flux du réel effiloche sans fin. Projetées sur un espace bidimensionnel, elles s'évertuent à assumer, dans leurs lois, leurs savoirs, leurs instruments physiques et mentaux, leurs conventions et leurs subdivisions, la tâche souvent contradictoire, décalée, de continuer la vie et d'assimiler les initiatives individuelles, d'amortir le choc des entreprises collectives qu'elles ordonnent, consolident, manifestent eu égard à une population et à un moment historique déterminés. Elles s'efforcent ainsi de remplir deux fonctions de base : l'une universelle, commune à toutes les espèces, visant à unir la matrice organique et la matrice physique, l'autre particulière, relative à une seule espèce, la nôtre, assurant la prédominance des liens collectifs, étant donné la substance des richesses distribuées et des pouvoirs exercés. 
Entre tous les dogmes concernant leur situation et leur marche, celui du dépérissement de la fonction universelle, et de la tension que son existence engendre, est le plus illustre. Cette conviction procède de plusieurs motifs. La difficulté qu'éprouvent les hommes à agir et à penser leur état social en tant qu'état quelconque parmi d'autres, soleil d'une galaxie formée d'un grand nombre de soleils, et à vivre cet état social objectivement quelconque comme leur état propre, est au cœur du mouvement qui se poursuit depuis des millénaires. On a tenté de la résoudre - les religions, les théories sociologiques, les idéologies laissant espérer la fin de l'histoire l'attestent - en adoptant l'idéal d'une sortie de la nature. On pouvait ainsi isoler une de ses dimensions et lui accorder un statut de faveur, en la soustrayant aux transformations imposées par la relation avec le monde matériel et biologique : face à la société active et vive, la nature était décrétée passive et morte. En mettant l'espoir de son devenir dans la négation de son lien à la nature, la société estompe son propre rôle dans la création de l'ordre naturel sous-jacent, et voit diminuer l'influence de celui-ci sur sa propre constitution. Elle ne conçoit plus que des rapports de violence et d'exploitation, son intervention raisonnée servant à limiter prétendument l'empire du désordre; elle justifie sa pratique de l'exploitation et de la violence en la magnifiant en conquête du monde extérieur. Au terme de celle-ci, elle aboutit à reconstituer son unité autour de la dimension particulière, privilégiée au départ, en se dégageant des servitudes qui accablent les espèces obligées de poursuivre leur besogne naturelle : « ... l'histoire se ferait toute seule, et la société placée en dehors, et au-dessus de l'histoire, pourrait, une fois encore, assumer cette structure régulière et comme cristalline ; dont les mieux préservées des sociétés primitives nous enseignent qu'elle n’est pas contradictoire à l'humanité ${ }^{189}$ ».

La seule cause que l'on imagine pour rendre compte de l'utilité et de la persistance de la fonction universelle est l'existence de la rareté. Pour la pallier, les collectivités sont contraintes de se livrer à un rude labeur: elles se soumettent à la dépendance du milieu afin de le contrôler, elles se donnent les institutions adéquates à mieux répartir la pénurie, tolèrent les inégalités tant qu'elles n’ont pas réussi à l'éliminer. Le jour où la rareté viendra à disparaître, les hiérarchies 
injustes disparaîtront avec elle. Le travail pénible perdra toute raison d'être, la pléthore de découvertes scientifiques et techniques suspendra l'action naturelle immédiate de l'homme. La société tournera le dos à la nature et se retirera de sa dépendance : "Certes, quels que soient les hommes et les événements, écrivait Jean-Paul Sartre ${ }^{190}$, ils apparaissent jusqu'ici dans le cadre de la rareté, c'est-à-dire dans une société encore (je souligne) incapable de s'affranchir de ses besoins, donc de la nature, et qui se définit par là même par ses techniques et ses outils. »

Une fois l'abondance instaurée, au règne de la nécessité commandé par la nature succédera le règne de la liberté, essence de la société, seule condition digne des hommes se retrouvant entre eux, affranchis du lien, devenu contingent, à la matérialité. Se réclamant de cette vision, toute progression annonce une société sans nature, de même que toute régression renvoie à une nature sans société, espoirs et menaces qui sont autant de signes de notre réussite ou de notre échec.

La maîtrise du milieu achevée, l’humanité soulagée de toute préoccupation le concernant tracent les linéaments d'une histoire dont les sujets, délivrés du spectre du manque, n'agissent plus dans son ombre. L'organisation sociale, contre nature dans sa conception même, sa sortie présumée de la nature, la règle qu'elle est censée opposer à la promiscuité (qui n’est qu'un autre nom de la rareté) y conduisent, préparent le dépérissement prévu et souhaité, et ne se justifient que dans cette éventualité. Là où se mêlent le leitmotiv du repliement sur soi et celui de la fin de la rareté, jaillit le thème, si prenant pour les défenseurs de la culture, de la rupture avec la nature. Savoir pourquoi elle a eu lieu et quelle en a été la teneur est une question à laquelle on n'a cessé de répondre. Suivant la réponse que l'on propose, la raison invoquée - la langue, la richesse, la répression des instincts, la résorption de la promiscuité animale, la technique, etc. — on assigne pour figure à la sociabilité humaine la capacité symbolique, la propriété, la famille, la production, etc., moyen qui est supposé assurer en chaque cas la domination finale de cette sociabilité.

190 J.-P. SARTRE : Critique de la raison dialectique, Paris, 1960, p. 202. 
Toutefois, la nature n'est pas sans nous, elle est avec nous et par nous ; on l'a voulue immuable et morte, alors qu'elle bouge et qu'elle a une histoire. Nous n'y reconnaissons pas seulement un objet mais aussi un sujet. A la lumière d'une riche expérience, il convient de s'inscrire en faux contre l'épuisement prétendu de nos rapports avec elle. Périodiquement, on croit accéder à un état ultime, rêve d'une humanité tranquille qui a gagné la partie, terminé son travail envers le monde, remporté grâce à la science ou à l'art un triomphe absolu sur les obstacles extérieurs, et qui peut toucher les dividendes de l'esprit et du labeur. On estime atteindre le palier où la collectivité a surmonté la rareté originelle. Un sociologue américain n’écrivait-il pas naguère : «Les hommes des sociétés surdéveloppées ont l'impression que la conquête manifeste de la nature, la victoire sur la pénurie est virtuellement achevée ${ }^{191}$. »

En réalité, aucune collectivité ne sort de l'histoire pour s'installer dans l'âge d'or, pas plus que l'humanité n'a vécu à l'âge d'or avant d'en être chassée vers l'histoire. Tant que nous restons actifs, tant que nous continuons à inventer, à produire des objets et des savoirs - et nous le faisons comme nous respirons - des ressources nouvelles sont créées, tandis que d'autres deviennent caduques et que certaines s’épuisent. L'invention est facteur de rareté : plus il y a d'inventions, de découvertes, plus il y a de rareté. La technique et la science ne la suppriment pas, elles l'engendrent. Les pierres rapportées de la lune, chargées d'histoire cosmique, valent plus cher que leur poids de diamants. Toujours et partout, spontanément, nous créons l'abondance et du même coup le manque, nous transformons l'une en l'autre. Qui se serait figuré, il y a quelques millénaires, que le temps, la vitesse, deviendraient des biens aussi précieux et aussi recherchés qu'ils le sont aujourd'hui ? Qui aurait pensé, il y a seulement un siècle, que l'eau et l'air seraient ce qu'ils commencent à être, des ressources qui s'amoindrissent ? S'ils le sont, c'est parce que des formes d'énergie différentes, des processus matériels nouveaux se sont multipliés, accroissant leur consommation d'une part, leur pollution de l'autre. Un coup d'œil jeté sur l'histoire nous convaincra qu'il n'y a là rien d'exceptionnel. La perspective que l'on puisse abolir radicalement la rareté, et avec elle la fonction universelle dans la nature, suivie de

191 C.W. Mills : The Sociological Imagination, New York, 1961., p. 15. 
l'émergence d'une organisation collective qui ne lui doive plus rien et s'en désintéresse, est illusoire ; les déductions pratiques et théoriques qu'on a voulu en tirer ne reposent sur aucun fondement.

Le changement corrélatif habite moins la réponse à donner à une rupture des deux ordres de réalité que la question qui se pose. Celle-ci pourrait s'énoncer ainsi : comment la fonction universelle de la société se relie-t-elle à sa fonction particulière ? Les solutions concrètes qu'elle a reçues diffèrent, certes, aux divers stades de l'évolution et de l'histoire. En retraçant leur genèse, en pénétrant les ressorts de chacune, les formes qu'elle a prises, on retrace et comprend la vie des collectivités qui les ont adoptées. La différenciation et la correspondance des deux fonctions, des prolongements sociaux inhérents, se placent au centre de gravité de la théorie. Et au centre de la pratique, dès lors que l'innocence cède devant la responsabilité, et que le devenir du milieu naturel n'est plus attribué à un pouvoir divin ou à un hasard mauvais calculateur. A juste titre, on incrimine un peu partout le décalage qui existe entre le développement des sciences, des techniques, et le faible pouvoir que nous avons de les orienter, de les raccorder à nos besoins et à nos buts. Ce décalage n'a pas pour cause, comme on le soutient, la rapidité et l'ampleur de leur essor, notre réussite trop éclatante dans ce domaine, notre maîtrise excessive des phénomènes objectifs qui nous pousse à perturber leur équilibre d'ensemble. Il est dû au fait que, conçue dans l'optique du provisoire, de l'accidentel, tel un mal nécessaire, notre fonction dans la nature a été laissée en friche ; nous ne l'avons pas développée consciemment, systématiquement, de sorte que son unité, sa signification nous échappent. Nous la voyons exercée de manière sporadique et dispersée, sans souci de la totalité, du lien qui embrasse l'écologique, l’industriel, le scientifique, le démographique : chacun de ces domaines est censé suivre ses règles et son dynamisme propres, comme s'il n'y avait pas de rapports entre eux, comme s'ils ne s'influençaient pas réciproquement, comme s'ils n'étaient pas les diverses facettes d'un seul et unique processus intéressant, en son entier, le corps social articulé avec les puissances de l'environnement.

En inversant les termes de l'équation usuelle, et en se tenant près des phénomènes réels, il est possible de concevoir une relation mutuelle : la société réagit aux changements de la nature dont elle est un 
des pôles, de même que la nature, l'englobant, répond à ce qui arrive dans les sociétés échelonnées sur les branches du monde vivant. A la place d'un lien unilatéral nous avons perçu un lien réciproque, à la place de l'hermétisme une communication, à la place d'un emboîtement mécanique une régulation organique. La fonction universelle des sociétés, en tant qu'elles sont des forces objectives, infléchissant les autres forces cosmiques, est donc une donnée permanente et non point transitoire, une dimension essentielle du système qu'elles constituent, et non pas un symptôme secondaire tant que ce système n'a pas atteint sa perfection, destiné à disparaître lorsqu'elle sera atteinte. Il importe, en vérité, en la retenant, de resocialiser la nature, de réapprendre à l'habiter, au lieu de vouloir la quitter. L'humanité est appelée probablement à disparaître, elle a aussi la capacité de se détruire ; toutefois, tant que ces deux événements ne se sont pas produits, cette vocation naturelle est à la fois inscrite dans la condition actuelle et marque la présence de l'espèce au sein de son véritable milieu évolutif et historique. Non pas, ainsi qu'on le suggère souvent, sur le mode des autres espèces, mais sur le mode qui est devenu le sien.

A ce sujet, on prétend toujours vaincre la matière, capter les puissances physiques et biologiques. On veut arracher au cosmos ses lois et ses secrets : on nous présente l'image de procédés et de produits jalousement thésaurisés, jaugeant les victoires de l'intelligence, richesses supputées d'une nation autant que l'encaisse métallique enfermée dans les chambres fortes. Les biens qu'il faudrait plutôt recenser et engendrer sont en réalité des facultés organiques, sociales et psychiques, en relation avec les éléments objectifs sur lesquels elles s'exercent, qui provoquent leur naissance et leur mort, et réciproquement, puisque aucun de ces éléments n'existe pour nous sans la faculté humaine qui le suscite et le parachève. Par l'intermédiaire des talents et des savoir-faire, nous communiquons avec l'univers matériel, nous l'ordonnons. L'enjeu constant est moins de conquérir la nature que de faire l'homme. Ce qui est pensé et vécu comme visée externe dissimule la visée authentique, interne, elle, qui nous concerne. La tradition appuie pareille inversion. La religion judéo-chrétienne statue que Dieu nous a accordé en gérance et concédé toutes les créatures, nos inférieures, et nous a donné licence de dominer la terre. La philosophie rationaliste a pris le relais, affirmant que le savoir est pouvoir, et que notre esprit nous rend maîtres et possesseurs de cette 
même terre. Toutes deux ont contribué à cette dissimulation, ont fixé l'exploitation pour modèle des rapports avec les êtres animés et inanimés, en réalité modèle des relations entre les hommes qui est à la fois consolidé et extrapolé, la loi sacrée et la propriété profane étant ainsi justifiées de concert.

Il se peut que cette justification et ce gouvernement du monde en tant qu'objet et de l'homme en tant que son exploiteur aient réussi à leur heure et enregistré des résultats positifs, même s’ils travestissaient la réalité. Maintenant, non seulement ils se voient démentis, ils sont devenus un obstacle à partir du moment où notre rôle déterminant dans le cours de la nature est apparent, où notre activité s'avère ruineuse, tant elle est teintée d'ambiguïté, d'irrationalité, et pétrie de violence, dès lors qu'il s'agit non plus de conquérir mais d'assurer la bonne marche d'une biosphère complexe, ayant évolué et qui évolue, exerçant une influence immédiate sur notre histoire comme nous en exerçons une sur la sienne. Si nous pouvons y intervenir, ce n'est pas du dehors, c'est du dedans, parce que nous y sommes. Friedrich Engels l'a déjà reconnu: "A chaque pas nouveau, nous sommes ainsi amenés à penser que nous ne dominons nullement la nature, à l'instar du conquérant d'un peuple étranger, comme si nous étions placés en dehors de la nature, mais qu'au contraire nous lui appartenons tout entier, par la chair, le sang, le cerveau, et en faisons partie ${ }^{192}$. »

Participants, nous y provoquons équilibres et déséquilibres, nous apparaissons régulateurs et constructeurs de son architecture. L'action, à cet égard, une fois replacée dans sa direction véritable, réduit la distance et l'étrangeté de notre rapport à l'ordre des choses, l'éloignement de l'organisme individuel et collectif à cet ordre, saisi uniquement en termes abstraits, marqué par la ségrégation, en fonction des qualités premières - espace, temps, lois, mesures, quantités. Elle établit la possibilité d'une familiarité, les qualités secondes, immédiates, du sensible, du perçu, de l'imaginaire remplissant le vide, entretenu, de l'homme à son univers. Instante est certes la recherche d'un retour, non pas retour à la nature, mais retour dans la nature.

$\underline{\text { Retour à la Table des Matières }}$

${ }^{192}$ F. ENGELS : La dialectique de la nature, Paris, 1950, p. 387. 
Retour à la Table des Matières

\section{Notes de la troisième partie}

\section{$\underline{\text { Retour au texte }}$}

Note 6 : Je ne parle à ce propos ni d'un déterminisme de l'environnement, ni d'un déterminisme des activités technico-matérielles, parce que, à l'époque envisagée, la correspondance entre l'effort dépensé par la société tournée vers la nature et l'effort qu'elle dépense pour se transformer elle-même apparaît relativement directe. Il serait intéressant d'examiner d'une manière plus générale la question de cette correspondance dans les sociétés qui ont succédé à la société de parenté. Mais, même sous cette forme, l'hypothèse d'une relation entre la chasse et la parenté qui, à maints égards, irait de soi, trébuche sur des obstacles d'importance. Le livre de Luc de Heusch, Pourquoi l'épouser ? où il reprend une thèse d'Engels, qui m’a incité à rédiger cette longue note, les a exposés en toute clarté. Selon lui, les sociétés archaïques connaîtraient deux séries de déterminismes : les uns procédant de la parenté, les autres du travail et de la technique. Les premiers auraient pour objet les facteurs organiques et instinctuels, les seconds les liens avec le milieu et les relations entre hommes concernant la production et la répartition des richesses. En d'autres termes, la parenté obéirait aux nécessités de la nature biologique interne, et l'organisation sociale globale aux nécessités de la nature matérielle externe. « Dans et par l'exogamie, écrit-il (p. 97), l’homme apporte une réponse différée, médiatisée par le groupe, aux exigences de sa propre nature biologique. La société à ce niveau n'est qu'une (souligné par moi) organisation de l'espèce homo. " A ce " niveau le plus profond », il faut envisager « la parenté, ainsi que les groupes et les classes d’âge comme une modalité particulière d'un système de transformations universel, définissant l'articulation première de l'homme en société avec sa propre nature biologique » (p. 105). Le processus de travail, par contre, dans ses effets sur les institutions et les échanges, ne jouerait qu'un rôle subordonné lorsqu’on analyse les sociétés dites primitives : «L’on pourrait dire que les décisions arbitraires relatives à l'alliance et à l'affiliation d'une part, à la répartition des générations à l'intérieur ou à l'extérieur du système de parenté d'autre part, constituent l'infrastructure dominante des sociétés technologiquement rudimentaires, dont l'ordre structural (à la fois praxis et pensée) relève davantage du premier degré d'articulation de la culture avec la nature. L'organisation du travail dépend de cet ordre structural, et non l'inverse. D'une certaine façon, les sociétés archaïques apparaissent plus proches de la nature, quand bien même elles seraient séparées d'elle par la distance du langage, qui 
impose à l'espèce son ordre symbolique et analytique décisif. Que cet ordre culturel véritablement premier — puisqu'il est associé à des économies de type paléolithique ou néolithique - véhicule ou non une sagesse idéale est une question philosophique étrangère à la recherche scientifique » (p. 133). La deuxième articulation avec la nature, médiée par les disciplines productives, prend le dessus et s'impose de plein droit à une phase ultérieure. « L'histoire générale de l'humanité, qui ne saurait être qu'une histoire des techniques, montre que l'exogamie cesse bientôt d'être un principe structurant (il ne figure plus dans notre code civil que sous sa forme négative rabougrie de prohibition de l'inceste) alors que la société tout entière s'organise pour le travail, c'est-à-dire l'exploitation de l'homme par l'homme. » (p. 136).

Ces textes précis excluent, on le voit, la possibilité de mettre en rapport les changements provoqués par la division naturelle de la chasse et de la cueillette, avec le passage des sociétés d'affiliation aux sociétés de parenté. A la fois parce qu'il s’agit de phénomènes indépendants, et parce que le premier précède le second. Malgré l'adhésion spontanée de la plupart à ces énoncés, j’hésite à souscrire à leurs conclusions ou à accepter les compromis que le bon sens conseille et dont on s'accommode tant bien que mal. A l'arrière-plan de ces propositions se retrouve l'opinion suivant laquelle la société a pris son départ en tant que modalité de maîtrise des pulsions et de la reproduction sexuelle. Celle-ci y remplit un rôle particulier, d'abord parce qu'elle commande l'évolution des espèces, et ensuite parce qu'elle a trait à un instinct qui est considéré comme social, par excellence. La maîtrise de la reproduction doit beaucoup à l'apparition du langage, introduisant un ordre symbolique dans le domaine fluctuant de l'organique. La parole et l'interdit ont conspiré, si l'on en croit la théorie, afin d'éloigner l'homme de sa nature, de tourner sa société contre la nature. La simultanéité de ces découvertes et de ces opérations, à l'instant des commencements décisifs, a imposé leurs répercussions (exogamie, prohibition de l'inceste, etc.) en tant que composantes permanentes de notre biologie et de notre culture, acquérant une sorte d'immunité à l'événement, à l’histoire. Là réside l'intérêt exceptionnel de ce moment, de nos sociétés passées, qui motive une recherche attentive de nos origines : nous décelons les signes de ce qui est permanent, inchangeable dans notre présent, de ce qui se conserve au-delà ou en deçà des remaniements intervenus par la suite. Bronislaw Malinowski a souligné tout ce que pouvait nous apporter une telle recherche, nous permettant de faire le départ entre les universels fondamentaux qui ne dépendent plus de nous et les processus variables, locaux, survenus depuis, et sur lesquels nous avons encore la latitude d'agir :

« Ce n'est pas, écrivait-il (A Scientific Theory of Culture, Chapell Hill (N.C.), 1944, p. 216), une question d' « origine » au sens quelque peu naïf de ce qui est arrivé au singe anthropoïde au commencement de la culture. Il s’agit plutôt de savoir si la guerre, comme la famille, le mariage, la loi et l'éducation, se trouve dans toutes les cultures humaines à chaque stade de 
développement, et plus particulièrement si elle a joué un rôle indispensable aux commencements les plus précoces de l'humanité. Car si l'on peut montrer que la guerre, c'est-à-dire le règlement collectif des problèmes intestinaux par la force armée, ne se trouve pas au commencement de la culture, cela prouve que la guerre n'est pas indispensable à la conduite des affaires humaines. "

Paradoxalement, cette conception, si cohérente par ailleurs, reprend de la main gauche ce qu'elle donne de la main droite. La distance de la société à la nature est bien rendue à l'aide de ces traits si l'on admet qu'elle soit relevée sur les dimensions choisies. Par ailleurs, que remarque-t-on ? A l'instar de ce qui se passe dans le règne animal, les sociétés archaïques continuent à être axées sur le phénomène principal de l'évolution — la reproduction sexuelle - et leur structure s'avère particulière à notre espèce, à cause des mutations génétiques qui l'ont individualisée, affectant les appareils neuro-physiologiques, les fonctions associées - la pensée et la communication linguistique - ainsi qu'il arrive dans toute spéciation sur l'échelle des êtres animés. Sans vouloir outrer l'argument, force est de conclure qu'il s'agit, en l'occurrence, d'une sorte d'état social de la nature, de sociétés naturelles. C'est de cette manière qu'on est en droit de comprendre le premier niveau indiqué par Luc de Heusch, où la société « n’est qu'une organisation de l'espèce homo ». Subrepticement on réintroduit une hétérogénéité «biologique » avec les sociétés qui sont fondées sur le travail, sur l'artificiel, selon le schème courant, qui est du même ordre que celle que l'on prétendait avoir annulée une fois pour toutes à l'aube de la préhistoire. Mais la volonté acharnée de conférer un poids si grand à l'instinct sexuel, au langage, de les dissocier des échanges écologiques, des pratiques connexes, de minorer ces échanges et ces pratiques, est-elle motivée ? Si l'on se rapporte à ce que nous en savons, il apparaît que ces instincts ne doivent pas être envisagés à part et que l'instinct sexuel n'est pas le seul à être social. Niko Tinbergen remarque à ce propos : «En d'autres termes, chez l'animal social, le membre de l'espèce fait partie du stimulant que l' " animal » cherche dans son comportement appétitif. Chez quelques espèces, tous les instincts, même l'instinct reproducteur et l'instinct du sommeil, ont des aspects sociaux. Chez beaucoup d'autres, l'aspect social, présent dans l'instinct alimentaire comme dans tous ceux étrangers à la reproduction, est absent de l'instinct reproducteur. » (N. TINBERGEN : L'Étude de l'instinct, Paris, 1971, p. 160.)

L'argument fondé sur l'exogamie ou sur la prohibition de l'inceste, en tant que points de rupture, assises profondes des couches successives de la culture, réclame, face à ces données de la biologie, une justification qui soit autre chose que la répétition d'une convention, œuvre de cette même culture. Et le sommeil, l'agressivité, la soif, etc., si l'on devait en reproduire le modèle, fourniraient autant d'appuis d'un pouvoir conceptuel équivalent, comme ils l'ont fait par le passé. Plus arbitraire encore m’apparaît la prétention à tenir pour négligeables les techniques qui étaient 
à la disposition des hommes primitifs, à soutenir qu'elles n'ont pas eu d'impact sur leurs systèmes de relations. Rien ne nous permet d'évaluer leur importance avec précision et d'affirmer qu'elles étaient subordonnées à l'exogamie, à la parenté. Certes, elles sont rudimentaires et d'un faible rendement énergétique. En portant ce jugement, nous les comparons aux techniques évoluées, aux puissantes forces productives artisanales ou mécaniques. Nous aurions avantage à les comparer à celles des chimpanzés et des babouins : nous les apprécierions alors à leur juste valeur. Si des variations écologiques après tout triviales engendrent des différenciations comme celles que nous avons notées entre les sociétés de clique et les sociétés de compagnonnage, on comprend qu'une population, ayant un volume identique, s'adonnant à la chasse et à la cueillette, possède des moyens aptes à révolutionner le milieu et l'organisation sociale. Si l'on poussait ce constat jusqu'au bout, on se mettrait à démontrer, le langage étant fils de la prédation, que les règles commandant aux instincts ont attendu d'abord qu'il y ait une nature humaine, que l'éloignement générateur de la culture, s'il a été tel qu'on l'a décrit, manifeste un éloignement de la parole par rapport au travail et non l'inverse.

Je ne veux toutefois pas aller si loin dans la voie de la spéculation. J’ai cru seulement utile de rappeler qu'en fondant la parenté sur la biologie, en interdisant de la rapporter à l'écologie et aux pratiques productives, on change les sociétés archaïques en sociétés à part, sans lien véritable avec celles qui les ont précédées ou leur ont succédé. Leurs membres ont beau chasser et cueillir, force est de penser qu'il s’agit là d'activités purement superfétatoires, tout juste bonnes à alimenter des mythes, mais qui ne marquent pas la vie collective pour l'essentiel. Si c'est le cas, on a le droit d'affirmer que «les lois d'airain de l'exogamie (et de la pensée livrée à elle-même à travers ses pratiques) gouvernent encore le travail. » (L. DE Heusch, op. cit., p. 138). Sinon, il est difficile de comprendre que ces activités aient changé notre biologie, nos rapports avec le milieu, bref tout, sans avoir un quelconque impact sur « les lois d'airain » de la parenté. Le hasard des lectures donne l'impression que l'on s'accommode fort bien d'un dualisme tranché entre la « culture matérielle » et la « culture culturelle », en déduisant les principes des sociétés archaïques tantôt de la première, tantôt de la seconde. Luc de Heusch a tenté une synthèse des deux attitudes à travers le marxisme et le structuralisme. Par là il les a aiguisées et a rendu impossible leur coexistence dans le bonheur éclectique. Mais il faut reconnaître que la mise eu commun de Freud et de Marx, de Claude Lévi-Strauss et de Jean-Paul Sartre, de la psychanalyse et de l'anthropologie structurale, du marxisme et de l'existentialisme, la conciliation des grands-pères, des pères et de leurs œuvres, ne sont que de piètres substituts à une recherche qui devrait s’intéresser au premier chef à la réalité. Peut-être y a-t-il d'autres connaissances à acquérir, d'autres interrogations à poser aujourd'hui, en partant, non de ce que d'autres ont su 
mais de ce qu'ils ont ignoré. C'est en nous plaçant au cœur de l'inconnu que nous avons le plus de chances de le découvrir. Retour au texte

\section{$\underline{\text { Retour au texte }}$}

Note 7 : Les conjectures sur la lutte des sexes et la prohibition de l'inceste découlent de la théorie plus générale de la division naturelle. Elles ont été formulées, pour le principal, avant la floraison récente d'une littérature d'inspiration féministe. Il n’y a donc pas lieu d'associer nécessairement ces conjectures aux thèmes qui prévalent dans cette littérature. Le problème de la libération des femmes ou de l'égalité des sexes mérite, certes, d’être analysé sur le plan théorique - et ce livre peut, à la rigueur, y contribuer — si l'on veut engager une action efficace afin de le résoudre. Le présent travail, comme bien d'autres, fait ressortir que ce problème s’inscrit dans la nature générale de notre société, plonge ses racines dans notre psychisme, l'organisation de notre environnement, de notre savoir et de nos techniques. Sa solution est une tâche de longue haleine ; elle présuppose une refonte profonde de nos institutions, vise les rapports entre les sexes pour autant que les sexes engagés dans ces rapports devraient euxmêmes changer socialement et psychiquement; le comportement sexuel proprement dit en subirait le contrecoup, mais il est en l'occurrence secondaire. L’égalité économique, politique, le réaménagement, forcément superficiel, des relations entre les sexes, dont on parle tant, sont souhaitables. Ils sont le cri du cœur et la voix de la justice. Ils ne répondent pas à l’essentiel, n’étant pas éclairés par une conception générale. Retour au texte

\section{$\underline{\text { Retour au texte }}$}

Note 8: Grande est l'incuriosité en ce qui concerne les raisons de la nonréciprocité des relations entre les hommes et les femmes, les règles qui perpétuent cette non-réciprocité, les conséquences et la place historique de ces relations. Commentant, dans un texte récent, les travaux de Marx et d’Engels consacrés aux sociétés primitives (Sur la société précapitaliste, Paris, 1970), Maurice Godelier se contente d'en reproduire les constats sans s'aventurer dans la théorie qui n’aurait eu de sens que si ces constats avaient été transformés en questions. «Enfin, il faut rappeler que dans les sociétés primitives la femme a une importance décisive pour le maintien des communautés par ses fonctions reproductives et économiques, et cette importance rend nécessaire le contrôle par la société de l'accès aux femmes. Mais ce contrôle, ce sont toujours les hommes qui l'exercent. La relation entre les sexes dans les sociétés primitives est donc fondamentalement asymétrique et non réciproque. La réciprocité n’existe qu’entre les hommes. » (p. 173).

L’incuriosité entraîne aussi à une utilisation irrégulière des guillemets 
et un emploi lâche des concepts. D’une part, quand il s'agit de biens, en général, la rareté apparaît « sociogène » et l'on constate l'existence de raretés qui semblent " artificielles », " instituées » (p. 119). D’autre part, à propos des femmes, on revient à une rareté naturelle : «La communauté garantit leur survie (de ses membres) et assure (la) continuité physique alors qu'en autorisant la compétition autour de biens rares donnant accès aux femmes et à l'autorité, elle assure son existence en tant que société » (p. 120).

Le caractère social d'un bien ou d'un rapport se fonde-t-il, selon le matérialisme dialectique, sur la rareté ou sur la prohibition?

« On constate que tout système de parenté suppose une forme quelconque de prohibition sexuelle et conjugale et que ceci démontre le caractère social des rapports de parenté. En renonçant à leurs droits sur certaines femmes (mères, sœurs, filles), les hommes d'un groupe les rendent disponibles, les offrent et acquièrent des droits sur les femmes d'autres groupes » (p. 112.) Qui leur a donné ces droits ? Comment ces droits se maintiennent-ils ? Pourquoi les hommes en usent-ils envers les membres féminins et non envers les membres masculins du groupe (fils, jeunes frères, etc.) ? Ces problèmes ne semblent pas appeler de solution.

En appliquant les instruments conceptuels de Karl Marx (au lieu de se borner à les rappeler) et aussi en s'inspirant de son courage intellectuel, les marxistes arriveraient peut-être à regarder les choses en face, au lieu de regarder toujours au-delà ou en deçà.

Faute de quoi, il n'est guère instructif de retrouver dans leurs écrits des paraphrases de ce que d'autres ont déjà exposé avec plus de talent et de force. $\underline{\text { Retour au texte }}$

\section{$\underline{\text { Retour au texte }}$}

Note 9 : La lutte des sexes façonne non seulement l'hétérosexualité mais aussi l'homosexualité. Dans les sociétés de primates, celle-ci constitue une solution positive à la tension qui oppose les générations. Le jeune mâle, le subordonné recherche et obtient la protection de l'adulte ou du supérieur par des cérémonies où il adopte une posture féminine et subit de la part de ce dernier un assaut sexuel symbolique ou réel. Les congrégations masculines y vivent obligatoirement, ouvertement, dans un cadre homosexuel requis par l'état de non reproducteur. La reprise de la cohabitation avec les femelles a lieu dès que l'occasion s'en présente.

L’initiation des garçons, dans les sociétés humaines, confère aux conduites homosexuelles une signification nouvelle en les dissimulant ou en les sublimant, moins aux yeux des hommes qu'à ceux des femmes. L'atmosphère d'une initiation est celle de la rupture, de la lutte, du passage. L'enfant est mis devant un choix, ou plutôt un choix lui est imposé entre deux groupes de parents, deux loyautés, deux modes d'existence. L'une des issues a probablement été la masculinité homosexuelle. Identifi- 
cation complète à la société des hommes, adhésion à ses contrats rigoureux et secrets, complicité dirigée contre les femmes, cet état idéal suppose la non-intelligence totale avec l'autre sexe. Il rejette le contact avec l'impur, l'inférieur, dispense du regret et de la culpabilité, introduit l'homme dans la compagnie de ses égaux. Le monde masculin se clôt sur lui-même ; il échappe à la dépendance, se soustrait au conflit, à l'interdit de l'inceste et à la raison qui l'a motivé.

L'autre issue, l'homosexualité masculine, laisse les hommes vivre dans le monde féminin sans contrevenir aux règles en vigueur ni en compromettre l'application. Elle se constitue en deçà de la rupture que représente l'initiation, dans le refus de la subir au prix d'une rupture, d'une sortie du groupe, d'un retournement contre les mères et les sœurs. L'individu déchiré par les sacrifices et les épreuves exigés de lui, haïssant le jeu de la ruse et de la mort qui fait de lui l'adversaire de celles pour et par lesquelles il est vivant, tend à un compromis, s'efforce d'unir les deux sexes en un seul.

Complétant l'hétérosexualité ou s'y opposant, l'homosexualité est le résultat des interdits, des rituels et des antagonismes qui les entourent. Elle instaure en quelque sorte un troisième sexe, synthèse, au sens chimique, des deux autres. On peut y voir une réponse normale à des conditions psychiques et sociales concrètes ; elle n’a ni disparu, ni " guéri », au cours de l'histoire des sociétés et des individus, comme le ferait une malformation ou une maladie que l'on peut diagnostiquer et soigner. Elle fait néanmoins l'objet d'une surveillance dont la direction confirme l'importance relative de la place occupée par chaque sexe. Étant donné que les hommes sont au centre de la société et les femmes à la périphérie, l’homosexualité de ceuxlà est plus localisée, et plus lourdement sanctionnée, le cas échéant, que l'homosexualité féminine, plus diffuse, et finalement tolérée. Pour l'homme, en effet, ne pas avoir de femme, c'est rompre les liens primordiaux avec les autres hommes, perturber la marche ordonnée du corps social, voir sa position dans la société abaissée. Les femmes, au contraire, ne sauraient, quels que soient leurs goûts et leurs conduites, troubler un ordre dont elles n’occupent que la partie domestique. L’hétérogénéité des sexes aboutit au contraste des sexualités. Ces diverses constellations ont dû être aménagées, travesties et embellies par les civilisations successives. Le mot amour les a couvertes de toute son ambiguïté, les images de la virilité et de la féminité ont forgé, sinon la réalité, à tout le moins les idéaux de l'espèce. Retour au texte 